\title{
SOLITARY WAVES IN THE LOWER ATMOSPHERE
}

\author{
by \\ Douglas Robert Christie
}

A thesis submitted for the degree of

Doctor of Philosophy

at the

Australian National University

January, 1991 
The published research papers which comprise this thesis were prepared over the period from 1976 to 1990 while I was a member of the staff of the Research School of Earth Sciences at the Australian National University. All of the research described herein is believed to be original. Part of the research described in these papers was performed in collaboration with Ms. S.S. Chen, Dr. R.H. Clarke, Professor R. J. Doviak, Professor A. L. Hales, Dr. K.J. Muirhead, and Mr. K.W. Thomas. This thesis has never been submitted to any other university or similar institution.

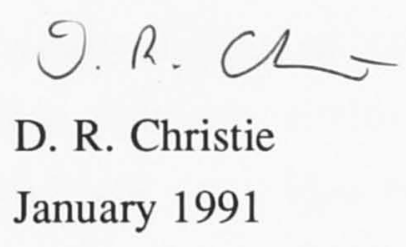


To my wife Mimi and daughters Nicole and Wendy 


\section{ACKNOWLEDGEMENTS}

I would like to express a deep sense of gratitude to a large number of people my family, friends and colleagues in the scientific community, and the staff at Earth Sciences - for their inspiration, support and encouragement over the years when these papers were written. I would especially like to thank Emeritus Professor Anton Hales, Foundation Director of the Research School of Earth Sciences, for his continuous encouragement and guidance and for his exceptionally perceptive comments on geophysical problems. I would also like to express my sincere thanks to Professor Kurt Lambeck, Director of the Research School of Earth Sciences, for his sustained interest and support and for his sound advice on numerous occasions. All of the material in this thesis reflects the strong influence of both Professor Hales and Professor Lambeck.

Special thanks is also due to Professor Stuart Turner, Head of the Geophysical Fluid Dynamics Group at R.S.E.S., Dr. Brian Kennett, Head of the Seismology Group at R.S.E.S. and Dr. Ken Muirhead, Head of the Australian Seismological Centre, for their encouragement, suggestions and support. Dr. Muirhead's enthusiasm and interest proved to be invaluable during the early stages of this work. I have also profited greatly from many valuable discussions with the late Dr. Reg Clarke of the University of Melbourne, Professor Tony Maxworthy of the University of Southern California, and Professor Roger Smith of the University of Munich and Monash University.

I would especially like to acknowledge the benefits of my collaboration with Professor Richard Doviak of the University of Oklahoma and the National Severe Storms Laboratory. His penetrating insight and original approach to scientific problems has greatly enhanced my understanding of atmospheric dynamics. I would also like to thank Professor Doviak for his generous hospitality during my visit in 1985 to the National Severe Storms Laboratory.

Finally, I would like to express my sincere appreciation to my wife, Mimi, for her patience, understanding and good humour over the many years when these papers were written. 


\section{ABSTRACT}

The publications which comprise this thesis are concerned with both an observational and theoretical description of solitary waves in the lower atmosphere. A description and interpretation of the first reported observations of solitary waves and related nonlinear wave disturbances in the nocturnal boundary layer over inland areas of the Northern Territory of Australia is presented in the first two papers. In the second paper, the morning glory of the Gulf of Carpentaria is identified for the first time as a visible manifestation of deep-fluid solitary waves propagating in a maritime inversion. The next paper is concerned with a more general description of the large scale coherence pattern of solitary waves over northern Australia and with the interpretation of unique acoustic radar observations of solitary atmospheric waves. Attention is focussed in the next three papers on the hypothesis that wind shear associated with solitary waves is a potentially serious hazard to aviation. The last paper in this series is devoted explicitly to the problem of wind shear generation and transport by solitary waves created in the interaction of a thunderstorm downdraft with an existing stable layer. It is estimated in this paper that solitary waves can plausibly be identified as the primary causal factor in at least one-third of all serious weather-related aircraft accidents. A more general description of the formation, propagation and decay of thunderstorm-generated solitary waves is given in the next paper. The final papers in this thesis are concerned with the theoretical description of finite-amplitude wave motions in the atmosphere and with the application of this theory to the interpretation of observations. The first of these papers is concerned with the results of a detailed study of the numerical solutions of the Benjamin-Davis-Ono equation, both with and without turbulent frictional damping, for a wide variety of initial perturbations; the final paper provides a thorough comparison of both weakly-nonlinear wave theory for a compressible sheared fluid and existing theory for highly nonlinear waves with exceptionally well-documented observations of a thunderstorm-generated solitary wave over Oklahoma. 


\section{CONTENTS}

1. Introduction

1.1 Summary 1-2

1.2 Significance of solitary atmospheric waves $1-6$ References $1-8$

\section{Publications}

2.1 Christie, D.R., Muirhead, K.J. and Hales, A.L. (1978). On solitary waves in the atmosphere. J. Atmos. Sci., 35, 805-825.

2.2 Christie, D.R., Muirhead, K.J. and Hales, A.L. (1979). Intrusive density flows in the lower troposphere: A source of atmospheric solitons. J. Geophys. Res., 84, 4959-4970.

2.3 Christie, D.R., Muirhead, K.J. and Clarke, R.H. (1981). Solitary waves in the lower atmosphere. Nature, 293, 46-49.

2.4 Christie, D.R. and Muirhead, K.J. (1983). Solitary waves: A hazard to aircraft operating at low altitudes. Aust. Met. Mag., 31, 97-109.

2.5 Christie, D.R. and Muirhead, K.J. (1983). Solitary waves: A low-level wind shear hazard to aviation. Int. J. Aviation Safety, 1, 169-190.

2.6 Doviak, R.J. and Christie, D.R. (1989). Thunderstorm-generated solitary waves: A wind shear hazard. A.I.A.A. J. of Aircraft, 46, 423-431.

2.7 Doviak, R.J., Thomas, K.W. and Christie, D.R. (1989). The wavefront shape, position, and evolution of a great solitary wave of translation. I.E.E.E. Trans. Geosci. Remote Sensing, 27, 658-665.

2.8 Christie, D.R. (1989). Long nonlinear waves in the lower atmosphere. J. Atmos. Sci., 46, 1462-1491.

2.9. Doviak, R.J., Chen, S.S. and Christie, D.R. (1991). A thunderstorm generated solitary wave observation compared with theory for nonlinear waves in sheared fluids. J. Atmos. Sci., 48, 87-111. 


\section{INTRODUCTION}

Solitary waves were first observed in 1834 by John Scott Russell in the form of localised single-crested waves of elevation which propagated steadily without change of form on the surface of the Union Canal linking Edinburgh and Glasgow (Russell, 1838). A particularly colourful, and often quoted, description of Russell's pursuit on horseback of a solitary wave generated by the abrupt halt of a canal barge is given in his 1844 'Report on Waves' to the British Association (Russell, 1845). Russell was fascinated by solitary waves and believed that in his 'great wave of translation' he had discovered a universal component in the general description of finite-amplitude wave motion. He conducted a number of controlled experiments which revealed many of the basic properties of solitary waves; however, despite his efforts and enthusiasm, solitary waves were dismissed as an interesting, but unimportant, curiosity by the scientific community during his lifetime.

The most important early theoretical work on solitary waves was that of Boussinesq (1872) who presented a general treatment of weakly nonlinear wave motions on the surface of a shallow fluid which yielded both right-running and left-running waves and which included Russell's solitary wave as a solution. This work was followed by the derivation of a single evolution equation for unidirectional finite-amplitude wave propagation by Korteweg and de Vries (1895). The Korteweg-de Vries, or KdV equation has become the paradigm evolution equation for nonlinear wave disturbances.

Interest in solitary waves from 1895 onwards was sporadic until the discovery of the soliton property of the KdV solitary waves by Zabusky and Kruskal (1965) and the ingenious work of Gardner et al. (1967) who demonstrated a method, which is now referred to as the inverse scattering transform, for linearising and thus exactly solving the nonlinear $\mathrm{KdV}$ equation. Zabusky and Kruskal showed that, despite significant nonlinear interaction, $\mathrm{KdV}$ solitary waves exhibit a particle-like behaviour in that they emerge completely unscathed from collision. They also showed that solitary waves are the asymptotic solution of the KdV equation. The discovery of solitons and the inverse scattering transform has had a profound impact on the development of many areas of mathematics and physics during the last two decades.

Studies of internal solitary waves were initiated by Keulegan (1953) who considered wave motions in a shallow (classical) 2-fluid system confined above and below by rigid boundaries. Benney (1966) and Benjamin (1966) extended this work to continuously stratified flow configurations and found that the evolution of finite-amplitude internal waves in shallow fluids is also governed to first 
order by the classical Korteweg-de Vries equation. A significant development occurred in 1967 when Benjamin (1967) and Davis and Acrivos (1967) independently presented the results of a theoretical and experimental investigation of a new class of solitary waves which propagate in density stratified layers embedded in a neutrally stable fluid of great depth. Further work on the theory of these deep-fluid waves was later presented by Ono (1975) and the governing evolution equation is usually referred to as the Benjamin-Davis-Ono or BDO equation. The BDO equation provides the basic theoretical foundation for the interpretation of solitary wave motions in the atmosphere.

Naturally occurring internal solitary waves have been identified in both marine and atmospheric environments. A thorough review of observations of solitary wave disturbances in the oceans and in inland lakes has recently been given by Ostrovsky and Stepanyants (1989). To the author's knowledge, the only direct evidence for the existence of solitary waves in the atmosphere prior to the publication of the first paper presented in this thesis (Christie et al., 1978; Section 2.1 ) is the description by Abdullah (1955) of a large scale localised disturbance with an effective wavelength of about $150 \mathrm{~km}$ which propagated over the mid-western United States during the early daylight hours of 29 June, 1951. This disturbance was interpreted by Abdullah on the basis of a shallow 2-fluid model as a classical KdV solitary wave. Since the publication of the initial solitary wave paper presented in Section 2.1, numerous reports of solitary wave activity in the lower atmosphere from a wide variety of locations around the world have appeared in the literature (see, e.g., Goncharov and Matveyev 1982; Doviak and Ge 1984; Drake 1985; Egger 1985; Noonan and Smith 1985; Physick 1986; Lin and Goff 1988; Smith 1988; Fulton et al. 1990; Ramamurthy et al. 1990; Cheung and Little 1991). A review of further work on solitary waves during the last two decades can be found in the introductory sections of the papers presented in Section 2 of this thesis.

The work by the author on atmospheric solitary waves with specific reference to the papers presented in this thesis is summarised briefly in the following section.

\subsection{Summary}

The first definitive observations of solitary waves in the atmospheric boundary layer were made at the Australian National University's Warramunga Research Station near Tennant Creek in the Northern Territory in late 1975 and described in a scientific report to the United States Air Force Office of Scientific Research (Christie et al., 1976). It was emphasised in this report that solitary atmospheric waves are a commonly occurring feature in the stable lower atmosphere. 
This initial work was extended over the next year and a full description and analysis of observations of atmospheric solitary wave phenomena over northern Australia, including a new class of waves of depression, was published in the Journal of the Atmospheric Sciences in 1978 (Christie et al., 1978; see Section 2.1). It was proposed in this paper that solitary waves which propagate on stable layers in the lower troposphere belong to the new class of internal finite amplitude deep fluid wave motions discovered by Benjamin (1967) and Davis and Acrivos (1967). The observations described in this paper were the first to show that solitary waves belonging to this fundamentally new class of internal wave motions can occur in a geophysical environment. Several source mechanisms for these disturbances were identified in this work, ranging from the transformation of deeply-penetrating sea-breeze fronts to katabatic flow. Evidence was also presented in this paper to show that solitary atmospheric waves occur on a wide variety of scales and propagate on occasion over distances in excess of 500 kilometres.

A study of the formation of atmospheric solitary waves in the transformation and evolution of intrusive density flows interacting with a stably-stratified lower atmosphere was then prepared and published in 1979 (Christie et al., 1979; see Section 2.2). Part of this paper was devoted to an interpretation of the morning glory phenomenon of the Gulf of Carpentaria. The morning glory is a spectacular propagating roll cloud formation or series of roll clouds which appears near dawn with considerable regularity during the spring months over the south-eastern Gulf of Carpentaria region. Morning glories were first brought to the attention of the scientific and meteorological communities in a pioneering paper by Clarke (1972). Prior to our work in 1979, it had been suggested that the morning glory was a katabatically produced hydraulic jump (Clarke, 1972), possibly 'some sort' of orographically induced gravity wave (Neal et. al., 1977), or a travelling sea-breeze vortex (R. H. Clarke, private communication, quoted in Neal et al., 1977). The morning glory roll cloud phenomenon was identified for the first time in the paper in Section 2.2 as a visible manifestation of deep-fluid solitary waves propagating in a stably-stratified maritime environment. This interpretation has proven to be in accord with all observations to date on morning glory waves (Smith, 1988; Christie, 1989).

Widely separated observations of large scale propagating waves were then made in both the Northern Territory and in northern Queensland. This material was described in considerable detail in a paper on solitary wave phenomena published in 1981 in Nature (Christie et al., 1981; see Section 2.3). The results presented in this paper provide considerable insight into the very large scale coherence pattern of nonlinear wave disturbances over the northern Australia region. The first explicit observations of solitary wave phenomena using an acoustic sounder were also reported in this paper. It is interesting to note that 
similar observations of solitary atmospheric waves using acoustic radar systems have only recently been described, nearly a decade after these original observations, from work which is currently being undertaken in the United States (Cheung and Little, 1990).

A long series of experimental observations of the formation and evolution of morning glory nonlinear waves was carried out between 1980 and 1983 using arrays of portable ultra-high sensitivity microbarometers which were specially designed and constructed for this purpose. A number of these experiments were undertaken as collaborative projects with scientists from Monash University, the University of Melbourne, the Commonwealth Scientific and Industrial Research Organisation and the Australian Bureau of Meteorology.

Solitary wave disturbances in the atmospheric boundary layer are accompanied by a localised transient wind squall which often produces strong horizontal and vertical shear components near the surface. Nonlinear atmospheric waves of this type were identified for the first time in an Australian National University Scientific Report prepared in 1982 (Christie and Muirhead, 1982) as a potentially serious hazard to aviation. This material and the results of further work on the aviation wind shear problem was published in two papers in 1983 (Christie and Muirhead, 1983a and 1983b; see Sections 2.4 and 2.5) and in a further brief article in 1985 (Christie and Muirhead, 1985). It is now recognised that solitary waves which occur as clear air disturbances represent a particularly insidious hazard to aircraft, especially during landing and take-off.

As a consequence of the work on solitary waves and the aviation wind shear problem, the author was invited by the National Severe Storms Laboratory in the United States in 1984 to participate in a collaborative experimental program to study the formation and propagation of thunderstorm-generated solitary wave disturbances over the Great Plains area. The work by Doviak and Ge (1984) and by Hasse and Smith (1984) had clearly demonstrated that thunderstorm-generated solitary waves do occur on occasion over Oklahoma. The frequency of occurrence of these disturbances was not, however, known at this time and the detailed mechanisms which led to the growth and decay of these disturbances were not well understood. Hence, an experiment was undertaken as part of the PRESTORM exercise during the months of June and July 1985 using the Australian National University's portable microbarometer array deployed in a long line over Oklahoma. This work led in part to the recognition that solitary waves are a commonly occurring feature in the thunderstorm environment over the mid-western United States and to the realisation that thunderstorm-generated solitary waves may be a particularly significant source of hazardous wind shear. Some aspects of this collaborative work were published in two articles in 1989 (Doviak and Christie, 1989 - see Section 2.6; Doviak et al., 1989 - see Section 2.7). 
A thorough theoretical study of the evolution of solitary waves in an unbounded fluid with turbulent frictional dissipation for both homogeneous and inhomogeneous waveguide conditions was also completed in 1989 and published in the Journal of the Atmospheric Sciences (Christie, 1989; see Section 2.8). This work on the solutions of the Benjamin-Davis-Ono-Burgers equation constitutes a major extension of an initial study of the general solutions of the BDO equation presented in a preliminary report by Christie and Muirhead (1981). The stability of deep-fluid solitary waves is illustrated in this paper by showing that the shape and speed of the solitary wave solutions of the BDO equation are preserved following a prolonged complex interaction with an extensive family of nonlinear dispersive waves. The evolution of internal deep-fluid bore waves of infinite extent as described by the BDO equation, both with and without turbulent frictional damping, are described for the first time in this paper. It is shown that, in the absence of dissipation, deep-fluid bore waves evolve continuously into an ever-increasing number of solitary waves. When turbulent dissipation is included, it is found that initial BDO bore waves of arbitrary form evolve rapidly into internal undular bores of constant form. Several models are proposed as a basis for the interpretation of the observed evolution of morning glory nonlinear wave disturbances. It is suggested that most of the observed features of morning glory disturbances can be accounted for by the evolution of a long, but finite-length, disturbance whose time evolution is governed to first order by the BDO-Burgers equation. The evolution process as described by the BDO equation is examined in detail for a wide variety of initial conditions and it is shown that most of the energy of an initial perturbation of the waveguide layer is focussed eventually into solitary wave components. The theory is extended to account, under specific conditions, for nonlinear wave propagation and decay when the upper atmosphere is both sheared and weakly stratified. It is also shown in this paper that atmospheric deep-fluid solitary waves can be created in the evolution process with amplitudes which are as much as four times the amplitude of the initial perturbation. This can be compared with the result from classical nonlinear wave theory which shows that solitary waves in shallow fluids are limited to maximum amplitudes which are at most no larger than twice that of the parent disturbance. This discovery provides considerable insight into the aviation wind shear problem and has an important bearing on our understanding of the role of these disturbances in the initiation and organisation of deep convection. Finally, model simulations are presented in this paper which illustrate the strong influence on the evolution pattern of both temporal and spatial variations in the degree of turbulent frictional damping.

The final paper presented in this thesis (Doviak et al., 1991; see Section 2.9) is concerned with a detailed comparison between nonlinear wave theory for a compressible fluid with shear and observations using a tall tower, a Doppler 
radar and a mesoscale surface network of a long-lived thunderstorm-generated solitary wave. A new model is proposed in this paper for the excitation of solitary waves by rapidly propagating storms. The analysis of these unique observations shows that trapped thunderstorm fluid is carried away from the storm over great distances along the axis of the solitary wave. This phenomenon is shown to be a manifestation of the influence of the Coriolis force on the dynamics of large amplitude solitary waves. It is emphasised in this paper that, while weakly nonlinear theory provides a fairly good description of the observed features of solitary waves of modest amplitude, an acceptable description of larger amplitude solitary atmospheric waves with recirculating flow can only be achieved within the framework of a fully nonlinear wave theory. It is also shown in this paper that highly nonlinear atmospheric solitary waves with closed circulation can deposit a thin layer of cold air, some tens of metres deep, in their wake at the surface. This result is significant because it implies that the density current interpretation which has often been used in the past to explain surface observations of propagating mesoscale disturbances may, in many cases, be incorrect.

\subsection{Significance of Solitary Atmospheric Waves}

It seems appropriate at this point to present a brief overview of the significance of solitary wave motions in the atmosphere.

Solitary waves are exceptionally stable isolated long-lived waves of large amplitude which evolve naturally as the long-lived components from quite general initial perturbations in stably stratified fluids (Maxworthy, 1980). As noted above, most of the energy of initial mesoscale disturbances will eventually be focussed into solitary waves provided suitable waveguide conditions exist. Large amplitude solitary waves will contain regions of recirculating trapped fluid which may have the properties of either the parent disturbance or of the environmental fluid immediately adjacent to the surface. Because of their stability and very large amplitude it must be expected that solitary waves play a significant role in the transport of mass, energy and momentum, in the initiation and organisation of deep convection, and in the production of turbulence in the atmosphere.

Solitary atmospheric waves can be excited by a wide variety of disturbances ranging from thunderstorm outflow density currents to mid-latitude cold frontal systems. Considerable insight into one of the most important source mechanisms for solitary waves in the lower atmosphere has recently been given by Hasse and Smith (1989) who showed from numerical simulations that a supercritical density current which is advancing into a stably stratified waveguide layer will transform 
directly into solitary waves.

It is now clear that solitary atmospheric waves occur commonly on a wide variety of scales ranging from effective wavelengths of some tens of metres to more than 100 kilometres. The wavefront may exceed $1000 \mathrm{~km}$ in length and some disturbances are observed to remain coherent over distances in excess of $1000 \mathrm{~km}$. These disturbances can have remarkably large amplitudes and can under some circumstances dominate the formation, growth and organisation of thunderstorms and squall lines. Even in the absence of deep convection, these waves can modulate the distribution of rainfall.

Large amplitude solitary atmospheric waves produce intense, rapidly varying horizontal and vertical winds. Waves of this type also provide an effective means for the redistribution and mixing of air at the surface. This results from the gradual depletion by surface friction of trapped recirculating fluid from within the wave and from a significant second-order transport process in which parcels of air near the surface are displaced over substantial distances along the direction of propagation by the passage of the wave. An understanding of the behaviour of solitary waves is therefore of considerable practical importance to the subject of aviation safety, the study of the mechanisms responsible for the dispersal of atmospheric pollutants and the problem of bush fire control.

The importance of solitary waves to our understanding of the fundamental dynamics of the atmosphere can be seen from the observations which have recently been described in Nature by Ramamurthy et al. (1990). These authors describe the morphology of two separate solitary wave disturbances which were observed to propagate for more than 1000 kilometres over the central and eastern United States with amplitudes of the order of the scale height of the atmosphere. These exceptionally large-amplitude waves effectively perturbed the whole of the troposphere and the lower stratosphere and completely dominated the weather, including the organisation of precipitation, throughout their lifetime. A remarkable feature of these waves is that they managed to maintain their identity over a long period of time while propagating through a seemingly chaotic background flow regime. These observations therefore have an important bearing on our understanding of the overall predictability of atmospheric motions (James, 1990). It has become increasingly fashionable in recent years, since the recognition of Lorenz's seminal paper (Lorenz, 1963), to assume that deterministic chaos is a dominating factor in the predictability of atmospheric motions. Observations of long-lived solitary atmospheric waves in a turbulent environment suggest that this may not necessarily be true. 


\section{References}

Abdullah, A.J. (1955). The atmospheric solitary wave. Bull. Amer. Meteor. Soc., 36, 515-518.

Benjamin, T.B. (1966). Internal waves of finite amplitude and permanent form. J. Fluid Mech., 25, 241-270.

Benjamin, T.B. (1967). Internal waves of permanent form in fluids of great depth. J. Fluid Mech., 29, 559-592.

Benney, D.J. (1966). Long non-linear waves in fluid flows. J. Math. \& Phys., 45, 52-63.

Boussinesq, J. (1872). Théorie des ondes et des remous qui se propagent le long d'un canal rectangulaire horizontal, en communiquant au liquide contenu dans ce canal des vitesses sensiblement pareilles de la surface au fond. $J$. Math. Pures Appl., 17, 55-108.

Cheung, T.K. and Little C.G. (1990). Meteorological tower, microbarograph array, and sodar observations of solitary-like waves on the nocturnal boundary layer. J. Atmos. Sci., 47, 2516-2536.

Christie, D.R. (1989). Long nonlinear waves in the lower atmosphere. J. Atmos. Sci., 46, 1462-1491.

Christie, D.R. and Muirhead, K.J. (1981). Observations of solitary atmospheric waves over northern Australia. Physics for Australia's Development, Second Applied Physics Conference, Australian Institute of Physics, Melbourne, 463-466.

Christie, D.R. and Muirhead, K.J. (1982). Solitary waves: A hazard to aircraft operating at low altitudes. Aust. Nat. Univ. Rpt., 1 September, 1982, 33 pp.

Christie, D.R. and Muirhead, K.J. (1983a). Solitary waves: A hazard to aircraft operating at low altitudes. Aust. Met. Mag., 31, 97-109.

Christie, D.R. and Muirhead, K.J. (1983b). Solitary waves: A low-level wind shear hazard to aviation. Int. J. Aviation Safety, 1, 169-190. 
Christie. D.R. and Muirhead, K.J. (1985). Solitary waves and low altitude wind shear over Australia. Aviation Safety Digest, 123, 3-8.

Christie, D.R., Muirhead, K.J. and Hales, A.L. (1976). Solitary waves in the atmosphere. AFOSR-76-1133TR, National Technical Information Service (NTIS:A031228) Springfield, VA., 22161. 59 pp.

Christie, D.R., Muirhead, K.J. and Hales, A.L. (1978). On solitary waves in the atmosphere. J. Atmos. Sci., 35, 805-825.

Christie, D.R., Muirhead, K.J. and Hales, A.L. (1979). Intrusive density flows in the lower troposphere: A source of atmospheric solitons. J. Geophys. Res., 84, 4959-4970.

Christie, D.R., Muirhead, K.J. and Clarke, R.H. (1981). Solitary waves in the lower atmosphere. Nature, 293, 46-49.

Clarke, R.H. (1972). The morning glory: An atmospheric hydraulic jump. $J$. Appl. Meterol., 11, 304-311.

Davis, R.E. and Acrivos, A. (1967). Solitary internal waves in deep water. $J$. Fluid Mech., 29, 593-607.

Doviak, R.J. and Christie, D.R. (1989). Thunderstorm-generated solitary waves: A wind shear hazard. A.I.A.A. J. of Aircraft, 46, 423-431.

Doviak, R.J. and Ge, R. (1984). An atmospheric solitary gust observed with a Doppler radar, a tall tower and a surface network. J. Atmos. Sci., 41, 2559-2573.

Doviak, R.J., Thomas, K.W. and Christie, D.R. (1989). The wavefront shape, position, and evolution of a great solitary wave of translation. I.E.E.E. Trans. Geosci. Remote Sensing, 27, 658-665.

Doviak, R.J., Chen, S.S. and Christie, D.R. (1991). A thunderstorm generated solitary wave observation compared with theory for nonlinear waves in sheared fluids. J. Atmos. Sci., 48, 87-111.

Drake, V.A. (1985). Solitary wave disturbances of the nocturnal boundary layer revealed by radar observations of migrating insects. Boundary Layer Meterol., 31, 269-286. 
Egger, J. (1985). Die Berliner Nebelwellen. Meteorol. Rdsch., 38, 103-107.

Fulton, R., Zrnic, D.S. and Doviak, R.J. (1990). Initiation of a solitary wave family in the demise of a nocturnal thunderstorm density current. $J$. Atmos. Sci., 47, 319-337.

Gardner, C.S., Greene, J.M., Kruskal, M.D. and Miura, R.M. (1967). Method for solving the Korteweg-de Vries equation. Phys. Rev. Lett., 19, 1095-1097.

Goncharov, V.P. and Matveyev, A.K. (1982). Observations of nonlinear waves on an atmospheric inversion. Izv. Acad. Sci. USSR Atmos. Oceanic Phys., Engl. Transl., 18, 61-64.

Hasse, S.P. and Smith, R.K. (1984). Morning glory wave-clouds in Oklahoma: a case study. Mon. Weather Rev., 112, 2078-2089.

Hasse, S.P. And Smith, R.K. (1989). The numerical simulation of atmospheric gravity currents, Part II. Environments with stable layers. Geophys. Astro. Phys. Fluid Dyn., 46, 35-51.

James, I. N. (1990). Order and chaos up in the air. Nature, 348, 283-284.

Korteweg, D. J. and de Vries, G. (1895). On the change of form of long waves advancing in a rectangular canal, and on a new type of long stationary waves. Phil Mag., 39, 422-443.

Keulegan, G.H. (1953). Characteristics of internal solitary waves. J. Res. Natl. Bur. Stand., 51, 133-140.

Lin, Y. -L. and Goff, R.C. (1988). A study of a thunderstorm generated mesoscale solitary wave in the atmosphere. J. Atmos. Sci., 45, 194-205.

Lorenz, E.N. (1963). Deterministic nonperiodic flow. J. Atmos. Sci., 20, $130-141$

Maxworthy, T. (1980). On the formation of nonlinear internal waves from gravitational collapse of mixed regions in two and three dimensions. $J$. Fluid Mech., 96, 47-64. 
Neal, A.B., Butterworth, I.J. and Murphy, K.M. (1977). The Morning Glory. Weather, 32, 176-183.

Noonan, J.A. and Smith, R.K. (1985). Linear and weakly nonlinear internal wave theories applied to 'morning glory' waves. Geophys. Astrophys. Fluid Dyn., 33, 123-143.

Ono, H. (1975). Algebraic solitary waves in stratified fluids. J. Phys. Soc. Jap., 39, 1082-1091.

Ostrovsky, L.A. and Stepanyants, Yu. A. (1989). Do internal solitons exist in the ocean? Rev. Geophys., 27, 293-310.

Physick, W. (1986). Observations of a solitary wave train in Melbourne, Australia. Aust. Meteor. Mag., 34, 163-172.

Ramamurthy, M.K., Collins, B.P., Rauber, R.M. and Kennedy, P.C. (1990). Evidence of very-large-amplitude solitary waves in the atmosphere. Nature, 348, 314-317.

Russell, J.S. (1838). Report of the committee on waves. Rep. Meet. Brit. Assoc. $A d v$. Sci., 7th, Liverpool, 1837, pp. 417-496. London: John Murray.

Russell, J.S. (1845). Report on waves. Rep. Meet. Brit. Assoc. Adv. Sci., 14th, York, 1844, pp. 311-390. London: John Murray.

Smith, R.K. (1988). Waves and bores in the lower atmosphere: The 'morning glory' and related phenomena. Earth-Sci. Rev., 25, 267-290.

Zabusky, N.J. and Kruskal, M.D. (1965). Interaction of "solitons" in a collisionless plasma and the recurrence of initial states. Phys Rev. Lett., 15, 240-243. 


\section{Section 2.1}

\section{On solitary waves in the atmosphere}

(D.R. Christie, K.J. Muirhead and A.L. Hales)

J. Atmos. Sci., 35, 805-825, 1978. 

Reprinted from Journal of the Atmospheric Sciences, Vol. 35, No. 5, May 1978 American Meteorological Society Printed in U. S. A.

\title{
On Solitary Waves in the Atmosphere
}

\author{
D. R. Christie, K. J. Mutrhead and A. L. Hales \\ Research School of Earth Sciences, The Australian National University, Canberra, A.C.T., 2600
}

(Manuscript received 20 January 1977, in final form 18 November 1977)

\section{ABSTRACT}

\begin{abstract}
This paper is concerned with a description and interpretation of two unusual types of isolated atmospheric gravity wave observed near Tennant Creek in central Australia. These waves occur in the form of solitary waves of elevation and solitary waves of depression. Comparison of experimental data with theory leads to the conclusion that the majority of the observed isolated waves of elevation belong to the class of deep-fluid internal solitary waves considered by Benjamin and by Davis and Acrivos. The second fundamentally different type of large-amplitude isolated wave is tentatively identified as a classical solitary wave of depression.

A brief discussion is given of a number of possible source mechanisms which may give rise to internal solitary atmospheric waves. It is proposed that the following two dynamical processes play an important role in the creation of solitary atmospheric waves in the arid interior of Australia: 1) the interaction of nocturnal katabatic density currents with an existing radiation inversion; and 2) the interaction of a propagating horizontal sea breeze vortex with the nocturnal inversion.
\end{abstract}

\section{Introduction}

It is well known that the creation, propagation and dissipation of gravity waves play an important role in the dynamics of the earth's atmosphere. The reasons for studying long-period waves of this type are therefore twofold: in the first place, an understanding of the initial disturbance which gives rise to these waves, the mechanisms by which they propagate and their dispersion characteristics provide insight into the fundamental nature of atmospheric fluid mechanics; second, it seems to be equally important to understand the influence that the passage of these waves has on the development of local meteorological conditions-for example, gravity waves may sometimes be correlated with the release of latent atmospheric instabilities which in turn give rise to such diverse phenomena as clear-air turbulence, severe convective storms and, possibly, in the extreme case the development of tornado activity.

The purpose of this paper is to present a description and interpretation of the results of an experimental investigation of large-amplitude isolated propagating atmospheric disturbances which have been observed, using an array of high-sensitivity microbarometers, at the Warramunga Seismic Station located near Tennant Creek in central Australia. It is thought that these unusual phenomena which occur as isolated waves of elevation and isolated waves of depression represent two new types of naturally occurring internal solitary wave. In particular, it will be shown that the properties of the waves of elevation are in accord with the class $0022-4928 / 78 / 0805-0825 \$ 10.50$

(C) 1978 American Meteorological Society of deep-fluid solitary waves considered by Benjamin (1967) and by Davis and Acrivos (1967) and that the internal waves of depression appear to be described by the classical solitary wave theories of Peters and Stoker (1960), Long (1965) and Benjamin (1966).

The properties of atmospheric waves have recently been reviewed by Gossard and Hooke (1975). In their most common form, horizontally propagating gravity waves in the troposphere occur as long-period, nearly sinusoidal wavetrains. This familiar form of atmospheric wave may be contrasted with an example of the specific type of unusual isolated wave considered in this paper as illustrated in the Tennant Creek microbarograph array record section shown in Fig. 1 for the late evening hours of 2 December 1976. Perhaps the most interesting feature of the observed isolated waves is their unusually large relative amplitude. A study of the properties of these waves therefore encompasses an investigation of the role of nonlinearity in largescale fluid motions and an investigation of the process of wave-induced turbulence. It must be anticipated that this class of waves will prove to be important in other areas of geophysical fluid mechanics.

\section{The internal solitary atmospheric wave}

The phenomenon of the solitary wave, by its essentially nonlinear nature, occupies a unique place in the development of the theory of wave propagation in fluids. A classical wave of this type is usually defined (Lamb, 1932, Section 252) as a wave of single elevation which propagates at uniform velocity without change 


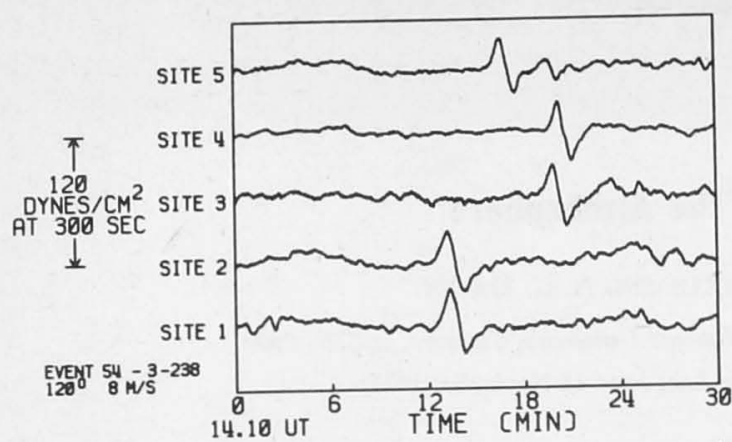

FIG. 1. An example of a microbarograph array record section corresponding to the passage of an internal solitary wave of elevation. The azimuth noted for this wave is the source direction measured from true north.

of form. The existence of these stationary waves may be viewed as being the consequence of equilibrium between the competing effects of nonlinearity and dispersion; that is, they arise from a balance between the tendency of waves to steepen ahead of their crests (amplitude dispersion) and the tendency of the longer period Fourier components to propagate at higher velocities (frequency dispersion).

The classical solitary wave of elevation was first observed by Scott-Russell $(1837,1844)$ on the free surface of shallow water of uniform depth. Subsequently, Boussinesq (1871) and Rayleigh (1876) independently derived approximations for the speed of propagation $c$ and the form $\eta(x)$ of the wave profile at the free surface. These authors found, to first order in the relative amplitude $\alpha=a / h$, a solitary wave solution described by

$$
\begin{aligned}
\eta(x) & =a \operatorname{sech}^{2}\left[\frac{(3 \alpha K)^{\frac{1}{2}} x}{2} \frac{x}{h}\right], \\
c & =[g h(1+\alpha)]^{\frac{1}{2}},
\end{aligned}
$$

where $h$ is the undisturbed fluid depth, $a$ the maximum amplitude of the wave and $g$ the acceleration due to gravity. A measure of the effective wavelength of these waves is given by the full width of the wave profile at half maximum, i.e.,

$$
w_{\frac{1}{2}}=\frac{4 \ln \left(1+2^{\frac{1}{2}}\right)}{(3 \alpha K)^{\frac{1}{3}}} h .
$$

In the Boussinesq approximation the factor $K$ in the argument of the hyperbolic secant takes the value $K=1$, while in the solution found by Rayleigh $K=(1+\alpha)^{-1}$.

These early investigations revealed an important property of solitary waves; that is, they are always supercritical-the speed of propagation always exceeds the speed

$$
c_{0}=(g h)^{\frac{1}{2}}
$$

of infinitesimal long waves. This property may be expressed to first order in the dimensionless amplitude as

$$
\mathrm{F}^{2}=1+\alpha>1,
$$

where $\mathrm{F}$ is the Froude number. It should be noted that all forms of the classical solitary wave are characterized by fluid systems in which the horizontal length scale of the motion is long compared to the total fluid depth.

Korteweg and de Vries (1895) showed that the time evolution of small but finite dispersive shallow-water waves is described, in the first approximation, by a nonlinear partial differential equation (the $\mathrm{KdV}$ equation) which may be written as

$$
\frac{\partial \eta}{\partial t}+c_{0}(1+\epsilon \eta) \frac{\partial \eta}{\partial x}+c_{0} \beta \frac{\partial^{3} \eta}{\partial x^{3}}=0
$$

where $\epsilon=\frac{3}{2}$ h and $\beta=h^{2} / 6$. From this equation they were able to demonstrate the existence of a class of periodic long waves of finite amplitude and permanent form which are described by the square of the Jacobi elliptic function $\mathrm{cn}$ of modulus $k$. In particular, they showed that these waves, which they called cnoidal waves, reduce to the classical solitary wave of finite extent in the limit as the wavelength goes to infinity. Despite this progress, it was not until relatively recently that Lavrent'ev (1964) and Friedrichs and Hyers (1954) succeeded in rigorously proving the mathematical existence of a solitary wave solution of the full nonlinear equations.

There have been a number of attempts to improve on the first-order approximation to the classical solitary wave. The second-order solution was found by Laitone (1960), the third-order by Grimshaw (1971), and a fifth-order expression for the wave speed has been given by Long (1956b). Much of the recent work on classical solitary waves has concentrated on the determination of the solitary wave of maximum height. Estimates have been obtained from an exact calculation of the position of points on the profile of the wave of maximum amplitude (Yamada, 1957; Yamada et al., 1968), from an extrapolation of a ninth-order series approximation for the profile of waves of less than maximum amplitude (Fenton, 1972), from an extrapolation of the results of a finite-difference formulation of the problem (Chan, 1974), and from the solution of an integral equation (Lenau, 1966; Byatt-Smith, 1970; Strelkoff, 1971). A puzzling aspect of these calculations has been the small, but significant, discrepancy between the various estimates for the wave of maximum amplitude. This problem has recently been resolved by Longuet-Higgins and Fenton (1974) who found that the wave of maximum height does not correspond-as had generally been assumed - to the wave of maximum speed. According to these authors the wave of maximum amplitude is specified by

$$
\alpha_{\max }=0.827, \quad F=1.286,
$$


in good agreement with the results of Yamada (1957) and Lenau (1966), while the wave of maximum speed corresponds to

$$
\alpha=0.790, \quad \mathrm{~F}_{\max }=1.294 .
$$

It is worth noting that the Boussinesq approximation (2.1) to the shape of the classical solitary wave compares favorably with the exact profiles computed by Yamada (1958) and by Byatt-Smith (1970) provided the nondimensional amplitude $\alpha$ is less than about 0.7 .

The theoretical study of internal solitary waves, i.e., waves which exist as a consequence of internal density stratification, was initiated by Keulegan (1953) who considered a system of two superimposed liquids of different constant densities, bounded above and below by rigid surfaces. Long (1956b) and Benjamin (1966) have also investigated this particular model. Using a systematic perturbation procedure developed by Friedrichs (1948) and Keller (1948), Abdullah (1956) obtained a first-order solution for the classical internal solitary wave at the interface of a two-layer atmosphere subject to the condition that the hydrostatic law holds for the upper layer; that is, that motion of the free surface can be neglected. This solution is a supercritical wave of elevation described by the Boussinesq approximation (2.1) with the expression for the phase velocity modified to

$$
c=c_{0}^{\prime}\left(1+\frac{1}{2} \alpha\right),
$$

where the infinitesimal internal wave critical speed is now given by the reduced form of the ordinary classical solitary wave of elevation; maximum amplitude at the interface much larger than the amplitude at the surface. The internal solitary wave may be either a wave of depression or a wave of elevation. If the difference in the two fluid densities is small, then the streamlines are lines of elevation at the interface if $R>1$ and lines of depression if $R<1$. This form of the two-fluid solution may be suited to a description of large-scale internal atmospheric solitary waves. For this particular model, the wave profile at the interface is given by the usual classical solitary waveform (2.1) with

$$
K=\left|\frac{(R-1)}{R^{2}}\right|
$$

and the corresponding internal critical speed reduces to

$$
c_{0}^{i}=\left[g\left(1-\frac{\rho_{2}}{\rho_{1}}\right) \frac{h_{1} h_{2}}{\left(h_{1}+h_{2}\right)}\right]^{\frac{1}{2}} .
$$

In the case of a fluid of finite depth with a density distribution which decreases exponentially upward, Peters and Stoker found an infinite number of possible internal solitary wave modes corresponding to an infinite spectrum of internal critical speeds. This problem has also been investigated by Long (1965), and in an elegant and very general treatment of the subject of internal solitary waves in shallow fluids by Benjamin (1966). The general solitary wave solutions in this case are complicated and will not be given here; it is noted, however, that according to Benjamin, for the case of a free upper boundary, the solitary wave mode corresponding to the highest critical speed-predominantly a wave of depression - is probably the most significant mode in fluids of this type.

Up to this point this discussion has been limited to a consideration of internal solitary waves which can exist in fluids of finite depth. Benjamin (1967) and Davis and Acrivos (1967) have independently presented the results of a theoretical and experimental investigation of an entirely new class of internal solitary waves which can exist in fluids of great depth. These new types of internal waves are shown to exist in regions where the fluid density varies only within a layer of thickness $h$ which is much smaller than the total fluid depth and smaller than the effective horizontal length scale $\lambda$ characteristic of the solitary wave. Thus, in stratified fluids of great depth, the fundamental scale against which wave dimensions are to be measured is the thickness of the region of significant density variation rather than the total fluid depth.

For a two-fluid system in which the upper fluid extends to infinity over a lower fluid of depth $h$ resting on a horizontal rigid surface, Benjamin finds a solitary wave solution of the form

$$
\eta(x)=\frac{a \lambda^{2}}{x^{2}+\lambda^{2}},
$$
the second describes an internal solitary wave with a 


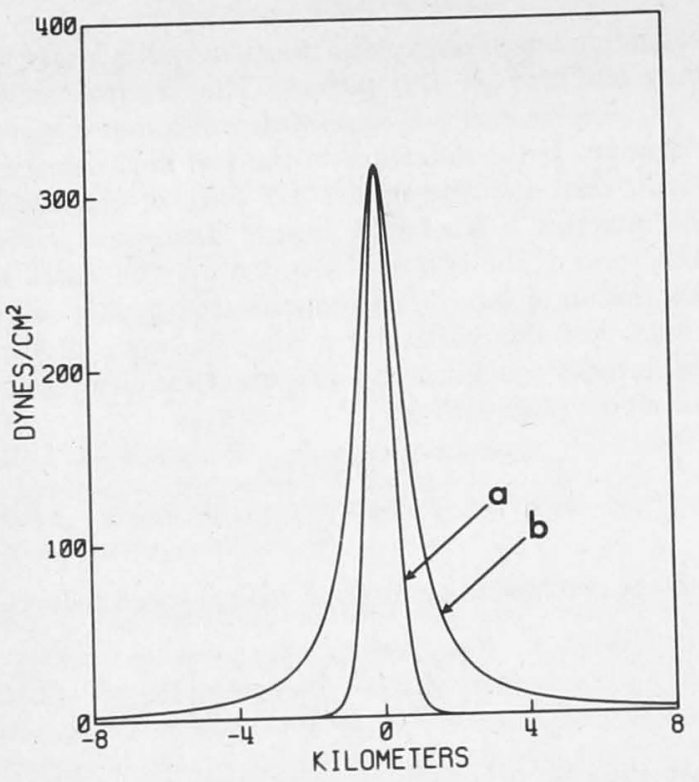

FIG. 2. Internal solitary wave surface pressure profiles correponding to a $5^{\circ} \mathrm{C}$ temperature inversion at the $980 \mathrm{mb}$ level. The dimensionless amplitude $\alpha$ is chosen to be 0.5 . (a) Classical internal solitary wave described by (2.1) with $K=1$. (b) Internal solitary wave described by Benjamin's theory (2.11) for a fluid of great depth.

with

where

$$
c=c_{0}^{\prime}\left(1+\frac{3}{4} \alpha\right)^{\frac{1}{2}},
$$

$$
2 \lambda=w_{\frac{1}{2}}=\frac{8 \rho_{1} h}{3 \rho_{2} \alpha},
$$

$c_{0}^{\prime}$ is the reduced internal wave critical speed given by (2.7), and $\rho_{2}$ and $\rho_{1}$ are the densities of the upper and lower fluids, respectively.

Benjamin has also considered the problem of a shallow fluid with an exponential density gradient in contact with a deep fluid of constant density and, further, the more general problem of solitary waves associated with a thin transition region contained between two homogeneous fluids of substantial depth. In direct analogy with the shallow fluid theory, the continuous variation in density is shown to give rise to an infinite set of solitary-wave modes. For the case of a thin region of sudden vertical density variation in a stably stratified system, the lowest order mode solitary-wave solution takes the form of a bulge which propagates along the interface. This interesting result has been confirmed in the laboratory experiments of Davis and Acrivos (1967) and Hurdis and Pao (1975). The experiments and numerical calculations of Davis and Acrivos are particularly interesting in that they show that solitary waves of this type can exist with dimensionless amplitude near 2.0 and that waves with large amplitudes exhibit an unusual closed circulation in the streamline pattern which suggests the presence of a propagating vortex pair.

It was long thought that solitary waves would only be produced as a consequence of rather specialized initial conditions. The discovery by Zabusky and Kruskal (1965) that the solitary wave or soliton solutions of the $\mathrm{KdV}$ equation (2.4) possess the remarkable linear property of preserving their form following nonlinear interaction has provided in recent years a great deal of impetus to the study of the general properties of the nonstationary solutions of the $\mathrm{KdV}$ equation and other nonlinear wave equations known to admit soliton solutions (see, e.g., Karpman 1975). The importance of these results to the study of atmospheric fluid mechanics stems principally from the fact that soliton production in density-stratified fluids is now known to occur under widely varying initial conditions.

We now consider the existence of solitary-wave motions in the atmosphere. It would seem, on the basis of the atmospheric scale involved, that the deep-fluid solitary-wave solution (2.11) found by Benjamin (1967) is best suited to a simple description of solitary waves in the planetary boundary layer, while classical internal solitary-wave theory should provide a reasonable description of higher altitude waves.

In order to clarify the interpretation of experimental data we consider the properties, as predicted by both the classical and deep-fluid theories, of internal solitary waves associated with a density discontinuity at the $980 \mathrm{mb}(280 \mathrm{~m})$ level corresponding to a temperature inversion of $5^{\circ} \mathrm{C}$. The air beneath the inversion is taken to have a mean temperature of $270 \mathrm{~K}$ and the dimensionless amplitude $\alpha$ of the wave is chosen to have a value of 0.5 , corresponding to a wave with amplitude less than maximum. With these conditions, a classical internal solitary wave as described by the theory of Abdullah [Eqs. (2.1) and (2.6) with $K=1$ ] would propagate along the inversion at a speed of $8.9 \mathrm{~m} \mathrm{~s}^{-1}$ with a full width at half maximum, $w_{\frac{1}{2}}$, of $0.81 \mathrm{~km}$ and would produce, assuming the hydrostatic approximation, a maximum pressure perturbation $\Delta P$ at ground level of 317 dyn $\mathrm{cm}^{-2}$; in contrast, the classical theory of Peters and Stoker [Eqs. (2.1) and (2.10) with $K \ll 0.1]$ describes an internal solitary wave which propagates with nearly the same velocity but with a much larger effective wavelength. Alternatively, for the same wave amplitude, Benjamin's deep-fluid theory (2.11) describes a solitary wave with $w_{\frac{1}{2}}=1.52 \mathrm{~km}$ propagating at a speed of $8.4 \mathrm{~m} \mathrm{~s}^{-1}$. It is, of course, unlikely that the classical treatment of the theory provides an accurate description of internal solitary waves whose characteristic horizontal dimensions, as in this example, are much smaller than the total fluid depth. A comparison of the classical and deep-fluid solitarywave profiles for an inversion at the $980 \mathrm{mb}$ level is shown in Fig. 2. Note that, as a consequence of an 
asymptotic exponential behavior, the influence of the classical wave is much more localized than that of the deep-fluid wave of elevation. The shape of both types of solitary wave depends on the value of the dimensionless amplitude $\alpha$; as $\alpha$ increases the wave profile narrows and the curvature at the crest increases until at maximum amplitude the peak is reduced in accordance with Stokes's (1880) conjecture ${ }^{1}$ to a cusp enclosing an angle of $120^{\circ}$.

The possible existence of atmospheric internal solitary waves was suggested by Abdullah (1949). The only direct evidence for atmospheric solitary waves appears to be the description by Abdullah (1955) of a large-amplitude propagating disturbance which appeared over Kansas during the early daylight hours of 29 June, 1951. This disturbance, which produced a ground level pressure perturbation of $3.4 \mathrm{mb}$, took the form of an elevated mass of cold air propagating on an inversion at a height of about $2 \mathrm{~km}$. The elevated disturbance appeared to extend over about $150 \mathrm{~km}$ and was observed to travel with approximately constant form at speeds between 18 and $24 \mathrm{~m} \mathrm{~s}^{-1}$ over a distance of about $800 \mathrm{~km}$. Abdullah concluded that this disturbance represented a classical internal solitary wave of elevation and attributed its formation to the impulsive movement of a quasi-stationary cold front into the layer of inversion. As can be seen from the following description, most of the atmospheric solitary waves observed at Tennant Creek which belong to the class of isolated waves of elevation have an effective wavelength of only a few kilometers; they therefore appear to be entirely different from the single observation described by Abdullah. It will be shown that the properties of this new form of isolated wave are best described by the deep-fluid solitary-wave theory. In addition, a description will be given of observations of large-scale solitary waves of depression. An examination of the properties of this second new type of solitary wave indicates that these waves are probably best described classical solitary waves which propagate in a stratified fluid whose density decreases continuously with height. Smart (1966) and Jordan (1972) have reported observations of "exponential pressure pulses" near Denver, Colo., which may represent a form of internal solitary wave. Additional evidence for the existence of solitary atmospheric waves based on observations of the pressure field is scarce. Phillips (1976) has described an interesting example of an isolated propagating disturbance which appeared as an exceptionally large pressure spike

${ }^{1}$ This conjecture, which has often been used as a limiting constraint in the mathematical development of large-amplitude wave theory, has recently been considered by Byatt-Smith and Longuet-Higgins (1976) as part of a study of the detailed shape of the profile of steep solitary waves. In all cases studied the maximum slope of the wave profile was less than $30^{\circ}$. However, the possibility that the maximum surface slope of the wave of highest amplitude exceeds the limit imposed by Stokes's criterion could not be excluded. of $4 \mathrm{mb}$ amplitude on barograph traces recorded on 25 March 1966 at El Adem and Tobruk in Libya. Apparently this disturbance was vigorous enough to trigger a seiche in Tobruk Harbor. It seems reasonable to assume that this disturbance and other smaller disturbances of a similar nature observed on the same day can be described as large-amplitude solitary waves of elevation.

At this point we would like to draw attention to two important acoustic radar studies which were carried out several years ago in areas of Australia with environments which are very similar to the semi-desert environment of the Warramunga Seismic Station. As will be seen, these studies provide considerable insight into the nature of the isolated propagating disturbances observed near Tennant Creek.

In a pioneering atmospheric acoustic sounding study McAllister et al. (1969) described an observation of a "weak, front-like" nocturnal disturbance characterized by the sudden appearance on the inversion level of a well-defined sharp spike in the sounder record. This initial pulse was followed by a rapid buildup of turbulence which, after a period of about $10 \mathrm{~min}$, evolved into a pattern of reflections from distinct slowly ascending strata. A further feature of this record are the clearly visible large-amplitude vertical oscillations of the atmosphere in the wake of the initial spike. It is worth noting that the ratio of the amplitude of the initial pulse-like disturbance to the depth of the inversion both ahead of and immediately behind the pulse is at least as high as 1.0. It should also be emphasized that evidence of large-scale vertical atmospheric motions associated explicitly with the spike in the sounder record are provided by the corresponding records from the $75 \mathrm{~m}$ level of an instrumented tower which show that the passage of the disturbance corresponding to the spike produced a simultaneous negative pulse of $4^{\circ} \mathrm{C}$ in the temperature field and a positive pulse of about $6 \mathrm{~m} \mathrm{~s}^{-1}$ in the wind speed at this level. These observations were carried out during the month of June at Ivy Tanks on the edge of the arid Nullarbor Plain in South Australia.

A very interesting description of a series of acoustic sounding experiments which were carried out, again during the month of June, at Julia Creek, Queensland, has been given by Reynolds and Gething (1970). They also observed, usually under clear conditions with light surface winds, several examples of well-defined, very large-amplitude spikes on the interface of the nocturnal inversion. These unusual disturbances appear on the sounder record in two different forms: 1) a single clearly defined pulse with dimensionless amplitude of the order of unity which occurs along or immediately behind the leading edge of a rise in the height of the inversion and which is usually followed by patterns which indicate further wave activity near the inversion level; and 2) clusters of particularly large-amplitude pulses on the 
inversion level which precede a pattern of lower amplitude wavetrains - in this case, the sounder records indicate little evidence for a change in the height of the inversion. Note that the profiles of all of these spikes on the inversion are very similar in form to the profile of a deep-fluid solitary wave.

Reynolds and Gething have also described the corresponding measurements of temperature, wind speed and wind direction recorded at $15 \mathrm{~m}$ intervals to a height of $75 \mathrm{~m}$ on three towers separated by distances of the order of $40 \mathrm{~km}$. A few of the essential features of these measurements are worth noting at this point. In the first place, the sounder measurement of the inversion height is in good agreement with the value determined from the tower measurements; there would therefore appear to be little doubt that the sounder records provide an accurate picture of the profile of disturbances on the inversion level. Second, an examination of the temperature structure associated with the isolatedpulse form of disturbance shows that by far the largest perturbation of the overall temperature field corresponds to the spike in the sounder record. This strongly suggests that this initial transient disturbance may be viewed as a distinct separate phenomenon and, as well, indicates that the dynamical processes associated with the pulse dominate the flow structure of the disturbance. At higher altitudes this initial perturbation takes the form of a strong negative temperature spike similar to that observed by McAllister et al.; at lower heights the amplitude of the spike decreases steadily with decreasing altitude and has almost disappeared from the trace recorded at $1.5 \mathrm{~m}$. Note that this temperature distribution is consistent with the perturbations that would occur during the passage of a single large-amplitude internal wave of elevation associated with a density profile - as in the examples considered herewhich decreases monotonically with height from the surface to the inversion level. Third, the observation of an apparently unattenuated disturbance at sites separated by about $40 \mathrm{~km}$ establishes that these pulses propagate over significant distances. These observations also reveal that the thermal structure associated with the spike in an isolated-pulse type of disturbance is identical in form to the structure associated with individual pulses in a cluster type of disturbance - this provides a convincing demonstration that the isolated pulses and the nonisolated pulses possess the same internal morphology, i.e., all of these events are manifestations of the same basic phenomena.

The essentially distinct nature of the pulselike disturbances is further indicated in the $75 \mathrm{~m}$ anemometer records which show a sudden temporary decrease in the wind speed from near $14 \mathrm{~m} \mathrm{~s}^{-1}$ to about $7 \mathrm{~m} \mathrm{~s}^{-1}$ during the passage of the pulse (in contrast to the observations of McAllister et al. where the pulse-associated wind showed an increase in speed) and a corresponding sudden temporary increase in the wind azimuth by about $60^{\circ}$.
Note that this perturbation of the wind vector is significantly larger than any of the perturbations which occurred further on the disturbance.

Finally, it is worth noting the acoustic radar observation described by Shaw (1971) of a transient isolated disturbance in the atmospheric boundary layer which occurred near Melbourne in the early morning hours of 9 June 1970. This disturbance, which takes the form of a single symmetrical wave of elevation, appears to be very similar to the deep-fluid solitary wave described by the theory of Benjamin (1967).

During the course of the experiments at Tennant Creek we have observed a wide variety of unusual propagating lower tropospheric disturbances, many of which bear a close resemblance to the series of observations reported by Reynolds and Gething (1970) and to the events described by McAllister et al. (1969) and Shaw (1971). Observations have been made of disturbances in the form of smooth shallow internal density currents, complex solitary-wave families superimposed along the leading edge of sustained internal density flows, isolated clusters of solitary waves of elevation, and disturbances in the form of individual solitary waves propagate along the nocturnal inversion. It is thought that all of these disturbances belong to the class of deep-fluid nonlinear propagating disturbance described by the theory of Benjamin (1967). In particular, we identify all of the boundary layer pulse-like disturbances noted in the acoustic radar records and all isolated waves in the microbarograph array records which correspond to a transient increase in surface pressure as deep-fluid internal solitary waves of elevation. Since this paper is primarily concerned with the phenomenon of the atmospheric solitary wave we include here only those observations which clearly illustrate the basic features of isolated solitary waves and isolated clusters of solitary waves which propagate along an atmospheric inversion. It should be emphasized, however, that the available experimental evidence indicates that the genesis of the deep-fluid solitary waves described in this paper may often be traced to the evolution of the observed density-flow disturbances. Since the properties of these complex density currents constitute a separate area of investigation, a detailed description and interpretation of these unusual flow phenomena will be published separately.

\section{Experimental arrangement and data processing techniques}

The Warramunga Seismic Station is situated $37 \mathrm{~km}$ south-southeast of Tennant Creek. The centered quadrilateral array of microbarometers is located on slowly undulating semi-desert terrain at an elevation of about $410 \mathrm{~m}$ MSL with relief in the area encompassed by the array rising to a maximum of about $6 \mathrm{~m}$. Probably the most important topographical features, insofar as the location of this array is concerned, are the 
$600 \mathrm{~m}$ Murchison and Davenport Ranges which run from 20 to $160 \mathrm{~km}$ to the south-southeast and the $1600 \mathrm{~m}$ Macdonnell Ranges, $450 \mathrm{~km}$ to the south. All of the area within $400 \mathrm{~km}$ to the west of the array can be described as semi-featureless stoney desert and an extensive dry steppe area known as the Barkly Tablelands forms the northeast quadrant.

Each element of the array consists of a National Bureau of Standards designed capacitor microphone which measures variations in pressure relative to a reference volume coupled through a high acoustic resistance to the atmosphere. The configuration of the infrasonic array along with the location of a vault containing a vertical long-period seismometer which is operated in conjunction with the infrasonic experiment are illustrated in Fig. 3. An array of Daniels noisereducing space filters arranged in the form of a cross with the microbarograph inlet port at the center has been installed at each site. These filters provide useful suppression of incoherent wind noise in the period range below about $20 \mathrm{~s}$. The measured amplitude response of the microbarometers is shown in Fig. 4.

The response of the differential pressure sensing array elements as a function of period is given by

$$
R(T)=\frac{A\left(T_{1}+T_{2}\right) i T}{T^{2}+\left(T_{1}+T_{2}\right) i T-T_{1} T_{2}},
$$

with $T_{1}=1.95 \mathrm{~s}, T_{2}=48.7 \mathrm{~s}$ and $A=77.99$ counts dyn $^{-1}$ $\mathrm{cm}^{-2}$. In this expression the coefficients $T_{1}, T_{2}$ and $A$ have been determined from a least-squares analysis of the measured instrumental response. An examination of this expression shows that waveforms with fundamental components near $T_{0}=\left(T_{1} T_{2}\right)^{\frac{1}{2}}=9.7 \mathrm{~s}$ pass through the infrasonic detection filters essentially undisturbed; in contrast, wave patterns with fundamental components of the order of $T_{2}$ undergo substantial differentiation in the detection process - this latter circumstance dominates the observation of the two

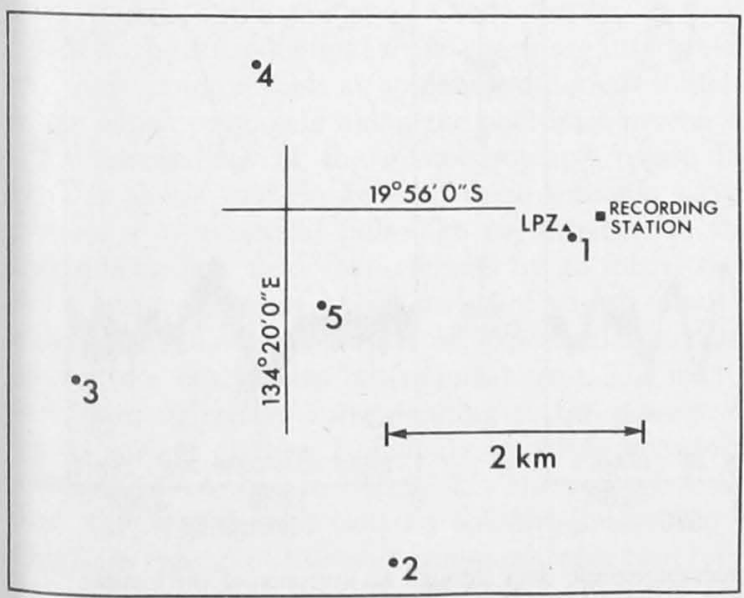

FIg. 3. Configuration of the five-component microbarograph array.

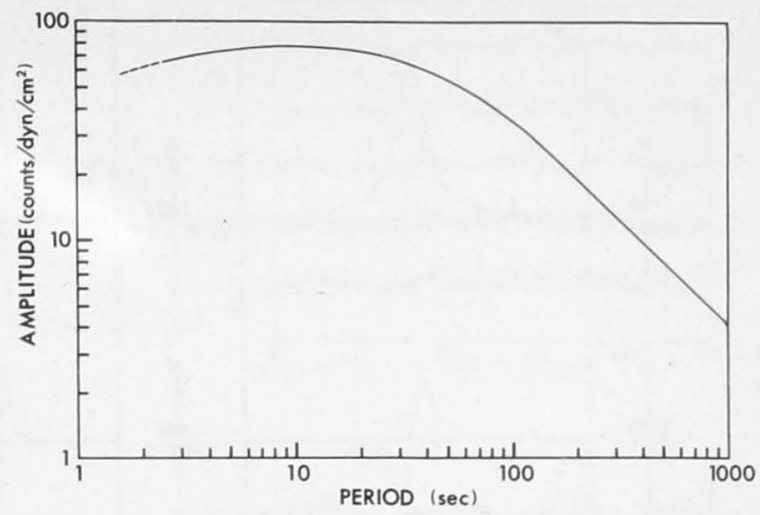

FIG. 4. Measured microbarograph amplitude response.

types of solitary-wave disturbance considered here. The influence of the instrumental response on the detection of solitary-wave surface pressure perturbations may be seen in the computed response to various forms of synthetic solitary wave data illustrated in Fig. 5. These patterns may be compared with the examples shown in Fig. 6 of the true surface pressure variation derived from recorded microbarograph data through an inversion of the instrumental response given by (3.1). As may be seen from these examples, solitary waves are easily identified in the output of the microbarograph array by their characteristic differential signature.

The five infrasonic channels and the vertical longperiod seismometer channel are sampled at a rate of two samples per second, digitized via a 14-bit ADC and recorded in IBM compatible format on 7-track, 556 bpi tape. At the Australian National University these tapes are converted to 9 -track, 800 bpi in a compact, two data word per 24-bit computer word format, which conserves magnetic tape and which is suitable for analysis on a $48 \mathrm{~K}$ Harris Datacraft $6024 / 4$ computer.

A SIAP S2000 meteorological station was installed at site 1 near the end of the recording period spanned by this work. This instrument provides a continuous record of surface temperature, wind speed, wind direction, rainfall and humidity. Accurate timing was achieved through the introduction of a chart marker activated by a pulse derived from the crystal-controlled digital time standard of the seismic station. The analysis of the meteorological data has not yet been completed. However, some of these data which are essential to the interpretation of the phenomena described here are included in this paper.

The main signal processing technique used on the array data consists of a beam-forming program which utilizes the nonlinear $N$-root method devised by Muirhead (1968). The output $e_{i}(t)$ of the $i$ th array element is digitally filtered in the bandpass region of interest, properly phased by time shifting to correspond to the passage of a plane wavefront across the array, and then reduced to the $N$ th root with sign preserved. 

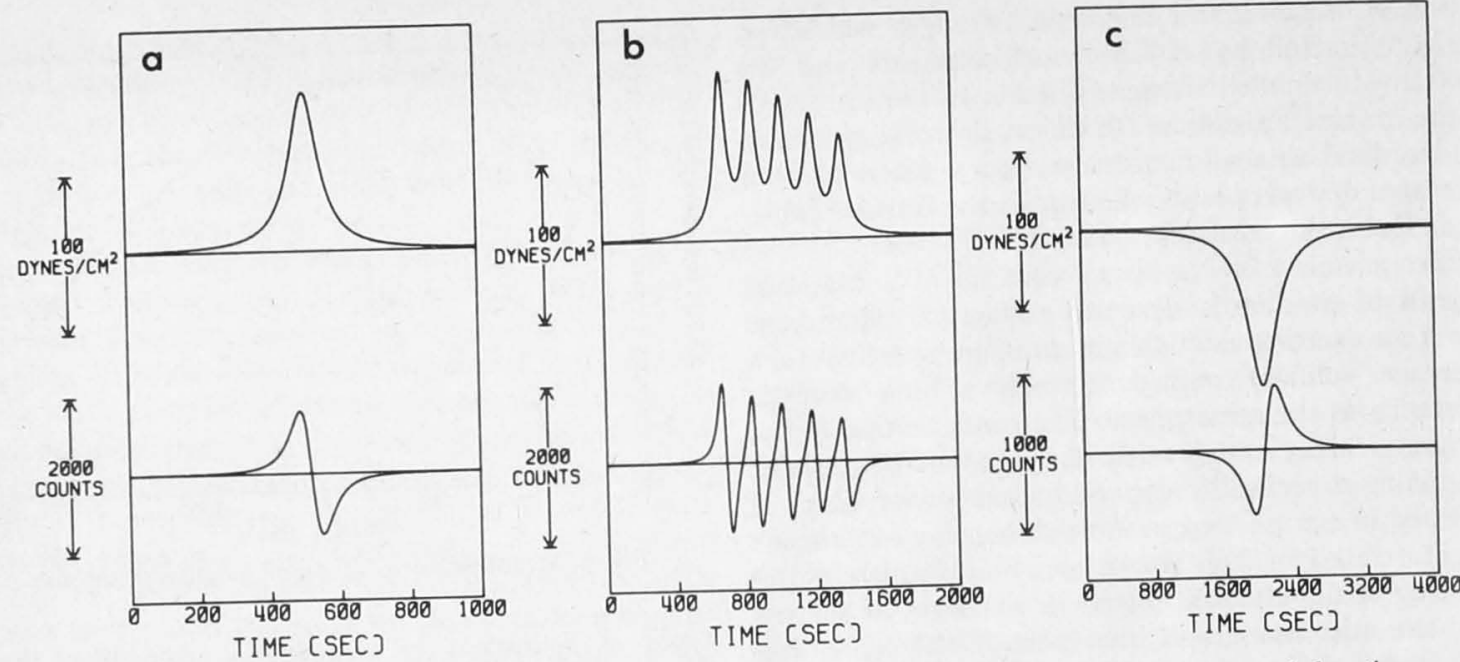

FIG. 5 . Computed microbarograph response to synthetic micropressure waveforms for (a) solitary wave of elevation, (b) solitary wave of depression.

The resulting product is summed over the $n$-element array to give

$$
R_{N}(t)=\frac{1}{n}-\sum_{i=1}^{n}\left|\bar{e}_{i}(t)\right|^{1 / N} \operatorname{sign}\left[\bar{e}_{i}(t)\right],
$$

where $\bar{e}_{i}(t)$ represents the filter output. This quantity is then raised to the $N$ th power with sign preserved to provide the signal statistic

$$
S_{N}(t)=\left|R_{N}(t)\right|^{N} \operatorname{sign}\left[R_{N}(t)\right] .
$$

The principal advantage of this type of automatic infrasonic array processing arises from the fact that this nonlinear beam-forming technique gives added weight to the presence of coherent energy in the spec- trum. This has proven to be of value in the treatment of data from Tennant Creek due to the prevalence in this semi-desert environment of incoherent, essentially nonGaussian noise such as that due to dust devils (small whirlwinds called willy-willies in Australia) which interact with only one sensor. It can be easily shown that large events of this type are suppressed in the output of the $N$ th root process by a factor of approximately $n^{-N}$.

All infrasonic data are processed for a value of $N=2$. This output is supplemented by a parallel determination of an integrated polarity stack over the phased array which corresponds to the $N$ th root process in the limit $N \rightarrow \infty$. Further details on the $N$ th root multichannel filter may be found in Kanasewich et al. (1973) and Muirhead and Ram Datt (1976).

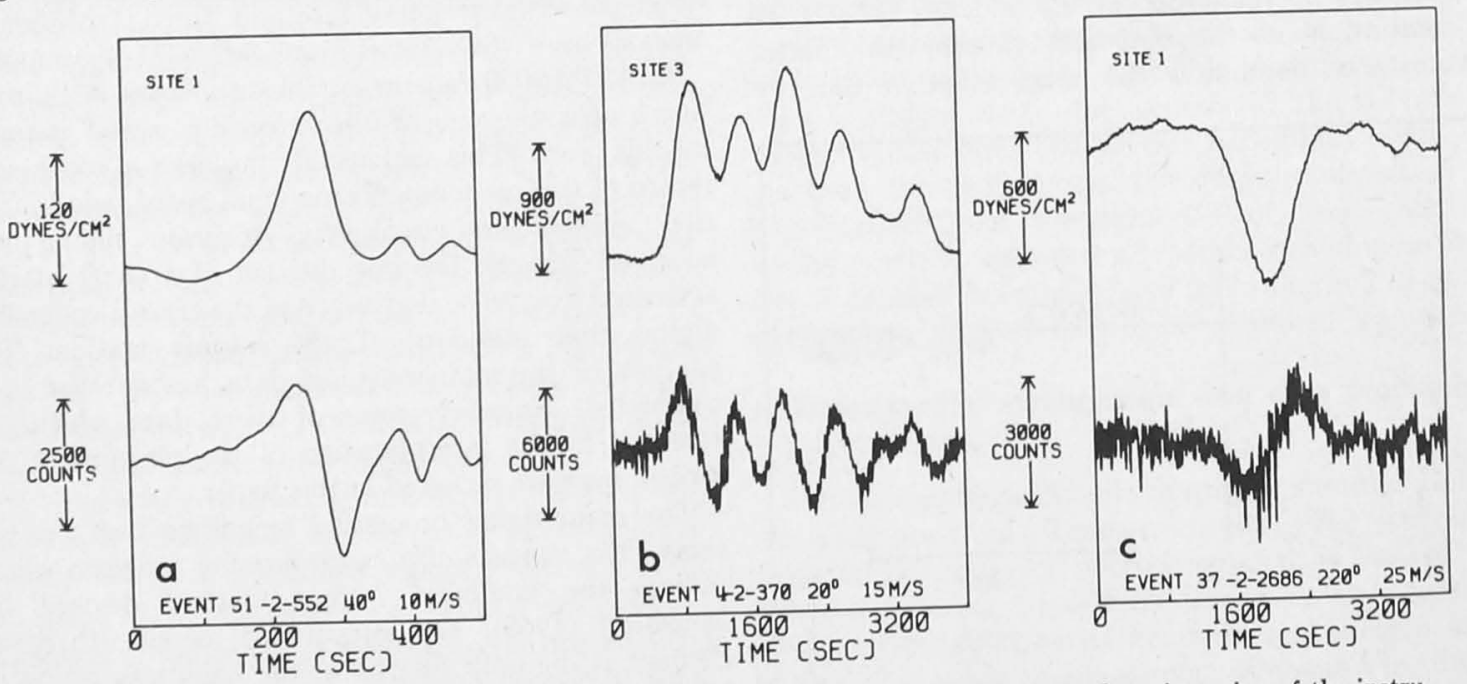

Fr. 6. Variation in surface pressure derived from recorded microbarograph data through an inversion of the instru(boliton wave packet and (c) solitary wave of depression. 
tment of ce in this ally nonils (small which inown that e output tely $n^{-1}$ of $N=2$. terminae phased rocess in ot multial. (1973
Power spectral estimates of the data are computed directly using the fast Fourier transform. In order to reduce the variance of the estimate the calculations are carried out using the method of time averaging over modified periodgrams described by Welch (1967). The digital time series $t_{r}$ is divided into $k$ portions $t_{r j}$ each of length $M=2^{10}$ points; the elements in each segment $j$ are then weighted according to a cosine taper data window $d_{r}$ effective over the $10 \%$ limits of the segments, as suggested by Bingham et al. (1967), and transformed to the frequency domain to give

$$
X_{j}(f)=\sum_{r=0}^{M-1} d_{r} t_{r j} \exp (-2 \pi i r f / M) .
$$

The power spectral density is then determined by averaging over the $k$ segments,

$$
\bar{P}(f)=(2 \Delta t / k M U) \sum_{j=1}^{k}\left|X_{j}(f)\right|^{2},
$$
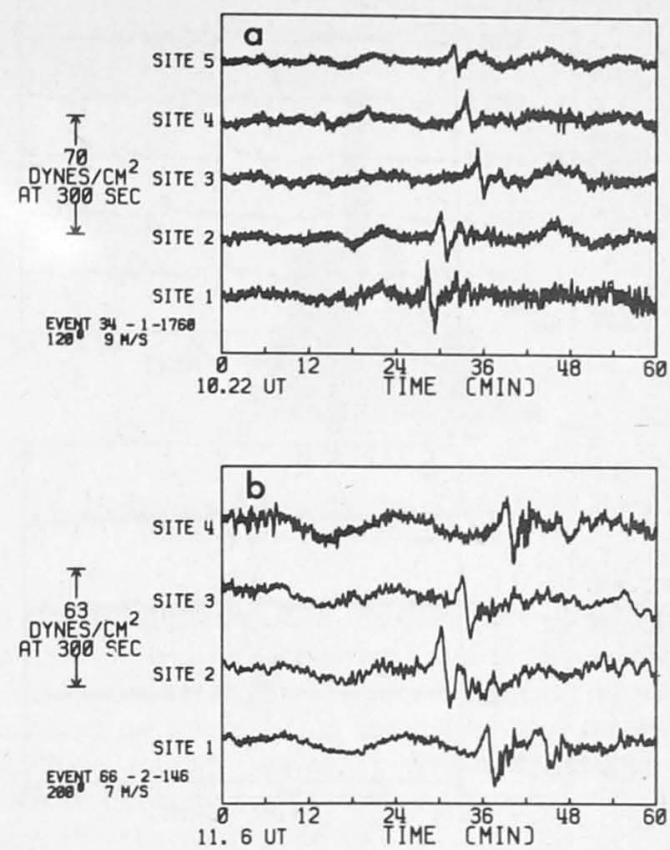

preserves the invariance of the area under the spectrum to the influence of the data taper window. The power spectral estimates obtained in this way are converted to true spectral estimates by dividing by the square of the microbarograph amplitude response shown in Fig. 4.

\section{Observations and interpretation}

We first consider the properties of the isolated waves of elevation. A large number of disturbances of this type have been observed in over two years of continuous recording at the infrasonic array near Tennant Creek. These unique waves, which only occur at night, are reasonably well described by the deep-fluid solitarywave theory outlined above. Consequently, as mentioned in the Introduction, these waves are interpreted as being examples of atmospheric internal solitary waves which propagate along the nocturnal inversion.

An examination of the microbarograph record inversions shows that, in general, these subsonic waves produce a symmetrical pulse-like perturbation in the atmospheric flow field characterized by an initial slow rise in surface pressure over a period of several minutes which gradually develops into an exponential increase leading to a fairly sharp but rounded crest. The microbarograph signatures corresponding to the passage of a wide variety of these commonly occurring buoyancy waves are illustrated in Figs. 7-12. The two relatively weak but well-defined isolated solitons illustrated in Fig. 7 are typical of the most commonly observed form waves. Occasionally (see Fig. 8b) large-amplitude isolated waves of elevation are observed to pass over the
FIG. 7. Examples of solitary waves of elevation observed at the Warramunga Seismic Station. Despite the relatively poor signalto-noise ratio these waves are clearly defined in the infrasonic records. The deep-fluid solidary-wave signals shown in this figure are typical of the large number of small-amplitude events of this type which have been observed.
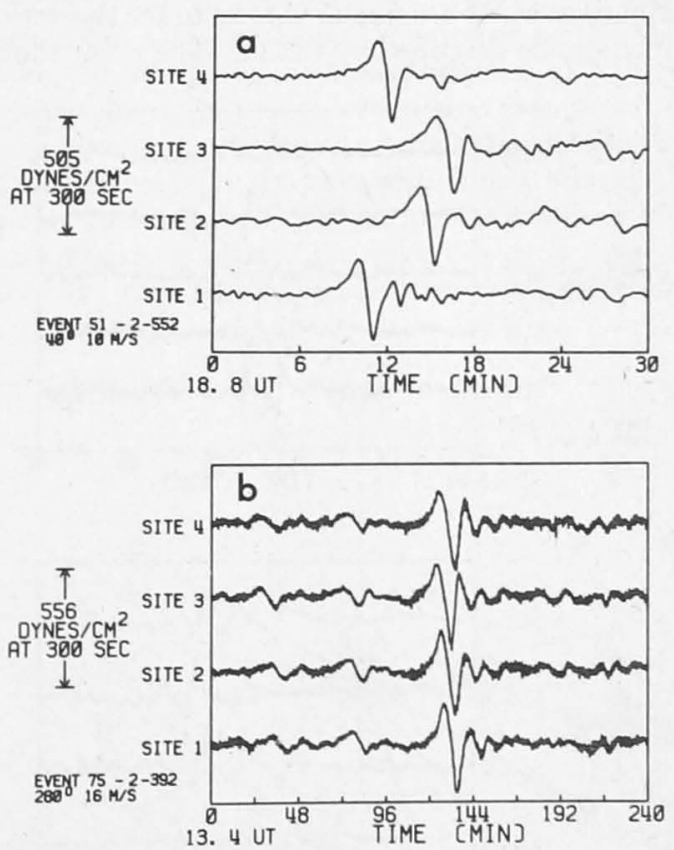

FIG, 8. Microbarometer array record sections which illustrate large-amplitude solitary waves of elevation. Note the almost complete absence of high-frequency components in the micropressure spectrum corresponding to the wake of the wave shown in (a). Two smaller solitary waves precede the unusually large-amplitude event shown in (b). 

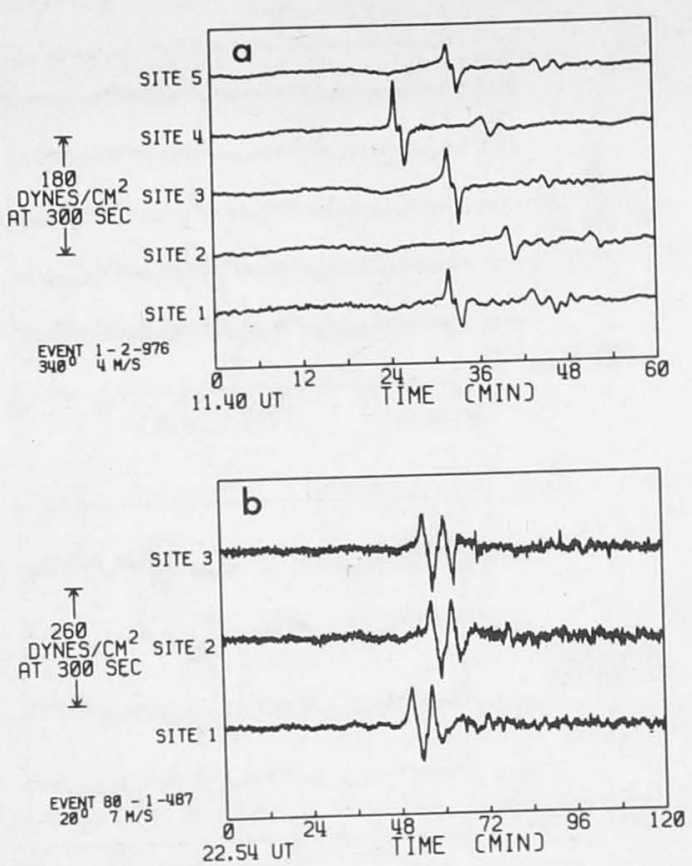

FIG. 9. Examples of unusual solitary wave characteristics for (a) an evolving solitary wave of elevation and (b), a solitary wave group consisting of two closely spaced large-amplitude solitons.

infrasonic array. Almost all of the observed solitarywave microbarometer signatures correspond to pressure distributions which are similar in form to the theoretical solution profile described by (2.11a). The event shown
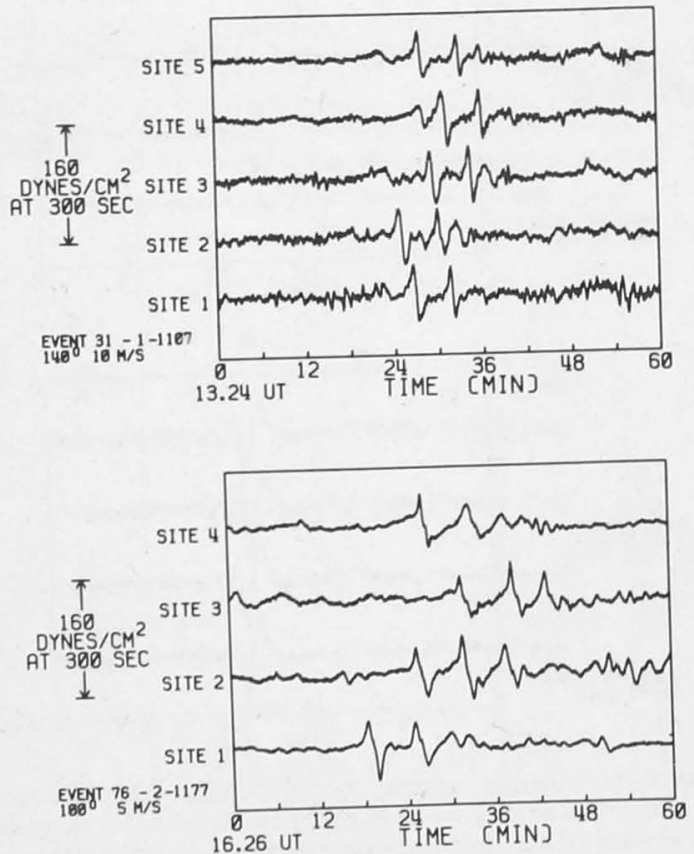

FIG. 10. Infrasonic array record sections containing isolated groups of well-separated solitary waves of elevation.
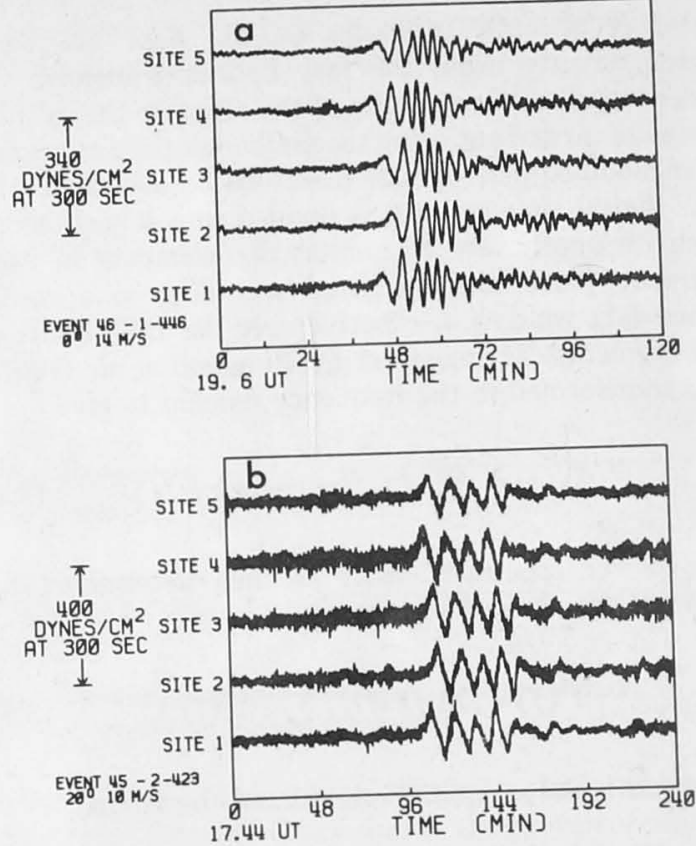

FIG. 11. Two examples of observations of largeamplitude solitary wave packets.

in Fig. 9a is unusual in that this wave of elevation produced, over part of the observational field, a surface produced, over pation in the form of a broad symmetrical pulse with a relatively flat crest. The significance of this peculiar wave pattern will be considered in more detail in the next section. Groups of waves consisting of well-separated individual solitons are sometimes observed as illustrated in the record sections shown in Fig. 10. In contrast, condensed "wave packets" consisting of two or more closely spaced solitons are frequently observed as shown in the examples in Figs. 9b, 11 and 12. It is worth noting that many of these soliton families are composed of waves of unusually large amplitude. These soliton wave packets may be very complex (see Fig. 12b) but they are usually ordered by amplitude with the higher velocity solitons appearing near the forward edge of the wave packet.

The salient features of these atmospheric solitary waves have been determined from an examination of the measured properties of 99 events recorded over a twoyear period. Waves of this type have been observed with amplitudes as high as $1100 \mathrm{dyn} \mathrm{cm}^{-2}$. They propagate at speeds between 4 and $18 \mathrm{~m} \mathrm{~s}^{-1}$, with an effective wavelength, as measured by the full width at half maximum, between 0.4 and $7.7 \mathrm{~km}$ and tend to occur sometimes for four nights in succession. Note that on a few occasions as many as three of these waves have been observed to pass over the experimental site from different directions within a period of $12 \mathrm{~h}$. Even though the available data span a period of only two years, it seems clear (see Fig. 13) that the frequency of 

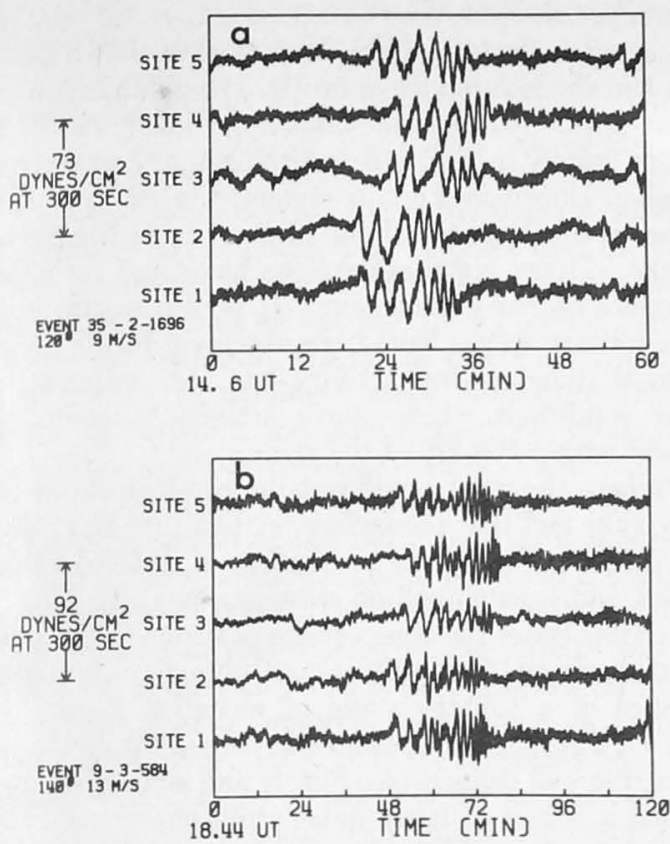

FIG. 12. Record sections which illustrate the signal characteristics of complex low-amplitude solitary-wave packets. In both of these examples the pronounced asymmetry of the differential signal indicates that the wave packet is formed from a family of well-separated solitons.

ation
urface trical ice of more isting es obwn in , cone fres. $9 \mathrm{~b}$ oliton large very dered earing

litary of the twoserved They ith an width end to Note waves al site Even y two ncy of

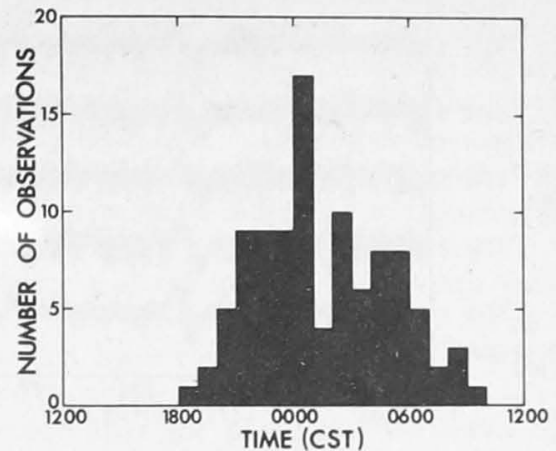

FIG. 14. Frequency of observation of deep fluid solitary waves as a function of time of day.

the north and northeast in the directions of the Timor Sea and the Gulf of Carpentaria and to the southeast in the direction of the Simpson Desert. It is worth remarking on the fact that the majority of the larger amplitude solitary waves of elevation detected at Tennant Creek arrive at the experimental site from azimuths between $20^{\circ}$ and $60^{\circ}$.

We now consider the infrasonic array observations of atmospheric solitary waves of depression. In contrast to the high frequency of occurrence of solitary waves of elevation only three waves of depression have been detected during the two-year observational period. An example of an isolated wave of depression may be seen in the microbarograph array record section shown in Fig. 16 for the early morning hours of 30 July 1976. Despite the scarcity of observations it seems clear that these phenomena represent a completely different form of atmospheric solitary-wave disturbance. An examination of the array records indicates that waves of this type propagate with phase velocities between 14 and $50 \mathrm{~m} \mathrm{~s}^{-1}$, that they have effective wavelengths in the

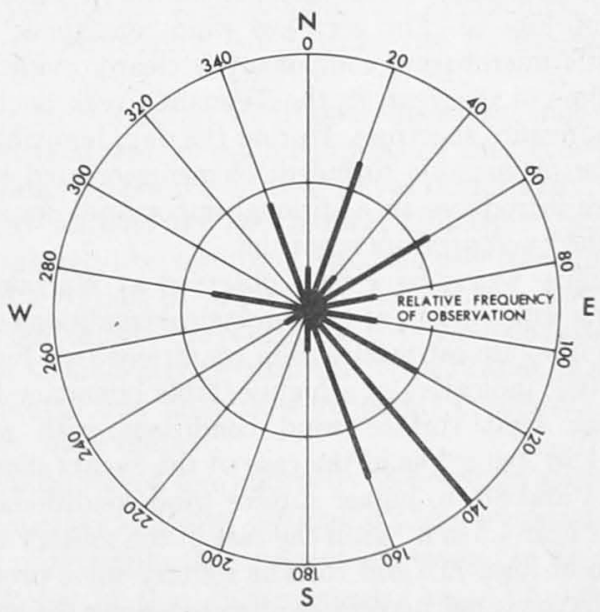

FIG. 15. Occurrence of solitary waves of elevation as a function of source azimuth. 


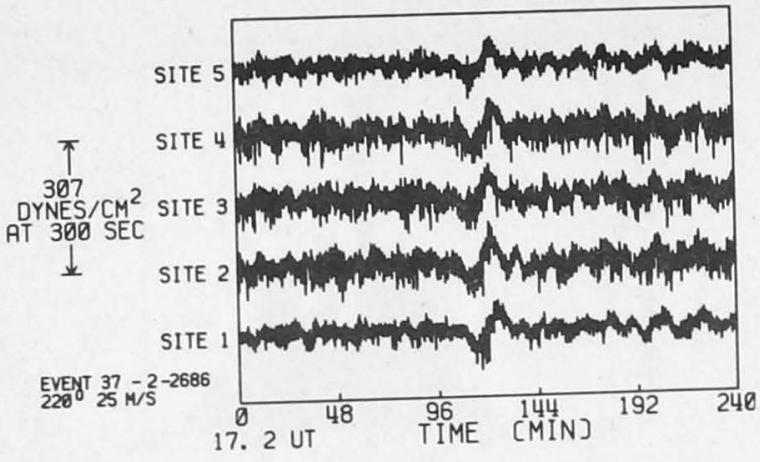

FIG. 16. Microbarograph array observation of a largeamplitude solitary wave of depression.

range from 8 to $30 \mathrm{~km}$, that they produce perturbations in the pressure field at the surface of up to $700 \mathrm{dyn} \mathrm{cm}^{-2}$, and that they originate at an azimuth of $220^{\circ}$ as measured from true north. Note that solitary waves of elevation have never been observed to originate in this direction. These properties suggest that the atmospheric scale of this type of isolated disturbance is substantially larger than the scale associated with the deep-fluid solitary waves of elevation. In particular, the unusually high phase velocities indicate that these disturbances are associated with higher altitude atmospheric structure. We therefore tentatively hypothesize that the observed isolated waves of depression properly belong to the classical solitary wave regime, and are in accord with the predictions of the theories of Peters and Stoker (1960), Long (1965) and Benjamin (1966) for classical solitary-wave propagation in a fluid whose density decreases exponentially with height.

In the absence of significant gravity wave activity the micropressure spectrum will be dominated by highfrequency pressure variations produced by turbulent eddies associated with winds near the surface. The influence of this component is evident in the microbarograph array record sections shown in Figs. 7-12 and in Fig. 16. For very low wind conditions, a $6 \mathrm{~s}$ acoustic microbarom component is clearly evident, at any time of the year, in the Tennant Creek nocturnal micropressure spectrum. During the day, buoyant convection of unstable turbulent elements created at the surface introduces an additional important component into the micropressure spectrum.

Solitary waves have been observed at Warramunga under a wide variety of meteorological conditions which range from almost totally calm conditions (see Figs. 8a and $10 \mathrm{~b}$ ), indicative of a highly stable boundary layer, through light surface wind conditions with speeds from 1 to $2 \mathrm{~m} \mathrm{~s}^{-1}$ as in the case of the events shown in Figs. 1 and $8 \mathrm{~b}$, to higher surface wind conditions with speeds near $4.5 \mathrm{~m} \mathrm{~s}^{-1}$ as in the case of the solitary waves shown in Figs. 11b and 16. The solitary wave propagation vector is not necessarily directed along the surface wind vector. For example, the meteorological measure- ments show that the surface wind vector and the propagation vectors of the wave packet shown in Fig. $12 \mathrm{a}$ and the isolated wave in Fig. 11b differ in azimuth by, about $15^{\circ}$, whereas the difference in azimuth for the soliton family in Fig. $11 \mathrm{~b}$ and the solitary wave of depression shown in Fig. 16 amounts to $90^{\circ}$ and $115^{\circ}$, respectively. Note that in all of these cases the passage of the solitary wave appears to have had no lasting influence on the surface wind. It is also worth noting that solitary waves have been observed during periods of local thunderstorm activity and also under totally clear conditions where storm activity is completely absent within $500 \mathrm{~km}$ of the array.

Perhaps the most significant meteorological observation is the fact that the surface temperature and humidity are not perturbed by the passage of isolated solitary waves and isolated soliton wave packets. An example which illustrates the observations of surface wind speed, wind direction, temperature and humidity during the passage of a solitary wave of elevation is given in Fig. 17. This particular event was chosen for illustration since it is well defined (see Fig. 1) and since it occurred during a period of fairly quiet atmospheric conditions near the surface. As can be seen from the diagram, the wind vector is virtually unchanged by the passage of the wave and no measurable perturbation in either the temperature or humidity can be associated with the main body of the wave or with the region in the wake of the wave. The dynamical properties of the atmosphere in the wake of the wave therefore appear to be almost

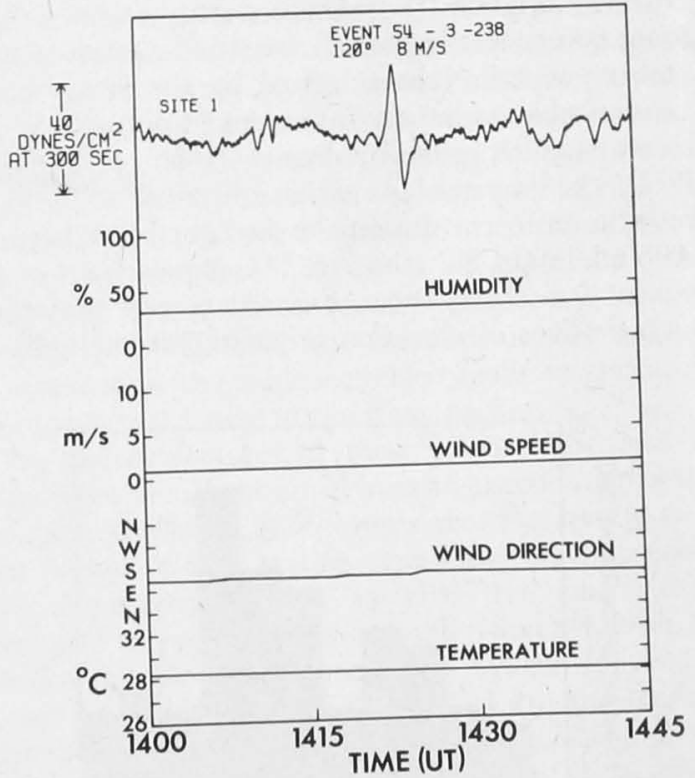

FIG. 17. Microbarograph record and corresponding measurements of surface temperature, humidity, wind speed and wind me solitary wave of elevation direction, recorded at site 1 , for the solitary 23.53 on the night of 2 December 1976 
the same as the conditions which prevailed prior to the arrival of the solitary wave.

\section{Discussion and conclusions}

A detailed comparison of the observed properties of atmospheric solitons with the predictions of solitarywave theory is complicated by the fact that the theoretical treatments have been restricted to static models with simple forms of the vertical density profile. Perhaps the most serious criticisms of the direct application of these models to nonlinear wave propagation in the earth's atmosphere are the neglect of wind shear (within the limits of dynamic stability) and the oversimplification of the fluid density structure.

In the discussion of the static theories it was noted that the classical solitary wave of elevation is stable to an amplitude of 0.827 . The stability limits for other types of classical waves, such as the solitary wave of depression, and for all forms of deep-fluid solitary wave are unknown; it is, however, clear from the work of Davis and Acrivos (1967) that the limits for deep-fluid solitary waves extend to much larger amplitudes. The fact that solitary waves can exist with very large amplitudes raises the possibility that waves of this type may significantly alter the dynamical properties of the atmosphere. This interaction could result from a tendency of very large-amplitude waves to break ${ }^{2}$ this complicated process can occur in either the forward or backward direction according to the specific form of the wind and density profiles (Long, 1956a, 1972)-or from a process of turbulent entrainment of fluid near the crest into the circulation pattern associated with the wave thus leading to turbulence in the wake and to further long-period oscillations corresponding to the motion of displaced buoyant fluid elements. A description of a rare observation of an evolving wave of elevation which may represent a breaking internal solitary wave is given below. If the atmospheric solitary waves described here possess an intrinsic turbulent wake then they are losing energy rapidly and it must be expected that they are of limited range.

Consider now the implications of the laboratory observations and numerical calculations of Davis and Acrivos (1967) on solitary waves propagating in a thin layer of fluid contained between two deep fluids comprising a stably stratified system. As has already been noted, these authors found that waves of large amplitude develop closed streamlines characteristic of the circulation in a vortex pair and, further, that these waves shed semi-periodic waves behind the main distur-

${ }^{2}$ The term "wave-breaking" is used here in the general sense of Long (1972) to denote any tendency for the isolated wave to steepen behind or ahead of the crest as it propagates. If this proves to be the case for large-amplitude atmospheric solitons then the dynamics of these waves may well be dominated by ampiitude dispersion and they must therefore be viewed as quasistationary phenomena. bance. It follows directly from an argument based on dynamic symmetry (Benjamin, 1967) that a single vortex occurs within large-amplitude solitary waves associated with an inhomogeneous shallow layer of fluid lying beneath a much deeper homogeneous fluid and that this internal circulation coupled with the entrainment hypothesis could lead to the development of a turbulent wake.

A measure of the influence of solitary wave propagation on the dynamical properties of the atmosphere is provided by a comparison (Fig. 18) of the power spectral densities of the surface pressure field evaluated before and after the passage of the wave. Since this technique includes contributions from fluid motions at high altitudes it should be particularly sensitive to changes in the atmosphere flow field induced by the main body of the wave - changes which may not be apparent in the observations of the flow field at the surface. An examination of all of the cases illustrated in Fig. 18 shows that the spectral character of the atmosphere is largely unaffected by the passage of the wave. In particular, the power spectra shown in Figs. 18a and $18 \mathrm{~b}$ for a solitary wave of elevation and in Fig. 18d for a solitary wave of depression indicate that the spectral characteristics both before and after the passage of the event are identical. In the case of the event shown in Fig. 18c, the wave of elevation appears to be associated with a slight increase in the amplitude of longer period spectral components. It is worth noting that in all of the three examples illustrated for solitary waves of elevation an identical 4-6 s peak due to microbarom activity appears prominently in both the spectrum evaluated ahead of the wave and the spectrum evaluated after the passage of the wave. The slight increase in the amplitude of the longer period components in the wake of the event in Fig. 18c-if at all significant-may be a measure of the irregular waves described by Davis and Acrivos (1967) which are shed by large-amplitude deep-fluid solitary waves or it may merely represent a small-amplitude component of the original disturbance which gave rise to the solitary wave.

The fact that an additional high-frequency component is absent in the micropressure spectrum corresponding to the wake of these waves suggests that these isolated solitons do not induce turbulence into the atmosphere. The results of this analysis coupled with the observation of an undisturbed surface flow field therefore leads to the conclusion that these disturbances do not significantly perturb the dynamical state of the atmosphere and that they represent essentially pure solitary-wave motion.

Solitary waves are normally observed to pass over the experimental site as highly coherent stationary linear wave fronts. The example illustrated in Fig. 9a is exceptional in that this particular wave of elevation was observed to evolve substantially over the $4 \mathrm{~km}$ 

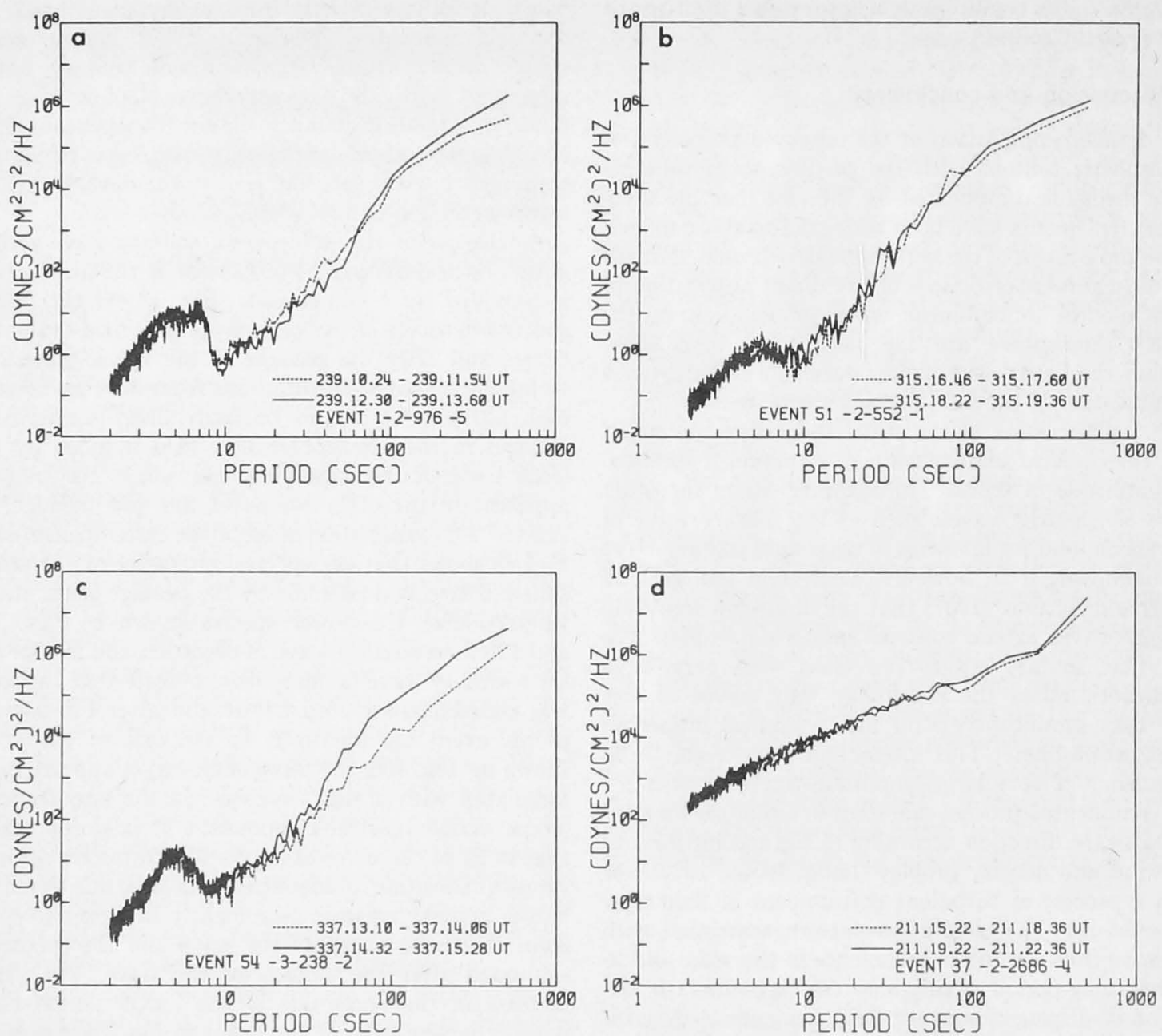

FIG. 18. Some comparisons of the micropressure power spectral density ahead of and in the wake of isolated waves which illustrate the influence of the passage of internal solitary waves on the atmospheric spectrum. High-frequency changes in the spectral estimates may be viewed as a measure of the degree of solitary wave-induced atmospheric turbulence. The analyses in (a), (b) and (c) correspond to solitary waves of elevation; the spectra shown in (d) correspond to a solitary wave of depression.

aperture of the infrasonic array. This is the only example of an evolving solution wave pattern to be observed during the two-year experimental period. The degree of evolution may be seen in the detailed diagrams presented in Fig. 19 which show both the microbarogram recordings at each array site and the corresponding true surface pressure variation as determined from the measured data through an inversion of the instrumental response specified by (3.1). This wave of elevation first appeared at site 4 and was then observed to propagate along the diagonal of the quadrilateral array to site 2 . The evolution of the solitary wave can therefore be followed by examining the sequence of wave patterns at sites 4,5 and 2 . As can be seen from the figure, the isolated wave steadily decreases in amplitude over the array and evolves from the unusual symmetric profile with a flattened crest observed at site 4 to the familiar deep-fluid Lorentz soliton profile observed at site 2. This pattern of evolution is further reflected in a steady decrease in the phase velocity from about $4.7 \mathrm{~m} \mathrm{~s}^{-1}$ to $4.2 \mathrm{~m} \mathrm{~s}^{-1}$.

There are a number of possible interpretations of this unusual evolving wave pattern. For example, as has already been noted, the form of the pressure distribution in the neighborhood of the crest of the initially observed wave could indicate an amplitude instability leading to a type of internal wave breaking which might be manifested in the production of turbulence at the crest through the Kelvin-Helmholtz mechanism. However, our current preferred explanation of this evolving wave pattern is that it represents the transition observed in the laboratory by Davis and Acrivos (1967) of $\mathrm{a}$ closed with a pure $\mathrm{x}$ standi 

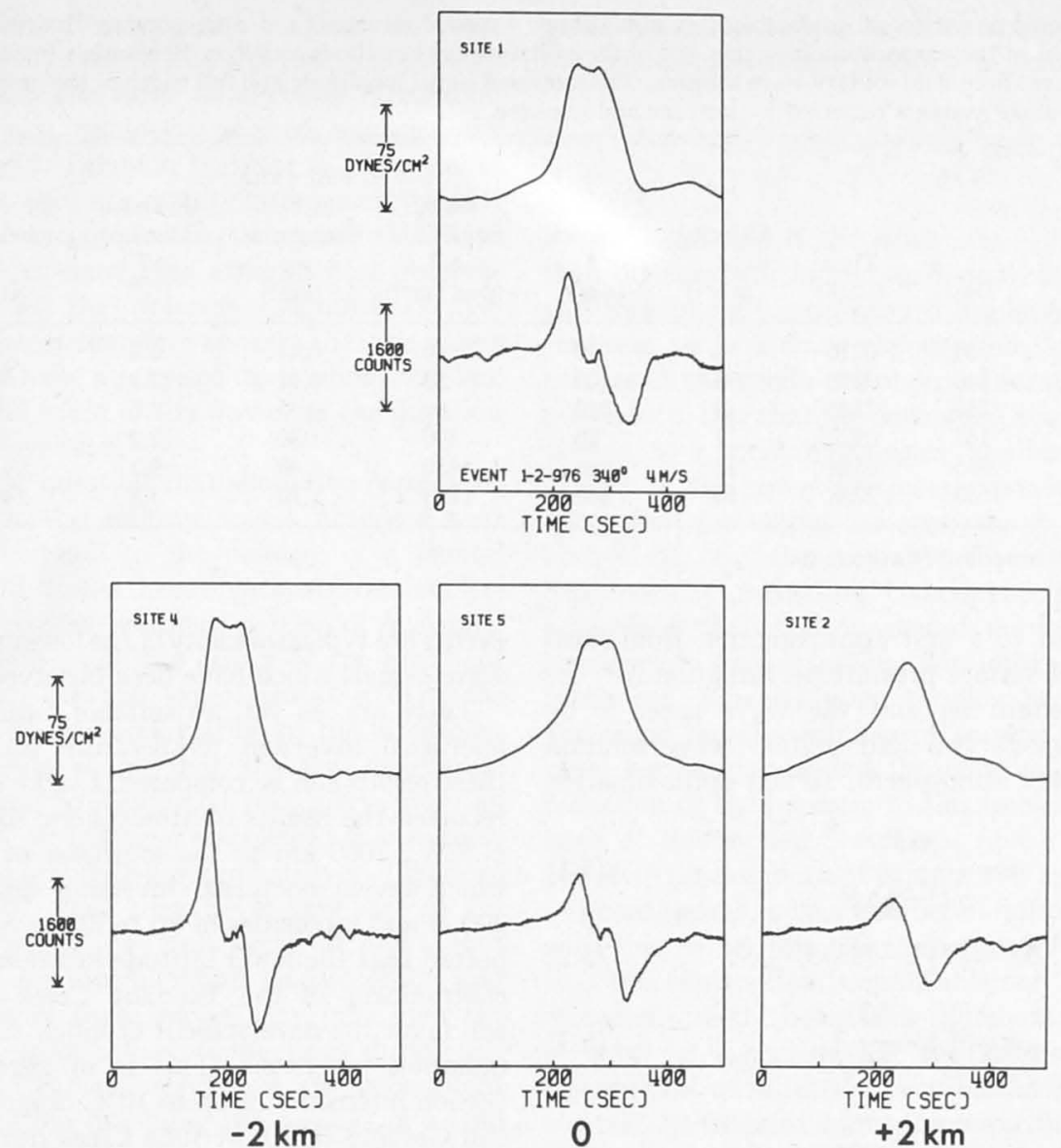

to the cved at ted in a about ions of iple, as ure disnitially tability $\mathrm{h}$ might at the 1. Howvolving ion ob(1967)

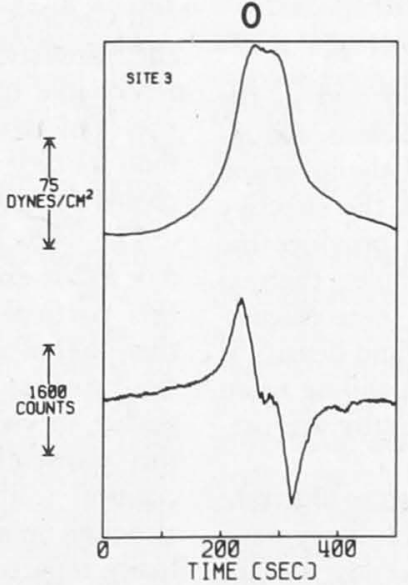

FIg. 19. Surface pressure characteristics produced by an unusual evolving solitary wave of elevation. Both the recorded microbarograph solitary wave signature (lower trace) and the derived absolute surface pressure perturbation (upper trace) are shown in detail for each site of the intrasonic array.

of a large-amplitude deep-fluid solitary wave with closed internal circulation to a smaller amplitude wave with an open streamline flow pattern characteristic of pure wave motion. It is evident that a complete understanding of the dynamical structure of these waves will require further extensive experimental observations of evolving soliton wave patterns.

We now consider some estimates of the intensity $\Delta T$ and height $h$ of the nocturnal inversion and a measure of the dimensionless solitary-wave amplitude $\alpha$ which 
TABLE 1. Measured properties of internal solitary atmospheric waves of elevation and corresponding theoretical estimates of the intensity and height of the temperature inversion and of the solitary-wave amplitude based on Benjamin's first-order approximation to the two-component deep-fluid solitary-wave solution. The measured signal amplitude and full width of the profile at half maximum are obtained from array averages corrected for instrumental response.

\begin{tabular}{|c|c|c|c|c|c|c|c|c|c|}
\hline Event & Day & $\begin{array}{c}\text { Time } \\
\text { (CST) }\end{array}$ & $\begin{array}{c}\text { Velocity } \\
c \\
\left(\mathrm{~m} \mathrm{~s}^{-1}\right)\end{array}$ & $\begin{array}{c}\text { Azimuth } \\
\theta \\
\text { (deg) }\end{array}$ & $\begin{array}{c}\text { Signal } \\
\text { amplitude } \\
\Delta P \\
\left(\text { dyn } \mathrm{cm}^{-2}\right)\end{array}$ & $\begin{array}{l}\text { Full width } \\
\text { at half } \\
\text { maximum } \\
w_{j} \\
(\mathrm{~km})\end{array}$ & $\begin{array}{c}\text { Inversion } \\
\text { intensity } \\
\Delta T \\
\left({ }^{\circ} \mathrm{C}\right)\end{array}$ & $\begin{array}{c}\text { Inversion } \\
\text { height } \\
h \\
(\mathrm{~m})\end{array}$ & $\begin{array}{c}\text { Solitary } \\
\text { wave } \\
\text { amplitude } \\
\alpha=a / h\end{array}$ \\
\hline $63-1-1233^{*}$ & 34 & 0216 & 4 & 20 & 50 & 0.47 & 6.8 & 55 & 0.32 \\
\hline $76-2-1177^{*}$ & 140 & 0228 & 5 & 100 & 50 & 0.51 & 12.2 & 42 & 0.23 \\
\hline $1-2-976$ & 238 & 2146 & 4 & 340 & 80 & 0.39 & 3.6 & 88 & 0.61 \\
\hline $80-1-487^{*}$ & 167 & 0920 & 7 & 20 & 300 & 1.30 & 2.2 & 390 & 0.83 \\
\hline $51-2-1318^{*}$ & 315 & 0524 & 13 & 280 & 450 & 4.40 & 9.2 & 430 & 0.27 \\
\hline $75-2-392$ & 133 & 2258 & 16 & 280 & 1100 & 7.70 & 3.8 & 1400 & 0.49 \\
\hline
\end{tabular}

* Leading soliton in solitary wave group.

may be obtained to a first approximation from measurements of the surface pressure perturbation $\Delta P$, the effective wavelength $w_{\frac{1}{2}}$ and the wave speed $c$ by applying Benjamin's two-fluid solitary wave solution (2.11) to the lower atmosphere. To this approximation

$$
\begin{gathered}
\alpha=\frac{\Delta P}{\rho_{1} c^{2}-\frac{3}{4} \Delta P}, \\
\Delta T=\frac{1}{2} T\left[1-(1-\delta)^{\frac{1}{2}}\right], \\
h=\beta\left(1-\frac{\Delta T}{T}\right),
\end{gathered}
$$

where

$$
\beta=\frac{3}{8} \alpha w_{\frac{1}{2}}, \quad \delta=4 \Delta P / g \beta \rho_{1} \alpha,
$$

$T$ is the temperature of the upper fluid and $\rho_{1}$ the density of the lower fluid. In these expressions, the intensity of the inversion and the height of the inversion above the surface are to be regarded as the effective parameters of a two-layer model which provides the simplest possible description of the complex thermal structure of the nocturnal inversion. In these calculations, $T$ is taken as $280 \mathrm{~K}$ and the lower fluid density is held constant at $1.2 \times 10^{-3} \mathrm{~g} \mathrm{~cm}^{-3}$ corresponding to an inversion near the $980 \mathrm{mb}$ level. The results are presented in Table 1.

The events listed in this table were chosen to illustrate the properties of a wide variety of isolated solitary wave forms. All of these events occurred under low surface wind conditions. It should be noted that the derived parameters corresponding to waves of large amplitude may be unreliable since the theory is valid only to first order in $\alpha$. As can be seen from the table, this model indicates that solitary-wave activity is usually associated with a temperature inversion with an effective depth between 40 to $400 \mathrm{~m}$ and with an effective intensity in the range from about 2 to $12^{\circ} \mathrm{C}$. The first entries in the table describe solitary-wave propagation along a relatively shallow inversion; these events are typical of many of the low-amplitude solitarywave signals which have been observed.

There are, as yet, no suitable measurements of the nocturnal inversion temperature profile with which these results can be compared. Clarke et al. (1971) have reported the results of atmospheric soundings at Hay, N.S.W., $2000 \mathrm{~km}$ to the southeast of Tennant Creek, which reveal nocturnal inversion depths from 80 to $200 \mathrm{~m}$ and intensities of up to about $5^{\circ} \mathrm{C}$. It can be expected that the lower latitude location and semi-desert environment of the Tennant Creek infrasonic array will favor the development of much stronger nocturnal radiation inversions. This is in agreement with inversion intensities of up to $10^{\circ} \mathrm{C}$ observed by Reynolds and Gething (1970) at Julia Creek during the course of their acoustic sounding experiments. It therefore seems reasonable to conclude that almost all of the isolated waves of elevation detected at Warramunga are deepfluid solitary waves which propagate along a nocturnally cooled surface layer.

The very large-amplitude event which occurred on day 392 is exceptional in that the scale associated with this particular solitary wave is significantly greater than that of any other wave of elevation detected during the two-year observational period. It is therefore suggested, in view of the atmospheric scale involved, that this particular wave may be better described as a classical solitary wave of elevation which is similar, although on a smaller scale, to the solitary-wave disturbance reported by Abdullah (1955).

It must be emphasized at this point that any discussion of the source mechanisms which excite these solitary waves is largely conjectural since the range and lateral extent of these waves are almost totally unknown. Existing evidence, such as the fact that these waves traverse the $4 \mathrm{~km}$ infrasonic array as highly coherent planar wave-fronts and the observation, noted above, by Reynolds and Gething (1970) that solitary wave associated disturbances appear to propagate essentially unattenuated over distances of about $40 \mathrm{~km}$, 
suggests that these waves originate at large distances from the array. However, if the internal morphology of the waves takes the form of a closed circulation leads to an intrinsic tendency to shed semi-periodic disturbances behind the main body or if these waves are breaking, then they are rapidly losing energy and it must therefore be anticipated that they are of limited range. On the other hand, the experimental observations do not indicate the presence of significant waveinduced turbulence; it therefore appears that the waves of elevation could have a range of up to about $600 \mathrm{~km}$, the limit set by the speed of the wave and the duration of the nocturnal inversion.

Perhaps the first question that should be considered is whether or not the solitary waves observed near Tennant Creek are due to the passage of a frontal system. Dry, cold fronts occur with reasonable frequency over the extreme southern portion of Australia. These fronts seldom reach the center of the continent and are very rare at the experimental site in the Northern Territory. An examination of the isochrones of these cold fronts shows that the few degenerate fronts which extend to Tennant Creek arrive from an azimuth near $220^{\circ}$. It has been established that solitary waves of elevation originate predominantly in directions near $20^{\circ}$ and $140^{\circ}$, that they never originate in the direction of $220^{\circ}$, that they only occur at night, that they often occur on several nights in succession and that two or more of these waves may pass over the experimental site from different directions during the same night. All of these observations are inconsistent with an interpretation involving the passage of a cold front. In addition, the microbarograph records of these well-defined isolated waves of elevation bear little resemblance to the complex micropressure pattern recorded by McDonald (1974) during the passage of a frontal system over an array located in northern Texas. It therefore seems very unlikely that cold fronts play an important role in the production of deep-fluid atmospheric solitons.

In contrast, the three classical solitary waves of depression detected at Tennant Creek do appear to originate in the same direction as atmospheric fronts. However, an examination of the synoptic patterns fails to confirm that these events coincided with the passage of a cold frontal system; nevertheless, the possibility that these large-amplitude waves occur in conjunction with weak frontal activity should not be ruled out at this point.

Smart (1966) and Jordan (1972) associate their observed exponential pressure pulses with thunderstorms. The organized, precipitation-initiated and precipitationmaintained downdraft of cold air in severe mature storms is well documented (Wallington, 1961; Browning and Ludlam, 1962; Spillane and McCarthy, 1969). Smart developed a model to describe these pressure pulses by considering the unstable solution of Brunt's well-known basic equation of motion (Brunt, 1927) for the vertical displacement $(d h)$ of a parcel of $\mathrm{air}^{3}$

$$
\frac{d^{2}(d h)}{d t^{2}}+\frac{g}{T}\left(\beta+\frac{d T}{d h}\right) d h=0 .
$$

Brunt noted that if the lapse rate $-d T / d h$ is greater than the adiabatic lapse rate $\beta$, no restoring force exists and consequently a downward displaced air parcel will continue to accelerate exponentially. Smart adapted this result to explain the observed form of the pressure pulses by noting that the downward descending parcel is subject to a constant pressure gradient and thus the density of the parcel increases exponentially with the result that (assuming the approximate validity of the hydrostatic law) the ground-level pressure contains an exponentially increasing component. Smart also hypothesized that the downward motion continues to accelerate in smooth laminar flow until a critical velocity is reached, at which point the flow becomes turbulent and mixes rapidly with nearby warmer air thus causing rapid oscillations behind the pulse and, eventually, a reduction of the pressure to the ambient level. On the basis of this model, Smart was able to account for 4 out of 10 observations of exponential pressure pulses.

There appear to be a number of difficulties associated with this model, the most serious of which is the fact that this explanation is applicable only to observations recorded directly beneath a thunderstorm downdraft; it must be expected that the ground-level pressure distribution outside this very localized region is of a completely different form. Consequently, the observations reported by Jordan (1972) of pressure pulses coming from thunderstorms at distances of the order of $100 \mathrm{~km}$ are not accounted for by this model as it stands.

It is an obvious extension of this model to include the influence of the gravity current of undercutting cold air that advances away from the thunderstorm as the downdraft spreads out at the surface. Wallington (1961) noted an example in which this density current extended over $50 \mathrm{~km}$ and the subject has recently been treated in detail by Charba (1974) and by Goff (1976). An interesting description of a Sudanese $h a b o o b$, a downdraft gravity current made visible by its high dust content, has been given by Lawson (1971). A reasonable explanation of long-range thunderstorm-generated pressure pulses is that they represent solitary waves produced by either the direct impulsive interaction of the

\footnotetext{
${ }^{3}$ Priestley (1953) has derived simultaneous equations which describe the vertical motion and temperature of buoyant fluid elements subject to turbulent mixing with the surroundings. A more general theoretical treatment which includes the effect of turbulent fluid entrainment has been given by Turner (1963). In the case of unstable environments solutions exist for both of these models in which the velocity of the fluid element ultimately increases exponentially with time. These models provide a firmer basis for a description of downdraft flow in thunderstorms.
} 
downdraft on an inversion or the concommitant interaction of the gravity current with an existing inversion. The latter mechanism is closely related to the phenomenon of soliton production in the developing frontal zone of an advancing bore as shown in the numerical studies of Peregrine (1966) and Vleigenthart (1971) on the evolution of nonstationary solutions of the $\mathrm{KdV}$ equation.

According to the comprehensive study of Goff (1976), the abrupt transition in surface atmospheric conditions which accompanies the passage of the frontal zone of a well-developed quasi-steady downdraft-produced density current is characterized by a sharp decrease in temperature in the range from 2 to $10^{\circ} \mathrm{C}$, a sudden onset of persistent winds and a rapid increase of about $3.4 \mathrm{mb}$ on the average in the surface pressure level. This transition is usually followed by the onset of precipitation. It is clear that a downdraft gravity current interpretation fails to account for the isolated nocturnal disturbances described in this paper since none of these perturbations of the atmospheric flow field are observed (see Fig. 17) to accompany these events.

It is possible that some of the solitary waves observed at Tennant Creek during the monsoon season from December to February are due to thunderstorm activity. Note, however, that only one solitary wave has been observed during the month of January. As can be seen from the monthly frequency analysis shown in Fig. 13, solitary waves are most commonly observed during the period from April to September, a period of very low storm activity. Furthermore, solitary waves have been observed to occur under completely clear conditions when storm activity is absent within several hundred kilometers of the array. We therefore conclude that the thunderstorm source mechanism is of minor importance.

The fact that solitary waves of elevation come from preferred directions suggest that they may be orographic in origin. First we note the large number of observations of isolated waves which arrive from an azimuth of $140^{\circ}$. These waves are usually detected near midnight but they also occur with reasonable frequency at other times during the night. As one proceeds in the direction of $140^{\circ}$ from the infrasonic array the terrain can be described, initially, as a more or less flat featureless plain. At about $10 \mathrm{~km}$ from the array a complicated pattern of hills comprising the Murchison Range begins to appear on the right at a distance of about $5 \mathrm{~km}$. This chain of hills, which rises to a maximum elevation of about $200 \mathrm{~m}$ over the surrounding plain, runs parallel to the $140^{\circ}$ direction for a distance of about $100 \mathrm{~km}$. Beyond this point the level plain slowly gives way to the Davenport Range which intersects the Murchison Range at the same altitude at about $140 \mathrm{~km}$ from the array. Further afield in this direction, the terrain descends to an arid plain which continues uninterrupted and finally merges with the Simpson Desert at a distance of about $460 \mathrm{~km}$.

The presence of these nearby ranges suggests that the origin of solitary waves coming from this direction is to be found in the interaction with an existing inversion of katabatic (downslope) nocturnal flow of surfacecooled air from these hills onto the surrounding plain. The hypothesis of supercritical katabatic drainage leading to an internal atmospheric hydraulic jump has been employed with considerable success by Ball (1965; see also Ball, 1957; Lied, 1964) to explain the sudden stationary discontinuity found in surface winds in the Antarctic. Similarly, Clarke (1972) has explained a frequently occurring, sudden, near-dawn squall, accompanied by a spectacular low horizontal roll cloud known as the "morning glory", on the south coast of the Gulf of Carpentaria by evoking the concept of a propagating undular gravity current created in the process of nocturnal surface flow down a 1:1000 slope from the $500 \mathrm{~m}$ highlands to the east. Clarke carried out a number of numerical experiments and concluded that internal atmospheric bores and propagating hydraulic jumps should commonly occur at low latitudes when katabatic flow discharges onto a plain under conditions of a stable radiation inversion.

This conclusion appears to be reinforced by the acoustic soundings at Julia Creek reported by Reynolds and Gething (1970) which revealed, as has already been noted, the occurrence of a jump in the height of the nocturnal inversions. These authors also hypothesized the existence of katabatic flow aided in part by the geostrophic wind and orographic convergence and concluded that the observed jumps in the inversion level could be attributed to either the head of a gravity current propagating as an internal bore or a traveling internal hydraulic jump.

Since the rise in topography in this direction appears to be sufficient to produce significant nocturnal boundary layer currents, it is proposed that the observed solitary waves coming from an azimuth of $140^{\circ}$ are generated in the interaction of katabatic flow with the nocturnal inversion and that they propagate along the inversion to the array. It is premature, without further experimental work, to examine this mechanism in detail but it probably takes one of the following forms:

1) The direct impulsive interaction of an advancing gravity current impinging on an established inversion.

2) The disturbance of the atmospheric flow field during the creation (or dissipation) of a hydraulic jump; this may occur at the point where supercritical downslope flow encounters the horizontal plane-this hydraulic jump may subsequently propagate upstream or downstream as flow conditions vary-or in conjunction with a supercritical-subcritical flow transition in the neighborhood of a topographic barrier on the horizontal plane. 
3) The momentary transition of a steady advancing bore to a nonstationary flow state upon encountering a topographic obstacle such as a ridge or valley.

4) The direct soliton production in the evolving frontal zone of a gravity current as described above for downdraft-associated density currents, probably the most important mechanism.

The interpretation in terms of katabatic flow at an orographic feature accounts for both the occurrence of waves coming from the direction of $140^{\circ}$ and for the scarcity of events originating in the southwest quadrant where orographic features suitable for the production of katabatic flow are absent. However, an attempt to apply an interactive katabatic wind interpretation to account for the source of the large number of solitary waves which originate in the northeast and northwest quadrants is less successful. Thus for waves which arrive from the direction of the Gulf of Carpentaria, the elevation decreases steadily from about $410 \mathrm{~m}$ MSL to $200 \mathrm{~m}$ at a distance of about $150 \mathrm{~km}$ from the array. From this point, the land rises slowly to form the Barkly Tablelands at an altitude of about $260 \mathrm{~m}$. At the edge of the Tablelands, about $340 \mathrm{~km}$ from the array, the land again rises to about $310 \mathrm{~m}$ and then falls continuously to the hot tropical coast of the Gulf $500 \mathrm{~km}$ from the array.

It is possible that solitary waves originating in this direction are produced in the dissipation of gravity currents flowing toward the array into the broad shallow valley which separates Warramunga and the Barkly Tablelands. However, in this case the topography would not appear to support significant katabatic flow. A katabatic density current interpretation for the source of events originating to the east and northeast can be ruled out for the same reason. A different type of source mechanism is therefore required to explain the solitary waves which originate in these directions.

An indication of the range of isolated waves which originate to the north of the array is given by the observation that the majority of these events are observed to occur between 0200 and 0700 CST. This suggests that the source of these waves is to be found at a considerable distance from the array. If it is assumed that these waves are created during or shortly after the formation of a stable inversion then the average measured speed of $10 \mathrm{~m} \mathrm{~s}^{-1}$ is consistent with a source in the neighborhood of the coastal edge of the Barkly Tablelands. This suggests that the solitary waves observed at Warramunga which originate in the directions of the Gulf of Carpentaria and the Timor Sea may represent the final form of deeply penetrating sea-breeze fronts. Solitary-wave production might proceed, as described above, through the formation of a solution wave packet along the leading edge of a shallow seabreeze current or simply through the evolution of the direct density current produced disturbance of the inversion layer. Insight into the nature of a new and probably more important mechanism is provided by the recent observations described by Simpson et al. (1977) of the formation of a horizontal vortex at the head of a sea-breeze front which separates from the sea breeze shortly before sunset and continues to propagate as an independent phenomenon. The unusual stability of a propagating semi-elliptical horizontal atmospheric vortex has been considered by Clarke (1965). Since closed circulation in the form of a propagating vortex is also a characteristic of large-amplitude deep-fluid solitary waves, it is reasonable to assume that a seabreeze generated vortex which interacts with the nocturnal inversion will eventually evolve into an internal atmospheric solitary wave.

This concludes the description and interpretation of atmospheric internal solitary waves which are observed in the arid interior of Australia. It is evident that further measurements of the properties of these waves are required if a full understanding of phenomena of this type is to be achieved. In particular, the evolution of the frontal zone of a shallow internal density current advancing into a deep stratified fluid and the role played by disturbances of this type in the production of solitary waves deserve further study. The experimental program at Warramunga is therefore being expanded to include further meteorological instrumentation. In addition, the infrasonic array is being enlarged, through the addition of portable array elements, to an aperture of about $100 \mathrm{~km}$. With the completion of this program it should be possible to carry out a detailed investigation of the degree of wave coherence over large distances and to establish with certainty the source mechanisms responsible for the creation of waves of this type.

Acknowledgments. The authors wish to express their gratitude to Professor J. S. Turner for helpful comments on a first draft of this paper and to Dr. J. F. Gettrust for several stimulating discussions during the course of this research. We thank also an unknown reviewer for comments on an earlier draft of this paper. These comments have led to the inclusion of some data from more recent observations at Tennant Creek. In addition, we wish to thank Mr. David Daffen and Mr. Blair Lade for their efforts in operating and maintaining the Warramunga Seismic Station. This research was supported by the Air Force Office of Scientific Research under Contract AFOSR 75.2759A.

\section{REFERENCES}

Abdullah, A. J., 1949: Cyclogenesis by a purely mechanical process. $J$. Meteor., 6, 86-97.

, 1955: The atmospheric solitary wave. Bull. Amer. Meteor. Soc., 36, 515-518.

1956: A note on the atmospheric solitary wave. $J$. Meteor., 13, 381-387.

Ball, F. K., 1956: The theory of strong katabatic winds. Aust. J. Phys., 9, 373-386. 
_ 1957: The katabatic winds of Adélie Land and King George V Land. Tellus, 9, 201-208.

Benjamin, T. B., 1966: Internal waves of finite amplitude and permanent form. J. Fluid Mech., 25, 241-270.

perm. depth. J. Fluid Mech., 29, 559-592.

Bingham, C., M. D. Godfrey and J. W. Tukey, 1967: Modern techniques of power spectrum estimation. Trans. IEEE, AU-15, No. 2, 56-66.

Boussinesq, J., 1871: Théorie de l'intumescence liquide appelée onde solitaire ou de translation se propageant dans un canal rectangulaire. Inst. France Acad. Sci. C. R., June 19, p. 755.

Browning, K. A., and F. H. Ludlam, 1962: Airflow in convective storms. Quart. J. Roy. Meteor. Soc., 88, 117-135.

Brunt, D., 1927: The period of simple vertical oscillations in the atmosphere. Quart. J. Roy. Meteor. Soc., 54, 30-32.

Byatt-Smith, J. G. B., 1970: An exact integral equation for . Roc. Roydon, A315, 405-418. steady surface waves. of steep solitary waves. Proc. Roy. Soc. London, A350, 175-189.

Chan, R. K.-C., 1974: A discretized solution for the solitary wave. J. Comput. Phys., 16, 32-48.

Charba, J. 1974: Application of gravity current model to analysis of squall-line gust front. Mon. Wea. Rev., 102, 140-155.

Clarke, R. H. 1965: Horizontal mesoscale vortices in the atmosphere. Aust. Meteor. Mag., 50, 1-25.

1972: The morning glory: an atmospheric hydraulic jump. J. Appl. Meteor., 11, 304-311.

A. J. Dyer, R. R. Brook, D. G. Reid, and A. J. Troup, 1971 The Wangara experiment: Boundary layer data. Tech. Pap. No. 19, Division of Meteor. Physics, CSIRO.

Davis, R. E., and A. Acrivos, 1967: Solitary internal waves in deep water. J. Fluid Mech., 29, 593-607

Fenton, J., 1972: A ninth-order solution for the solitary wave. J. Fliid Mech., 53, 257-271.

Friedrich K. O, 1948: On the derivation of the shallow water theory. Appendix to J. J. Stoker, The formation of breaker and bores. Comm. Pure A ppl. Math., 1, 81-87.

, and D. H. Hyers, 1954: The existence of solitary waves. Comm. Pure Appl. Math., 7, 517-550.

Goff R C 1976. Vertical structure of thunderstorm outflows. Mon. Wea. Rev., 104, 1429-1440.

Gossard, E. E., and W. H. Hooke, 1975: Waves in the Atmosphere. Elsevier, $456 \mathrm{pp}$.

Grimshaw, R., 1971: The solitary wave in water of variable depth. Part 2. J. Fluid Mech., 46, 611-622.

Hurdis, D. A., and H. Pao, 1975: Experimental observation of internal solitary waves in a stratified fluid. Phys. Fluids, 18, 385-386.

Jordan, A. R., 1972: Atmospheric gravity waves from winds and storms. $J$. Atmos. Sci., 29, 445-456.

Kanasewich, C. D., C. D. Hemmings and T. Alpaslan, 1973 $N$-th root stack nonlinear multichannel filter. Geophysics, 38 , $327-338$

Karpman, V. I., 1975: Non-linear Wind Dispersive Media. Pergamon Press, [Trans. from Russian]. hallow water Comm. Pure A ppl. Math., 1, 323-339.

Keulegan, G. H 1953. Characteristics of internal solitary waves. J. Res. Nat. Bur. Stnd., 51, 133-140.

Korteweg, D. J., and G. de Vries, 1895: On the change of form f long waves advancing in a rectantular canal and on a new type of long stationary waves. Phil. Mag., 39, 422-443.

Laitone, E. V., 1960: The second approximation to cnoidal and solitary waves. J. Fluid Mech., 9, 430-444.

Lamb, H., 1932: Hydrodynamics, 6th ed. Cambridge University Press, 423-426.
Lavrent'ev, M. A., 1947: On the theory of long waves. Akad. Nauk. Ukrain. RSR, Zbornik Prac. Inst. Mat., 1946, No. 8 13-69. [Trans. in Amer. Math. Soc. Transl., Ser. 1, 11, 273 $321,1962]$.

Lawson, T. J., 1971: Haboob structure at Khartoum. Weather, 26, $105-112$.

Lenau, C. W., 1966: The solitary wave of maximum amplitude. J. Fluid Mech., 26, 309-320.

Lied, N. T., 1964: Stationary hydraulic jumps in a katabatic flow near Davis, Antarctica, 1961. Aust. Meteor. Mag., 47, 40-51.

Long, R. L., 1956a: Long waves in a two-fluid system. J. Meteor. 13, 70-74

1956b: Solitary waves in one- and two-fluid systems. Tellus, $8,460-471$.

1965: On the Boussinesq approximation and its role in the theory of internal waves. Tellus, 17, 46-52.

1972: The steepening of long, internal waves. Tellus, 24, $88-99$.

Longuet-Higgins, M. S., and J. D. Fenton, 1974: On the mass, momentume. II momentum energy and circulation of a

Trallister, L. G., J. R. Pollard, A. R. Mahoney and P. J. R. Shaw 1969: A new approach to the Shaw, 1969: Acoustic sounding a

(1974: Naturally occurring atmospheric acoustical signals. J. Acoust. Soc. Amer., 56, 338-351.

Muirhead, K. J., 1968: Eliminating false alarms when detecting seismic events automatically. Nature, 186, 704.

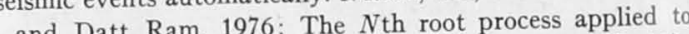
, seismic array data. Geophys. J. Roy. Astron. Soc., 47, 197210 .

Peregrine, D. H., 1966: Calculations of the development of an undular bore. J. Fhid Mech., 25, 321-330.

A Solitary waves in liquid having non-constant density. Comm. Pure Appl. Math., 13 115-164.

Philips, D. H, 1976: A pressure jump and an associated seich at Tobruk. Meteor. Mag., 105, 110-122.

Priestley, C. H. B., 1953: Buoyant motion in a turbulent environment. Aust. J. Phys., 6, 279-290.

Rayleigh, Lord, 1876: On waves. Phil. Mag., 1, 257-279.

Reynolds, R. M and J. T. Gething, 1970: Acoustic sounding at

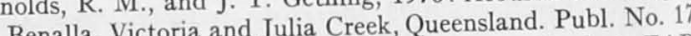
Benalla, Victoria and Julia Creek, Queensland. Pubect EAR, Meteor. Dept., University of Melbourne, Project EA Reps. V-VII.

Scott-Russell, J., 1837: Report on Waves. Brit. Assoc. Rep., No. 417.

1844: Report on Waves. Brit. Assoc. Rep., No. 311.

Shaw, 1971

Simpson, J. E., D. A. Mansfield and J. R. Milford, 1977 : Inland penetration of sea-breeze fronts. Quart. J. Roy. Meteor. Soc 103, 47-76.

Smart, E. 1966: An examination of atmospheric pressure pulses , M.Sc. thesis, Dept. of Physics, Colorado School of Mines, Golden.

illane, K. T., and M. J. McCarthy, 1969: Downdraft of the . Meteor. Mag., 17, 3-24.

thes, G. G., 1880: On the theory of oscillatory waves, Matheingical Papers, Vol. 1, Cambridge University Press, 197-229, 314

Strelkoff T. 1971. An exact numerical solution of the solitary wave Proc. 2nd Int. Conf. Numerical Methods Fhid Dy namics, Springer, 441-446.

Turner J S. 1963: The motion of buoyant elements in turbulent surroundings. J. Fluid Mech., 16, 1-16.

Vliegenthart, A. C., 1971: On finite-difference methods for the Korteweg-de Vries equation. J. Eng. Math., 5, 137-155 
ng waves. Akad. at., 1946, No. 8 , Ser. 1, 11, 273-

artoum. Weather, imum amplitude a katabatic flow Mag., 47, 40-51. stem. J. Meteor.,

d systems. Tellus, nd its role in the raves. Tellus, 24 ,

74: On the mass, litary wave. II

hey and P. J. R. aproach to the E, 57, 579-587.

mospheric acous-351 .

$\mathrm{s}$ when detecting 04.

rocess applied to n. Soc., 47, 197

velopment of an waves in liquids Appl. Math., 13, associated seiche urbulent environ257-279.

ustic sounding at nd. Publ. No. 17, Project EAR,

rit. Assoc. Rep.,

No. 311 .

ord, 1977 : Inland Roy. Meteor. Soc.,

c pressure pulses Dept. of Physics,

owndraft of the ., 17, 3-24.

ry waves. Matheridge University

n of the solitary ethods Fluid Dy

ients in turbulen methods for the 5, 137-155.
Wallington, C. E., 1961 : Observations of the effects of precipitation downdraughts. Weather, 16, 35-43.

Welch, P. D., 1967: The use of fast Fourier transforms for the estimation of power spectra: a method based on time averaging over short modified periodograms. Trans. IEEE, AU-15, No. 2, 70-73.

Yamada, H., 1957: On the highest solitary wave. Rep. Res. Inst. Appl. Mech. Kyushu Univ., 5, 53-67.
1958: On approximate expressions of solitary wave. Rep. Res. Inst. Appl. Mech. Kyushu Univ., 6, 35-47.

G. Kimura and J. Okabe, 1968: Precise determination of the solitary wave of extreme height on water of a uniform depth. Rep. Res. Inst. Appl. Mech. Kyushu Univ., 16, 15-32.

Zabusky, N. J., and M. D. Kruskal, 1965: Interaction of "solitons" in a collisionless plasma and the recurrence of initial states. Phys. Rev. Lett., 15, 240-243. 
Section 2.2

Intrusive density flows in the lower troposphere: A source of atmospheric solitons

(D.R.Christie, K.J. Muirhead and A.L. Hales)

J. Geophys. Res., 84, 4959-4970, 1979. 


\title{
Intrusive Density Flows in the Lower Troposphere: A Source of A tmospheric Solitons
}

\author{
D. R. Christie, K. J. Muirhead, and A. L. Hales \\ Research School of Earth Sciences, A ustralian National University \\ Canberra, A.C.T. 2600
}

\begin{abstract}
This paper is concerned with the properties of complex propagating nonlinear tropospheric disturbances observed near Tennant Creek in the arid interior of the Northern Territory of A ustralia. Many of these unusual atmospheric disturbances resemble the well-known internal undular surges observed in the oceans and in inland stratified bodies of water. A description is presented of a wide variety of observations of solitary-wave-dominated evolving density intrusions, and it is shown that many of the features of these unique disturbances are in qualitative agreement with the predictions of nonlinear dispersive wave theory. In particular, it is shown that the evolutionary behavior of these disturbances is governed by the Benjamin-Ono equation and that intrusive motions of this type play an important role in the generation of boundary layer solitary atmospheric waves. Existing experimental evidence indicates that these disturbances originate primarily in the interaction of katabatic flows, propagating sea breeze vortices, and 'morning glory' phenomena with the stably stratified nocturnal radiation inversion.
\end{abstract}

\section{INTRODUCTION}

Solitary waves are isolated finite amplitude waves of permanent form and constant velocity which arise from an equilibrium between the competing processes of higher-frequency harmonic generation through amplitude dispersion and the transfer of energy to the lower-frequency modes through normal frequency dispersion. These waves have been widely stud ied ever since they were first observed and described by Scott Russell in 1837 [Scott Russell, 1837]. However, despite this long history, solitary waves were regarded for many years as a mathematical and physical curiosity which appeared to require rather specialized conditions for their creation and which were therefore expected to be of minor importance in the initial value problem for nonlinear dispersive wave systems. It is only recently that it has been recognized that solitary waves play a fundamental role in the evolution of a wide variety of physically interesting problems. More specifically, for a large class of physically relevant nonlinear evolution equations the initial value problem for arbitrary data condenses into a pure solitary wave solution in the asymptotic $(t \rightarrow \infty)$ limit. For a good discussion of general solitary wave theory and an account of recent developments in the area the interested reader is referred to the reviews by Scott et al. [1973] and Cercignani [1977].

Many geophysical wave phenomena can be described by nonlinear dispersive wave theory. For example, on a planetary scale, solitary wave models explain the wavelike structure in the magnetosphere-solar wind interaction [Kikuchi, 1976] and describe the properties of finite amplitude Rossby waves in the oceans and atmosphere [Long, 1964; Clarke, 1971; Redekopp. 1977]. An interesting example of the latter type of wave has been given by Maxworthy and Redekopp [1976], who suggest that the Great Red Spot of Jupiter is a manifestation of a solitary Rossby wave on a horizontally sheared zonal flow. On a smaller scale, nonlinear dispersive wave theory describes many of the features of tsunami propagation [Hammack, 1973; Hammack and Segur, 1978a] and provides the framework for the interpretation of nonlinear internal wave observations in

\footnotetext{
${ }^{1}$ Now with the Geosciences Program, University of Texas at Dallas, Richardson, Texas 75080.
}

Copyright (c) 1979 by the American Geophysical Union Paper number $9 \mathrm{C} 0469$ $0148-0227 / 79 / 009 \mathrm{C}-0469 \$ 01.00$ the oceans and in thermally stratified inland bodies of water.

The latter observations are particularly relevant to the interpretation of the new type of complex propagating atmospheric disturbance considered in this paper. Nonlinear internal long water waves have been observed to occur as single isolated solitary wave disturbances [Gaul, 1961; Ziegenbein, 1970; Brekhouskikh et al., 1975] or as complex families of solitary waves in association with the leading edge of an evolving intrusive density flow or internal surge [Halpern, 1971; Thorpe et al., 1972; Hunkins and Fliegel, 1973; Lee and Beardsley, 1974; Ivanov and Konyayev, 1976; Gargett, 1976].

In a recent paper [Christie et al., 1978] we presented a detailed description of large-amplitude atmospheric waves in the form of isolated solitary waves and isolated solitary wave packets which were observed over a 2 -year period, using an array of high-sensitivity microbarometers, at the Warramunga seismic station located near Tennant Creek in central A ustralia. Three distinct types of isolated nonlinear atmospheric wave phenomena were observed: (1) large-scale waves of elevation which belong to the class of classical shallow-fluid internal solitary waves, (2) large-scale internal solitary waves of depression, and (3) solitary waves associated with the atmospheric boundary layer which are described by the deep-fluid nonlinear wave theory of Benjamin [1967].

The primary objectives of this paper are to present a description of a series of unique observations of a variety of commonly occurring complex propagating atmospheric boundary layer disturbances, to show that the morphology of these disturbances is consistent with an interpretation within the framework of Benjamin's deep-fluid nonlinear dispersive wave theory, and to demonstrate that the genesis of the observed deep-fluid solitary atmospheric waves and wave packets may plausibly be traced to these complex intrusive flows. An example of the specific type of propagating long-wave disturbance considered in this paper is shown in Figure 1. It will be seen that disturbances of this type are similar in many respects to the complex solitary-wave-associated intrusive disturbances observed in the oceans and in stratified inland lakes.

\section{Nonlinear Dispersive Wave Theory}

During the past decade the concept of the soliton [Zabusky and Kruskal, 1965] has emerged as one of the most important elements in the description of nonlinear wave propagation in 


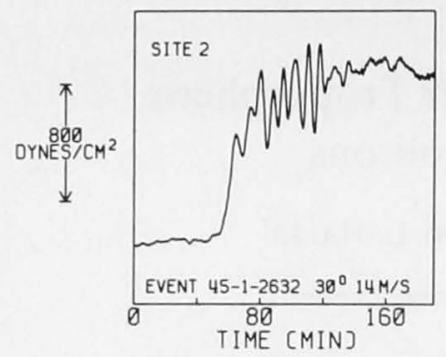

Fig. 1. An example of the typical morphology of a solitary-wavedominated intrusive atmospheric disturbance. The azimuth noted in this diagram corresponds to the source direction measured from true north.

dispersive media. Solitons are exceptionally stable solitary waves which preserve their identity under nonlinear interaction. Since the discovery of the soliton, a number of ingenious methods have been developed for obtaining exact solutions to many physically interesting nonlinear evolution equations [Gardner et al., 1967; Lamb, 1974; Hirota and Satsuma, 1976]. In this section we shall briefly review those aspects of nonlinear dispersive wave theory which are particularly relevant to the interpretation of evolving nonlinear wave phenomena in the atmosphere. For further details the reader is referred to the lucid treatment of the subject by Whitham [1974] and to the recent account by Ablowitz [1978] of developments in inverse scattering transform theory.

The propagation of small- but finite-amplitude internal long waves in a shallow fluid is described by the classical Korteweg and de Vries [1895] ( $\mathrm{KdV}$ ) evolution equation,

$$
\eta_{t}+c_{0} \eta_{x}+\alpha \eta \eta_{x}+c_{0} \beta \eta_{x x x}=0
$$

where $c_{0}$ is the maximum speed of infinitesimal waves, $\alpha$ is a constant which describes the nonlinear effects of finite amplitude, and $\beta$ is a parameter which characterizes the phase speed dispersion for shallow fluids in the long-wavelength limit. In an elegant study of internal waves in stratified fluids, Benjamin [1967] discovered a new class of finite amplitude waves of permanent form which, in contrast to classical nonlinear waves, can propagate in fluids of infinite depth. The properties of these waves have also been studied both experimentally and theoretically by Davis and Acrivos [1967]. Internal wave motions which belong to this new class occur in systems where the density of the stratified fluid varies significantly only in a region whose depth $h$ is small in comparison to the total depth and to the wavelength of the wave. On the basis of a heuristic argument, Benjamin proposed that the infinitely deep fluid counterpart to the $\mathrm{KdV}$ equation is given by an evolution equation in the form

$$
\eta_{t}+c_{0} \eta_{x}+\alpha \eta \eta_{x}-c_{0} \gamma \mathcal{F}\left(\eta_{x}\right)=0
$$

where the constant $\gamma$ characterizes the linear dispersion relation for deep fluids and the operator $\mathcal{F}$ is defined by the Hilbert transform

$$
\mathcal{F}[f(x)]=-\frac{1}{\pi} \rho \int_{-\infty}^{\infty} \frac{1}{z-x}\left\{\frac{\partial f(z)}{\partial z}\right\} d z
$$

This equation has been rigorously derived by Ono [1975] and in a different context by Gargett [1976] and by Levikov [1977]. Joseph [1977b] has found a solution in the long-wavelength limit to Whitham's generalized evolution equation [Whitham, 1967] which describes solitary wave propagation along a thin thermocline in a fluid of arbitrary depth. This generalization to a finite depth fluid represents the natural connection between the classical shallow-fluid $\mathrm{KdV}$ theory and the infinitely deep fluid theory of Benjamin.

The steady state solutions $\eta=\eta(x-c t)$ of (1) and (2) are well known. For the $\mathrm{KdV}$ equation the solitary wave is given by

$$
\begin{gathered}
\eta(x, t)=\eta_{0} \operatorname{sech}^{2}[\Delta(x-c t)] \\
c=c_{0}+\frac{1}{5} \alpha \eta_{0}
\end{gathered}
$$

with $\Delta=\left(\eta_{0} \alpha / 12 c_{0} \beta\right)^{1 / 2}$, and for the Benjamin-Ono equation the spatially localized solution takes the form of a Lorentzian

$$
\begin{gathered}
\eta(x, t)=\eta_{0} \lambda^{2} /\left[(x-c t)^{2}+\lambda^{2}\right] \\
c=c_{0}+\frac{1}{4} \alpha \eta_{0}
\end{gathered}
$$

with $\lambda=4 c_{0} \gamma / \alpha \eta_{0}$. Note that the classical solitary wave disturbance vanishes exponentially and is therefore of limited range, while the Benjamin solitary wave, which decays algebraically, extends over a relatively long range.

This paper is mainly concerned with the nonstationary solutions of the evolution equations; in particular, since the propagating disturbances considered here are associated with the atmospheric boundary layer, we are primarily interested in the form of the solutions to the initial value problem near the infinitely deep fluid limit described by the Benjamin-Ono equation (2).

The precise behavior of the general nonstationary solution to the Benjamin-Ono equation is not nearly as well understood at the present time as that of the general solution to the classical $\mathrm{KdV}$ equation. However, exact $\mathrm{N}$-soliton solutions have been found for both the infinitely deep fluid problem and the generalized thin-thermocline finite depth problem [Joseph, 1977a; Joseph and Egri, 1978]. Furthermore, it is now known from recent experimental observations (T. Maxworthy, private communication, 1978) and from the results of numerical calculations [Meiss and Pereira, 1978] that Lorentzian deep-fluid solitary waves exhibit soliton behavior and that the general solution to the Benjamin-Ono initial value problem is qualitatively very similar to that of the classical $\mathrm{KdV}$ problem. We can therefore gain insight into the properties of an evolving nonlinear disturbance propagating in a suitably stratified deep fluid by examining the well-known general characteristics of the corresponding classical problem defined by the $\mathrm{KdV}$ equation. The mathematical problem has been widely studied [Gardner et al., 1967, 1974; Berezin and Karpman, 1967; Hirota, 1971; Wadati and Toda, 1972; Segur, 1973; Ablowitz and Newell, 1973; Ablowitz and Segur, 1977], and the results confirmed both numerically [Zabusky, 1967; Vliegenthart, 1971] and through a series of detailed laboratory experiments [Zabusky and Galvin, 1971; Hammack and Segur, 1974, 1978b; Weidman and Maxworthy, 1978]. The salient features of these investigations may be summarized as follows:

1. A general initial disturbance of finite extent will evolve into a finite discrete set of solitons and an independent dispersive wave train.

2. The group velocity of the dispersing waves is less than or equal to the critical wave speed $c_{0}$. Since the speed $c$ of each soliton is supercritical and since the deviation of $c$ from $c_{0}$ is proportional to wave amplitude, the solution evolves into a family of solitons ordered by amplitude, followed by a train of dispersing waves.

3. The amplitude of the solitons will not exceed twice the maximum amplitude of the initial disturbance.

4. The existence of at least one soliton in the asymptotic solution is guaranteed if the net volume of the initial distur- 
tely deep
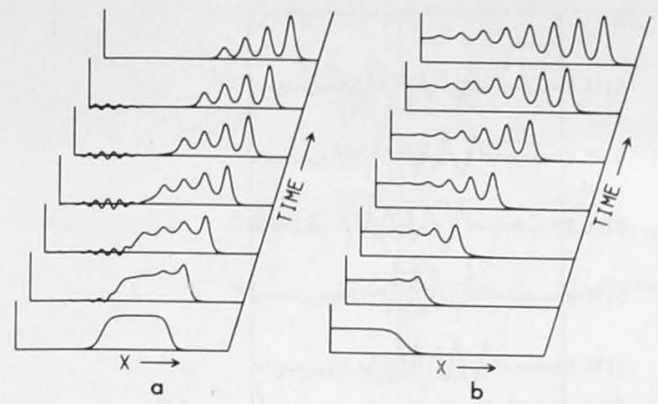

Fig. 2. Schematic representation, based on the predictions of nonlinear dispersive wave theory, of the evolutionary behavior pattern of $(a)$ a finite length wave of elevation and $(b)$ the leading edge of a long wave. The initial disturbance in Figure $2 a$ evolves into a supercritical solitary wave family followed by a weak dispersing subcritical oscillatory wave train. Asymptotically, this disturbance is reduced to four solitary waves which are ordered by amplitude. In Figure $2 b$ the evolutionary pattern is characterized by the formation of a long train of amplitude-ordered solitary waves along the leading edge of the disturbance.

bance is positive or zero. If the initial disturbance is predominantly positive, most of the wave energy goes into the production of solitons. Soliton production when the net initial volume is less than zero depends on the detailed form of the disturbance. If the initial disturbance is everywhere negative, no solitons emerge, and thus the solution evolves into a pure dispersive state.

It must be emphasized that the behavior of the nonstationary solutions to the deep-fluid Benjamin-Ono equation will be only qualitatively similar to the classical behavior pattern outlined above. In particular, we note that little is known at the present time about the exact behavior of the dispersing wave train portion of the deep-fluid solution. However, since the atmospheric disturbances described in this paper correspond to initially positive disturbances in the form of intrusive density flows, almost all of the wave energy is expected to appear in the asymptotic soliton state, and thus the exact nature of the dispersive state is of only minor importance in the interpretation of these experimental observations.

To summarize this discussion, the well-known properties of the solutions to the classical nonlinear dispersive wave evolution problem coupled with the presently understood behavior of the nonstationary solutions of the Benjamin-Ono equation clearly indicate that a long smooth atmospheric disturbance propagating along the boundary layer inversion will steepen in the regions of negative slope under the influence of amplitude dispersion and eventually condense into a distinct family of solitary waves and a small dispersing oscillatory tail. This characteristic evolution pattern is shown in detail in the extensive series of laboratory experiments described by Hammack and Segur [1974] on the evolution of a wide range of finite amplitude disturbances and in the numerical calculations presented by Vliegenthart [1971] of the development of a soliton family along the leading edge of an initially smooth bore. Figure 2 shows a schematic representation of the principal features in the evolutionary behavior of both a finite length wave of elevation and the frontal zone of a long wave of elevation. It will be shown that the morphology of the complex propagating atmospheric disturbances observed at Tennant Creek is in reasonable agreement with this predicted behavior pattern, and thus it may be concluded that these observations represent various stages in the development of a finite amplitude disturbance as described in a first approximation by the

Benjamin-Ono evolution equation and that the final asymp- totic form of these disturbances consists of a family of independent solitary atmospheric waves.

\section{EXPERIMENTAL A RRANGEMENT}

The complex nonlinear atmospheric gravity wave disturbances described in this paper were observed by using an array of National Bureau of Standards designed high-sensitivity microbarometers located at the Warramunga seismic station, 37 $\mathrm{km}$ SSE of Tennant Creek, at a latitude of $19^{\circ} 56^{\prime} \mathrm{S}$ in the arid interior of Australia. It is thought that the high frequency of observation of these non inear waves and the excellent resolution of the wave patterns result primarily from the unique location of the infrasonic array, a location which favors a high degree of stability in the lower atmosphere due to the absence of the perturbing influence of both the oceans and large-scale orographic features. The development of intense nocturnal radiation inversions, which provide an ideal medium for the propagation of nonlinear dispersive waves, may be attributed to this unusual atmospheric stability coupled with the lowlatitude semidesert environment of the array.

The infrasonic array is configured in the form of a fiveelement centered quadrilateral with a net aperture of $4 \mathrm{~km}$. Signals from the five infrasonic channels are transmitted to a central recording station, sampled at a rate of 2 samples per second, digitized, and recorded in IBM compatible format on magnetic tape. Further details of the digital recording and data processing techniques are given by Christie et al. [1978].

The microbarometer array elements are designed to effectively filter out those components in the surface micropressure spectrum which lie beyond the useful passband extending from about 1 to $1000 \mathrm{~s}$. The phase and modulus of the microbarograph transfer function $R$ are given as a function of period by

$$
\begin{gathered}
\phi=\tan ^{-1}\left(T^{2}-T_{0}{ }^{2}\right) /\left(T_{1}+T_{2}\right) T \\
|R|=A \cos \phi
\end{gathered}
$$

where $T_{1}=1.95 \mathrm{~s}, T_{2}=48.7 \mathrm{~s}, T_{0}=\left(T_{1} T_{2}\right)^{1 / 2}=9.7 \mathrm{~s}$, and $A=$ 77.9 counts/dyn $/ \mathrm{cm}^{2}$.

Since the fundamental spectral components of the atmospheric gravity waves considered here are large in comparison with $T_{0}$, the array records provide an essentially differential micropressure signature of propagating wave phenomena. Two examples are given in Figure 3 which illustrate inversions of array record sections corresponding to the leading edge of a fairly smooth density intrusion and a complex soliton-dominated disturbance to provide the true micropressure trace at the surface. The microbarograph transfer function has been deconvolved from all experimental array data presented in the remainder of this paper.

\section{OBSERVATIONS AND INTERPRETATION OF COMPLEX PROPAGATING ATMOSPHERIC BOUNDARY} LAYER Disturbances

The infrasonic array has been operating continuously at Tennant Creek since August 1975. During this period, observations have been made of a wide variety of unusual nocturnal nonlinear wave disturbances ranging from extensive perturbations in the form of smooth intrusive density flows, complex evolving nonlinear wave disturbances, and fully developed undular intrusions to localized soliton wave packets and observations of isolated single solitary waves of elevation. Since these disturbances are only observed at night and since the observed phase speeds and amplitudes are consistent with asymptotic itial distur- 

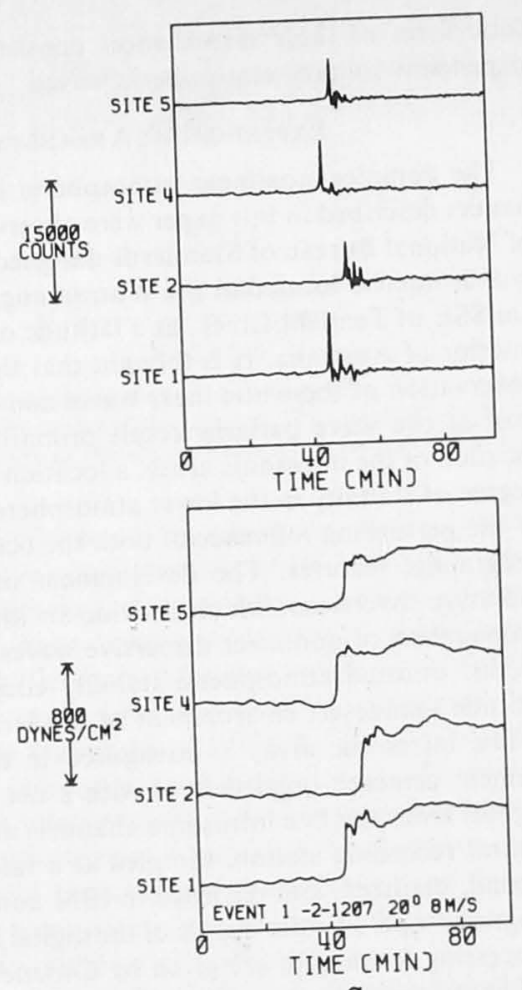
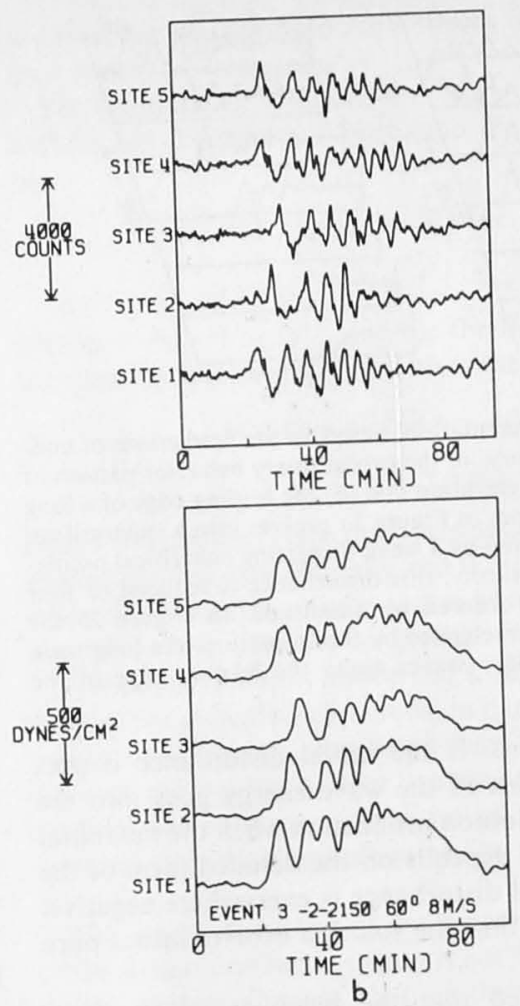

Fig. 3. Two examples of the inversion (lower figures) of microbarograph array record sections (upper figures) cor列 responding to $(a)$ a relatively smooth intrusive disturbance and $(b)$ a solitaryal array configuration where the four sites site numbering shown in these diagrams corresponds to a centered quadrilateral ar located at the center.

a boundary layer disturbance, it may be concluded that these observations correspond to disturbances that propagate along the nocturnal radiation inversion. As was noted in the inmajor objectives of this paper are to show that of these observations represent various stages in the evoluall of the disturbances and that the wave patterns are consistent with an interpretation observed the Benjamin-Ono infinitely deep fluid evolution equation. A detailed meteorological study of the influence of these disturbances on the ambient atmospheric flow field has also been carried out and will be published separately along with the implications of a statistical study of the diurnal and seasonal frequency of observation and the variations in amplitude, azimuth, and phase velocity. How- ever, it is worth noting here that the azimuthal distribution and the frequency of occurrence of the complex nonlinear disturbances are qualitatively similar to those of the isolated solitary waves and solitary wave packets. It is also worth remarking on the fact that almost all of the observed complex intrusive disturbances produce only minor perturbations in the tmospheric flow field at the surface. Thus it may be concluded me energy of these intrusive disturbances is that most of the energy of these intrusive disturbances is concentrated near the nocturnal boundary layer interface and the soliton-producing interfacial density intrusions observed on the ocean thermocline and in inland stratified bodies of water. Nevertheless, it should be emphasized that the experimow that these intrusive disturbances pertur they $\mathrm{p}$ linear waves of quas propag and $w$ microp

At th tant stu sient di observe Julia C these co associat version, lies of $n$ turbed unusual Gething bance as Let us propaga appear t nonlinea first be evolve a array; th viewed as agreemen
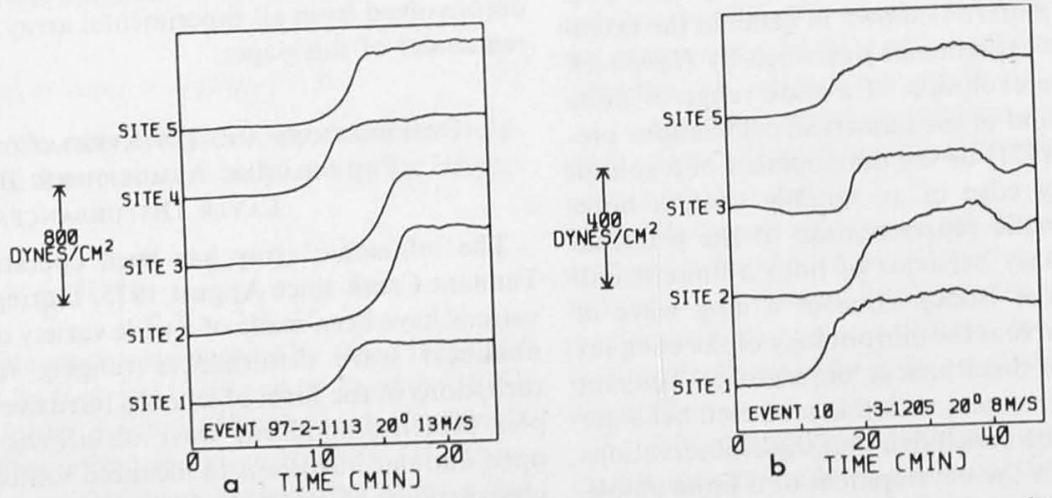
Fig. 4. Array record sections illustrating the frontal zone of two relatively smooth solitary-wave-free int
spheric disturbances. Disturbances of this type appear to represent a primary evolutionary state 

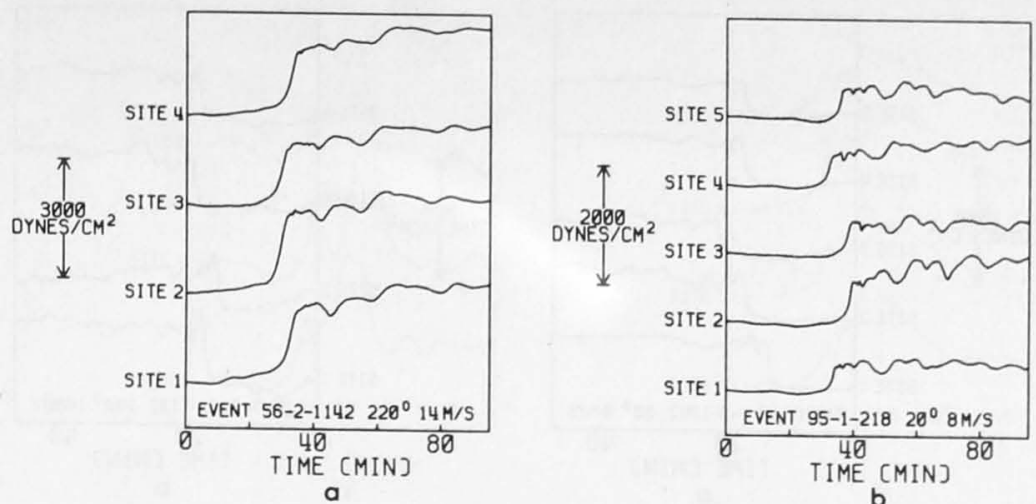

Fig. 5. Large-amplitude intrusive disturbances which illustrate an early stage in the development of solitary waves.

perturb the motion of the boundary layer as a whole in that they preferentially excite the lowest-order asymmetrical nonlinear wave mode in the form of a sequence of internal solitary waves of elevation. The next lowest-order mode takes the form of quasi-symmetrical interfacial waves [Benjamin, 1967] which propagate at speeds which are much less than those observed and which produce only second-order perturbations in the micropressure field at the surface.

At this point we would like to draw attention to the important study described by Reynolds and Gething [1970] of transient disturbances on the nocturnal boundary layer which were observed, using an acoustic radar, during the month of June at Julia Creek, $800 \mathrm{~km}$ to the east of Tennant Creek. Some of these complex perturbations occur as 'spikelike' disturbances associated with an increase in the depth of the radiation inversion, while others appear to take the form of isolated families of nonlinear waves propagating along the relatively undisturbed boundary layer interface. It seems clear that the unusual atmospheric phenomena described by Reynolds and Gething belong to the same class of nonlinear wave disturbance as the soliton-dominated disturbances considered here.

Let us now examine a series of observations of complex propagating atmospheric disturbances whose morphologies appear to exhibit the evolutionary pattern predicted by the nonlinear dispersive wave theory outlined above. It should first be emphasized that these disturbances do not usually evolve appreciably over the 4-km aperture of the infrasonic array; the individual array observations must therefore be viewed as various stages in the evolutionary process, and thus agreement with the predicted behavior pattern can only be inferred from the observations. A measure of the degree of evolution over an array of aperture $A$ may be obtained by considering two Lorentzian deep-fluid solitary waves with dimensionless amplitudes $a_{1}=\eta_{0}{ }^{1} / h$ and $a_{2}=\eta_{0}{ }^{2} / h$ which propagate in the same direction along a nocturnal boundary layer represented, in a two-fluid description, by an inversion of depth $h$ and intensity $\Delta T$. The relative speed of the two Benjamin solitons is given by

$$
s \simeq{ }_{8}^{3} c_{0}\left(a_{2}-a_{1}\right)
$$

the relative shift in the centroidal positions of the solitary waves during the traverse of the array may be expressed to first order by

$$
\Delta x \simeq \frac{s A}{c_{0}}=\frac{3}{8} A\left(a_{2}-a_{1}\right)
$$

and the effective soliton wavelengths (full width at half maximum) are given by

$$
w=2 \lambda=\frac{8}{3}\left(1+\frac{\Delta T}{T}\right) \frac{h}{a}
$$

where $T$ is the temperature of the lower fluid. Assuming a typical boundary layer specified by $T=300 \mathrm{~K}, \Delta T=5 \mathrm{~K}$, and $h=300 \mathrm{~m}$ and taking $a_{1}=0.2, a_{2}=0.4$ as representative amplitudes, the soliton wavelengths are $w_{1} \simeq 4000 \mathrm{~m}, w_{2} \simeq$ $2000 \mathrm{~m}$, and the relative shift $\Delta x$ over the array is $300 \mathrm{~m}$, which is about $10 \%$ of the spatial shift required to resolve fully the two-soliton wave pattern. The individual array observations do not therefore provide a direct measure of the evolutionary
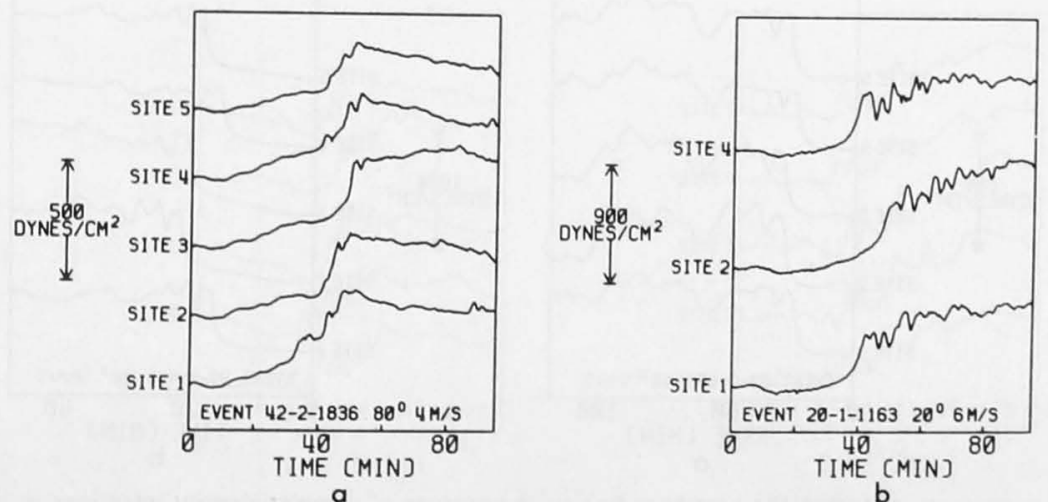

Fig. 6. Record sections corresponding to two relatively weak evolving disturbances in an early evolutionary state 

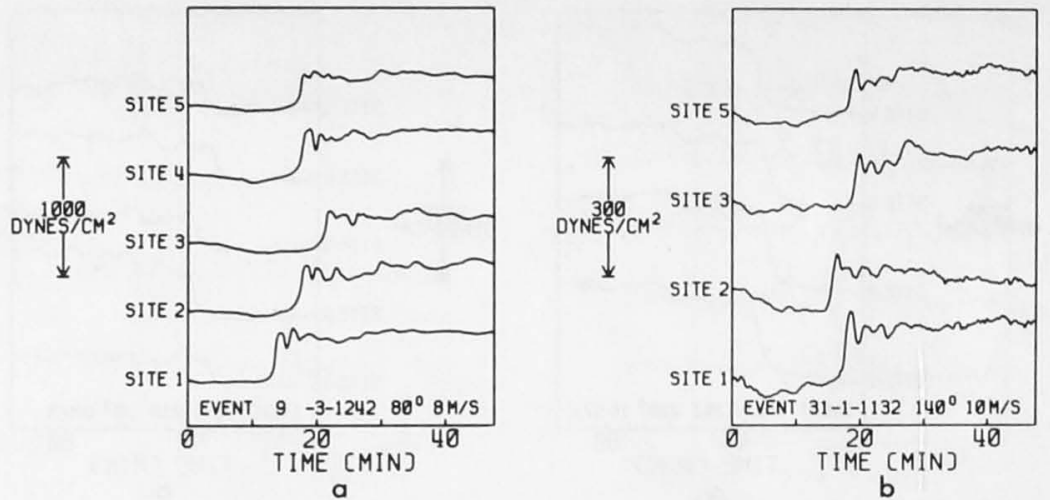

Fig. 7. Two relatively simple disturbances which illustrate the initial formation of solitary waves along the leading edge of intrusive flows.

pattern; they do, however, provide sufficient data for an accurate determination of the wave propagation vector.

The following observations are therefore presented in an ordered sequence which is in qualitative agreement with the theoretically predicted finite amplitude long-wave evolutionary pattern.

\section{Soliton-Free Intrusive Disturbances}

Only a few examples of long smooth solitary-wave-free intrusive disturbances have been observed at Warramunga over the 3-year observational period. Two examples which illustrate the typical morphology of these events are illustrated in Figure 4. As can be seen, there appears to be no evidence for soliton formation in the frontal zones of these propagating disturbances; it therefore seems reasonable to assume that these smooth disturbances correspond to the initial state in the evolutionary process.

\section{Initial Soliton Development}

A large number of observations have been made of intrusive disturbances whose structure is indicative of the early stages of soliton formation. Two examples of large-amplitude intrusive flows with weak long-period undulations corresponding to the initial condensation of large-amplitude solitary waves are shown in Figure 5. The form of these disturbances may be contrasted with that of the smaller-scale events illustrated in the record sections presented in Figure 6. Both of the disturbances shown in Figure 6 are interesting in that they propagate at low velocities and thus a noticeable degree of evolution occurs over the 4-km array. The relatively weak event shown in Figure $6 a$ provides direct observational evidence for the influence of nonlinear steepening in the frontal zone of the disturbance. This unusually slow disturbance first appears at site 1 and then proceeds to traverse the array as a planar wave front directed along the axis defined by sites 1,5 , and 3. As can be seen, the slope of the leading edge of the disturbance increases continuously as the wave progresses over the array, and at site 3 there is primary evidence for initial soliton formation. The two relatively simple disturbances shown in Figure 7 and the more complex intrusions illustrated in Figure 8 appear to be in a more advanced evolutionary state in that several distinct solitary waves are beginning to form in the frontal zone of the disturbance. It is possible, but by no means clear, that some of the oscillations in these early evolutionary states represent the formation of a subcritical dispersive wave component.

\section{Soliton-Dominated Intrusive Disturbances}

Most of the propagating boundary layer intrusive disturbances observed at Tennant Creek belong to this category. Consider first a series of observations of relatively localized short-lived disturbances with a spatial extent of about $20 \mathrm{~km}$. The record sections shown in Figure 9 are chosen to illustrate the process of soliton formation along the leading edge of clearly localized propagating intrusive disturbances. These wave patterns may be compared with the expected behavior pattern shown in Figure $2 a$. The more complicated disturbances shown in Figures 10-12 illustrate a number of inter-
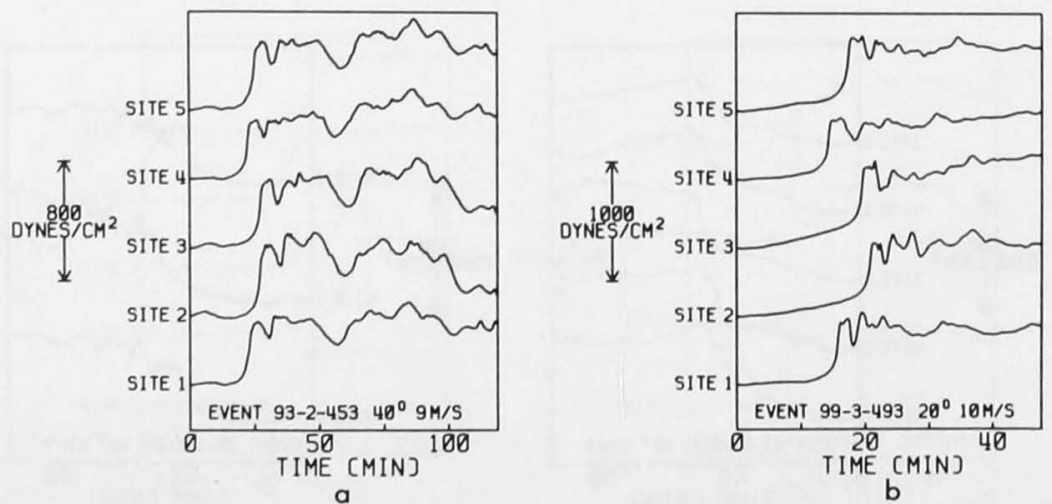

Fig. 8. Surface pressure perturbations corresponding to the passage of complex density intrusions in an intermediate developmental stage. 

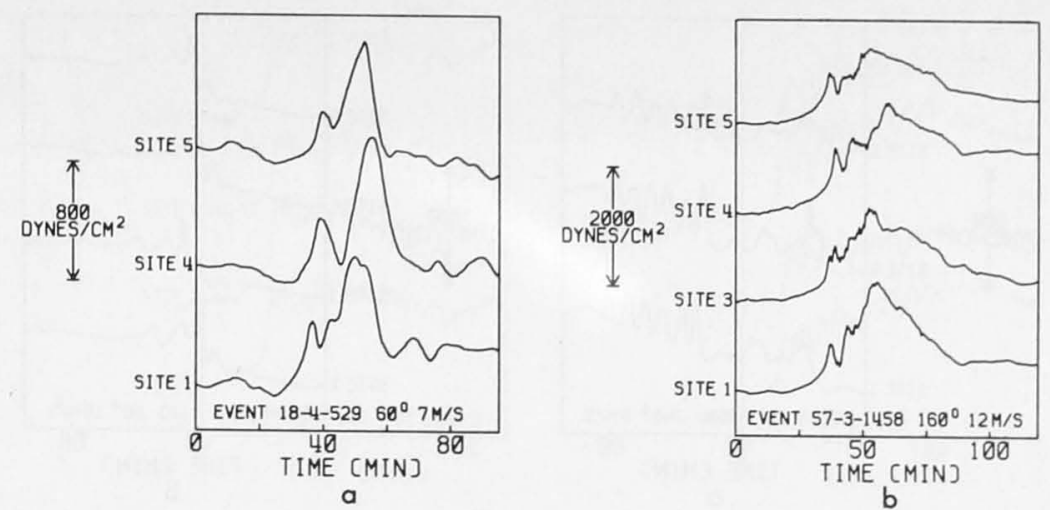

Fig. 9. Microbarograph array record sections of two localized propagating intrusive atmospheric disturbances. Solitary wave formation is evident along the leading edge of these intrusions.

e disturcategory. localized ut $20 \mathrm{~km}$. illustrate edge of es. These behavior ed disturof inter-

esting evolutionary features. The fact that none of the solitarywave-like components in the patterns shown in Figures 10 and 11 are completely ordered by amplitude may indicate that these actively evolving wave forms originate in unusually complex initial disturbances. The wave pattern illustrated in Figure $11 a$ is particularly interesting in that an essentially pure solitary wave packet consisting of six distinct components has condensed out well behind the leading edge of the disturbance. The essentially asymptotic character of this solitary wave family indicates that the wave packet originated in a completely independent disturbance.

Figure 12 provides a comparison of two fairly similar intrusive disturbances whose asymptotic states apparently correspond to the excitation of three solitary waves. As can be seen, the morphologies of these events differ in that the profiles of the solitons in event 43-1-1894 are flattened at the crest; in fact, it might appear that the leading soliton is about to fission into two solitary waves. However, an isolated solitary wave disturbance with a similarly distorted profile has been observed [Christie et al., 1978] to evolve into the normal Lorentzian wave form; it therefore seems likely that the disturbance illustrated in Figure $12 a$ represents a fairly early stage in the evolutionary process and that this disturbance will eventually evolve into a soliton pattern which is similar to that shown in Figure $12 b$. This distortion of the profile of higher-amplitude leading solitary waves, which may be related to a form of amplitude instability, has been observed on several occasions (e.g., Figures $6 b$ and $8 b$ ) and will be discussed briefly in the next section.
Finally, consider the six examples shown in Figures 1, 3b, 13 , and 14 of observations of extensive, well-developed families of solitary waves associated with the leading edge of intrusive density flows. As can be seen from the diagrams, all of these disturbances are characterized by the excitation of a large number of solitary waves. The observation shown in Figure $13 a$ is particularly noteworthy in that this disturbance, which took over 3 hours to pass over the infrasonic array, consists of at least 17 individual solitary waves and extends over a spatial distance of about $130 \mathrm{~km}$. At first glance, it might appear that the morphology of these extended disturbances is in good agreement with the behavior pattern shown in Figure $2 b$ for the evolution of the frontal zone of an initially smooth long wave of elevation. However, in the case of all of these observations the solitary waves are not completely ordered by amplitude, and thus it may be concluded that while these patterns are in fairly good agreement with the representation of Figure 2, these disturbances have apparently evolved from a more complex initial state.

\section{Asymptotic Disturbances}

An extensive series of observations of asymptotic boundary layer disturbances in the form of pure solitary wave packets, families of well-separated solitons, and isolated solitary waves has been described by Christie et al. [1978]. An example of a typical large-scale amplitude-ordered pure six-component solitary wave packet and an event consisting of two low-amplitude spatially separated solitons on a noisy background are shown
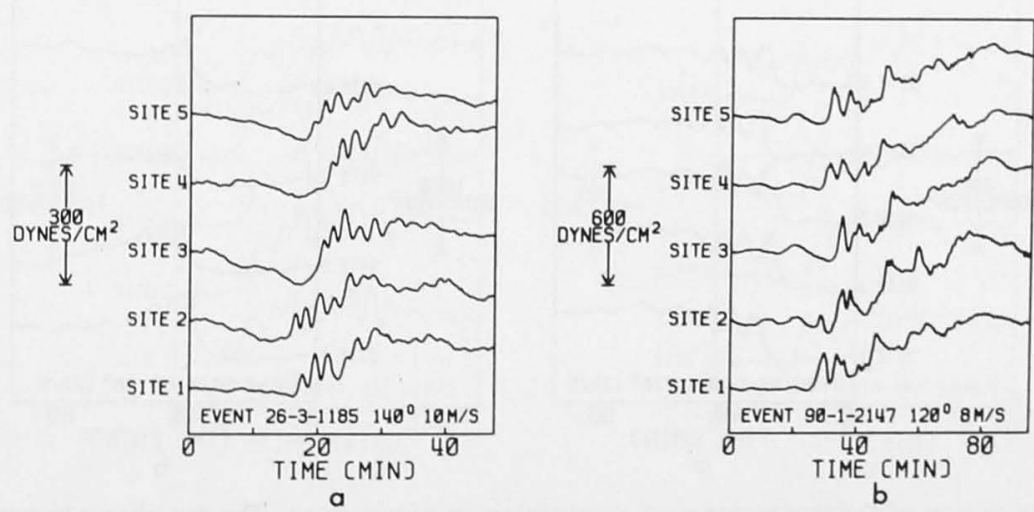

Fig. 10. Examples of complex solitary-wave-dominated intrusive disturbances. 

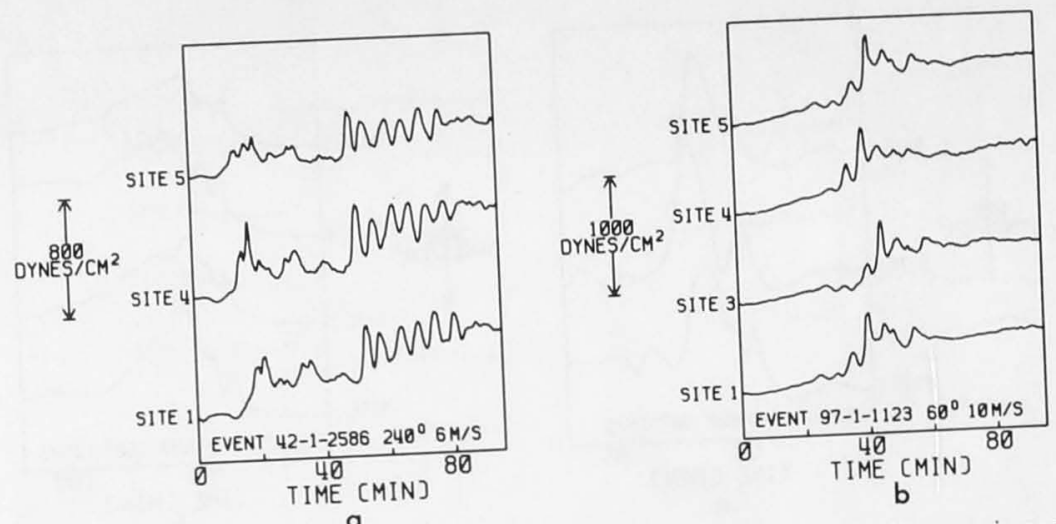

11. Unusual complex solitary-wave-associated density intrusions. Note the essentially pure six-component solitary Fig. 11. Unusual complex solitary-wave-associated density intrusions.
wave family which appears in the wake of the disturbance shown in Figure $11 a$. This wave packet probably represents the asymptotic state of an independent disturbance. The inter of the disturbance.

amplitude solitary wave has condensed out near the center of

in Figures $15 a$ and $15 b$, respectively. The solitary wave packet may be interpreted as the asymptotic state of a fairly extensive dissipating soliton-dominated intrusion similar to the exan in Figures 14, while the two-component soliton family represents the asymptotic form of a more localized smaller-scale disturbance.

\section{Discussion AND CONCLUSIONS}

The detailed sequence of observations presented in the last arly indicates that initially smooth long propagating ection asymptotatmospheric boundary lates ically into a finite number by qualitative agreement with the benjamin-Ono nonlinear the nonstationary solutions of the dispersive wave equation. It should be not the formation of a observations provide a clear indication of the formain. This, prominent coherent dispersive oscillatory wave traine these however, is to be expected since, as was noted above, these waves appear to originate in initially positive disturily into the therefore the wave energy is expected to go primarily into state production of solitons with the result that the dispersive stainly should appear only as a second-order effect. possible that some of the weak irregular oscillations in the wake of these disturbances do in fact represent the dispersing part of the solution.

It has been emphased that only a relatively few observaions have been made of soliton-dominated intrusive disturbances in which the solitary waves are ordered by amplitude.

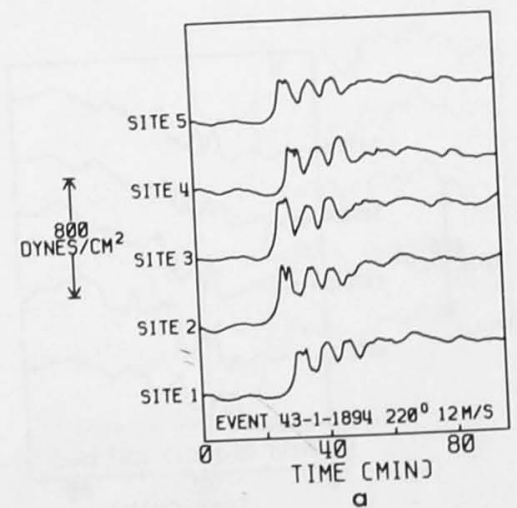

This then indicates that the initial disturbances are more complicated than those of the simple models shown in Figure 2. ively, perturbations along the propagation path, such Alternatively, perturbations alo depth, may lead to the excitation as variations in the inver [Kaup and Newell, 1978; Ko and Kuehl, of new solitarion of the original pattern, thus masking the 1978] and a distortion of tisturbance.

structure of the original disturbance.

It might seem reasonable to expect that for very dispersion tude disturbances, nonlinearity will dominate over and eventually and therefore the disturbance will stepe break in the forward direction, thus leading to the formation of a turbulent internal bore. A measure of the relative im classitance of nonlinearity and dispersion in the evolution of cumber [Ursell, 1953]

$$
U=\eta_{0} \lambda^{2} / h^{3}
$$

where $\lambda$ is the effective wavelength. If $U \gg 1$, nonlinearity dominates the fluid motion; if $U \ll 1$, the evolutionary character is determined by dispersion. Solitary waves correspond to a balance between nonlinearity and dispersion and are hereferesented by $U=0(1)$. This definition of the Ursell number is only suitable for a description of classical finite propagation in shallow fluids. For fluids of amplitude wave propagation in great depth
jamin, 1967]

$$
U=\eta_{0} \lambda / h^{2}
$$

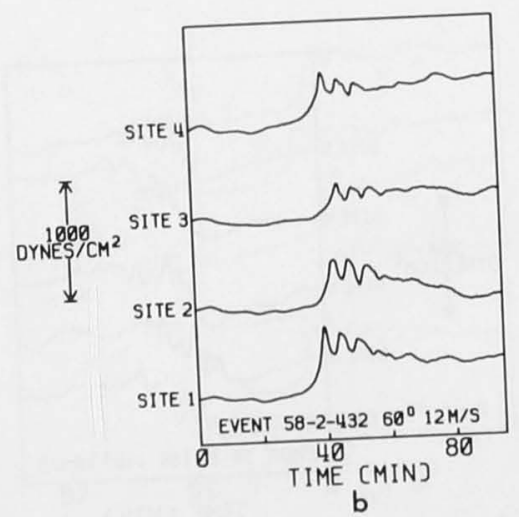

The fac dispersic waves ir motions than the An ex gating values therefore in the da appear to bility in case of evidence festation distorted tude dist tal obser patterns presence line patte numerica and Acriv tern at $h$ region of tion of str theless, in required however, Fig. 12. A comparison of the morphologies of simple intrusive density flows. The disturbance shown in
appears to represent an earlier stage in the evolutionary process than that shown in Figure $12 b$. 

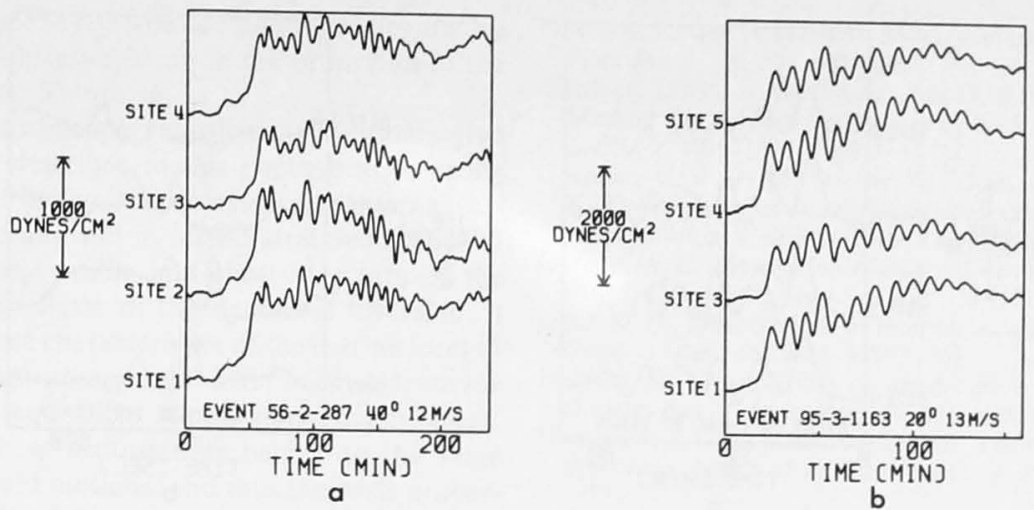

Fig. 13. Two excellent examples of microbarograph array record sections which illustrate the development of a very extensive family of solitary waves along the leading edge of intrusive density flows.

shear, strong nonlinearity will not lead to internal wave breaking in these deep-fluid solitary waves.

Very little is known at the present time about the precise nature of the source mechanism which leads to the production of these solitary wave generating disturbances. Nevertheless, it is clear that many of these disturbances must originate at distances of several hundred kilometers from the array. This can be seen from the following simple estimates of the distance required for an initially smooth disturbance to evolve into the extensive asymptotic wave forms observed at Warramunga. Suppose that a long smooth wave of elevation is created on an established boundary layer inversion at time $t=0$ at a distance $X$ from the infrasonic array. Ignoring phase shifts and assuming that the initial perturbation evolves over $X$ into an ordered disturbance, the minimum arrival time at the array of the leading solitary wave in the disturbance is given to first order by $t_{1}=X / c$, where $c$ is the Benjamin solitary wave phase speed,

$$
c=c_{0}(1+K)
$$

with $K=\frac{3}{8} a$. The arrival time of the minimum amplitude solitary wave produced by the disturbance is given approximately by $t_{2}=X / c_{0}$. Hence a first-order estimate of the minimum source distance is

$$
X_{\min }=[(1+K) / K] c_{0} \Delta t
$$

where $\Delta t=t_{2}-t_{1}$ is the observed difference in the arrival time at the array of the solitary waves of minimum and maximum amplitude. Equation (13) probably underestimates the true

Fig. 14. Examples of extensive soliton-dominated intrusions. Note the low degree of amplitude ordering in the solitary wave pattern associated with the disturbance shown in Figure $14 a$ 


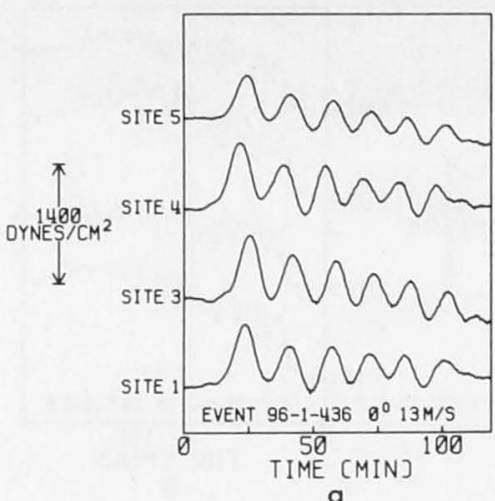

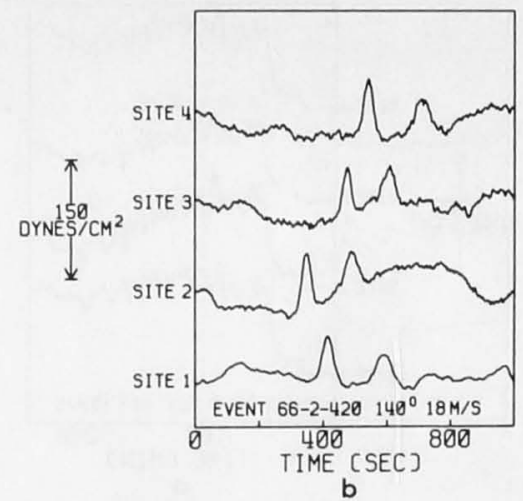

Fig. 15. Asymptotic soliton states. (a) A pure six-component solitary wave packet. (b) Two asymptotic, spatially separated low-amplitude solitary waves on a noisy micropressure background.

source distance since it is based on the assumption that the leading soliton observed at the array propagates over the distance $X$ at the constant velocity $c$. A rough upper bound on the source distance, $X_{\max }$, can therefore be obtained by assuming that the amplitude of the leading solitary wave varies linearly with distance from the origin and thus the maximum estimate of the wave speed used to calculate $t_{1}$ should be replaced by the minimum mean value

$$
\bar{c}=c_{0}[1+(K / 2)]
$$

Hence

$$
X_{\max }=[(2+K) / K] c_{0} \Delta t
$$

Consider now an application of (13) and (15) to observations of extensive well-developed solitary-wave-dominated disturbances. An estimate of the dimensionless amplitude $a$ of the leading soliton may be obtained from the two-fluid boundary layer inversion model as

$$
a=\Delta P /\left(\rho c^{2}-\frac{3}{4} \Delta P\right)
$$

where $\Delta P$ is the measured pressure perturbation and $\rho$ is the mean density of the boundary layer. Typically, the values of the parameters are $a=0.6, c_{0}=10 \mathrm{~m} / \mathrm{s}$, and $\Delta t=6000 \mathrm{~s}$, and hence some of the observed large-amplitude extensive solitary wave disturbances appear to originate at distances between 330 and $590 \mathrm{~km}$ from the array. It should be emphasized that these estimates are only approximate and that considerable uncertainty is introduced by the possibility that the properties of the atmospheric boundary layer vary along the propagation path.

It seems likely that some of the density intrusions observed at Tennant Creek are produced by thunderstorm-generated downdraft outflows at the surface. However, this does not appear to be the most important source mechanism since many of the soliton-associated events are observed during periods when totally clear conditions prevail throughout the Northern Territory. Furthermore, as was noted above, these disturbances only occur at night, and, as well, they originate in preferred directions.

A possible explanation for the large number of relatively weak disturbances which arrive at the array from an azimuth of $140^{\circ}$ (e.g., Figure $7 b$ ) is that they originate in the interaction of katabatic flow from the range of small hills to the southeast of the array with the developing stably stratified nocturnal boundary layer on the featureless plain to the north. The possible significance of katabatic drainage in the low-latitude regions of Australia has been discussed by Reynolds and Gething [1970] and by Clarke [1972].

The most probable explanation of the large-amplitude solitary-wave-dominated disturbances (e.g., Figure 13) which originate in the direction of the Gulf of Carpentaria, $550 \mathrm{~km}$ to the north, is that they are generated in the interaction of a sea breeze vortex or in the interaction of the 'morning glory' phenomenon with the boundary layer radiation inversion. The morning glory is a spectacular propagating isolated roll cloud formation which is frequently observed along the southern and eastern coasts of the Gulf of Carpentaria. It has been suggested that this unusual phenomenon may represent a propagating katabatically produced internal hydraulic jump [Clarke, 1972], a traveling sea breeze vortex (R. H. Clarke, private communication, quoted by Neal et al. [1977]), or an orographically produced propagating gravity wave disturbance [Neal et al., 1977]. In view of the observations presented in this paper it seems very likely that the morning glory phenomenon is in fact a manifestation of a fairly well developed large-amplitude isolated solitary wave or group of solitary waves. In this interpretation the roll clouds are associated with the closed circulation cells in the streamline pattern of largeamplitude deep-fluid internal solitary waves propagating along a marine inversion.

Regardless of the exact nature of the source disturbance it is clear that the production of solitary waves will depend on the existence of a suitably stratified boundary layer. This accounts for the failure to observe solitary waves in the thoroughly mixed turbulent daytime boundary layer. In general, the interaction of a propagating intrusion with a density-stratified deep fluid will result, initially, in the formation of a long smooth wave at the head of the intrusion; this wave will subsequently evolve according to the Benjamin-Ono equation. A significant portion of the energy of the intrusion is therefore dissipated directly into the production of long waves and into the production of closed circulation cells in the larger-amplitude solitons. In the asymptotic state, all trace of the original intrusive fluid body is lost, and the disturbance is reduced to a finite number of stable, independent, solitary atmospheric waves.

The main conclusion of this investigation is that intrusive density flows in the atmosphere provide a primary source of solitary atmospheric waves. It should be noted, however, that other types of disturbances (T. Maxworthy, unpublished manuscript, 1978) may play an equally important role in soliton production. For example, solitary waves may evolve from the disturbance associated with the dissipation of a Kelvin-
Heln

wave

flow

ntru:

resem

ges o

water

therm

must 1

these

charac

Nevert

fundan

ties of

either

regard,

detailec

atmosp

through

examine

spheric

This $i$

cal fluid

little is

nisms th

role play

topogray

these lar

of the $1 \mathrm{c}$

tually le:

totic sol

further e

achieve

phenome

Acknow

J. S. Turr

tions on tl

authors ha

on the nat

wish to th

ating the $\mathrm{V}$

the Air Fi

$75.2759 \mathrm{~A}$.

Ablowitz,

Appl. M.

Ablowitz,

spectrum

Phys., 14

Ablowitz,

de Vries

Benjamin,

depth, $J$

Berezin, $Y_{1}$

bances ir

Engl. Tr

Brekhovski

Serikov, :

856-864,

Cercignani,

mento, 7 .

Christie, D.

the atmos

Clarke, R.

$D_{y n}, 2,3$

Clarke, R. F

Appl. Met 
Helmholtz instability or the collapse of standing eddies and lee waves induced by a sudden variation in the properties of the flow over an orographic feature.

It is evident that the atmospheric solitary-wave-dominated intrusive disturbances described in this paper bear a strong resemblance to the well-known soliton-associated internal surges observed in the oceans and in inland stratified bodies of water. Since the density profile and shear structure of the thermocline differ from those of the nocturnal inversion, it must be expected that the characteristics of the marine form of these nonlinear wave disturbances will differ in detail from the characteristics of the equivalent atmospheric disturbances. Nevertheless, all of these disturbances belong to the same fundamental class of fluid motions, and thus the basic properties of these large-scale waves may be profitably studied in either the marine or the atmospheric environment. In this regard, it is worth noting that it is easier to obtain accurate detailed experimental observations of these disturbances in the atmospheric boundary layer. Moreover, it should be possible, through the use of portable microbarograph array elements, to examine the complete evolutionary pattern of these atmospheric disturbances.

This investigation leaves a number of interesting geophysical fluid dynamical problems unanswered. In particular, very little is known about the detailed form of the source mechanisms that excite these disturbances, the exact nature of the role played by strong nonlinearity, the relative importance of topographic perturbations, the influence of the passage of these large-amplitude disturbances on the dynamical structure of the lower troposphere, and the mechanisms which eventually lead to the destruction of the extremely stable asymptotic solitary wave component. It is therefore evident that further experimental observations will be required in order to achieve a thorough understanding of these nonlinear wave phenomena.

Acknowledgments. The authors wish to express their gratitude to J. S. Turner for many valuable discussions and perceptive observations on the interpretation of complex geophysical fluid motions. The
authors have also benefited greatly from many stimulating discussions on the nature of solitary waves with T. Maxworthy. In addition, we wish to thank Blair Lade and Phillip Taylor for their efforts in operating the Warramunga seismic station. This research was supported by the Air Force Office of Scientific Research under contract AFOSR$75.2759 \mathrm{~A}$.

\section{REFERENCES}

Ablowitz, M. J., Lectures on the inverse scattering transform, Stud. Appl. Math., 58, 17-94, 1978

Ablowitz, M. J., and A. C. Newell, The decay of the continuous spectrum for solutions of the Korteweg-de Vries equation, J. Math. Phys., 14, 1277-1284, 1973.

Ablowitz, M. J., and H. Segur, Asymptotic solutions of the Kortewegde V ries equation, Stud. Appl. Math., 57, 13-44, 1977.

Benjamin, T. B., Internal waves of permanent form in fluids of great depth, J. Fluid Mech., 29. 559-592, 1967

Berezin, Yu. A., and V. I. Karpman, Nonlinear evolution of disturbances in plasmas and other dispersive media, Sov. Phys. JETP, Engl. Transl., 24, 1049-1056, 1967.

Brekhovskikh, L. M., K. V. Konjaev, K. D. Sabinin, and A. N Serikov, Short-period internal waves in the sea, J. Geophys. Res., 80 856-864, 1975.

Cercignani, C., Solitons: Theory and applications, Riv. Nuovo Cimento, 7, 429-469, 1977.

Christie, D. R., K. J. Muirhead, and A. L. Hales, On solitary waves in the atmosphere, J. Atmos. Sci., 35, 805-825, 1978.

Clarke, R. A., Solitary and cnoidal planetary waves, Geophys. Fluid Dyn., 2, 343-354, 1971.

Clarke, R. H., The morning glory: An atmospheric hydraulic jump, $J$ Appl. Meterol., 11, 304-311, 1972.
Davis, R. E., and A. Acrivos, Solitary internal waves in deep water, $J$ Fluid Mech., 29, 593-607, 1967

Gardner, C. S., J. M. Greene, M. D. Kruskal, and R. M. Miura, Method for solving the Korteweg-de V ries equation, Phys. Rev. Lett., 19, 1095-1097, 1967.

Gardner, C. S., J. M. Greene, M. D. Kruskal, and R. M. Miura, Korteweg-de Vries equation and generalizations, VI, Methods for exact solution, Commun. Pure Appl. Math., 27, 97-133, 1974.

Gargett, A. E., Generation of internal waves in the Strait of Georgia, British Columbia, Deep Sea Res., 23, 17-32, 1976

Gaul, R. D., Observations of internal waves near Hudson Canyon, $J$. Geophys. Res., 66, 3821-3830, 1961

Halpern, D., Observations on short-period internal waves in Massachusetts Bay, J. Mar. Res., 29, 116-132, 1971.

Hammack, J. L., A note on tsunamis: Their generation and propagation in an ocean of uniform depth, J. Fluid Mech., 60, 769-799, 1973

Hammack, J. L., and H. Segur, The Korteweg-de Vries equation and water waves, 2, Comparison with experiments, J. Fluid Mech., 65. 289-314, 1974 .

Hammack, J. L., and H. Segur, Modelling criteria for long water waves, J. Fluid Mech., 84, 359-373, 1978 a.

Hammack, J. L., and H. Segur, The Korteweg-de Vries equation and water waves, 3, Oscillatory waves, J. Fluid Mech., 84, 337-358,
$1978 b$.

Hirota, R., Exact solution of the Korteweg-de Vries equation for multiple collisions of solitons, Phys. Rev. Lett., 27, 1192-1194, 1971.

Hirota, R., and J. Satsuma, N-soliton solutions of model equations for shallow water waves, J. Phys. Soc. Jap., 40, 611-612, 1976.

Hunkins, K., and M. Fliegel, Internal undular surges in Seneca Lake: A natural occurrence of solitons, J. Geophys. Res., 78, 539-548, 1973.

Ivanov, V. A., and K. V. Konyayev, A bore on the thermocline (in Russian), Izv. A kad. Nauk SSSR Fiz. Atmos. Okeana, 12(4), 416423, 1976. (Izv. Acad. Sci. USSR Atmos. Oceanic Phys., Engl.
Transl., 12(4), 251-255, 1976.)

Joseph, R. I., Multi-soliton-like solutions to the Benjamin-Ono equation, J. Math. Phys., 18, 2251-2258, 1977a.

Joseph, R. I., Solitary waves in a finite depth fluid, J. Phys. A, 10 , L225-L227, 1977b.

Joseph, R. I., and R. Egri, Multi-soliton solutions in a finite depth fluid, J. Phys. A, II, L97-L102, 1978.

Kaup, D. J., and A. C. Newell, Solitons as particles, oscillators, and in slowly changing media: A singular perturbation theory, Proc. Roy. Soc. London, Ser. A, 361, 413-446, 1978.

Kikuchi, H., Shocks, solitons and the plasmapause, J. Atmos. Terr. Phys., 38, 1055-1060, 1976.

Ko, K., and H. H. Kuehl, Korteweg-de Vries soliton in a slowly varying medium, Phys. Rev. Lett., 40, 233-236, 1978.

Korteweg, D. J., and G. de Vries, On the change of form of long waves advancing in a rectangular canal and on a new type of long stationary waves, Phil. Mag., Ser. 5, 39, 422-443, 1895.

Lamb, G. L., Jr., Bäcklund transformations for certain nonlinear evolution equations, J. Math. Phys., 15, 2157-2165, 1974.

Lee, C.-Y., and R. C. Beardsley, The generation of long nonlinear internal waves in a weakly stratified shear flow, J. Geophys. Res., 79, 453-462, 1974.

Levikov, S. P., Nonstationary and slightly nonlinear internal waves in a deep ocean, Oceanology. Engl. Transl., 16, 551-554, 1977.

Long, R. R., Solitary waves in the westerlies, J. Atmos. Sci., 2I, 197$200,1964$.

Maxworthy, T., On the formation of internal solitary waves from the gravitational collapse of mixed regions in two and three dimensions, submitted to J. Fluid Mech., 1978.

Maxworthy, T., and L. G. Redekopp, A solitary wave theory of the Great Red Spot and other observed features of the Jovian atmosphere, Icarus, 29, 261-271, 1976.

Meiss, J. D., and N. R. Pereira, Internal wave solitons, Phys. Fluids, 21, 700-702, 1978

Neal, A. B., I. J. Butterworth, and K. M. Murphy, The morning glory, Weather, 32, 176-183, 1977.

Ono, H., Algebraic solitary waves in stratified fluids, J. Phys. Soc. Jap., 39, 1082-1091, 1975.

Redekopp, L. G., On the theory of solitary Rossby waves, J. Fluid Mech., 82, 725-745, 1977

Reynolds, R. M., and J. T. Gething, Acoustic sounding at Benalla, Victoria and Julia Creek, Queensland, Publ. 17, Rep. V-VII, Project volve from the of a Kelvin- 
EAR, Meteorol. Dep., Univ, of Melbourne, Melbourne, Australia, 1970.

Scott, A C. F Y F. Chu, and D. W. McLaughlin, The soliton: A new concept in applied science, Proc. IEEE, 61, 1443-1483, 1973.

concting of the British Associatience, pp. 417-496, London, 1837

Solutions of the equation, 1, J. Fluid Mech., 59, 721-736, 1973.

Thorpe, S. A. A. Hall, and I. Crofts, The internal surge in Loch Ness, Nature, 237, 96-98, 1972.

Ursell, F., The long-wave paradox in the theory of gravity waves, Proc. Cambridge Phil. Soc., 49, 685-694, 1953.

Vliegenthart, A. C., On finite-difference methods for the Korteweg-de Vries equation, J. Eng. Math., 5, 137-155, 1971.

Wadat $M$, and M. Toda, The exact Noliton solution of the Korteweg-de Vries equation, J. Phys. Soc. Jap., 32, 1403-1411, 1972

Weidman, P. D., and T. Maxworthy, Experiments on strong interactions between solitary waves, J. Fluid Mech., 85, 417-431, 1978.

Whitham, G. B., Variational methods and applications to water waves, Proc. Roy. Soc. London, Ser. A, 299, 6-25, 1967.
Whitham, G. B., Linear and Nonlinear Waves, Wiley-Interscience, New York, 1974

Zabusky, N. J., A synergetic approach to problems of nonlinear dispersive wave propagation and interaction, Proceedings of the Symposium on Nonlinear Partial Differential Equations, edited by W. F Ames, pp 223-258, Academic, New York, 1967.

W. F. Ames, Zabusky, N. J., and C. J. Galvin, Shallow-water waves, the Korteweg. de Vries equation and solitons, J. Fluid Mech., 47, 811-824, 1971. collisionless plasma and the recurrence of initial states, Phys. Rev collisionless plasma and $15,240-243,1965$.

Ziegenbein, J. Spatial observations of short internal waves in the Strait of Gibraltar, Deep Sea Res., 17, 867-875, 1970.

(Received November 1, 1978 revised February 28, 1979 accepted March 23, 1979.) 


\section{Section 2.3}

\section{Solitary waves in the lower atmosphere}

(D.R. Christie, K.J. Muirhead and R.H. Clarke)

Nature, 293, 46-49, 1981. 


\section{Solitary waves in the lower atmosphere}

\section{R. Christie \& K. J. Muirhead}

Research School of Earth Sciences, Institute of Advanced Studies, Australian National University, Canberra, ACT 2600, Australia

\section{R. H. Clarke}

Department of Meteorology, School of Earth Sciences

University of Melbourne, Parkville, Victoria 3052, Australia

Solitary waves are of intense interest in the physical and mathematical sciences'. These nonlinear waves often seem to have a primary role in the asymptotic description of propagating disturbances in inland lakes and coastal waters ${ }^{2-4}$, in the thermocline of the open sea $^{5}$ and in the lower atmosphere ${ }^{6,7}$. We present here new acoustic sounder observations of complex tropospheric solitary-wave disturbances at Tennant Creek in the arid interior of Australia (Fig. 1), a description and interpretation of a new type of visible wave phenomena over northern Australia which appear as thin propagating cumulus cloud lines, and a discussion of observations at Burketown on the Gulf of Carpentaria of a new class of low-altitude propagating solitary-wave roll clouds which originate to the south. These observations, when correlated with observations at Tennant Creek, indicate that solitary-wave-generating disturbances in the form of internal bores ${ }^{8,9}$ propagate over large distances.

Nonlinear internal waves on a boundary layer waveguide in an infinitely deep fluid are described to first order by the BenjaminOno equation ${ }^{10,11}$. The general properties of the solutions of nonlinear evolution equations of this type are well known ${ }^{1.12-22}$ Arbitrarily long, but finite, initial disturbances in the form of long waves of elevation or bores of limited spatial extent evolve into a finite number of solitary waves followed by a complex dispersing oscillatory wave train ${ }^{14.17 .18}$. Infinitely long smooth bores or waves of elevation develop undulations along the leading edge which evolve asymptotically into solitary waves ${ }^{22}$ These characteristic patterns provide a guide for the interpretation of evolving long internal wave disturbances in the atmosphere.

The properties of solitary waves in the lower troposphere have been determined from surface micropressure observations recorded continuously over a $5-\mathrm{yr}$ period on a high-sensitivity infrasonic array ${ }^{6}$ located at Warramunga near Tennant Creek (Fig. 1), and from observations ${ }^{7}$ of solitary waves associated with the Morning Glory phenomenon ${ }^{23.24}$ of the Gulf of Carpentaria. Observations have been made of a wide variety of nonlinear wave phenomena ranging in form from essentially pure asymptotic solitary-wave states to complex evolving solitary-wavedominated disturbances and undular internal bores which seem to represent early stages in the production of solitary waves. The detailed structure of two extensive solitary-wave-dominated disturbances may be seen in the acoustic facsimile records from Warramunga (Figs 2,3). These particular events are of only modest amplitude compared with many of the observations recorded on the infrasonic array. In both cases the solitary wave component appears along the leading edge of a more extensive disturbance in the form of a complex internal bore.

A noteworthy feature of internal solitary waves is the closed circulation cell which develops in the interior of large-amplitude solitary waves ${ }^{11.17 .25}$. If sufficient moisture is present solitary waves may appear as horizontal propagating retrograde lowlevel roll clouds. The cloud formation accompanying the Morning Glory of the Gulf of Carpentaria is a spectacular phenomenon of this type ${ }^{7}$. Robin ${ }^{26}$ has described a propagating roll cloud family over Spencer Gulf in South Australia which may also be interpreted as a visible manifestation of solitary waves propagating on a marine inversion.
Fig. 1 Map of northern Australia showing the location (O) of the infrasonic array and acoustic sounder installations at the Warramunga Infrasonic Array near Tennant Creek and the positions ( $)$ and orientations of three observations of unusual low-altitude propagating cloud line events which correlate with events at 03.50 CST $(5$ October 1979) (A) 23.25 CST $(24$ July 1979) (B) and 00.50 cst (6 October 1979) (C) detected during the preceding night at Warramunga. The measured propagation speeds and source azimuths determined from the phased array records are: $A: c=5.6 \mathrm{~m} \mathrm{~s}^{-1}, \quad \theta=215^{\circ} ; \quad \mathrm{B}: \mathrm{c}=$ $11.7 \mathrm{~m} \mathrm{~s}^{-1}, \theta=235^{\circ} ; \quad \mathrm{C}: \mathrm{c}=9.8 \mathrm{~m} \mathrm{~s}^{-1}$ $\theta=180^{\circ}$

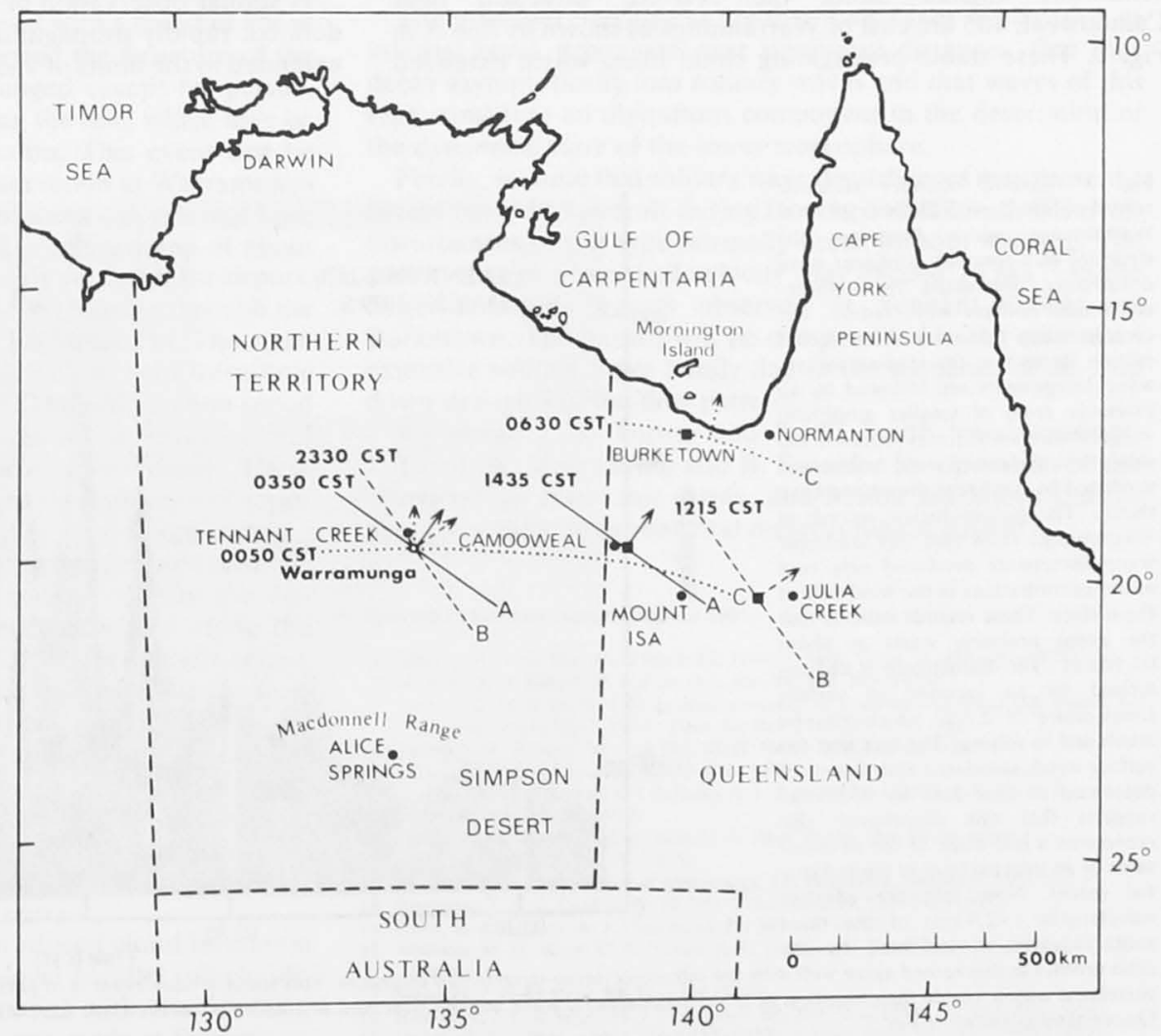


Fig. 2 Monostatic acoustic sounder facsimile record obtained at Warramunga for the period from 0350 to mut 15 c si ton 22 October 1979 which phology of a well defined solitary-wave-dominated complex disturbance which arrived at the array shorlly before the transition of transition of laver to a convecting davtime boundary ayer. The dark portions of the record cepresent regions of enhanced echo returns from small-scale temperature inhomogeneitics. The corresponding micropressure and surface wind records icreate that the major nonlinear wave disturbance in this record persisted for

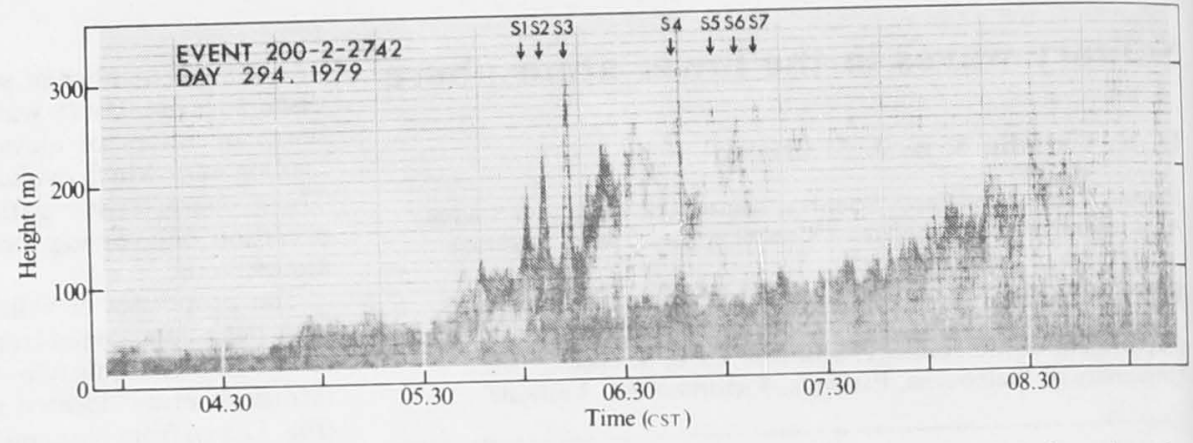

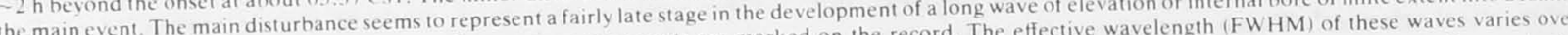
he maine (1.4-2.4km Sunrise $4-2.4 \mathrm{~km}$. Sunrise occurred at 06 cord bevond $(17.30$ cs

The structure of the boundary layer over northern Australia is typically characterized ${ }^{27}$ by a persistent marked elevated inversion at an altitude of several kilometres overlying, during the night time and early daylight hours, an intense surface-based ration the onset of convective activity the radiation inversion. Wis eroded away from its base b. $^{28.29}$ and usually vanishes before noon. It must be expected that relatively smallvarimarily with the scale nonlinear wave disturbances asso influence of convection, nocturnal inversion will, under the day. Larger-amplitude disdecay and disappear during the day. Larger-amplitude disturbances which involve the elevated inversion

expected to survive for longer periods of time.

The following observations indicate that long nonlinear wave disturbances propagate over substantial distances along these boundary layer waveguides. On 5 October 1979, at 14.35 CST, two closely spaced propagating quasicontinuous thin cumulus cloud lines were observed from the ground in totally clear conditions aligned along the NW-SE direction near Camooweal, $405 \mathrm{~km}$ east of Warramunga as shown by line $\mathrm{A}$ in Fig. 1. These stable propagating cloud lines, which extended form horizon to horizon, had a width and depth of some hundreds of metres and a spacing of some kilometres. This observation is correlated in direction and in time, assuming a mean propagation speed of about $6 \mathrm{~m} \mathrm{~s}^{-1}$, with the nonlinear wave disturbance shown in Fig. 3, observed about $11 \mathrm{~h}$ earlier at Whe disturbance apparently dissolved during the afternoon of 5 October.

The preferred explanation of the two coherent propagating cloud lines observed near Camooweal is that they represent capping clouds created by the lifting of moist air to the condencapping clouds created by the lifting of moist air to the condensation level during the passage of solitary waves associated wh the leading edge of a dissipating finite-length internal bore. The character of the solitary wave pattern can be expected to evolve char nonlinear wave disturbance adjusts to the 列 evolving directly to any unlikely that the observed cloud lines

A similar observation of a single, almost continuous, clearly Aefined, rapidly propagating cumulus cloud line, which again extended to the limits of visibility, was made in otherwise totally Warramunga which illustrates the structure of a complex nonlinear wave disturbance dominated bi a threeomponent solitary wave family. The corresponding infrasonic array record
section shows that the leading solitary section shows that the leading solitary cxtensive train of smaller amplitude irregular waves which may represent the subcritical dispersing wave component predicted by nonlinear dispersive wave theory. The meteorological records at Warramunga show that this nonlinear wave disturbance produced only very miner perturbations in the wind field at the surface. These records indicate that the event probably starts at about $03.10 \mathrm{CST}$. The disturbance is characterized by an increase in surface temperature of $2.5^{\circ} \mathrm{C}$ which may be lemperature of $2.5^{\circ} \mathrm{C}$ which may be The fact that the surface winds associated with this even decreased to near zero by $05.00 \mathrm{CST}$ suggests that this disturbance also represents a late stage in the developrepresents a late stage in the developtial extent. Note that the effective wavelengths $(\sim 2.9 \mathrm{~km})$ of the three echo profiles in this record agree well with the infrasonic-array-determined estimates. This solitary wave event is of particular interest because it seems to be well correlated with a visible manifesta

Queensland as shown in Fig. 1.

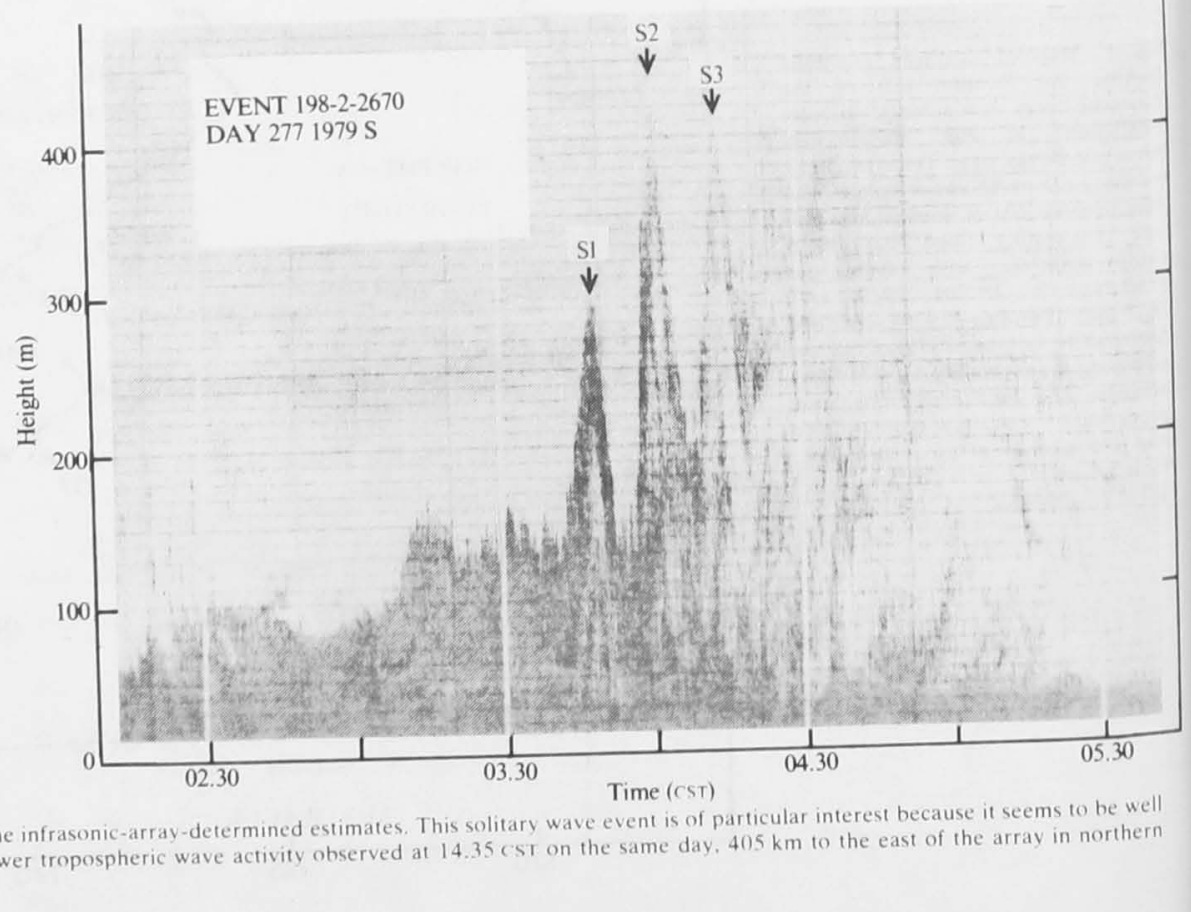

$135^{\circ}$

Fig. 4

Octob

paralle

bounda

vations

Fig.

were

clear cor

of Julia

event w:

the clou

$12 \mathrm{~m} \mathrm{~s}^{-}$

cloud lis

bations

attribute
correlate

at 23,30

with an

$1.5 \mathrm{mba}$

and with

anemom

nocturna

decrease

then inct

variatior

boundar

available

turbance

direction

suggestir

level der

single cld

a large a

of a fini

advancin

this case.

with the

A diff

Consistin

similar i

Glory ${ }^{7}$ 


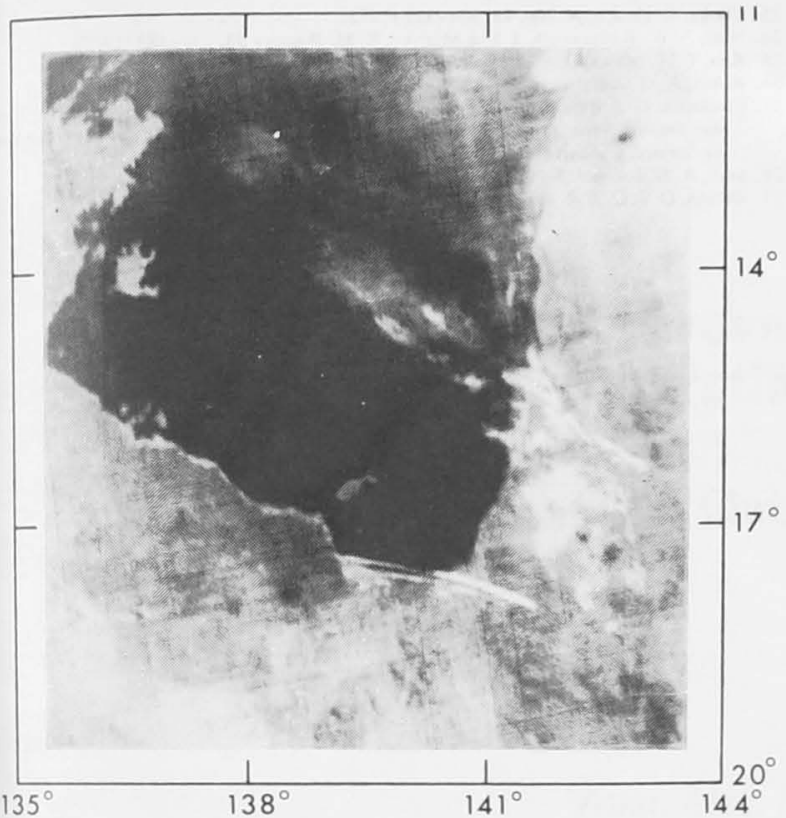

Fig. 4 Visual imagery of the Gulf of Carpentaria area from the DMSP Meteorological Satellite Program) satellite at 07.07 CST on 6 1979 , showing the position and orientation of two slightly curved October 1979, showing the position and orientation of two slightly curved
parallel low-altitude roll clouds produced by solitary wave activity in the boundary-layer waveguide. These cloud lines can be correlated with obserations of nonlinear wave pressure perturbations and surface wind shear during the preceding night at Warramunga and Mount Isa as shown in The cloud lines are $285 \mathrm{~km}$ long, $\sim 13 \mathrm{~km}$ apart and $\sim 6 \mathrm{~km}$ wide and observed to propagate towards an azimuth of $10^{\circ}$ with a speed of $15 \mathrm{~ms}$

ore. The to evolve sts to the fore ver tly to any

s, clearly ich again se totally

clear conditions over an essentially featureless plain, $75 \mathrm{~km}$ west of Julia Creek (Fig. 1, line B) at 12.15 CST on 25 July 1979. This event was tracked by vehicle for $2 \mathrm{~h}$ and it was established that the cloud line was propagating north-east with a speed of about $12 \mathrm{~m} \mathrm{~s}^{-1}$. During this observational period the structure of the cloud line remained essentially unchanged except for perturbations in the modulation pattern along the line, which may be attributed to the influence of convection. This event can be correlated with an infrasonic array observation at Warramunga at 23.30 CST on 24 July, in the form of a smooth internal bore with an amplitude of $0.8 \mathrm{mbar}$, with a pressure jump of about 1.5 mbar observed at 07.00 CST on 25 July at Mount Isa airport, and with a marked wind shift (NNE to SW) moving through the anemometer array operated by Mount Isa Mines Ltd. The mean nocturnal speed of $13 \mathrm{~m} \mathrm{~s}^{-1}$ agrees fairly well with the infrasonic array estimate of $11.7 \mathrm{~m} \mathrm{~s}^{-1}$ from $235^{\circ}$. The propagation speed decreased to an average of $\sim 8 \mathrm{~m} \mathrm{~s}^{-1}$ during the morning and then increased to $\sim 12 \mathrm{~m} \mathrm{~s}^{-1}$ during the early afternoon. These variations in speed may be attributed to the influence of topography in the neighbourhood of Mount Isa and to the evolving boundary layer density structure. In this particular event the available wind soundings behind the leading edge of the disturbance show a low-level wind speed component along the direction of motion nearly equal to the propagation speed, suggesting that this disturbance must be associated with a lowlevel density current. The preferred explanation of this unusual single cloud line is that it represents a capping cloud produced by arge amplitude solitary wave associated with the leading edge of a finite-length internal bore initiated by a density current advancing into the stratified boundary layer. Alternatively, in this case, the propagating cloud line may be due to lift associated with the head of an advancing gravity current

A different form of solitary-wave-produced cloud formation consisting of two well developed low-level parallel roll clouds, Similar in all respects to those associated with the Morning Glory but originating from the south, was made at Burketown
(Fig. 1, line C) at 06.30 CST on 6 October 1979. Visual satellite imagery of these waves is shown in Fig. 4. Remnants of three cloud lines further north with similar orientation were also seen in the visual imagery at 09.30 CST. The cloud lines appeared to propagate with a phase speed of $\sim 15 \mathrm{~m} \mathrm{~s}^{-1}$ from $190^{\circ}$ and were coherent with pressure jumps on the barographs at Normanton (07.00 CST), Mornington Island (08.15 CST) and Mount Isa (0000 CST). Wind data from the Mount Isa Mines Ltd mesoscale array reveal sharp changes from very light southwesterly to fresh southerly winds, gusting above $13 \mathrm{~m} \mathrm{~s}^{-1}$, unaccompanied by any sign of solitary wave activity. The disturbance was observed at Warramunga at 00.50 CST in the form of an internal bore of amplitude 0.7 mbar. The array-determined speed $\left(9.8 \mathrm{~m} \mathrm{~s}^{-}\right.$ and azimuth $\left(180^{\circ}\right)$ imply a curvature in the wavefront in agreement with the pattern defined by the cloud lines shown in Fig. 4. Again, these observations may be attributed to the passage of a disturbance in the form of an internal bore of finite extent which evolves asymptotically in time into a family of solitary waves.

On all occasions the propagating cloud lines and coherent pressure signatures seem to be closely related to synoptic developments. On the early morning of 5 October a trough lay $\sim 400 \mathrm{~km}$ south-west of Warramunga with a NW-SE orientation. Over the next $36 \mathrm{~h}$ this feature, with a following high pressure ridge, moved east across the Australian continent at a speed of about $7 \mathrm{~m} \mathrm{~s}^{-1}$ (at lat. $20^{\circ}$ ). The observations presented above indicate that on the evening of 5 October, on the southwest side of this trough, evidently as a result of deformation of the nocturnal boundary layer, a propagating internal bore developed in the area south of Tennant Creek and moved NNE at over $15 \mathrm{~m} \mathrm{~s}^{-1}$ on 6 October for at least $700 \mathrm{~km}$. It developed solitary waves, made visible by propagating roll cloud lines in the Gulf area as shown in Fig. 4. The sequence observed on 24-25 July was similar, except that in this case the trough, pressure jump line and wind shear line were coincident throughout their known history, progressing steadily eastward at about $12 \mathrm{~m} \mathrm{~s}$ for at least $8 \mathrm{~h}$ and, in the case of the trough and shear line, for a further $21 \mathrm{~h}$ after the cloud line had been seen near Julia Creek.

The principal conclusions are that long waves of elevation or internal bores propagate over significant distances, that they decay asymptotically into solitary waves and that waves of this type constitute an ubiquitous component in the description of the dynamical state of the lower troposphere

Finally, we note that solitary wave disturbances may present a severe hazard to aircraft during landing and take-off. Clear-air disturbances of this type normally occur without warning. The vector change in vertical velocity may exceed $16 \mathrm{~m} \mathrm{~s}^{-1}$ in the larger-amplitude events observed at Tennant Creek and Burketown. The hazard will be compounded in the case of an extensive solitary wave family due to the sequence of up- and down-draughts in the flow pattern.

We thank J. S. Turner and R. K. Smith for discussions, J. Tarca, A. Mainwaring and B. Schaefer for operation of the Warramunga Infrasonic Array, and Mount Isa Mines Ltd for data from their environmental meteorological network.

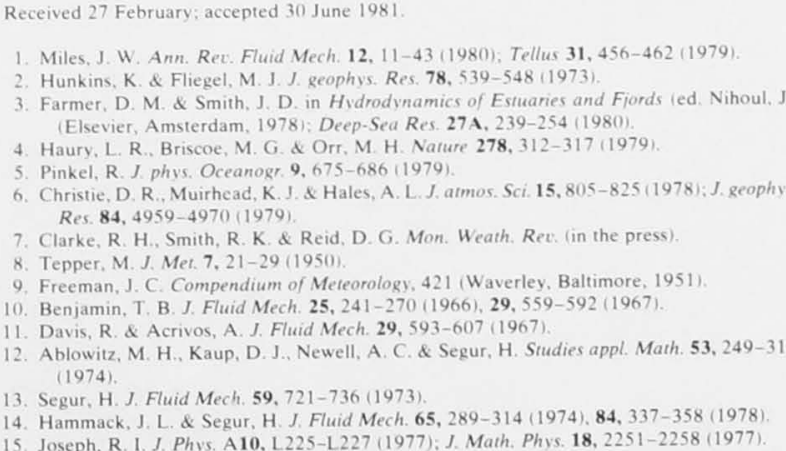


16. Kubota, T., Ko, D. R. S. \& Dobbs, L. J. Hydron.

$12,157-165(1978)$

7. Maxworthy, T. J. Fluid Mech. 96, 47-64 (1980).

18. Meiss, J. D.. d Percira, N. R. Phys. Fle

19. Maslowe, S. A. \& Redekopp, L2 G. Geopho)

(1979): J. Fluid Mech 101, 321 348 (1980) 1909 ) Math Res. Rep 44 University of

0. Grimshaw, R. Proc. R. Soc. A368, 359-375 (1979); Mart. Res. Rep. (in the press).

Melbourne, 1980); Studies appl. Mart. (in the press, 74A. 233-235 (1979).

21. Matsuno, Y. J. Phys. A12, 619-621 (1979); Phys. Letl.
23. Clarke, R. H. J. appl. Met. 11, 304-311 (1972).

. Weather 32, 176-183,1977).

24. Neal, A. B., Butterworth, 1. J. \& Murphy, K, 115-127 (1979)

25. Kao, T. W. \& Pao, H.-P. Fluid Mech, 978 )

26. Robin, A. G. Aust. Met. Mag. 26, 125 (1978). Clarke, R. H. \& Brook, R. R. The Koorin Expedition. Armostralian Government Publish. ver Tropical Savannah Land

28. Stull, R. B. J. atmos. Sci. 30, $1092-1099-467(1973)$ 


\section{Section 2.4}

\section{Solitary waves: A hazard to aircraft operating at low altitudes}

(D.R. Christie and K.J. Muirhead)

Aust. Met.Mag., 31, 97-109, 1983. 


\title{
Solitary waves: a hazard to aircraft operating at low altitudes
}

\author{
D. R. Christie and K. J. Muirhead, Research School of Earth \\ Sciences, Institute of Advanced Studies, Australian National \\ University, Canberra \\ (Manuscript received September 1982; revised November 1982) \\ This paper is concerned with the serious hazard to the operation of aircraft at low \\ altitudes created by solitary waves in the atmospheric boundary layer. A thorough \\ investigation of these non-linear waves has shown that they occur frequently at all \\ times of the year over northern Australia, that they often occur without warning as \\ sudden clear-air disturbances and that they produce intense transient horizontal and \\ vertical wind-shear zones near the surface. A brief description is given of the properties \\ of these disturbances which relate directly to the problem of air safety. Solitary wave \\ disturbances are found to be implicated as the primary causal factor in a significant \\ number of wind-shear related aircraft accidents.
}

\section{Introduction}

ary atmospheric waves are remarkably large litude, single-crested waves of elevation which agate along horizontal wave-guides formed by sions in the stably stratified, atmospheric dary layer. It is becoming increasingly evident he intense, transient shear zones associated with recently discovered class of non-linear, spheric wave disturbance (Christie et al. 1978, constitute a serious hazard to the operation of aft at lower altitudes. Aircraft may suddenly inter severe, horizontal wind-shear conditions ounded by strong up and downdraughts ciated with the leading and trailing edges of idual solitary waves which, if they occur during anding or take-off stage, may result in large tures from the glide path leading to undershoot ershoot of the runway, or to insufficient lift take-off or possibly even to in-flight structural re. It is worth noting that modern jet aircraft be subject to particularly serious hazards under conditions. We emphasise that these essentially inear wave disturbances are known to occur ently throughout the northern Australian region probably elsewhere, that they usually occur as clear-air disturbances, and that they are seldom detected by conventional meteorological instrumentation. Under present aviation conditions, aircraft operating at low altitudes may encounte these localised, complex, clear-air disturbances completely without warning.

The purpose of this paper is to draw attention to the potentially serious hazard created by boundary layer solitary waves to the operation of aircraft at low altitudes and to present a brief description of some specific properties of solitary waves which bear directly on our understanding of the problem of low level, transient wind-shear. More work is needed to establish the basic parameters which determine the degree of hazard associated with individual disturbances and to determine with certainty the underlying factors which govern the genesis and propagation of solitary wave disturbances in the lower atmosphere.

Detailed studies of the fundamental properties of atmospheric solitary wave phenomena have recently been carried out by the Australian National University at the Warramunga Infrasonic Array located near Tennant Creek in the arid interior of the Northern Territory and, under tropical maritime conditions, at Burketown in northern Queensland. One of the principal results of these investigations is the observation that solitary wave associated disturbances maintain a coherent identity over large distances and therefore it should be possible, using suitably located sensors, to predict the occurrence of these non-linear waves with sufficient accuracy to allow Air Traffic Controllers to issue warnings and to undertake other precautionary measures. This aspect and some preliminary recommendations will be considered briefly in the last two sections of this paper

\section{Solitary atmospheric waves}

Only in the last few years has it been recognised that solitary waves play an important fundamental role in the description of a wide variety of geophysical fluid dynamical phenomena (the reader is referred to the excellent review articles by Scott et al. (1973) and Miles (1980) for background material on solitary waves). This importance stems from the observation that arbitrary, initial long wave disturbances in a density-stratified fluid evolve into a finite number of internal solitary waves followed by a weak dispersing, non-linear wave train. The solitary waves, or solitons, which usually dominate the asymptotic disturbance, are supercritical waves of permanent form which maintain their identity under non-linear interaction.* 
Solitary waves in the atmospheric boundary layer belong to the class of deep fluid, internal solitary waves first considered by Benjamin (1967) and Davis and Acrivos (1967). The properties of these internal non-linear waves have been studied in detail in the laboratory (Davis and Acrivos 1967; Maxworthy 1979, 1980; Kao and Pao 1979; Koop and Butler 1981 ) and the theory of waves of this type is currently a subject of intense interest. Joseph (1977a) and Kubota et al. (1978) have derived a general evolution equation for weakly non-linear waves in a finitedepth fluid which reduces in the deep-fluid limit to the Benjamin-Ono equation (Benjamin 1967; Ono 1975), the appropriate governing equation for finite amplitude, long wave disturbances in the atmospheric boundary layer. Other significant developments include the confirmation of the nonlinear stability or soliton property for the solitary wave solutions of the Benjamin-Ono equation (Joseph 1977b; Meiss and Pereira 1978), the generalisation of the Benjamin-Ono equation in two dimensions (Ablowitz and Segur 1980; Redekopp 1980), the extension of weakly non-linear theory to higher order (Grimshaw 1981b; Joseph and Adams 1982) and the work by Benney and Grimshaw (1982) and Tung et al. (1982) on the fully non-linear problem.

A number of recent studies have focused on the detailed properties of non-linear waves in a sheared environment; these papers provide the basic theoretical framework for the interpretation of solitary wave disturbances in the atmospheric boundary layer. Maslowe and Redekopp $(1979,1980)$ and Tung et al. (1981) have presented thorough treatments of the theory of non-linear waves in stratified shear flows, including flows with critical layers. Maslowe and Redekopp $(1979,1980)$ and Pereira and Redekopp (1980) consider as well the influence of radiation damping on solitary waves propagating in a waveguide embedded in a weakly stratified, ambient environment. A general theory of non-linear waves in stratified shear flows, which includes both the effects of radiation and frictional damping and as well the influence of temporal and spatial perturbations, has been presented by Grimshaw (1981a, 1981c). Grimshaw has also derived (Grimshaw 1980) the general evolution equations which describe non-linear wave propagation in both dry and moist atmospheres. Grimshaw compares the results with incompressible theory and finds that the main influence of compressibility and moisture can be included in the theoretical description through an appropriate redefinition of the Brunt-Väisälä frequency.

* Strictly speaking, solitons do not occur in nature since waves in real fluids are always subject to perturbations such as frictional damping. Nevertheless, in most circumstances the soliton description holds to a good approximation over reasonable periods of time. Furthermore, it is convenient to retain this term when therefore be used throughout this paper with this qualification.
Observations of solitary waves over northern Australia

It is now accepted that internal solitary waves of large amplitude are a commonly occurring feature in both the oceans and the atmosphere. The ultrasensitive, infrasonic array at Warramunga was established in 1975 to study atmospheric wave activity over the northern Australian region. A survey of the long period, digital records from this facility led to the initial discovery (Christie et al. 1976) of interna solitary waves in the lower atmosphere. Following this discovery it was soon realised that under certain specific conditions solitary waves in the atmospheric boundary layer would appear as isolated, horizontal, low level, propagating roll clouds. Generally speaking, visual observations of this type comparatively rare. A noteworthy exception to thi rule is the area in the neighbourhood of Burketown on the tropical south coast of the Gulf of Carpentaria where spectacular transverse roll clouds associated with solitary waves propagating along a maritime inversion may be observed in the early morning hours during the months of September and October (Clarke 1972; Neal et al. 1977; Christie et al. 1979; Christie et al. 1981). These unusual propagating roll cloud formations are known locally as the 'Morning Glory'. The results of detailed experimental investigations of the morphology and origin of these visible, non-linear wave disturbances have recently been presented by Clarke et al. (1981) and Clarke (1983).

The photograph shown in Fig. 1 of a solitary wave produced roll cloud observed at Burketown during the spring of 1981 graphically illustrates the scale of the non-linear wave disturbances considered in this paper. The base of this propagating roll cloud is estimated to be about $0.3 \mathrm{~km}$ and the top of the cloud lies at about $1.5 \mathrm{~km}$. Note that the position of the cloud line marks the centre of a localised region of intense vertical and horizontal wind shear near the surface and that the disturbance is believed to extend above the visible cloud roll for several kilometres. Solitary wave produced roll clouds are also observed on occasion in other coastal regions of Australia. Robin (1978) has given a description of a spectacular roll cloud formation which appears to be a visible manifestation of a relatively small solitary wave disturbance propagating - probably as a pre-frontal disturbance - on a marine inversion over Spencer Gulf

Over one thousand significant, solitary wave associated, clear-air disturbances have been recorded during the seven-year observational period at the Warramunga Infrasonic Array. These non-linear, boundary layer waves clearly represent a commonly occurring, ubiquitous, component in the description of the dynamical state of the lower atmosphere. Despite this high frequency of occurrence there have only been a few isolated reports of visible, solitary wave activity over the central Australian region. Even individual, solitary wa dominated, propagate a 
ver northern

waves of large eature in both ultrasensitive established in ivity over the $y$ of the long lity led to 6) of internal re. Following under certain e atmospheric d, horizonta

Is. Generally his type ception to this of Burketown of Carpentaria Ids associa ed g a maritime morning hours ctober (Clarke 1979; Christie ing roll cloud the 'Morning experimental origin of these have recently 1) and Clarke

a solitary wave ketown during tes the scale of sidered in this g roll cloud is op of the cloud osition of the lised region of shear near the ieved to extend cal kilometres. also observed of Australia. f a spectacular to be a visible solitary wave as a pre-frontal over Spencer

solitary wave been recorded period at the se non-linear, nt a commonly the description r atmosphere ence there have isible, solitary an region. Even

Fig. 1 Approaching Morning Glory roll cloud formation produced by a solitary wave disturbance propagating towards the southwest over saline coastal flats, $5 \mathrm{~km}$ north of Burketown, Queensland at 0730 LST (local standard time), 1 October 1981.

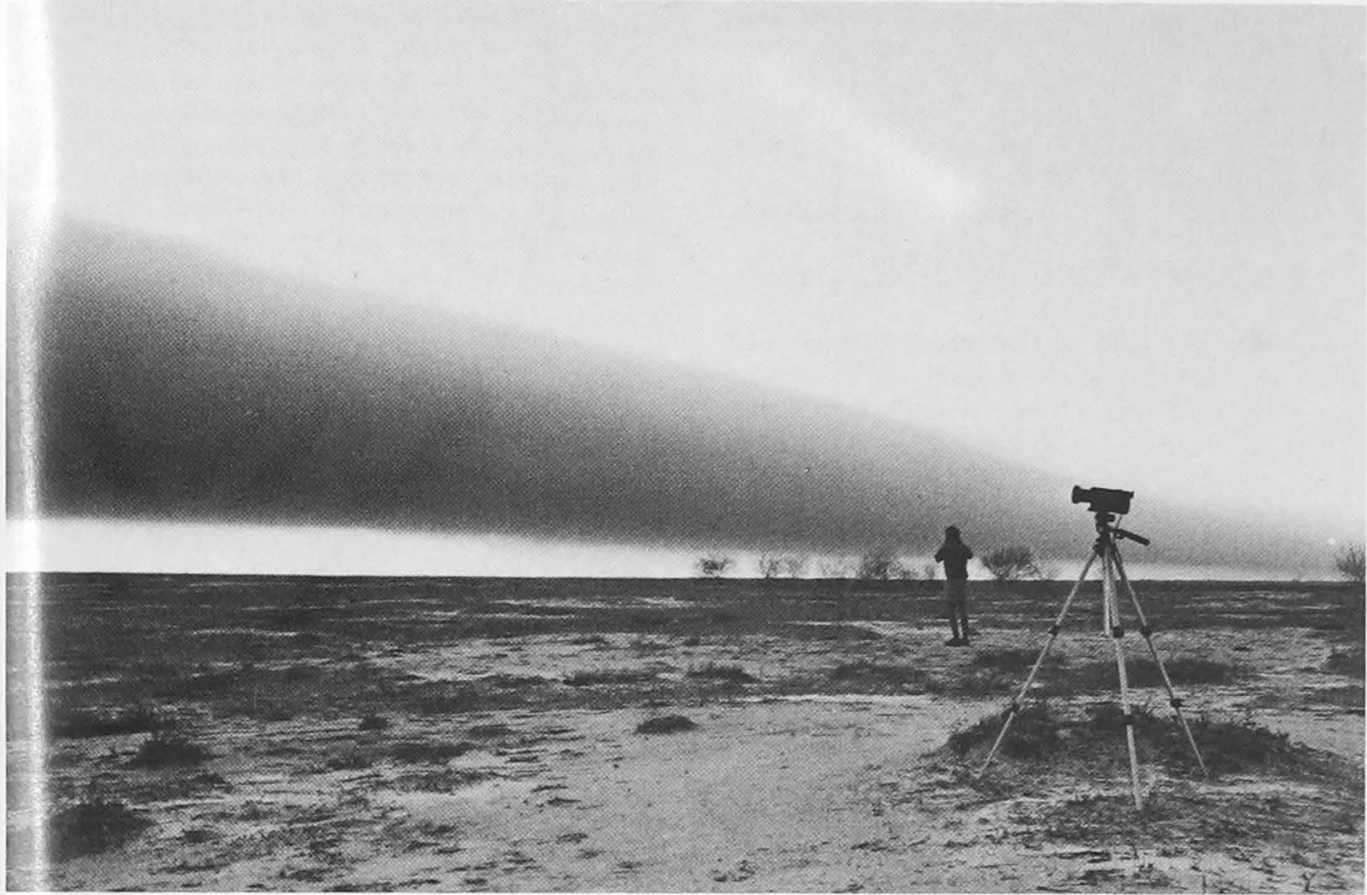

under the humid conditions prevailing along the outhern margin of the Gulf of Carpentaria, where phenomena are observed visually with some regularity at certain times of the year, it has now been established that many solitary wave disturbances occur as cloud-free, or nearly cloud-free, disturbances. For example, a total of 17 largeamplitude, non-linear wave disturbances were recorded on infrasonic microbarometers during a preliminary experimental study carried out by the Australian National University at Burketown between 23 September and 3 October 1980. Of these events, only one occurred under sufficiently ideal conditions of low level humidity to produce an associated, poorly developed, quasi-continuous roll cloud. All of these observations underline the fact that in the arid interior of Australia solitary wave disturbances almost invariably occur as sudden, clear-air disturbances and that even in coastal areas a substantial number of these frequently occurring, transient shear events will be cloud-free and thus generally undetectable with currently employed observational techniques.

The detailed studies of solitary wave phenomena at Tennant Creek and Burketown have shown that these non-linear wave disturbances occur as individual, isolated solitons; as amplitude-ordered, solitary wave packets; and as complex, solitondominated, evolving, long wave disturbances which propagate as predominately interfacial disturbances along boundary layer inversions. Typical examples of the absolute micropressure signature of solitary wave dominated, clear-air disturbances recorded on the infrasonic array $37 \mathrm{~km}$ south-southeast of Tennant Creek are shown in Fig. 2. Some further examples of complex, soliton-dominated disturbances are presented in the selection, shown in Fig. 3, of essentially differential micropressure records from the recently installed high sensitivity, infrasonic instrument at the Tennant Creek Airport. The events shown in these two diagrams illustrate various stages in the evolution of long, non-linear, dispersive wave disturbances in the lower atmosphere. Solitary waves in the atmospheric boundary layer occur only rarely as isolated individual solitons. This is to be expected since most initial disturbances give rise to more than one solitary wave component and, as well, individual solitary waves eventually fission into two or more components under the influence of perturbations such as spatial variations in the waveguide density and shear structure. Solitary waves are usually observed in the form of essentially asymptotic soliton families preceding a dispersing remnant of the original disturbance or, more frequently, as partially developed solitary wave groups condensing out along the leading edge of the parent long wave disturbance.

The solitary wave dominated disturbances observed at Burketown appear to be similar in many respects to those observed at Tennant Creek. This 
Fig. 2 Surface micropressure signatures of typical solitary wave dominated disturbances recorded at the Warramunga Infrasonic Array near Tennant Creek. The onset time, propagation speed c, source azimuth $\theta$, and effective wavelength $\lambda$ (FWHM) for the leading soliton in each event is as follows:

(a) 0546 LST 8 October $1980 ; c=12.5 \mathrm{~m} / \mathrm{s}$, $\theta=20^{\circ}, \lambda=5.0 \mathrm{~km}$

(b) 0252 LST 7 December $1978 ; c=6.5 \mathrm{~m} / \mathrm{s}$, $\theta=4^{\circ}, \lambda=3.1 \mathrm{~km}$

(c) 0544 LST 15 October $1980 ; \mathrm{c}=7.6 \mathrm{~m} / \mathrm{s}$, $\theta=7^{\circ}, \lambda=1.7 \mathrm{~km}$

(d) 0214 LST 9 December 1980; $c=14.8$ $\mathrm{m} / \mathrm{s}, \theta=58^{\circ}, \lambda=4.5 \mathrm{~km}$

(e) 2258 LST 11 August $1980 ; c=8.9 \mathrm{~m} / \mathrm{s}$, $\theta=187^{\circ}, \lambda=1.8 \mathrm{~km}$.

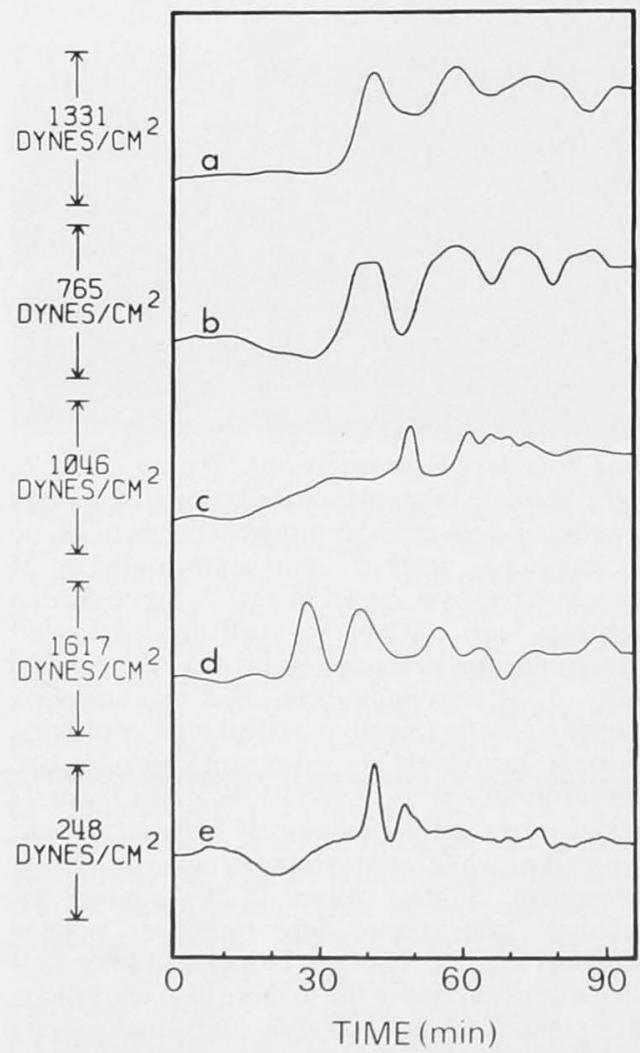

is illustrated by the absolute micropressure records from Armraynald Station near Burketown (Fig. 4) which show the structure of a typical, relatively localised, multiple, solitary wave disturbance and that of a more extensive, complex, evolving, soliton dominated disturbance.

Detailed information on the morphology of boundary layer solitary waves is provided by a monostatic acoustic sounder (see Christie et al. 1981) operated continuously in conjuction with the infrasonic array at Tennant Creek. Useful high
Fig. 3 Complex non-linear wave disturbances detected on an infrasonic microbarometer installed at the Tennant Creek Airport. Note that this high-sensitivity instrument produces an essentially differential record of longerperiod atmospheric waves. For comparison, the absolute micropressure signature observed at the Warramunga array, $37 \mathbf{~ k m}$ to the south-southeast corresponding to event (d) is shown in trace (d) of Fig. 2. Event onset times, speeds and azimuths are as follows:

(a) 0552 LST 22 December 1980; $c=10.7$ $\mathrm{m} / \mathrm{s}, \theta=28^{\circ}$

(b) 0214 LST 17 October 1980; c=10.7 $\mathrm{m} / \mathrm{s}, \theta=343^{\circ}$

(c) 2117 LST 9 December $1980 ; c=7.5 \mathrm{~m} / \mathrm{s}$, $\theta=22^{\circ}$

(d) 0136 LST 9 December 1980; $c=14.8$ $\mathbf{m} / \mathbf{s}, \theta=5^{\circ}$.

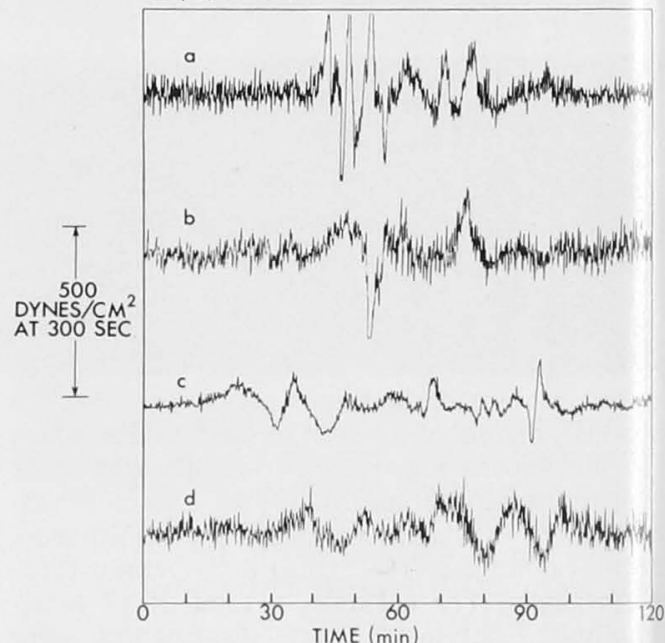

resolution, acoustic sounding records can only be obtained for relatively small-scale, solitary wave events. However, as can be seen in the typical facsimile record presented in Fig. 5, the acoustic sounder at Tennant Creek does provide a detailed measure of the structure of solitary wave disturbances. The disturbance shown in this diagram (see Fig. 2(b) for the corresponding micropressure record) is dominated by four, fairly well-developed, solitary waves along the leading edge. Note the flattened crest in the surface pressure profile of the leading solitons in this disturbance. This can be explained by the presence of a recirculating region of higher density fluid which propagates with the solitary wave as described below. The acoustic sounder records are particularly interesting in that they illustrate the remarkably large amplitudes, relative to the characteristic vertical scale of the waveguide, which typically distinguish disturbances belonging to this unique class of long internal waves.

The salient features of solitary atmospheric wave disturbances as revealed by the investigations in
Fig. 4 H

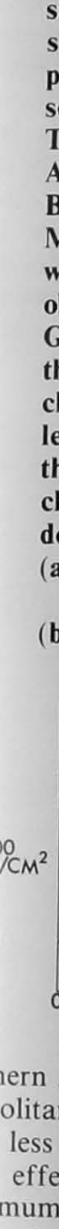

undred $r$

ucture,

first or

) To a

solitary $\mathrm{w}$

speed by a

(Benjami

Supercriti

to propag;

on occasic

observed

(c) The

disturbans

Benjamin.

disturbanc

number

suberitical pattern is Benjaminbe seen $\mathrm{fr}$ wave of $e$ amplitude 
Fig. 4 Examples of the absolute micropressure signature corresponding to typical complex solitary wave dominated disturbances propagating in a maritime inversion along the southern margin of the Gulf of Carpentaria. The observations shown here were made at Armraynald Station, $32 \mathrm{~km}$ southeast of Burketown, Queensland. Several well defined Morning Glory-type roll clouds associated with the leading solitons in event (b) were observed in the Burketown area and at Gregory Downs Station, $130 \mathrm{~km}$ inland from the Gulf of Carpentaria. Note that the roll cloud formations accompanied only the leading solitons in this disturbance and that the residual disturbance was completely cloud-free. The parameters for each event (as defined for Fig. 2) are as follows:

(a) 2246 LST 21 October $1981 ; c=9.7 \mathrm{~m} / \mathrm{s}$, $\theta=139^{\circ}, \lambda=5.2 \mathrm{~km}$

(b) 0528 LST 28 October 1981; c=14.5 $\mathrm{m} / \mathrm{s}, \theta=77^{\circ}, \lambda=3.7 \mathrm{~km}$.

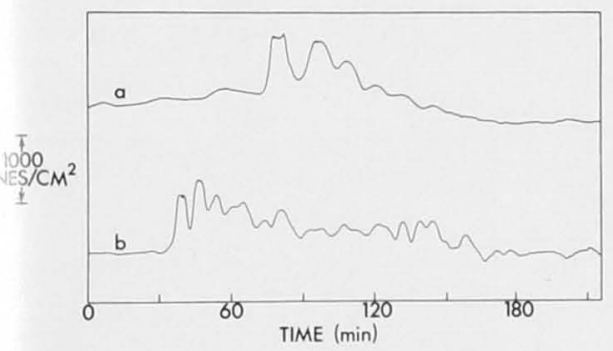

thern Australia are as follows:

Solitary waves occur with amplitudes ranging

$\mathrm{m}$ less than $0.1 \mathrm{~km}$ to greater than $1.0 \mathrm{~km}$ and effective wavelengths (full width at half imum amplitude (FWHM)) between a few dred metres and $10 \mathrm{~km}$. For a given waveguide cture, the effective wavelength varies inversely, ïrst order (Benjamin 1967), with amplitude.

b) To a first approximation, the phase speed of tary waves exceeds the linear, long wave, phase eed by an amount proportional to wave amplitude (Benjamin 1967; Davis and Acrivos 1967). Supercritical waves of this type are usually observed to propagate with speeds between 6 and $16 \mathrm{~m} \mathrm{~s}^{-1}$ but on occasion, particularly-large-amplitude waves are observed with speeds exceeding $20 \mathrm{~m} \mathrm{~s}^{-1}$.

(c) The evolution of long non-linear wave disturbances in the atmosphere is governed by the Benjamin-Ono equation. Arbitrary long wave disturbances evolve into an amplitude ordered, finite number of solitons followed by a dispersing, subcritical, oscillatory wave train. This characteristic pattern is illustrated in the numerical solution of the Benjamin-Ono equation presented in Fig. 6. As can be seen from this figure, an initially smooth long wave of elevation evolves under the influence of amplitude and frequency dispersion into an ordered
Fig. 5 Monostatic acoustic sounder facsimile record of a relatively small-amplitude solitary wave dominated disturbance observed during the early morning hours at Warramunga near Tennant Creek. Four solitons may be distinguished in this record and in the corresponding surface micropressure record shown in Fig. 2(b). Time is shown in GMT (LST $=$ GMT $+9^{1 / 2}$ hours). The array-determined propagation speed and source azimuth are $c=6.5 \mathrm{~m} / \mathrm{s}$ and $\theta=4^{\circ}$.

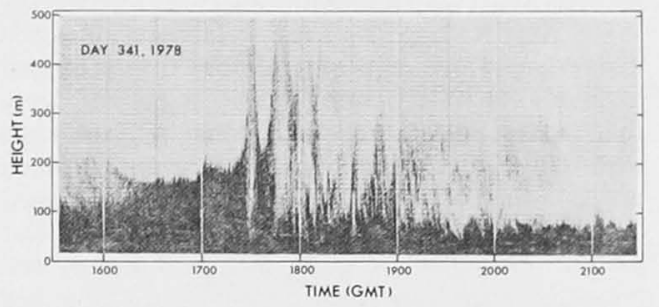

Fig. 6 Numerical solution of the Benjamin-Ono equation showing the evolution of a finitelength long wave of elevation into an amplitude-ordered set of solitary waves followed by a weak dispersing wave train. Units are non-dimensional. Coordinate system moves to the right at the critical or linear phase speed. In this frame of reference the solitary waves propagate to the right while the subcritical dispersive component propagates to the left. For clarity, the last trace is offset to show the detailed form of the asymptotic disturbance.

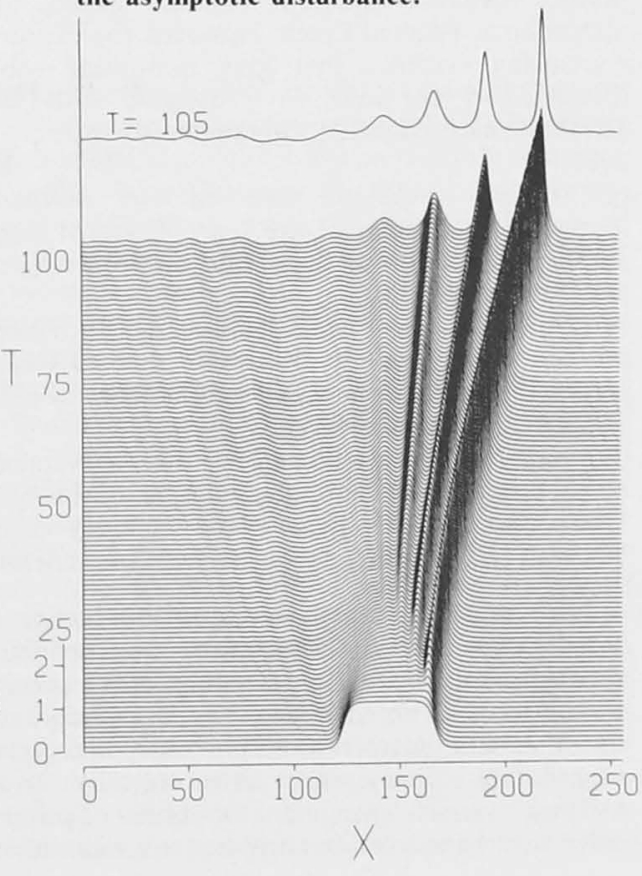


set of solitary waves followed by an almost negligible dispersive wave train. This example illustrates the general observation that the solitary wave component will almost completely dominate the asymptotic disturbance derived from long waves of elevation. Other examples of the numerical integration of the Benjamin-Ono equation from general initial conditions may be found in Christie and Muirhead (1981).

(d) Solitary waves of large amplitude exhibit closed circulation in the relative streamline pattern and take the form of a horizontal propagating vortex (Davis and Acrivos 1967; Kao and Pao 1979; Maxworthy 1980; Tung et al. 1982). The development of closed streamline flow appears to be the principal manifestation of large non-linearity in internal, deepfluid waves of this type. This behaviour contrasts with that of large-amplitude surface waves which break in the forward direction. Waves with closed circulation have an unusually intense wind shear pattern (examples are given in Figs 8 and 9) and present a particularly severe hazard to aircraft at low altitudes.

(e) The leading updraught and trailing downdraught in the solitary wave flow field may exceed $8 \mathrm{~m} \mathrm{~s}^{-}$ as shown in the direct measurements described by Clarke et al. (1981). Maximum horizontal wind speeds are typically in the range from 10 to $15 \mathrm{~m} \mathrm{~s}^{-1}$, but may on occasion be much higher. Horizontal winds near the surface may substantially exceed the speed of propagation in waves with closed circulation.

(f) Solitary atmospheric waves are usually observed during the night time and early morning daylight hours. Waves are seldom detected during the afternoon at Tennant Creek. However, there is now convincing evidence that long non-linear wave disturbances continue to propagate into the afternoon along elevated waveguides (Christie et al. 1981)

(g) Solitary waves are observed over northern Australia at all times of the year. Waves of large amplitude occur most frequently between Augus and November.

(h) Although solitary wave dominated disturbances are observed from all directions, they originate predominantly to the northeast and southeast at both Tennant Creek and Burketown.

(i) Solitary waves may gain energy from irreversible moist processes such as precipitation (Grimshaw 1980). Under suitable circumstances solitary waves may also trigger deep convection and thus generate thunderstorms.

The mechanisms which lead to the production of solitary waves in the atmosphere are, generally speaking, not at all well understood at the present time. Since, as noted above, any large-amplitude disturbance in the lower atmosphere can be expected to generate solitary waves asymptotically on an existing inversion waveguide, the genesis of solitary wave disturbances can be attributed to a wide variety
Fig. 7 Calculated properties of a relatively smallamplitude solitary wave propagating in a surface based, inversion waveguide of effective depth $\mathbf{H}=500 \mathrm{~m}$ and intensity $\Delta \theta=\theta_{0}-\theta_{\mathrm{s}}=10^{\circ} \mathrm{C} \quad\left(\theta_{\mathrm{s}}\right.$ is the potential temperature at the surface and $\theta_{0}$ is the potential temperature of the neutrally stable atmosphere above the inversion). Brunt$V$ aisala frequency in the inversion layer varies with altitude as $N^{2}=N_{0}{ }^{2} \operatorname{sech}^{2}(z / H$ (a) Relative streamline pattern. Values of the non-dimensional stream function are shown at left.

(b) Horizontal wind components.

(c) Vertical wind components.
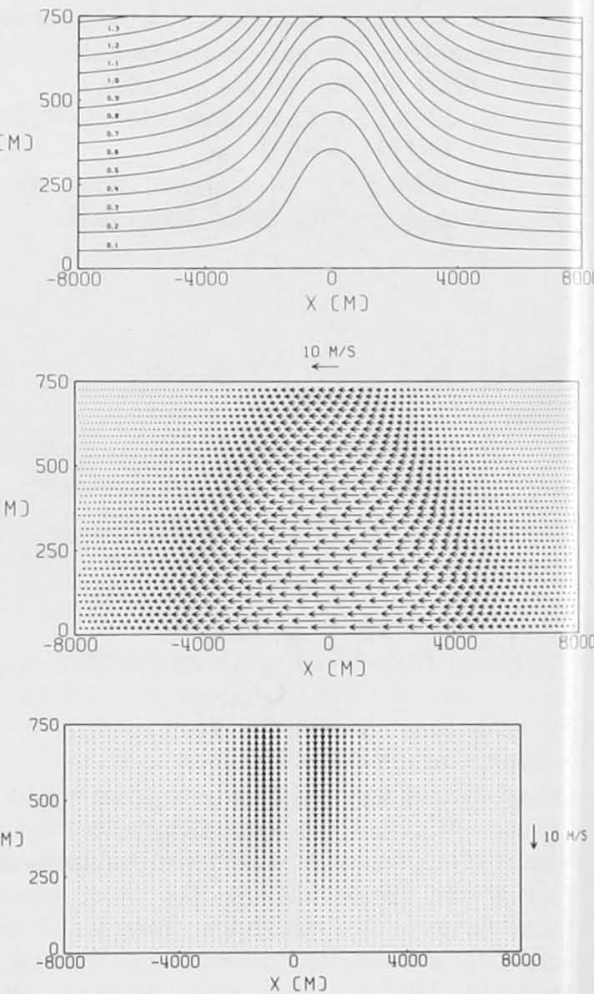

of processes including: deeply penetrating, sea-breeze fronts; intense thunderstorm activity; katabatic flows; mid-latitude cold frontal activity; the collapse of orographically-produced standing waves and eddies; and on a larger, and perhaps more important, scale to geostrophic adjustment. In a recent study, Clarke (1982) presents evidence to show that substantial number of the asymptotic non-linear wave disturbances observed at Burketown originate some $500 \mathrm{~km}$ to the northeast in the interaction of easterly and westerly sea-breeze fronts over the Cape York Peninsula. It is also worth drawing attention at this point to an observation described by Rust and Doviak (1982) of a propagating 'solitary gust' over 
vely smallating in a eguide of intensity potential $\theta_{0}$ is the ally stable ). Bruntsion layer $h^{2}(\mathbf{z} / \mathbf{H})$ lues of the are shown
Fig. 8 Properties of a solitary wave with closed circulation propagating in a relatively shallow boundary layer inversion of effective depth $\mathbf{H}=300 \mathrm{~m}$ and intensity $\Delta \theta=10^{\circ} \mathrm{C}$. See Fig. 7 for definition of the waveguide model used in these calculations.

(a) Relative streamline pattern.

(b) Horizontal wind components.

(c) Vertical wind components.
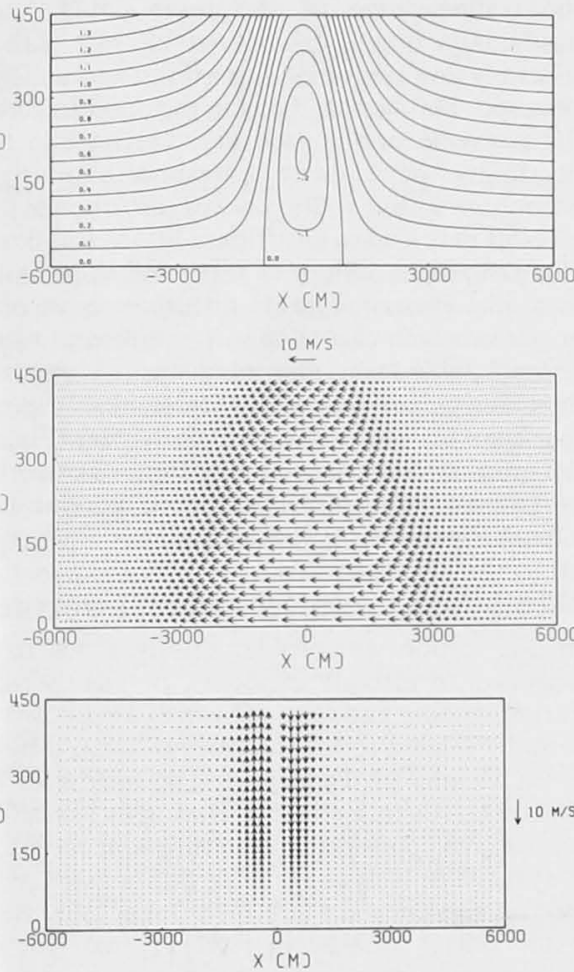

central Oklahoma which was tracked with the aid of a sensitive Doppler radar for over $80 \mathrm{~km}$ as it detached and propagated away from its origin along the leading edge of a dissipating, thunderstorm produced, downdraught density current. Winds with maximum updraught and downdraught components of about $6 \mathrm{~m} \mathrm{~s}^{-1}$ were measured directly as the transient disturbance passed the 444-m KTVY television tower instrumented by the National Severe Storms Laboratory. The streamline pattern and observed scale of this disturbance show that this isolated, propagating, transient shear disturbance can be interpreted as an essentially asymptotic, thunderstorm produced, solitary wave with closed circulation propagating in a stably stratified, boundary layer waveguide.

\section{Wind-shear and aircraft performance}

In order to summarise this discussion and to bring the problem of aircraft safety into focus, we consider
Fig. 9 Model calculation of (a) the relative streamline pattern, (b) the corresponding horizontal wind components and (c) the vertical wind field for an asymptotic nonlinear atmospheric wave disturbance consisting of three spatially separated, amplitude-ordered solitary waves propagating in an inversion (as in Fig. 7) of depth $\mathrm{H}=300 \mathrm{~m}$ and intensity $\Delta \theta=10^{\circ} \mathrm{C}$.
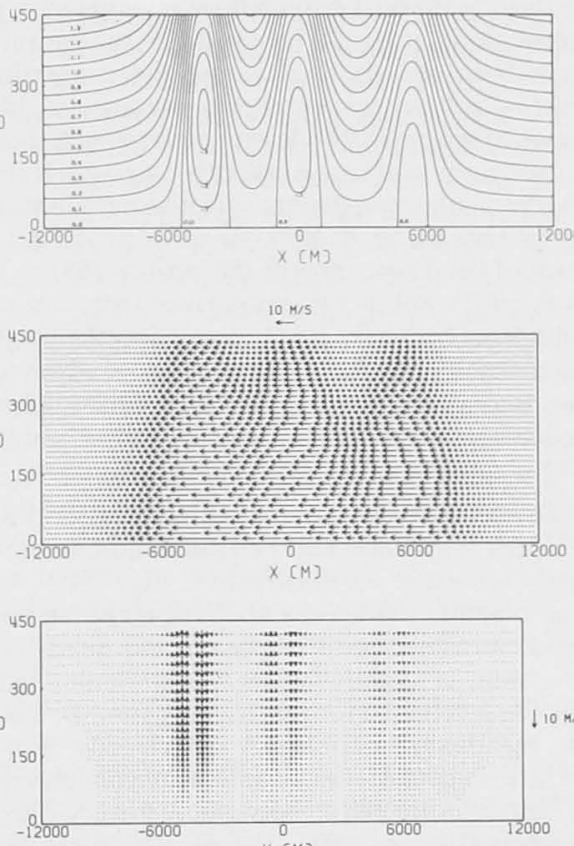

the results of a calculation of the streamline patterns and associated shear structures for (a) a relatively small-amplitude solitary wave propagating on a deep, boundary layer inverson, (b) a more intense, largeramplitude solitary wave disturbance with closed circulation propagating along a relatively shallow waveguide, and (c) a complex multi-soliton disturbance. A simple but realistic model of the atmospheric boundary layer is assumed in which the Brunt-Väisälä frequency, $\mathrm{N}^{2}=\operatorname{gd}(\ln \theta) / \mathrm{dz}(\Theta$ is the potential temperature), of the stably-stratified fluid near the surface varies with altitude $\mathrm{z}$ as $\mathrm{N}^{2}=\mathrm{N}^{2}$ $\operatorname{sech}^{2}(\mathrm{z} / \mathrm{H})$ where $\mathrm{H}$ is a measure of the depth of the surface-based inversion. The solitary wave solutions for this model have been derived for weakly non-linear waves in the Boussinesq approximation by Benjamin (1967) and more recently by Grimshaw (1981b) who extended the theory to second order in wave amplitude. The calculated streamline patterns and wind-fields presented here are based on a semiempirical analytic solution for the relative stream function which exhibits closed circulation in waves of large amplitude and which reduces, at lower amplitudes, to the second order solution derived by 
Grimshaw. A description of this solution will be presented elsewhere. The solitary wave profiles determined from this approximation are in good agreement, up to values of dimensionless amplitude of about 2.2, with the exact numerical solutions to the full non-linear eigenvalue problem presented by Tung et al. (1982). For the sake of simplicity, the effects of both surface friction and radiation of waves into the upper atmosphere and the influence of shear in the background flow are ignored in these calculations. It should be noted that the parameters have been chosen to illustrate the properties of internal solitary waves of only average amplitude; waves of larger amplitude with correspondingly more intense shear zones are frequently observed. It should also be emphasised that the effective horizontal scale of solitary waves is quite sensitive to the detailed form of the density profile (Grimshaw 1981b; Tung et al. 1982). Solitary waves derived from the model considered here have effective wavelengths which are comparable with the average of those observed in the atmosphere; waves with shorter horizontal scales and proportionally more intense vertical winds are also commonly observed.

The calculated relative streamline patterns along with the horizontal and vertical wind components near the surface are illustrated in Figs 7 to 9 . In the first example, shown in Fig. 7, the amplitude of the wave, measured with respect to the depth of the inversion, is slightly below the minimum relative amplitude required for recirculating flow. In this case the maximum horizontal wind velocity near the surface is about $13.1 \mathrm{~m} \mathrm{~s}^{-1}$ and the maximum vertical velocity is estimated to be about $2.3 \mathrm{~m} \mathrm{~s}^{-1}$. The next example (Fig. 8) shows the calculated streamline pattern and velocity field for a relatively largeamplitude, isolated, solitary wave with closed circulation propagating in a fairly shallow, boundary layer waveguide. Waves of this type with regions of recirculating flow produce particularly intense, transient, wind shear zones. In this example, the maximum horizontal and vertical wind components are estimated to be $18.7 \mathrm{~m} \mathrm{~s}^{-1}$ and $4.3 \mathrm{~m} \mathrm{~s}^{-}$ respectively. As can be seen from the figures, the effective horizontal scale of the transient shear zones in these representative solitary waves is comparable with the horizontal scale of the final critical stage during landing and the initial stage during take-off The final example shown in Fig. 9 illustrates the relative streamline pattern and associated variable wind field for a more complex amplitude ordered, multi-soliton disturbance, a form of disturbance which is typical of many of the well-developed solitary wave disturbances observed in the lower atmosphere.

Solitary waves may affect aircraft performance in a variety of ways. Aircraft which encounter solitary waves from the front at low altitudes will first experience an increase in lift due to the sudden onset of horizontal head wind shear and the vertical updraught along the leading edge of the wave. This is followed directly by a sudden reduction in lift due to the combined effects of rapidly decreasing head wind and the downdraught along the trailing edge of the disturbance. The normal reaction of a pilot to the initial increase in lift during a landing approach (see Fig. 10) would be to decrease air speed in attempt to return to the normal glide path and this action coupled with the sudden decrease in lift along the trailing edge of the wave could lead particularly in the case of jet aircraft with their relatively slow response - to a disastrous undersho situation. For aircraft flying in the opposite direction the converse occurs (see Fig. 11) leading to possibility of runway overshoot.Similarly, encounter with a solitary wave disturbance duri take-off may lead to insufficient lift to clear obstacles along the flight path or to stall conditions. Aircraft may also encounter these disturbances at oblicue angles in which case they will be subject to sudden unpredictable transverse wind-shears compounced by strong up and downdraughts. It must be expected that complex multi-soliton disturbances (Fig. 9) the most commonly occurring form of disturbar - present even more hazardous conditions aircraft operating at low altitudes.

\section{Wind-shear related aircraft accidents}

In this section we consider briefly a few relevant examples of aircraft accidents, or near accidents, during landing and take-off which are known to be causally related to low level wind-shear conditions in the vicinity of the airport. The subject of windshear hazards to aircraft operation at low altitudes has received considerable attention in recent years (Fujita 1976; Hall et al. 1976; Fujita and Caracen 1977; Anderson and Clark 1978; Jones 1978; Bell an Tsui 1981; Membery 1982). Most of thes

Fig. 10 Illustration of the possible undershoot behaviour of an aircraft during a head-on encounter with a solitary wave on final approach to runway. The solitary wave is propagating to the left at velocity c Unexpected lift associated with the increasing head wind and updraught along the leading edge of the wave could also lead, in this case, to a runway overshoot situation if encountered during the final stage in th landing approach.

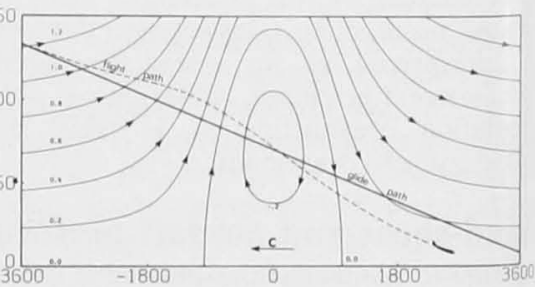

Fig. 11 Runw: solitar of the and $d \mathrm{c}$ wave o encoul
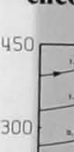

300

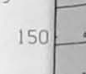

stigations iscussion nse win ndraughts ents, terrai ude cold sient win erstandabl ertheless, lable to in produced inated dist d-wide pre take-off thorough conditions in A by Anderson

note that whil e the best kr wind conditio related wind-st only 15 were at activity. The incidents were horizontal win reports). On tl outlined above incidents (and c plausibly be at the boundary la shear incidents accord with th Only a few reported in th Survey by Al however, been serious, civil ransient wind ubstantial dan The following wich seem to of this paper. FH227 crash Airport at 1743 
ve. This

lift due ng head ng edgs f a pilo pproac ed in and th ift alon: lead

Fig. 11 Runway overshoot conditions produced by a solitary wave propagating along the direction of the flight path. The increasing tail wind and downdraft along the trailing edge of the wave could also lead to runway undershoot if encountered during the final approach stage.

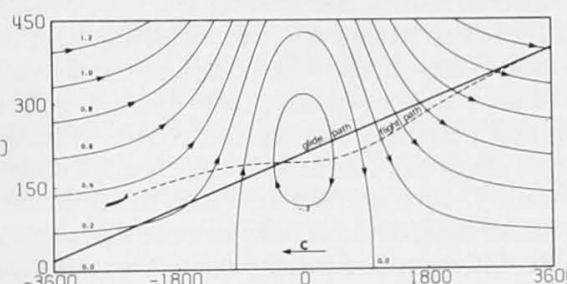

investigations have been primarily concerned with a discussion of fairly-well understeod sources of intense wind-shear such as thunderstorm downdraughts, downdraught generated density currents, terrain induced shear, sea-breezes and midfitude cold frontal systems. By comparison, ransient wind-shear of unknown origin has derstandably received relatively little attention. evertheless, we believe there is sufficient evidence va lable to indicate that localised wind-shear of the pe produced by the passage of a solitary wave dominated disturbance does present a very significant world-wide problem for aircraft during the landing and take-off phase.

thorough survey investigation of wind-shear conditions in Australia has recently been carried out by Anderson and Clark (1978). It is interesting to note that while thunderstorms and frontal systems are the best known sources of hazardous low level wind conditions, of a total of 93 meteorologically related wind-shear incidents compiled in this survey, only 15 were attributed to frontal and thunderstorm activity. The remaining low-level wind-shear incidents were categorised as vertical shear of horizontal wind (31 reports) and downdraught (47 reports). On the basis of the solitary wave studies outlined above we believe that many of these latter incidents (and quite possibly some of the former) can plausibly be attributed to solitary wave activity in the boundary layer. Several examples of typical windshear incidents cited in Anderson and Clark are in accord with this conclusion.

Only a few cases of relatively minor damage were reported in the Pilot and Air Traffic Controller Survey by Anderson and Clark. There have, however, been a number of well documented, serious, civil airliner crashes related directiy to transient wind-shear conditions which resulted in substantial damage and in some cases loss of life. The following list contains only a few examples which seem to be particularly relevant to the subject of this paper.

1. FH227 crashed on landing approach to St Louis Airport at 1743 local time (Department of Transport
1976). Aircraft totally destroyed, 38 fatalities. Observations of intense up and downdraughts and roll clouds preceding a thunderstorm strongly suggest that solitary wave disturbances were a contributing factor in this accident.

2. B-707 crashed on final approach at night to Pago Pago International Airport (Departure of Transport 1978). Aircraft destroyed, 97 fatalities. The probable cause of this accident has been attributed to failure on the part of the flight crew to correct an excessive rate of descent resulting from destabilising wind changes associated with thunderstorm produced flow over uneven terrain near the final approach path. The aircraft first encountered an increasing head wind and/or updraught condition well out to sea, at which point thrust was reduced to return the aircraft to the normal glide path. Subsequently, the positive effect of the head wind or updraught condition disappeared and the aircraft, under low thrust conditions, developed a high descent rate and crashed $1200 \mathrm{~m}$ short of the runway. This is precisely the behaviour that one would expect from a head-on encounter with a solitary wave disturbance. The apparent horizontal scale of the varying wind field is in agreement with this interpretation. Non-linear wave produced windshear should therefore be considered a possible causal factor in this accident. The analysis is complicated in this case by variations in terrain along the approach path which undoubtedly influenced the wind-shear pattern to some extent.

3. DC-9 landed $192 \mathrm{~m}$ short of threshold at 1342 local time at Melbourne Airport (Department of Transport 1980). Minor damage to aircraft. This accident occurred during the onset of a 'frontal' system characterised by a propagating roll cioud. During the approach, the aircraft first deviated above the glide path under the influence of the leading edge of the disturbance and subsequently descended below the glide path to near the minimum allowed altitude. At this point the descent was halted and the aircraft returned to the normal glide path. Shortly after, the aircraft developed a further loss of lift, dropped rapidly below the glide path and landed short. Reduced visibility due to heavy precipitation was a definite contributing factor to this accident. The frontal passage at the airport was somewhat earlier than predicted which suggests the formation of a prefrontal long wave disturbance. The strong variable shear pattern indicated by the behaviour of the aircraft, the observed propagating roll cloud, and the sudden change in surface pressure are all consistent with an interpretation based on a family of supercritical solitary waves (see Fig. 9) developing along the leading edge of a pre-frontal evolving, long, non-linear wave of elevation.

4. B-747 experienced strong variable wind-shear on approach to Bahrain Airport at 1023 local time (Membery 1982). This incident occurred during the passage of a weak trough/cold frontal system over the airport area. During the approach the aircraft encountered an increasing head wind accompanied 
by a strong updraught of nearly $5 \mathrm{~m} \mathrm{~s}^{-1}$ which led to a deviation of some $40 \mathrm{~m}$ above the glide path. This was followed immediately by a rapid decrease in head wind coupled with a very strong downdraught approaching $20 \mathrm{~m} \mathrm{~s}^{-1}$. As a result, the aircraft rapidly descended below the normal glide path. An increase in power to the 92 per cent takeoff thrust level led to an arrest in the descent at an altitude of about $250 \mathrm{~m}$, some $180 \mathrm{~m}$ below the glide path. The aircraft subsequently returned to a reasonable altitude and landed normally. Again, the observed behaviour of the aircraft is consistent with an encounter with a large-amplitude solitary wave propagating ahead of an advancing cold frontal system.

We emphasise at this point that we have not undertaken an exhaustive search of the aviation literature and that the above examples were chosen primarily because they appear to be consistent with typical, solitary wave produced, wind-shear conditions. It must also be emphasised that a solitary wave interpretation of known wind-shear related aircraft accidents is difficult, and necessarily somewhat speculative at the present time, since the available meteorological observations are usually too sparse, and lack the required sensitivity and resolution, to demonstrate with absolute certainty the presence of a non-linear wave disturbance at the time of the accident.

\section{Discussion}

Solitary atmospheric waves have only been studied in detail in northern Australia. It is clear however, from isolated reports, that they are not unique to tropical areas but occur world-wide. The climatology of these non-linear wave disturbances is almost completely unknown at the present time and even in the case of the extensively studied waves over northern Australia, the origin and conditions under which they propagate remain poorly understood. Some areas of Australia will be more favourable for large-amplitude solitary wave activity than others. At the moment it appears that these waves predominate over areas of level terrain and thus they probably occur over much of the Australian region. Waves of this type are known to be unstable to topographic perturbations and are therefore less likely to be found in areas with significant orography such as the highlands of southeast Australia.

Attention should be drawn at this point to the southerly buster (or burster), a strong southerly wind squall which frequently occurs during the spring and summer along the coast of New South Wales. Comprehensive descriptions of the southerly buster maybe found in Gentilli (1969) and Colquhoun (1981). It seems that this phenomenon is invariably associated with a topographically produced distortion of a cold frontal system whereby the advancing front progressively develops an S-shaped deformation, speeding up to form a southerly coastal jet on the eastern side of the coastal ranges, and slowing down on the western side. The southerly buster is often accompanied by convective storms; it also frequently occurs as a sudden clear-air disturbance (Colquhoun 1981) with surface winds in excess of $20 \mathrm{~m} \mathrm{~s}^{-1}$ and may present a serious hazard to aircraft during the landing and take-off stage at Sydney Airport and at other airports along the New South Wales coast. On occasion the disturbarce produces a spectacular, propagating roll cloud aligned perpendicular to the coast. The detailed structure of the southerly buster is not known with certainty. In a number of cases the observational evidence indicates that the phenomenon is essentially a coastally trapped density current as described by the dynamical model proposed by Baines (1980). On many occasions, however, the near-surface wind field is observed to exhibit a more complex variable structure which implies (Colquhoun 1981) the existence of a closed circulation cell or series of cells in the streamline pattern similar to that found in large-amplitude solitary wave disturbances (see for example Fig. 9). The southerly buster therefore of en appears to be a manifestation of a large-amplitude solitary wave family propagating in a maritime inversion. In this interpretation, the solitary waves are generated along the leading edge of a dissipating, coastally trapped, density current and emerge as stable entities in the form of large-amplitude solitons with regions of recirculating flow containing entrained fluid from the parent density flow. This is the expected asymptotic behaviour for a dissipating density current advancing at sub-critical or critical velocities into a density-stratified fluid and is consistent with the laboratory studies of Kao and $\mathrm{PaO}$ (1979) and Maxworthy (1980).

It would clearly be an important contribution to air safety if Air Traffic Controllers could predict and observe the passage of solitary wave disturbances over the airport region. This is certainly feasible. Since these disturbances maintain a coherent identity over large distances and since they are easily and reliably detected on sensitive microbarometers, data from an array of high resolution micropressure sensors in the neighbourhood of an airport could be telemetred to a central recording site and analysed in real time under microprocessor control using standard array, beam forming techniques to provide a continuous display of location, intensity, propagation characteristics and expected time of arrival in a practical form suited to routine use by Air Traffic Controllers. In this regard it is worth noting some practical aspects of the wave produced, transient, wind-shear detection problem. Solitary wave activity can be detected by surface based anemometers, analogue and Doppler acoustic sounders, Doppler microwave radars and sensitive microbarometers. It has been our experience that the surface wind record is often very complex and difficult to interpret and is frequently insensitive to litary

vated

areliable

ssor in

pensive

ted to

ited re serious

ids, rai

acoust

instru

tary w

ustic L

w et al

ic wi

portin

h-sens

iak 19

detect

d-shea

require

pler $r$

urban

e acti

erthel

orts $\mathrm{s}$

-sensi

e to

sient,

view

and 1

airpor

itivity

tively

nstalle

vay a

ide a

ar wa

ed to

se instr

vel, sh

downdra

Microbard

suited to $a$

airports. E

performal

based on a

detectors

neighbour

Washingt

thunderst simple, i designed a solitary these disc no infor disturban complete discrimina and would of hazard 
iges, and souther] e storm clear-a : winds i is hazar f stage ; the Ne sturbanc oll clouc detailed own with rvation cribed

1980).

wind field variable

981)

es of cel

found

(see

ore of en

mplitu

maritin

ry wa

ssipatin

merge

e soliton

ontaining

low. T

issipati

or critica

bution to litary wave activity in both surface based and vated inversions. Anemometers are therefore reliable and should not be used as the primary sor in a detection network. Acoustic sounders are pensive to install and maintain and are not at all ted to array measurements. These devices have ited resolution and range and their performance seriously degraded by the influence of surface ds, rain, and environmental noise. Furthermore, acoustic echo record is often difficult to interpret 1 instruments of this type do not reliably detect itary wave disturbances. On the other hand, an ustic Doppler sounder (Bourne and Brann 1978; w et al. 1981) would provide a direct measure of atic wind-shear and would play a valuable porting role in a wind-shear detection system. igh-sensitivity Doppler microwave radars (Rust and viak 1982) represent a promising development for detection of propagating, low level, clear-air d-shear disturbances. Two widely spaced radars required to map the three-dimensional wind field. opler radars are relatively insensitive to clear-air sturbances and may not reliably detect solitary e activity in the atmospheric boundary layer. vertheless, in many circumstances, particularly at morts sited in extensive areas of level terrain, a high-sensitivity Doppler radar installation could rove to be a valuable component in a low level, ransient, wind-shear detection system.

view of these observations we suggest that the best and by far the most reliable detection system for airport use should be based on an array of highsensitivity microbarometers. These instruments are relatively inexpensive, reliable and compact and can be installed in any location including alongside the runway and under the approach path and they provide a definitive measure of propagating nonlinear wave disturbances in a form which is well suited to real time signal processing. In addition, these instruments provide a sensitive measure of low level, shear-wave activity and thunderstorm, downdraught produced, density currents. Microbarometers are therefore particularly well suited to a transient wind-shear detection system at airports. Bedard et al. (1977) describe the design and performance of a closely related detection system based on a dense network of pressure-jump threshold detectors which has been installed in the neighbourhood of Dulles International Airport in Washington, D.C., to monitor the approach of thunderstorm produced density currents. These simple, inexpensive detectors could probabiy be designed to trigger reliably on the leading edge of a solitary wave dominated disturbance. However, these discrete threshold detectors provide virtually no information on the morphology of the disturbance. An on-line time series analysis of the complete detailed micro-pressure pattern would discriminate between various types of disturbances and would provide a useful appraisal of the degree of hazard associated with individual disturbances.
National Bureau of Standards MkII infrasonic microbarometers are used as the primary array element at the Warramunga Infrasonic Array. A simplified wide-band version of these ultra-sensitive instruments could easily be adapted for use in a practical prediction and warning system at airports.

This paper has been primarily concerned with the potential hazard of boundary layer solitary waves to aircraft operating at low altitudes. Solitary waves will also dominate non-linear wave disturbances propagating in elevated waveguides and some episodes of higher altitude, clear-air turbulence at longer periods can probably be attributed to waves of this type.

\section{Conclusions and recommendations}

The principal conclusion of this study is that solitary waves in the atmospheric boundary layer constitute a potentially serious hazard to aircraft operating at low altitudes. It has been emphasised that these largeamplitude waves occur frequently over the northern Australian region, that they nearly always occur as sudden, clear-air disturbances and that aircraft may encounter these transient wind-shear disturbances completely without warning.

Pilots operating in northern Queensland are generally well aware of the hazards of flying through the propagating, solitary wave produced, roll cloud formations that frequent the southern margin of the Gulf of Carpentaria. It is likely that the commonly occurring clear-air solitary wave disturbances observed in inland regions of Australia produce equally intense, low level, transient wind-shear zones; they should therefore be regarded as equally hazardous to the operation of aircraft at low altitudes.

The authors wish to make the following recommendations based on the investigation of the properties of solitary atmospheric waves:

1. Pilots and Air Traffic Controllers should be made aware of the potential hazard of solitary waves and should become familiar with the influence of these propagating, localised, shear disturbances on the behaviour of aircraft during the landing and takeoff stage.

2. No attempt should be made to land or take off through a propagating, low level, roll cloud. Considerable caution should also be exercised when flying behind cloud lines of this type as a residual cloud-free disturbance with intense shear-zones may extend for some tens of kilometres in the wake of the leading solitons.

3. Pilots should exercise caution when reducing thrust or increasing drag to counter a sudden onset of head wind shear or updraught during the landing approach as this may be followed by a sudden decrease in lift which could lead to a disastrous undershoot situation.

4. Reports of horizontal shear and strong up and downdraughts in the vicinity of airports should be 
promptly relayed to incoming and outgoing flights. 5. The frequency of occurrence and intensity of solitary wave disturbances should be established throughout Australia. Initially, a limited survey should be undertaken to delineate high risk areas by installing individual recording, high-sensitivity microbarometers, set to operate at periods up to at least $1000 \mathrm{~s}$, at selected, heavily used, airports. Data from a large aperture array of this type would also provide the basic observational material required to develop an operational predictive model and to determine the detailed evolutionary behaviour of solitary atmospheric waves over the Australian region.

6. Airports should be protected by an array of sensitive wideband microbarometers connected to an on-line central processor with capabilities for providing a suitable warning to Air Traffic Controllers of potentially hazardous, solitary wave disturbances approaching the airport area.

\section{Acknowledgments}

The authors wish to express their gratitude to Professors A. L. Hales, K. Lambeck and J. S. Turner for valuable discussions of this work and for several helpful suggestions. One of the authors (DRC) would like to thank R. H. Clarke of the Department of Meteorology, University of Melbourne, for many stimulating discussions of nonlinear wave phenomena. We particularly wish to thank the managers of stations in the Burketown area for their generous assistance during the 1980 and 1981 field projects. We are also grateful to Andrea and Tony Bowring and Joy and Terry Arnold of Burketown for providing observational material and to John Tarca, Alan Mainwaring and Brian Schaefer for their efforts in operating the Warramunga Infrasonic Array and for their valuable assistance in the field experiments.

\section{References}

Anderson, K. W. and Clark, B. A. J. 1978. A questionnaire survey of opinions of pilots and air traffic controllers on wind shear in Australia. Aeronautical Research Laboratory, Department of Defence, Melbourne.

Ablowitz, M. J and Segur, H. 1980. Long internal waves in fluids of great depth. Studies in Appl. Math. 62, 249-62.

Baines, P. G. 1980. The dynamics of the southerly buster Aust. Met. Mag. 28, 175-200.

Bedard, A. J. Jr, Hooke, W. H. and Beran, D. W. 1977 The Dulles Airport pressure jump detector array for gust front detection. Bull. Am. met. Soc. 58, 920-6.

Bell, G. J. and Tsui, K. S. 1981. A low-level wind shear detection system. Weather 36, 42-6.

Benjamin, T. B. 1967. Internal waves of permanent form in fluids of great depth. J. Fluid Mech. 29, 559-92.

Benney, D. J and Grimshaw, R. 1982. Large amplitude solitary waves in unbounded stratified fluids. Studies in Appl. Math. 66, 181-7.
Bourne, I. A. and Brann, H. N. 1978. The remote sensing of low level wind shears near airports. AIRMET Conference, Royal Meteorological Society, Canberra, 59-64.

Christie, D. R., Muirhead, K. J. and Hales, A. L. 1976. Solitary waves in the atmosphere. AFOSR-76-1133TR, National Technical Information Service (NTIS:A A031228) Springfield, Va, 22161

Christic, D. Muirhead, K. J and Hales, A. L. 19 On solitary waves in the atmosphere. J. Atmos. Sci. 805-25

Christie, D. R. Muirhead, K. J. and Hales, A. L. 1979. Intrusive density flows in the lower troposphere: A source of atmospheric solitons. J. geophys. Res. 84, 4959-70. Cl atme, R. H. 19

Christie, D. R., Muirhead, K. J. and Clarke, R. H. 19

Christie, D. R. and Muirhead, K. J. 1981. Observations of solitary atmospheric waves over Northern Australia. Physics for Australia's Development, Second Applied Physics Conference, Australian Institute of Physics, Melbourne, 463-6.

Clarke, R. H, 1972. The morning glory: an atmospheric hydraulic jump. Jnl appl. Met. 11, 304-11.

Clarke, R. H. 1983. Fair weather nocturnal inland wind surges and atmospheric bores. Aust. Met. Mag. (in press). Clarke, R. H., Smith, R. K. and Reid, D. G. 1981. The Morning Glory of the Gulf of Carpentaria: an atmospheric undular bore. Mon. Weath. Rev. 109, atmospheric $1726-50$.

Colquhoun, J. R. 1981. The origin, evolution and structure of some southerly bursters. Tech. Report 40, Bur. Met., Australia.

Davis, R. E and Acrivos, A. 1967. Solitary internal waves in deep water. J. Fluid Mech. 29, 593-607.

Department of Transport 1976. Approach through a thunderstorm. Aviation Safety Digest 94, 10-13.

Department of Transport 1978. An accident report revised. Aviation Safety Digest 103, 8-11.

Department of Transport 1980. DC-9 lands short of runway. Aviation Safety Digest 110, 24-8.

Fujita, T. T. 1976. Spearhead echo and downburst nea the approach end of a John F. Kennedy Airport runway, New York City. Univ. of Chicago, SMRP Res. Paper 137, $51 \mathrm{pp}$.

Fujita, T. T and Caracena, F. 1977. An analysis of three weather-related aircraft accidents. Univ. of Chicago, SMRP Res. Paper 145, 40 pp.

Gentilli, J 1969 . Some regional aspects of southerly buster phenomena. Weather 24, 173-80.

Grimshaw, R. 1980/81. Solitary waves in a compressible fluid Pageoph. 119, 780-97.

Grimshaw, R. 1981a. Evolution equations for long, nonlinear internal waves in stratified shear flows. Studies in Appl. Math. 65, 159-88.

Grimshaw, R. 1981b. A second-order theory for solitary waves in deep fluids. Physics Fluids 24, 1611-18.

Grimshaw, R. 1981c. Slowly varying solitary waves in deep fluids. Proc. Roy. Soc. Lond. A376, 319-32.

Hall, F. F. Jr, Neff, W. D. and Frazier, T. V. 1976. Wind shear observations in thunderstorm density currents. Nature, 264, 408-11

Jones, D 1978. Operational problems: jet blast, wake turbulence and wind shear. AIRMET Conference, Royal Meteorological Society, Canberra, 49-54

Joseph, R. I. 1977a. Solitary waves in a finite depth fluid. I. Phys, A1O, L225-L227. oseph, R.

Benjamir

oseph, R.

nonlineas

Fluids 24

T. W (ermocl 115-?

C.

ternal s

lech. II

Kubota, T opagat atified

157-6

Maslowe, S stratif namic.

Maslowe, S ives in $1-48$.

Maxworthy duced Geoph cions ir $-64$. 
emote sensing ts. AIRMET ty, Canberra

A. L. 1976 $R-76-1133 T R$ e (NTIS:AD.

A. L. 1978 tmos. Sci.

A. L. 1979 here: A source $84,4959-7$

e, R. H. 19

Nature 2

Observations

ern Australia. cond Applied e of Physics

atmospheric 11.

inland wind Mag. (in press) G. 1981. The rpentaria: an th. Rev. 109,

and structure 40, Bur. Met.

internal waves 507.

ch through

4. 10-13.

report revised

ands short of -8 .

ownburst near irport runway, RP Res. Paper ralysis of three - of Chicago, outherly buster a compressible ons for long, - flows. Studies

ry for solitary 1611-18.

waves in deep 9-32.

V. 1976. Wind nsity currents.

et blast, wake iference, Royal 4. ite depth fluid
Joseph, R. I., 1977b. Multi-soliton-like solutions to the Benjamin-Ono equation. J. Math. Phys, 18, 2251-8.

Joseph, R. I. and Adams, R. C. 1981. Extension of weakly nonlinear theory of solitary wave propagation. Physics Fluids 24, 15-22.

Kao, T. W. and Pao, H.-P. 1979. Wake collapse in the thermocline and internal solitary waves. J. Fluid. Mech. , 115-27.

Koop, C. G. and Butler, G. 1981. An investigation of internal solitary waves in a two-fluid system. J. Fluid Mech. 112, 225-51.

Kubota, T., Ko, D. R. S. and Dobbs, L. D. 1978. Propagation of weakly nonlinear internal waves in a ratified fluid of finite depth. A.I.A.A.J. Hydronautics $157-65$

Maslowe, S. A. and Redekopp, L. G. 1979. Solitary waves in stratified shear flows. Geophys. Astrophys. Fluid Dynamics 13, 185-96.

Maslowe, S. A. and Redekopp, L. G. 1980. Long nonlinear waves in stratified shear flows. J. Fluid Mech. 101. $321-48$.

Maxworthy, T. 1979. A note on the internal solitary waves produced by tidal flow over a three-dimensional ridge. J. Geophys. Res. 84, C1, 338-46.

Maxworthy, T. 1980. On the formation of nonlinear internal waves from the gravitational collapse of mixed regions in two and three dimensions. J. Fluid Mech. 96. 47-64.

Meiss, J. D. and Pereira, N. R. 1978. Internal wave solitons. Phusics Fluids 21, 700-2; 22, 201.

Membery, D. A. 1982. A documented example of strong wind-shear. Weather 37, 19-22.
Miles, J. W. 1980. Solitary waves. Ann. Rev. Fluid Mech., $12,11-43$.

Neal, A. B., Butterworth, I. J. and Murphy, K. M. 1977 The Morning Glory. Weather 32, 176-83.

Ono, H. 1975. Algebraic solitary waves in stratified fluids. J. Phys. Soc. Japan 39, 1082-91.

Periera, N. R. and Redekopp, L. G. 1980. Radiation damping of long, finite-amplitude internal waves. Physics Fluids 23, 2182-3.

Redekopp, L. G. 1980. Similarity solutions of some twospace-dimensional nonlinear wave evolution equations. Studies in Appl. Math. 63, 185-207.

Robin, A. G. 1978. Roll cloud over Spencer Gulf. Aust. Met. Mag. 26, 125.

Rust, W. D. and Doviak, R. J. 1982. Radar research on thunderstorms and lightning. Nature 297, 461-8

Scott, A. C., Chu, F. Y. F. and McLaughlin, D. W. 1973. The soliton: a new concept in applied science. Proc. $I$. E. E. E. $61,1443-83$.

Shaw, N. A., Bourne, I. A., Mace, O, and Robinson, G. S. 1981. Acoustic radar for monitoring wind and turbulence in the atmosphere. Physics for Australia's Development, Second Applied Physics Conference, Australian Institute of Physics, Melbourne, 467.

Tung, K.-K., Chan, T. F, and Kubota, T. 1982. Large amplitude internal waves of permanent form. Studies in Appl. Math. 66, 1-44.

Tung, K.-K., Ko, D. R. S. and Chang, J. J. 1981. Weakly nonlinear internal waves in shear. Studies in Appl. Math. $65,189-221$. 
Section 2.5

Solitary waves: A low-level wind shear hazard to aviation

(D.R. Christie and K.J. Muirhead)

Int. J. Aviation Safety, 1, 169-190, 1983. 


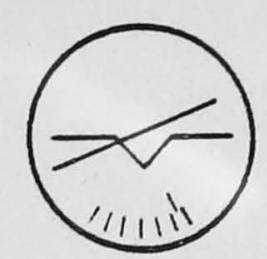

\section{International Journal of}

\section{Aviation Safety}

Reprinted by The International Journal of Aviation Safety from Volume 1, September 1983. 


\section{Solitary Waves}

\section{A Low-Level Wind Shear Hazard To Aviation}

By D. R. Christie and K. J. Muirhead, Research School of Earth Sciences, Institute of Advanced Studies, Australian National University

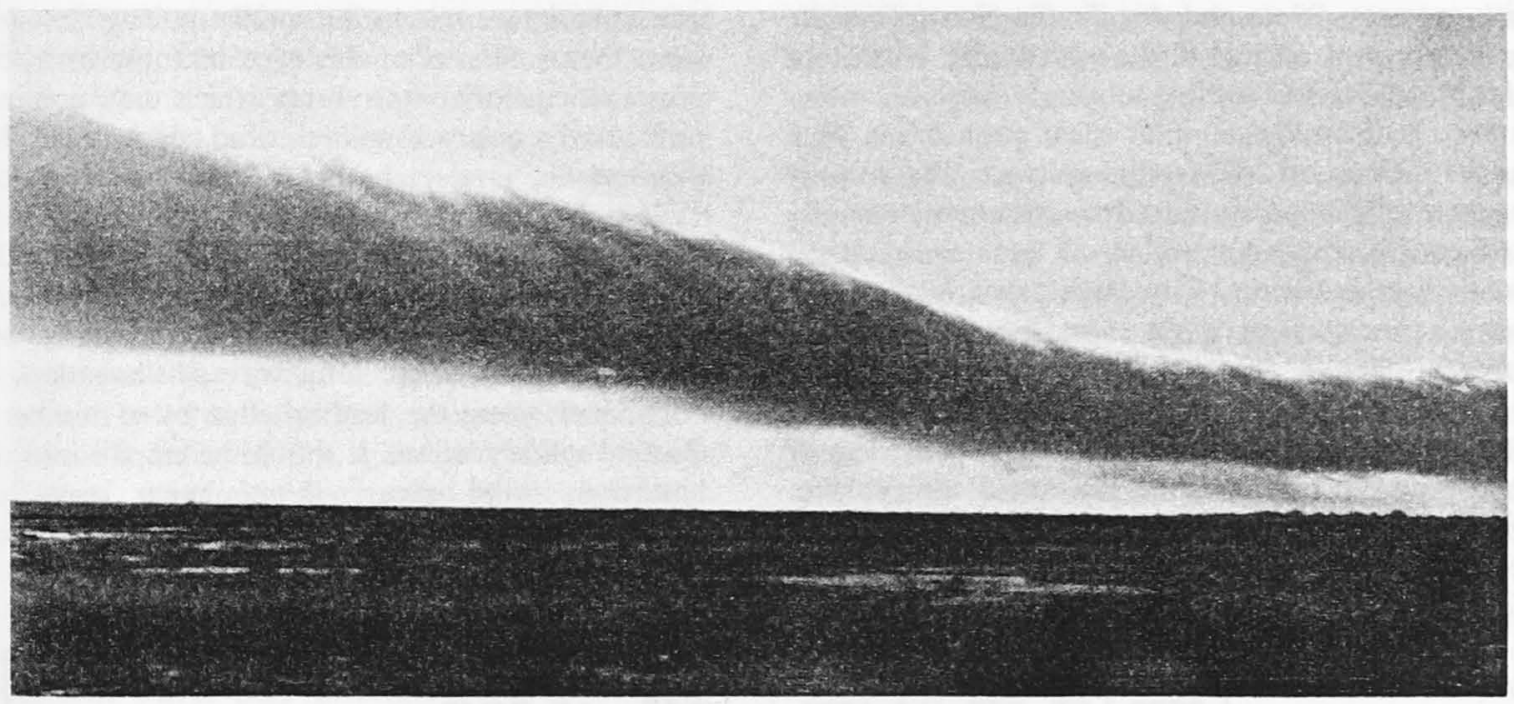

Australia`s Morning Glory cloud formation.

This paper is concerned with the potentially serious hazard to aviation created by solitary waves and related nonlinear wave disturbances in the atmospheric boundary layer. Solitary waves are remarkably large amplitude single-created waves of elevation which propagate in waveguides formed by inversions in the lower atmosphere. They arise naturally as the stable component in the evolution of a wide variety of long nonlinear wave disturbances. Atmospheric solitary waves produce strong horizontal and vertical low-level wind shears which are comparable in intensity with other well-known forms of hazardous wind shear. A thorough study of the properties of these commonly-occurring waves has been carried out at the Warramunga Infrasonic Array, located in the arid interior of Australia's Northern Territory, and, under humid maritime conditions, along the tropical southern margin of the Gulf of Carpentaria. This investigation has shown that solitary-wave disturbances are produced by a wide variety of atmospheric phenomena, including sea-breeze and cold frontal systems, katabatic flow and intense thunderstorms. Solitary wave dominated disturbances have been observed to propagate over great distances. Waves of this type almost invariably occur in inland areas as sudden, unexpected, transient clear-air disturbances. Even in maritime regions these ubiquitous waves are found to occur more often than not as clear-air disturbances. A description is given of the basic properties of boundary-layer solitary waves which relate directly to the problem of low-level wind shear.

Copyright C 1983 The Capstan Press. All Rights Reserved.

International Journal of Aviation Safety, pp. 129-256 
Transient low-level wind shear has been recognized for some time as a serious, but poorly understood, hazard to aircraft in the airport environment. In recent years wind shear has been directly implicated as the primary casual factor in a significant number of aircraft accidents. Recent studies of transient wind shear (Fujita and Caracena, 1977; Bedard et al., 1977; Bedard, 1982; McCarthy et al., 1982) have been primarily concerned with intense, localized thunderstorm down-draughts-or microbursts-and with thunderstorm-produced density currents. Pilots and Air Traffic Controllers are generally well aware of the potentially hazardous shears associated with thunderstorms and other known meteorological wind shear phenomena such as sea-breeze and cold frontal systems. The primary purpose of this paper is to draw attention to a new and equally important source of dynamic low-level wind shear associated with large amplitude solitary waves propagating in the atmospheric boundary layer.

Solitary waves of elevation arise as the stable component in the disintegration of a wide variety of long wave disturbances in the lower atmosphere. Nonlinear wave disturbances of this type propagate over great distances and often appear without warning as sudden unexpected clear-air disturbances. A noteworthy feature of solitary waves in a boundary layer inversion waveguide is their remarkably large amplitudes which may on occasions exceed one kilometre. In contrast to surface waves which break in the forward direction, the principal manifestation of nonlinearity in larger amplitude internal solitary waves is the region of recirculating flow in the relative streamline pattern (Davis and Acrivos, 1967; Tung et al., 1982) which leads, in the case of atmospheric waves, to unusually intense low-level wind shears. Waves of this type take the form of a propagating horizontal vortex which may present a particularly severe low-level wind shear hazard to aviation.

The characteristic morphology and scale of the nonlinear wave disturbances considered here is illustrated by the typical well-resolved surface micropressure signature shown in Fig. 1. As can be seen in this example, these long wave disturbances are dominated along the leading edge by a number of discrete solitary waves. It should be emphasized that hazardous wind shears in nonlinear wave disturbances of this type are almost invariably associ-

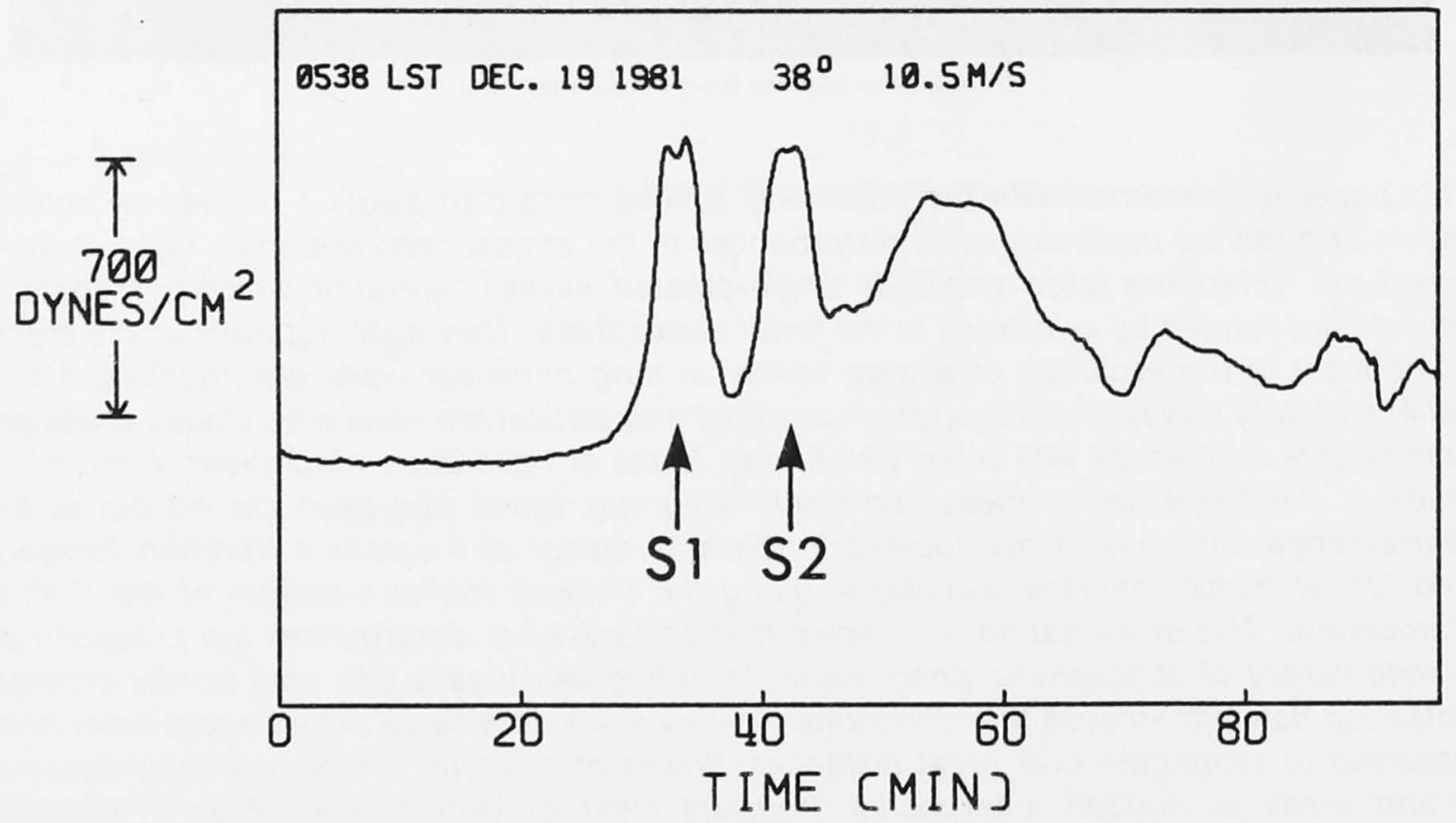

Figure 1. Surface micropressure record from the Warramunga Infrasonic Array near Tennant Creek in central Australia which illustrates the morphology of a complex evolving nonlinear wave disturbance dominated along the leading edge by two well-resolved large amplitude solitary waves. The azimuth noted is the source direction measured from true north. Time is given in local standard time (LST $=\mathrm{GMT}+9.5 \mathrm{~h}$ in the Northern Territory). 
ated with the leading solitary waves. Low-level wind shears in the residual disturbance behind the solitary wave components tend to be small by comparison and are probably of little significance to the subject of air safety.

The wind shear pattern in individual solitary waves is characterized by strong vertical and horizontal shears in the form of an up-draught along the leading edge and a down-draught along the trailing edge accompanied by rapidly varying horizontal winds with maximum intensity at the centre of the wave near the surface. Complex, transient wind disturbances of this type can effect the performance of aircraft in a variety of ways. Perhaps the most serious situation arises when an aircraft, on final approach, encounters a boundary-layer solitary wave from the front. Under these conditions, the aircraft will be subject to a temporary period of positive lift followed by a sudden disastrous loss of lift which may leave the aircraft dangerously close to the ground, well short of the runway threshold. A behaviour pattern of this type, i.e., a brief period of positive lift followed by a sudden loss of lift, is typical of many low-level wind shear incidents. Under present aviation conditions at most airports, aircraft may encounter these sudden transient shear disturbances completely without warning. Wind shear associated with these commonly occurring ubiquitous waves may therefore account for some hitherto unexplained aircraft accidents.

Solitary waves in the atmospheric boundary layer were first identified in 1976 during a survey of the long-period micropressure records from the Australian National University's Warramunga Infrasonic Array near Tennant Creek in the arid interior of the Northern Territory of Australia (Christie et al., 1978). This initial solitary wave study was followed (Christie et al., 1979) by a more general investigation of the properties of evolving long nonlinear wave disturbances. It was proposed in this latter paper that the Morning Glory phenomenon - a remarkable low-level propagating roll cloud formation which frequents the southern coast of the Gulf of Carpentaria (Clarke, 1972) - can be interpreted as a spectacular visible manifestation of a solitary wave disturbance propagating in a marine inversion. This interpretation has been confirmed in a number of recent investigations (Christie et al., 1981; Clarke et al., 1981a; Christie and Muirhead, 1981). The significance of these solitary wave roll clouds to the subject of air safety will be discussed in some detail later in this paper.

Although extensive well-documented records are lacking, the existing evidence suggests that solitary wave disturbances occur very commonly and are particularly well-developed over much of the Australian region. This regional feature can be attributed primarily to the featureless, semi-desert terrain which characterizes much of the Australian continent. It should be emphasized however, that solitary-waveassociated disturbances are frequently observed elsewhere and are a potentially serious world-wide wind shear hazard to aviation. Disturbances of this type are created naturally by a wide variety of atmospheric phenomena ranging from intense thunderstorms to frontal systems and can be expected to occur wherever conditions of low-level stability prevail.

A preliminary investigation of solitary-waveproduced wind shear, based on a study of solitary wave activity over northern Australia, has been given by Christie and Muirhead (1983). In this paper we present further observational material on solitary wave disturbances including some material from an experimental investigation of the evolution of nonlinear wave disturbances over long distances. In addition, a brief summary of the most relevant results of a recent investigation of a new class of topographically-trapped solitary wave disturbances observed along the south-east coast of Australia will be presented.

This paper is organized as follows. A summary of the properties of solitary wave and other closelyrelated nonlinear wave phenomena is given, followed by a description of nonlinear wave wind shear patterns and the influence of these transient shear disturbances on the performance of aircraft. Finally, a brief discussion of the relative importance of solitary-wave wind shear to the general low-level wind shear problem is presented along with some preliminary recommendations.

\section{Properties of Solitary Waves}

Solitary waves are isolated single-crested waves of permanent form which propagate in horizontal waveguides. Waves of this type were first discovered by John Scott Russell on the surface of a canal in Scotland in 1834 (Russell, 1838). Russell clearly recognized that solitary waves play a fundamental role in the description of long waves on the surface of water. Nevertheless, the significance of these observations was not appreciated at the time and, even though they attracted some theoretical interest, solitary waves eventually came to be regarded as merely an elegant curiosity of no real importance. 


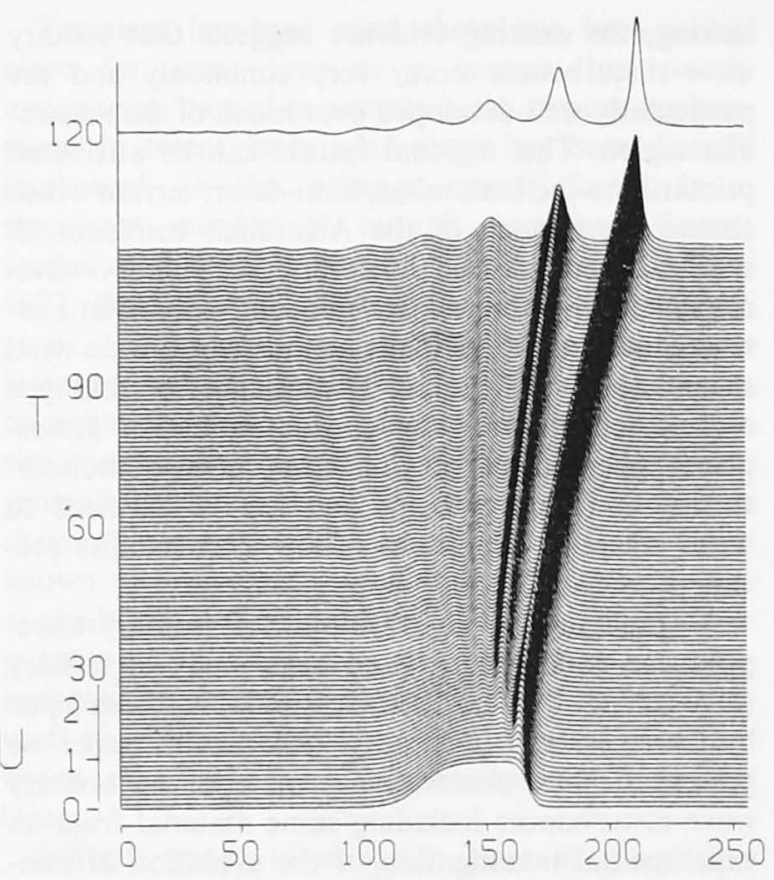

Figure 2. Numerical solution of the Benjamin-Ono evolution equation illustrating the disintegration of an arbitrary finite-length long wave of elevation into a discrete set of amplitude-ordered permanent solitary waves followed by a weak complex dispersing subcritical wave train. The coordinate system in this illustration and in Fig. 3 moves at the critical or linear phase speed. Solitary waves, which propagate at supercritical speeds, more to the right in this frame of reference; the dispersive wave component moves to the left. Units are nondimensional. The last trace is offset for clarity to illustrate the detailed structure of the essentially asymptotic form of the disturbance.

Only in recent years has it been recognized that solitary waves are unusually stable entities which describe a wide variety of dynamical phenomena found throughout the physical and biological sciences. The reader is referred to the excellent review article by Miles (1980) for background material on solitary waves in fluid systems.

Solitary waves in the atmospheric boundary layer belong to the class of nonlinear wave disturbances in infinitely deep fluids first considered by Benjamin (1967) and Davis and Acrivos (1967). The properties of this new class of internal wave motion has attracted a great deal of attention in recent years. These waves have been studied extensively in the laboratory (see, e.g. Maxworthy 1979, 1980 and Koop and Butler, 1981) and are currently a subject of intense theoretical interest. To first order, the evolution of finite-amplitude long wave disturbances of this type is described by the Benjamin-Ono equation (Benjamin, 1967; Ono, 1975). The basic theoretical framework for the description of solitary wave disturbances in a sheared inversion waveguide may be found in Maslowe and Redekopp (1980), Grimshaw (1980, 1981a, b, c 1982) and Tung et al. (1981). The most important recent developments have been the extension of the weakly nonlinear theory to higher order by Grimshaw (1981b) and the numerical and analytical work by Tung et al. (1982) on the fully nonlinear problem.

It is now accepted that internal solitary waves are a commonly occurring ubiquitous feature in both the oceans and the atmosphere. The importance of these waves in geophysical fluid systems stems from the observation that arbitrary positive disturbances in suitably stratified fluids, as the stable lower atmosphere, evolve into a finite number of internal solitary waves followed by a dispersive oscillatory wave train. This characteristic evolution pattern for waves in the atmospheric boundary layer is illustrated by the numerical solutions of the BenjaminOno equation presented in Fig. 2. In this example, an arbitrary initially smooth long wave of elevation evolves under the influence of amplitude and frequency dispersion into an amplitude-ordered family of solitary waves followed by a complex dispersing wave train. Note that most of the energy in positive initial wave disturbances of this type goes into the production of solitary waves.

Long waves of depression do not produce solitary waves. They may however give rise to another class of large amplitude dispersive waves which are also a source of potentially hazardous low-level wind shears. The genesis of waves of this type may be seen in the evolution of an arbitrary long wave of depression as shown in the numerical experiment illustrated in Fig. 3. In this case, a family of large amplitude waves is produced along the trailing edge of a long wave of depression. It should be noted that large-amplitude nonlinear waves which arise under these conditions are (in contrast to solitary waves) dispersive and propagate at speeds which are less than the linear phase speed. Nevertheless, these unusual large amplitude waves, which we shall call "quasi-solitary" waves in accordance with Yoshimura and Watanabe (1979), may exist for significant periods of time and may on occasion be a source of hazardous dynamic wind shear. Some further examples of the numerical integration of the Benjamin-Ono equation from general initial disturbances may be found in Christie and Muirhead (1981). 


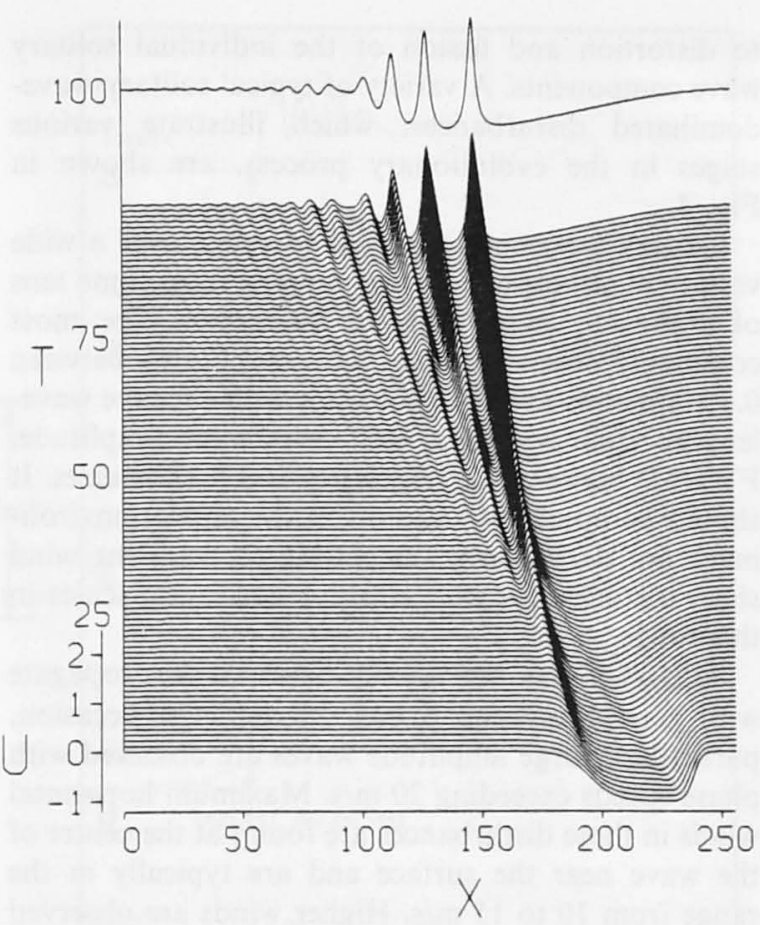

Figure 3. Numerical integration of the Benjamin-Ono equation showing the disintegration of a long wave of depression into a complex family of large amplitude "quasi-solitary" waves. Note that these sub-critical waves are not waves of permanent form; they are dispersive and therefore relatively short-lived. Observations of waves of this type over northern Australia are fairly rare. They usually occur in association with nearby thunderstorm activity.

The solitary wave solutions of the Benjamin-Ono equation are known to exhibit the nonlinear stability or solition property (Joseph, 1977). That is, despite a strongly nonlinear interaction, deep-fluid solitary waves emerge unscathed from collision. Solitary waves in the stably-stratified boundary layer are therefore unusually stable waves and can be regarded, at least to this approximation, as solitons.

The general evolution of long wave disturbances in the lower atmosphere may be summarized as follows. Since the speed of solitary waves increases with amplitude, long wave disturbances in a homogeneous boundary-layer waveguide must eventually be dominated by an amplitude-ordered family of solitary waves. If the initial disturbance is largely a wave of depression, a family of large amplitude quasisolitary waves may be created. These waves are however dispersive and their lifetime is limited. This aspect is reflected in the fact that waves in this latter class are only occasionally observed. Thus, it is the frequently observed long-lived solitary waves, with their associated localized shear zones, which are of primary concern to the subject of air safety.

\section{Observations of Solitary Waves in the Atmospheric Boundary Layer}

Solitary atmospheric waves have been studied in detail only near Tennant Creek in the arid interior of northern Australia (Christie et al., 1978; 1979), in the area between Tennant Creek and the Gulf of Carpentaria (Christie et al., 1981) and along the south-eastern margin of the Gulf of Carpentaria (Christie and Muirhead, 1981; Clarke et al., 1981; Clarke, 1983). There is now ample evidence to show that waves of this type are not all unique to northern Australia; they certainly occur over most inland areas of the Australian continent. Since these ubiquitous waves are the natural asymptotic product in the disintegration of a wide variety of atmospheric phenomena, transient low-level wind shear disturbances of this type must be expected to occur world-wide wherever suitable waveguide inversion conditions exist.

\section{Observations in Australia}

A through investigation of the properties of solitary atmospheric wave disturbances has been carried out over an eight-year period at the Warramunga Infrasonic Array, 37 kilometres southsoutheast of Tennant Creek in the centre of the Northern Territory. Over one thousand large amplitude solitary-wave dominated disturbances have been recorded at Warramunga during this period. One important conclusion from these observations, which is clearly of some concern to aviation, is that waves of this type almost invariably occur over inland areas as sudden unexpected clear-air disturbances. Visible solitary waves in the form of low-level propagating roll clouds are however observed with some regularity during the spring in the neighbourhood of Burketown on the tropical south coast of the Gulf of Carpentaria. Cloud formations of this type are also seen occasionally in other coastal areas of Australia. A description of the spectacular solitary wave roll clouds seen at Burketown is given later in this section.

The extensive observations at Warramunga have shown that solitary atmospheric waves occur as isolated, individual solitons, as essentially asymptotic, amplitude-ordered solitary wave packets and, in their most frequently observed form, as partially resolved 


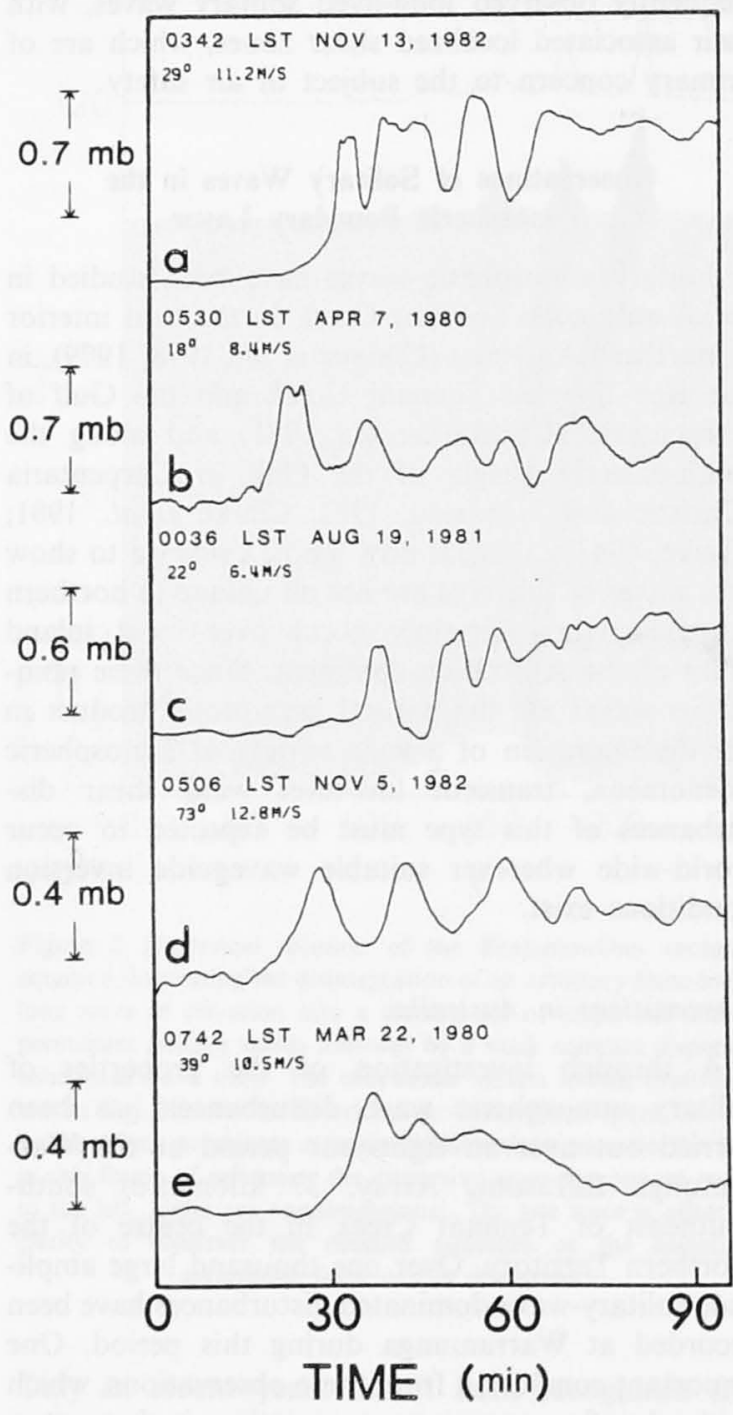

Figure 4. Examples of typical solitary-wave-dominated disturbances recorded at the Warramunga Infrasonic Array near Tennant Creek. The effective wavelength (FWHM) of the leading soliton in each of these disturbances is approximately (a) $3.0 \mathrm{~km}$, (b) $3.0 \mathrm{~km}$, (c) $1.5 \mathrm{~km}$, (d) $5.4 \mathrm{~km}$, (e) $4.0 \mathrm{~km}$. These typical micropressure records have been chosen to illustrate a variety of stages in the evolution of long nonlinear wave disturbances in the atmospheric boundary layer.

solitary wave families associated with the leading edge of evolving long-wave disturbances. Completely isolated solitary waves are only rarely observed in the atmospheric boundary layer. This is to be expected since most initial long-wave disturbances decay into more than one solitary wave component. In addition, solitary waves in a boundary-layer waveguide are usually subject to perturbations which eventually lead to distortion and fission of the individual solitary wave components. A variety of typical solitary-wavedominated disturbances, which illustrate various stages in the evolutionary process, are shown in Fig. 4.

Solitary waves are observed to occur over a wide variety of atmospheric scales ranging from some tens of metres to more than 10 kilometres. The most commonly observed waves have amplitudes between 0.3 kilometres and 1.0 kilometres with effective wavelengths (full width at half maximum amplitude, FWHM) between 0.5 kilometres and 6 kilometres. It should be noted that aircraft in the airport environment are particularly susceptible to transient wind shear disturbances with effective horizontal scales in this range.

Solitary waves are usually observed to propagate with speeds between 6 and $16 \mathrm{~m} / \mathrm{s}$. On occasion, particularly large amplitude waves are observed with phase speeds exceeding $20 \mathrm{~m} / \mathrm{s}$. Maximum horizontal winds in these disturbances are found at the centre of the wave near the surface and are typically in the range from 10 to $15 \mathrm{~m} / \mathrm{s}$. Higher winds are observed in waves with closed circulation where the low-level winds exceed the speed of propagation. Waves with recirculating flow are frequently observed and can be distinguished in the micropressure record (see Figures 4 and 5) by the characteristic flattened crest in the solitary wave surface pressure signature. This distortion in the micropressure profile can be explained by the contribution from the region of higher density recirculating fluid which propagates with the solitary wave. A calculation of the relative streamline pattern and associated wind field in a typical large amplitude solitary wave disturbance with closed circulation is presented below

Up until now the detailed study of solitary wave activity along the largely uninhabited southern margin of the Gulf of Carpentaria has been limited to the period from mid-September to early November when conditions are most favourable for roll cloud formation. Despite this relatively limited observational period, more than 100 large-amplitude, well-resolved solitary-wave-dominated disturbances have been recorded on a microbarometer network deployed by the Australian National University in the neighbourhood of Burketown during a series of experiments carried out between 1980 and 1982. The morphology of these maritime nonlinear wave disturbances seems to be very similar to those seen inland near Tennant Creek as can be seen in the selection of typical micropressure signatures shown in Fig. 5. 


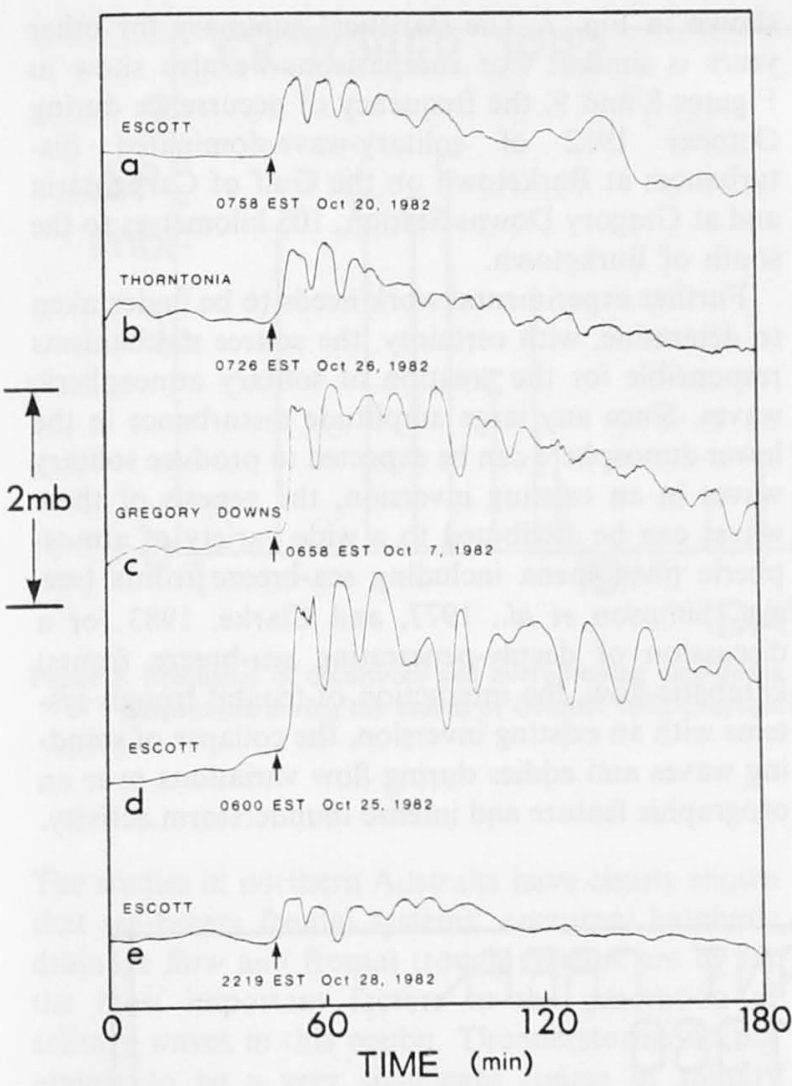

Figure 5. Surface micropressure signatures of typical complex solitary-wave-dominated long wave disturbances recorded at various stations in northern Queensland. Escott station is $15 \mathrm{~km}$ west, Gregory Downs $105 \mathrm{~km}$ south, and Thorntonia $205 \mathrm{~km}$ south of Burketown on the Gulf of Carpentaria. Note that all of these disturbances were clear-air disturbances except for the extensive nonlinear-wave disturbance on 25 October which was accompanied by several well-defined roll cloud formations. Traces of rapidly dissolving cumulus accompanied the event on the 20 October in the area to the north of Burketown. The propagation speed, source azimuth and effective wavelength of the leading solitons are respectively (a) $8.9 \mathrm{~m} / \mathrm{s}, 50^{\circ}, 3.2 \mathrm{~km}$; (b) $11.7 \mathrm{~m} / \mathrm{s}$, $68.4 .2 \mathrm{~km}$; (c) $6.0 \mathrm{~m} / \mathrm{s}, 90^{\circ}, 2.2 \mathrm{~km}$; (d) $12.1 \mathrm{~m} / \mathrm{s}, 60^{\circ}, 5.1 \mathrm{~km}$ and (e) $9.7 \mathrm{~m} / \mathrm{s}, 25,3.5 \mathrm{~km}$

A detailed investigation of the coherence and evolution of long nonlinear wave disturbances over the area between Burketown and Tennant Creek was also carried out during the 1981 and 1982 experimental seasons. This investigation has provided an abundance of new and interesting scientific material on disturbances of this type and has led to a number of conclusions which are directly relevant to the subject of air safety. Perhaps the most important result from these experiments is the observation that seemingly unimportant long wave disturbances can generate,

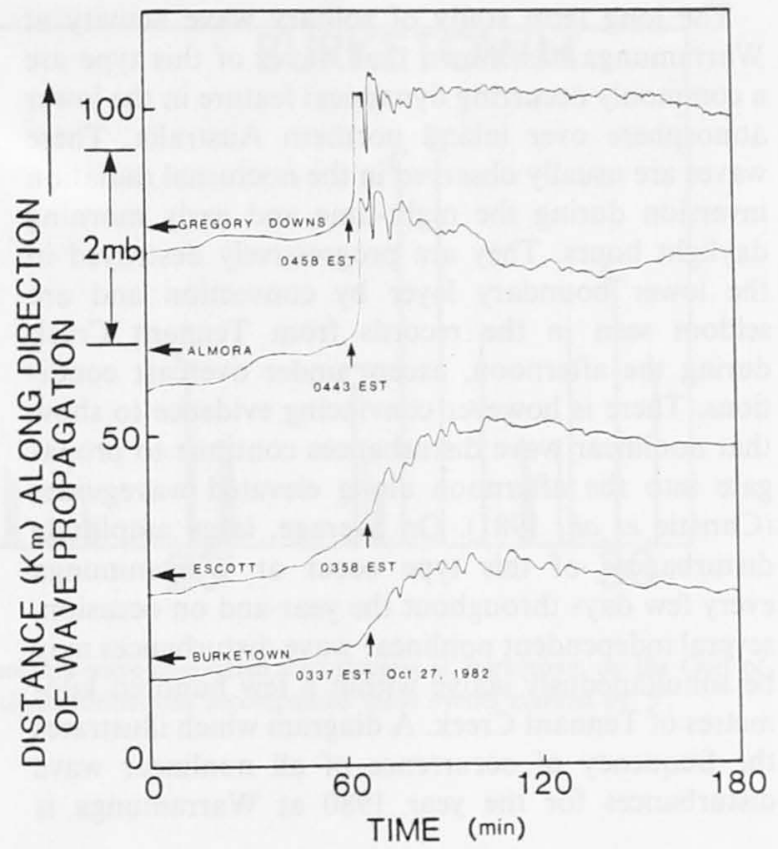

Figure 6. Observed evolution of a long nonlinear wave of elevation between Burketown on the Gulf of Carpentaria and Gregory Downs station, $105 \mathrm{~km}$ to the south. The wave is propagating towards the southwest $\left(\theta=249^{\circ}\right)$ as a clear-air disturbance with an average speed of $14.1 \mathrm{~m} / \mathrm{s}$. When first detected the wave was associated with only very minor perturbations in the surface wind field. Over the next $30 \mathrm{~km}$ the wavefront steepened under the influence of nonlinear dispersion and a family of large amplitude solitary waves with strong closed circulation wind shear zones developed along the leading edge of the disturbance. These observations demonstrate that solitary wave wind shear disturbances can evolve rapidly from apparently benign long wave disturbances.

over relatively short periods of time, a series of large amplitude solitary wave disturbances. An example of this rapid evolution, which is in qualitative agreement with the general predictions of first order nonlinear dispersive wave theory, is shown in Fig. 6. The observation that large amplitude solitary waves can be created under the influence of nonlinear dispersion over short periods of time and over relatively short distances clearly represents an important complication in the prediction problem for wind shear disturbances of this type. A second important result from these large-scale coherent experiments is the observation that long nonlinear wave disturbances in the lower atmosphere often propagate over great distances, sometimes in excess of 700 kilometres, and often have wavefronts which extend for many hundreds of kilometres. 
The long term study of solitary wave activity at Warramunga has shown that waves of this type are a commonly occurring dynamical feature in the lower atmosphere over inland northern Australia. These waves are usually observed in the nocturnal radiation inversion during the night-time and early morning daylight hours. They are progressively destroyed in the lower boundary layer by convection and are seldom seen in the records from Tennant Creek during the afternoon, except under overcast conditions. There is however convincing evidence to show that nonlinear wave disturbances continue to propagate into the afternoon along elevated waveguides (Christie et al., 1981). On average, large amplitude disturbances of this type occur at Warranmunga every few days throughout the year and on occasion, several independent nonlinear wave disturbances may be simultaneously active within a few hundred kilometres of Tennant Creek. A diagram which illustrates the frequency of occurrence of all nonlinear wave disturbances for the year 1980 at Warramunga is shown in Fig. 7. The statistical summary for other years is similar. For comparison, we also show in Figures 8 and 9 , the frequency of occurrence during October 1982 of solitary-wave-dominated disturbances at Burketown on the Gulf of Carpentaria and at Gregory Downs Station, 105 kilometres to the south of Burketown.

Further experimental work needs to be undertaken to determine, with certainty, the source mechanisms responsible for the creation of solitary atmospheric waves. Since any large amplitude disturbance in the lower atmosphere can be expected to produce solitary waves in an existing inversion, the genesis of these waves can be attributed to a wide variety of atmospheric phenomena including sea-breeze fronts (see. e.g., Simpson et al., 1977, and Clarke, 1983 for a discussion of deeply-penetrating sea-breeze fronts) katabatic flow, the interaction of frontal trough systems with an existing inversion, the collapse of standing waves and eddies during flow variations over an orographic feature and intense thunderstorm activity.

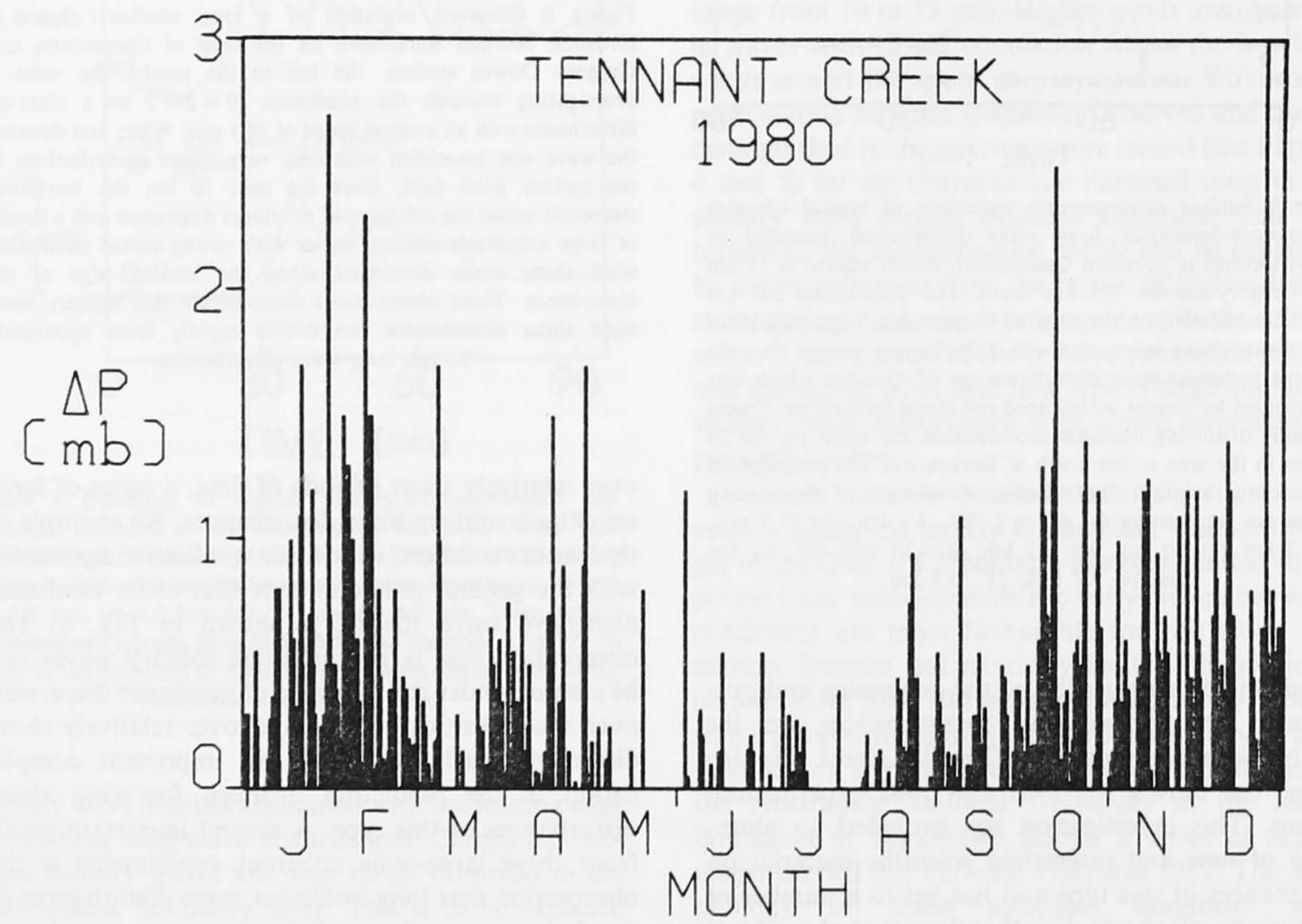

Figure 7. Frequency of occurrence and micropressure amplitudes of all nonlinear wave disturbances detected at the Warramunga Infrasonic Array during 1980. All of these waves occurred as clear-air disturbances 


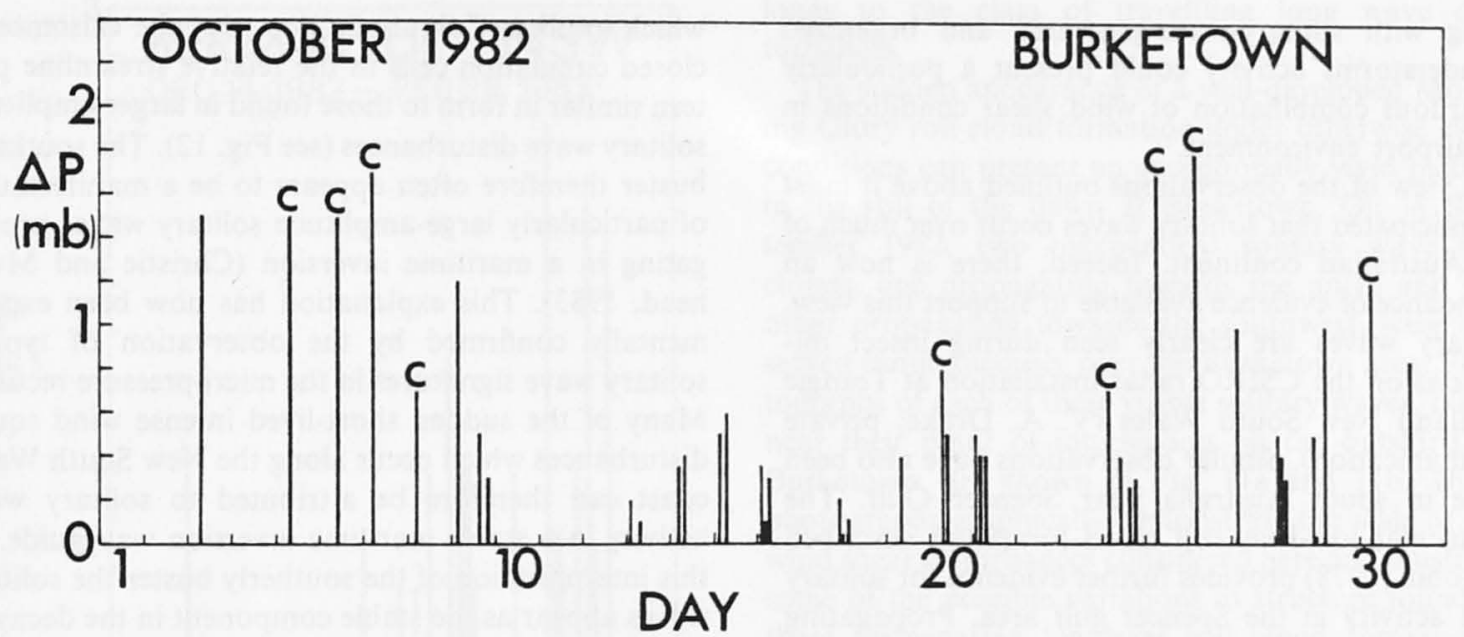

Figure 8. Frequency of occurrence and micropressure amplitudes of solitary-wave-dominated disturbances at Burketown on the Gulf of Carpentaria during the month of October 1982. Propagating cloud formations accompanied those events marked by ' $\mathrm{c}$ '.

The studies in northern Australia have clearly shown that sea-breeze frontal systems, nocturnal katabatic drainage flow and frontal trough systems are by far the most important factors in the generation of solitary waves in this region. Thunderstorms do not appear to be a very significant source of solitary waves in this area. Nevertheless, intense storm systems are probably an important factor in the production of solitary waves in other areas and should be viewed as a potential source of hazardous solitary wave wind shear, even at distances of up to several hundred kilometres.

It should also be pointed out that solitary waves may produce thunderstorms. Waves of this type, with their large amplitudes, are an effective trigger for deep convection and thus they may leave a line of thunderstorms in their wake when propagating into a region of conditional instability. Solitary wave wind shear

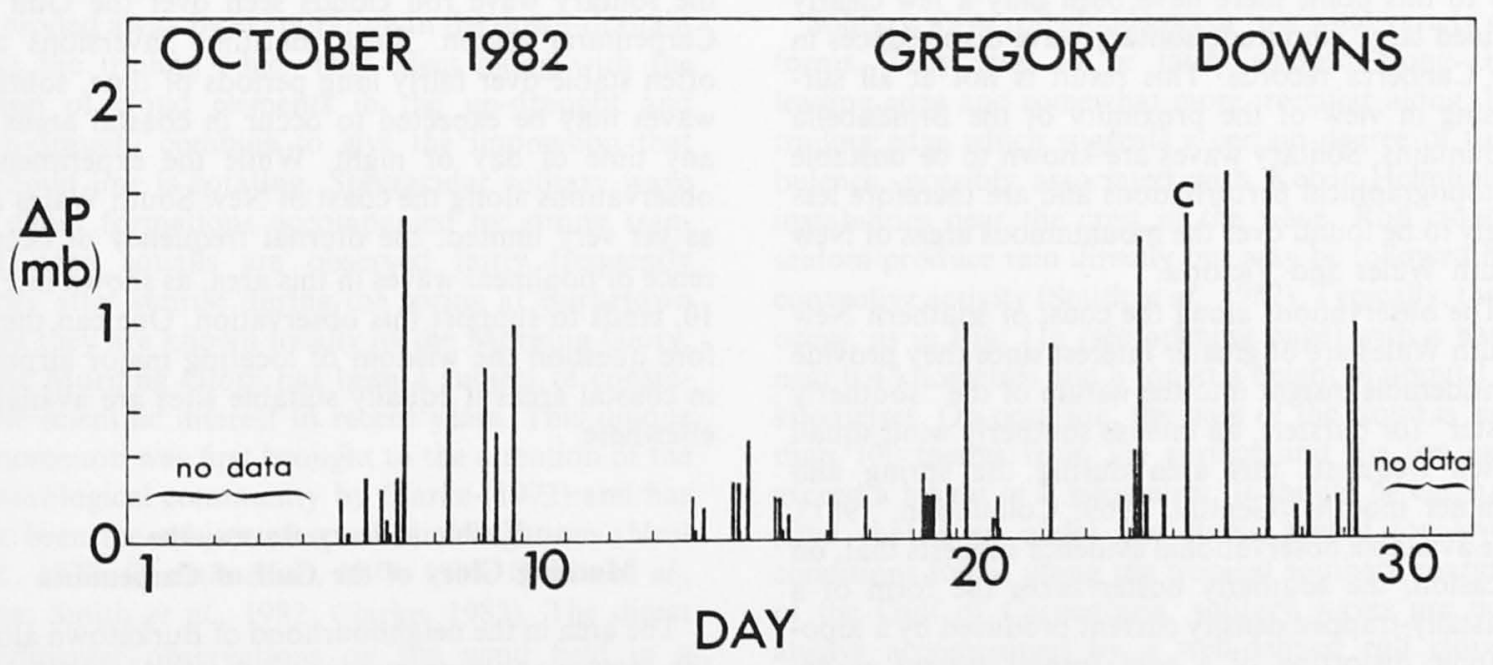

Figure 9. Frequency of occurrence and amplitudes of nonlinear wave disturbances at Gregory Downs station, $105 \mathrm{~km}$ south of Burketown All of these disturbances with the exception of the event on 25 October were clear-air disturbances. 
along with solitary-wave-generated and organized thunderstorms activity could present a particularly hazardous combination of wind shear conditions in the airport environment.

In view of the observations outlined above it must be anticipated that solitary waves occur over much of the Australian continent. Indeed, there is now an abundance of evidence available to support this view. Solitary waves are clearly seen during insect migrations on the CSIRO radar installation at Trangie in inland New South Wales (V. A. Drake, private communication). Similar observations have also been made in south Australia near Spencer Gulf. The spectacular low-level roll cloud formation described by Robin (1978) provides further evidence for solitary wave activity in the Spencer gulf area. Propagating cloud lines which resemble the solitary wave roll cloud formations over the Gulf of Carpentaria are occasionally seen in the GMS satellite imagery near the northwestern Australian coast. These disturbances appear to originate over the Kimberley Plateau (Clarke et al., (1981a). There is also direct observational evidence from the Australian Cold Fronts Research Programme, Phase 1 (Physick and Troup, 1981; Clarke et al., 1981b) to show that long nonlinear wave disturbances are created ahead of advancing cold frontal systems over south eastern Australia.

Recently, we installed sensitive recording microbarometers at Canberra in the Australian Capital Territory and along the southern New South Wales coast to monitor solitary wave activity in these areas. $\mathrm{Up}$ to this point there have been only a few clearly defined large amplitude solitary wave disturbances in the Canberra records. This result is not at all surprising in view of the proximity of the Brindabella Mountains. Solitary waves are known to be unstable to topographical perturbations and are therefore less likely to be found over the mountainous areas of New South Wales and Victoria.

The observations along the coast of southern New South Wales are of greater interest since they provide considerable insight into the nature of the "southerly buster" (or burster), an intense southerly wind squall which frequents this area during the spring and summer months (Gentilli, 1969; Colquhoun, 1981). The available observational evidence suggests that, on occasion, the southerly buster takes the form of a coastally-trapped density current produced by a topographic distortion of a cold frontal system as described by the elegant dynamical model proposed by Baines (1980). On many occasions, however, the observed winds exhibit a more complex structure which implies (Colquhoun, loc. cit.) the existence of closed circulation cells in the relative streamline pattern similar in form to those found in larger amplitude solitary wave disturbances (see Fig. 12). The southerly buster therefore often appears to be a manifestation of particularly large-amplitude solitary waves propagating in a maritime inversion (Christie and Muirhead, 1983). This explanation has now been experimentally confirmed by the observation of typical solitary wave signatures in the micropressure records. Many of the sudden short-lived intense wind squall disturbances which occur along the New South Wales coast can therefore be attributed to solitary wave activity in a stable maritime inversion waveguide. In this interpretation of the southerly buster the solitary waves appear as the stable component in the decay of a dissipating coastally-trapped density current. Largeamplitude solitary waves produced in this way have regions of closed circulation containing entrained fluid from the parent density flow. The generation of solitary waves by a density current advancing into a density-stratified fluid (see Maxworthy, 1980) is probably the most important mechanism involved in the genesis of solitary waves in the lower atmosphere.

The southerly buster is often accompanied by severe weather; it also frequently occurs as a sudden clear-air disturbance with low-level winds in excess of $20 \mathrm{~m} / \mathrm{s}$ which may present a serious wind shear hazard to aviation at Sydney Airport and at all other airports along the New South Wales coast. Occasionally, the onset of these disturbances is marked by a spectacular propagating roll cloud formation similar in form to the solitary wave roll clouds seen over the Gulf of Carpentaria region. Since maritime inversions are often stable over fairly long periods of time, solitary waves may be expected to occur in coastal areas at any time of day or night. While the experimental observations along the coast of New South Wales are as yet very limited, the diurnal frequency of occurrence of nonlinear waves in this area, as shown in Fig. 10 , tends to support this observation. One can therefore question the wisdom of locating major airports in coastal areas if equally suitable sites are available elsewhere.

\section{Visible solitary waves - the Morning Glory of the Gulf of Carpentaria}

The area in the neighbourhood of Burketown along the remote southern margin of the Gulf of Carpentaria is unique in that it is the only known location where solitary wave roll cloud formations can be seen with any regularity. Cloud formations of this type are 


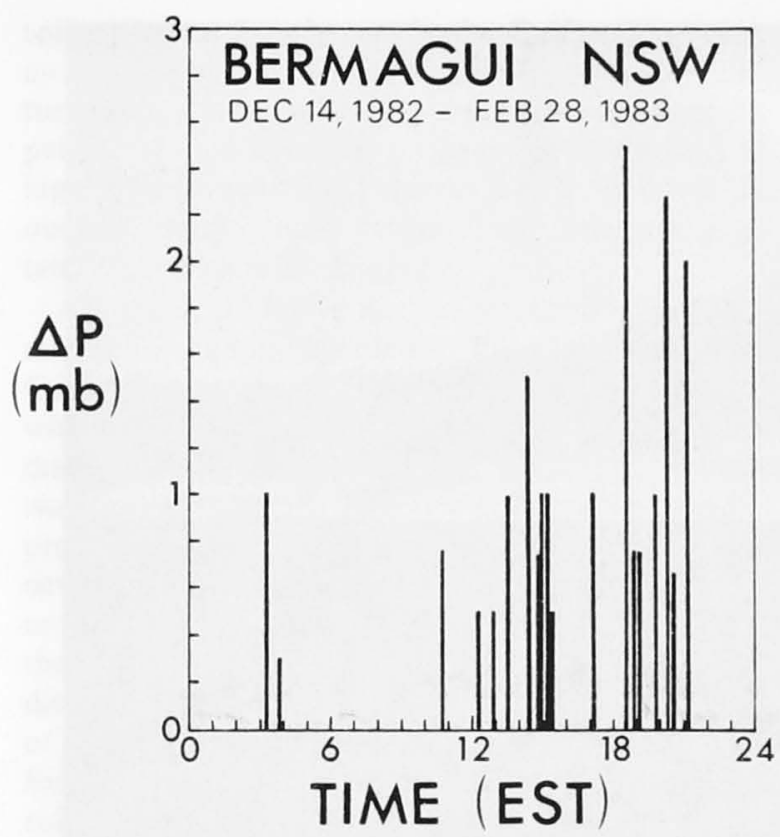

Figure 10. Diurnal frequency of occurrence and amplitude of long nonlinear maritime wave disturbances observed during the summer of 1982/83 at Bermagui on the south coast of New South Wales.

often observed in the form of a rapidly advancing horizontal low-level roll of dense cloud of remarkably uniform thickness which extends in a long arch from horizon to horizon. The cloud in these disturbances is created continually by condensation in the updraught along the leading edge of the solitary wave and eroded away by evaporation in the down-draught along the trailing edge. This effect along with the motion of cloud elements in the up-draught and down-draught combine to give the impression that the cloud line is rotating. Spectacular solitary wave roll cloud formations accompanied by strong transient wind squalls are observed fairly frequently shortly after sunrise during the spring at Burketown where they are known locally as the Morning Glory.

The Morning Glory has been a subject of considerable scientific interest in recent years. This unique phenomenon was first brought to the attention of the meteorological community by Clarke (1972) and has since been the subject of several investigations (Neal et al., 1977; Christie et al., 1979, 1981; Clarke et al., 1981a; Smith et al., 1982; Clarke, 1983). The direct experimental observations on the wind field in a number of Morning Glory disturbances reported by Clarke et al. (1981a) are particularly important since they show conclusively that the Morning Glory be- longs to the class of travellling long wave disturbances.

The sudden appearance of a well-developed Morning Glory roll cloud formation under otherwise clear conditions can present an exceptionally vivid picture in the still of the dawn at Burketown. On 18 September 1982, two independent solitary wave roll clouds, one propagating towards the north and the other propagating towards the southwest, were observed simultaneously over the Burketown area. Photographs of each of these visible solitary waves, taken near their point of intersection on the outskirts of Burketown, are shown in Fig. 11a and 11b. These photographs graphically demonstrate the scale of the wind shear disturbances considered here and illustrate some of the possible variations in structure found in these propagating low-level roll cloud formations. The base of these clouds is estimated to be at about 400 metres. The cloud in the larger amplitude disturbance from the south extends to a height of about 1500 metres above the surface and the top of the smaller disturbance lies at about 800 metres. The apparent distortion along the wavefront of the smaller disturbance can be attributed to the strong nonlinear interaction near the point of intersection of the two solitary wave disturbances. Note that these propagating roll clouds mark, in each case, the position of a low-level zone of intense vertical and horizontal wind shear. The vertical extent of solitary wave disturbances is not as yet known with certainty (Clarke et al., 1981a). It is believed however that disturbances of this type may extend for several kilometres above the cloud formations.

Solitary wave roll clouds occur in a wide variety of forms. They tend to be fairly smooth along the leading edge and somewhat more irregular along the trailing edge which suggests a certain degree of turbulence, possibly associated with Kelvin-Helmholtz instabilities near the crest of the wave. Roll clouds seldom produce rain directly but may be followed by convective activity (Smith et al., 1982). Typically, they occur, as in Fig. 11a (see opening page) with a base near 0.4 kilometres and a top at a height of about 1.5 kilometres. On occasion, the base of the cloud is less than 100 metres from the surface and the top can exceed a height of 2 kilometres. It should be emphasized that even under the stable humid low-level conditions found along the tropical southern margin of the Gulf of Carpentaria, solitary waves are not always accompanied by a well-defined roll cloud. Some disturbances produce only a poorly-defined disconnected roll cloud while others are marked by only a thin line of scattered irregular cumulus. Many 
D. R. Christie and K. J. Muirhead
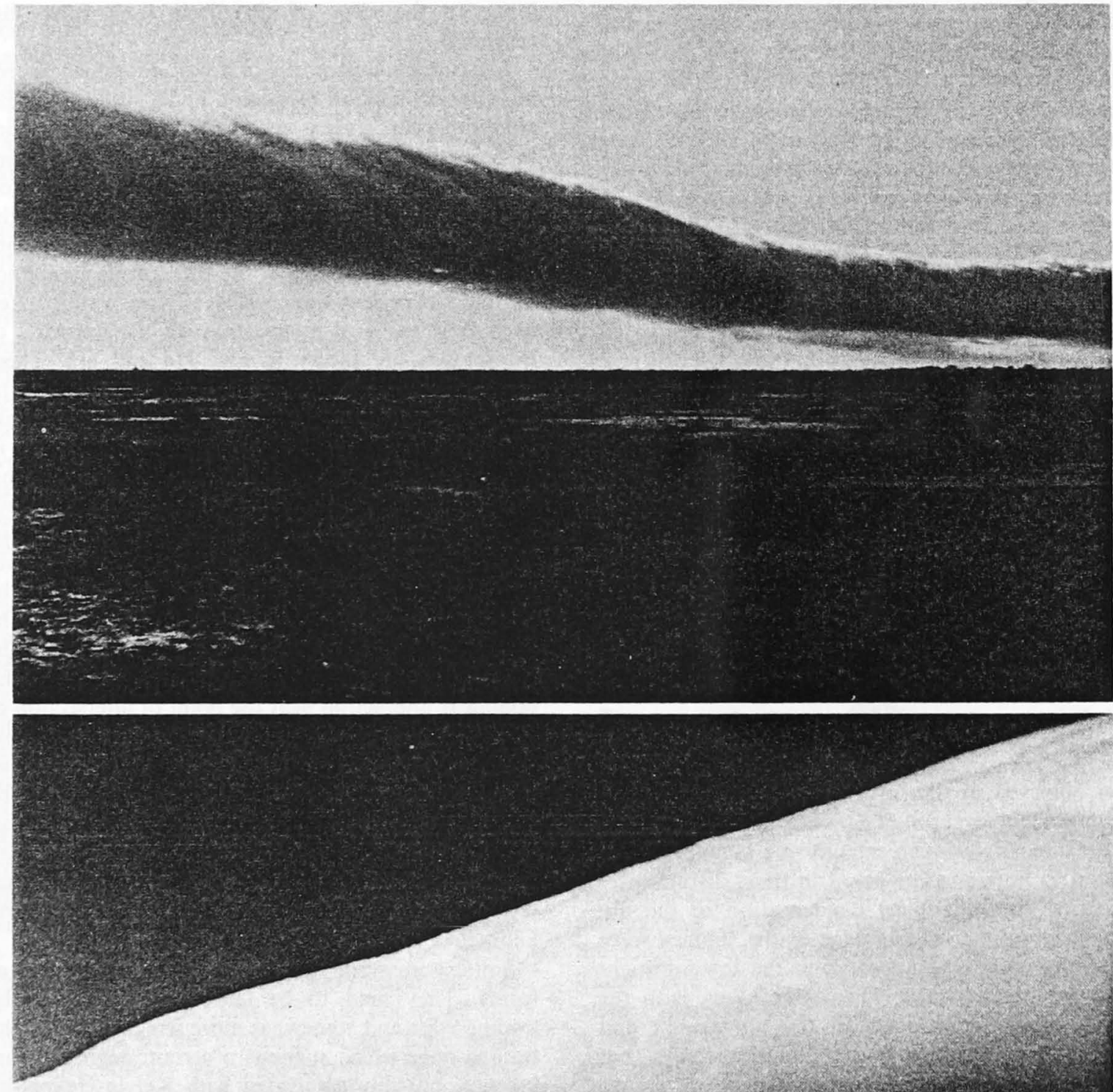

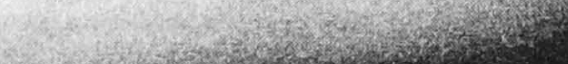

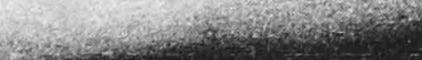

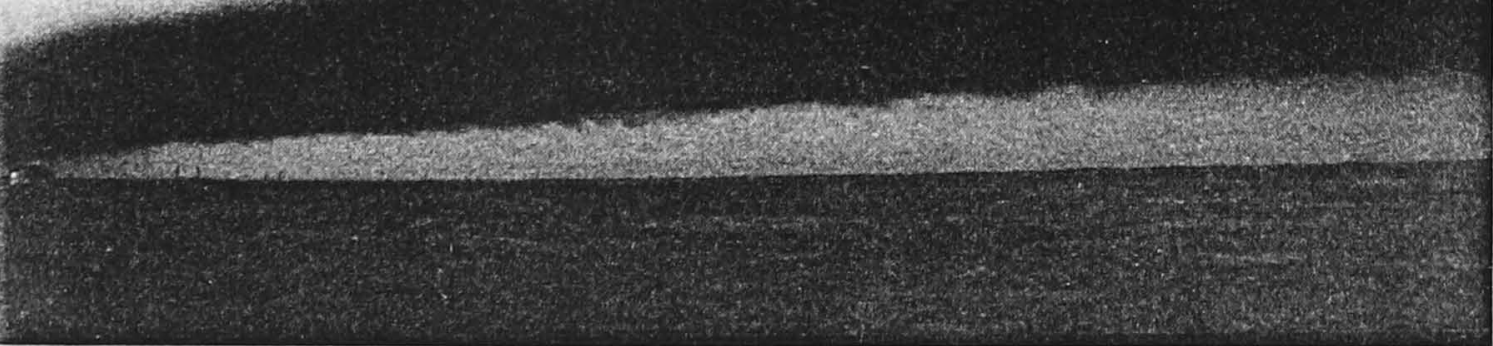

Figure 11. Approaching Morning Glory roll cloud formation produced by independent solitary wave disturbances propagating (a) towards the south-west and (b) towards the north, photographed near their point of intersection on the outskirts of Burketown, Queensland, at about 0730 LST on 18 September 1982. 
solitary wave disturbances in the Gulf region occur, as in inland areas, as completely clear-air disturbances. Solitary waves are sometimes accompanied by thin coherent propagating cloud lines at higher levels near 3 kilometres which supports the observation that on occasion these disturbances extend to considerable height.

All types of solitary-wave-produced cloud formations should be regarded by Pilots and Air Traffic Controllers as a positive indication of severe low-level wind shear conditions. In most nonlinear long wave disturbances seen at Burketown the leading solitary wave usually has the largest amplitude and usually produces, when conditions are suitable, the bestdeveloped roll cloud formation. The locations of one or more solitary wave roll clouds do not always define the extent of hazardous low-level shear zones in these disturbances. In many disturbances, only the position of the leading solitary wave is marked by a wellformed roll cloud; solitons in the wake of this leading roll cloud may produce only poorly-defined cloud lines or they may, as is often the case, be completely cloud free. The observation of a low-level roll cloud cannot even be taken as a reliable indication of the leading edge of these disturbances since clear-air solitary waves have, on occasion, been detected ahead of the first visible wave. Considerable caution should therefore be exercised when flying either ahead of or behind low-level propagating roll clouds.

\section{Observations in Other Areas}

An examination of the meteorological literature shows that long nonlinear wave disturbances of the type considered here are not unique to Australia. They appear to occur commonly world-wide in most maritime areas and in areas of relatively featureless terrain where suitable conditions of low-level stability prevail. As examples, we note the historically interesting large amplitude propagating wave disturbance observed on 14 August 1914 over southern England (Geophysical Memoirs, 1914), the description by Pothecary (1954) of the propagation of a long wave disturbance over south and central England, and the extensive observations reported by Stilke (1972) of internal gravity wave disturbances over northern Germany. Many of these observations clearly belong to the class of nonlinear boundary-layer waves considered here. Remarkably similar disturbances are also frequently seen at any time of day or night and at all times of year over Malta (see e.g., Kirk, 1963 and Perry, 1976). Perry's observations of the roll cloud formations which sometimes accompany these dis- turbances are of particular interest. Perry also documents two aircraft reports of severe turbulence associated with one of these visible disturbances. In one case severe clear-air turbulence was reported by a British Airways Trident between 2000 feet and 3000 feet; in the other, an Antonov 24 encountered severe turbulence on approach to Luqua airport and was forced to make a second approach. Large amplitude disturbance which strongly resemble the long nonlinear waves considered here have also been reported over the eastern Mediterranean by Hardy (1971) and, at Khartoum, by Matthews (1951). Another observation which illustrates the ubiquity of solitary atmospheric waves is the report by Novozhilov (1969) of a propagating low-level roll cloud, accompanied by a transient wind squall, observed in the early morning over eastern Russia.

Long nonlinear wave disturbances in the lower atmosphere appear to be a fairly common feature in many areas of the United States. Williams (1953) has given a thorough description of a wide variety of propagating "pressure wave" disturbances observed over Nebraska, Kansas and northern Oklahoma which bear a remarkable resemblance to the nonlinear boundary layer waves seen over northern Australia. Other clearly related events, observed in the same area, have been studied in detail by Tepper (1950). The properties of a variety of nonlinear long-period internal waves in a maritime inversion at San Diego have been investigated by Gossard et al. (1970) using an FM-CW radar. The results of this study provide clear evidence for solitary wave activity over southern California. More recent reports include the observations described by Schreffler and Binkowski (1981) of two clearly-defined partially resolved nonlinear wave disturbances in the St Louis area and the interesting description by Rust and Doviak (1982) of a clear-air "solitary gust" which was monitored using a sensitive Doppler microwave radar as it propagated for more than 80 kilometres over central Oklahoma from its origin along the leading edge of a thunderstorm down-draught outflow. This latter observation appears to be a particularly well-documented example of the creation of a solitary wave in the decay of a thunderstorm-produced density current. Finally we note the observation described by Bedard et al. (1979) of a gravity wave disturbance detected on an array of discrete pressure jump sensors installed as a wind shear warning system at Dulles International Airport in Washington D.C.. A developing family of solitary waves with closed circulation can clearly be seen in the surface micropressure records of this nonlinear wave disturbance. 


\section{Solitary Wave Wind Shear}

Solitary waves produce rapidly varying horizontal winds near the surface accompanied by strong updraughts along the leading edge and down-draughts along the trailing edge of the wave. The up-draughts and down-draughts in solitary wave flow fields are known (from aircraft and pilot balloon measurements carried out in visible solitary wave disturbances) to exceed $8 \mathrm{~m} / \mathrm{s}$ on occasion (Clarke et al., 1981a). Maximum horizontal wind speeds in waves with recirculating flow exceed the propagation speed and may be greater than $15 \mathrm{~m} / \mathrm{s}$ near the surface. In order to illustrate the complex morphology of the solitary wave wind field and to focus attention on the aviation wind shear problem we have carried out a calculation of the relative streamline pattern and low-level wind shear pattern for typical solitary wave disturbances propagating in a simple, but realistic, boundary layer waveguide in which the Brunt-Väisällä frequency, $\mathrm{N}^{2}=\operatorname{gd}(\ln \theta) / \mathrm{dz}$, varies with altitude as $\mathrm{N}^{2}=\mathrm{N}_{0}^{2} \operatorname{sech}^{2}(\mathrm{z} / \mathrm{H})$. Here, $\theta$ is the potential temperature and $\mathrm{H}$ is a measure of the effective depth of the surface-based inversion. Benjamin (1967) has derived the first order solitary wave solutions in the Boussinesq approximation for this particular model. A complicated solution, correct to second order in wave amplitude, has recently been given by Grimshaw (1981b). Since the properties of large amplitude internal solitary waves are known to depart significantly from those predicted by the weakly nonlinear theories (Tung et al., 1982) we have based the streamline and wind field calculations on a semiempirical analytic solution to the problem which exhibits the recirculating flow pattern found in waves of large amplitude (Davis and Acrivos, 1967) and which reduces at lower amplitudes to the second order solitary wave solution derived by Grimshaw. The relative streamlines determined in this approximation are in good agreement for dimensionless amplitudes up to about 2.2 with the exact numerical solutions of the fully nonlinear wave problem presented by Tung et al. (1982). The calculations therefore span the range of amplitudes normally found for waves in the atmospheric boundary layer. In order to keep the treatment as simple as possible, the influence of surface friction, radiation damping and shear in the background flow field have been ignored. The calculations are intended to illustrate the properties of typical large-amplitude solitary atmospheric waves. It should be emphasized that the effective wavelengths of solitary waves are known to depend sensitively on the precise form of the waveguide density profile (Grimshaw, 1981b;
Tung et al., 1982). Waves with proportionally smaller horizontal scales and correspondingly more intense vertical wind shears are frequently observed over northern Australia.

The results of a calculation of the relative streamline and the horizontal and vertical wind components near the surface for a fairly large amplitude solitary wave propagating in a relatively deep boundary-layer waveguide are shown in Fig. 12. Note the region of recirculating fluid in the interior of this large amplitude wave. As noted above, propagating horizontal vortices of this type produce unusually intense lowlevel winds which may present a particularly severe hazard to aviation. In this example, the maximum horizontal and vertical wind components are about 22 $\mathrm{m} / \mathrm{s}$ and $6 \mathrm{~m} / \mathrm{s}$ respectively. Similar calculations of the wind shear patterns of solitary waves in both deep and shallow boundary-layer inversions may be found in Christie and Muirhead (1983).

A model calculation of the relative streamline pattern for a more complicated amplitude-ordered three-component solitary wave family is shown in Fig. 13. In this case the wave parameters were chosen to illustrate the morphology and scale of the most commonly observed well-resolved form of these long nonlinear wave disturbance. No attempt has been made, for the sake of simplicity, to include the complex residual sub-critical which trail behind the leading solitary wave family. It can be seen from both Figures 12 and 13 that the effective scale of solitary wave disturbances is comparable with the critical scale involved during the initial take-off stage or the final approach stage.

\section{Solitary Waves and Aircraft Performance}

Wind shear in the aviation context may be viewed as any rapid change in either wind speed or wind direction, including vertical wind components, which results in a significant displacement of an aircraft from its intended flight path. Jet-powered aircraft, with their relatively slow response, are particularly susceptable to a sudden encounter with low-level shear. Unexpected wind shear can, however, have a serious adverse influence on the performance of all types of aircraft.

Low-level wind shear in the airport environment has been recognized for some time as a serious problem for aviation and has been the subject of several detailed investigations (see e.g., Fujita, 1976; Fujita and Caracena, 1977; Bedard et al., 1979; Bedard, 1982; McCarthy et al., 1982). In recent years a substantial number of fatal aircraft accidents have 
naller tense over

ream-

nents

litary

layer

on of

mpli-

ontal

low-

evere

mum

ut 22

of the

deep

ound

nline

dered

on in

osen

most

long

been

the

$d$ the

both

itary

itical

$r$ the

ewed

wind

hich

craft

raft,

larly

level

ve a

$f$ all

nent

ious

$t$ of

976 ;

979;

ears

have

\section{DIRECTION OF}

WAVE PROPAGATION
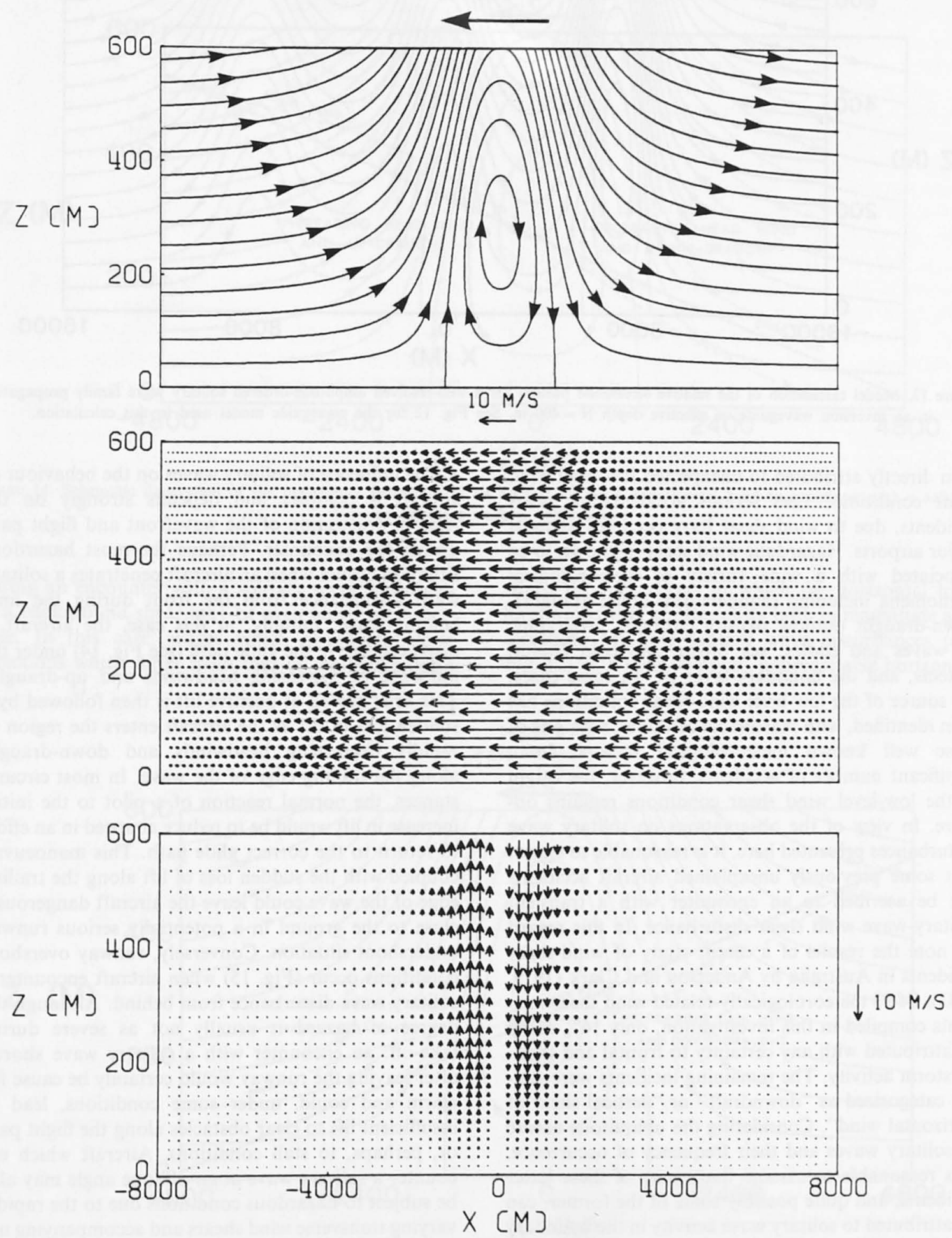

Figure 12. Calculated relative streamline pattern and wind components for a fairly large amplitude solitary wave with recirculating flow propagating in a surface-based inversion of effective depth $\mathrm{H}=400 \mathrm{~m}$ and intensity $\Delta \theta=\theta_{0}-\theta_{\mathrm{S}}=10^{\circ} \mathrm{C}\left(\theta_{\mathrm{S}}\right.$ is potential temperature at the surface, $\theta_{0}$ is the potential temperature of the neutrally stable atmosphere above the inversion waveguide). Brunt-Väisällä frequency in the waveguide model varies as $\mathrm{N}^{2}=\mathrm{N}_{0}^{2} \operatorname{sech}^{2}(z / H)$. Wind components are relative to the ground. 
DIRECTION OF WAVE PROPAGATION

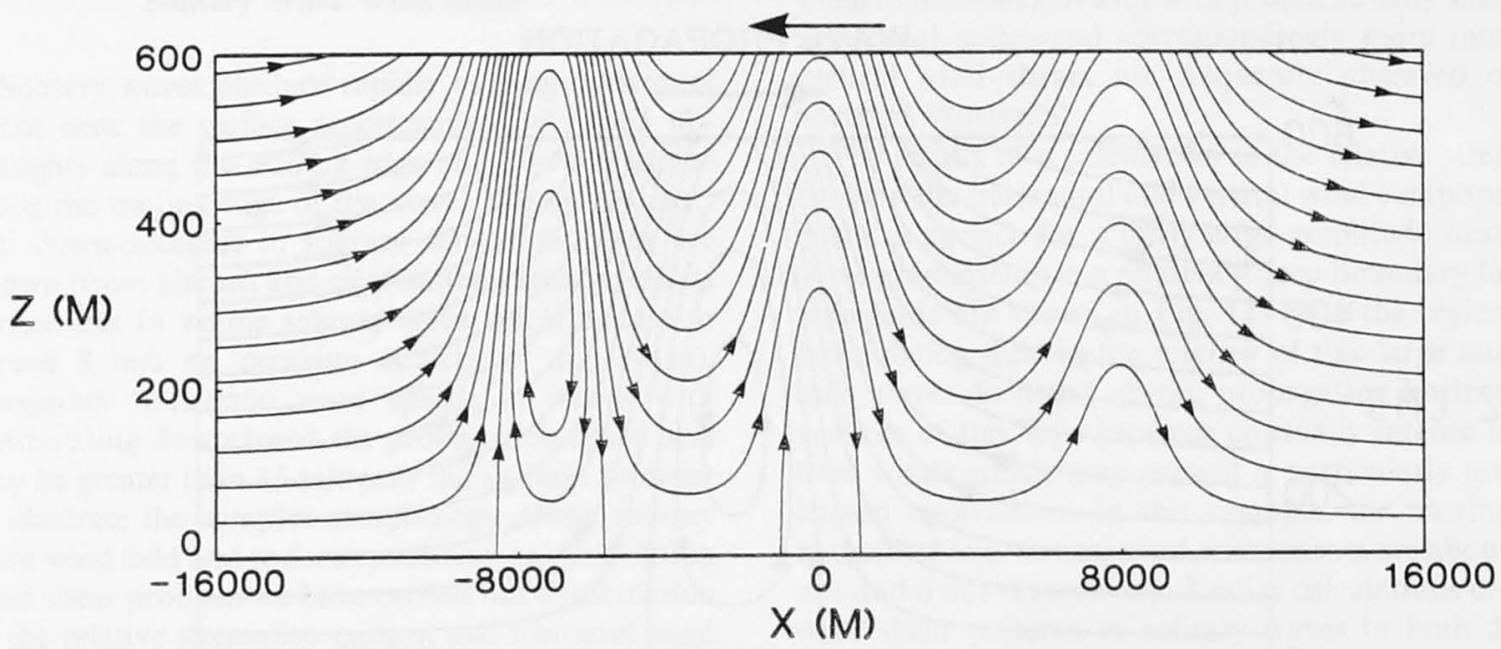

Figure 13. Model calculation of the relative streamline pattern for a well-resolved amplitude-ordered solitary wave family propagating in an inversion waveguide of effective depth $\mathrm{H}=400 \mathrm{~m}$. See Fig. 12 for the waveguide model used in this calculation.

been directly attributed to unexpected low-level wind shear conditions. Less serious accidents, or nearaccidents, due to wind shear have occurred at most major airports. Hazardous wind shear is known to be associated with a wide variety of meteorological phenomena including thunderstorm down-draughts, down-draught outflow density currents, orographic lee waves and eddies, sea breeze and cold frontal systems, and the boundary-layer jet. In some cases, the source of the low-level wind shear conditions has been identified, with reasonable certainty, with one of these well known meteorological factors. In a significant number of accidents, however, the origin of the low-level wind shear conditions remains obscure. In view of the observations on solitary wave disturbances prèsented here, it is reasonable to expect that some previously unexplained aircraft accidents can be ascribed to an encounter with a transient solitary-wave wind shear disturbance. In this regard we note the results of a recent study of wind-shear incidents in Australia by Anderson and Clark (1979, 1981). Of 93 meteorologically-related wind shear incidents compiled in this investigation, only $16 \%$ could be attributed with any certainty to frontal and thunderstorm activity. The remaining incidents were simply categorized as "downdraft" or "vertical shear of horizontal wind". Considering the ubiquitous nature of solitary waves and their frequency of occurrence, it is reasonable to assume that many of these latter incidents, and quite possibly some of the former, can be attributed to solitary wave activity in the boundary layer.
The influence of solitary waves on the behaviour of aircraft is complex and depends strongly on the relative orientation of the wavefront and flight path during the encounter. Perhaps the most hazardous situation occurs when an aircraft penetrates a solitary wave disturbance from the front during the final landing stage. Initially, in this case, the aircraft is displaced above the glide path (see Fig. 14) under the influence of increasing headwinds and up-draught. This brief period of positive lift is then followed by a sudden loss of lift as the aircraft enters the region of rapidly decreasing headwinds and down-draught along the trailing edge of the wave. In most circumstances, the normal reaction of a pilot to the initial increase in lift would be to reduce airspeed in an effort to return to the correct glide path. This manoeuvre, coupled with the sudden loss of lift along the trailing edge of the wave could leave the aircraft dangerously close to the ground in a potentially serious runway undershoot situation. Conversely, runway overshoot conditions occur (Fig. 15) when aircraft encounter a solitary wave disturbance from behind. Although the degree of hazard is usually not as severe during take-off, an encounter with a solitary wave shortly after leaving the runway would certainly be cause for alarm and could, under some conditions, lead to insufficient lift to clear obstacles along the flight path or, perhaps, to stall conditions. Aircraft which encounter a solitary wave at an oblique angle may also be subject to hazardous conditions due to the rapidly varying transverse wind shears and accompanying upand down-draughts. The behaviour of an aircraft 


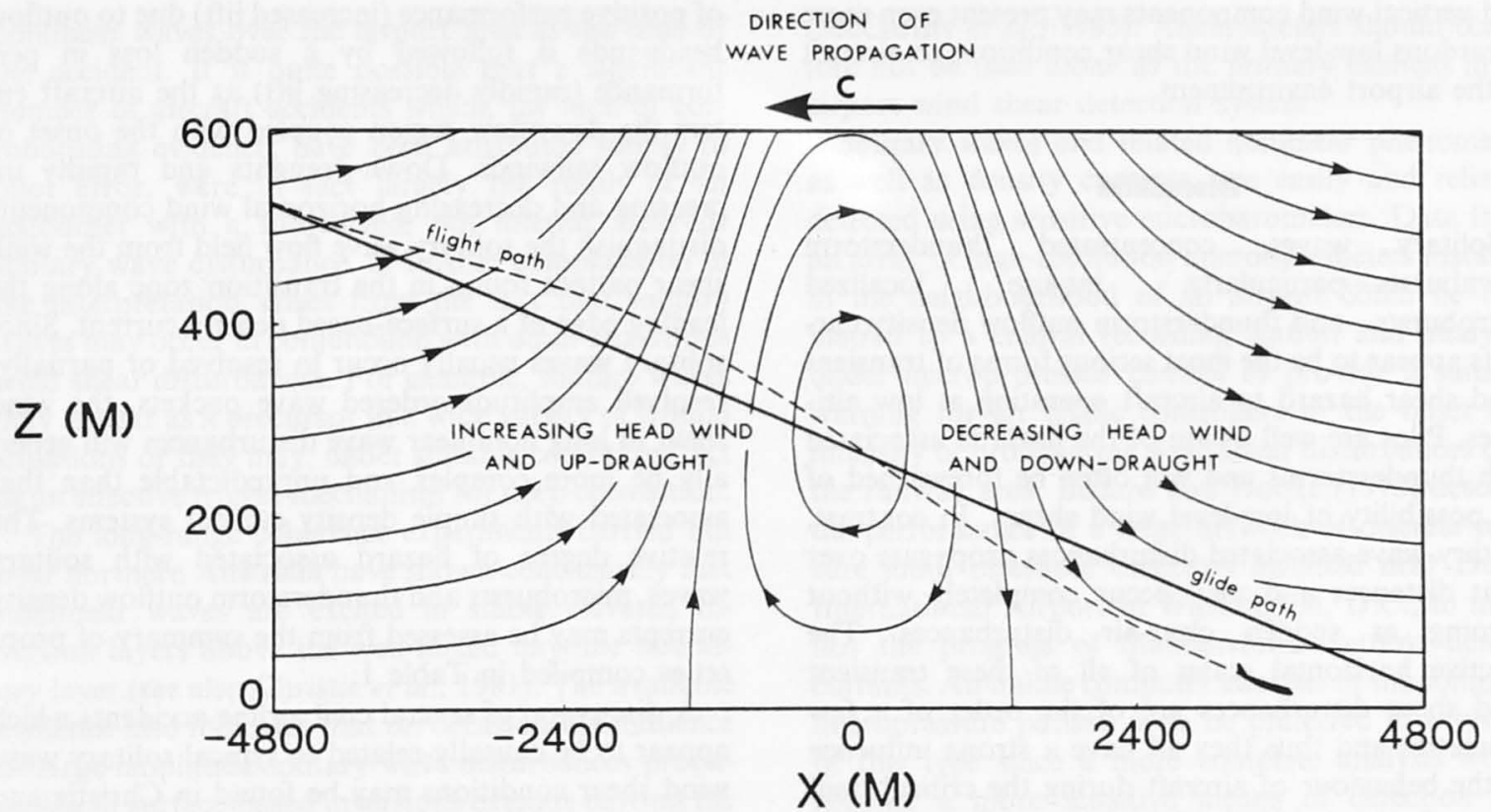

Figure 14. Schematic illustration of the undershoot behaviour of an aircraft during a hypothetical head-on encounter with a solitary wave on final approach to the runway.

during an encounter with a solitary wave embedded in a background shear may be even more unpredictable. In this case, waves which propagate along directions which differ from that of the background winds produce shears in which the horizontal lowlevel winds vary continuously both in magnitude and direction. Multiple solitary wave disturbances (see Fig. 13) with their complex, unpredictable horizontal

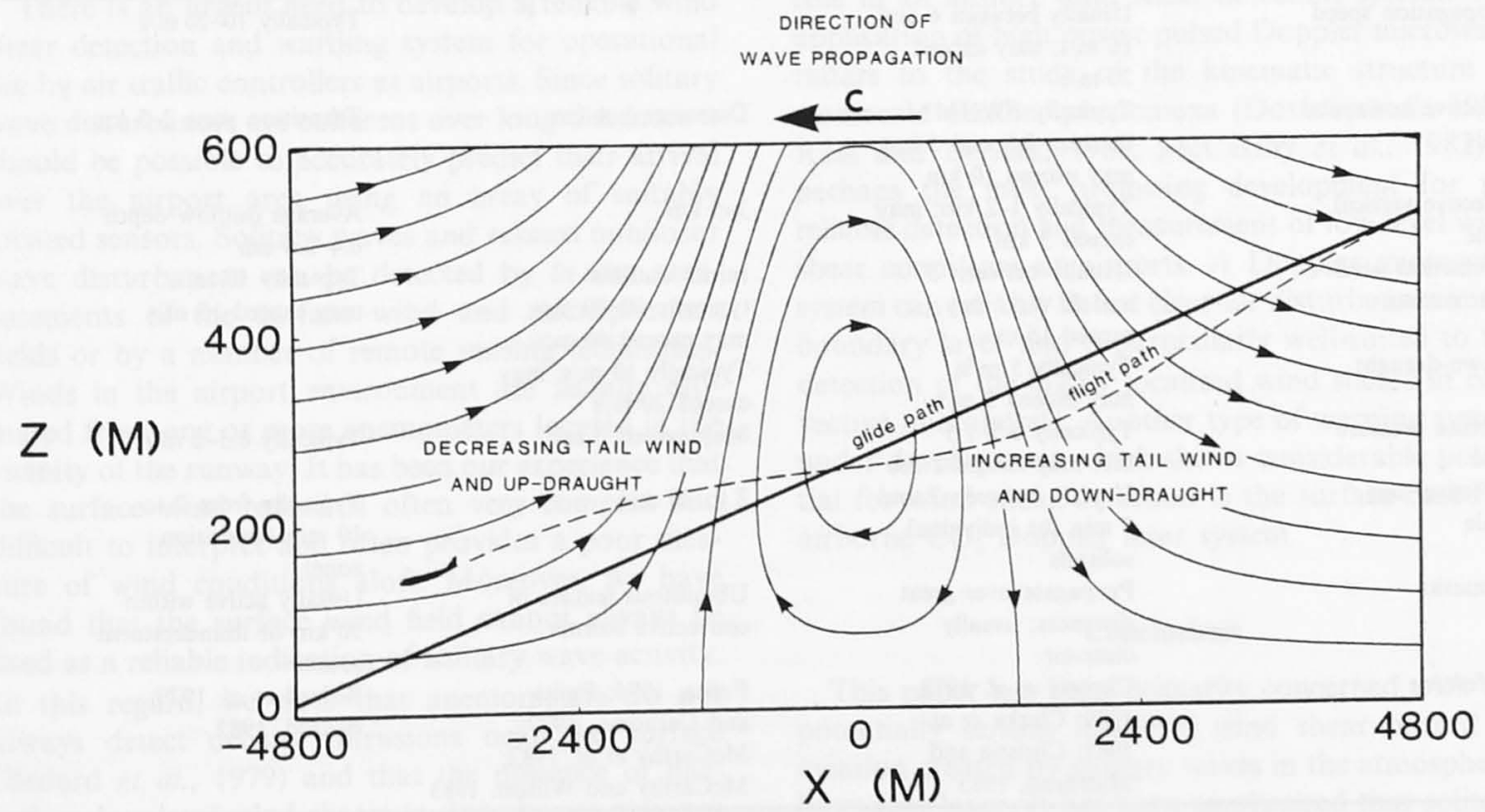

Figure 15. Runway overshoot conditions encountered by an aircraft landing in a solitary wave propagating along the direction of flight. 
and vertical wind components may present even more hazardous low-level wind shear conditions to aircraft in the airport environment.

\section{Discussion}

Solitary waves, concentrated thunderstorm downbursts - particularly intense localized microbursts-and thunderstorm outflow density currents appear to be the most serious forms of transient wind shear hazard to aircraft operating at low altitudes. Pilot are well aware of the hazards associated with thunderstorms and will often be forewarned of the possibility of low-level wind shears. In contrast, solitary-wave-associated disturbances propagate over great distances and may occur completely without warning as sudden clear-air disturbances. The effective horizontal scales of all of these transient wind shear disturbances are of the order of a few kilometres and thus they all have a strong influence on the behaviour of aircraft during the critical final approach stage and the initial take-off stage. It is worth noting that the performance of an aircraft during a head-on encounter on final approach with a solitary wave (Fig. 14) is remarkably similar to the behaviour of an aircraft during an unexpected encounter with a microburst. In the latter case a period of positive performance (increased lift) due to outflow headwinds is followed by a sudden loss in performance (rapidly decreasing lift) as the aircraft enters the downflow region coupled with the onset of outflow tailwinds. Down-draughts and rapidly increasing and decreasing horizontal wind components distinguish the solitary wave flow field from the wind shear pattern found in the transition zone along the leading edge of a surface-based density current. Since solitary waves usually occur in resolved or partiallyresolved amplitude-ordered wave packets, the wind shear in long nonlinear wave disturbances will generally be more complex and unpredictable than that associated with simple density current systems. The relative degree of hazard associated with solitary waves, microbursts and thunderstorm outflow density currents may be assessed from the summary of properties compiled in Table 1.

A discussion of several civil airline accidents which appear to be causally-related to typical solitary wave wind shear conditions may be found in Christie and Muirhead (1983). It should be emphasized that a solitary-wave explanation of many wind shear related accidents is difficult and necessarily speculative at the present time since the available meteorological record almost always lacks sufficient resolution and sensitivity to show with certainty the presence of long

Table 1. Properties of solitary waves, thunderstorm microbursts and thunderstorm outflow density currents.

\begin{tabular}{|c|c|c|c|}
\hline Parameter & Solitary wave & Convective microburst & Density current \\
\hline Propagation speed & $\begin{array}{l}\text { Usually between } 6 \text { and } \\
16 \mathrm{~m} / \mathrm{s} ; \text { may exceed } \\
20 \mathrm{~m} / \mathrm{s}\end{array}$ & & Typically $10-20 \mathrm{~m} / \mathrm{s}$ \\
\hline $\begin{array}{l}\text { Effective horizontal } \\
\text { scale }\end{array}$ & $\begin{array}{l}\text { Typically FWHM* } \\
\text { between } 0.5 \text { and } 6 \mathrm{~km} \text {; } \\
\text { may exceed } 10 \mathrm{~km}\end{array}$ & Diameter $1-4 \mathrm{~km}$ & Transition zone $2-5 \mathrm{~km}$ \\
\hline $\begin{array}{l}\text { Effective vertical } \\
\text { scale }\end{array}$ & $\begin{array}{l}\text { Typically } 1-2 \mathrm{~km} \text {; may } \\
\text { exceed } 3 \mathrm{~km}\end{array}$ & $3-5 \mathrm{~km}$ & $\begin{array}{l}\text { Average outflow depth } \\
0.4-0.6 \mathrm{~km}\end{array}$ \\
\hline $\begin{array}{l}\text { Horizontal winds } \\
\text { near surface }\end{array}$ & $\begin{array}{l}\text { Usually between } 5 \\
\text { and } 12 \mathrm{~m} / \mathrm{s} ; \text { may } \\
\text { exceed } 15 \mathrm{~m} / \mathrm{s}\end{array}$ & $\begin{array}{l}\text { Initial outflow } \\
\text { typically } 10-30 \mathrm{~m} / \mathrm{s} \\
\text { may exceed } 40 \mathrm{~m} / \mathrm{s}\end{array}$ & $\begin{array}{l}\text { Typically } 10 \mathrm{~m} / \mathrm{s} \\
\text { may exceed } 15 \mathrm{~m} / \mathrm{s}\end{array}$ \\
\hline Down-draught & $\begin{array}{l}\text { Typically } 5 \mathrm{~m} / \mathrm{s} \\
\text { may exceed } 8 \mathrm{~m} / \mathrm{s}\end{array}$ & $\begin{array}{l}\text { Typically } 10 \mathrm{~m} / \mathrm{s} \text {; may } \\
\text { exceed } 20 \mathrm{~m} / \mathrm{s}\end{array}$ & \\
\hline $\begin{array}{l}\text { Surface pressure } \\
\text { field }\end{array}$ & $\begin{array}{l}\text { Typically } 0.5-1.5 \\
\text { mb; may exceed } 2 \mathrm{mb}\end{array}$ & May exceed $5 \mathrm{mb}$ & Typically $0.5-2 \mathrm{mb}$ \\
\hline $\begin{array}{l}\text { Effective time } \\
\text { scale }\end{array}$ & $\begin{array}{l}\text { Usually between } 2 \text { and } \\
7 \text { min for individual } \\
\text { solitons }\end{array}$ & 2 to $20 \mathrm{~min}$ & $\begin{array}{l}\text { Typically from } 2 \text { to } \\
10 \text { min (transition } \\
\text { zone) }\end{array}$ \\
\hline Remarks & $\begin{array}{l}\text { Propagate over great } \\
\text { distances; usually } \\
\text { clear-air }\end{array}$ & $\begin{array}{l}\text { Ubiquitous feature of } \\
\text { convective storms }\end{array}$ & $\begin{array}{l}\text { Usually active within } \\
30 \mathrm{~km} \text { of thunderstorm } \\
\text { source }\end{array}$ \\
\hline Reference & $\begin{array}{l}\text { Christie et al. 1978, } \\
\text { 1979; Clarke et al. } \\
\text { 1981; Christie and } \\
\text { Muirhead, } 1983\end{array}$ & $\begin{array}{l}\text { Fujita, 1976; Fujita } \\
\text { and Caracena, 1977; } \\
\text { McCarthy et al. 1982; } \\
\text { McCarthy and Wilson, } 1983\end{array}$ & $\begin{array}{l}\text { Bedard et al. 1979; } \\
\text { Bedard, } 1982\end{array}$ \\
\hline
\end{tabular}

${ }^{*}$ Full width at half-maximum amplitude 
nonlinear waves over the airport area at the time of the accident. It is quite possible that a significant number of aircraft accidents which, for lack of corroborating evidence, have been attributed simply to pilot error, were in fact largely the result of an encounter with a short-lived, but intense, clear-air solitary wave disturbance. A further complication in the interpretation arises from the fact that solitary waves may occur in conjunction with other hazardous wind shear disturbances. For example, solitary waves may appear as a precursor in a wide variety of frontal situations or they may, under suitable conditions, act as an effective trigger mechanism for deep convection.

The long-range coherence experiments carried out over northern Australia have shown conclusively that nonlinear waves are excited in stable elevated inversion layers above the well-mixed daytime boundary layer (see also Christie et al., 1981). The available evidence also indicates that on occasion the influence of large-amplitude solitary wave disturbances propagating in surface-based inversions extends beyond the lower few kilometres. Solitary wave activity in the lower troposphere may therefore play a significant role in the production of clear-air turbulence (CAT) at altitudes up to about 4 kilometres. It is also possible that long nonlinear waves occur in higher inversion waveguides. This may provide an explanation for some aircraft reports of long-period highlevel turbulence such as the 'gravity wave' incidents over the mid-Atlantic described by Roach (1969).

There is an urgent need to develop a reliable wind shear detection and warning system for operational use by air traffic controllers at airports. Since solitary wave disturbances are coherent over long distances it should be possible to accurately predict their arrival over the airport area using an array of suitably located sensors. Solitary waves and related nonlinear wave disturbances can be detected by in situ measurements of the surface wind and micropressure fields or by a number of remote sensing techniques. Winds in the airport environment are usually estimated from one or more anemometers located in the vicinity of the runway. It has been our experience that the surface wind record is often very complex and difficult to interpret and often provides a poor measure of wind conditions aloft. Moreover, we have found that the surface wind field cannot always be used as a reliable indication of solitary wave activity. In this regard, we note that anemometers do not always detect density intrusions near the surface (Bedard et al., 1979) and that the presence of hazardous low-level wind shears in downbursts may not be indicated immediately in the surface wind records
(McCarthy et al., 1983). Anemometers should therefore not be used alone as the primary element in an airport wind shear detection system.

Solitary waves and related nonlinear phenomena, as well as density currents, are easily and reliably detected using sensitive microbarometers. Data from an array of high-resolution microbarometers installed in the neighbourhood of an airport could be telemetred to a central recording station and analysed under microprocessor control to provide a suitable warning for air traffic controllers of the onset and intensity of propagating wind shear disturbances over the runway area. Bedard and Hooke (1978) describe the performance of a large network of discrete pressure jump threshold detectors installed near Dulles International Airport in Washington, D.C., to monitor the progress of thunderstorm outflow density currents. An online computer analysis of the complete micropressure pattern is to be preferred in a system of this type since a more complete analysis would provide a more sensitive means of detection and would distinguish between the various types of boundary-layer wind shear phenomena and thus provide a measure of the degree of wind-shear hazard associated with individual disturbances.

Winds in the boundary layer can also be determined using a wide variety of Doppler radar techniques. Acoustic Doppler sounders (Shaw et al., 1981) provide a direct measure of static wind shear under clear conditions and could play a valuable supporting role in an airport wind shear detection system. The application of high power pulsed Doppler microwave radars to the study of the kinematic structure of mesoscale weather phenomena (Doviak et al., 1979; Rust and Doviak, 1982; McCarthy et al., 1982) is perhaps the most promising development for the reliable detection and measurement of low-level wind shear conditions at airports. A Doppler microwave system can reliably detect clear-air disturbances in the boundary layer and is particularly well-suited to the detection of the highly localized wind shears in convective microbursts. Another type of warning system under development which shows considerable potential for wind shear detection is the surface-based or airborne $\mathrm{CO}_{2}$ Doppler laser system.

\section{Conclusions}

This paper has been primarily concerned with the potentially serious low-level wind shear hazard to aviation created by solitary waves in the atmospheric boundary layer. It has been emphasized that solitary waves are a commonly occurring ubiquitous feature 
in the lower atmosphere. Solitary-wave-dominated disturbances propagate over great distances and may be encountered by aircraft without warning as sudden transient clear-air wind-shear disturbances. Further work needs to be undertaken to determine with certainty the precise nature of the source mechanisms involved and the specific meteorological factors which lead to the formation and long-range propagation of these disturbances. Further work is also required to determine the precise meteorological conditions which favour particularly severe low-level solitary wave wind-shear conditions.

The following conclusions and recommendations are based on the study of solitary waves in the lower atmosphere.

1. Solitary wave roll clouds are dangerous. No attempt should be made to land or take-off through a low-level propagating roll cloud. Pilots should exercise considerable caution when flying at low levels within 40 kilometres on either side of a low-level roll cloud since these visible solitary waves may be accompanied by other clear-air solitary wave disturbances.

2. Pilots and Air Traffic Controllers should become familiar with the properties of solitary waves and their influence on the performance of aircraft during the landing and take-off stage.

3. All reports of wind shear in the vicinity of airports should be promptly relayed to incoming and outgoing flights. Air Traffic Controllers should be aware that the detection of one solitary wave wind squall over the airport area may well be an indication of the onset of a sequence of identical wind squalls separated in time by some 10 to 20 minutes.

4. The frequency of occurrence and intensity of solitary waves should be established in areas where traffiic density is high.

5. Internationally recognized, acceptable limits need to be established for all forms of wind shear.

6. A reliable wind-shear detection and warning system should be developed and installed at all airports so that Air Traffic Controllers can undertake suitable preventative measures when acceptable windshear limits are exceeded.

\section{ACKNOWLEDGEMENTS}

The authors wish to express their gratitude to Professors A. L. Hales, K. Lambeck and J. S. Turner for their valuable advice and support during the course of this work. One of the authors (DRC) would particularly like to thank $\operatorname{Dr} R$. H. Clarke of the Department of Meteorology, University of Melbourne for many stimulating discussions. We are extremely grateful to the managers of stations in the Gulf of Carpentaria area and in inland Queensland for their generous assistance. We particularly wish to thank Joy and Terry Arnold of Burketown for providing observational material, Lyn and Len Stolk and Bob Silcott of Escott Station for their generous support, Martin Haemmerle and Noel Henderson of Burketown for their expert photography, and John Tarca, Alan Mainwaring and Brian Schaefer for their skillful efforts in operating the Warramunga Infrasonic Array and for their valuable assistance in the field experiments. We also wish to express our gratitude to Robert Dabrowski for his expert assistance with the data reduction for this project.

This research was supported by the Air Force Office of Scientific Research under Contract AFOSR-830045 .

\section{References}

Anderson, K. W. and Clark, B. A. J.: 1979, 'Wind Shear in Australia', Aviation Safety Digest 106, 14-20.

Anderson, K. W. and Clark, B. A. J.: 'A Study of Wind Shear Effects on Aircraft Operations and Safety in Australia', Systems Report 24, Aeronautical Research Laboratories, Department of Defence, Melbourne, Victoria.

Baines, P. G.: 1980: 'The Dynamics of the Southerly Buster', Aust. Met. Mag. 28, 175-200

Bedard, A. J., Jr.: 1982. 'Sources and Detection of Atmospheric Wind Shear', A.I.A.A. Journal 20, 940-945.

Bedard, A. J., Jr., Hooke, W. H. and Beran, D. W.: 1977, 'The Dulles Airport Pressure Jump Detector Array For Guest Front Detection' Bull. Amer. Meteor. Soc. 58, 920-926.

Bedard, A. J., Jr. and Hooke, W. H., 1978, 'The Dulles Airport Pressure-Sensor Array For Gust-Front Detection-System Design and Preliminary Results', Fourth Symposium on Meteorological Observations and Instrumentation, American Meteorological Society, Denver, Colorado, 115-124

Bedard. A. J., Jr., Merrem, F. H., Simms, D. and Cairns, M. M.: 1979. 'A Thunderstorm Gust-Front Detection System. Part 1. System Operation and Significant Case Studies. Part II. Statistical Results.' Federal Aviation Administration Report No. FAA-RD-79-55. National Technical Information service, Springfield, Virginia, 22161

Benjamin, T. B.: 1967, 'Internal Waves of Permanent Form in Fluids of Great Depth', J. Fluid Mech. 29, 559-592

Christie, D. R. and Muirhead, K. J.: 1981, 'Observations of Solitary Waves Over Northern Australia'. Physics for Australia's Development, Second Applied Physics Conference, Australian Institute of Physics, Melbourne, 463-466

Christie, D. R. and Muirhead, K. J.: 1983, 'Solitary Waves: A Hazard to Aircraft Operating at Low Altitudes', Aust. Met. Mag. 31, No. 2. 
Christie, D. R.: Muirhead, K. J. and Clarke, R. H.: 1981, 'Solitary Waves in the Lower Atmosphere', Nature 293, 46-49.

Christie, D. R., Muirhead, K. J. and Hales, A. L.: 1978, 'On Solitary Waves in the Atmosphere', J. Atmos. Sci. 35, 805-825.

Christie, D. R., Muirhead, K. J. and Hales, A. L.: 1979, 'Intrusive Density Flows in the Lower Troposphere: A Source of Atmospheric

Solitons', J. Geophys. Res. 84, 4959-4970.

Clarke, R. H.: 1972, 'The Morning Glory: An Atmospheric Hydraulic Jump', J. Appl. Meteor. 11, 304-311.

Clarke R. H.: 1983, 'Fair Weather Nocturnal Inland Wind Surges and Atmospheric Bores', In Press, Aust. Met. Mag. 31

Clarke, R. H., Smith, R. K. and Reid, D. G.: 1981a, 'The Morning Glory of the Gulf of Carpentaria: An Atmospheric Undular Bore', Mon. Wea. Rev. 109, 1726-1750.

Clarke, R. H., Coulman, C. E. and Ryan, B. F., 1981b, Aircraft Penetrations, Cold Fronts Research Programme, Phase 1. Aust. Bureau of Met. Tech. Rep. No. 46, (ed. B. F. Ryan), 52-56.

Colquhoun, J. R.: 1981, 'The Origin, Evolution and Structure of Some Southerly Bursters', Aust. Bureau of Met. Tech. Rep., No. 40, 57 pp.

Davis, R. E. and Acrivos, A.: 1967, 'Solitary Internal Waves in Deep Water', J. Fluid Mech. 29, 593-607.

Doviak, R. J., Zrnić, D. S. and Sirmans, D. S.: 1979, 'Doppler Weather Radar', Proc. IEEE 67, $1522-1553$.

Fujita, T. T.: 1976, 'Spearhead Echo and Downburst Near the Approach End of John F. Kennedy Airport Runway, New York City', SMRP Res. Paper 137, University of Chicago, Ill., 51 pp.

Fujita, T. T. and Caracena, F.: 1977, 'An Analysis of Three Weather-Related Aircraft Accidents', Bull. Amer. Met. Soc. 58, 1164-1181. Gentilli, J.: 1969, 'Some Regional Aspects of Southerly Buster Phenomena', Weather 24, 173-180.

Geophysical Memoirs: 1914, 'The South Wales Tornado of October 27, 1913, With a Note on Remarkable Pressure Oscillations Observed on August 14, 1914', Meteorological Office, No. 11, Neill and Co., Edinburgh, 1-17.

Grimshaw, R.: 1980/81, 'Solitary Waves in a Compressible Fluid', Pageoph. 119, 780-797.

Grimshaw, R.: 1981a, 'Evolution Equations for Long, Nonlinear Internal Waves in Stratified Shear Flows', Studies in Appl. Math. 65, 159-188.

Grimshaw, R.: 1981b, 'A Second-Order Theory For Solitary Waves in Deep Fluids', Phys. Fluids 24, 1611-1618.

Grimshaw, R.: 1981c, 'Slowly Varying Solitary Waves in Deep Fluids', Proc. R. Soc. Lond. A376, 319-332.

Grimshaw, R.: 1982, 'Solitary Waves in Density Stratified Fluids', Math. Res. Rep. 21, University of Melbourne, Victoria, 17 pp.

Gossard, E. E., Richter, J. H. and Atlas, D.: 1970, 'Internal Waves in the Atmosphere from High-Resolution Radar Measurements', J Geophys. Res. 75, 3523-3536

Hardy, R. N.: 1971, 'Gravity Wave Severe Turbulence Near Cyprus', Met. Mag. 100, 209-220.

Joseph, R. I.: 1977, 'Multi-Soliton-Like Solutions to the Benjamin-Ono Equation', J. Math. Phys. 18, $2251-2258$.

Kirk, T. H.: 1963, 'An Oscillatory Pressure Jump at Malta, 2 September 1960', Met. Mag. 92, 89-92.

Koop, C. G. and Butler, G.: 1981, 'An Investigation of Internal Solitary Waves in a Two-Fluid System', J. Fluid Mech. 112, 225-251

Maslowe, S. A. and Redekopp, L. G.: 1980, 'Long Nonlinear Waves in Stratified Shear Flows', J. Fluid Mech. 101, 321-348.

Matthews, L. S.: 1951, 'Pressure and Wind Oscillation at Khartoum', Weather 6, 184-185.

Maxworthy, T.: 1979, 'A Note on the Interna! Solitary Waves Produced by Tidal Flow Over a Three-Dimensional Ridge', J. Geophys. Res. $84, \mathrm{Cl}, 338-346$

Maxworthy, T.: 1980, 'On the Formation of Nonlinear Internal Waves From the Gravitational Collapse of Mixed Regions in Two and Three Dimensions', J. Fluid Mech. 96, 47-64

McCarthy, J., Wilson, J. W. and Fujita, T. T.: 1982, 'The Joint Airport Weather Studies Project', Bull. Amer. Met. Soc. 63, 15-22.

McCarthy, J. and Wilson, J. W.: 1983, 'Applications of Doppler Radar to Aviation Operations-JAWS Experiences', A.I.A.A. 21st Aerospace Sciences Meeting, American Institute of Aeronautics and Astronautics, Reno, Nevada, AIAA-83-0205.

Miles, J. W.: 1980, 'Solitary Waves', Ann. Rev. Fluid Mech. 12, 11-43.

Neal, A. B., Butterworth, I. J. and Murphy, K. M.: 'The Morning Glory', Weather 32, 176-183.

Novozhilov, N. I.: 1969, 'Wind and Cloud at Sunrise', Priroda, Moscow, 3, 69-70 (In Russian, English translation available from Dr R.

K. Smith, Monash University, Clayton, Victoria, Australia 3168).

Ono, H.: 1975, 'Algebraic Solitary Waves in Stratified Fluids, J. Phys. Soc. Japan 39, 1082-1091

Perry, J. D.: 1976, 'An Analysis of Pressure Jumps at Luqa, Malta in the years 1968-72', Met. Mag. 105, 166-184.

Physick, W. and Troup, A. J.: 1981, Surface Network Interpretation, Cold Fronts Research Programme, Phase 1, Aust. Bureau of Met. Tech. Rep. No. 46, (ed. B. F. Ryan), 43-47.

Pothecary, I. J. W.: 1954, 'Short-Period Variations in Surface Pressure and Wind', Quart. J. Roy. Meteor. Soc., 80, 395-401.

Roach, W. T.: 1969, 'Some Aircraft Reports of High-level Turbulence', Met. Mag. 98, 65-78.

Robin, A. G.: 1978, 'Roll Cloud Over Spencer Gulf', Aust. Met. Mag. 26, 125.

Russell, J. S.: 1838, Report of the Committee on Waves, Rep. Meet. Brit. Assoc. Adv. Sci., 7th, Liverpool, 1837, London: John Murray, $417-496$

Rust, W. D. and Doviak, R. J.: 1982, 'Radar Research on Thunderstorms and Lightning', Nature 297, 461-468.

Shreffler, J. H. and Binkowski, F. S.: 1981, 'Observations of Pressure Jump Lines in the Midwest, 10-12 August 1976', Mon. Wea. Rev $109,1713-1725$.

Shaw, N. A., Bourne, I. A., Mace, O. and Robinson, G. S.: 1981, 'Acoustic Radar for Monitoring Wind and Turbulence in the Atmosphere', Physics for Australia's Development, Second Applied Physics Conference, Australian Institute for Physics, Melbourne, Victoria, 467. Simpson, J. E., Mansfield, D. A. and Milford, J. R.: 1977, 'Inland Penetration of Sea-Breeze Fronts', Quart. J. R. Met. Soc. 103, 47-76. Smith, R. K., Crook, N. and Roff, G.: 1982, 'The Morning Glory: An Extraordinary Atmospheric Undular Bore', Quart. J. R. Met. Soc. $108,937-956$.

Stilke, G.: 1973, 'Occurrence and Features of Ducted Modes of Internal Gravity Waves Over Western Europe and Their Influence on Microwave Propagation', Boundary-Layer Meteorol. 4, 493-509. 
D. R. Christie and K. J. Muirhead

Tepper, M.: 1950, 'A Proposed Mechanism of Squall Lines: The Pressure Jump Line', J. Meteor. 7, 21-29.

Tubota, T.: 1982, 'Large Amplitude Internal Waves of Permanent Form', Studies Appl. Math. 66, 1-44. Tung, K.-K., Ko, D. R. S. and Chang, J. J,: 1981, 'Weakly Nonlinear Internal Waves in Shear', Studies Appl. Math. 65, 189-221.

Will

Yoshimura, K and Watanabe, S.: 1979, 'Formation of Quasi-Solitary Wave in Korteweg-deVries Equation from Initial Wave without Bound State', J. Phys. Soc. Japan 47, 998-1002. 
Section 2.6

\section{Thunderstorm-generated solitary waves: A wind shear hazard}

(R.J. Doviak and D.R. Christie)

A.I.A.A. J. of Aircraft, 46, 423-431, 1989. 


\section{Thunderstorm-Generated Solitary Waves: A Wind Shear Hazard \\ R. J. Doviak and D. R. Christie}

Reprinted from 


\title{
Thunderstorm-Generated Solitary Waves: A Wind Shear Hazard
}

\author{
R. J. Doviak* \\ National Oceanic and Atmospheric Administration, Norman, Oklahoma \\ and \\ D. R. Christie† \\ Australian National University, Canberra, Australia
}

\begin{abstract}
Observations of a boundary-layer solitary wave sensed with the National Severe Storms Laboratory's Doppler weather radar and a 444-m-tall instrumented tower suggest that solitary and other nonlinear waves are a source of significant wind shear hazard to safe flight and thus should be studied both experimentally and theoretically. Wave transport of the horizontal momentum of the vertically sheared ambient air contributed much to the observed wind perturbations and horizontal wind shear. Observations are compared with, and shown to agree fairly closely with, the waveform predicted by steady state, weakly nonlinear, internal wave theory.
\end{abstract}

\section{Introduction}

$\mathbf{T}$

HE introduction of new observational devices (e.g., Doppler radar, ${ }^{1}$ airborne Doppler lidar $\left.^{2}\right)$ and improvements in conventional techniques (e.g., meteorological instruments on tall towers ${ }^{3}$ ) are leading to new insights and an understanding of a variety of thunderstorm phenomena that are hazardous to safe flight. Thunderstorm outflows are particularly dangerous to jet aircraft because of their high wing loading, and because of the delay in thrust of the engines after power is applied. ${ }^{4}$

Research at the National Center for Atmospheric Research and the University of Chicago has made the aviation community aware of wind shear hazards accompanying damaging downdrafts, recently called downbursts or, if of small diameter (i.e., 0.4-4 km; 1300-13,000 ft), microbursts. ${ }^{5,6}$ However, these are not the only weather phenomena that can contain wind shear dangerous to an aircraft on its ascent from or descent into air terminals. Tornadoes and/or their larger diameter parent circulations, called mesocyclones, have caused crashes. On October 6, 1981, a Fokker F-28 commercial aircraft was observed exiting a thunderstorm cloud base at the location of a tornado that apparently sheared a wing from its fuselage.

Gust fronts, usually associated with the leading edge of thunderstorm-generated density currents, have been identified for a long time as a region of low altitude wind shear dangerous to safe flight. ${ }^{7}$ It has also been known for many years that thunderstorm downdrafts, the source of these ground-level currents, harbor winds that are dangerous to aircraft. The principal hazard of the microburst, however, is not necessarily confined to the direct effects of the downdraft. As Lee et al. ${ }^{8}$ pointed out, the aircraft is passing beneath a downdraft "experiences first a strong headwind, then roughly no horizontal wind at all as it enters the downflow area, and finally experiences a strong tailwind. These wind shifts from headwind to

Received Dec. 14, 1987; presented as Paper 88-0695 at the AIAA 26th Aerospace Sciences Meeting, Reno, NV, Jan. 11-14, 1988; revi23, 1988. This paper is declared a work of the U.S. Government and is not subject to copyright protection in the United Govern

*Senior Scientist, Environmental Research Laboratories, National Severe Storms Laboratory.

†Research School of Earth Science, Institute of Advanced Studies. tailwind, with a vector difference of $40 \mathrm{~m} / \mathrm{s}$ or more in $4 \mathrm{~km}$ in the horizontal, may be more dangerous than the well-organized gust front.'”

Much of the earlier research on wind shear focused attention on the gust front of the density current. However, it is now known that gust fronts and associated thin lines of reflectivity are observed at the leading edge of bores ${ }^{9}$ and solitary waves ${ }^{10}$ as well as density currents. The Federal Aviation Administration has deployed a network of anemometers (i.e., the Low Level Wind Shear Alert System, LLWAS) to detect these traveling gusts and to estimate their horizontal shear. ${ }^{11}$ In its simplest form, a density current is a shallow layer of laminar flow having a leading edge that moves at a velocity dependent upon the depth of the current as well as the difference in density (or temperature) of the ambient fluid and that within the current. ${ }^{12}$ If this description applied to atmosphéric density currents, the wind shear hazards would be minimal because aircraft would experience only a net increase in headwind However, currents are not necessarily laminar and they often have at their leading edge a mass of recirculating air (called a density or gravity current head) that is somewhat deeper than the current behind it; this propagating horizontal rotor with its pattern of increasing and decreasing head or tail wind is the primary source of hazardous wind shear. Wind gusts generated by these rotors can have their vorticity (shear) magnified as it is being stretched by the radially diverging outflow. ${ }^{13}$ Evidence suggests that this zone of shear could be more dangerous to safe flight than the wind change encountered across the microburst itself.

One of the earliest accidents that has been attributed to changes in head wind is that reported in 1958 by Stewart, who described a "microburst-type" wind shear event (Fig. 1) that downed a BOAC Argonaut on its takeoff from the Kano Airport in Nigeria in June 1956. ${ }^{14}$ The investigating board concluded that, "The accident was the result of the loss of height and airspeed caused by the aircraft encountering, at approximately $250 \mathrm{ft}$ after take-off, an unpredictable thunderstorm cell that gave rise to a sudden reversal of wing direction, heavy rain, and possible downdraft condition.'

Hazardous low-altitude shear has been associated with mountain lee waves, sea breezes, cold frontal passages, and more recently, with large-amplitude gravity (or buoyancy) wave disturbances. ${ }^{15}$ Gossard observed gravity waves that had wind speed changes as much as $20 \mathrm{~m} / \mathrm{s}$ ( $40 \mathrm{knots}$ ) in a distance of $5 \mathrm{~km}(3 \mathrm{mi}){ }^{16}$ 


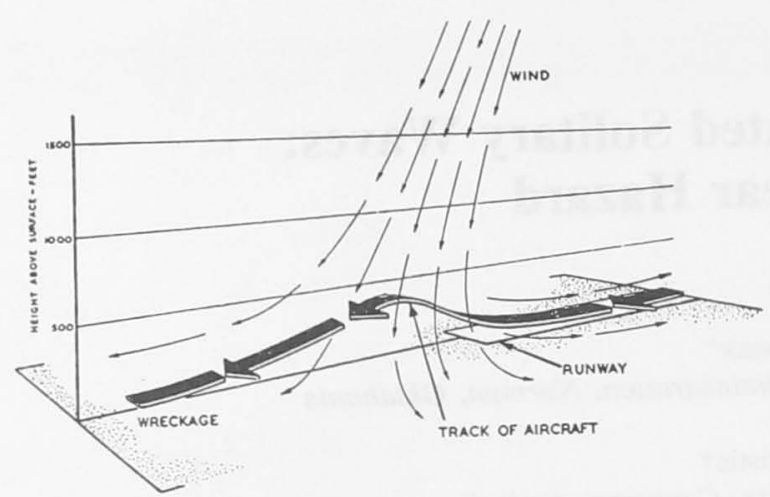

Fig. 1 Unusual conditions that led to the Kano accident in $\mathbf{1 9 5 6 .}$

In this paper, we focus our attention on gravity waves that In this paper, we focus our attent width (wavelength) and, consehuve lare exceptionally stable and can quently, strong shear, but that are with relatively travel hundreds of kilometers from the source with relatively little attenuation of the possibly hazardous shear. These gravity waves, known as solitary waves, ${ }^{17}$ have recently been idenity waves, known as solitude shear. ${ }^{18-20}$

ified as a significant source of low-altitude shear.

The ease with which solitary wat large-amplitude nonlinear in the laboratory suggests the lowest waves might be a commonly occurring feature in the lowest part of the troposphere whenever suitable boundary-layer conditions exist and sources are active. ${ }^{21}$ The first definitive observations of short wavelength atmospheric solitary waves in the planetary boundary layer were made in 1976 at the Australian National University's Warramunga Infrasonic Array near The origin of many of Tennant Creek in Northern Australia. The orion a deeply penetrating Gulf of Carpentaria sea breeze front with an in tense nocturnal inversion.

Solitary waves are most commonly observed with ampliSolitar $300-1000 \mathrm{~m}(900-3000 \mathrm{ft})$ and effective horizontal they usually propagate with speeds between 6 and $16 \mathrm{~m} / \mathrm{s}$ ( 12 and 32 knots) but, on occa-

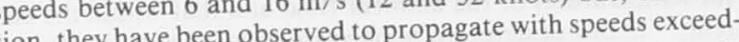
ng $30 \mathrm{~m} / \mathrm{s}$ (60 knots). Atmospheric solitary waves propagate ing strong temperature in amplitude as their width decreases, thereby increasing shear, the speed of long-wavelength weak and their speed exceeds the speed of long-wavelength weak disturbances by an amount proportional speeds. Wind shear in Thus, solitary waves have supercritical speeds. Wind the presence of large-amplitude solitary waves is enhanced by the pre-induced recirculating flow within the wave that exceed the speed of the horizont

Long-wavelength disturbances of very small amplitude exerience little dispersion and accompanying change in shape, perulting in long lifetimes. Short-wavelength disturbances of resull and experience significant small amplitude, on the other hate quickly. However, short wavelength disturbances of large amplitude can evolve into wavelength disturbances of large amplitude can evolve into waves of permanent form under the influence of amplitude dispersion. In other words, the tendency of a large-amplitude disturbance to steepen and break because of nonlinearity is offset by normal frequency dispersion, which tends to smooth the disturbance. Solitary waves are therefore unusually stable large-amplitude disturbances that can carry intense shear long distances.

We show results of observations suggesting that shear generWe show results of obser air ated by solitary waves can have a significant effect on aircraft performance. Solitary waves that produce low-altitude wind shear require strong stability of the atmosphere close to the Earth's surface and near-neutral stability above. ${ }^{23}$ Thus, it is likely that many significant events may pass unobserved because such stable conditions usually occur in the late night or early morning hours when observations are sparse. On the ther hand, thunderstorm outflows can form stable layers at any time and subsequent thunderstorm downdrafts, impinging on large-amplitude gravity waves, as observed by Doviak and Ge. ${ }^{10}$ It must be expected therefore that shear may be encountered at any time over the airport region when widespread thunderstorm conditions prevail. In this context, it is worth noting that some pilots and other members of the aviation community have long recognized that new, and seemingly benign, cell formations are ften more violent than the present storm. ${ }^{24} \mathrm{~A}$ good example where conditions of this type have lead to tragedy is the DallasFort Worth storm that caused the crash of Delta 191 in 1985 (e. see Fig 1.20 of Fujita ${ }^{25}$ ). It has been recommended in the Safety Digest of the 1960 issue of Skyways that "if it begins to rain in the direct take-off flight path, close to but separated from the main storm, it is prudent to remain on the ground until it has passed away.", 24

Solitary waves might be one of the most insidious forms of Solitary waves might be occur, without warning, as wind shear because they usually occur, without warning, as unexpected clear-air disturbances, invisible to the naked and far from any storms. Yet they can harbor shears that might be destructive to aircraft and crew. Although there is no mith conclusive evidence linking solitary waves documentation with conclusive evidence linking solitary waves be dismissed as the cause. For example, a study by Anderson 列 incidents in Australia, only $16 \%$ could be attributed with any incidents in Australia, only $16 \%$ ctivity. 26,27 Thus, concertainty to frontal and thunders of solitary waves and their frequency of occurrence, Christie and Muirhead ${ }^{18}$ inferred that it 列 is reasonable to assume that many of the remaining incident and quite possibly some of the former, can be attributed to solitary wave activity in the first few hundred meters that the atmospheric boundary layer. It is to be emphasized that the horizontal wind shear associated with the wave is

near the ground where aircraft are most vulnerable.

The effect of a solitary wave on aircraft performance is complicated because it depends on the pilot's response to physical stimuli. Furthermore, the response depends on the direction of approach into the wave. For example, Fig. reproduces the streamlines of a numerically modeled and an observed solitary wave on which is superimposed a 3 deg glide path for an aircraft approaching the wave propagating toward The wind relative to a coordinate frame that moves with the wave. For this case, it can be deduced that the aircraft would first experience an increase in deduced by a decrease in head wind and downdraft. The short-dashed line suggests the flight path hat the opposite effects of shear. For aircraft approaching from the opposite direction, the flight path would initially be below the glide

IRECTION OF WAVE PROPAGATION $\longrightarrow$

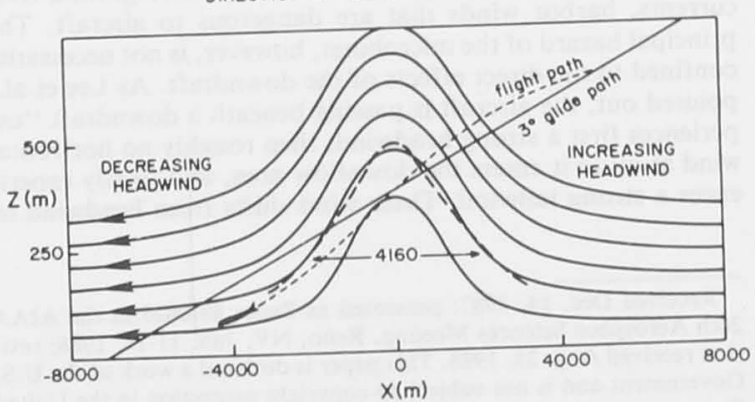

ig. 2 Hypothetical aircraft encounter with a solitary wave (solid 列 servation ${ }^{10,15}$. 
path and then (if the aircraft is still airborne) above, with the possibility that the aircraft would overshoot the runway.

The wind profile along a glide slope through a solitary wave in a shearless environment differs from that encountered in a microburst because a single-crested solitary wave produces only an increase in head wind (or tail wind) and then decreases to the initial wind state, whereas the microburst generates both head and tail wind components. That is, the horizontal wind shift is unimodal in a solitary wave, whereas it is bimodal in a microburst. However, a sequence of two or more solitary waves can evolve from an undular bore (to be shown later in Fig. 10) and this can give the pilot the appearance of alternating head and tail wind components, thus making control of the aircraft more difficult. On the other hand, wind shear effects along glide slopes can resemble those associated with a microburst, if flights are made through a solitary wave in a vertically sheared environmental wind.

\section{Observations}

Doviak and $\mathrm{Ge}^{10}$ presented evidence that a thin line of reflectivity commonly associated with gust fronts was in fact a solitary wave generated by the interaction of thunderstorm outflows. However, they did not make quantitative comparisons of data with theory. In the following sections, we examine in greater detail data from a tall $(444 \mathrm{~m})$ television (KTVY) tower, instrumented by the National Severe Storms Laboratory, to determine the waveform to be compared with that deduced from two-dimensional weakly nonlinear theory of solitary waves.

\section{The Ambient Environment}

It can be shown that it is the virtual potential temperature $\theta_{v 0}$ of the unsaturated ambient atmosphere that determines its stability and hence its period of a buoyancy oscillation. A strongly stable layer embedded in an atmosphere having a

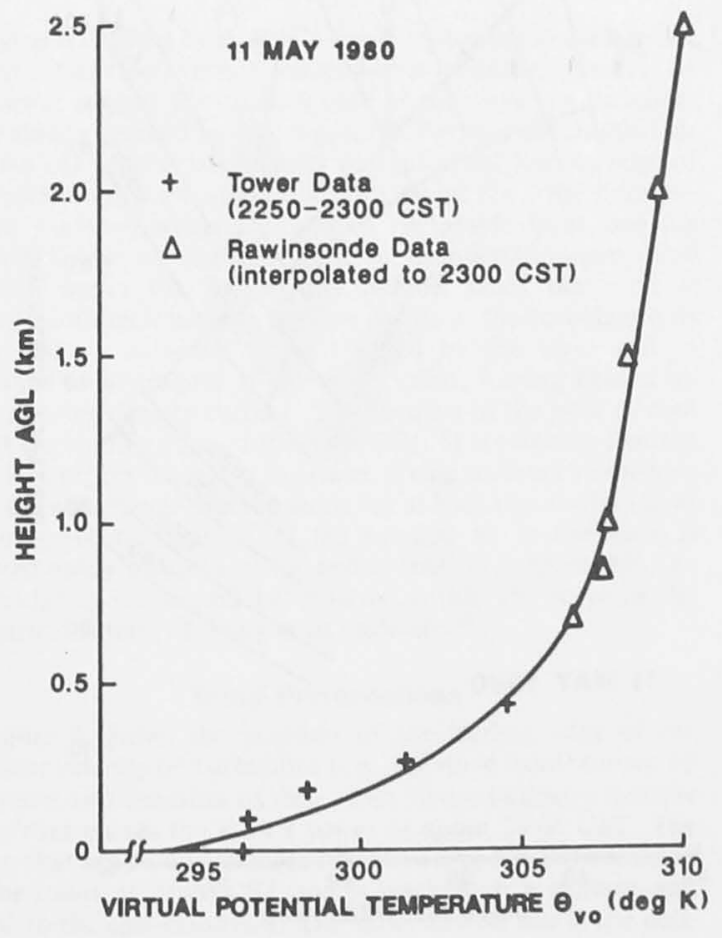

Fig. 3 Virtual potential temperature vs height above ground leve (AGL1) circa 22:55 CST on May 11, 1980. much longer period of buoyancy oscillation conducts largeamplitude gravity wave disturbances long distances from their source. The $\theta_{v 0}$ is related to the sensible temperature $T$ (i.e., the dry bulb temperature in $\mathrm{K}$ ) and the ratio $w$ of water vapor to dry air density (i.e., the mixing ratio in grams per gram) by the equation

$$
\theta_{\nu 0}=\left(\frac{1+1.61 w}{1+w}\right) T\left(\frac{1000}{p}\right)^{0.286(1-0.23 w)}
$$

where $p$ is pressure in millibar units. To compute the vertical profile of $\theta_{10}$ (Fig. 3), the measured $T$ and pressure were used to relate the pressure heights of the rawinsondes to real heights in meters above the ground. It was noted that if a standard atmosphere was used to obtain this relation, height errors larger than $100 \mathrm{~m}$ above $2 \mathrm{~km}$ and thus temperature errors of about $1 \mathrm{~K}$ or more would have occurred.

The profile (Fig. 3) in advance of the wave is calculated from data obtained with tall tower instruments up to $444 \mathrm{~m}$ and from rawinsondes for higher altitudes by interpolating data at the 18:00 CST (Central Standard Time) 5/11/80 and 06:00 CST 5/12/80 sounding times to the 23:00 CST time of wave passage at the tower. The $\theta_{t 0}$ computed from tower data show the presence of an intensely stable surface-based layer having a thickness of at least several hundred meters topped by a weakly stratified region.

Figure 4 shows the vertical profiles of the ambient wind components normal to $\left(u_{c}\right)$ and parallel to $\left(v_{e}\right)$ the wave front before the tiem (23:05-23:15) the wave passes the tower. The estimates from anemometers $(\Delta)$ on the tower $(36 \mathrm{~km}$ from the radar at azimuth $356 \mathrm{deg}$ ) are $10 \mathrm{~min}$ averages of the data spaced 10s apart about 22:45, the time Doppler radar data were also used to compute $u$ and $v_{a}$.

The ambient winds measured with radar (o in Fig. 4) are obtained from estimated Doppler velocities for an assumed horizontally uniform wind model, least-squares fitted to the Doppler velocities measured over an azimuthal sector from 330 to $30 \mathrm{deg}$ at ranges near 30 to $40 \mathrm{~km}$ where data were free from ground clutter and range-aliased overlying echoes. The horizontal bars indicate the $95 \%$ confidence limits, assuming errors are Gaussian distributed and using the computed rms values of the data about the model velocity. The vertical bars denote the uncertainty in beam height for a 0.1 deg uncertainty in elevation angle and also the variation in beam height because radar data from a $4 \mathrm{~km}$ range interval were used in the fitting. The solid lines in Fig. 4 are the inferred profiles of the ambient wind, $u_{a}$ and $v_{a}$.

The winds measured by rawinsonde ( + in Fig. 4 ) are values interpolated from sounding data at 18:00 on May 11 and 06:00 on May 12. Although the interpolated temperature profile appears to reasonably estimate the ambient temperature above the stable layer because there is less than a $2^{\circ} \mathrm{C}$ change over the $12 \mathrm{~h}$ interval between soundings, the wind field changes con siderably during this interval and the interpolated wind differs significantly from the radar-derived wind. Nevertheless, there is good agreement between tower- and radar-measured winds that are from data close to the same time.

The difference in wind measured by the radar and tower at the common height of $400 \mathrm{~m}$ is most likely due to the combined effects of reflectivity gradients, beamwidth, beam blockage, and nonuniformity of the wind that was assumed in the model used to fit the Doppler data. For example, Fig. 4 shows the ambient wind profile $v_{0}$ averaged for the 2 min period just preceding the solitary wave at the tower. We assume that this $v_{0}$ profile represents the values that the ambient wind would have if the wave were not present.

\section{Equivalent Potential Temperature}

The equivalent potential temperature $\theta_{e}$ is the final temperature of an air parcel after it is lifted dry adiabatically to its lifting condensation level, then pseudowet adiabatically to a 
great height to condense water as it is formed, and finally brought down dry adiabatically to a reference pressure of 1000 mbar 28 The $\theta_{e}$ is a conserved property of the air parcel even with the change of moisture phase as would occur if a cloud is formed when nearly saturated air is lifted by the wave.

The $\theta$ is not so easy to compute. An approximate formula ${ }^{29}$ is

$$
\theta_{e} \simeq \theta \mathrm{e}^{L\left(T_{s}\right) w_{s} / c_{p} T_{s}}
$$

where $\theta$ is the potential temperature that a parcel of unsatuif brought adiabatically to a reference rated air would have if brought adiabatically to a referen at $T_{s}$,
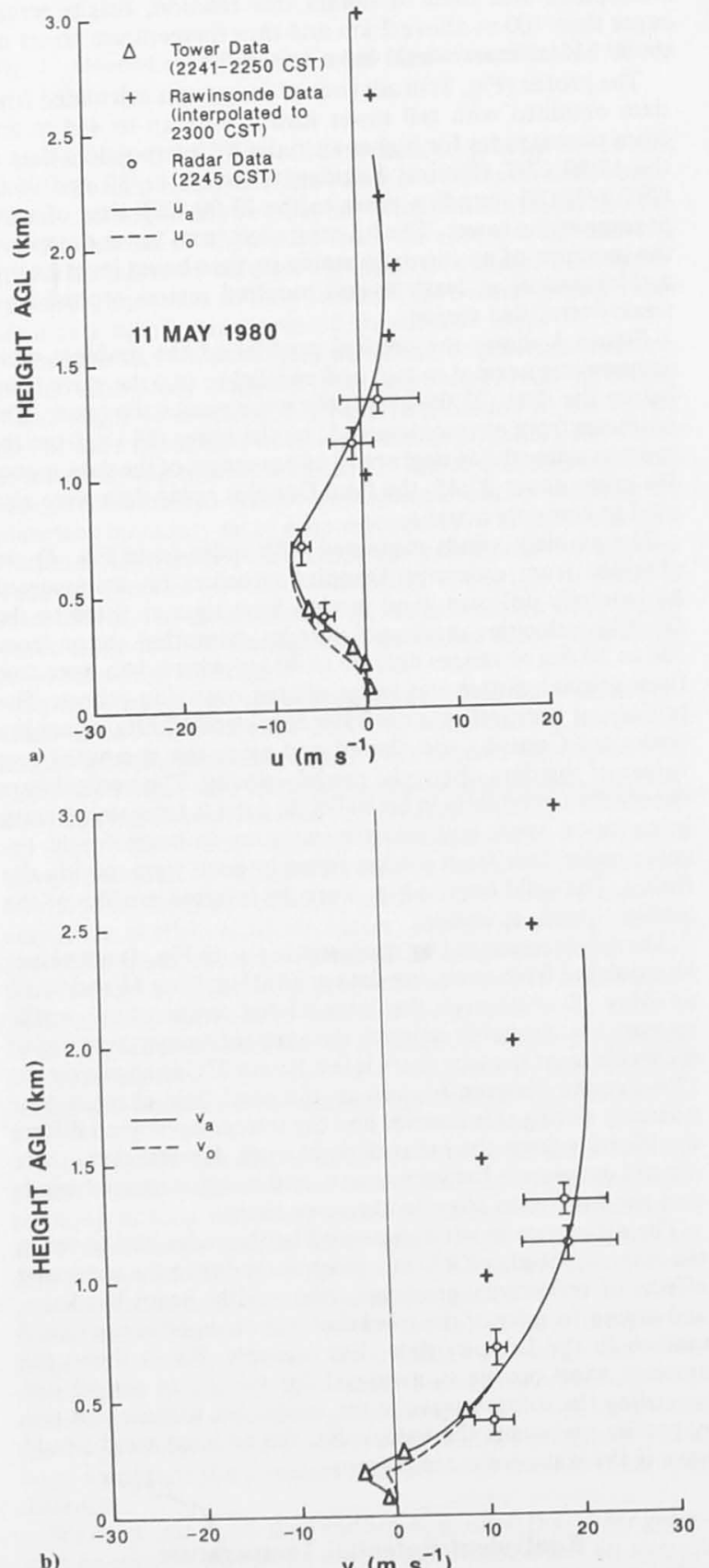

Ambient wind components perpendicular $(u)$ and parallel $(v)$ to the front.
$T_{s}$ the temperature $(\mathrm{K})$ of the parcel when adiabatically cooled to saturation, $w_{s}$ the saturation mixing ratio equal to the to saturation, $w_{s}$ the parcel since $w$ is a conserved quantity, mixing ratio $w$ of the parcel since $w$ is a conserved quantity, and $c_{p}$ the specific heat of dry air. The error in $\theta_{e}$ computed
using this approximate formula can be as large as a few degrees compared to more exact formulas. ${ }^{28}$ Although $\theta_{e}$ values computed using this approximate formula are not exact, contours of constant $\theta_{e}$ can still estimate well the parcel trajectories.

Thus, if the wave is steady and the ambient atmosphere vertically stratified, air parcels travel along surfaces of convertial temperature (Fig. 5) stant $\theta_{e}$. Contours of equiler air (shaded area), which potentially cooler air (shaded area), which suggests that trapped thunderstorm outflow might be recirculating within the wave, whereas the environmental air passes through the wave (or the wave passes through the environ-

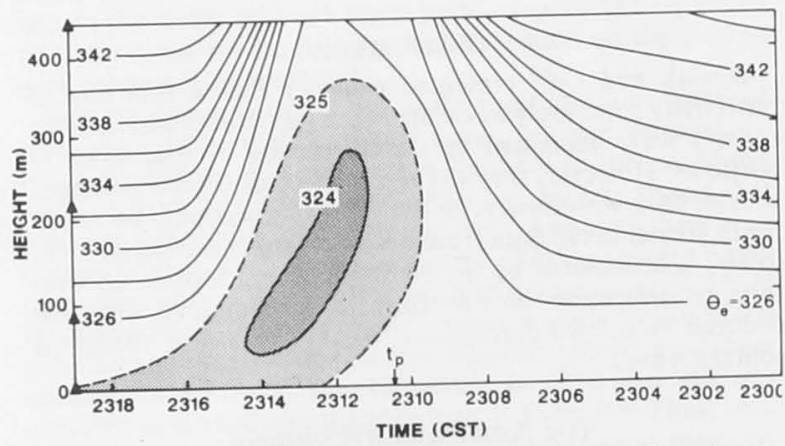

Fig, 5 Equivalent potential temperature in $K\left(t_{p}\right.$ is the time of peak in the $u$ wind component).

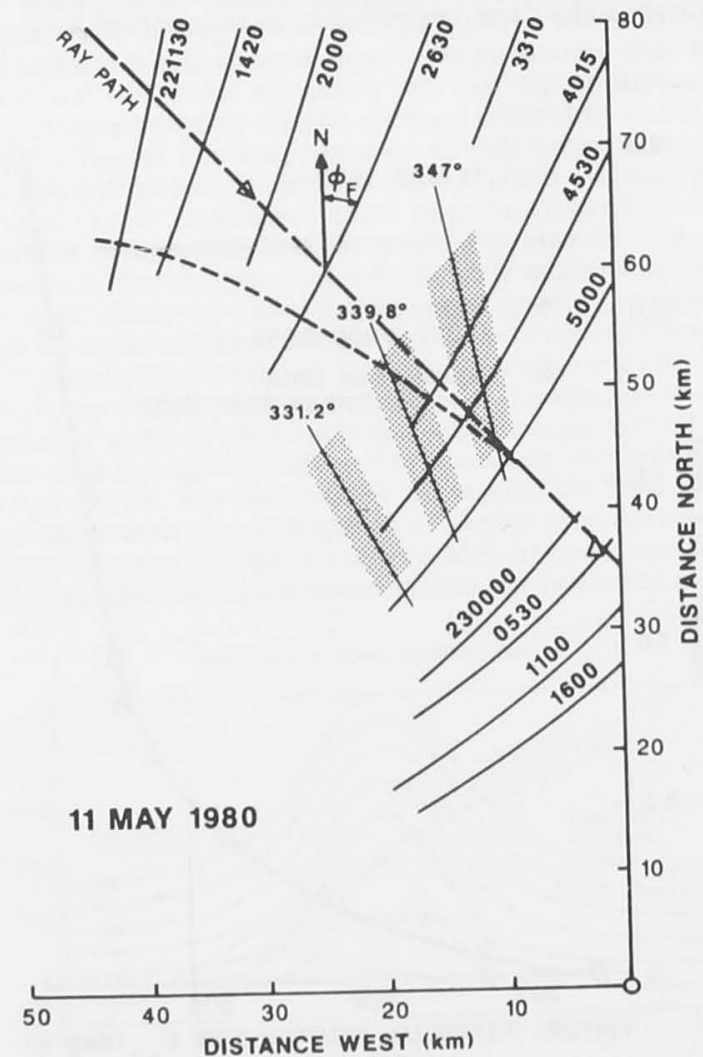

Fig, 6 Isochrones of the leading edge of the solitary wave (KTVY Fig which Doppler data were extracted to determine wave characteristics) 

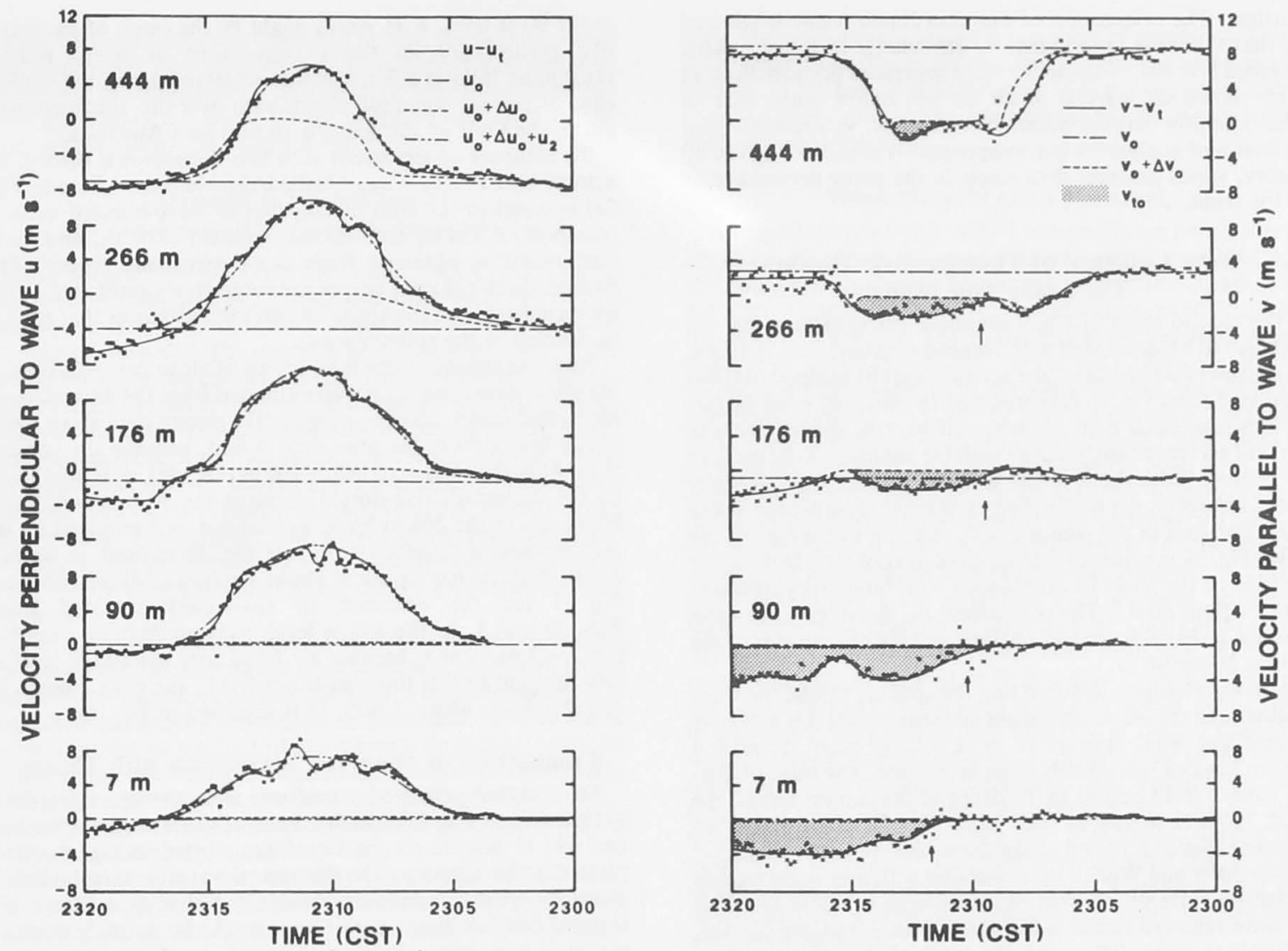

Fig. 7 Samples of wind components at five tower levels, taken at $10 \mathrm{~s}$ intervals.

ment) as it is lifted by it. While the $\theta_{e}$ contours show a laminar recirculating flow, vertical velocity data from anemometers on the tower suggest the outflow core of the wave is a turbulent blob slowly descending to the ground. Perhaps the trapped air is a cut-off vo-tex that initially formed at the leading edge of the horizontally spreading density current (i.e., the thunderstorm outflow) when it impacted the stable layer (see the section below on the evolution of thunderstorm-generated solitary waves for further discussion). Once the wave is formed with recirculating outflow inside it, the turbulent outflow eddy is assumed to be trapped by the wave and to continue to propagate at the wave speed, leaving behind an ever-slowing density current. The position of the pool of cool air at the trailing edge of the wave (Fig. 5) is evidence that the heavier outflow is lagging the wave, giving credence to the idea that the wave drags the trapped air or at least that the two have some symbiotic relation. If the trapped air in the wave is formed solely of ambient air and if friction is negligible, the recirculating air should be centered within the wave as the numerical results of Tung et al. indicate. ${ }^{30}$

\section{Wind Perturbations}

Figure 6 shows the position of the leading edge of the Doppler velocity perturbations (i.e., the wave front) caused by the wave as a function of time. This figure indicates that the wavefront passes the KTVY tower at about 23:05 CST. The storm that supposedly created this wave is about $100 \mathrm{~km}$ north of the radar at 23:00 CST and is tracking at a supercritical speed to the east-northeast. The short dashed line is the path of the wave front normals through the KTVY tower and the long dashed line is the ray path. ${ }^{43} \mathrm{~A}$ time series of data spaced $10 \mathrm{~s}$ apart has been plotted in Fig. 7 to show the temporal dependence of wind components $u$ and $v$ orthogonal to and along the front at five heights. If we ignore the contribution of storm outflow wind (i.e., the shaded areas in Fig. 7), these data exhibit at lower altitudes (i.e., $z<176 \mathrm{~m}$ ) a definite short-wavelength solitary perturbation that is nearly wholly in the NW-SE cross section and consequently exhibits the characteristics of a two-dimensional wave. However, at the highest anemometer levels (i.e., 266 and $444 \mathrm{~m}$ ) pronounced wave-like perturbations appear in both the $u$ and $v$ components.

\section{Components of Wind Change}

A close examination of the data suggests that the wind field can be analyzed in terms of six components,

$$
v=v_{0}+\Delta v_{0}+v_{2}+v_{t 0}+v_{\mathrm{KH}}+v_{t}
$$

where $v_{0}$ is the background flow assumed to be horizontal in absence of the wave, $\Delta \boldsymbol{v}_{0}$ the change caused by the wave when it vertically transports different amounts of $v_{0}$ momentum because of $v_{0}$ shear, $v_{2}$ the wave component associated with perturbations confined to the NW-SE vertical cross section (and, for large amplitude waves, can include recirculating flow as demonstrated by Tung et al. ${ }^{30}$ ), $v_{t 0}$ the axial (i.e., parallel to the wavefront) component of the trapped thunderstorm outflow, $v_{K H}$ the velocity of well-defined, short, wavelike perturbations assumed to be Kelvin-Helmholtz $(\mathrm{K}-\mathrm{H})$ waves, and $v_{t}$ the contribution due to turbulence and other ill-defined, small-scale wind perturbations.

For the sake of argument, we have separated the solitary wave component into two parts: $v_{2}$ applicable to regions both within and outside the trapped flow, and a component $v_{f 0}$ assumed perpendicular to $v_{2}$ associated only with the trapped 
outflow. The orientation of our coordinate frame is selected so that the wave component $v_{2}$ lies wholly in the $x, z$ plane oriented NW-SE. Because the wave generated perturbations in three directions, parcels of air do not follow paths that lie wholly in any vertical plane. Nevertheless, $v_{2}$ deduced from estimates of analyzed wind components is equal to $\boldsymbol{v}_{2}$ given by theory, which assumes flow solely in the plane perpendicular to the front.

\section{Wave Guidance of Thunderstorm Outflow: The Leaky Pipe Model}

The shaded areas in Fig. 7 are those perturbations that are assumed to be associated with trapped thunderstorm outflow The arrows there indicate the arrival times of trapped outflow estimated from Fig. 5. It is seen that the onset of wind perturbations associated with trapped outflow (the shaded areas in Fig. 7b) agrees reasonably well with the location of the trapped air inferred from the $\theta_{e}=325 \mathrm{~K}$ contour (Fig. 5) at all tower levels. However, the trapped outflow barely reaches the $444 \mathrm{~m}$ level. The spur in the contour of $\theta_{e}$, trailing to the rear of the wave (Fig. 5), is evidence that the pool of cool air is leaking out the rear of the wave in accordance with laboratory observations of fluid flow. ${ }^{21}$ The component $v_{t 0}$, lingering after wave passage, gives evidence that outflow leaking out the rear of the wave is below $90 \mathrm{~m}$.

Although we have deduced that the cool air behind the wave originates in the wave, one might conjecture that it is a density current and what appears to be a solitary wave is really a current head of remarkably large amplitude. The ratio of density current head heights to the depth of the current behind the head, recently measured with radar for 25 cases, shows an average value of 1.14 and a maximum observed ratio of $1.35 .^{31}$ Droegemeier and Wilhelmson, working with numerical models of density currents, showed ratios as large as about two. ${ }^{32}$ If the wave reported herein is the head of an advancing current, its height is about $400 \mathrm{~m}$ and the depth of the current behind it is less than $100 \mathrm{~m}$; it has a ratio larger than 4 , an unusual value. Although this observation alone cannot preclude this shallow layer of cool air from being a density current, more evidence against this hypothesis is provided by the observation that the relative winds in this layer are away from the front at all levels. Further support is derived from the disparity between the observed speed of the wavefront and that theoretically deduced for density current fronts. That is, because the depth of the current is less than $100 \mathrm{~m}$ and the difference in virtual potential temperature is, at most, $0.8^{\circ} \mathrm{C}$, the speed $c_{g}=\sqrt{g h \Delta \theta_{v} / \theta_{v}(\mathrm{o})}$ of the current's front computes to be less than $2 \mathrm{~m} / \mathrm{s}$. This is markedly less than the observed speeds that are larger than $12 \mathrm{~m} / \mathrm{s}$

\section{Wind Perturbations Produced by Vertical Transport of Horizontal Momentum}

The $\Delta u_{0}$ and $\Delta v_{0}$ components in Fig. 7 are estimated by assuming that parcels of air, traveling along lines of constant $\theta_{e}$ (Fig. 5), do not change their horizontal momentum. Contours of $\theta_{e}$ should give an accurate depiction of parcel trajectories if the flow is steady and if the atmosphere is horizontally homogeneous. For example, Fig. 5 suggests that air from a height of about $100 \mathrm{~m}$ above ground is lifted by the wave to the $444 \mathrm{~m}$ altitude of the topmost anemometer. The pair of negative peaks seen in the $\left(v_{0}+\Delta v_{0}\right)$ trace (Fig. $7 \mathrm{~b}$, top) is most likely generated as air, having the peak negative $v_{0}$ from the $176 \mathrm{~m}$ level (see Fig. 4b), passes twice through the anemometer at the $444 \mathrm{~m}$ level. Moreover, the computed shape and amplitude of the waveform $\left(v_{0}+\Delta v_{0}\right)$, at the $444 \mathrm{~m}$ level, where the only contributions to changes in $\left(v-v_{t}\right)$ are from the vertical transport of momentum, agree remarkably well with the observed shape and amplitude. The good agreement of the observed data $v$ and the wind change deduced from Figs. 4 and 5 suggest that turbulence is weak (i.e., rms values are less than about $0.5 \mathrm{~m} / \mathrm{s}$ ) and that $v_{\mathrm{KH}}=0$ at the $444 \mathrm{~m}$ level. However, at the $90 \mathrm{~m}$ level, $\mathrm{K}-\mathrm{H}$ waves might be the cause of the wavelike perturbation in the $v$ component of wind. KelvinHelmholtz billows are frequently observed along the trailing edge of solitary wave roll clouds seen over the southern margin of the Gulf of Carpentaria in northern Australia. ${ }^{19}$

To compare observations with two-dimensional theory, we assume that $u-u_{t}-u_{\mathrm{KH}}$ (dashed/double-dotted lines in Fig. $7 \mathrm{a})$ is equal to the sum of $\Delta u_{0}$ due to wave-induced vertical transport of the $u_{0}$ momentum, ambient flow $u_{0}$, and wave component $u_{2}$ obtained from two-dimensional theory. The dashed/double-dotted curves are subjectively estimated to filter turbulence and the apparent wave disturbances that appear to develop in the solitary wave.

Near the ground, there is no lifting of air so $\Delta u_{0}=0$. Hence, the wave component $u_{2}$ is well estimated from the dashed/double-dotted curve. Moreover, vertical velocity data show weak lifting below $90 \mathrm{~m}$ (as does Fig. 5) and, because the vertical shear of $u_{0}$ is nearly zero below $100 \mathrm{~m}$ (Fig. 4a), $u_{2}$ is also given by the dashed/double-dotted curve at the $90 \mathrm{~m}$ tower level. However, at the $266 \mathrm{~m}$ level, $u_{0}$ is about $-4 \mathrm{~m} / \mathrm{s}$ and air of zero momentum is lifted from near the $100 \mathrm{~m}$ level, so we can expect that $\Delta u_{0}$ has a peak of about $4 \mathrm{~m} / \mathrm{s}$. The dashed lines on Fig. 7 are the estimates of $\left(u_{0}+\Delta u_{0}\right)$ obtained from Figs. $4 \mathrm{a}$ and 5 . At the $444 \mathrm{~m}$ level, $u_{0}$ is much more negative (i.e., $-7 \mathrm{~m} / \mathrm{s}$ ) and, because air from near the $100 \mathrm{~m}$ level is also brought to this topmost tower level, the peak change in $\Delta u_{0}$ should be about $7 \mathrm{~m} / \mathrm{s}$, as is sketched in Fig. $7 \mathrm{a}$.

\section{Comparison of Observed Waveforms with Theory}

To compare observed waveform with theory, $u_{2}(t)$ data obtained from Fig. 7 for each minute of observation at the 266 and $444 \mathrm{~m}$ heights on the tower are plotted in Fig. 8. Also plotted in this figure are the theoretical waveforms (solid lines) given by weakly nonlinear theory. ${ }^{33}$ Although evidence of trapped outflow suggests the wave might be strongly nonlinear, it is interesting that the observed waveforms agree well with the analytical expression (solid line) deduced from nonlinear theory. Chen compared vertical wave velocities with those determined by theory and found very good agreement. ${ }^{34}$ However, we notice that the wind data at $444 \mathrm{~m}$ above ground level suggest a faster decrease of $u_{2}$ at the extremities of the wave. At the height of $266 \mathrm{~m}$ (and also at lower heights), $u_{2}(t)$ data show the wave crest to be more rounded and the sides to be steeper than indicated by theory. This could be anticipated because the numerical results of Tung et al. ${ }^{30}$ show that strongly nonlinear waves do have more rounded crests and steeper sides. Furthermore, Cummins and Le Blond ${ }^{35}$ compared waveforms of observed oceanic solitary waves with those shapes produced by weakly nonlinear theories and they also found, in all cases, that the observed waveforms exhibited more rounded crests and steeper sides. As in the case of the
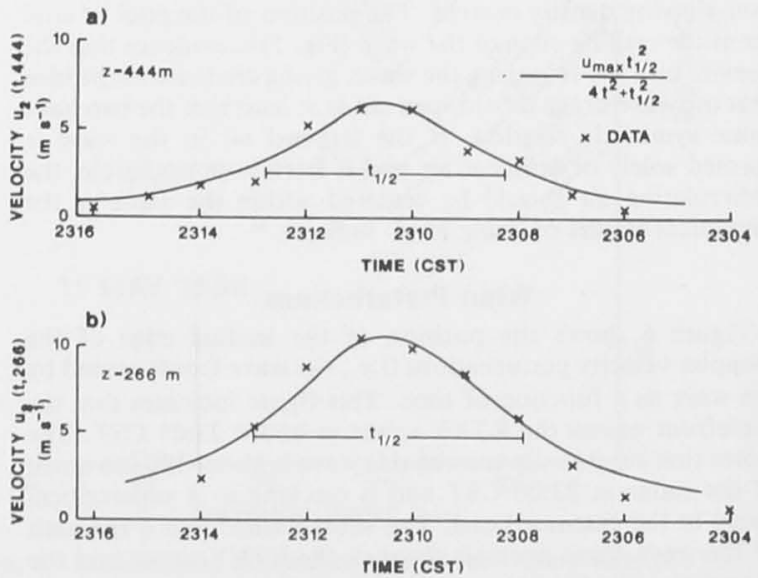

Fig. 8 Comparison of weakly nonlinear theory and observed waveform. 
atmospheric solitary wave examined herein, the oceanic waves had amplitudes that violated weakly nonlinear assumptions. Therefore, the departure of the observed waveform from that given by weakly nonlinear theory might be explained by the large amplitude of the observed wave.

\section{Scenario for Evolution of}

Thunderstorm-Generated Solitary Waves

Data suggest that the observed solitary wave had trapped thunderstorm air that was cooler and drier than the wave's ambient environment and thus, being more dense, leaked out the rear of the wave. The presence of this cool pool of air left behind the wave can complicate the interpretation of measurements if they are made only with instruments near the ground, because temperature drop and wind shift data might be misinterpreted as evidence of an advancing density current. However, observations with the tall tower and Doppler radar show that this pool of very shallow air (less than $100 \mathrm{~m}$ ) leaked out of the wave. Furthermore, the parent storm provides unexpected support for the solitary wave as outflow is guided by the wave and flows continuously along the wave's axis away from the storm.

It is remarkable that the storm outflow remained in the wave for distances of at least $60 \mathrm{~km}$ from the storm. Although storm outflows propagate as density currents to large distances, it is suggested that gravity waves (and, in particular, solitary waves formed by the interaction of the storm's downdraft with the stable layer) can transport the outflow faster and probably, with greater momentum, farther. Thus, hazardous shear, normally confined to regions beneath downdrafts, might indeed be found at large distances from them.

The results of this study and the observations of Linden and Simpson ${ }^{13}$ suggest a scenario for the evolution of the solitary wave (Fig. 9). A thunderstorm downdraft generates a density current and a horizontal rotor at its leading edge. If the current and its rotor are also imbedded in an inversion layer, they launch one or more wave-like disturbances that grow and propagate at speeds determined by the wave amplitude and vertical profile of the wind and the virtual potential temperature of the ambient environment. The density current front has a speed that is dependent on the depth of the current and, because this depth is continually decreasing, the wave eventually propagates ahead, leaving the residual current behind. As the wave propagates away from the current, it drags along a horizontal vortex of thunderstorm outflow, separating it from its source. The wave and circulating outflow air continue to propagate away at velocities faster than the current's front, thus leaving it farther and farther behind. The recirculating trapped air in the wave creates strong shear near the Earth's surface. However, as the trapped outflow slowly leaks out the rear of the wave, leaving behind a shallow pool of cool air, the wave amplitude decreases. After the outflow air is completely

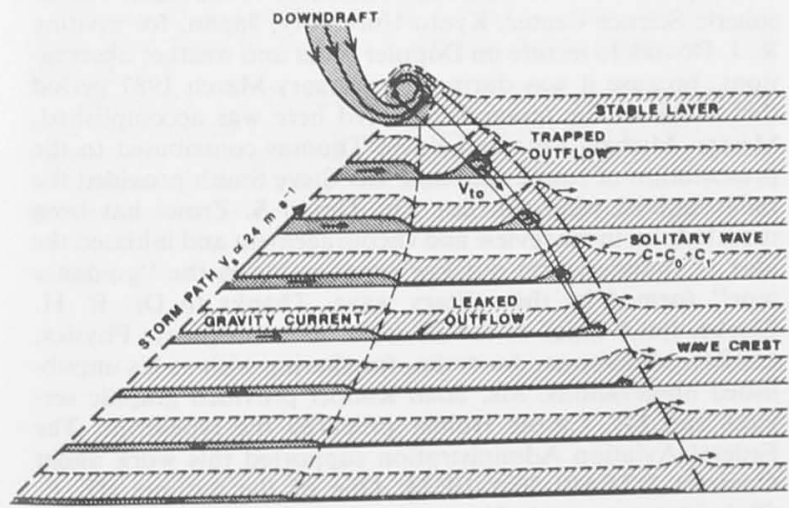

Fig. 9 Scenario for the evolution of the thunderstorm-generated solitary wave. gone, the wave can continue on as a wave of permanent form, if the energy loss due to ground friction and radiation can be ignored. If the part of the troposphere overlying the inversion layer is not neutrally stable, wave energy will be radiated away from the inversion layer. ${ }^{23}$

\section{Wind Shear Hazard Potential}

Although the observed solitary wave reported herein was 60 $\mathrm{km}(32 \mathrm{n}$. mi.) from the storm that apparently generated it, it still contained significant horizontal and vertical shears (e.g., $4.4 \times 10^{-3}$ and $\left.60 \times 10^{-3} \mathrm{~s}\right)$ of the horizontal wind $90 \mathrm{~m}(300$ ft) above the ground. The horizontal shear persisted over a distance of about $1500 \mathrm{~m}$ (i.e., $\simeq \frac{3}{4} \mathrm{n}$. mi.). Short-wavelength waves, which developed atop this solitary wave, intensified the horizontal shear to $7.6 \times 10^{-3}$ s over a distance of $700 \mathrm{~m}(0.4$ $\mathrm{n}$. mi.). For a perspective on the significance of such shears on aircraft performance, note that a decrease in head wind along a southeastward $3 \mathrm{deg}$ glide slope into the solitary wave is larger $(38 \mathrm{knots}$ or $19 \mathrm{~m} / \mathrm{s})$ than the one $(35 \mathrm{knots}$ or $17.5 \mathrm{~m} / \mathrm{s})$ that caused a $50 \mathrm{~m}(150 \mathrm{ft})$ drop in altitude of a Boeing 747 aircraft on its approach into Melbourne, Australia. ${ }^{36}$ Turbulence behind the wave possessed even stronger horizontal shears with wind changes as large as $6 \mathrm{~m} / \mathrm{s}$ (12 knots) over a $130 \mathrm{~m}(400 \mathrm{ft})$ distance or a shear value near $50 \times 10^{-3} \mathrm{~s}$. An aircraft landing at a speed of $72 \mathrm{~m} / \mathrm{s}$ (140 knots could experience a $6.2 \mathrm{~m} / \mathrm{s}(12 \mathrm{knots})$ head wind decrease in $\sim 2 \mathrm{~s}$. These large solitary wave shears were observed at the tall tower site when the storm was $60 \mathrm{~km}$ away. At points closer to the storm, the shear should be significantly larger. For example, the wave amplitude observed by Doppler radar at 22:45 CST for locations $45 \mathrm{~km}$ from the storm's edge was almost double that observed at the tower. ${ }^{37}$ It should be emphasized that thunderstorm-generated waves of much larger amplitude than that described here and with proportionally more intense shear have been observed. 9

The only documented flights of an aircraft through largeamplitude solitary waves occurred on Sept. 29 and Oct. 4, 1979. ${ }^{38}$ The aircraft, a Beechcraft Travelair, was directed to make measurements in morning glorys over the Gulf of Carpentaria off the northern coast of Australia. The pilot was instructed to fly at constant altitude and power through these glorys and often found himself on first an upward and then a downward current causing the aircraft to ascend and descend

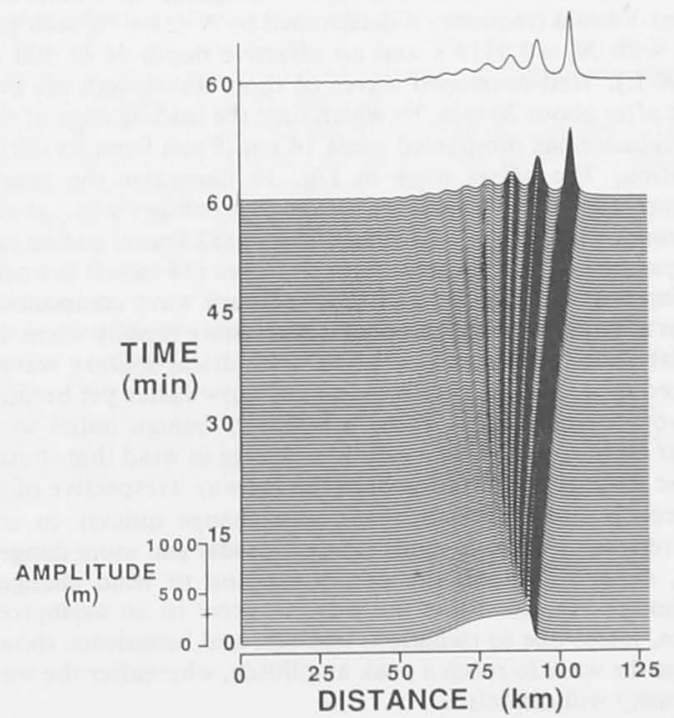

Fig. 10 Wave-induced vertical displacement of air vs position along the direction of propagation with time as a parameter. The coordinate system moves at the speed $C_{0}(11.1 \mathrm{~m} / \mathrm{s}$ or $22.2 \mathrm{knots})$, the fastest that waves of infinitesimal amplitude can propagate. 
at rates as high as $600 \mathrm{~m} / \min (2000 \mathrm{ft} / \mathrm{min})$. During the traverse of the leading solitary wave on Oct. 4 , the pilot was forced to pull out when he was within $50 \mathrm{~m}(150 \mathrm{ft})$ of the sea. This wave originated in the collision of opposing sea breeze fronts over the Cape York peninsula ${ }^{39,40}$ and not in thunderstorm activity. Yet it produced significant shear that was hazardous to safe flight, even though it was more than $300 \mathrm{~km}$ from its source. It should be noted, however, that the wave on Oct. 4 is not a particularly large-amplitude disturbance. For example, the surface pressure perturbation corresponding to the leading disturbance amounted to only about $0.8 \mathrm{mbar}$. Morning glory solitary waves of much larger amplitude $(>2.5$ mbar) have been observed. In this regard, the flight through the disturbance on Sept. 29 is of interest in that it was a somewhat larger disturbance $(1.4 \mathrm{mbar})$ with significantly more intense shear. The minimum altitude traverse through this disturbance was, however, carried out at a height of 600 $\mathrm{m}$. Even so, the aircraft suffered a drop in altitude of more than $200 \mathrm{~m}$, which is hardly trivial. A later attempt (1982) to fly through one of these phenomena was frustrated by the pilot who refused to fly into the wave after he had seen it (R.H. Clarke, private communication).

Christie and Muirhead ${ }^{18}$ showed how an initially long wave with relatively benign shear can evolve under the influence of nonlinearities and frequency dispersion into an amplitudeordered family of solitary waves of large-amplitude and shorter half-amplitude widths, in which the shear hazard is markedly increased. Their work suggests that the intense, transient shear zones generated with these waves constitute a serious hazard to the safety of flight at low altitudes.

A recent theoretical study ${ }^{22}$ of nonlinear dispersive wave disturbances has shown that, under ideal atmospheric waveguide conditions and when the initial disturbance has large volume, the ratio of the amplitude of the leading solitary wave to the amplitude of the initial disturbance approaches a limiting value of 4.0. This investigation has also shown that the formation of solitary waves is a surprisingly rapid process; well-developed solitary waves with their characteristic intense wind shear patterns can be created over a distance of some tens of kilometers. As an illustration, Fig. 10 shows the results of a numerical integration of the Benjamin-Davis-Ono equation, the governing equation for the evolution of long nonlinear waves in the atmosphere, for a relatively small-amplitude and longer-wave initial disturbance propagating as a bore in a simple, but realistic, boundary-layer waveguide in which the Brunt-Väisälä frequency is determined by $N^{2}(z)=N_{0}^{2} \operatorname{sech}^{2}(z)$ $H$ ), with $N_{0}=0.0314 \mathrm{~s}$ and an effective depth $H$ of $500 \mathrm{~m}$ $(1500 \mathrm{ft})$. Well-developed waves of short wavelength are evident after about $20 \mathrm{~min}$, by which time the leading edge of the disturbance has progressed some $14 \mathrm{~km}(9 \mathrm{mi})$ from its initial position. The offset trace in Fig. 10 illustrates the nearly asymptotic form attained by the leading solitary wave at the $1 \mathrm{~h}$ mark when its speed is nearly $16 \mathrm{~m} / \mathrm{s}$ ( 32 knots) and its has propagated a total distance close to $55 \mathrm{~km}(34 \mathrm{mi})$. It is worth noting that the evolution of short solitary wave components from a longer-wave disturbance occurs more rapidly when the initial wave has larger amplitude. Cylindrical solitary waves, forced by a divergent outflow, might grow faster yet because of vortex stretching. ${ }^{13}$ Thus, a relatively benign initial wind shear event, one having a step-like change in wind that should cause aircraft only to overshoot the runway irrespective of its approach into the disturbance, can change quickly to one whereby wind shear is significantly bimodal and more dangerous, depending upon the pilot's reaction to wind changes. Although Fig. 10 shows the wave to grow to an asymptotic form, losses due to radiation, leakage, and turbulence should cause the wave to reach a peak amplitude, whereafter the wave intensity will diminish.

To determine whether thunderstorm generated gravity waves have caused any U.S. aircraft accidents in the past, we now review the paper of Rudich, ${ }^{41}$ who summarized the weather conditions leading to 33 weather-involved air carrier accidents that have been investigated and formally reported on by the National Transportation Safety Board for incidents that occurred during the period 1962-1984. In 11 of the 18 fatal crashes, wind shear was implicated, but altogether there were 26 accidents causing injuries and/or damage to the aircraft in which wind shear may have been the cause or the contributing cause of the accident. In nine of the fatal cases (seven of the nonfatal ones), the aircraft either penetrated thunderstorm rain or was in the area of thunderstorm rain. In one case, the aircraft flew through heavy rain, but thunder was not reported. If thunderstorm-generated, large-amplitude gravity waves were involved in these accidents, they would likely be in their earliest stage of formation; but, of course, it is at the time when these waves might have the largest amplitude. For example, a large amplitude, wave-like perturbation or rotor is deduced to be in the outflow of the thunderstorm that caused the crash of Delta flight 191 at the Dallas/Fort Worth International Airport on Aug. 2, $1985 .{ }^{42}$ In only one fatal accident was wind shear noted as a possible cause without rain being mentioned, so waves might have been present. In two of the fatal cases, it was reported that the aircraft was near, or had entered, a roll cloud that was in advance of a squall line producing moderate rain. The presence of a roll cloud is strong evidence for the existence of a horizontal rotor and the formation of a solitary wave. In eight of the nonfatal accidents, there were no reported thunderstorms in the vicinity of the accident, and thus gravity waves or wave-induced turbulence might have been the cause or contributing cause of the accidents. Therefore, ignoring the cases involving thunderstorm rain in which microbursts are likely to be the cause, gravity waves might be implicated in at least 11 of the 33 investigated accidents.

\section{Conclusions}

Although we have no conclusive evidence to date that solitary or gravity waves have been a cause or a contributing cause of fatal accidents, the data, as meager as they are, suggest that horizontal rotors at the leading edge of thunderstorm gravity currents (often called gust fronts) and strongly nonlinear waves can pose a hazard to safe flight and may have caused accidents. Large-amplitude, short-wavelength solitary waves can propagate far distances with little loss in amplitude and might also pose a wind shear hazard in areas far removed from thunderstorm activity. Thus, internal gravity waves should be studied both experimentally and theoretically. Furthermore, because it is the strongly nonlinear waves that pose the threat, theoretical studies should resort to numerical models. Experiments must employ equipment, such as tall towers, Doppler radar, and aircraft, that can provide the unambiguous observations far above the ground.

\section{Acknowledgments}

Many thanks to Susumu Kato, Director of the Radio Atmospheric Science Center, Kyoto University, Japan, for inviting R. J. Doviak to lecture on Doppler radar and weather observations, because it was during the January-March 1987 period that much of the research reported here was accomplished. Messrs. Michael Eilts and Kevin Thomas contributed to the presentation of tower data and Mr. Steve Smith provided the radar-derived wind profiles. Dr. Dusan S. Zrnic' has been most helpful in his review and encouragement and initiated the idea of thunderstorm outflow propagating in the "guidance pipe" formed by the solitary wave. Thanks to Dr. R. H. Clarke, (ret.) Chief of the Division of Atmospheric Physics, CSIRO, Melbourne, Australia, for sharing with us his unpublished observations. Ms. Joan Kimpel provided graphic services and Ms. Carole Holder prepared the typescript. The Federal Aviation Administration supported this work under Contract DTFA01-80-Y-10524. One of the authors (D. R Christie) was supported in part by the U.S. Air Force Office of Scientific Research under Contract AFOSR-83-0045. 


\section{References}

${ }^{1}$ Doviak, R. J. and Zrnic', D. S., Doppler Radar and Weather Observations, Academic Press, Orlando, FL, 1984.

${ }^{2}$ McCaul, E. W., Bluestein, H. B., and Doviak, R. J., "Airborne Doppler Lidar Observations of Convective Phenomena in Oklahoma," Journal of Atmospheric and Oceanic Technology, Vol. 4, No. 3, 1987, pp. 479-497.

${ }^{3}$ Carter, J. K., "The Meteorological Instrumented WKY-TV Tower Facility," Oper. Div., National Technical Information Service, Springfield, VA, NSSL Tech. Memo. 50, 1970 (NTIS COM-7100100).

${ }^{4}$ Kessler, E., "'Wind Shear and Aviation Safety," Nature, Vol. 315 No. 6016, 1985, pp. 179-180.

5Fujita, T. T., "The Downburst," Dept. of Geophysical Science, University of Chicago, Chicago, SMRP Rept. 210, 1985.

${ }^{6}$ McCarthy, J., Wilson, J. W., and Fujita, T. T., "The Joint Airport Weather Studies Project," Bulletin of the American Meteorological Society, Vol. 63, 1982, p. 15-22.

Byers, H. R. and Braham, R. R., Jr., "The Thunderstorm," Report of the Thunderstorm Project, U.S. Government Printing Office, Washington, DC, 1949.

${ }^{8}$ Lee, J. T., Stokes, J., Sasaki, Y., and Baxter, T., "Thunderstorm Gust Fronts-Observations and Modeling," Systems Research and Development Service, Federal Aviation Administration, Washington, DC, 1978

${ }^{9}$ Fulton, R., "Observations of the Interactions of a Nocturnal Thunderstorm Outflow with a Stable Environment," M.S. Thesis in Meteorology, University of Oklahoma, Norman, 1987.

${ }^{0}$ Doviak, R. J., and Ge, R. S., "An Atmospheric Solitary Gust Observed with a Doppler Radar, a Tall Tower and a Surface Network," Journal of the Atmospheric Sciences, Vol. 41, 1984, pp $2559-2573$.

"Goff, R. C. "'Low Level Wind Shear Alert System (LLWAS)," National Aviation Facilities Experimental Center, Atlantic City, NJ, Rept. FAA-RD-80-45, 1980.

12 von Kármán, T., "The Engineer Grapples with Nonlinear Problems," Bulletin of the American Mathematical Society, Vol. 46, 1940 pp. 615-683.

${ }^{13}$ Linden, P. F., and Simpson J. E. "Microbursts: A Hazard for Aircraft," Nature, Vol. 317, Oct. 1985, pp. 601-602.

${ }^{14}$ Stewart, O., Danger in the Air, Philosophical Library, New York, 1958 , pp. 53-63.

${ }^{15}$ Christie, D. R, and Muirhead, K. J., "Solitary Waves: A Hazard to Aircraft Operating at Low Altitudes," Research School of Earth Sciences, Australian National University, Rept., 1982.

${ }^{16}$ Gossard, E. E., "Aircraft Hazard Assessment from a Clear-Ai Radar and Meteorological Tower Study of Gravity Wave Events," U.S. National Oceanic and Atmospheric Admin., Boulder, CO (Doc. PB83-257139, National Technical Information Service, Springfield, VA 22161), 1983

${ }^{17}$ Christie, D. R., Muirhead, K. J, and Clarke, R. H. "Solitary Waves in the Lower Atmosphere," Nature, Vol. 293, No. 5827, 1981 , pp. 46-49.

${ }^{18}$ Christie, D. R, and Muirhead, K. J., "Solitary Waves: A Hazard to Aircraft Operating at Low Altitudes," Australian Meteorological Magazine, Vol. 31, 1983, pp. 97-109.

${ }^{19}$ Christie, D. R. and Muirhead, K. J., "Solitary Waves, a Low Level Wind Shear Hazard to Aviation," International Journal of Aviation Safety, Vol. 1, Sept. 1983, pp. 169-190.

${ }^{20}$ Christie, D. R. and Muirhead, K. J. "Solitary Waves and Low-Altitude Wind Shear in Australia," Aviation Safety Digest, No. 123, 1985, pp. 3-8.

${ }^{21}$ Maxworthy, T., " On the Formation of Nonlinear Internal Waves from Gravitational Collapse of Mixed Regions in Two and Three Dimensions," Journal of Fluid Mechanics, Vol, 96, 1980, pp, 47-64.

${ }^{22}$ Christie, D. R. "Long Nonlinear Waves in the Lower Atmosphere," Journal of the Atmospheric Sciences, to be published March or April 1989.

${ }^{23}$ Crook, N. A., "The Effect of Ambient Stratification and Moisture on the Motion of Atmospheric Undular Bores," Journal of the Atmospheric Sciences, Vol. 43, No. 2, 1986, pp. 171-181.

${ }^{24}$ Farnsworth, T. H., "Discussing Wind Shear, Causes and Effect of Rough Air Phenomenon," Skyways, Henry Publications, New York, 1960, pp. 42-43.

${ }^{25}$ Fujita, T. T., “DFW Microburst,"' Dept. of Geophysical Science, University of Chicago, Chicago, SMRP Research Paper 217, 1986.

${ }^{26}$ Anderson, K. W. and Clark, B. A. J., "Wind Shear in Australia," Aviation Safety Digest, Vol. 106, 1979, pp. 14-20.

${ }^{27}$ Anderson, K. W. and Clark, B. A. J., "A Study of Wind Shear Effects on Aircraft Operations and Safety in Australia," Aeronautical Research Lab., Dept. of Defense, Melbourne, Victoria, Aust., Systems Rept. 24, 1981

${ }^{28}$ Bolton, D. "The Computation of Equivalent Potential Temperature," Monthly Weather Review, Vol. 108, 1980, pp. 1046-1053.

${ }^{29}$ Holton, J. R., An Introduction to Dynamic Meteorology, Academic Press, New York, 1972.

${ }^{30}$ Tung, K. K., Chan, T. F., and Kubota, T., "Large Amplitude Internal Waves of Permanent Form," Studies in Applied Mathematics, Vol. 66, 1982, pp. 1-44.

${ }^{31}$ Mahoney, W. P. III, "Gust Front Characteristics and the Kinematics Associated with Interacting Thunderstorm Outflows," Monthly Weather Review, Vol. 116, No. 5, 1988.

${ }^{32}$ Droegemeier, K. K and Wilhelmson, R. B. "Numerical Simulation of Thunderstorm Outflow Dynamics," Journal of the Atmo spheric Sciences, Vol. 44, No. 8, 1987, pp. 1180-1210.

${ }^{33}$ Benjamin, T. B., "Internal Waves of Permanent Form in Fluids of Great Depth," Journal of Fluid Mechanics, Vol. 29, 1967, pp. 559-592.

${ }^{34}$ Chen, S., "A Theoretical Analysis and Comparison of the Theory and Observation of Wind and Pressure Fields in an Atmospheric Solitary Wave," M.S. Thesis, Dept. of Meteorology, University of Oklahoma, Norman, 1985

${ }^{35}$ Cummins, P. F. and Le Blond, P. H., "Analysis of Internal Solitary Waves Observed in Davis Strait," Atmosphere-Ocean, Vol. 22, No. 2, 1984, pp. 173-192 (Canadian Meterological and Oceanic Society)

${ }^{36}$ Woodfield, A. A., "Wind Shear and Vortex Wake Research in U.K. 1982," Proceedings of Sixth Annual Workshop on Meteorological and Environmental Inputs to Aviation Systems, edited by W. Frost and D. W. Camp, NASA Rept. CP-2274 and U.S. Dept. of Transportation Rept. DOT/FAA/RD-82/72, 1983, pp. 66-83.

${ }^{37}$ Doviak, R. J and Chen, S., "Observations of a Thunderstorm Generated Gust Compared with Solitary Wave Theory,'”Systems Res. and Dev. Service, Federal Aviation Administration, Washington, DC FAA Rept. DOT F1700.7, 1988

${ }^{38}$ Clarke, R. H., Smith, R. K., and Reid, D. G., "The Morning Glory of the Gulf of Carpentaria: An Atmospheric Undular Bore," Monthly Weather Review, Vol. 109, 1981, pp. 1726-1750

${ }^{9}$ Noonan, J. A and Smith, R. K., "The Generation of North Australian Cloud Lines and the 'Morning Glory',"' Australian Meteorological Magazine, Vol. 35, 1987, pp. 31-45

${ }^{40}$ Clarke, R. H., "Colliding Sea-Breezes and the Creation of Internal Atmospheric Bore Waves: Two-Dimensional Numerical Studies," Australian Meteorological Magazine, Vol. 1984, pp. 207-226.

${ }^{41}$ Rudich, R. D., "Weather-Involved U.S. Air Carriers Accidents 1962-84-A Compendium and Brief Summary," AIAA Paper 860327. Jan. 1986

${ }^{42}$ Caracena, F., "The Crash of Delta Flight 191 at Dallas-For Worth International Airport on 2 August 1985: Multiscale Analyses of Weather Conditions," National Oceanic and Atmospheric Administration, Tech. Rept. ERL 430-ESG 2, 1986.

${ }^{43}$ Doviak, R. J. and Thomas, K. W., "The Wave Front Shape and Position of a Great Solitary Wave of Translation," Proceedings of International Geo-Science and Remote Sensing Symposium, Sept. 1988 
Section 2.7

The wavefront shape, position, and evolution of a great solitary wave of translation

(R.J. Doviak, K.W. Thomas and D.R. Christie)

I.E.E.E. Trans. Geosci. Remote Sensing, 27, 658-665, 1989. 


\section{The Wavefront Shape, Position, and Evolution of a Great Solitary Wave of Translation}

Richard J. Doviak

Kevin W. Thomas

Douglas R. Christie 


\title{
The Wavefront Shape, Position, and Evolution of a Great Solitary Wave of Translation
}

\author{
RICHARD J. DOVIAK, SENIOR MEMBER, IEEE, KEVIN W. THOMAS, AND DOUGLAS R. CHRISTIE
}

\begin{abstract}
Doppler radar mapped the evolution of a pair of atmospheric solitary waves emanating from a thunderstorm complex. The $100-\mathrm{km}$-long curved wavefront and position of these few-kilometer-wide waves are hypothesized to be a result of an amplitude-dependent wave speed. The observed unfolding is compared with a solution of the nonlinear integro-differential equation governing the evolution of finite amplitude disturbances in stratified media.
\end{abstract}

\section{INTRODUCTION}

$\mathrm{I}_{\mathrm{t}}^{\mathrm{T}}$ was in the summer of 1834 that John Scott Russell, a fellow of the Royal Society at Edinburgh, discovered what he later described as a great wave of translation [1]. This wave of first order is a single-crested displacement of the water surface that propagates with remarkably little change of form or amplitude. His first encounter with these unique waves of solitary form is said to have been near the Hermiston Experimental Station on the Union Canal [2], only several miles from the center of Edinburgh and thus close to the site of the 1988 International Geoscience and Remote Sensing Symposium. Subsequent studies led to a Report of the Committee on Waves [3], in which Russell states that "there are wide fields of novel and important science opening up in this direction, which will furnish an ample harvest of rich knowledge for the labor of several succeeding seasons."' Such far-reaching implications and importance of this perceptive statement were not appreciated until the significance of the solitary wave as an important stable state of systems in such diverse fields as meteorology, quantum mechanics, and plasma and laser physics was realized in the mid-1960's [2]

It was more than a century after Russell's discovery that the first observations of an atmospheric solitary wave were reported [4], but the first definitive measurements which established the existence of these very large amplitude waves of translation were made in northern Australia by Christie et al. during the 1970's [5]. The advances made in radar technology since World War II led to the Doppler

Manuscript received March 15, 1989; revised July 18, 1989. This work was partially supported by the FAA under Contract No. DTFA01-80-Y10524

R. J. Doviak is with the National Severe Storms Laboratory, $1313 \mathrm{Hal}$ ey Circle, Norman, OK 73069.

K. W. Thomas is with the Cooperative Institute for Mesoscale Meteorological Studies, University of Oklahoma, Norman, OK 73019.

D. R. Christie is with the Research School of Earth Sciences, Australian National University, Canberra, ACT, 2601 Australia.

IEEE Log Number 8930935 weather radar observation of a thunderstorm-generated solitary wave in 1980 [6]. The close similarity in form of solitary waves on the surface of water and the waves internal to the atmosphere is illustrated in Fig. 1.

The top configuration in Fig. 1(a), taken from the original work of Russell [7], gives the initial condition wherein water of a larger depth is dammed at the beginning of a long channel. The second configuration shows the wave propagating to the right, along the channel, at some time after the barrier is lifted. It is of importance that Russell realized from his observations that the solitary wave "be not regarded as an arbitrary phenomenon deriving all its characteristics from the conditions in which it was first generated, but that it is a phenomenon 'sui generis,' assuming to itself that form and those dimensions under which alone it continues to exist as a wave. The existence of a moving heap of water of arbitrary shape or magnitude is not sufficient to entitle it the designation of a wave of the first order" [7]. The final configuration in Fig. 1(a) shows the wave at the end of the channel, at which instant the barrier at this end is closed in order to catch the wave and measure its volume.

In Fig. 1(b) we display a Doppler radar measurement of the radial velocity of air (i.e., motion toward or away from the radar) as a function of the radial distance along the radar beam whose direction is near the horizon (i.e., the elevation angle is $0.4^{\circ}$ ). This huge perturbation of the ambient wind is caused by a wave that emerged from the outflow of a thunderstorm. The unimodal pattern of velocity demonstrates that air is permanently translated after the passage of the wave

Although the physics and mathematical expressions that describe solitary waves in these two diverse media are different, the basic mechanisms which allow these waves to achieve great amplitude and longevity are the same. In contrast to sinusoidal waves of small amplitude, which cause parcels of fluid to orbit in closed paths, solitary waves permanently displace or translate fluid a finite distance in the direction of propagation.

Solitary atmospheric waves are not merely spectacular and interesting (for example, the descriptive term "morning glory" has been given to these waves observed in northern Australia) or just a scientific curiosity; they have been targeted as phenomena that are responsible for generating wind-shear hazards to aircraft [8]-[10]. Furthermore, because they are exceptionally large amplitude 


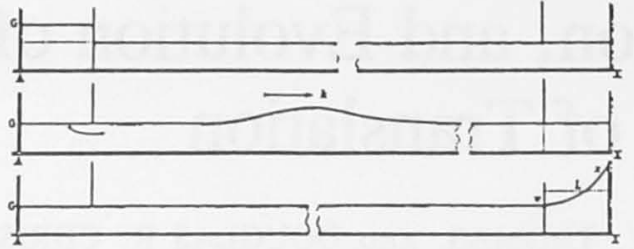

(a)

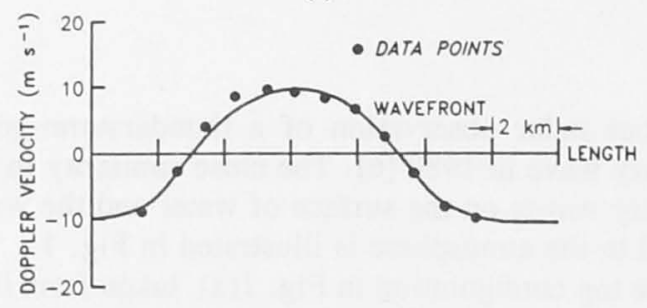

(b)

Fig. 1. (a) Configuration of a great solitary wave of translation on the surface of water observed by Russell (reference [7]), and (b) one observed in the atmosphere (reference [23]) with Doppler radar. Photocopy of (a) was supplied by the Trustees of the National Library of Scotland. The vertical axis in (a) is in the direction of displacement of the water above its ambient level of repose, whereas in (b) it is the component of air velocity toward the radar. The ambient air has a Doppler velocity of -10 $\mathrm{m} \mathrm{s}^{-1}$

waves of elevation (i.e., those that lift air), they might be instrumental in propagating disturbances long distances to where atmospheric conditions are suitable for the initiation of convective storms. Under certain meteorological conditions, thunderstorm outflows have been identified as principal initiators of other thunderstorms and can account for most observed summertime air-mass storms [11]. However, it is commonly thought that the leading edge of density currents, the cool dense air flowing out of storms and along the ground, can directly initiate other storms. We suggest that, at least in some instances of widespread activity, air in a stable layer often formed by density currents serves as the waveguide (see Sections II and III) in which solitary waves might telecommunicate, to long distances, the storm-initiating disturbances.

Remote sensors permit the observer to sample a large area and obtain an almost instantaneous picture of these naturally occurring phenomena, even though they might propagate long distances across the earth. We have taken advantage of this capability to observe, over large areas, the curvilinear shape of an advancing wave. We offer a theory to explain the curvature of the wavefront, and compare observations with the numerical solutions of the Benjamin-Davis-Ono equation [12], which describes the evolution of weakly nonlinear gravity (buoyancy) waves.

\section{The Characteristics of Solitary Waves}

Through numerous observations on rivers and tidal waves in estuaries such as the Firth of Forth in Scotland, and extensive series of laboratory experiments contrived with ingenuity and conducted with a high degree of accuracy, Russell [7] was able to prove that the rather simple expression

$$
c=\sqrt{g(h+a)}
$$

gives the propagation velocity $c$ of the solitary water wave, where $g$ is the acceleration of gravity, $h$ is the depth throughout the fluid in repose, and $a$ is the height of the crest of the wave above the plane of repose.

A key feature distinguishing a solitary wave from a myriad of other waves, including electromagnetic, propagating in media whose properties are uniform in the direction of travel, is the strong dependence of wave speed on wave amplitude. Furthermore, and most importantly, these waves of large amplitude can travel long distances in dispersive media without change of form. This is crudely explained by the fact that nonlinear effects in the peak of the wave generate higher harmonics whereas, for water and atmospheric waves at least, dispersion causes shorter wavelength components to propagate more slowly and thus lag farther behind, causing a decrease in the harmonic content [13]. Thus, the two effects can balance one another, allowing all components to travel as a secure group.

For the solitary wave to develop and propagate in the atmosphere with little or no attenuation, there must be a waveguide formed by a layer of air overlaid with potentially warmer air having negligible stratification [14], [15]. This condition can arise in thunderstorm environments because thunderstorms usually deposit on the surface a large volume of cold air that slowly decreases in depth and expands horizontally to form a thin layer of air having a potential temperature much less than the air above it. Furthermore, thunderstorms often develop under conditions in which air in the lowest few kilometers is well mixed by shallow convective activity, which forms a layer of air of near-neutral stability.

This layer of air is lifted above the intruding denser air.

\section{Observations of a Solitary Disturbance}

Surface observations of large-amplitude solitary waves generated by thunderstorms are handicapped by the fact that these waves can trap and advect the cold thunderstorm downdraft air which leaks out of the rear of the wave. Leakage is also seen in laboratory-generated waves [16], and leaked thunderstorm air can mislead the observer into believing that the phenomenon being examined is a density current [17]. A Doppler radar, measuring the air velocity above this layer of leaked air, will show that the air in advance of and the air behind the solitary gust have the same ambient velocity, whereas surface instruments will show a sustained wind shift and temperature drop that characterize the passage of a density current front.

On June 1, 1985 the Doppler radar operated by the staff of the National Severe Storms Laboratory (NSSL) in Norman, Oklahoma, detected a density current emanating from a thunderstorm, and then showed it to evolve into two isolated disturbances (Fig. 2). The evolution of this pair of wave-like gusts is most clearly depicted in a rapid playback of a sequence of computer generated PPI's. At the time (2211 central standard time (CST)) of the data presented in Fig. 2, the density current seen earlier is no 


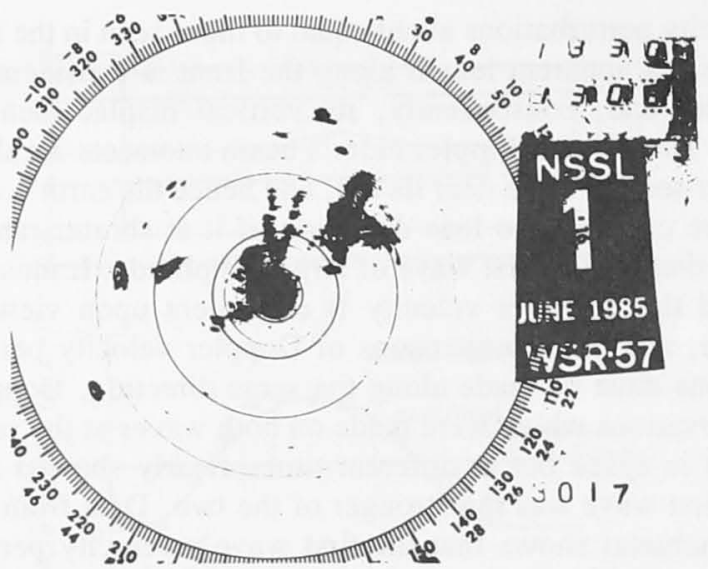

Fig. 3. Display of storms at 1830 CST showing a large storm, with its nearest border at a $115-\mathrm{km}$ range northeast of the radar, that passed 90 min earlier $60-\mathrm{km}$ north of the radar. The bold range mark circles are at $100-\mathrm{km}$ intervals. Anomalous echoes are seen from southwest to northnortheast and to ranges of about $170 \mathrm{~km}$. The storm that later generated the great solitary wave of translation is centered at about $180-\mathrm{km}$ west.

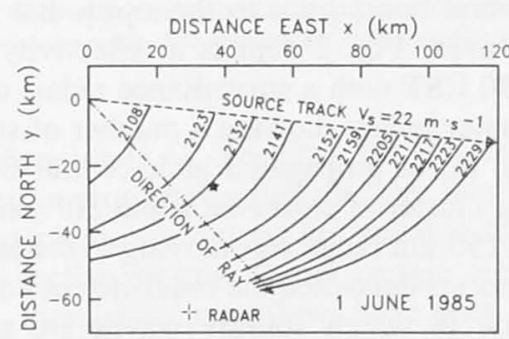

Fig. 4. Isopleths of wavefront locations of a solitary wave observed on June 1, 1985. Time is in hours and minutes CST. The reference time at the origin is approximately 2058 . The cross locates the radar whose data were used to locate the wave. The "**" symbol locates the site of an anemometer.

thought that the strongly curved front is due to inhomogeneities in the properties of the waveguide, causing the wave to have speeds that differ from place to place. Although we cannot discount the effects of inhomogeneities, we offer an alternative explanation based on the fact that a solitary wave's speed is a function of its amplitude, similar to (1).

Because the wave has a larger amplitude near the storm, it more likely has recirculating cold thunderstorm air which settles out of the wave, decreasing its amplitude. Radiation into the upper atmosphere [19] as well as the mechanical turbulence generated by shear and air flowing over rough ground can also cause wave attenuation. Thus, in general, it is expected that wave amplitude and consequently wave speed will depend upon time, or equivalently upon distance from the storm. We shall show that wave-speed change does account for strongly curved wavefronts.

\section{Wavefront Position Relative to a Moving SOURCE}

The thunderstorm downdraft and outflowing density current at the ground disturbs the surface layer of cooler air (i.e., cooler than the potential temperature of the air above it) laid down by the earlier density currents. We hypothesize that the intruding density current generated by the passing storm raises the height of the preexisting stratified layer over a limited area and for a limited period of time. This finite disturbance of long wavelength then propagates outward, away from the storm path, and evolves into a finite number of solitary waves, as is qualitatively shown by the theoretical description presented in Section V. In other words, the interaction of the rapidly moving outflow density current with the stable layer results in a transformation, over a relatively short period of time, of the initial long, but finite, wavelength density perturbation into a sequence of solitary waves. Support for a model of this type has recently been provided by the results of a very thorough numerical study reported by Haase and Smith [20], which show that when the environment is stable-stratified, the frontal zone of a rapidly moving sustained density current is transformed directly into a sequence of solitary waves.

Because the downdraft source moves along a straight line at a constant speed $V_{s}$ from the direction $\delta_{s}$, each short wave that evolves out of this long-wave disturbance is a quasi-two-dimensional wave packet which propagates along rays confined to the horizontal because of waveguidance. In a homogeneous medium, as was assumed in this case, theory [21] determines that rays are straight. But because they emanate from a source moving along the straight track of the storm, successively generated rays must be parallel to one another.

The theoretical speed of localized large-amplitude disturbances is the speed of wave energy propagating along a ray. Therefore, the wavefront speed, measured along a ray, should equal that given by theory. If wave speed were constant, then the wavefront would form a straight line emanating from the source at an angle $\beta$ to its track, and rays would be perpendicular to the wavefront as well as being parallel to one another. Then we could use the Mach relation,

$$
c=V_{s} \sin \beta
$$

to derive an estimate of $c$. Because this relation is derived for rays perpendicular to straight wavefronts, whereas the front observed here is curved, the ray paths need to be determined. Although it is natural to construct rays orthogonal to wavefronts, this construction would result in curved rays. But theory shows that rays in horizontally homogeneous media are straight, although not necessarily orthogonal to a wavefront [21]. Thus, even though the wavefront is curved, rays must be straight and parallel and must intersect the source path at an, as yet, unknown angle. A modified form of the Mach relation has been derived to account for changes in $c$ and as given by Doviak and Chen [23].

The direction of rays is not easy to determine, but it might be controlled in some way by the displacement of air at the source, and/or it could depend upon the value of Scorer's parameter $l[24]$ above the layer of waveguiding air. It can be shown that Scorer's parameter determines which spectral components of the horizontally propag. the proc the atm and alt be zero Scorer': tion of , tential $\mathrm{t}$ ally mo the vert inate. A it can ca Thus, w $l$ to be $z$ have ne waves v directior obtain v solitary eters in and the any arbi in those disturba form.

Using ometry deduce

where

and $d_{r}$ is of a ray' came, $t$ $0, r(t)$ $c(t)$ is $\mathrm{t}$ rection $\delta$ Althol $\delta_{r}$ and $\mathrm{tl}$ detailed (444 m) wave ob: and Che though value fol direction approxin natively, served d mean. N theoretic as we sh locations 
its. We nerated existing 1 period th then th, and is qualented in rapidly ayer reeriod of density Support 1 by the rted by ie envirapidly directly

straight ch short nce is a pagates f wave imed in straight. long the ed rays

ude disig along along a ed were ght line ck, and well as re Mach

derived reas the ed to be rays orresult in zontally cessarily ugh the parallel nknown las been by Dov-

e, but it ment of he value aveguider deterzontally propagating disturbance will leave the waveguide and, in the process, leak disturbance energy upward and away into the atmosphere, destroying the effectiveness of the guide and altering the waveform. Scorer's parameter needs to be zero or imaginary for waveguidance to be perfect [14]. Scorer's parameter depends upon both wind in the direction of wave propagation and the vertical gradients of potential temperature. But in the upper atmosphere $l$ is usually more dependent upon wind, whereas near the earth the vertical gradients of potential temperature often dominate. Although temperature is a scalar, wind is not, and it can cause the waveguide to have anisotropic properties. Thus, waves would favor propagation directions that cause $l$ to be zero or imaginary, or sufficiently small positive to have negligible attenuation. As a consequence, solitary waves would propagate over long distances in preferred directions. To verify this hypothesis, it is necessary to obtain vertical profiles of temperature and wind during a solitary wave episode, because these specify the parameters in the Taylor-Goldstein wave equation [12], [25], and the solution of this equation determines $c$ and $l$ along any arbitrarily selected direction of propagation. But only in those directions for which $l$ is zero or imaginary will disturbances evolve into solitary waves of permanent form.

Using the ideas presented above, we construct the geometry presented in Fig. 5, and from this we are able to deduce that the position of the wave is given by

$$
\begin{aligned}
& x(t)=-d_{r} \sin \delta_{s}-r(t) \sin \delta_{r} \\
& y(t)=-d_{r} \cos \delta_{s}-r(t) \cos \delta_{r}
\end{aligned}
$$

where

$$
r(t)=\int_{d_{r} / V_{s}}^{t} c(t) d t, \quad \frac{d_{r}}{V_{s}} \leq t
$$

and $d_{r}$ is the distance along the storm track to the location of a ray's origin, $\delta_{s}$ is the direction from which the storm came, $t=0$ is the time at which the source is at $x=y=$ $0, r(t)$ gives the wavefront position along the ray, and $c(t)$ is the speed of the wave along the ray from the direction $\delta_{r}$.

Although we did not have the data to reliably estimate $\delta_{r}$ and thus the speed of the wave observed on June 1, detailed measurements made, using instruments on a tall $(444 \mathrm{~m})$ tower and at many surface sites, on a solitary wave observed on another day (May 11, 1980) led Doviak and Chen [23] to deduce a ray direction of $315^{\circ}$. Although this by itself is not a sound reason to select this value for $\delta_{r}$, both waves propagated in roughly the same direction (i.e., southeastward), and we assume for a first approximation that $\delta_{r}$ was the same in both cases. Alternatively, we could determine the $\delta_{r}$ that best fits the observed data along a selected front, or all the fronts in the mean. Nevertheless, the assumed value of $315^{\circ}$ results in theoretically estimated wavefront positions that fit well, as we shall see, the data for most of the observed frontal locations.

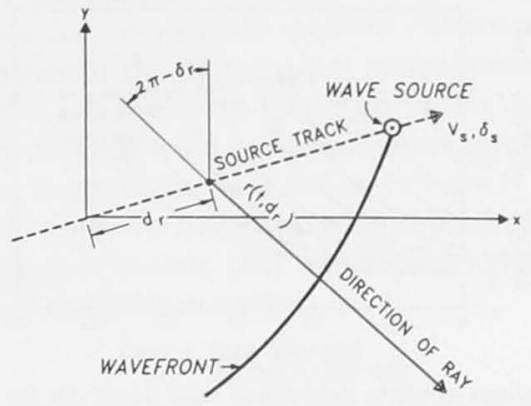

Fig. 5. Geometry used to determine the wavefront position.

The data of Doviak and Chen [23, fig. 11] shows that $c(t)$ had a time dependence that can be well described by an equation of the form:

$$
c\left(t^{\prime}\right)=c_{\infty}+c_{1} \exp \left(-\gamma t^{\prime}\right)
$$

where $c_{\infty}$ is a constant asymptotic speed attained by the wave (presumably after all recirculating outflow has leaked away and radiation into the upper atmosphere has ceased), $t^{\prime}$ is the time after a solitary wave is initiated, and $\gamma$ is the rate at which the speed is exponentially decreasing. If the wave does not reach a finite amplitude in the asymptotic limit, but continues to attenuate under the influence of frictional dissipation or radiation losses until the wave becomes infinitesimally small, then $c_{\infty}$ is the wave speed of linear waves. Assuming a ray direction on June 1 that is equal to $315^{\circ}$, we were able to estimate the parameters of (4) by fitting the integral of (4) to the observed position of the wave along the arbitrarily selected ray shown on Fig. 4. The integral of (4) fitted the data with an average error of less than $0.1 \mathrm{~km}$ and a standard error of about $2 \mathrm{~km}$ over the ray path length of $90 \mathrm{~km}$. Thus (4) fits our data as well as those of Doviak and Chen [23]. From this fit we estimated that $c_{1}=27 \mathrm{~m} \mathrm{~s}^{-1}, c_{\infty}$ $=0 \mathrm{~m} \mathrm{~s}^{-1}$, and $\gamma=2.33 * 10^{-4} \mathrm{~s}^{-1}$. We hypothesize that $c_{\infty}$ vanishes because ambient winds advected the medium at the wavespeed toward the direction from which the waves propagated. A rawinsonding at 2054 CST for a site $40-\mathrm{km}$ south of Oklahoma City showed that winds, below 1-km AGL, were about $9 \mathrm{~m} \mathrm{~s}^{-1}$ in a direction opposite to that of wave propagation. Furthermore, the wave speed calculated from (4), when the wave was at the radar site, is $5 \mathrm{~m} \mathrm{~s}^{-1}$, which is in close agreement to the $4 \mathrm{~m} \mathrm{~s}^{-1}$ deduced earlier from anemometer and Doppler radar data. Source speed and direction $\left(V_{s}=22 \mathrm{~m} \mathrm{~s}^{-1}\right.$ and $\delta_{s}=275^{\circ}$ ) were obtained by tracking the intersection of the thin line of weak reflectivity factor $(<20 \mathrm{dBZ})$ caused by the wave, with the high reflectivity of the storm's precipitation. Using these parameter values in (3), we obtained the theoretically estimated wavefront isopleths sketched in Fig. 6. These show at least qualitative agreement with those observed in Fig. 4. For example, in the ambient air outside the storm (beyond about $20 \mathrm{~km}$ of the storm track) the wavefront position determined by theory is within a few kilometers of the observed position for most of the 100-km-long wavefront. The largest departure 


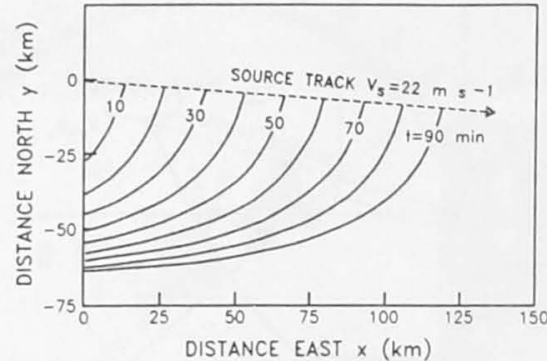

Fig. 6. Wavefront isopleths determined from theory for the data derived from observations on June 1, 1985. Time $t$ is in minutes after the reference time at $x=y=0$.

of theory from observations is at wavefront locations near the storm track, where it is difficult to discriminate velocity perturbations caused by the wave from those attributed to internal motions in the storm.

Anemometer data from the surface site (Fig. 4) shows that both waves produced a wind perturbation having a direction from about $325^{\circ}$, which also estimates $\delta_{r}$ [23]. The wave perturbation wind observed with the anemometer at the radar site appeared to be corrupted by another disturbance and could not be used to give a reliable estimate of $\delta_{r}$. Nevertheless, if the $\delta_{r}=325^{\circ}$ estimate was used and wavefront position data were fitted along the $325^{\circ}$ ray instead of the $315^{\circ}$ one, the parameters of (4) would take on the values of $c_{1}=25.8 \mathrm{~m} \mathrm{~s}^{-1}, c_{\infty}=0$ $\mathrm{m} \mathrm{s}^{-1}$, and $\gamma=2.97 * 10^{-1} \mathrm{~s}^{-1}$, being nearly equal to those derived earlier. Furthermore, the theoretically estimated wavefront position would be, at most, $5 \mathrm{~km}$ from those positions shown in Fig. 6 and for the most part be within a couple of kilometers of the observed front. We need data from several anemometers to determine whether the direction of wave perturbation winds along the front is nearly constant to verify our hypothesis that rays are parallel and from the direction of the perturbation wind.

In order to test (3) in a more quantitative manner, we also applied it to data collected on May 11, 1980 for which we had independent estimates of all parameters, including the ray direction [23]. Fig. 7 compares the observed wavefront and that given by (3). There is a quantitative agreement between the two: The theory shows nearly the same rotation of the wavefront as it propagates southeastward, as well as giving the correct inclination of the front.

\section{COMParison With THEORY}

Theoretical studies by Benjamin [26], Davis and Acrivos [27], and Ono [28] have led to the specification of the integro-differential equation,

$$
\frac{\partial f}{\partial \tau}+\alpha f \frac{\partial f}{\partial \xi}-\beta \frac{\partial^{2}}{\partial \xi^{2}} H\{f\}=0
$$

which, given an initial disturbance, governs solitary wave evolution in horizontally stratified fluids overlaid with an infinitely deep layer of neutral stability. In this equation, $f$ is the distance-time-dependent factor of the stream function that determines the motions of air perturbed by the

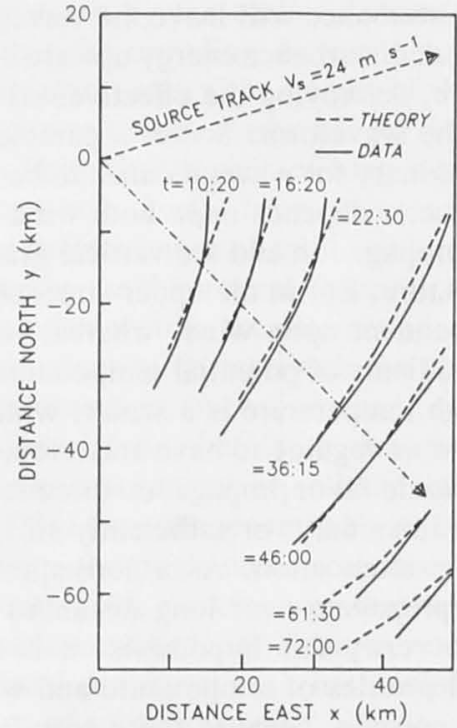

Fig. 7. Comparison of the wavefront location and shape estimated from theory with that observed on May 11, 1980. Time is given in minutes and seconds after a reference time at 2204 CST.

wave; $H\{f\}$ denotes the Hilbert transform of $f$, and $\tau$ and $\xi$ are scaled time and space coordinates. The constants $\alpha$ and $\beta$ are specified by the vertical profiles of ambient wind and temperature. Equation (5) has been named the Benjamin-Davis-Ono (BDO) equation.

Fig. 8 gives the result of a numerical solution of (5) in which a transient disturbance is applied at the left-hand boundary of the domain, and the waves evolve on the halfline along a ray direction. To simulate the initial influence of thunderstorm outflow, the waveguide depth is forced to increase linearly from its ambient level for a period of $1.5 \mathrm{~min}$, for a total displacement of $350 \mathrm{~m}$. Thereafter, the traveling storm is assumed to maintain this displacement at the origin for a period of $7.0 \mathrm{~min}$. Finally, the perturbing influence is removed linearly over a period of $1.5 \mathrm{~min}$ as the storm departs from the position of the ray origin. Since the observed storm speed was $22 \mathrm{~m} \mathrm{~s}^{-1}$, this time period suggests that the perturbing source had a length of about $13 \mathrm{~km}$. This is less than, as expected, the roughly $40-\mathrm{km}$ diameter of the storm, since the storm diameter gives only the extent of the rain region and not the smaller area of intense downdrafts (e.g., [18, fig. 9.6a]).

The waveguide that is used in this case is simply a windless lower layer stratified according to $N=$ $N_{0} \operatorname{sech}(z / h)$, where $N$ is the Brunt-Väisälä frequency, $z$ is the height variable, and $N_{0}=0.0338 \mathrm{~s}^{-1}$. This corresponds to a maximum potential temperature gradient of about $4.5^{\circ} \mathrm{C}$ per $100 \mathrm{~m}$, or a sensible temperature gradient of about $3.5^{\circ} \mathrm{C}$ per $100 \mathrm{~m}$ of height. The effective waveguide depth $h$ was selected to be $500 \mathrm{~m}$. Unfortunately, profiles of temperature and wind at the time and location of the disturbance were not available for determining whether these parameters are in accord with the environment on June 1, 1985. Although a temperature sounding from Oklahoma City nearly 4 -hr before wave passage 


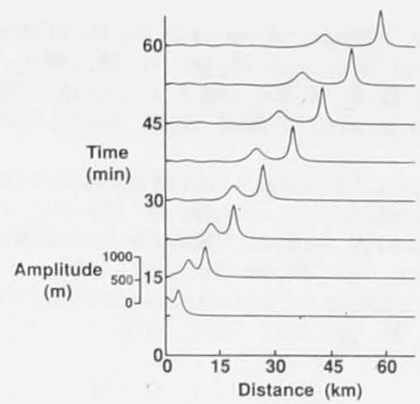

Fig. 8. Results of a numerical integration of the Benjamin-Davis-Ono equation illustrating the generation of two solitary waves. Shown is the wave amplitude (i.e., vertical displacement of air) versus distance from a source with time as a parameter. The depth of the stable layer in repose is $500 \mathrm{~m}$. nored in the theoretical treatment of (5), could acco for this discrepancy. Since wave speed is a function of the wave speed derived from Fig. 8 is nearly constant at $16 \mathrm{~m} \mathrm{~s}^{-1}$, whereas observations suggest that speed is exponentially decreasing from a maximum of $27 \mathrm{~m} \mathrm{~s}^{-1}$. It is thought that attenuation and environmental winds, ig- amplitude, attenuation will cause the speed to decrease. A more detailed comparison requires environmental data and a measure of the perturbation temperature generated by the wave, and is beyond the scope of this paper. However, when detailed wind and temperature information derived from instruments on a 444-m-tall tower during the passage of another solitary wave were used in the analysis, Doviak and Christie [10] found good agreement with solutions of the BDO equation.

\section{Conclusions}

We have observed that the atmosphere supports solitary waves with properties similar to those on water observed more than 100 years ago by John Scott Russell in the canals of Edinburgh, Scotland. We have shown that the wavefront shape, position, and evolution of a large amplitude solitary atmospheric wave of translation, generated by a moving thunderstorm, can be estimated, given the single direction along which the disturbance propagates with an amplitude-dependent speed. Solutions of the integro-differential equation, which governs the evolution of the waves, show that a pair of solitary waves can be generated as observed and that these numerically generated waves have time and space scales that compare fairly well with observations.

In order to determine the horizontal homogeneity of the wind and temperature that characterizes the propagation medium, and to determine the direction in which the anisotropic waveguide has the most favorable trapping properties and to verify the theory, future experiments would benefit from having a network of profilers (4 or more) with a lower height $(₹ 1 \mathrm{~km})$ resolution of about $100 \mathrm{~m}$ or better and a spacing of no more than several tens of kilometers. If, in addition, these profilers can record data with a time resolution of about $1 \mathrm{~min}$ or less, they could also be used to determine the height dependence of the wave, well above the altitudes where Doppler weather radars lose detection capability. Since it has been suggested [23] that energy propagates along the direction (ray direction) of velocity perturbations caused by the wave near the surface, a network of surface stations that are no more than several kilometers apart are needed to sample the wave perturbation along the $100-\mathrm{km}$ or longer curved wavefront to determine whether indeed rays are straight and parallel but not necessarily perpendicular to the front. Finally, a Doppler weather radar, of sufficient capability to observe echoes from precipitation-free areas around thunderstorms, is required to map the position, shape, and progress of the wave. Most importantly, we need the cooperation of nature.

\section{ACKNOWLEDGMENT}

The authors thank the engineering staff, led by D. Sirmans, of the NSSL for the fine data collection system they designed and operated with the utmost care. J. Wardius of the NSSL provided data from surface-band meteorological instruments. We also appreciate the constructive comments of Dr. R. K. Smith of Monash University, 
Clayton, Australia, and Dr. N. A. Crook of the National Center for Atmospheric Research, whose review helped to improve the original manuscript.

\section{REFERENCES}

1] J S. Russell, "Experimental researches into the laws of certain hymotion of floating bodies a ies, and have not the Resistance of Fluids," Trans. Roy. Soc. Edinknown laws of the Resistance of Fluids, 1840

burgh, Solitons and Nonlinear Wave Equations. Orlando, FL: Academic, 1982, $630 \mathrm{pp}$.

[3] J. S. Russell, "Seventh report on the committee on waves," in Trans. Brit. Assoc. Adv. Sci., vol. 6. London: John Murray, 1838, pp. 417-496.

[4] A. J Abdullah, "The atmospheric solitary wave," Bull. Amer. Meteorol. Soc., vol. 36, pp. 515-518, 1955.

[5] D. R. Christie, K. J. Muirhead, and A. L. Hales, "On solitary waves in the atmosphere," J. Atmos. Sci., vol. 35, pp. 805-825, 1978

G R. J. Doviak and R. S. Ge, "An atmospheric solitary gust observed with a Doppler radar, a tall tower and a surface network," J. Atmos. Sci, vol. 41, pp. 2559-2573, 1984

Scit. Assoc. Adv. Sci. (York, Eng.), Sept. 1844. London: John Murray, 1845, pp. 311390 (and plates 47-56).

[8] D. R. Christie and K. J. Muirhead, "Solitary waves: A hazard to aircraft operating at low altitudes," Aust. Meteorol. Mag., vol. 31, pp. $97-109,1983$

[9] R . H Clarke, R. K. Smith, and D. G. Reid, "The morning glory of the Gulf of Carpentaria: An atmospheric undular bore,' Mon. Weath. Rev., vol. 109, pp. 1726-1750, 1981

[10] R. J Doviak and D. R. Christie, "Thunderstorm-generated solitary waves: A wind shear hazard," J. Aircrafi, vol. 26, pp. 423-431, 1989.

[11] J. F W Purdom, "Subjective interpretation of geostationary satellite data for nowcasting," in Nowcasting, K. A. Browning, Ed. New York: Academic, 1982, chap. 3.4, pp. 149-166.

[12] D. R. Christie, "Long nonlinear waves in the lower atmosphere," $J$. Atmos. Sci. vol 46, pp. 1462-1491, 1989.

[13] J. Lighthill, Waves in Fluids. London: Cambridge Univ. Press, 1979, 504pp.

"The effect of ambient stratification and moisture on the motion of atmospheric undular bores," J. Atmos. Sci., vol. 43, pp. 171-181, 1986.

[15] N. A . Crook, "Trapping of low-level internal gravity waves," J. Atmos. Sci., vol. 45, pp. 1533-1541, 1986.

16] T. Maxworthy, "On the formation of nonlinear internal waves from gravitational collapse of mixed regions in two and three dimensions," J. Fluid Mech., vol. 96, pp. 47-64, 1980.

[17] R. Fulton, D. S. Zrnić, and R. J. Doviak, "Initiation of a solitary wave family in the demise of a nocturnal thunderstorm density current," J. Atmos. Sci., to be published.

[18] R. J. Doviak and D. S Zmić, Doppler Radar and Weather Observa tions Orlando, FL: Academic, 1984, sec. 2.2.3.2

[19] S. A. Maslowe and L. G. Redekopp, "Long nonlinear waves in stratified shear flows," J. Fluid Mech., vol. 101, pp. 321-348, 1980.

[20] S. P. Haase and R. K. Smith, "The numerical simulation of atmosphere gravity currents, Part II: Environments with stable layers, Geophys. Astrophys. Fluid Dyn., to be published.

[21] G. B. Whitham, Linear and Nonlinear Waves. New York: Wiley, $1974,636 p p$

122] R. J. Doviak, "Gravity wavefront curvature and a modified Mach relation," in Joint Remote Sensing Conf., 4th Symp. Intern. Soc. Acoust. Remote Sensing and the 2 nd Aust. Conf. Phys. Remote Sensing Atmos. and Oceans. Canberra, ACT, Aust.: Aust. Defense Acad., 1988, pp. 75 (1-6)

23] R. J Doviak and S. Chen, "Observations of a thunderstorm generated gust compared with solitary wave theory," Adv. Sys. Acquisition Serv., Washington, DC, FAA Rep. DOT/FAA/SA-88/1, 1988 $135 \mathrm{pp}$.
24] R. S. Scorer, "Theory of waves in the lee of mountains,"

Quart. J. Roy. Meteorol Soc, vol. 75, pp. 41-56, 1949

[25] K. K. Tung, D. R. S. Ko, and J. J. Chang, "Weakly nonlinear in ternal waves in shear," Stud. Appl. Math., vol. 65, pp. 189-221, 1981

[26] T. B. Benjamin, "Internal waves of permanent form in fluids of great depth," J. Fluid Mech., vol. 29, pp. 559-592, 1967.

27] R. E. Davis and A. Acrivos, "Solitary internal waves in deep water, J. Fluid Mech, vol. 29, pp. 593-607, 1967

28] H. Ono, "Algebraic solitary waves in stratified fluids," J. Phys. Soc. Japan., vol. 39, pp. 1082-1091, 1975

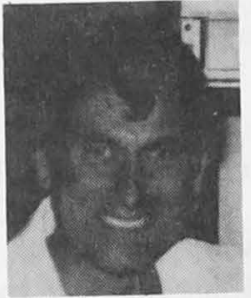

Richard J. Doviak (S'56-M'57-SM'72) received the B.S.E.E. degree (1956) from Rensse laer Polytechnic Institute, Troy, NY, and the M.S.E.E. (1959) and Ph.D. (1963) degrees from the University of Pennsylvania, Philadelphia.

$\mathrm{He}$ is a Senior Scientist at the National Severe Storms Laboratory (NSSL) and Adjunct Professor of Electrical Engineering and Meteorology at the University of Oklahoma, Norman. At NSSL he has, for 15 years, led Doppler radar and storm electricity research. In 1981 he was PI in a joint NASA, NSSL, and OU project to study airborne Doppler lidar measurements of wind as a first step toward the surveillance of winds from space. mentrical Engineering (January 1-March 31 1987) at the University of Kyoto, Kyoto, Japan. He is an Associate Ed, 1. IEEE Editor The numerous jour Observations (Orlando, co-author of the book

FL: Academic, 1984).

Dr. Doviak is the co-recipient of the IEEE's 1988 Harry Diamond Award or contributions to, and applications of, weather radar science. $\mathrm{He}$ merican Meteorological Society, the American Geophys(union, and Commission F of URSI

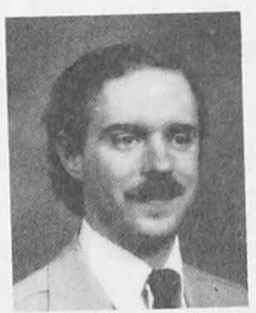

Kevin W. Thomas was born in Akron, $\mathrm{OH}$, on March 16, 1956. He attended Kent State University, Kent, OH, from 1974 to 1976 . He attended Penn State University, University Park, from 1976 to 1978 , where he received the B.S. degree in meteorology. He did his M.S. work in meteorology from 1979 to 1983 at the University of Oklahoma, Norman.

Since 1983 he has been a staff member at the University of Oklahoma, where he became Computer Manager for the School of Meteorology. He currently works at the National Severe Storms Laboratory and is Manager currently works at the National as being a Computer Meteorologis developing a real-time code to process Doppler radar.

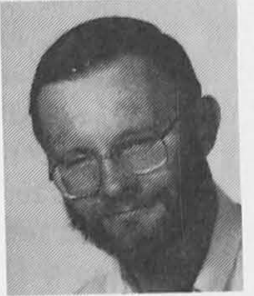

Douglas R. Christie received the B.A.Sc. degree in engineering physics, and the M.A. degree in physics from the University of Toronto, Toronto ON, Can., in 1962 and 1964, respectively

He has been with the Research School of Earth Sciences of The Australian National University for the last 14 years. His principal research interest in recent years has been in the area of nonlinear wave motions in the atmosphere. 
Section 2.8

ids," J. Phys. Soc.

\title{
Long nonlinear waves in the lower atmosphere
}

956) from Rensse-

oy, NY, and the

963) degrees from

, Philadelphia

he National Severe

Adjunct Professor

Meteorology at the

man. At NSSL he

$r$ radar and storm

was PI in a joint

ler lidar measure

winds from space.

(January 1-March

Ie is an Associate

litor of the IEEE

has contributed to

hysics, and is the

vations (Orlando,

y Diamond Award science. He is a merican Geophy

\author{
(D.R. Christie)
}

J. Atmos. Sci., 46, 1462-1491, 1989.

n Akron, $\mathrm{OH}$, on Kent State Univer1976. He attended y Park, from 1976 3.S. degree in merk in meteorology sity of Oklahoma, aff member at the he became ComMeteorology. He $y$ and is Manager ter Meteorologist

B.A.Sc. degree M.A. degree in oronto, Toronto, spectively.

h School of Earth tional University

al research inter le area of nonliniere. 
Reprinted from Journal OF THE ATMOSPHERIC SCIENCES, Vol. 46, No. 11 , 1 June 1989 American Meteorological Society

Long Nonlinear Waves in the Lower Atmosphere

D. R. CHRISTIE 


\title{
Long Nonlinear Waves in the Lower Atmosphere
}

\author{
D. R. CHRISTIE \\ Research School of Earth Sciences, The Australian National University, Canberra, Australia
}

(Manuscript received 1 June 1987, in final form 7 October 1988)

\section{ABSTRACT}

\begin{abstract}
This paper is concerned with the theoretical description of long, finite-amplitude waves in the stably stratified lower atmosphere. The time evolution of these waves is governed to first order by the Benjamin-Davis-Ono (BDO) equation when frictional processes are negligible or by the BDO-Burgers equation when turbulent dissipation is significant. Numerical solutions of both of these model equations are presented for a wide variety of initial conditions ranging from long waves of finite volume to internal deep-fluid bore waves of infinite spatial extent. It is shown that initially smooth long wave disturbances evolve rapidly under ideal homogeneous waveguide conditions into solitary waves of exceptionally large amplitude. The BDO-Burgers equation is found to have highly stable, time-independent, deep-fluid internal bore wave solutions which may be either oscillatory or monotonic depending upon the degree of frictional dissipation. A number of specific models for the time evolution of long nonlinear atmospheric waves are proposed and discussed in detail. Explicit formulae are given for the wave propagation parameters, surface perturbation pressure, and wind components and these are illustrated for a simple, but realistic, boundary-layer waveguide model. A study has also been made of the influence on nonlinear wave propagation of either spatial or temporal variations in the degree of turbulent dissipation. It is shown that an increase or decrease in the frictional damping coefficient, such as might be encountered at a land-sea boundary, can induce a significant variation in the speed of propagation and a substantial change in the morphology of finite-amplitude boundary layer wave disturbances. Finally, it is shown that wave induced turbulence plays an important role in the evolution of long nonlinear atmospheric waves.
\end{abstract}

\section{Introduction}

It has long been recognized that internal gravity waves are an important dynamical feature in the lower atmosphere. The significance of these ubiquitous waves arises from their ability to transfer energy and momentum and from the role they play in the generation of turbulence and in the initiation and organization of deep convection. In the last few years it has become increasingly clear that long gravity waves which propagate on stable layers in the lower troposphere are often highly nonlinear in nature. A good illustration of the importance of nonlinearity in the description of long atmospheric wave disturbances is given by observations of solitary waves on nocturnal inversions over northern Australia (Christie et al. 1978, 1979) and over central Oklahoma (Doviak and Ge 1984). Waves of this type often have amplitudes which are comparable to the effective depth of the waveguide; they are an essentially nonlinear phenomenon and the successful description of their properties can only be given within the framework of nonlinear dispersive wave theory. Although linear long wave theory appears to give a reasonable estimate of phase speed, an analysis based on the linearized equations completely fails to describe the morphology and evolution of finite-amplitude long internal wave disturbances. This paper will be concerned with

Corresponding author address: Douglas R. Christie, Australian National University, Research School of Earth Science, Institute of Advanced Studies, P.O. Box 4, Canberra 2600, Australia. a general theoretical description of nonlinear wave propagation in the stably stratified lower atmosphere.

A particularly interesting and exceptionally welldocumented example of nonlinear wave activity in the lower atmosphere is given by the "morning glory" phenomenon of northeastern Australia. The morning glory is a strong wind squall or series of wind squalls, often accompanied by one or more spectacular solitarywave roll-cloud formations, occurring commonly near dawn with remarkable regularity during the spring months over the southern Gulf of Carpentaria region and the adjacent Cape York Peninsula. A number of studies carried out in recent years (Christie et al. 1981; Clarke et al. 1981; Christie and Muirhead 1981, 1983a,b; Clarke 1983b; Egger 1983, 1984; Smith and Morton 1984; Noonan and Smith 1985; Smith et al. 1986 ) suggest that the morning glory is, in essence, a long nonlinear internal boundary-layer wave disturbance which evolves asymptotically in time into a family of large amplitude solitary waves. It is now widely recognized that similar large-amplitude propagating wave phenomena occur commonly, but usually without cloud, over much of the Australian region (Christie et al. 1978, 1979; Christie and Muirhead 1983a, 1985; Drake 1984, 1985; Mulroney 1984; Smith 1986; Clarke 1986; Physick 1986) and elsewhere (Shreffler and Binkowski 1981; Goncharov and Matveyev 1982; Hasse and Smith 1984; Doviak and Ge 1984; additional references may be found in Christie and Muirhead 1983b and in the recent review of the subject by Smith 1988). 
In the present paper we will be concerned with a general description of the evolution of morning glory waves and other closely related nonlinear wave phenomena which are confined to the lower few kilometers of the atmosphere. Christie et al. $(1978,1979)$ proposed, on the basis of the atmospheric scales involved, that the evolution of boundary-layer disturbances of this type is governed, to first order, by the deep-fluid Benjamin-Davis-Ono (BDO) equation (Benjamin 1967). Until now, detailed theoretical modeling of atmospheric waves of this type has been limited largely to a study of the stationary solitary wave components. Solitary wave solutions of the BDO equation for a simple two-fluid boundary layer model were used by Christie et al. (1978) to interpret observations of nonlinear waves over the arid interior of northern Australia. A similar solitary wave analysis has been used by Goncharov and Matveyev (1982) to estimate the depth and intensity of boundary layer temperature inversions from nonlinear wave observations. Solitary wave solutions corresponding to a realistic analytical model for potential temperature and shear profiles in the ambient environment have been derived by Clarke et al. (1981) and compared with morning glory observations. Recently, Noonan and Smith (1985) have reported the results of a very detailed study of morning glory solitary waves based on a numerical treatment of the waveguide eigenvalue problem. In both of these latter investigations the calculated morning glory wave speeds were found to compare favorably with observed propagation speeds. ${ }^{1}$ Clarke et al. found, however, that the predicted effective wavelengths of the solitary wave components were significantly smaller than those indicated by the observations, while in the investigation reported by Noonan and Smith, the predicted wavelengths were substantially larger than those observed.

It is assumed in all of these relatively simple model calculations that the time-independent BenjaminDavis-Ono equation provides a valid description of these disturbances. Strictly speaking, the steady-state $\mathrm{BDO}$ equation is applicable only to completely noninteracting deep-fluid solitary waves of modest amplitude which propagate in an inviscid horizontally homogeneous waveguide embedded in a neutrally stable fluid of infinite extent. Since atmospheric solitary waves seldom, if ever, occur as effectively isolated phenomena independent of other solitary wave components, calculations of wave parameters based on the time-independent solutions of the BDO equation may be unreliable. Furthermore, the evolution of long nonlinear wave disturbances in the lower atmosphere is always subject to dissipative processes and may also be influenced by variations in waveguide structure. Thus, a

'Noonan and Smith (1985) emphasize that calculated phase speeds are sensitive to the precise value of the waveguide depth and the appropriate value for this depth may differ from the obvious choice based on a visual assessment of the stability profile. more realistic description of these disturbances should be based on the time-dependent solution and should include the effects of spatial and temporal inhomogeneities in the waveguide structure, turbulent dissipation, and perhaps, damping due to the radiation of waves into the upper atmosphere. Since, in many cases of interest, the waveguide may be viewed as essentially uniform, the present paper will be primarily concerned with a theoretical study of the time-evolution of nonlinear wave disturbances under ideal homogeneous inviscid waveguide conditions and under conditions where turbulent dissipation plays a significant role in the evolution process.

A preliminary step in the study of the evolution of nonlinear boundary layer waves has been described by Christie and Muirhead ( 1981 ) who numerically solved the Benjamin-Davis-Ono equation for a variety of finite-volume initial long wave disturbances. Egger $(1983,1984)$ has also considered the development of morning glory nonlinear waves in a simple two-fluid model based on the internal bore-wave solution of the classical shallow-fluid Korteweg-de Vries (KdV)Burgers equation. It is clear, however, from the discussion in Benjamin (1967), that the Korteweg-de Vries equation does not provide a proper description of nonlinear waves in a surface-based waveguide beneath an unbounded fluid. This investigation will therefore focus on a study of the evolution of long nonlinear wave disturbances as given within the framework of a generalized deep-fluid Benjamin-Davis-Ono equation with turbulent dissipation.

A brief review of the observed properties of long nonlinear atmospheric wave disturbances, as illustrated by the morning glory of the Gulf of Carpentaria, is given in section 2 . This is followed in section 3 by a discussion of the application of nonlinear dispersive wave theory to a description of finite-amplitude wave motions in the stably stratified lower atmosphere. The Benjamin-Davis-Ono-Burgers equation is introduced at this point as a model equation for the dynamics of these nonlinear wave disturbances. Explicit expressions are given for both the surface perturbation pressure and wind components and these results are applied to a simple, but realistic, waveguide model to illustrate the principal features of long nonlinear waves in the atmospheric boundary layer. The main results of the present paper are presented in section 4. Numerical solutions of the BDO equation are given in section $4 \mathrm{a}$ for a wide variety of initial conditions ranging from smooth internal deep-fluid bore waves of infinite extent to long waves of finite volume. One of the most important results to emerge from this investigation is the discovery that relatively benign long-wave disturbances in the atmospheric boundary layer can evolve rapidly under ideal uniform waveguide conditions into solitary waves of exceptionally large amplitude. This result has obvious implications for the subject of hazardous aviation wind shear (Christie and Muirhead 1983a,b, 1985; 
Doviak and Christie 1989) and raises a number of interesting questions regarding the role of these disturbances in the initiation and organization of deep convection. The results of a numerical investigation of both the stationary and time-dependent solutions of the BDO-Burgers equation are given in section $4 \mathrm{~b}$. Solutions corresponding to deep-fluid time-independent internal bore waves and to decaying spatially localized long wave disturbances are presented in detail and a number of specific models for nonlinear atmospheric waves are proposed, based on these results. Section 5 is devoted to a study of nonlinear wave propagation under conditions where variations in the degree of turbulent friction occur either at a boundary or as a result of the onset of shear instability in the leading solitary wave components.

\section{Properties of nonlinear waves}

The morning glory of the Gulf of Carpentaria provides a particularly good illustration of the properties of long nonlinear wave disturbances in the lower atmosphere. The morning glory originates (Clarke 1983b; Clarke 1984; Crook and Miller 1985; Noonan and Smith 1986, 1987) during the early evening over the central highlands of the Cape York Peninsula in the transformation of a sea-breeze surge from the Coral Sea into an internal long wave disturbance. This disturbance continues to propagate during the following morning towards the southwest over the southern Gulf of Carpentaria on a nocturnal/maritime stable layer and then inland over the arid interior of northern Australia. The initially smooth long wave disturbance develops undulations along the leading edge which evolve rapidly into discrete solitary waves with amplitudes comparable to the effective depth of the surface-based waveguide. Under suitable conditions, the leading solitary wave components produce roll-cloud formations which may extend from a base at a few hundred meters to a height of more than $2 \mathrm{~km}$. These solitary-wave roll-cloud formations, which typically have a width of about $4 \mathrm{~km}$, seldom produce precipitation; however, large amplitude disturbances of this type do initiate deep convection and thunderstorms are frequently observed to develop over the Gulf of Carpentaria in the wake of these disturbances.

The salient features of morning glory wave disturbances may be seen in the selection of surface micropressure records shown in Figs. 1-3. These records have been chosen to illustrate the principal features of the most commonly observed forms of these disturbances. In many cases, the disturbance appears to have a welldefined horizontal length in the direction of propagation. On occasion, however, these disturbances extend over considerable distances and the form of the disturbance is reminiscent, to some extent, of a classical bore wave on the surface of shallow water. It should be emphasized, however, that an analogy with the clas-

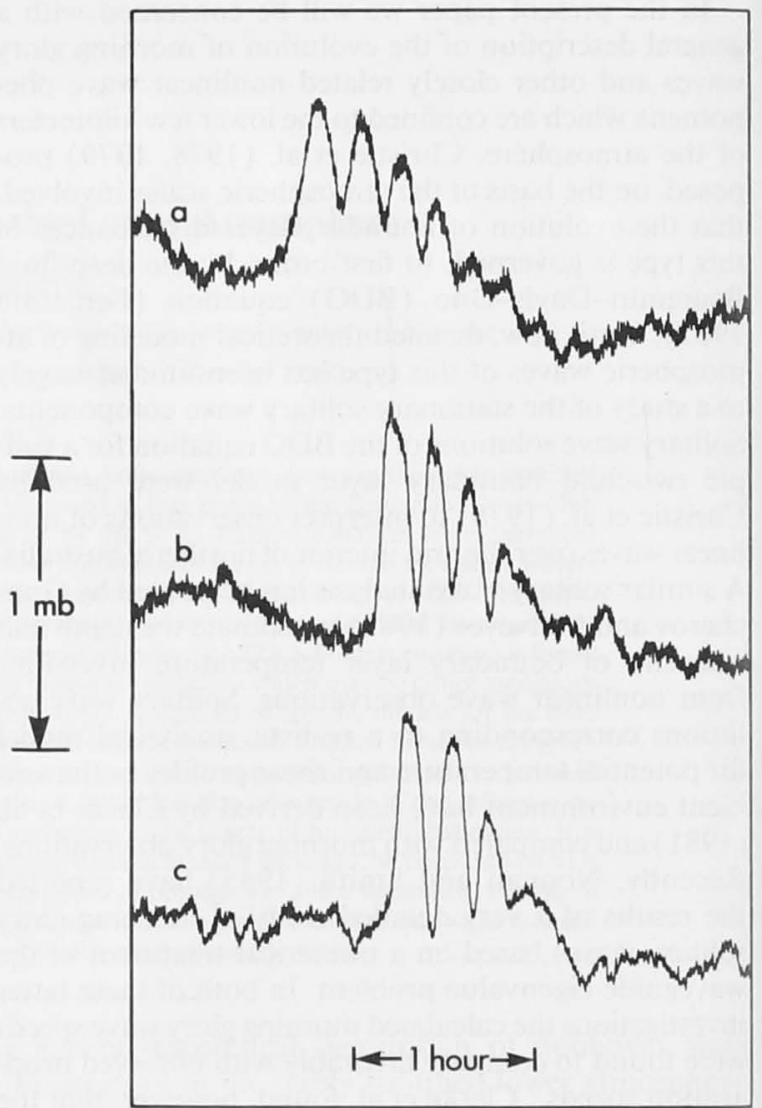

FIG. 1. Surface micropressure records corresponding to amplitudeordered morning-glory solitary-wave disturbances at Edward River on the eastern side of the Gulf of Carpentaria. Onset times, source azimuths and propagation speeds are as follows: (a) 2152 EST 15 September $1983,28^{\circ}, 5.9 \mathrm{~m} \mathrm{~s}^{-1}$; (b) 2120 EST 18 September 1983 , $31^{\circ}, 6.3 \mathrm{~m} \mathrm{~s}^{-1}$; (c) 2117 EST 24 September 1983, 35, $7.2 \mathrm{~m} \mathrm{~s}^{-1}$

sical bore wave phenomenon should not be taken too literally. A careful investigation of the surface pressure signature of morning glory disturbances has shown that the pressure perturbation due to these disturbances is not usually maintained at a constant level over prolonged periods of time. This implies that the depth of the stable layer does not remain elevated for long periods of time behind the leading edge of these disturbances. The observed decay in surface wind speed behind the leading solitary wave components in these disturbances is also consistent with this interpretation.

On average, most morning glory disturbances have three or four well-developed solitary wave components. In some cases, these disturbances appear to occur in the form of an essentially asymptotic amplitude-ordered family of solitary waves (see Fig. 1), while other morning glory disturbances are observed with only one well-defined solitary wave component along the leading edge (see Figs. 2b and 3d) ahead of the main disturbance. In contrast, on occasion, particularly extensive 


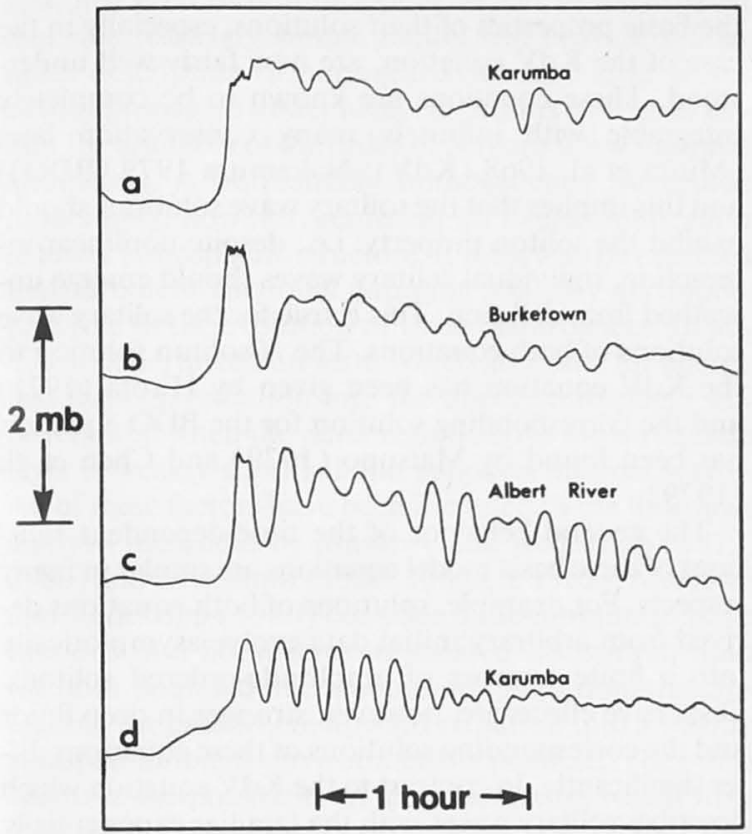

FIG. 2. Typical surface micropressure signatures of extensive morning-glory disturbances recorded at various locations around the southeastern margin of the Gulf of Carpentaria. Onset times, source azimuths and propagation speeds are as follows: (a) 0323 EST 3 October $1983,77^{\circ}, 10.2 \mathrm{~m} \mathrm{~s}^{-1}$; (b) 0650 EST 3 October $1983,79^{\circ}$, $10.6 \mathrm{~m} \mathrm{~s}^{-1}$; (c) 0649 EST 9 October $1983,51^{\circ}, 17.1 \mathrm{~m} \mathrm{~s}^{-1}$; (d) 0539 EST 15 October $1983,33^{\circ}, 8.8 \mathrm{~m} \mathrm{~s}^{-1}$. The event shown in panel b represents a later stage in the development of the event illustrated in panel a. Events (panels b and c) were accompanied by solitary-wave roll-cloud formations. The flattened crest in the micropressure profile of the leading solitary wave in events shown in panels $b$ and $c$ is a manifestation of closed circulation in the relative streamline pattern.

disturbances are observed with eight or more clearly defined solitary wave components (see Fig. 2d and also Fig. 20 in Smith and Morton 1984). In well-developed disturbances the horizontal separation between the leading solitary wave components is typically between 3 and $14 \mathrm{~km}$ and the amplitude of the leading solitary wave is usually within the range from 300 to $1000 \mathrm{~m}$. Morning glories which originate over the Cape York Peninsula propagate towards the southwest with speeds between 5 and $18 \mathrm{~m} \mathrm{~s}^{-1}$. Similar nonlinear wave disturbances which originate to the south and southeast are also observed on occasion along the southern margin of the Gulf of Carpentaria (Christie et al. 1981; Smith et al. 1982; Smith et al. 1986).

The extensive aerological observations reported by Clarke et al. (1981), Smith et al. (1982), Clark (1983b), and Smith and Morton (1984) have provided an abundance of data on the environmental waveguide conditions over the southern Gulf of Carpentaria region. Morning glory waves propagate on a stably stratified boundary layer underlying an almost neutral elevated layer which extends for several kilometers above the waveguide height. The depth of the stable layer is typically between 400 and $1200 \mathrm{~m}$ and the average Brunt-Väisälä period in this waveguide layer is usually in the range from 4 to $6 \mathrm{~min}$. The environmental wind component normal to the wavefront is usually towards the disturbance in the lower layer and along the direction of propagation at higher levels. The ambient normal winds tend to be light at lower levels and are always less than the observed propagation speed of the wave front at higher levels. Clarke (1983b) and Smith and Morton (1984) have emphasized that critical levels in the environmental wind structure are apparently absent in the case of morning glory wave disturbances.

\section{Evolution equations for long nonlinear internal waves in the atmosphere}

The theoretical description of long finite-amplitude internal waves in stratified fluids has received a great deal of attention in recent years. For waves of modest amplitude in shallow homogeneous fluids, the appropriate equation (Benney 1966; Benjamin 1966) which describes the time evolution of the vertical displacement at any point in the fluid,

$$
\eta(x, z, t)=A(x, t) \varphi(z),
$$

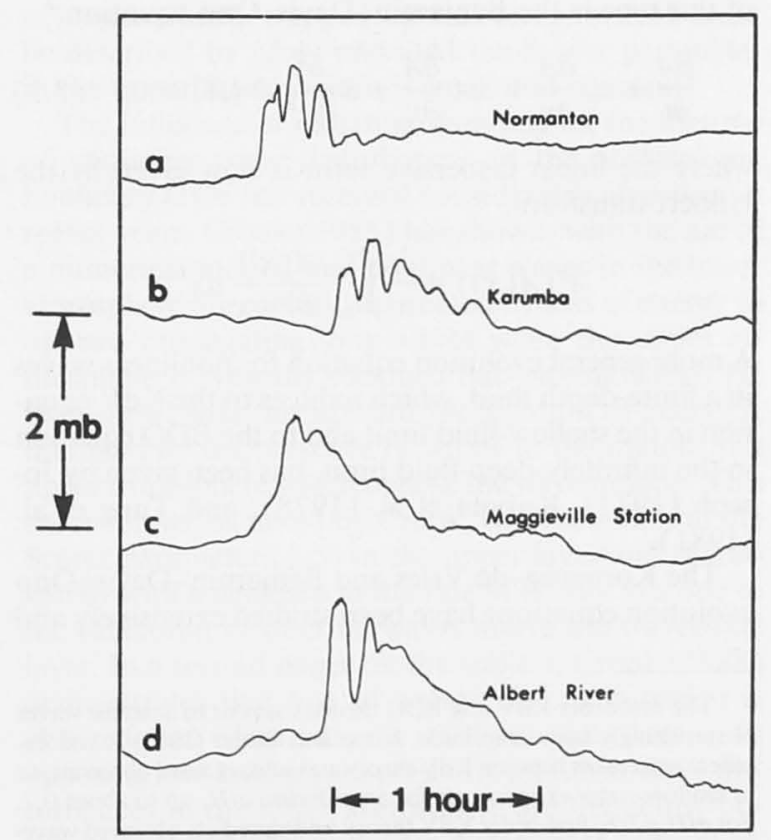

FIG. 3. Examples of typical micropressure records corresponding to relatively short-lived morning glory nonlinear wave disturbances. Onset times, source azimuths and propagation speeds are respectively: (a) 0140 EST, 26 September 1983, $92^{\circ}, 11.3 \mathrm{~m} \mathrm{~s}^{-1}$; (b) 0020 EST 28 September $1983,94^{\circ}, 10.0 \mathrm{~m} \mathrm{~s}^{-1}$; (c) 0028 EST 23 October 1983 , $96^{\circ}, 11.7 \mathrm{~m} \mathrm{~s}^{-1}$; (d) 0741 EST 27 October 1983, 34, $14.7 \mathrm{~m} \mathrm{~s}^{-1}$. All of these events occurred as clear-air disturbances with the exception of the event shown in panel (d), which was accompanied by a single spectacular solitary-wave roll-cloud formation in the Burketown area. 
is the familiar Korteweg-de Vries equation,

$$
\frac{\partial A}{\partial t}+c_{0} \frac{\partial A}{\partial x}+\alpha A \frac{\partial A}{\partial x}+\beta \frac{\partial^{3} A}{\partial x^{3}}=0 .
$$

Here, $x$ is the horizontal coordinate in the direction of propagation, $c_{0}$ the linear long wave phase speed, and the coefficients of the nonlinear and dispersive terms, $\alpha$ and $\beta$, respectively, along with the vertical modal function, $\varphi(z)$, are determined by the environmental waveguide density profile $\rho_{0}(z)$ and shear distribution $u_{0}(z)$, where $z$ is the streamline height in the unperturbed flow. As suggested by Christie et al. (1978), the $\mathrm{KdV}$ equation may provide a reasonable first-order model for larger scale atmospheric wave disturbances with horizontal wavelengths comparable to the depth of the troposphere. Here, however, we are concerned primarily with the description of nonlinear wave disturbances which are confined to surface-based waveguides with effective vertical scales of the order of 1 $\mathrm{km}$. These waves therefore belong to the class of nonlinear wave disturbances in deep fluids first considered by Benjamin (1967), Davis and Acrivos (1967), and later by Ono (1975). In this case the appropriate scale is determined by the effective depth, $h$, of the embedded waveguide rather than by the overall effective fluid depth, $H$. The governing evolution equation for waves of this type is the Benjamin-Davis-Ono equation. ${ }^{2}$

$$
\frac{\partial A}{\partial t}+c_{0} \frac{\partial A}{\partial x}+\alpha A \frac{\partial A}{\partial x}+\delta \frac{\partial^{2}}{\partial x^{2}} \mathscr{H}(A)=0,
$$

where the linear dispersive term is now given by the Hilbert transform,

$$
\mathscr{H}(A(x))=\frac{1}{\pi} \int_{-\infty}^{\infty} \frac{A\left(x^{\prime}\right)}{x^{\prime}-x} d x^{\prime} .
$$

A more general evolution equation for nonlinear waves in a finite-depth fluid, which reduces to the $\mathrm{KdV}$ equation in the shallow-fluid limit and to the BDO equation in the infinitely-deep-fluid limit, has been given by Joseph (1977), Kubota et al. (1978), and Tung et al (1981)

The Korteweg-de Vries and Benjamin-Davis-Ono evolution equations have been studied extensively and

The first order $\mathrm{KdV}$ and $\mathrm{BDO}$ theories appear to describe waves of surprisingly large amplitude. Koop and Butler (1981) found ex cellent agreement between $\mathrm{KdV}$ theory and solitary wave observation in shallow-water experiments for amplitudes, $a / H$, up to about 0.2 For $a / H=0.6$, first-order $\mathrm{KdV}$ theory underpredicts observed wavelengths by about $15 \%$. Deep-fluid BDO theory for wave speed has been compared by Tung et al. (1982) with fully nonlinear solitary wave calculations and with the experimental observations of Davis and Acrivos (1967). First-order theory for wave speed begins to fail noticeably when $a / h$ exceeds about 0.8 . Agreement with first-order predictions for the contraction of wavelength with increasing amplitude probably deteriorates when $a / h$ exceeds 0.5 . For $a / h \approx 1.0$, the weakly nonlinear amplitude/wavelength scaling breaks down completely as recirculation regions appear in the flow and the width of solitary waves begins to expand with increasing amplitude. the basic properties of their solutions, especially in the case of the $\mathrm{KdV}$ equation, are now fairly well understood. These equations are known to be completely integrable with infinitely many conservation laws [Miura et al. 1968 (KdV); Nakamura 1979 (BDO)] and this implies that the solitary wave solutions should exhibit the soliton property; i.e., despite nonlinear interaction, individual solitary waves should emerge unscathed from collision. This is true for the solitary wave solutions of both equations. The $\mathrm{N}$-soliton solution to the $\mathrm{KdV}$ equation has been given by Hirota (1971) and the corresponding solution for the BDO equation has been found by Matsuno (1979) and Chen et al. (1979).

The general behavior of the time-dependent solutions of these basic model equations are similar in many respects. For example, solutions of both equations derived from arbitrary initial data evolve asymptotically into a finite number of amplitude-ordered solitons. Dispersive effects are, however, stronger in deep fluids and the corresponding solutions of these equations differ significantly. In contrast to the $\mathrm{KdV}$ equation which describes solitary waves with the familiar exponentially decaying $\operatorname{sech}^{2}$ profile, the BDO solitons are algebraic. Furthermore, as a property in common with most soliton-generating equations, the $\mathrm{KdV}$ equation describes solitary waves which suffer a phase shift on collision. In this regard, the BDO solitons are somewhat exceptional in that they emerge from interaction along their precollision trajectories (Case 1978; Matsuno 1979). In addition, as emphasized by Koop and Butler (1981), the wavelength-amplitude scaling of BDO solitary waves differs fundamentally from that described by the $\mathrm{KdV}$ theory. For waves in unbounded fluids the appropriate wavelength-amplitude relationship is given by

$$
\left(\frac{\lambda}{h}\right)=\mathrm{O}\left(\frac{a}{h}\right)^{-1} \text {, }
$$

while the corresponding scaling requirement for classical shallow-fluid internal solitary waves is determined by

$$
\left(\frac{\lambda}{H}\right)^{2}=\mathrm{O}\left(\frac{a}{H}\right)^{-1}
$$

where $a$ is the amplitude and $\lambda$ a measure of the effective horizontal extent of the wave. Finally it should be noted that the amplitude of solitary waves generated in the decay of arbitrary disturbances in infinitely deep fluids may be substantially larger than the amplitude of $\mathrm{KdV}$ solitons created under similar initial conditions in shallow fluids. This important aspect will be examined further in the numerical experiments described below.

The unperturbed Benjamin-Davis-Ono Eq. (3.3) provides a description of waves of modest amplitude in a homogeneous waveguide embedded in a neutrally 
stratified fluid of infinite extent. It can be anticipated that this relatively simple model will provide a firstorder description of nonlinear boundary layer waves as they propagate under ideal conditions over level terrain for distances of the order of perhaps a few hundred kilometers. A horizontally homogeneous waveguide model may, however, represent an oversimplification of lower atmospheric structure. It must also be expected that turbulent frictional damping will play an important role in the evolution of these disturbances. In addition, a perturbation term which accounts for energy loss through radiation into the upper atmosphere will be required when the outer region above the waveguide layer is weakly stratified and supports internal waves. All of these factors have been included in the thorough analysis presented by Maslowe and Redekopp (1979, 1980 ) and Grimshaw (1980, 1981a,b, 1982). Maslowe and Redekopp ( 1980 ) consider finite-amplitude wave motions in a homogeneous sheared Boussinesq fluid for both bounded and unbounded fluid domains, including the case where upward radiation into a weakly stratified ambient environment is possible. They also examine the possibility of singular modes which may exist when critical layers occur where the phase speed is equal to the ambient flow speed. The theoretical treatment presented by Grimshaw (1981a) is also concerned with long nonlinear internal waves in both shallow and deep fluids and includes the influence of temporal and spatial variations in the waveguide structure along with the effects of both radiation and frictional damping. The appropriate evolution equation for nonlinear wave propagation in an inhomogeneous waveguide subject to turbulent frictional dissipation and radiation is given by a generalization of the $\mathrm{BDO}$ equation:

$$
\begin{aligned}
\frac{\partial A}{\partial t}+c_{0} \frac{\partial A}{\partial x}+\alpha(x, t) A \frac{\partial A}{\partial x}+\delta(x, t) \frac{\partial^{2}}{\partial x^{2}} \mathscr{H}(A) \\
=\mu(x, t) \frac{\partial^{2} A}{\partial x^{2}}-\frac{\delta(x, t) \operatorname{sgn}\left(u_{\infty}-c_{0}\right)}{\pi} \int_{-\infty}^{\infty} A\left(x^{\prime}\right) \\
\times d x^{\prime} \int_{0}^{\hat{\alpha}} k\left(\hat{\alpha}^{2}-k^{2}\right)^{1 / 2} \cos \left[k\left(x-x^{\prime}\right)\right] d k,
\end{aligned}
$$

where $\mu$ is a Burgers eddy diffusivity coefficient and $\hat{\alpha}$ the cut-off wavenumber for internal waves in the weakly stratified outer region above the boundary-layer waveguide. The variation in the coefficients, $\alpha, \delta$ and $\mu$, of this generalized equation reflect the temporal and spatial variations in waveguide structure. We shall refer to Eq. (3.4) as the BDO-Burgers equation when energy loss due to radiation is negligible and the last term vanishes.

The analysis presented by Maslowe and Redekopp (1980) and Grimshaw (1981a) of ducted wave propagation when radiation of long internal waves is pos- sible neglects the influence of shear in the outer flow domain. This is likely to be a fairly severe restriction when applied to nonlinear waves in the atmosphere since shear is generally present in the middle and upper troposphere and this may greatly alter the cutoff wavenumber in the upper fluid. Internal wave motions in an unsheared weakly stratified outer layer are described to leading order by a Helmholtz equation with a cutoff wavenumber given by $\hat{\alpha}=N_{\infty} /\left|u_{\infty}-c_{0}\right|$, where $N_{\infty}$ is the constant Brunt-Väisälä frequency in the upper fluid and $u_{\infty}$ the constant background wind. As a first step towards a more general treatment of this problem, we note that the results of Maslowe and Redekopp (1980) and Grimshaw (1981a) can be extended to include variations in the upper wind profile by recognizing that the governing Boussinesq equations (see, e.g., Maslowe and Redekopp 1980, p. 324) for internal wave propagation in the stratified fluid above the waveguide layer again reduce, when shear is included, to a Helmholtz equation with $\hat{\alpha}$ determined by $\hat{\alpha}^{2}=l^{2}$, where

$$
l^{2}(z)=\frac{N^{2}}{\left(u_{0}-c_{0}\right)^{2}}-\frac{\partial^{2} u_{0} / \partial z^{2}}{\left(u_{0}-c_{0}\right)}
$$

is the Scorer parameter (Scorer 1949). The evolution of long nonlinear trapped wave disturbances will again be described by (3.4) provided the Scorer parameter in the upper fluid domain is small and constant.

The influence of radiation damping on the lifetime of nonlinear wave disturbances in the atmospheric boundary layer has received considerable attention in recent years. Crook (1986) has shown, with the aid of a numerical model, that nonlinear waves in the lower atmosphere are rapidly attenuated by loss of energy to upward propagating long waves when the upper atmosphere is typically stratified but without shear. He then shows that this upward propagation of energy is inhibited by the presence of winds in the middle and upper troposphere which oppose the wave motion. This shear results, as noted by Crook, in a reduction in the Scorer parameter (3.5) in the upper layer and is consistent with a decrease in the rate of energy loss due to the excitation of internal waves above the waveguide layer. In a second paper on the subject, Crook (1988) demonstrates that loss of energy due to radiation is also reduced when either a midlevel inversion or an opposing low-level jet are present. Nonlinear wave disturbances in the lower atmosphere are frequently observed to propagate over large distances (Clarke et al. 1981; Clarke 1983b; Doviak and Ge 1984; Hasse and Smith 1984; Smith and Morton 1984). It is reasonable to assume that observations of long-lived nonlinear wave disturbances such as these can be attributed to one or more of the mechanisms discussed by Crook (1986, 1988) for inhibiting energy loss through radiation damping. 
The influence of energy loss due to radiation into the upper atmosphere on the evolution of long nonlinear wave disturbances will not be considered further in this paper. Since frictional damping is likely to be important under most boundary-layer conditions, we shall focus our attention in this initial evolution study on a detailed investigation of finite-amplitude long wave propagation in a horizontally homogenous inversion waveguide under both ideal conditions where dissipative processes may be neglected and under conditions where turbulent frictional damping has a significant influence on the evolution pattern. In both cases, we shall assume that the Scorer parameter for the outer layer is small enough to ensure that losses due to upward propagation of energy are negligible. As will be seen, the results of this investigation provide a fairly satisfactory description of the principal features of long-wave disturbances in the lower atmosphere.

Following Grimshaw (1980), for compressible fluids of infinite extent, the vertical modal function, $\varphi(z)$, satisfies the eigenvalue problem:

$$
\begin{aligned}
\frac{\partial}{\partial z}\left(\rho_{0}\left(c_{0}-u_{0}\right)^{2} \frac{\partial \varphi}{\partial z}\right) & +\rho_{0} N^{2} \varphi \\
& =v \frac{\partial}{\partial z}\left(\frac{\left(c_{0}-u_{0}\right)^{2}}{c_{s}^{2}}\right) \varphi \\
\varphi & =0 \text { on } z=0, \\
\frac{\partial \varphi}{\partial z} \rightarrow 0 & \text { as } z \rightarrow \infty
\end{aligned}
$$

where $c_{s}$ is the velocity of sound, $N$ the Brunt-Väisälä frequency defined by $N^{2}=g d\left(\ln \theta_{v}\right) / d z$, where $\theta_{\mathrm{v}}$ is the virtual potential temperature. The compressibility parameter, $v$, is given by $v=\gamma g h / c_{s}^{2}$, where $\gamma$ is the ratio of the specific heats. It will be assumed that the eigenfunctions, $\varphi(z)$, have been normalized to a maximum absolute value of 1 . This Sturm-Liouville problem generally admits an infinite number of vertical modal functions, each corresponding to a distinct infinitesimal long-wave phase speed, $c_{0}$. The lowest mode solution will usually be the only solution of interest since higher modes propagate much slower and are not generally observed in the atmosphere.

It is worth noting that the non-Boussinesq vertical modal equation (3.6a) can be rewritten as a long wavelength form of the Taylor-Goldstein equation for a compressible fluid through the transformation $\bar{\varphi}=\left(u_{0}-c_{0}\right) \varphi$; thus

$$
\begin{aligned}
\frac{\partial}{\partial z}\left(\rho_{0} \frac{\partial \bar{\varphi}}{\partial z}\right)+ & \left\{\frac{\rho_{0} N^{2}}{\left(u_{0}-c_{0}\right)^{2}}-\frac{\partial}{\partial z}\left(\rho_{0} \frac{\partial u_{0}}{\partial z}\right) /\left(u_{0}-c_{0}\right)\right. \\
& \left.-\frac{v}{\left(u_{0}-c_{0}\right)^{2}} \frac{\partial}{\partial z}\left(\frac{\left(c_{0}-u_{0}\right)^{2}}{c_{s}^{2}}\right)\right\} \bar{\varphi}=0 .
\end{aligned}
$$

In the Boussinesq approximation, the effect of density variations on the inertial terms may be neglected and for an incompressible fluid, Eq. (3.7) becomes the familiar form of the Taylor-Goldstein equation for zero wavenumber:

$$
\frac{\partial^{2} \bar{\varphi}}{\partial z^{2}}+l^{2}(z) \bar{\varphi}=0
$$

where $l^{2}(z)$ is given by $(3.5)$.

For a homogeneous compressible fluid, the coefficients $\alpha$ and $\delta$ in the BDO equation are given by (Grimshaw 1980):

$$
\begin{gathered}
\alpha=\frac{1}{I} \int_{0}^{\infty}\left\{3 \rho_{0}\left(c_{0}-u_{0}\right)^{2}\left(\frac{\partial \varphi}{\partial z}\right)^{3}-\frac{7}{2} v \rho_{0} \frac{N^{2} \varphi^{3}}{c_{s}^{2}}\right\} d z \\
\delta=\frac{1}{I}\left\{\rho_{0}\left(c_{0}-u_{0}\right)^{2} \varphi^{2}\right\}_{z \rightarrow \infty}
\end{gathered}
$$

where

$$
\begin{aligned}
I=2 \int_{0}^{\infty}\left\{\rho _ { 0 } \left(c_{0}\right.\right. & \left.-u_{0}\right)\left(\frac{\partial \varphi}{\partial z}\right)^{2} \\
& \left.+v \rho_{0} \varphi^{2} \frac{\partial}{\partial z}\left(\frac{c_{0}-u_{0}}{c_{s}^{2}}\right)\right\} d z .
\end{aligned}
$$

In most cases, as noted by Grimshaw (1980), the effects due to compressibility are small and the lower atmosphere may be taken to be incompressible provided the appropriate Brunt-Väisälä frequency for a dry or moist atmosphere is used in the equations.

The evolution of the streamline pattern within a stably stratified atmospheric waveguide may be calculated from the vertical displacement given by (3.1). Above this region, the amplitude of the disturbance in a neutrally stratified fluid decays algebraically and the vertical displacement may be obtained, as described by Benjamin (1967), using Fourier transforms:

$\eta(x, z, t)=\int_{-\infty}^{\infty} \mathcal{F}(A) \exp [-i k x-|k|(z-h)] d k$,

where

$$
\mathcal{F}(A)=\frac{1}{2 \pi} \int_{-\infty}^{\infty} A(x, t) \exp (i k x) d x .
$$

The outer streamline pattern can also be determined using Fourier methods (see Maslowe and Redekopp 1980 ) when the upper atmosphere is stratified and energy is radiated away from the trapped disturbance in the lower layer. We also note that streamline displacements should be multiplied by a factor $[\rho(z) / \rho(0)]^{1 / 2}$. This factor, which has been ignored in the treatment of boundary-layer waves, will be significant at higher altitudes. 
Long nonlinear waves in the atmospheric boundary layer are often detected using an array of microbarometers. It can be easily shown by applying Bernoulli's equation to the surface streamline and using the modal Eq. (3.6) that the surface perturbation pressure associated with the passage of a stationary nonlinear wave disturbance is given by

$$
\Delta P(x, t)=g A(x, t)\left[\int_{0}^{h} \rho(z)\left(\frac{\partial \varphi}{\partial z}\right) d z-\rho_{2}\right],
$$

where $\rho(z)$ is the ambient density profile,

$$
\begin{array}{ll}
\rho(z)=\rho_{0}(z), & z \leqslant h, \\
\rho(z)=\rho_{2}, & z>h .
\end{array}
$$

This expression is consistent with the leading order perturbation pressure term which appears in the derivation of the BDO equation (Ono 1975). It therefore appears that (3.11) provides a general first-order description of the surface perturbation pressure produced by nonlinear wave disturbances in the lower atmosphere.

The horizontal and vertical wind components in the stratified inversion layer are given, respectively, by

$$
u^{i}(x, z, t)=u_{0}(z)+c A(x, t) \frac{\partial \varphi(z)}{\partial z}
$$

and

$$
w^{i}(x, z, t)=-\left(c-u_{0}(z)\right) \frac{\partial A(x, t)}{\partial x} \varphi(z) .
$$

In the outer neutrally stratified region above the inversion waveguide, the wind components may be determined by evaluating

$$
\begin{aligned}
& u^{0}(x, z, t)=u_{0}(z)-c \int_{\infty}^{\infty}|k| \\
& \quad \times \exp [-i k x-|k|(z-h)] \mathcal{F}(A) d k,
\end{aligned}
$$

and

$$
\begin{aligned}
& w^{0}(x, z, t)=-\left(c-u_{0}(z)\right) \int_{-\infty}^{\infty} i k \\
& \quad \times \exp [-i k x-|k|(z-h)] \mathcal{F}(A) d k .
\end{aligned}
$$

The Benjamin-Davis-Ono equation has time-independent solutions, $A=f(x-c t)$, in the form of algebraic solitary waves:

$$
\begin{aligned}
A(x, t) & =\frac{a \lambda^{2}}{(x-c t)^{2}+\lambda^{2}}, \\
c-c_{0} & =\frac{1}{4} \alpha a=\frac{\delta}{\lambda} .
\end{aligned}
$$

For stationary waves of this type, expressions for the vertical displacement ${ }^{3}$ and wind components in the upper neutrally stratified region may be determined explicitly from (3.10) and (3.13) as

$$
\eta(x, z, t)=\frac{a \lambda(\lambda+z-h)}{(x-c t)^{2}+(\lambda+z-h)^{2}},
$$

$u^{0}(x, z, t)=u_{0}(t)$

$$
+\frac{c a \lambda\left[(x-c t)^{2}-(\lambda+z-h)^{2}\right]}{\left[(x-c t)^{2}+(\lambda+z-h)^{2}\right]^{2}},
$$

and

$w^{0}(x, z, t)=\frac{4 a \lambda\left(c-u_{0}(z)\right)(\lambda+z-h)(x-c t)}{\left[(x-c t)^{2}+(\lambda+z-h)^{2}\right]^{2}}$

The Benjamin-Davis-Ono equation also has periodic long-wave stationary solutions (Benjamin 1967) in the form of an infinite train of supercritical constant amplitude waves which represent the deep-fluid counterpart to the well-known elliptic cosine or "cnoidal" wave solutions of the classical Korteweg-de Vries equation. It should be emphasized that long nonlinear guided wave disturbances in the lower atmosphere seldom, if ever, occur in the form of a long sequence of periodic constant amplitude waves. This is as expected since "cnoidal" waves cannot be generated in the evolution of any realistic physical disturbance of limited spatial extent. These stationary periodic wave solutions will therefore not be considered further as a possible model for nonlinear boundary layer waves. In contrast, the solitary wave components play a fundamentally important role in the description of the evolution of long-wave disturbances of this type.

General solutions to the vertical modal eigenvalue problem (3.6) for arbitrary shear and stratification are not known, and, for many cases of interest, this system must be treated numerically (Noonan and Smith 1985 ). Under some conditions, however, the boundary-layer waveguide can be approximated by a simple model and useful analytical solutions corresponding to realistic density and shear profiles can be found. The results of a detailed study of a reasonably realistic boundary-layer flow model, including expressions in closed form for the evolution equation coefficients, surface perturbation pressure, and wind components, are given in appendix A. Figures 4 and 5, illustrate the essential features of long nonlinear waves: the relative streamline patterns, surface perturbation pressures, and wind fields for both a solitary wave and a nearly asymptotic amplitude-ordered family of solitary waves. These waves propagate as disturbances in the lowest

\footnotetext{
${ }^{3}$ The expression for the outer solitary wave solution given in (3.15) agrees with the dimensionless expression in Davis and Acrivos (1967) and (after correction for a misprint) with Eq. (3.83) in Benjamin (1967).
} 

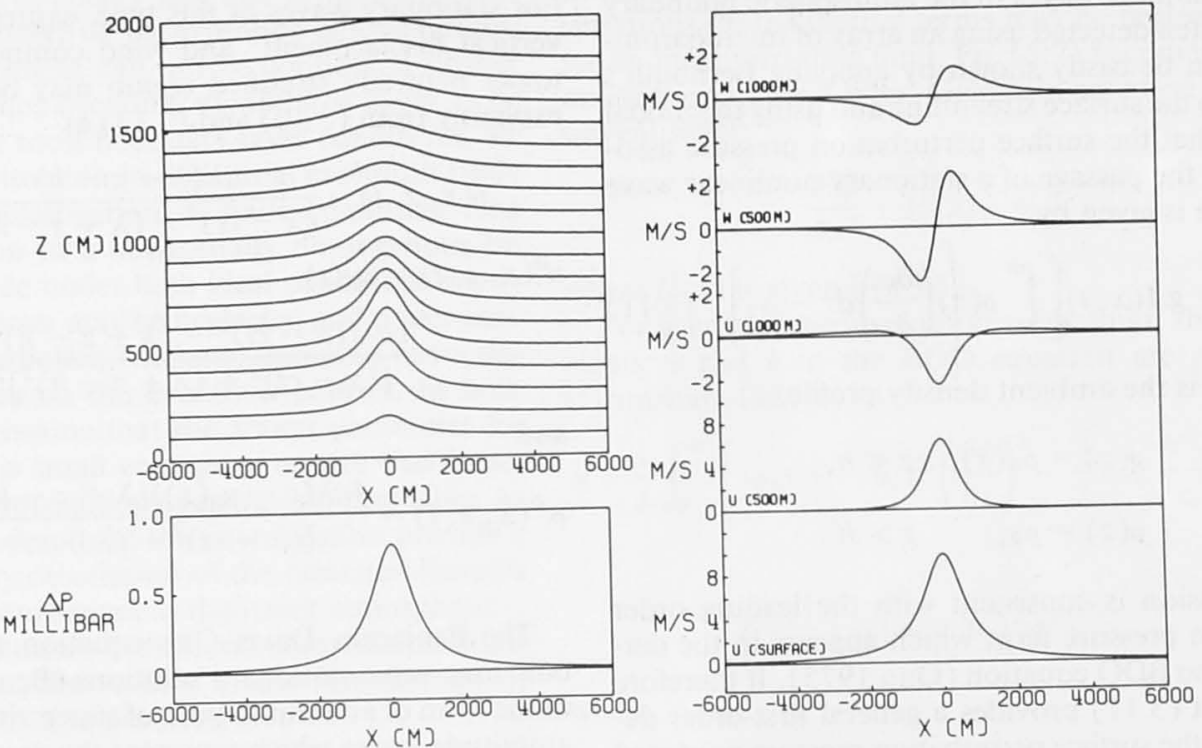

FIG 4. Inner and outer relative streamline patterns, surface perturbation pressure, and wind components FIG. 4. Inner and outer relative streamite corresponding to a solitary waver $u_{2}=0, \Delta \rho=0, h=500 \mathrm{~m}$, and constant Brunt-Väisälä period $T_{N}=4.0$ $\min$.

mode $(n=1)$ in surface-based waveguides, described by the simplest form of the model given in appendix A, with $\Delta \rho=u_{0}=0$ and constant Brunt-Väisälä frequency in the stable layer.

Equation (3.4) may be regarded as a general model equation for the description of nonlinear waves of modest amplitude in the atmospheric boundary layer. The observed properties of morning glory waves suggest that they are essentially a one-dimensional disturbance. Under some conditions, however, such as when transverse variations in waveguide structure occur, the description of these disturbances may require an exten-
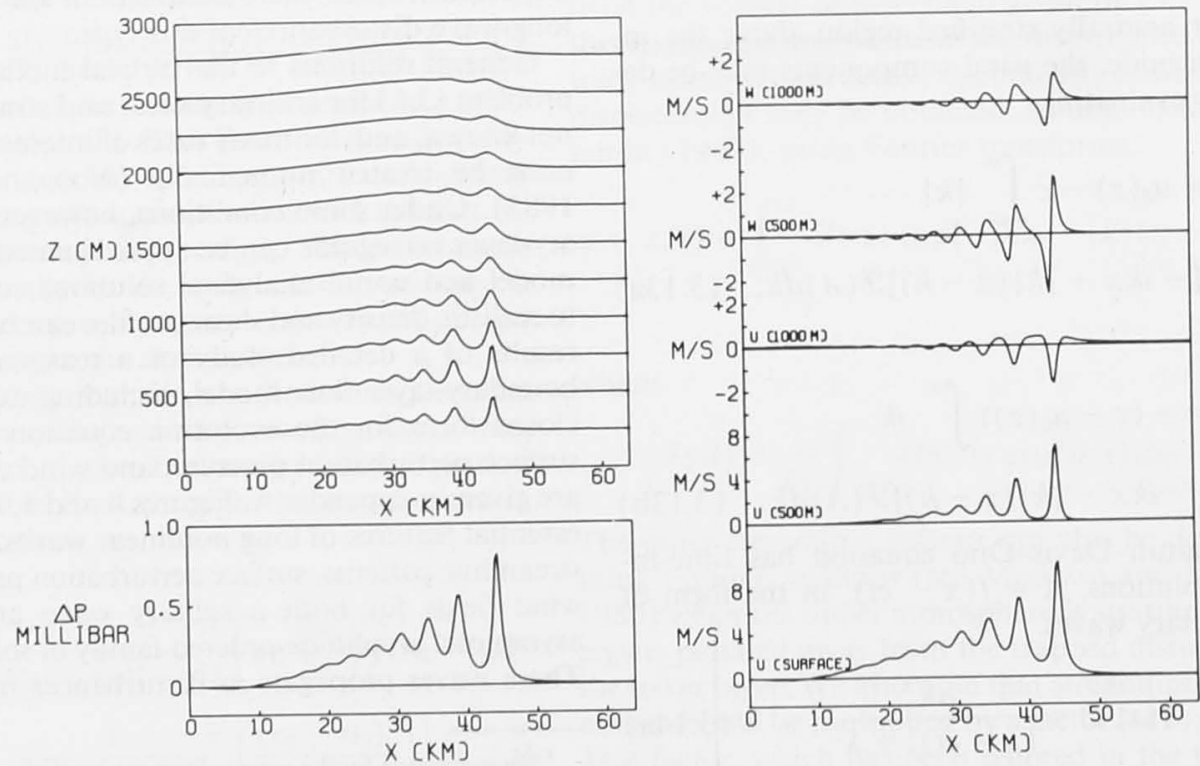

FIG 5. Relative streamline pattern, surface perturbation pressure and wind components for an amplitudeordered family of solitary waves propagating in a boundary layer waveguide (as in Fig. 4) with $n=1, u_{1}$ $=u_{2}=0, \Delta \rho=0, h=500 \mathrm{~m}$, and $T_{N}=4.1 \mathrm{~min}$. 
sion of the BDO theory to two spatial dimensions (see, e.g., Ablowitz and Segur 1980; Redekopp 1980). Furthermore, a theoretical treatment which includes the effects of the earth's rotation (Grimshaw 1985) may be required in some instances, especially at higher latitudes, when nonlinear wave disturbances are subject to the influence of a topographic boundary.

\section{Numerical solutions}

In this section we shall consider a number of models for the evolution of nonlinear atmospheric waves which are based on either the inviscid Benjamin-Davis-Ono Eq. (3.3) or the Benjamin-Davis-Ono-Burgers Eq. (3.4) (when $\hat{\alpha}=0$ ) with turbulent frictional damping. In this initial study we shall suppose that the environmental waveguide is horizontally homogeneous; i.e., the ambient density, $\rho_{0}$, and wind component, $u_{0}$, along the direction of propagation are both functions of height only and thus the coefficients $\alpha$ and $\delta$ in the evolution equation are constant. We shall, however, in some of the numerical experiments (see section 5) examine the effect of a temporally and spatially varying frictional term.

It will be convenient to study the solutions of the BDO-Burgers equation in a coordinate system which moves with the linear phase speed $c_{0}$. The evolution equation may then be rewritten in terms of nondimensional variables as

$$
\frac{\partial U}{\partial T}+U \frac{\partial U}{\partial X}+\mathscr{H}\left(\frac{\partial^{2} U}{\partial X^{2}}\right)-\epsilon \frac{\partial^{2} U}{\partial X^{2}}=0,
$$

where

$$
\begin{gathered}
X=\frac{1}{h}\left(x-c_{0} t\right), \\
T=\frac{\delta}{h^{2}} t, \\
U(X, T)=\frac{\alpha h}{\delta} A(x, t),
\end{gathered}
$$

and $\epsilon$ is related to the eddy diffusivity coefficient, $\mu$, by $\epsilon=\mu / \delta$.

The accuracy of the numerical solutions described below has been checked by comparing the theoretical and numerical values of the lower order conserved polynomial invariants. The first three conservation laws of the Benjamin-Davis-Ono equation (4.1a) (with $\epsilon$ $=0$ ) are as follows (Ono 1975):

$$
\begin{aligned}
Q_{1} & =\int_{-\infty}^{\infty} U d X, \\
Q_{2} & =\int_{-\infty}^{\infty} \frac{1}{2} U^{2} d X, \\
Q_{3} & =\int_{-\infty}^{\infty}\left\{\frac{1}{3} U^{3}+U \mathscr{H}\left(\frac{\partial U}{\partial X}\right)\right\} d X .
\end{aligned}
$$

The quantities $Q_{1}$ and $Q_{2}$ are also integral invariants of the KdV equation. The BDO-Burgers equation exhibits only one time-invariant quantity, $Q_{1}$. In all cases where these laws apply, these integral invariants were found to vary by less than $0.1 \%$ over the duration of the numerical experiment.

\section{a. Solutions of the Benjamin-Davis-Ono equation.}

We shall first consider the time evolution of nonlinear wave disturbances in a horizontally homogeneous inviscid boundary-layer waveguide as described by the unperturbed constant-coefficient Benjamin-DavisOno equation. As noted above, BDO solitary waves retain their identity following collision. Interacting BDO solitons have been studied in a series of numerical experiments by Meiss and Pereira (1978) who also showed that both solitary and dispersive waves are created in the evolution of an arbitrary initial disturbance with sech ${ }^{2}$ profile. A very detailed analysis of the interaction of BDO solitons has been given by Matsuno (1980), who shows that the nature of the interaction is governed by the ratio of initial amplitudes, $U_{1}$ and $U_{2}$, of the interactions solitons. An illustration of the interaction of solitary waves governed by the BDO equation is presented in Fig. 6 for $U_{1} / U_{2}=2.8$. In this example the solitons exchange identities at the instant of collision without passing through each other. For higher amplitude ratios $\left(U_{1} / U_{2} \geqslant 3+2 \sqrt{2}\right)$, the disturbance at the center of interaction is single crested and the solitons pass through each other.

The solitary wave solutions of the BDO equation are also stable under nonlinear interaction with large amplitude dispersive wave components. This is shown by the results of the numerical experiment presented in Fig. 7 where a solitary wave is observed to emerge unscathed (and apparently with zero phase shift) following a complex interaction with subcritical dispersive waves created in the decay of a long wave of depression.

The behavior of the general time-dependent solutions of the BDO equation is similar in many respects to that of the corresponding solutions of the classical $\mathrm{KdV}$ equation. An excellent description, based on both a theoretical and experimental investigation, of the time evolution of long nonlinear shallow-water waves governed by the KdV equation may be found in Segur (1973) and Hammack and Segur (1974). The general properties of the time-dependent solutions of the BDO equation which are most relevant to the interpretation of atmospheric wave phenomena may be seen in the numerical solutions presented in Fig. 8 for the evolution of an arbitrary initial disturbance in the form of a long wave of elevation. The salient features of the general solutions to the BDO equation may be summarized as follows:

1) Arbitrary initial disturbances of finite volume evolve asymptotically into a finite number of perma- 


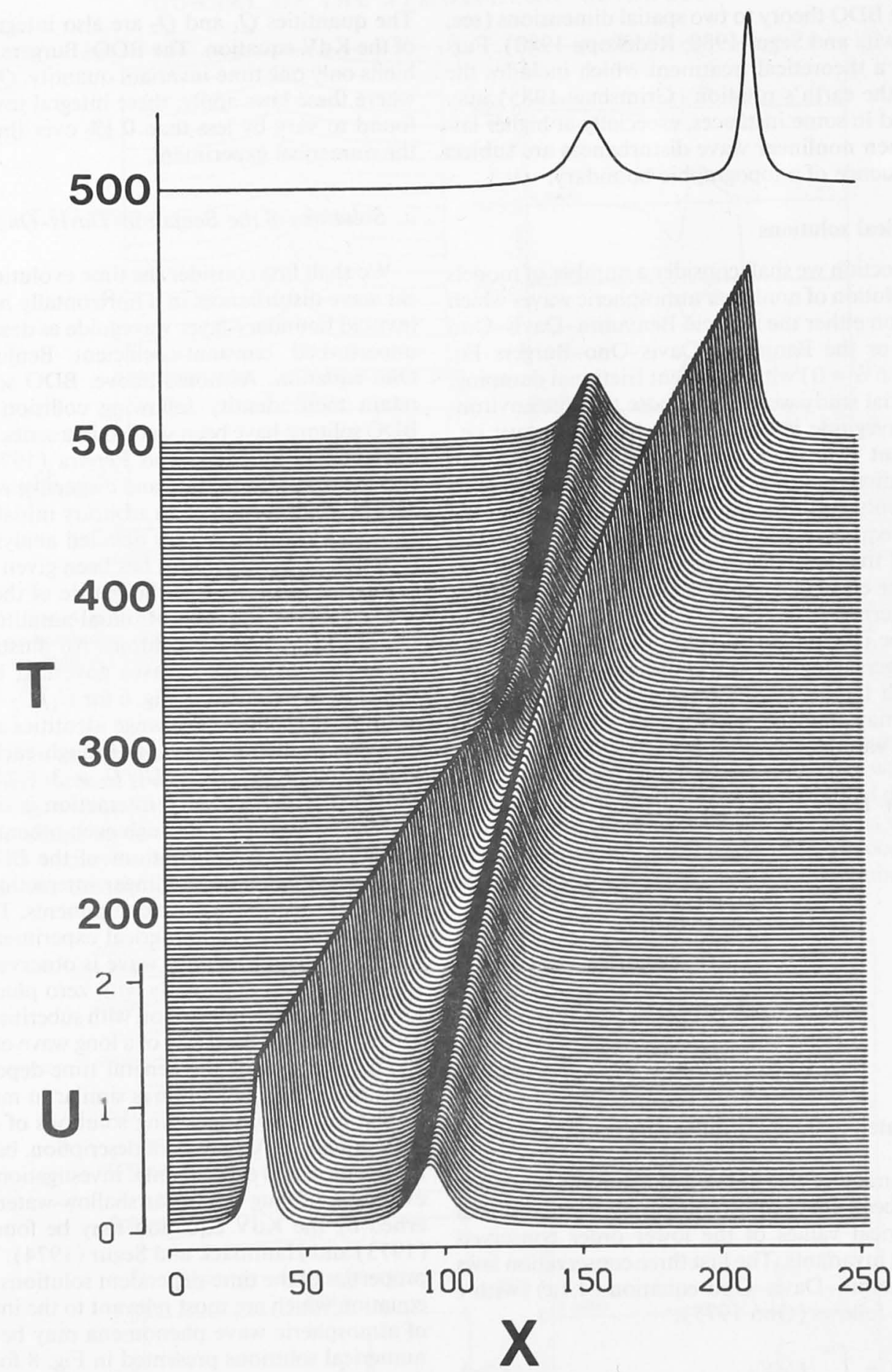

FIG. 6. Interaction of solitary waves governed by the Benjamin-Davis-Ono equation. The last trace has been reproduced separately for clarity. The coordinate system in this illustration and in all subsequent illustrations moves at the critical or linear phase speed. In this frame of reference, solitary waves propagate to the right and subcritical dispersive wave components propagate to the left. Units are nondimensional. 


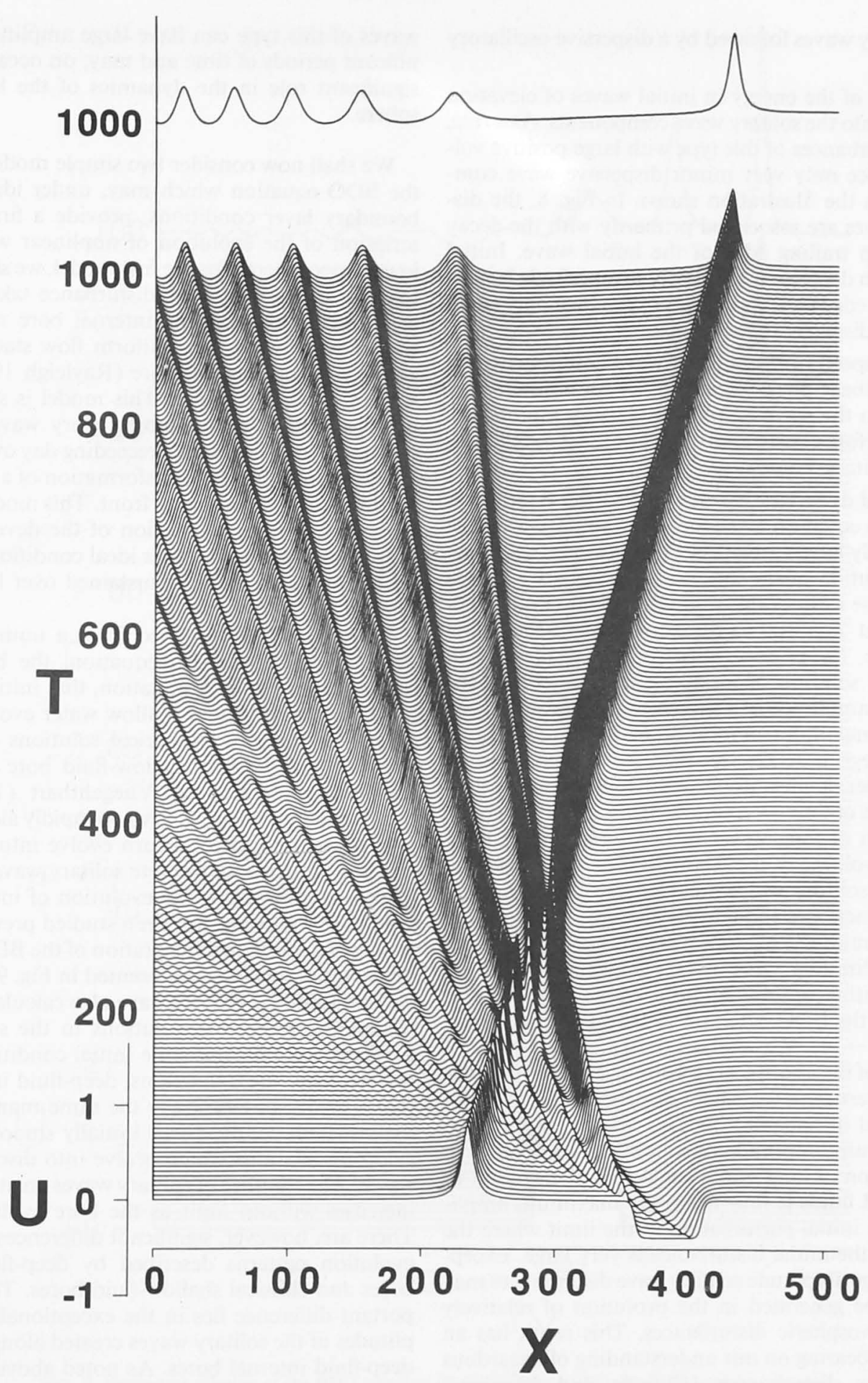

FIG. 7. Numerical integration of the Benjamin-Davis-Ono equation illustrating the stability of a solitary wave under strongly nonlinear interaction with large amplitude subcritical dispersive waves created in the evolution of an initially smooth long wave of depression. 
nent solitary waves followed by a dispersive oscillatory wave train.

2) Most of the energy in initial waves of elevation is focused into the solitary wave components. As a rule, initial disturbances of this type with large positive volume produce only very minor dispersive wave components. In the illustration shown in Fig. 8, the dispersive waves are associated primarily with the decay of the steep trailing edge of the initial wave. Initial waves which decrease more slowly in amplitude behind the leading edge (see, e.g., Fig. 11) produce only small amplitude dispersive wave trains.

3) The speed of the solitary wave components exceeds the linear phase speed, $c_{0}$, by an amount proportional to the wave amplitude. The asymptotic solution therefore consists only of a family of amplitudeordered solitary waves.

4) Initial disturbances governed by the BenjaminDavis-Ono equation can evolve into solitary waves of exceptionally large amplitude. For example, in the case of the evolution of the long-wave disturbance shown in Fig. 8, the ratio of the amplitude of the leading soliton, $U_{s}$, at $T=100$ to the amplitude of the initial disturbance, $U_{0}$, is 2.58 . This can be compared with the general solution of the $\mathrm{KdV}$ equation where the maximum amplitude of a solitary wave cannot exceed twice the maximum amplitude of the initial disturbance (Peregrine 1972; Segur 1973). We have carried out a number of integrations of the BDO equation over long periods of time for a variety of initial perturbations in the form of long waves of elevation. In the limit where the volume of the disturbance is very large, the number of solitons which emerge from the initial perturbation increases indefinitely and the value of the amplitude ratio $U_{s} / U_{0}$ appears to slowly approach, as $T \rightarrow \infty$, a limiting value of 4.0 . This ratio is the same as the limiting amplitude ratio found by Matsuno (1984) for the BDO equation in the limit of zero dispersion $(\delta \rightarrow 0)$. An analysis, based on the work of Matsuno, of the asymptotic solution of the BDO equation for internal waves where dispersion is significant is presented in appendix B. This analysis shows that the maximum amplitude of solitary waves created in the evolution of long nonlinear waves of elevation in unbounded fluids is four times the maximum amplitude of the initial perturbation in the limit where the volume of the initial disturbance is very large. Exceptionally large amplitude solitary wave disturbances may therefore be generated in the evolution of relatively benign atmospheric disturbances. This result has an important bearing on our understanding of hazardous wind shear disturbances (Christie and Muirhead 1983a,b; 1985; Doviak and Christie 1989) and is clearly of interest to the subject of nonlinear wave-induced deep convection.

5) Initial waves of depression, i.e., disturbances which are everywhere negative, decay only into dispersive wave components (see Fig. 7). Subcritical waves of this type can have large amplitudes for significant periods of time and may, on occasion, play a significant role in the dynamics of the lower atmosphere.

We shall now consider two simple models based on the BDO equation which may, under ideal uniform boundary layer conditions, provide a first-order description of the evolution of nonlinear waves in the lower troposphere. For the first model, we shall suppose that the initial long-wave disturbance takes the form of a smooth propagating internal bore marking the transition between two uniform flow states in direct analogy to the classical bore (Rayleigh 1914) on the surface of shallow water. This model is suggested by the observation that morning glory waves originate during the evening of the preceeding day over the Cape York Peninsula in the transformation of a deeply penetrating tropical sea-breeze front. This model may provide a reasonable description of the development of long nonlinear waves under ideal conditions when the generating disturbance is sustained over long periods of time.

Peregrine (1966) showed from a numerical treatment of the Boussinesq equation, the bidirectional equivalent of the $\mathrm{KdV}$ equation, that initially smooth bores on the surface of shallow water evolve into undular disturbances. Numerical solutions of the KdV equation for classical shallow-fluid bore waves have also been presented by Vliegenthart (1971), who showed that undulations develop rapidly along the edge of the bore and these in turn evolve into an ever-increasing sequence of discrete solitary waves.

To our knowledge, the evolution of internal deepfluid bore waves has not been studied previously. The results of a numerical integration of the BDO equation for waves of this type is presented in Fig. 9a. For comparison (see Fig. 9b), we have also calculated the corresponding bore-wave solutions to the shallow-fluid $\mathrm{KdV}$ equation for the same initial conditions. As can be seen from these diagrams, deep-fluid internal bore waves evolve in essentially the same manner as their shallow-fluid counterparts: initially smooth bores develop undulations which evolve into discrete solitary waves. The number of solitary waves created evidently increases without limit as the bore evolves in time. There are, however, significant differences in the early evolution patterns described by deep-fluid internal bores and classical shallow-fluid bores. The most important difference lies in the exceptionally large amplitudes of the solitary waves created along the edge of deep-fluid internal bores. As noted above, the amplitudes of solitary waves generated in the evolution of shallow-fluid KdV bores do not exceed twice the amplitude of the parent bore. In contrast, the leading soliton produced in the evolution of an initially smooth deep-fluid bore eventually attains an amplitude which is a factor of 4 larger than the amplitude, $A_{\infty}$, of the undisturbed bore. These disturbances also differ in 


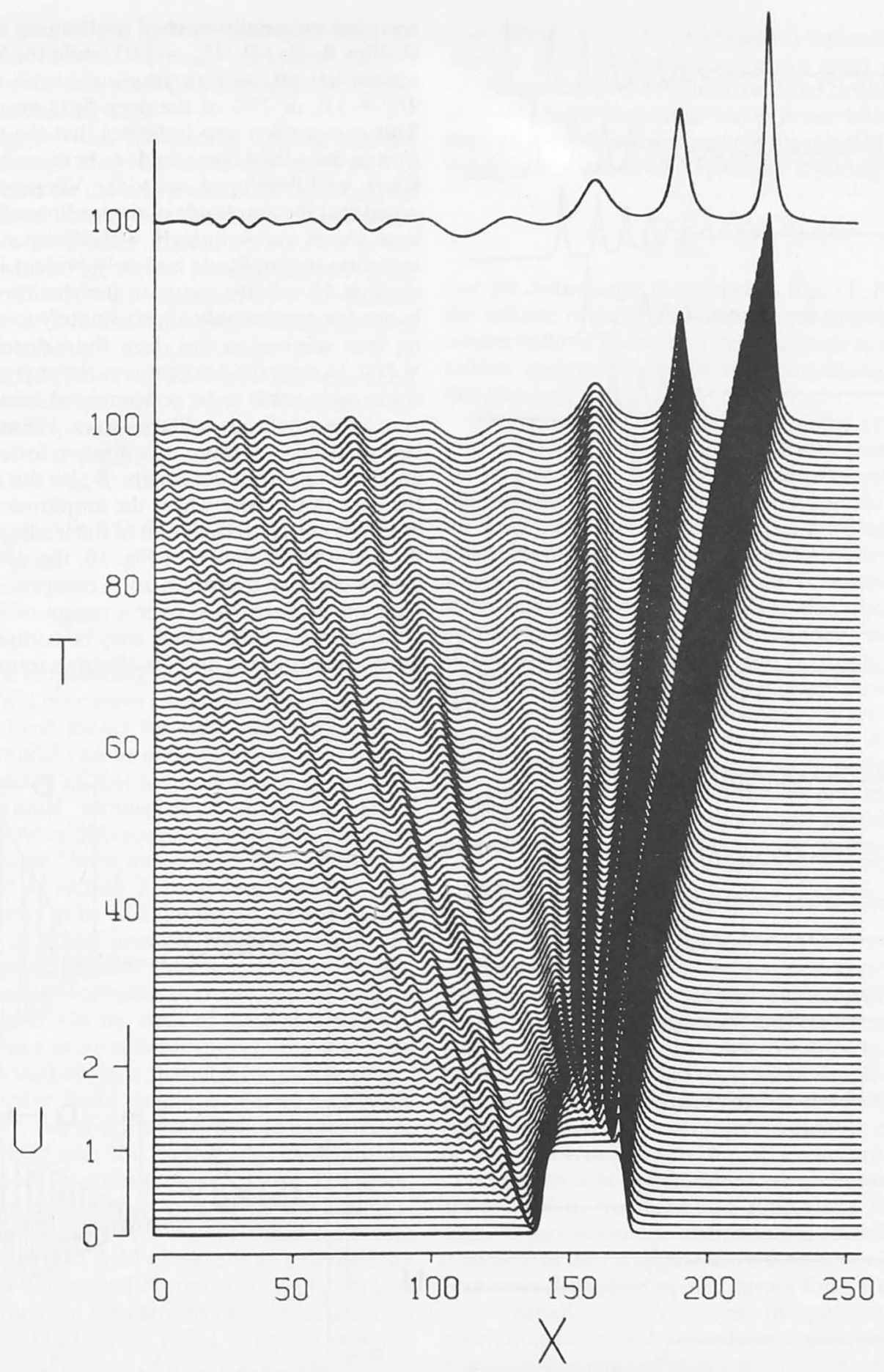

FIG. 8. Numerical solution of the BDO equation illustrating the evolution of a long square wave of elevation into a finite number of amplitude-ordered solitons followed by a subcritical dispersive wave train.

morphology. Consider, for example, a comparison of the detailed evolution patterns, as shown in Fig. 10, at a time $T=100$. Solitary waves produced along the leading edge of $\mathrm{KdV}$ bores approach the limiting amplitude ratio more rapidly than their BDO counterparts in deep fluids. In this example, the leading $\mathrm{KdV}$ solitary 
a

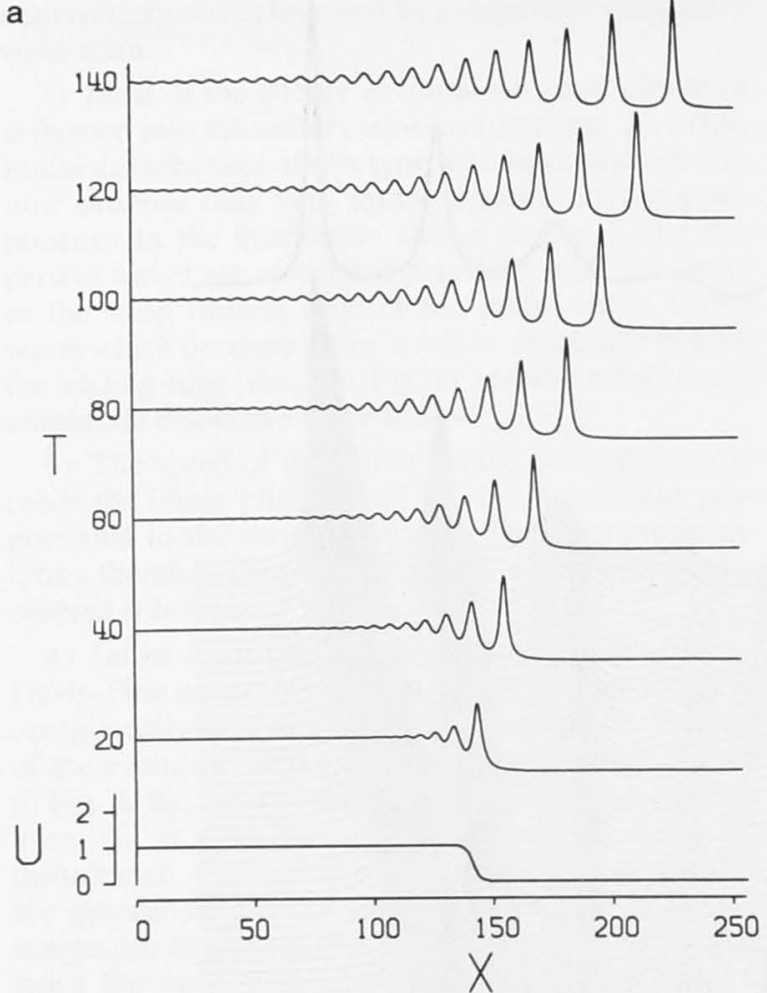

b

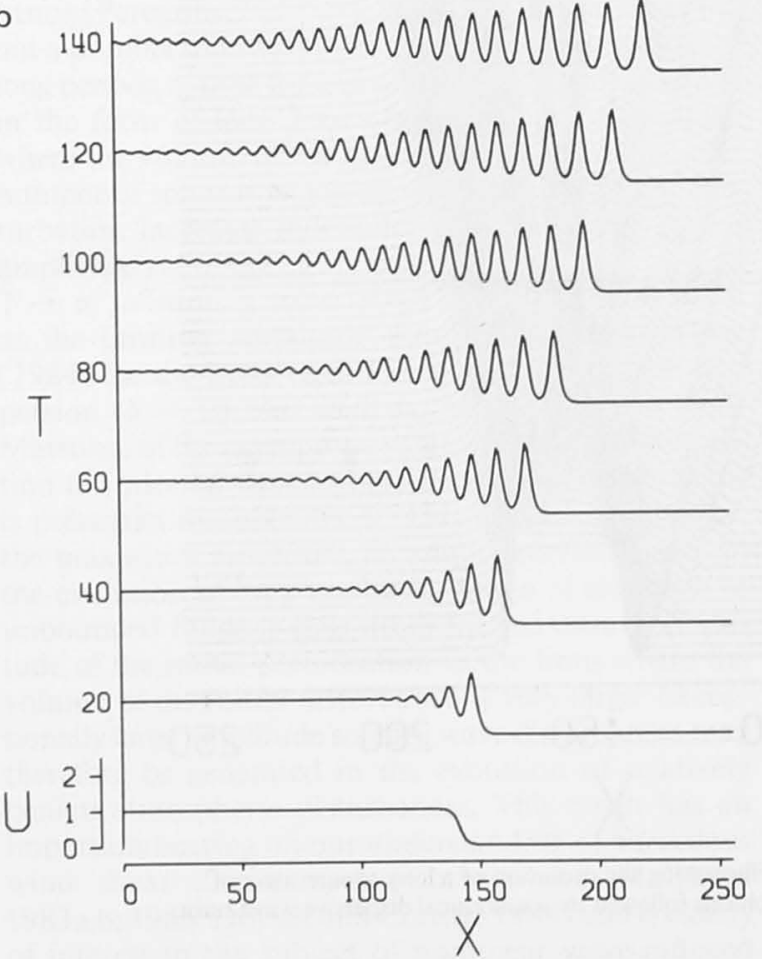

FIG. 9. (a) Numerical integration of the BDO equation for an initially smooth deep-fluid internal bore wave, (b) Corresponding solution of the shallow-fluid $\mathrm{KdV}$ equation for the same initial bore wave condition used in the calculations shown in (a). wave has essentially reached the limiting amplitude for shallow fluids ( $U_{s} / U_{\infty}=2.0$ ) while the leading BDO soliton has attained an amplitude ratio of about $U_{s} /$ $U_{\infty}=3.0$, or $75 \%$ of the deep fluid amplitude limit. This comparison also indicates that the transition region in deep-fluid bores tends to be more localized with fewer well-developed solitons. Vliegenthart (1971) noted that the amplitude of the leading solitons in $\mathrm{KdV}$ bore waves varies linearly with distance. This linear variation in amplitude is clearly evident in Fig. 10 for the first 12 solitary waves in the shallow-fluid disturbance but applies only approximately to the first three or four solitons in the deep fluid disturbance at $T$ $=100$. In deep-fluid bore waves the energy in the transition zone tends to be concentrated initially in a few very large amplitude solitary waves. These remarks can be expressed in a more quantitative form by defining an effective transition length, $D$, by the region along the edge of the bore where the amplitude of the oscillations is at least $10 \%$ of that of the leading soliton. For the disturbance shown in Fig. 10, the deep-fluid bore has an effective transition zone comprising seven solitary waves distributed over a region of length 97 , in dimensionless units, which may be compared with 14 developing solitons and an effective transition length

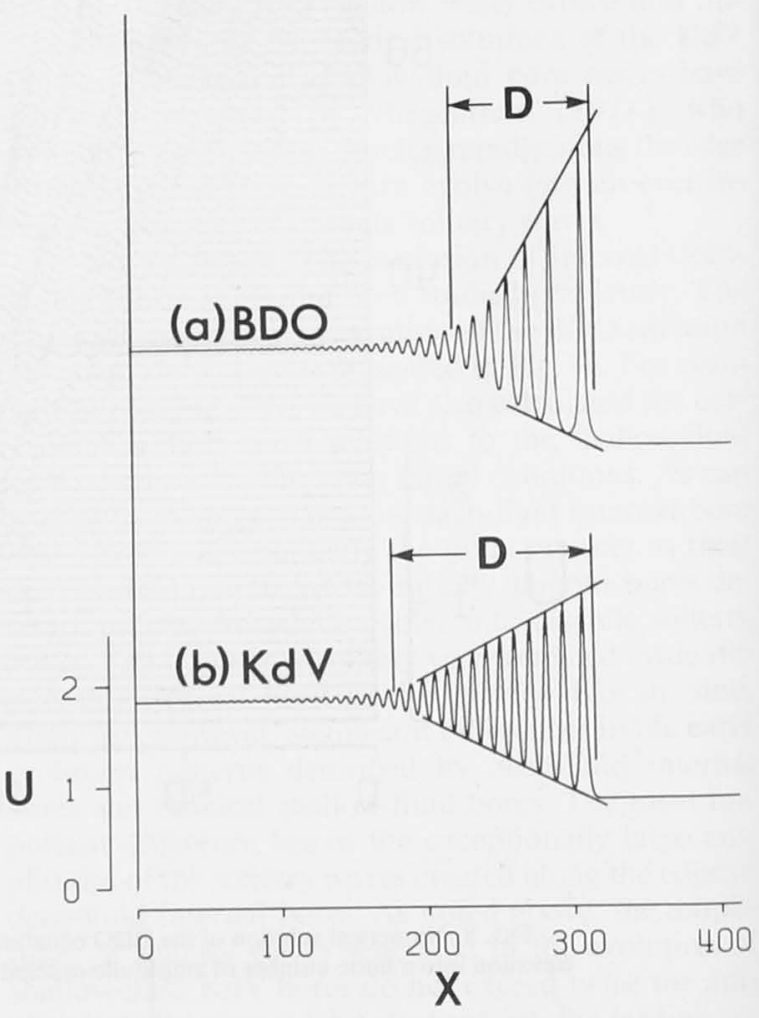

FIG. 10. Detailed comparison of (a) the structure of a deep-fluid internal bore wave with (b) the structure of a shallow-fluid bore wave corresponding to the same solutions as those shown in Fig. 9 at $T$ $=100$. 
of 137 in the case of the $\mathrm{KdV}$ shallow-fluid bore. In both cases, the distance between individual solitary waves in the transition zone decreases monotonically away from the leading edge.

The spacing between the leading solitons and the average wavelength over the transition zone will be useful parameters for the interpretation of experimental data. The scale of both of these parameters is substantially larger for deep-fluid internal bore waves. In the example shown in Fig. 10, the leading solitons in the BDO bore at $T=100$ are separated by a nondimensional distance of 21.9 and the average wavelength over the effective transition zone is estimated to be 14.0. In the case of the $\mathrm{KdV}$ bore, the leading soliton separation and average wavelength are 13.2 and 9.7, respectively.

The asymptotic propagation speed, $c_{b}$, of bore waves in inviscid fluids is simply given by the speed of the leading soliton. Thus, for BDO bore waves,

$$
c_{b}=c_{0}+\alpha A_{\infty},
$$

and for $\mathrm{KdV}$ bores,

$$
c_{b}=c_{0}+\frac{2}{3} \alpha A_{\infty} .
$$

It should be emphasized that the infinitely long internal deep-fluid bore wave considered here represents a highly idealized model for nonlinear atmospheric waves. This model can be expected to provide an accurate description of disturbances which occur only under exceptional atmospheric conditions. Some morning glory wave disturbances certainly do resemble extensive sustained bore waves of this type. However, as emphasized in section 2 , most morning glory disturbances appear to be of finite length. In most cases, the surface winds and pressure recorded behind the main disturbance indicate that the inversion height does not remain elevated but decreases slowly back to the ambient level. On the basis of these observations, we propose that a more suitable model for initial nonlinear wave disturbances is that of a long wave of elevation of effective finite length, $L$, whose amplitude decreases slowly but continuously behind a maximum amplitude located near the leading edge of the disturbance. This model is consistent with a long, but finite, unsteady wave disturbance created in the transformation during the early evening of a cutoff sea-breeze surge, as indicated in field observations described by Clarke (1965, 1983a) and Simpson et al. (1977) and in detailed numerical simulations of sea-breeze surges recently reported by Clarke (1984). A typical example of the evolution of a wave of this type as described by the BDO equation is shown in Fig. 11. In this case, the initial disturbance evolves almost entirely into a finite number of amplitude-ordered solitary waves. The dispersive wave components have very small amplitudes and play an almost insignificant role in the description of these disturbances. As the wave evolves, the overall length of the disturbance increases continuously as dis- crete solitary waves form and slowly separate from one another along the leading edge. An exact expression for the number, $N_{s}$, of solitons created in the evolution of finite length deep-fluid waves is not known. However, an approximate estimate of the number of solitary waves can be made ( see appendix B) using

$$
N_{s}=\frac{1}{4 \pi} \int_{-\infty}^{\infty} U(X, 0) d X .
$$

For the disturbance illustrated in Fig. $11, N_{s} \approx 5$. As the volume of the initial disturbance increases, $N_{s}$ increases without bound and the amplitude of the leading soliton approaches the limiting amplitude for BDO disturbances, $4 U_{0}$.

The inviscid BDO description of the evolution of long finite length nonlinear wave disturbances outlined above provides a reasonable description of many of the features of long nonlinear atmospheric wave disturbances, especially during the earlier stages of evolution involving the formation and growth of solitary waves. This relatively simple model is, however, subject to serious criticism since it fails to describe the decay and disintegration of these disturbances. Furthermore, in some cases (see Figs. 2b and 3d) only one large amplitude solitary wave is observed to develop along the leading edge of the disturbance contrary to the predictions of this relatively simple model. As we shall see, turbulent dissipation plays an important role in the evolution of these disturbances and must be included in a more realistic theoretical treatment of the properties of these guided nonlinear waves.

\section{b. Solutions of the Benjamin-Davis-Ono-Burgers equation.}

The description of the evolution of deep-fluid longwave disturbances is modified substantially when a term for turbulent dissipation is included in the governing equation. We shall first consider the effect of turbulent friction on the properties of simple deep-fluid internal bore waves as described by the BDO-Burgers evolution equation (4.1). Internal bores in either deep or shallow inviscid fluids (Fig. 9) evolve continuously in time as an ever-increasing number of solitary waves are created in the transition zone along the leading edge. When turbulent dissipation is included in the treatment of the classical shallow fluid bore wave, initial bore wave disturbances of arbitrary form are found to evolve rapidly to a stable time-independent solution which may be smooth or undular depending upon the degree of frictional dissipation (Canosa and Gazdag 1977). This classical stationary bore or "shock" wave solution to the steady state $\mathrm{KdV}$-Burgers equation has been studied in the phase plane as a model for weak shock waves in plasmas by Grad and Hu (1967), and also by Johnson $(1970,1972)$ as a model for undular bores on a shallow viscous fluid. Johnson numerically integrated the steady state $\mathrm{KdV}$-Burgers equation, and 


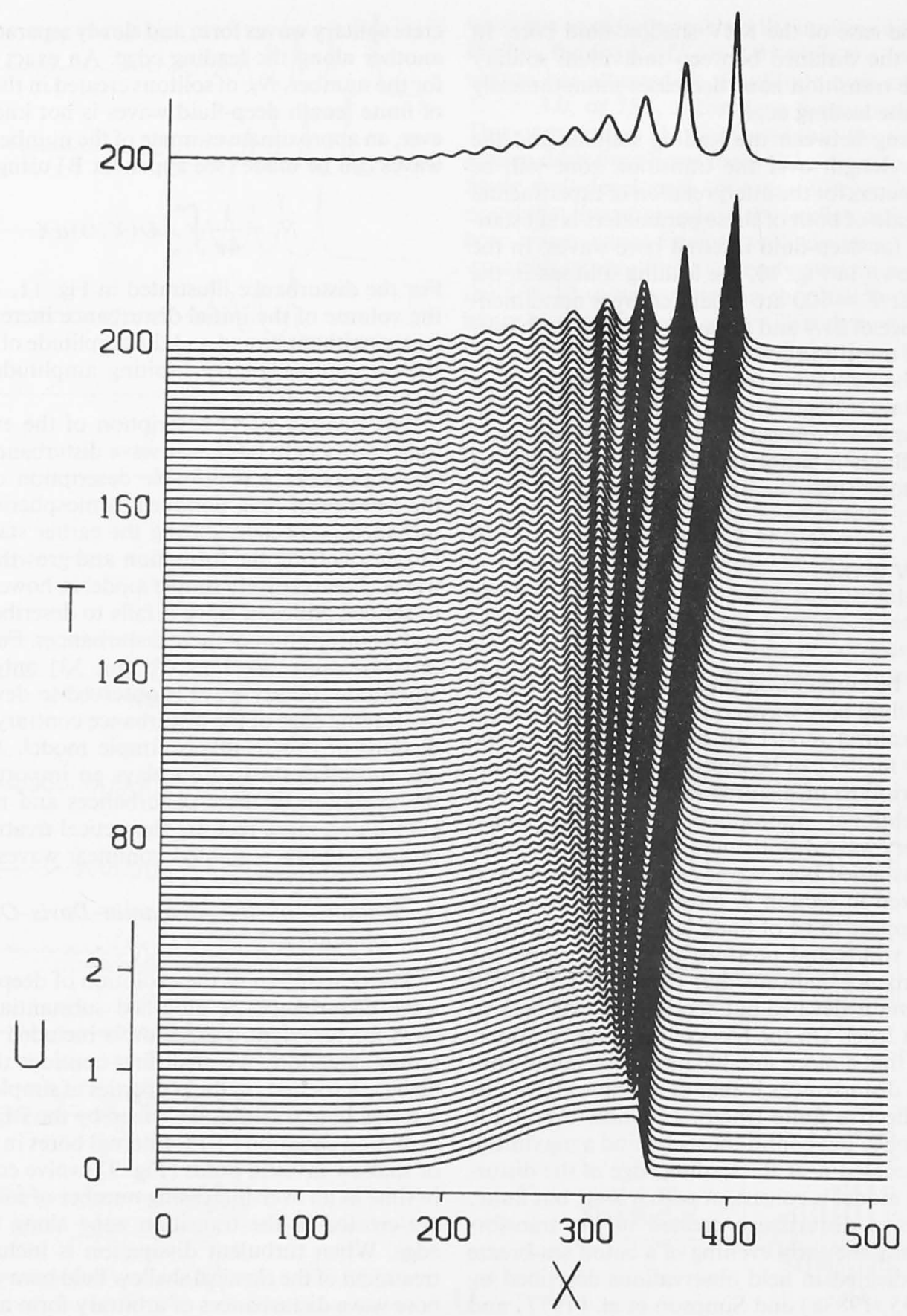

FIG. 11. Solution of the BDO equation for an initial model disturbance for atmospheric waves in the form of a long, but finite-length, wave of elevation. The inversion height behind the leading edge of the disturbance at $T=0$ decreases slowly, but continuously, to the ambient inversion level.

also derived asymptotic solutions, corresponding to either weak or strong damping, for the stationary borewave transition between uniform upstream and downstream flow states. Detailed accounts of the time-in- dependent solution of the $\mathrm{KdV}$-Burgers equation have also been given by Jeffrey and Kakutani (1972), Whitham (1974), Karpman (1975), and, as a proposed model for the morning glory, by Egger (1983, 1984).
1 JUN

Foll

tion

tion.

are d

wher

distu

domi

undu

excee

monc

neglig

Taylo

Th

$\mathrm{BDO}$.

ously.

ceptic

turbar

bound

deep-1

for ld

which

patior

depth

for su

It

deep-

equat

spond

$\mathrm{KdV}-$

BDO-

meric

functi

dition

sults

and 1.

turbul

these

wave

lution

the da

bore $y$

and th

latory

domir

mono

(4.7) i

We ha

solutid

noting 
Following Johnson (1970), the properties of the stationary bore wave solution of the $\mathrm{KdV}$-Burgers equation,

$$
\frac{\partial U}{\partial T}+U \frac{\partial U}{\partial X}+\frac{\partial^{3} U}{\partial X^{3}}-\epsilon \frac{\partial^{2} U}{\partial X^{2}}=0
$$

are determined by the value of the parameter

$$
m=2 \epsilon \sqrt{2 / U_{\infty}},
$$

where $U_{\infty}$ is the dimensionless amplitude of the undisturbed bore at $X \rightarrow-\infty$. When $m<4$, dispersion dominates and the resulting stationary bore wave is undular. If $m>4$, the influence of turbulent dissipation exceeds that of dispersion and the bore profile is monotonic. In the limit where dispersive effects are negligible, the stationary bore solution is given by the Taylor shock profile (Taylor 1910),

$$
U(X)=\frac{U_{\infty}}{2}\left[1-\tanh \left(\frac{U_{\infty} X}{4 \epsilon}\right)\right] .
$$

The corresponding stationary bore solutions for the BDO-Burgers equation have not been studied previously. As will be seen, these steady solutions are exceptionally stable, even under strongly nonlinear perturbation, provided the upstream and downstream boundary conditions are constant. These stationary deep-fluid bore waves may provide a reasonable model for long nonlinear atmospheric wave disturbances which occur under conditions where turbulent dissipation is significant and where the increased inversion depth behind the leading edge of the bore is sustained for substantial periods of time.

It can be anticipated that the stationary internal deep-fluid bore waves described by the BDO-Burgers equation are similar in many respects to the corresponding well-known solutions of the shallow-fluid $\mathrm{KdV}$-Burgers equation. The steady solutions to the BDO-Burgers equation have been determined by numerically integrating (4.1) for an initial positive step function of amplitude $U_{0}$ subject to the boundary conditions: $U(-\infty)=U_{0}, U(+\infty)=0$. Some typical results of these calculations are presented in Figs. 12a and $12 \mathrm{~b}$ for both weak $(\epsilon=0.1)$ and strong $(\epsilon=2.0)$ turbulent dissipation coefficients. It can be seen from these diagrams that the initially time-dependent borewave solution rapidly converges to the steady state solution. As in the case of $\mathrm{KdV}$-Burgers equation, when the damping coefficient is small, the stationary internal bore wave solution is dominated by dispersive effects and the solution (Fig. 9a) takes the form of an oscillatory bore. If, on the other hand, turbulent dissipation dominates over dispersion, the stationary solution is monotonic and approaches the Taylor shock profile (4.7) in the limit where dispersive effects are negligible. We have also evaluated the corresponding steady state solutions to the $\mathrm{KdV}$-Burgers equation. It is worth noting that the effects of dispersion are much stronger
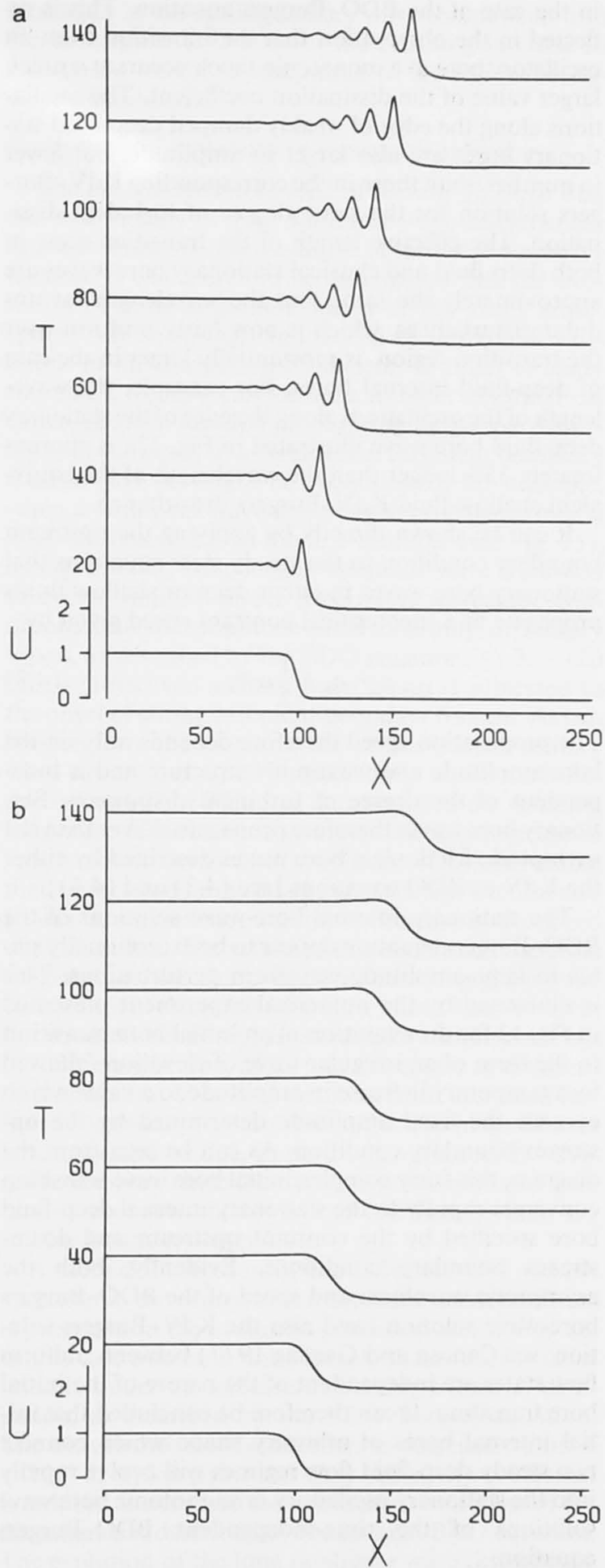

FIG. 12. Numerical integration of the BDO-Burgers equation for an initially smooth deep-fluid internal bore with (a) weak turbulent dissipation, $\epsilon=0.1$ and (b) strong turbulent dissipation, $\epsilon=2.0$. Stationary internal bore wave solutions are well established by $T$ $=80$ in both cases. 
in the case of the BDO-Burgers equation. This is reflected in the observation that the transition from an oscillatory bore to a monotonic shock occurs at a much larger value of the dissipation coefficient. The oscillations along the edge of weakly damped deep-fluid stationary bores are also larger in amplitude, but fewer in number, than those in the corresponding $\mathrm{KdV}$-Burgers solution for the same degree of turbulent dissipation. The effective length of the transition zone in both deep-fluid and classical stationary bore waves are approximately the same, but the wavelength for undular disturbances, which is now fairly uniform over the transition region, is substantially larger in the case of deep-fluid internal bores. For example, the wavelength of the oscillations along the edge of the stationary deep-fluid bore wave illustrated in Fig. 12a is approximately $35 \%$ longer than the wavelength of the equivalent shallow-fluid $\mathrm{KdV}$-Burgers disturbance.

It can be shown directly by applying the upstream boundary condition to the steady state equations that stationary bore waves in either deep or shallow fluids propagate at a supercritical constant speed given by

$$
c=c_{0}+\frac{1}{2} \alpha A_{\infty}
$$

The propagation speed therefore depends only on the bore amplitude and waveguide structure and is independent of the degree of turbulent dissipation. Stationary bore waves therefore propagate slower than the asymptotic frictionless bore waves described by either the $\mathrm{KdV}$ or BDO equations [see (4.3) and (4.4)]

The stationary internal bore-wave solutions of the BDO-Burgers equation appear to be exceptionally stable to large-amplitude waveform perturbations. This is illustrated by the numerical experiment presented in Fig. 13 for the evolution of an initial bore transition in the form of an irregular wave of elevation followed by a temporary increase in amplitude to a value which exceeds the fixed amplitude determined by the upstream boundary condition. As can be seen from the diagram, this fairly complex initial bore wave transition converges rapidly to the stationary internal deep-fluid bore specified by the constant upstream and downstream boundary conditions. Evidently, both the asymptotic waveform and speed of the BDO-Burgers bore-wave solution (and also the KdV-Burgers solution; see Canosa and Gazdag 1977) between uniform flow states are independent of the nature of the initial bore transition. It can therefore be concluded that initial internal bores of arbitrary shape which connect two steady deep-fluid flow regimes will evolve rapidly into the stationary oscillatory or monotonic bore wave solutions of the time-independent BDO-Burgers equation.

On occasion, very extensive nonlinear wave disturbances (see Fig. 2), with eight or more partially resolved solitary waves, are observed over northern Australia. Relatively smooth, quasi-stationary disturbances in the

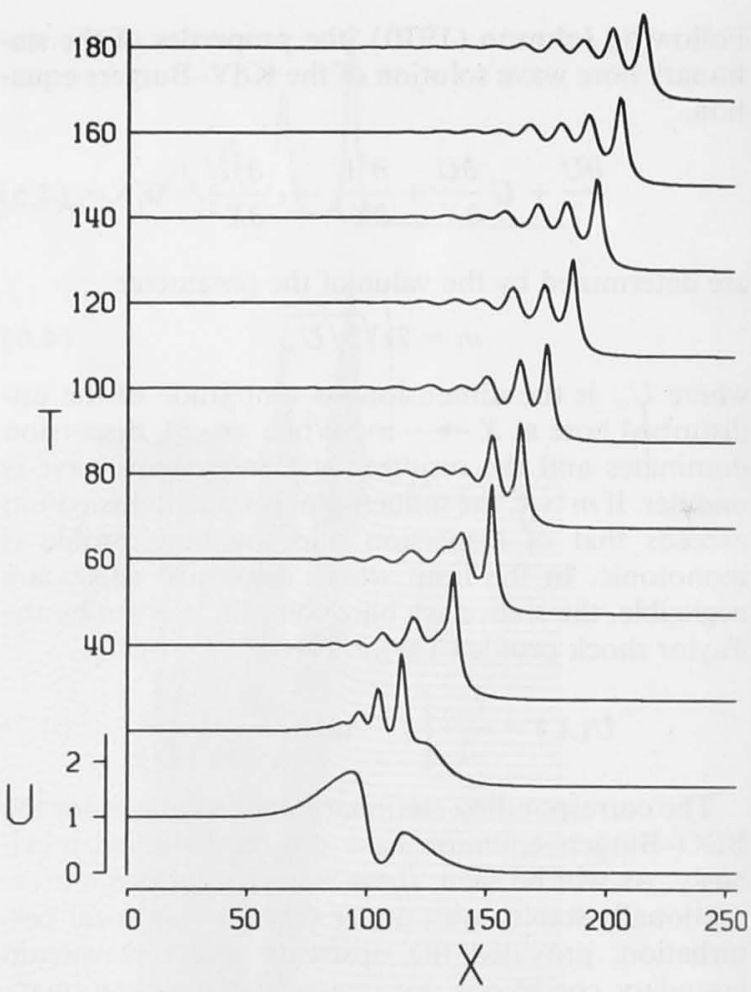

FIG. 13. Illustration of the stability of the BDO-Burgers stationary internal bore wave solution to large amplitude perturbations. This calculation shows that arbitrary transition wave forms connecting different uniform flow states converge rapidly to the unique timeindependent bore wave solution specified by the uniform boundary conditions and the degree of turbulent dissipation. In this example $\epsilon=0.1$. The stationary wave solution is established by $T=140$.

form of monotonic bore waves are also seen on occasion. Both of these types of nonlinear wave disturbances have many features in common with the class of internal deep-fluid bore waves described by the steady state BDO-Burgers equation. It has been emphasized, however, that nonlinear wave disturbances often appear to have limited spatial extent. In this case a description of the properties of these commonly occurring disturbances in terms of a stationary internal bore wave model is no longer possible. We therefore propose that a more suitable model for nonlinear wave disturbances of this type, which are subject to turbulent dissipation, is that described by the time-evolution of an initially smooth, long, but finite-length wave of elevation, as governed by the deep fluid BDO-Burgers equation.

The propagation characteristics of finite-length initial wave disturbances differ substantially from those of infinitely long internal bore wave disturbances when turbulent frictional dissipation is significant. Solitary waves may also form along the leading edge of these finite-volume disturbances and the degree to which they develop depends again on the relative influence of tur- bulen

tures gover in the the sa trast $v$ long displa For $\mathrm{m}$ along in am descri solutic From conste decays bound disper and th as it de ampli wave c length dissipa uated 1 The le turban earlier are co speed initial cous $\mathrm{f}$ ponen its earl expres: 
bulent dissipation and dispersion. The principal features in the evolution of finite-length disturbances, as governed by the BDO-Burgers equation, may be seen in the numerical experiments presented in Fig. 14 for the same initial disturbance as that in Fig. 11. In contrast with the steady solution for an idealized infinitely long internal bore wave, the solution for the vertical displacement in this case is always time dependent. For modest dissipation, solitary waves develop rapidly along the leading edge of the disturbance and increase in amplitude to a maximum which is less than that described by the corresponding stationary bore wave solution for the same degree of turbulent dissipation. From this point on, the undular wave profile has almost constant form but the amplitude of the disturbance decays continuously as energy is dissipated by turbulent boundary friction. When dissipation dominates over dispersion, the formation of solitary waves is suppressed and the initial disturbance slowly increases in length as it decays in amplitude. It is worth noting that smaller amplitude solitary waves and the oscillatory dispersive wave components which arise in the evolution of finitelength waves of elevation in the absence of frictional dissipation (e.g., see Figs. 8 and 11 ) are strongly attenuated by a relatively small degree of turbulent damping. The leading wave components in finite volume disturbances of this type propagate, at least during the earlier stages of evolution, at supercritical speeds which are comparable to, though slightly smaller than, the speed of stationary internal bore waves of the same initial amplitude. Since long waves of this type in viscous fluids produce negligible dispersive wave components, the overall length of the disturbance during its early development is determined by the approximate expression

$$
L(t) \approx L(0)+\frac{1}{2} \alpha A_{0} t
$$

\section{Nonlinear wave propagation in an inhomogeneous waveguide}

The BDO-Burgers equation with constant coefficients appears to provide a reasonably satisfactory description of many of the essential features in the evolution of long nonlinear wave disturbances in the lower atmosphere. Conditions can occur, however, where the assumption of a uniformly constant eddy diffusivity coefficient is no longer justified in the real atmosphere. In this section we shall therefore examine the influence on nonlinear wave propagation of spatial and temporal variations in the degree of turbulent frictional dissipation. Two specific problems which have a direct bearing on the interpretation of experimental observations will be addressed. In section 5 a we present the results of a numerical study of nonlinear wave propagation under conditions where the degree of turbulent dissipation varies along the propagation path. This is followed in section $5 \mathrm{~b}$ by a brief discussion of shear instability in the leading solitary wave components and the subsequent influence of the resulting increase in turbulence on the development of the residual longwave disturbance.

\section{a. Horizontal variations in waveguide structure}

The calculations presented in section $4 \mathrm{~b}$ show that the morphology of long nonlinear wave disturbances depends sensitively on the degree of turbulent boundary friction. It can therefore be anticipated that the evolution of finite-amplitude waves in the lower atmosphere will be strongly influenced by horizontal variations in the dissipation coefficient. In order to study this effect, we have carried out a number of numerical experiments in which a propagating long-wave disturbance is subjected to a variation in turbulent boundary friction such as might be encountered, for example, when a nonlinear wave disturbance propagates over a land-sea boundary. In the first example (see Fig. 15), a long, smooth, finite-length, nonlinear wave is allowed to evolve initially under frictionless waveguide conditions into an amplitude-ordered family of solitary waves, as described by the BDO equation. At $T=120$ this well-resolved solitary wave group is subjected to the onset of strong turbulent boundary friction. As can be seen from the diagram, the well-developed solitary wave structure is rapidly eroded away under the influence of turbulent frictional damping and the resulting long-wave disturbance continues to slowly increase in length as it decays away in amplitude. It is worth noting that an increase in the degree of turbulent friction also results in a significant and rapid decrease in the speed of propagation. The results of this calculation therefore suggest that the sharp bend in morning-glory-wave cloud-lines at the point where the cloud lines intersect the sea-land boundary, as described by Smith and Page (1985), may be due, in part, to a decrease in the speed of propagation over land resulting from increased turbulent boundary friction. The reduction in speed over land may also be due, in part, to a variation in waveguide structure since the stratified boundary layer over land is probably shallower and less intense than the marine boundary layer.

A second illustration of the influence of a sudden variation in the degree of turbulent frictional damping is presented in the evolution pattern shown in Fig. 16. In this case a smooth finite-length disturbance evolves initially under the influence of strong frictional dissipation, as described by the BDO-Burgers equation. The disturbance slowly decreases in amplitude until $T$ $=120$, at which point the eddy diffusivity coefficient is reduced to zero and the subsequent evolution of the disturbance is described by the inviscid BDO equation. The evolution of the long nonlinear wave disturbance proceeds in this case as expected. Prior to the sudden decrease in boundary friction, the disturbance evolves only slowly, as described in section $5 \mathrm{~b}$, and when frictional damping is removed the disturbance rapidly in- 


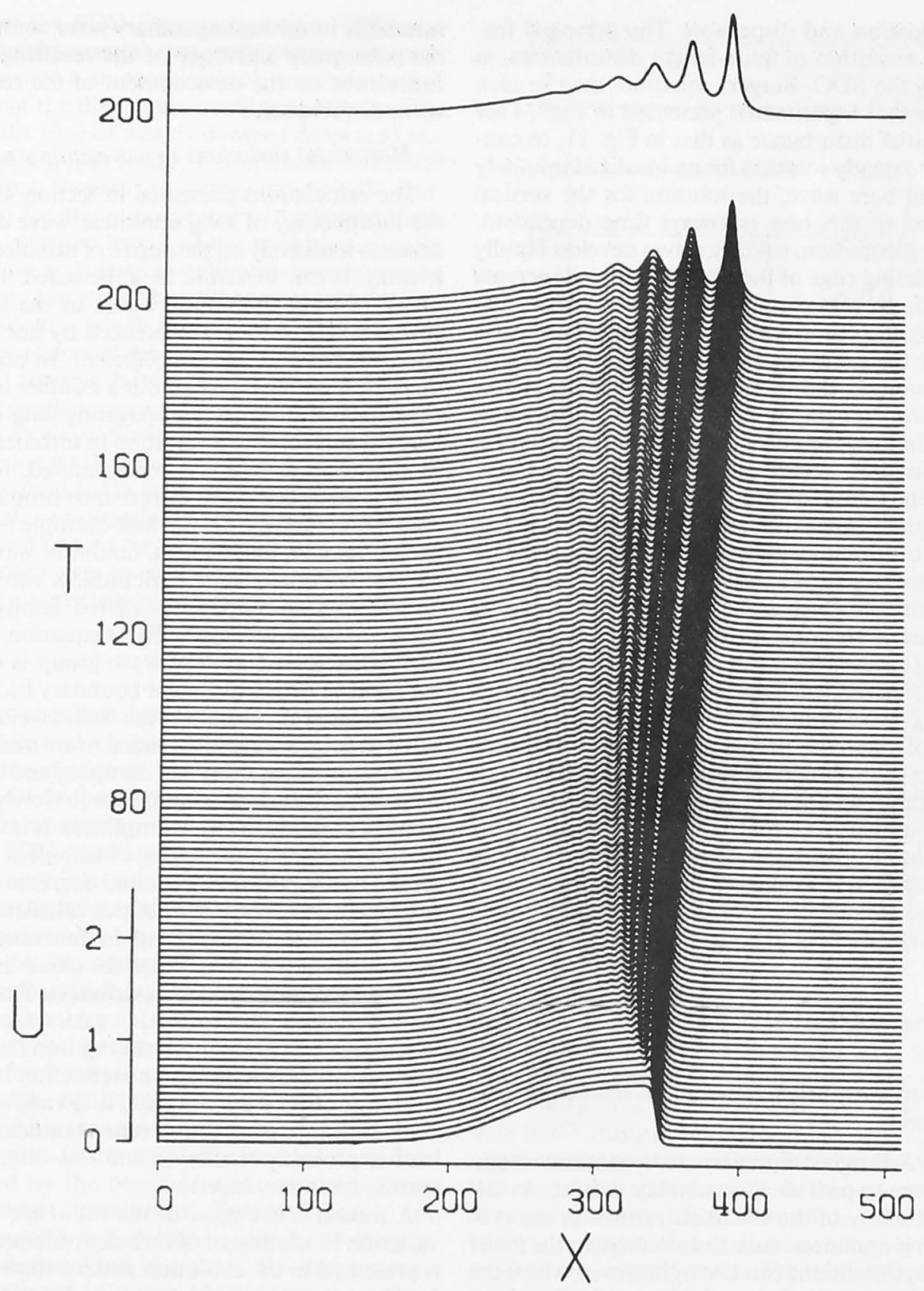

FIG. 14. Numerical integration of the BDO-Burgers equation for an initial model disturbance for finite-length atmospheric waves with $(a) \epsilon=0.05$.

creases in speed as solitary waves develop along the leading edge.

The two examples of nonlinear wave propagation in an inhomogeneous waveguide presented here serve to illustrate the principal effects which result from horizontal variations in the degree of turbulent frictional dissipation. We have also carried out a number of numerical experiments in which the eddy diffusivity coef- ficient varies continuously over the course of the experiment. These results will not be presented in detail as they are similar in most respects to those described above.

\section{b. Solitary wave-induced turbulence}

Up to this point, all of the calculations have been based on the assumption that the turbulent friction 


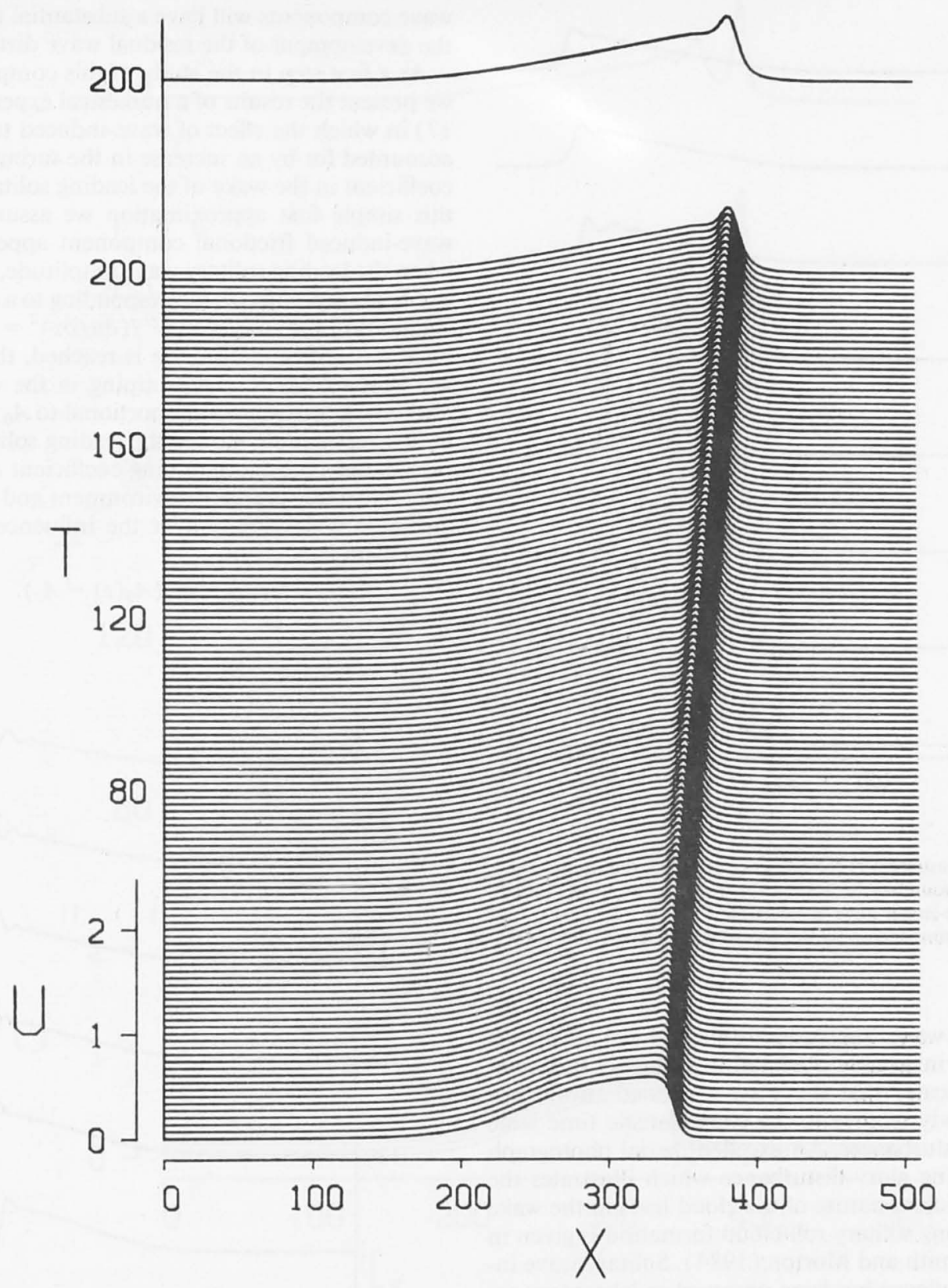

FG 14. $($ Continued $)(\mathrm{b}) \epsilon=0.5$

f the ex-

in detail lescribed

ave been t friction coefficient is independent of the nature of the nonlinear wave motions. This may prove to be a reasonable approximation when the Richardson number throughout the environmental shear flow is large and when the amplitude of the waves is small. It must, however, be expected that the degree of frictional dissipation will depend on both the amplitude and period of the wave motion and that wave-induced variations in the dissipation rate may have a significant influence on the evolution of these disturbances. A nonlinear wave-in- duced variation in the damping coefficient is clearly indicated by observations of morning glory wave phenomena and other closely related nonlinear wave disturbances. For example, the leading solitary wave roll cloud formation in visible morning glory disturbances is often observed (Christie et al. 1983b; Smith and Morton 1984) to be extremely smooth along the leading edge and highly turbulent along the trailing edge. This wave-induced turbulence is apparently the result of the onset of Kelvin-Helmholtz instability near the 


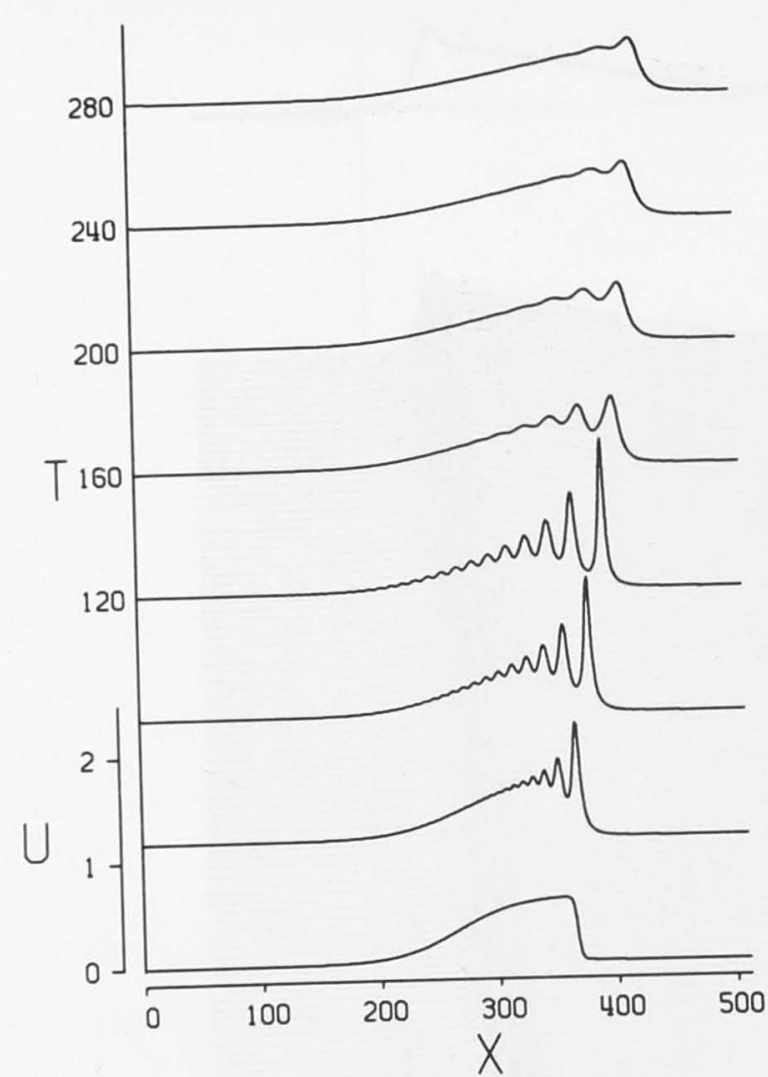

FIG 15. Illustration of the influence of an increase in the degree of turbulent boundary friction on the evolution of a long, initially smooth, finite-length wave of elevation. For $T<120, \epsilon=0$; for $T$ $>120$, the disturbance is subject to strong dissipation with $\epsilon=0.5$.

crest of the wave. Subsequent solitary-wave roll clouds are almost invariably observed to be highly turbulent which indicates that the wave-generated turbulence decays slowly relative to the characteristic time scale of these disturbances. An excellent aerial photograph of a morning glory disturbance which illustrates the highly turbulent nature of the cloud lines in the wake of the leading solitary roll-cloud formation is given in Fig. 2 of Smith and Morton (1984). Solitary wave induced turbulence has been observed in laboratory experiments [J. Simpson, personal communication (Smith and Morton 1984); also see Maxworthy (1980)] and has been directly observed in the atmosphere by Doviak and Ge (1984) using the KTVY tower instrumented by the National Severe Storms Laboratory in Norman, Oklahoma. Further evidence for wave-induced turbulence is provided by the observation of an increase in surface temperature due to mixing which almost invariably occurs in the wake of the leading solitary wave in well-developed morning glory disturbances. It must be expected that wave-induced turbulence associated with the leading solitary wave components will have a substantial influence on the development of the residual wave disturbance.

As a first step in the study of this complex process, we present the results of a numerical experiment (Fig. 17) in which the effect of wave-induced turbulence is accounted for by an increase in the turbulent friction coefficient in the wake of the leading solitary wave. In this simple first approximation we assume that the wave-induced frictional component appears initially when the leading solitary wave amplitude, $A_{0}$, reaches a critical amplitude, $A_{c}$, corresponding to a critical local Richardson number, $J=N^{2} /(\partial u / \partial z)^{2}=1 / 4$, and that once this critical amplitude is reached, the degree of wave-induced frictional damping in the wake of the leading solitary wave is proportional to $A_{0}-A_{c}$. Thus, in this very simple model, the leading solitary wave is subject to a constant damping coefficient $\epsilon=\epsilon_{0}$, characteristic of the ambient environment and the residual disturbance develops under the influence of a timedependent coefficient,

$$
\epsilon(t)=\epsilon_{0}+\epsilon_{1}\left(A_{0}(t)-A_{c}\right) .
$$

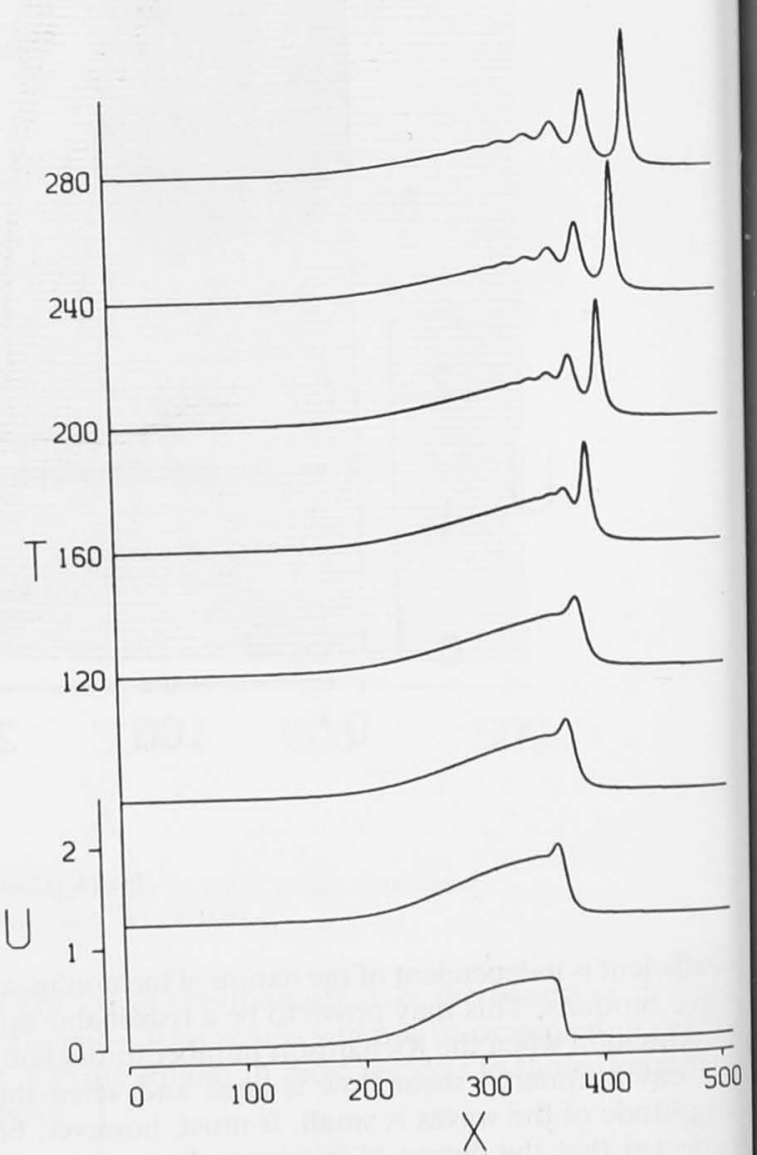

FIG 16. Numerical integration of the BDO-Burgers equation itlustrating the effect of a decrease in turbulent dissipation on the evolustrating the effect of a decrease in turbulen, long wave of elevation lution of an initially smooth, $\epsilon=0.5$ for $t<120$. For $T>120, \epsilon=0$.
The res show tl of the 1 pletely residua 
luence on bance.

x process, ment (Fig. bulence is at friction wave. In that the s initially 0 , reaches itical local , and that degree of ake of the $A_{c}$. Thus, ry wave is $\epsilon_{0}$, chare residual of a time-

(5.1)

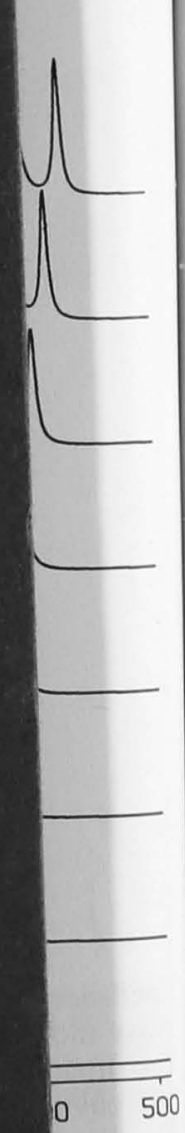

equation il on the evo of elevation

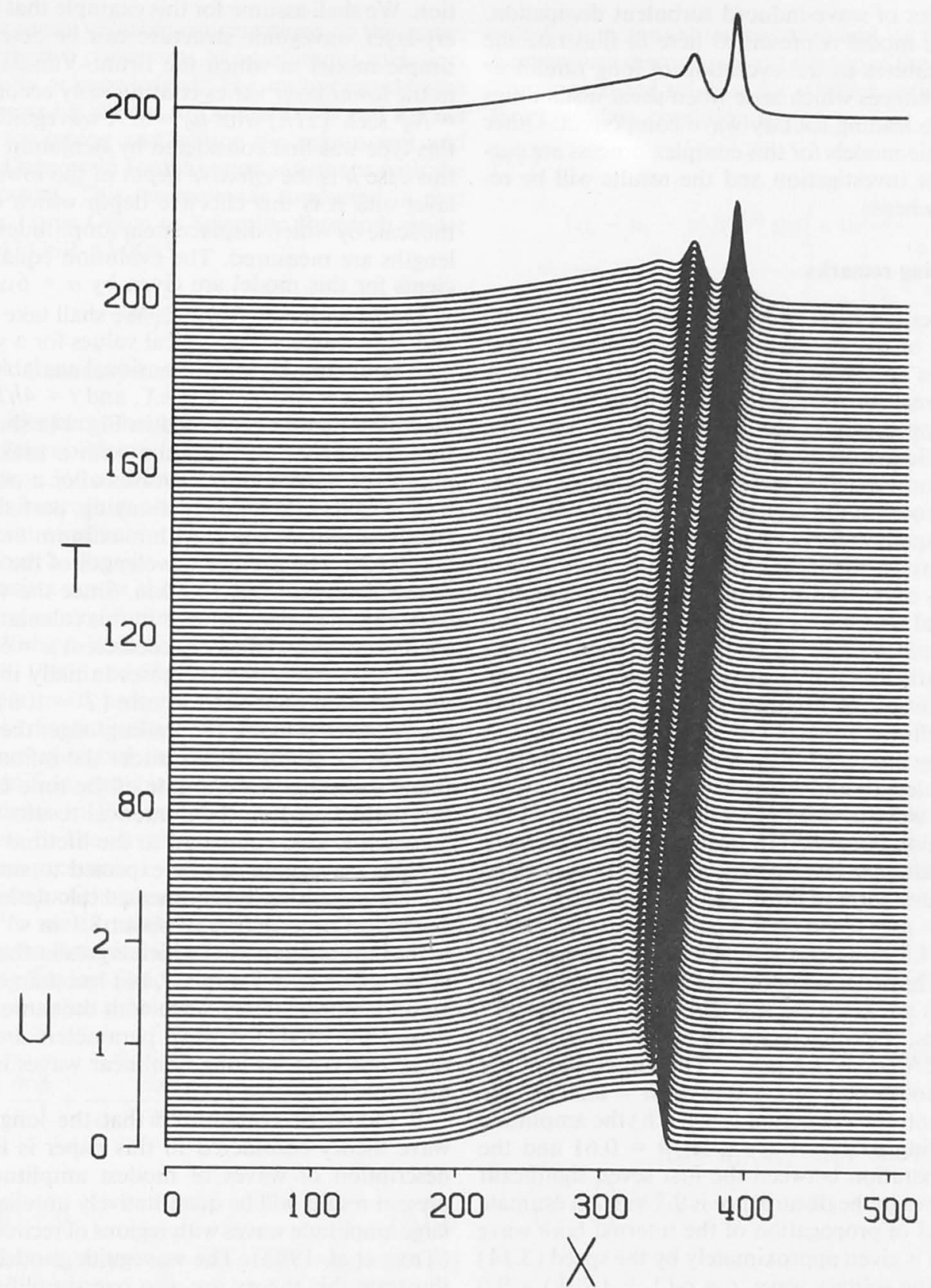

FIG. 17. Model calculation illustrating the influence of wave-induced turbulent dissipation on the evolution of long-wave disturbances in the lower atmosphere. In this example the turbulent dissipation coefficient in the BDO-Burgers equation is given by the spatially and temporally varying coefficient specified by $(6.1)$ with $\epsilon_{0}=0.05, \epsilon_{1}=0.5$ and a critical amplitude, $A_{c}$, for the onset of wave-induced turbulence which is $20 \%$ larger than the maximum amplitude of the initial disturbance.

The results of this calculation, as illustrated in Fig. 17, show that the turbulence field produced in the wake of the leading solitary wave can limit or inhibit completely the development of further solitary waves in the residual disturbance. This model therefore provides a possible explanation for observations of nonlinear wave phenomena (Figs. 2b and 3d) in which only one large amplitude solitary wave develops along the leading edge of the parent disturbance. It should be emphasized, however, that this model considerably oversimplifies 
the dynamics of wave-induced turbulent dissipation. This simple model is presented here to illustrate the principal features in the evolution of long nonlinear wave disturbances which arise when shear instabilities occur in the leading solitary wave component. Other more realistic models for this complex process are currently under investigation and the results will be reported elsewhere.

\section{Concluding remarks}

This paper has been concerned with the theoretical description of the evolution of long nonlinear wave disturbances in the atmospheric boundary layer. A number of realistic models for these disturbances have been developed within the framework of either the deep-fluid Benjamin-Davis-Ono evolution equation for ideal homogeneous waveguide conditions where frictional processes are negligible, or the BDO-Burgers evolution equation for conditions where turbulent dissipation plays a significant role. The evolution of a wide variety of initial disturbances, including infinitely long internal bore waves and long waves of finite volume has been studied in considerable detail. These models clearly explain the essential features in the observed evolution of morning glory waves and other similar nonlinear atmospheric wave phenomena.

As an illustration of these results, consider the predicted wavelength and speed of propagation of an internal bore wave as determined by the solution of the BDO equation illustrated in Fig. 9a. We shall suppose that the boundary layer waveguide can be described by the simplest form of the model outlined in appendix A with $\Delta \rho=u_{0}=0$ and typical parameters, $N=0.0233$ $\mathrm{s}^{-1}\left(T_{N}=4.5 \mathrm{~min}\right)$ and $h=500 \mathrm{~m}$. In this case, the amplitude, horizontal coordinate and time in dimensional units are given by $A=2 h U / \pi^{2}, x=h X$ and $t$ $=\pi^{2} h T / 4 c_{0}$, respectively, for the lowest mode $(n=1)$ with $c_{0}=2 \mathrm{Nh} / \pi=7.4 \mathrm{~m} \mathrm{~s}^{-1}$. The amplitude of the initially smooth bore at $t=0$ is $A_{0} / h=0.20$. At the end of the integration period ( $t=6.4 \mathrm{~h}$ ) the amplitude of the leading solitary wave is $A_{s} / h=0.61$ and the average separation between the first seven significant solitary waves in the disturbance is $9.2 \mathrm{~km}$. An estimate of the speed of propagation of the internal bore wave at $t=6.4 \mathrm{~h}$ is given approximately by the speed (3.14) of the leading solitary wave, $c=c_{0}\left(1+A_{s} / 2 h\right)=9.6$ $\mathrm{m} \mathrm{s}^{-1}$. All of these wave parameters are in the typical range of those observed for nonlinear waves in the atmospheric boundary layer.

It was assumed in the above example that the long nonlinear wave disturbance evolved under ideal waveguide conditions where the influence of frictional dissipation could be ignored. As a second more realistic illustration, consider the time evolution of a finitelength initial disturbance (Fig. 14a) under boundarylayer conditions where turbulent damping is significant and the governing equation is the BDO-Burgers equa- tion. We shall assume for this example that the boundary-layer waveguide structure can be described by a simple model in which the Brunt-Väisälä frequency in the lower layer varies continuously according to $N^{2}$ $=N_{0}^{2} \operatorname{sech}^{2}(z / h)$ with $u_{0}=0$. A waveguide model of this type was first considered by Benjamin (1967). In this case $h$ is the effective depth of the lower stratified layer and it is this effective depth which determines the scale by which displacement amplitudes and wavelengths are measured. The evolution equation coefficients for this model are given by $\alpha=6 c_{0} / 5 h$ and $\delta$ $=3 h c_{0} / 4$ with $c_{0}=N_{0} h / \sqrt{2}$. We shall take $h=400 \mathrm{~m}$ and $N_{0}=0.026 \mathrm{~s}^{-1}$ as typical values for a stably stratified boundary layer. Dimensional variables are now given by $A=5 h U / 8, x=h X$, and $t=4 h T / 3 c_{0}$. The evolution pattern presented in Fig. 14a shows that an initially smooth perturbation with a maximum am plitude of $A_{0} / h=0.375$ evolves after a period of 4.0 $\mathrm{h}(T=200)$ into a slowly decaying, partially resolved family of solitary waves with maximum amplitude $A$ / $h=0.625$. The average wavelength of the oscillations in this disturbance is $10.7 \mathrm{~km}$. Since the value of the damping coefficient, $\epsilon$, used in this calculation is 0.05 , we find an eddy diffusivity coefficient $\mu=\delta \epsilon=110 \mathrm{~m}^{2}$ $\mathrm{s}^{-1}$. The perturbation increases initially in amplitude over a period of about $130 \mathrm{~min}(T=108)$ as solitary waves develop along the leading edge; thereafter, the disturbance slowly decays under the influence of frictional damping. An estimate of the time constant for this decay based on the numerical results is about 14 $\mathrm{h}$, which is long compared to the lifetime over which a stable waveguide can be expected to survive in the lower atmosphere. The numerical calculations also give a speed of propagation of about $8.1 \mathrm{~m} \mathrm{~s}^{-1}$ at the end of the integration period which is greater than the linear phase speed, $c_{0}=7.4 \mathrm{~m} \mathrm{~s}^{-1}$, but less than the speed of an undamped solitary wave with the same amplitude. Again, the predicted wave parameters are typical of those observed for long nonlinear waves in the lower atmosphere.

It should be emphasized that the long, nonlinear wave theory considered in this paper is limited to a description of waves of modest amplitude and the present results will be quantitatively unreliable for very large amplitude waves with regions of recirculating fluid (Tung et al. 1982). The waveguide models chosen to illustrate this theory are also oversimplified in many respects. A more complete description of the evolution of these disturbances should include a detailed analysis of the influence of spatial and temporal variations in the waveguide density and shear structure.

Acknowledgments. It is a pleasure to acknowledge many stimulating discussions with Professors A. L. Hales and K. Lambeck, and Dr. K. J. Muirhead during the course of this work. The author is especially grateful to Dr. R. H. Clarke and Dr. R. K. Smith for their critical reviews of the manuscript, and to Dr. R. J. 
Doviak for many valuable conversations on this topic. The constructive comments of anonymous reviewers are gratefully acknowledged. The author would also like to express his thanks to Denise Devir and Rachel Colombo for typing and editing the manuscript and to Robert Dabrowski for his expert assistance with the data processing. This research was supported in part by the Air Force Office of Scientific Research under Contract AFOSR-83-0045.

\section{APPENDIX A}

\section{Boundary-Layer Flow Model}

We consider here a boundary-layer waveguide model in which a layer of depth $h$, with constant Brunt-Väisälä frequency, $N_{1}$, and linear wind profile, $u_{0}(z)=u_{1}$ $+u_{2}^{\prime} z$, lies beneath a neutrally stable layer $(N=0)$ with constant wind component, $u_{\infty}$. We shall suppose, as well, that a density discontinuity, $\Delta \rho=\rho_{1}(h)$ $-\rho_{2}(h)$, exists across the interface $z=h$. Solutions for this flow configuration when shear is present but with $\Delta \rho=0$ have been found by Maslowe and Redekopp $(1979,1980)$ and Clarke et al. (1981). We extend these results to include the influence of a potential temperature discontinuity at the interface between the lower shear layer and the upper neutrally stratified layer. In addition, explicit expressions are obtained for the coefficients in the evolution equation, the wind components in the inversion layer, and the surface perturbation pressure.

The solution of the eigenvalue problem for this configuration is most readily achieved by treating the problem in the Boussinesq approximation and solving the resulting Taylor-Goldstein equation (3.8). In this case the eigenvalue problem for $\bar{\varphi}=\left(u_{0}(z)-c_{0}\right) \varphi$ is given by

$$
\frac{\partial^{2} \bar{\varphi}}{\partial z^{2}}+\frac{J u_{2}^{\prime 2}}{\left(c_{0}-u_{1}-u_{2}^{\prime} z\right)^{2}} \bar{\varphi}=0
$$

to a d the r very $\mathrm{g}$ fluid sen to many lution alysis ons in

vledge A. L. during rateful their R. J.

and the dynamic boundary condition at the interface (continuity of pressure, cf., Benjamin 1966)

$$
\frac{\partial \bar{\varphi}(h)}{\partial z}=\frac{\partial u_{0}(h)}{\partial z}+\frac{K}{u_{0}(h)-c_{0}}
$$

where $J=N_{1}{ }^{2} / u_{2}{ }^{2}$ is the Richardson number for the inversion layer and

$$
K=\frac{g\left(\rho_{1}(h)-\rho_{2}(h)\right)}{\rho_{1}(h)} \approx \frac{g \Delta \theta}{\theta},
$$

with $\theta$ the potential temperature and $\Delta \theta$ the potential temperature discontinuity at the top of the stable layer. The normalized solution for $\varphi$ which satisfies the surface boundary condition is

$\varphi(z)$

$$
=\frac{\left|c_{0}-u_{1}-u_{2}^{\prime} h\right|^{1 / 2} \sin \left(\sigma \ln \left|\frac{c_{0}-u_{1}-u_{2}^{\prime} z}{c_{0}-u_{1}}\right|\right)}{\left|c_{0}-u_{1}-u_{2}^{\prime} z\right|^{1 / 2} \sin \left(\sigma \ln \left|\frac{c_{0}-u_{1}-u_{2}^{\prime} h}{c_{0}-u_{1}}\right|\right)},
$$

where $\sigma=(J-1 / 4)^{1 / 2}$. It will be assumed that $\sigma$ is real and thus the ambient stratified shear flow is linearly stable. Substitution of the solution for $\varphi(z)$ into (A1d) yields the eigenvalue condition

$$
c_{0}=u_{1}+\frac{u_{2}^{\prime} h}{1-e^{k}}
$$

with

$$
\begin{array}{r}
\kappa=\frac{1}{\sigma} \tan ^{-1}\left[2 \sigma /\left(1-\frac{2 K}{u_{2}^{\prime}\left(c_{0}-u_{1}-u_{2}^{\prime} h\right)}\right)\right]-\frac{n \pi}{\sigma} \\
n=0, \pm 1, \pm 2, \cdots
\end{array}
$$

The coefficients appearing in the BDO equation can now be evaluated from the formulae given in (3.9). After a lengthy, but straightforward computation, we find, for $\Delta \theta / \theta \ll 1$,

$$
\begin{aligned}
& \alpha=\frac{2 J u_{2} \sin \xi\left\{\left|c_{0}-u_{1}\right|^{3 / 2} J^{1 / 2} S+\left|c_{0}-u_{1}-u_{2}^{\prime} h\right|^{3 / 2}\right\}}{(2+J)\left|c_{0}-u_{1}\right|^{1 / 2}} \\
& \times\left[\sigma\left\{\left|c_{0}-u_{1}\right|(1-R)-\left|c_{0}-u_{1}-u_{2}^{\prime} h\right|\right\}\right]^{-1}
\end{aligned}
$$

and

$$
\delta=\frac{\left|c_{0}-u_{1}\right|\left(u_{\infty}-c_{0}\right)^{2} \sin ^{2} \xi}{\left(J-\frac{1}{4}\right) u_{2}^{\prime}\left\{\left|c_{0}-u_{1}\right|(1-R)-\left|c_{0}-u_{1}-u_{2}^{\prime} h\right|\right\}}
$$

where

$$
\begin{aligned}
R & =\frac{2 K^{2} \sin ^{2} \xi}{\left(J-\frac{1}{4}\right) u_{2}^{\prime 2}\left(c_{0}-u_{1}-u_{2}^{\prime} h\right)^{2}(2 \sigma \cot \xi-1)}, \\
S= & \frac{K \sin \xi}{u_{2}\left(c_{0}-u_{1}-u_{2}^{\prime} h\right) \sigma J^{1 / 2}}[1-J \\
& \left.\times \frac{(2+J) K^{2} \sin ^{2} \xi+2 u_{2}^{\prime 2}\left(c_{0}-u_{1}-u_{2}^{\prime} h\right)^{2} \sigma^{2} J}{u_{2}^{2}\left(c_{0}-u_{1}-u_{2}^{\prime} h\right)^{2}(2 \sigma \cot \xi-1) \sigma^{2} J}\right],
\end{aligned}
$$


and

$$
\xi=\sigma \ln \left|\frac{c_{0}-u_{1}-u_{2}^{\prime} h}{c_{0}-u_{1}}\right|
$$

Explicit expressions for the wind components in the shear layer as described by this fairly general waveguide model can also be obtained. The substitution of (A2) into (3.12) gives

$$
u^{i}(x, z, t)=\frac{\left(u_{1}+u_{2}^{\prime} z\right)|r-z|^{3 / 2} \sin \xi+c A(x, t) \operatorname{sgn}(z-r)|r-h|^{1 / 2} J^{1 / 2} \sin \chi(z)}{|r-z|^{3 / 2} \sin \xi}
$$

and

$$
w^{i}(x, z, t)=\frac{\left(u_{1}+u_{2}^{\prime} z-c\right) \frac{\partial A(x, t)}{\partial x} \sin \left(\sigma \ln \left|\frac{r-z}{r}\right|\right)|r-h|^{1 / 2}}{|r-z|^{1 / 2} \sin \xi},
$$

where

$$
r=\left(c_{0}-u_{1}\right) / u_{2}^{\prime},
$$

and

$$
\chi(z)=-\sigma \ln \left|\frac{r-z}{r}\right|+\tan ^{-1}(2 \sigma) .
$$

Since $N_{1}{ }^{2} h / g \ll 1$, we shall approximate the density distribution in the lower layer by $\rho(z)=\rho_{s}\left(1-N_{1}^{2} z\right.$ / $g)$. Equation (3.11) can then be evaluated to give the surface perturbation pressure,

$$
\begin{aligned}
& \Delta P(x, t)=g A(x, t)\left[\rho_{s}+\frac{\rho_{s} N_{1}^{2} r \sigma}{g J^{1 / 2}}\left\{G \left[F+\left|\frac{r-h}{r}\right|\right.\right.\right. \\
& \left.\left.\left.\times\left(\frac{1}{\sigma}-F\right)\right]-\frac{1}{\sigma}\left|\frac{r-h}{r}\right|^{1 / 2} \frac{\sin \chi(0)}{\sin \xi}\right\}-\rho_{2}\right], \quad(\mathrm{A} 8)
\end{aligned}
$$

with

$$
F=\frac{4 J}{2 \sigma(1-2 \sigma \cot \xi)}
$$

and

$$
G=\frac{K}{N_{1}\left(u_{1}+u_{2}^{\prime} h-c_{0}\right)} .
$$

If we now take the limit of the above results as $u_{2}^{\prime} \rightarrow 0$ we obtain the evolution equation coefficients, waveguide wind components and surface perturbation pressure for a flow model with a capping inversion above a bottom layer with constant Brunt-Väisälä frequency and constant ambient winds. Thus, the solution of the eigenvalue problem for this simplified boundarylayer flow model is given by

$$
\varphi(z)=\frac{\sin [\zeta z]}{\sin [\zeta h]}
$$

with the eigenvalue condition

$$
\begin{aligned}
\zeta h & =\tan ^{-1}\left[\frac{\left(c_{0}-u_{1}\right) N_{1}}{K}\right]+n \pi, \\
n & =0, \pm 1, \pm 2, \cdots,
\end{aligned}
$$

where $\zeta=N_{1} /\left(c_{0}-u_{1}\right)$. In the limit $u_{2}^{\prime} \rightarrow 0$, expressions (A4) to (A8) for the BDO coefficients, wind components, and perturbation pressure reduce to

$$
\begin{gathered}
\alpha=\frac{2 N_{1}\left\{3-\sin ^{2}[\zeta h]\right\}}{2 \zeta h+\sin [2 \zeta h]}, \\
\delta=\frac{2\left(u_{\infty}-c_{0}\right)^{2} \sin ^{2}[\zeta h]}{N_{1}\{2 \zeta h+\sin [2 \zeta h]\}}, \\
u^{i}(x, z, t)=\frac{u_{1} \sin [\zeta h]+c A(x, t) \zeta \cos [\zeta z]}{\sin [\zeta h]}, \\
w^{i}(x, z, t)=\frac{\left(u_{1}-c\right)[\partial A(x, t) / \partial x] \sin [\zeta z]}{\sin [\zeta h]},
\end{gathered}
$$

and

$$
\Delta P(x, t)=\frac{N_{1} \rho_{s} A(x, t)\left(c_{0}-u_{1}\right)}{\sin [\zeta h]} .
$$

Finally, if we also let $\Delta \rho \rightarrow 0$ in the eigenvalue condition (A10) we find the solution for the simplest form of this waveguide model corresponding to a flow configuration with constant Brunt-Väisälä frequency in the lower layer and constant winds. In this case,

$$
\begin{gathered}
\varphi(z)=(-1)^{n+1} \sin \left(\frac{2 n-1}{2 h} \pi z\right), \quad n=1,2,3 \cdots, \\
c_{0}=u_{1} \pm \frac{N_{1} h}{\left(n-\frac{1}{2}\right) \pi},
\end{gathered}
$$

and the coefficients in the BDO equation are

$$
\alpha=\frac{2\left(c_{0}-u_{1}\right)}{h},
$$

and

$$
\delta=\frac{4 h\left(c_{0}-u_{\infty}\right)^{2}}{[(2 n-1) \pi]^{2}\left(c_{0}-u_{1}\right)} .
$$

The wavegu turbatic $u^{i}(x, z$

BDO equ in the lir the num of a finit without $t$ solitary four time turbance is applica sion is si analysis $\mathrm{t}$ solution tial interr embedde dispersior properties

The nu have shov disturban ergy is for sume whe dispersive totic solut of individ served int and the su can then variant for $n$th conse

we have $\int_{-\infty}^{\infty}$ 
The substitution of (A16) into the formulae for the waveguide wind components (3.12) and surface perturbation pressure (3.11) leads immediately to

$$
\begin{aligned}
& u^{i}(x, z, t)=u_{1}+(-1)^{n+1} \\
& \quad \times \frac{c A(x, t)(2 n-1) \pi}{2 h} \cos \left(\frac{(2 n-1) \pi z}{2 h}\right), \\
& w^{i}(x, z, t)=(-1)^{n+1} \\
& \quad \times\left(u_{1}-c\right) \sin \left(\frac{(2 n-1) \pi z}{2 h}\right) \frac{\partial A(x, t)}{\partial x},
\end{aligned}
$$

(A7)

0 , expresnts, wind ice to

(A11)

(A12) quency in case,

$2,3 \cdots$

(A16)

(A17)

$$
\Delta P(x, t)=(-1)^{n+1} \frac{2 h A(x, t) \rho_{s} N_{1}^{2}}{(2 n-1) \pi} .
$$

\section{APPENDIX B}

\section{Asymptotic Solution of the Benjamin- Davis-Ono Equation}

A detailed analysis of the asymptotic solution of the BDO equation (3.3) has been given by Matsuno (1984) in the limit of zero dispersion $(\delta \rightarrow 0)$. In this limit, the number of solitary waves created in the evolution of a finite volume initial wave of elevation increases without bound and Matsuno shows that the maximum solitary wave amplitude in the asymptotic solution is four times the maximum amplitude of the initial disturbance. It is not immediately obvious that this result is applicable to deep-fluid internal waves when dispersion is significant. Here, we examine, using a similar analysis to that presented by Matsuno, the asymptotic solution of the BDO equation corresponding to an initial internal wave perturbation of large volume on an embedded waveguide in an unbounded fluid where the dispersion coefficient, $\delta(\neq 0)$, is determined by the properties of the ambient shear flow.

The numerical experiments described in section $4 \mathrm{a}$ have shown that when the volume, $V_{0}$, of the initial disturbance is large and positive, almost all of the energy is focused into solitary waves. Thus, we shall assume when $V_{0}$ is large the energy carried away by the dispersive components can be ignored and the asymptotic solution comprises only an amplitude-ordered set of individual solitary waves. Each solitary wave conserved integral invariant can be evaluated separately and the sum over all solitons in the asymptotic solution can then be equated with the conserved integral invariant for the initial parent disturbance. Denoting the $n$th conserved invariant by

$$
I_{n}[A(x, t)]=\int_{-\infty}^{\infty} W_{n}[A(x, t)] d x,
$$

we have

$$
\int_{-\infty}^{\infty} W_{n}[A(x, 0)] d x=\sum_{j=1}^{N_{s}} I_{n}\left[A_{s}^{j}(x, t)\right],
$$

where $A(x, 0)$ is the initial perturbation and $A_{s}{ }^{j}(x, t)$ is the $j$ th solitary wave (3.14) with amplitude $a_{j}$ in the asymptotic solution. Expressions for the integral invariants of the BDO equation are given in Meiss and Pereira (1978) and Nakamura (1979) (up to $n=5$ ) and in Case (1979) (to $n=6$ ). The first three conserved invariants are listed in (4.2). Substituting the general expression (Case 1979) for the $n$th conserved invariant corresponding to the solitary wave solution of the dimensional BDO equation (3.3),

$$
I_{n}\left[A_{s}(x, t)\right]=\frac{4 \pi \delta}{\alpha}\left(\frac{a}{4}\right)^{n-1}
$$

into (B2) gives

$$
\int_{-\infty}^{\infty} W_{n}[A(x, 0)] d x=\frac{4 \pi \delta}{\alpha} \sum_{j=1}^{N_{s}}\left(\frac{a_{j}}{4}\right)^{n-1} .
$$

The total number of solitary waves, $N_{s}$, created in the evolution of an initial perturbation is now given by (B4) when $n=1$ as

$$
N_{s}=\frac{\alpha}{4 \pi \delta} \int_{-\infty}^{\infty} A(x, 0) d x,
$$

and the amplitudes, $a_{j}$, of the solitary waves are determined by the solution of the set of equations defined by (B4) for $2 \leqslant n \leqslant N_{s}+1$ (Matsuno 1984).

We now consider the form of the asymptotic solution in the limit where the volume of the initial perturbation is very large, i.e., $V_{0} \rightarrow \infty$ and the number of solitary waves which are created (B5) increases without bound. Thus, following Matsuno (1984), a number density function, $F(a)$, for solitary waves in the asymptotic solution can be defined where $F(a) d a$ gives the number of solitons, $d N_{s}$, with amplitudes in the interval $a$ and $a+d a$. The set of equations (B4) can then be approximated by

$$
\int_{-\infty}^{\infty} W_{n}[A(x, 0)] d x=\frac{4 \pi \delta}{\alpha} \int_{0}^{\infty}\left(\frac{a}{4}\right)^{n-1} F(a) d a .
$$

In order to proceed, we note that $W_{n}[A(x, t)]$ in the general expression (B1) for the $n$th conserved invariant can be written as the sum of a leading term $[A(x, t)]^{n}$ / $n$ and a complex polynomial expression $R_{n}$ (Nakamura 1979). Matsuno (1984) has shown that in the limit of zero dispersion ( $\delta \rightarrow 0$ ), contributions to the integral invariant from $R_{n}$ vanish. We have evaluated numerically the first four nontrivial $(2<n \leqslant 6)$ conserved invariants of the BDO equation for dispersive $(\delta \neq 0)$ nonlinear waves. In each case, it is found that the relative contribution of the $R_{n}$ terms to the integral invariant is negligibly small in the limit of large positive perturbation volume. It seems very likely that this result holds in general. Accordingly, in the limit $V_{0} \rightarrow \infty$, we ignore contributions from $R_{n}$ and write (B6) as a set of integral equations 


$$
\frac{1}{n} \int_{-\infty}^{\infty}[A(x, 0)]^{n} d x=\frac{4 \pi \delta}{\alpha} \int_{0}^{\infty}\left(\frac{a}{4}\right)^{n-1} F(a) d a,
$$$$
n=1,2,3, \cdots \text {. }
$$

The solution of (B7) has been found by Matsuno (1984) to be

$$
F(a)=\frac{\alpha}{16 \pi \delta} \int_{4 A(x, 0)>a} d x,
$$

where the integration interval is subject to the condition $a<4 A(x, 0)$. As a simple example, consider an initial perturbation in the form of a wave of elevation of length $L_{0}$ and constant amplitude $A_{0}$. In this case, the solution for the number density function is given by

$$
\begin{aligned}
& F(a)=\frac{\alpha L_{0}}{16 \pi \delta}, \quad a<4 A_{0}, \\
& F(a)=0, \quad a \geqslant 4 A_{0},
\end{aligned}
$$

and the total number of solitary waves that emerge from the initial perturbation is $N_{s}=\alpha A_{0} L_{0} / 4 \pi \delta$. The maximum amplitude of solitary waves in the asymptotic solution in the limit $V_{0} \rightarrow \infty$ is therefore four times the maximum amplitude of the initial disturbance, in agreement with the limiting amplitude ratio indicated by the numerical experiments described in section $4 \mathrm{a}$ when the number of solitons is large.

\section{REFERENCES}

Ablowitz, M. J., and H. Segur, 1980: Long internal waves in fluids of great depth. Stud. Appl. Math., 62, 249-262.

Benjamin, T. B., 1966: Internal waves of finite amplitude and permanent form. J. Fluid Mech., 25, 241-270.

1967: Internal waves of permanent form in fluids of great depth J. Fluid Mech., 29, 559-592.

Benney, D. J., 1966: Long nonlinear waves in fluid flows. J. Math Phys. 45, 52-63.

Canosa, J., and J. Gazdag, 1977: The Korteweg-de Vries-Burgers equation. J. Comput. Phys., 23, 393-403.

Case, K. M., 1978: The $N$-soliton solution of the Benjamin-Ono equation. Proc. Nat. Acad. Sci. U.S.A., 75, 3562-3563.

Chen, H. H., Y. C. Lee and N. R. Pereira, 1979: Algebraic internal wave solitons and the integrable Calogero-Moser-Sutherland $\mathrm{N}$ body problem. Phys. Fluids, 22, 187-188.

Christie, D. R., and K. J. Muirhead, 1981: Observations of solitary atmospheric waves over Northern Australia. Physics for Australia's Development. Second Applied Physics Conf., Australian Institute of Physics, Melbourne, 463-466.

, and - 1983a: Solitary waves: A hazard to aircraft operating at low altitudes. Aust. Meteor. Mag., 31, 97-109.

- and $-1983 \mathrm{~b}$ : Solitary waves: A low-level wind shear hazard to aviation. Int. J. Aviat. Safety, 1, 169-190.

, and -1985 : Solitary waves and low-altitude wind shear in Australia. Aviat. Safety Dig., 123, 3-9.

and A. L. Hales, 1978: On solitary waves in the atmosphere. J. Atmos. Sci., 35, 805-825.

- 1 - 1979: Intrusive density flows in the lower troposphere: A source of atmospheric solitons. J. Geophys. Res. 84, 4959-4970.

and R. H Clarke, 1981. Solitary waves in the lower atmosphere. Nature, 293, 46-49.

Clarke, R. H., 1965: Horizontal mesoscale vortices in the atmosphere. Aust. Meteor. Mag., 50, 1-25. 1983a: Fair weather nocturnal inland wind surges and atmospheric bores: Part I: Nocturnal wind surges. Aust. Meteor. Mag. 31, 133-145.

, 1983b: Fair weather nocturnal inland wind surges and atmospheric bores: Part II: Internal atmospheric bores in northern Australia. Aust. Meteor. Mag., 31, 147-160. (Corrigenda, 32, 53.)

1984: Colliding seabreezes and atmospheric bores: Two-dimensional numerical studies. Aust. Meteor. Mag., 32, 207-226. 1986: Several atmospheric bores and a cold front over southern Australia. Aust. Meteor. Mag., 34, 65-76.

_ R. K. Smith and D. G. Reid, 1981: The morning glory of the Gulf of Carpentaria: An atmospheric undular bore. Mon. Wea. Rev., 109, 1726-1750.

Crook, N. A., 1986: The effect of ambient stratification and moisture on the motion of atmospheric undular bores. J. Atmos. Sci. 43, 171-181

1988: Trapping of low-level internal gravity waves. J. Atmos. Sci. 45, 1533-1541.

and M. J. Miller, 1985: A numerical and analytical study of atmospheric undular bores. Quart. J. Roy. Meteor. Soc., 111, 225-242.

Davis, R. E., and A. Acrivos, 1967: Solitary internal waves in deep water. J. Fluid Mech., 29, 593-607.

Doviak, R. J., and R. Ge, 1984: An atmospheric solitary gust observed with a Doppler radar, a tall tower and a surface network. $J$ Atmos. Sci., 41, 2559-2573.

, and D. R. Christie, 1989: Thunderstorm generated solitary waves: A wind shear hazard. J. of Aircraft., in press.

Drake, V. A. 1984: A solitary wave disturbance of the marine boundary layer over Spencer Gulf revealed by radar observations of migrating insects. Aust. Meteor. Mag., 32, 131-135.

1985: Solitary wave disturbances of the nocturnal boundary layer revealed by radar observations of migrating insects. Bound. Layer Meteor., 31, 269-286.

Egger, J., 1983: The morning glory: A nonlinear wave phenomenon. Mesoscale Meteorology - Theories, Observations and Models, D. K. Lilly and T. Gal-Chen, Eds., Reidel, 339-353. 1984: On the theory of the morning glory. Beitr. Phys. Atmos., 57, 123-134.

Goncharov, V. P., and A. K. Matveyev, 1982: Observations of nonlinear waves on an atmospheric inversion. Izv. Acad. Sci. USSR Atmos. Oceanic Phys., Engl. Transl., 18, 61-64.

Grad, H., and P. N. Hu, 1967: Unified shock profile in a plasma. Phys. Fluids, 10, 2596-2602.

Grimshaw, R., 1980/81: Solitary waves in a compressible fluid. $\mathrm{Pa}$ geoph., 119, 780-797.

, 1981a: Evolution equations for long, nonlinear internal waves in stratified shear flows. Stud. Appl. Math., 65, 159-188.

198 lb: Slowly varying solitary waves in deep fluids. Proc. Roy. Soc. London, 376, A 319-332.

1982: Solitary waves in density stratified fluids. Nonlinear Deformation Waves, U. Nigul and J. Engelbrecht, Eds., IUTAM Symposium, Tallinn, Springer, 431-447.

1985: Evolution equations for weakly nonlinear, long internal waves in a rotating fluid. Stud. Appl. Math. 73, 1-33.

Haase, S. P., and R. K. Smith, 1984: Morning glory wave clouds in Oklahoma: A case study. Mon. Wea. Rev., 112, 2078-2089.

Hammack, J. L., and H. Segur, 1974: The Korteweg-de Vries equation and water waves. Part 2: Comparison with experiments. J. Fluid Mech., 65, 289-314.

Hirota, R, 1971: Exact solution of the Korteweg-de Vries equation for multiple collisions of solitons. Phys. Rev. Lett., 27, 11921194.

Jeffrey, A., and T. Kakutani, 1972: Weak nonlinear dispersive waves: A discussion centered around the Korteweg-de Vries equation. SIAM Rev., 14, 582-643.

Johnson, R. S., 1970: A non-linear equation incorporating damping and dispersion. J. Fluid Mech., 42, 49-60.

, 1972: Shallow water waves on a viscous fluid - the undular bore. Phys. Fluids, 15, 1693-1699.
I JUNE 1

Joseph, ]

$A: 1$
Karpma

Karpma
gam

Koop, C.

Kubota,

wea

dep

Maslowe

sheo
- , and

$J . F$
atsuno

Ono
,- 19

Mal

$-, 19$

pp.

Maxwort

from
dim

Meiss, J.

Flui

Miura, $\mathbf{R}$

de $Y$
vatic

1209

Mulrones

Met

Nakamur

the $\mathrm{F}$

Noonan,

inter

Astr

insu

distu

anc

no, H.,

Soc.

Peregrine,

bore 
Joseph, R. J., 1977: Solitary waves in a finite depth fluid. J. Phys. A: Math. Gen., 10, 225-227.

Karpman, V. I., 1975: Non-linear Waves in Dispersive Media. Pergamon, $186 \mathrm{pp}$

Koop, C. G., and G. Butler, 1981: An investigation of internal solitary waves in a two-fluid system. J. Fluid Mech., 112, 225-251.

Kubota, T., D. R. S. Ko and L. D. Dobbs, 1978: Propagation of weakly nonlinear internal waves in a stratified fluid of finite depth. A.I.A.A.J. Hydronautics, 12, 157-165.

Maslowe, S. A., and L. G. Redekopp, 1979: Solitary waves in stratified shear flows. Geophys. Astrophys. Fluid Dyn., 13, 185-196.

, and 1980: Long nonlinear waves in stratified shear flows. J. Fluid Mech., 101, 321-348.

Matsuno, Y., 1979: Exact multi-soliton solution of the BenjaminOno equation. J. Phys. A: Math. Gen., 12, 619-621.

- 1980: Interaction of the Benjamin-Ono solitons. J. Phys. A. Math. Gen., 13, 1519-1536.

study of

oc., 111,

$\mathrm{s}$ in deep

observed

twork. J.

d solitary

marine

ervations

oundary

Bound.

omenon.

Models,

Atmos,

s of non-

ci. USSR

plasma.

luid. $P a$ -

al waves

88.

roc. Roy.

inear De.

IUTAM

internal

clouds in -2089.

equation

J. Fluid

equation 7, 1192 pp.

Maxworthy, T., 1980: On the formation of nonlinear internal waves from the gravitational collapse of mixed regions in two and three dimensions. J. Fluid Mech., 96, 47-64.

Meiss, J. D., and N. R. Pereira, 1978: Internal wave solitons. Phys. Fluids, 21, 700-702; 22, 201

Miura, R. M., C. S. Gardner and M. D. Kruskal, 1968: Kortewegde Vries equation and generalizations. II: Existence of conservation laws and constants of motion. J. Math. Phys., 9, 12041209.

Mulroney, D. T., 1984: Roll clouds over the Somerville-Tyabb area. Meteor. Aust., 3, 4-5.

Nakamura, A., 1979: Bäcklund tranform and conservation laws of the Benjamin-Ono equation. J. Phys. Soc. Jpn., 47, 1335-1340.

Noonan, J. A., and R. K. Smith, 1985: Linear and weakly nonlinear internal wave theories applied to 'morning glory' waves. Geophys. Astrophys. Fluid Dyn., 33, 123-143.

$\longrightarrow$, and - 1986: Sea breeze circulations over Cape York Peninsula and the generation of the Gulf of Carpentaria cloud line disturbances. J. Atmos, Sci., 43, 1679-1693.

, and - 1987: The generation of north Australian cloud lines and the 'morning glory.' Aust. Meteor. Mag., 35, 31-45.

Ono, H., 1975: Algebraic solitary waves in stratified fluids. J. Phys. Soc. Jpn., 39, 1082-1091.

Peregrine, D. H., 1966: Calculations of the development of an undular bore. J. Fluid Mech., 25, 321-330.
, 1972: Long waves in two and three dimensions. Proc. Symp. on Long Waves, University of Delaware, 63-90.

Physick, W., 1986: Observations of a solitary wave train in Melbourne, Australia. Aust. Meteor. Mag., 34, 163-172.

Lord Rayleigh, 1914: On the theory of long waves and bores. Proc. Roy. Soc. London, Ser. A, 90, 324-328.

Redekopp, L. G., 1980: Similarity solutions of some two-space-dimensional nonlinear wave evolution equations. Stud. Appl. Math., 63, 185-207.

Scorer, R. S., 1949: Theory of waves in the lee of mountains, Quart. J. Roy. Meteor. Soc., 75, 41-56.

Segur, H., 1973: The Korteweg-de Vries equation and water waves: Solutions of the equation, 1. J. Fluid Mech., 59, 721-736.

Shreffler, J. H., and F. S. Binkowski, 1981: Observations of pressure jump lines in the Midwest, 10-12 August 1976. Mon. Wea. Rev., 109, 1713-1725.

Simpson, J. E., D. A. Mansfield and J. R. Milford, 1977: Inland penetration of sea breeze fronts. Quart. J. Roy. Meteor. Soc.
103, 47-76.

Smith, R. K., 1986: Evening glory wave-cloud lines in northwestern Australia. Aust. Meteor. Mag., 34, 27-33.

- 1988: Waves and bores in the lower atmosphere: The 'morning glory' and related phenomena. Earth-Sci. Rev., 25, 267-290.

, and B. R. Morton, 1984: An observational study of northeasterly 'morning glory' wing surges. Aust. Meteor. Mag., 32, 155-175.

and M. A. Page, 1985: Morning glory wind surges and the Gulf of Carpentaria cloud line of 25-26 October 1984. Aust. Meteor. Mag., 33, 185-194.

, N. Crook and G. Roff, 1982: The Morning Glory: An extraordinary atmospheric undular bore. Quart. J. Roy. Meteor. Soc. 108, 937-956

- M. J. Coughlan and J.-L. Lopez, 1986: Southerly noctural wind surges and bores in northeastern Australia. Mon. Wea Rev., 114, 1501-1518

Taylor, G. I., 1910: The conditions necessary for discontinuous motion in gases. Proc. Roy. Soc. London Ser. A, 84, 371-377.

Tung, K.-K., D. R. S. Ko and J. J. Chang, 1981: Weakly nonlinear internal waves in shear. Stud. Appl. Math., 65, 189-221.

- T. F. Chan and T. Kubota, 1982: Large amplitude internal waves of permanent form. Stud. Appl. Math., 66, 1-44.

Vliegenthart, A. C., 1971: On finite difference methods for the Korteweg-de Vries equation. J. Eng. Math., 5, 137-155.

Whitham, G. B., 1974: Linear and Nonlinear Waves. Wiley \& Sons, $636 \mathrm{pp}$ 
Section 2.9

\section{A thunderstorm generated solitary wave observation compared with theory for nonlinear waves in sheared fluids}

(R.J. Doviak, S.S. Chen and D.R. Christie)

J. Atmos. Sci., 48, 87-111, 1991. 


\title{
A Thunderstorm Generated Solitary Wave Observation Compared with Theory for Nonlinear Waves in Sheared Fluids.
}

\author{
Richard J. Doviak, Shuyi S. Chen ${ }^{1}$, and Douglas R. Christie ${ }^{2}$ \\ NOAA, Environmental Research Laboratories \\ National Severe Storms Laboratory \\ 1313 Halley Circle \\ Norman, OK 73069
}

July 1990

(To appear J. Atmos. Sci. 48, 87-111, 1991)

1 Pennsylvania State University, Department of Meteorology, 503 Walker building, University Park, PA 16802

2 The Australian National University, Research School of Earth Sciences, Institute of Advanced Studies, G.P.O. Box 4, Canberra, ACT 2601 Australia 


\section{Contents}

(This table is a guide for the referees and not a part of the paper) ABSTRACT

1. Introduction

2. Theory . . . . . . . . . . . . . . . . . . . 3

a. The Eigenfunction equation . . . . . . . . . . . . . . . . . . 4

b. The second-order perturbations for sheared flow . . . . . . . . 7

c. The upperllayer equations . . . . . . . . . . . . . . . . . . 10

d. The equation of wave evolution and the steady-state solution $\quad 12$

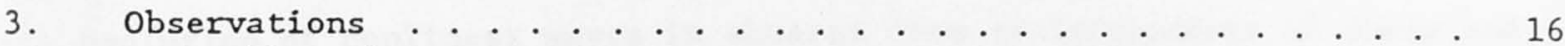

a. The ambient environment . . . . . . . . . . . . . . . . . . 17

b. Wind perturbations observed with tower and surface

instruments . . . . . . . . . . . . . . . . . . . . . . . . 21

1) THE COMPONENTS OF WIND CHANGE AND THE PLANE OF CIRCULATION . . . . . . . . . . . . . . . . . . . 22

2) DYNAMICS OF TRAPPED THUNDERSTORM OUTFLOW . . . . 24 i. Guided Outflow . . . . . . . . . . . . . . 24 ii. Leaking of trapped outflow . . . . . . . . 26

3) EROSION OF THE WAVE GUIDE . . . . . . . . . . . . . 27

4) WAVE CHARACTERISTICS FROM SURFACE AND TOWER DATA . . . . . . . . . . . . . . . . . . . 28

c. Wave characteristics observed using Doppler radar . . . . . . 29

1) THE DIRECTION OF WAVE TRAVEL . . . . . . . . . . 30

2) WAVE SPEED ...................... . . . 31

3) DOPPLER VELOCITY PERTURBATIONS . . . . . . . . . . 32

4) ESTIMATES OF WAVE AMPLITUDE AND HALF WIDTH . . . 33

5) TIME DEPENDENCE OF WAVE AMPLITUDE . . . . . . . . 34

4. Comparison with theory . . . . . . . . . . . . . . . . . . . 36

a. Indirect approach . . . . . . . . . . . . . . . . 36

b. Direct approach . . . . . . . . . . . . . . . . 41

c. Comparison with strongly nonlinear theory . . . . . . . . . . 43

d. An evolution model for the generation of solitary waves by rapidly moving storms . . . . . . . . . . . . . . . 48

5. Summary and conclusions . . . . . . . . . . . . . . . . . . . . 51

6. Acknowledgments. . . . . . . . . . . . . . . . . . . . . . 56

Appendix: Conditions for solitary waves to have permanent form in presence of vertical propagation . . . . . . . . . . . . 57

REFERENCES . . . . . . . . . . . . . . . . . . . . . . . . . . . . . . . 62

List of Figures . . . . . . . . . . . . . . . . . . . . . . . . 67 


\section{ABSTRACT}

The theory of internal nonlinear waves in an incompressible motionless medium is extended to waves in compressible, sheared flow to provide a basis for the interpretation of atmospheric solitary waves. We show that if the Scorer parameter is zero in a semi-infinite upper region, the integrodifferential equation (i.e., the Benjamin-Davis-Ono (BDO) equation) defining the evolution of nonlinear waves in sheared flow is independent of shear and stability in the upper layer. We present a new type of evolution model, based on the numerical solution of the BDO equation, for the generation of solitary waves by a thunderstorm moving at supercritical speeds. The results of a thorough study of observations of a thunderstorm-generated solitary wave are presented in detail. These observations show that some of the storm's outflow, which was denser than the environment through which the wave propagated, was trapped in the interior of the wave. We hypothesize that the Coriolis force then caused this denser air to flow along the axis of the wave away from the storm's southern flank for distances in excess of $100 \mathrm{~km}$. Analysis reveals that the temporarily trapped recirculating air in the leading solitary wave is gradually deposited along the ground, forming an advancing shallow layer of denser air behind the wave. The derived wave parameters are then compared with theory. A new type of analysis, based on the separation of the wind change due to vertical transport of horizontal momentum from the observed wind perturbations, results in improved agreement between weakly nonlinear theory and observations. This analysis supports the deduction that wave energy propagates along straight rays, parallel to the plane of recirculating flow, but oblique to the curved wavefront. The failure of weakly nonlinear theory to account for all the observed wave characteristics 
is shown to be caused by the presence of recirculating flow. Comparison with numerical results for strongly nonlinear waves shows reasonably good agreement for all the wave characteristics except wave speed which, is significantly less than that predicted when wave amplitudes are large. Finally, we develop relations that govern the minimum wave amplitude threshold required for the propagation of solitary waves in waveguides bordered by weakly. stratified sheared flow. 
Recent advances in remote sensing techniques have given atmospheric scientists an x-ray view of the internal morphology and dynamics of a wide variety of atmospheric phenomena that are transparent in clear weather and invisible at night. The use of modern Doppler weather radar, in particular, has led to answers to long-standing questions in meteorology and revealed in unprecedented detail the structure of unusual phenomena, one of which is the atmospheric solitary wave. Its oceanic counterpart, an internal solitary wave in the deep-sea thermocline, was widely studied during the last decade (Ostrovsky and Stepanyants, 1989). Solitary waves are large-amplitude singlecrested, buoyancy waves with amplitude-dependent speeds, which exceed the speed of long waves of infinitesimal amplitude. Waves of this type are exceptionally stable entities that propagate over long distances with remarkably little change in form or amplitude.

The radar image of a thunderstorm-generated solitary wave typically takes the form of a thin line of Doppler-shifted reflectivity propagating away from the storm. The frequent observation of propagating reflectivity lines in the neighborhood of thunderstorms and the ease with which solitary waves can be generated (Maxworthy, 1980) suggest that solitary waves, and the closely related phenomenon of an undular bore, might be a commonly occurring feature in the thunderstorm environment whenever suitable atmospheric stratification exists and sources are active. Solitary atmospheric waves and undular bore waves occur frequently over northern Australia (Christie et al., 1978; Clarke et al., 1981; Smith, 1988). Waves of this type have large amplitudes and play a significant role in the initiation and organization of deep convection (Zhang and Fritsch, 1987; Carbone, et al. 1990). They are also a source of 
intense localized wind shear, which can be hazardous to aircraft operating near the surface (Christie and Muirhead, 1983; Doviak and Christie, 1989). Doviak and Ge (1984) show an observed isolated propagating disturbance to be a solitary atmospheric wave generated when a thunderstorm downflow impinges on a preexisting pool of stable air that was deposited at the surface by the outflow from an earlier storm. A different interpretation of these observations given by Chimonas and Nappo (1987). They conclude that all the well-defined characteristics of the wave can be understood through a bow-wave model based on linear wave theory. However, linear theory cannot describe the observed formation and propagation of at least two well-separated, amplitudeordered waves of elevation containing regions of recirculating flow. Recirculation confirms the wave is strongly nonlinear (Tung et al., 1982), and the appearance of two amplitude-ordered waves is a natural consequence in the evolution of sufficiently long nonlinear disturbances, a result given by nonlinear wave theory (Christie, 1989) and also observed with Doppler radar (Doviak, et al. 1989) under circumstances similar to those reported here. Furthermore, Doppler radar and surface data presented here show, as nonlinear wave theory demonstrates, that the second weaker wave lags farther behind the first as time progresses because its amplitude-dependent speed is less than the speed of the leading wave.

In this paper we also develop a theory for nonlinear waves in compressible and sheared flow, introduce surface and Doppler data not presented in Doviak and Ge (1984), provide new and detailed analysis of all available data, and make a quantitative comparison of theory and observation. This comparison should provide a better understanding of nonlinear wave properties and a basis for determining whether observed isolated gusts and 
thin lines of radar reflectivity are manifestations of evolving solitary waves. This more complete analysis and comparison with nonlinear buoyancy wave theories should help resolve some of the features of the data that puzzled Chimonas and Nappo, as well as the present authors.

\section{Theory}

We follow Ono's (1975) development of weakly nonlinear wave theory for incompressible fluids in a motionless state, but we extend his analytic equations to a moist unsaturated atmosphere in sheared flow. Weakly nonlinear waves displace air vertically a finite distance, but much less than the scale thickness $h_{s}$ of the stable atmospheric layer that guides the wave. Ono's equations apply to the shearless atmosphere if the Coriolis force can be neglected and if the incompressible fluid and the atmosphere are dynamically similar. It can be shown that for the disturbance wavelengths (approximately a few kilometers) considered here, the Coriolis force is negligible if trapped air is not present in the wave, a valid assumption for weakly nonlinear waves. The conditions under which the equations governing motions in the atmosphere are equivalent to those for an incompressible fluid are given by Spiegel and Veronis (1960). The most important requirement is that both media should have equivalent Brunt-Väisälä (B-V) frequencies $N$ (i.e., identical static stability). The principal defect in treating the compressible atmosphere this way is the absence of acoustic waves (Gossard and Hooke, 1975), but they are unimportant to this analysis. Thus we can adapt Ono's equations to the atmosphere by replacing the incompressible fluid density $R_{0}(z)$ in his equations with 


$$
R_{0}(z)=\frac{R_{0}(0) \theta_{v_{0}}(0)}{\theta_{v_{0}}(z)}
$$

where $\theta_{\text {vo }}$ is the height $(z)$ dependent virtual potential temperature of the ambient atmosphere. A more formal treatment of nonlinear wave theory for a compressible fluid, which leads to essentially the same results, can be found in Grimshaw (1980). More recently, Miesen (1990) shows conditions under which compressibility can significantly alter these results.

We consider an atmosphere separated into a lower region of depth $h$, in which the air has strong static stability and moves with a wind that may be strongly curved with height and a semi-infinitely deep, weakly stable, upper region with weakly curved flow.

\section{a. The Eigenfunction equation}

Consider two-dimensional flow in the above-described horizontally homogeneous atmosphere with dependent variables expanded in a series ordered in powers of the perturbation parameter $\varepsilon-\delta_{m} / h_{s} \ll 1$, where $\delta_{m}$ is the maximum vertical displacement caused by the wave. When this expansion is inserted into the Navier-Stokes and mass continuity equations, we find that the height-dependent part $\phi(z)$ of the first-order perturbation streamfunction

$$
\psi=\varepsilon \psi_{1}-\varepsilon f(\xi, \tau) \phi(z)
$$

satisfies the eigenfunction equation

$$
-\left[\left(u_{0}-c_{0}\right) \phi^{\prime} / \theta_{v o}\right]^{\prime}+\left(\phi u_{0}^{\prime} / \theta_{v o}\right)^{\prime}-g \phi \theta_{v o}^{\prime} /\left[\theta_{v o}^{2}\left(u_{o}-c_{0}\right)\right]-0,
$$


where primes denote differentiation with respect to $z$, the linear long-wave phase speed $c_{0}$ is the eigenvalue, and

$$
\xi-\varepsilon\left(x-c_{0} t\right), \quad \tau-\varepsilon^{2} t, \quad z-z,
$$

transform the independent variables into a stretched coordinate system. We transform the scales of space and time to extract from the Navier-Stokes formulations simplified equations that describe single-crested disturbances of half-amplitude width, $2 \lambda$, large compared with $h_{s}$. This is accomplished in the stretched coordinate system in which horizontal and vertical scales are roughly matched, and the scaling in time results from application of the dispersion relation, $\omega-k c_{0}(1-\beta|k|)$, appropriate for long waves in fluids of infinite depth (Dodd et al., 1982). We attach the dimensions of $\psi$ to $f(\xi, t)$ so that we can set $\phi(h)-1$ without loss of generality.

By defining the relation

$$
\tilde{\phi} \equiv\left[\theta_{v_{0}}(0) / \theta_{v_{0}}\right]^{1 / 2} \phi,
$$


(2.3) is transformed to

$$
\begin{gathered}
\tilde{\phi}^{\prime \prime}\left\{\frac{1}{2} \frac{\theta_{\text {vo }}^{\prime \prime}}{\theta_{\text {vo }}}-\frac{3}{4}\left[\frac{\theta_{\text {vo }}^{\prime}}{\theta_{\text {vo }}}\right]^{2}+\frac{\theta_{\text {vo }}^{\prime} u_{0}^{\prime}}{\theta_{\text {vo }}\left(u_{0}-c_{0}\right)}-\frac{u_{0}^{\prime \prime}}{\left(u_{0}-c_{0}\right)}\right. \\
\left.+\frac{g \theta_{\text {vo }}^{\prime}}{\theta_{\text {vo }}\left(u_{0}-c_{o}\right)^{2}}\right\} \tilde{\phi}=0 .
\end{gathered}
$$

In this form, all terms containing the ambient variables $\theta_{\mathrm{vo}}, u_{0}$ appear in the coefficient of $\tilde{\phi}$ and thus we can compare relative magnitudes. For the data we consider, the first three terms in this coefficient are negligible compared with the other two. Hence, (2.6) reduces to

$$
\tilde{\phi}^{\prime \prime}+\ell^{2} \tilde{\phi} \simeq 0
$$

where

$$
l^{2}-\frac{N^{2}}{\left(u_{0}-c_{0}\right)^{2}}-\frac{u_{0}^{\prime \prime}}{\left(u_{0}-c_{0}\right)}
$$

Here, $\mathrm{N}^{2}-g \theta^{\prime}{ }_{\mathrm{vo}} / \theta_{\text {vo }}$ is the $\mathrm{B}-\mathrm{V}$ frequency squared, and $\ell$ is the vertical wave number for waves of long horizontal length. These simplifications are consistent with the Boussinesq approximation, and (2.7a) is the TaylorGoldstein equation for waves of extreme length. (i.e., horizontal wave number $k$ $\rightarrow 0$ ) in a Boussinesq fluid. Because changes in $\theta_{\text {vo }}$ are small compared with $\theta_{\mathrm{vo}}(0), \phi \simeq \tilde{\phi}$ is a good approximation.

Scorer $(1949,1954)$ was the first to recognize the importance of the vertical profile of $\ell$ in explaining the behavior of airstreams and, hence, $\ell$ 
is referred to as the Scorer parameter. We note that a given vertical profile of the Scorer profile can correspond to an infinite variety of wind and temperature profiles. It was recently shown by Crook $(1986 ; 1988)$ that the essential condition for the trapping of buoyancy waves is for $l$ to vanish in the upper region. Since the atmosphere rarely has an upper region in which $\ell$ is zero throughout, we determine, in the Appendix, the general conditions under which solitary waves are not significantly affected by an upper layer in which $\ell \neq 0$.

b. The second-order perturbations for sheared flow

Proceeding to the next-order terms of the expansion, we find, after some tedious but straightforward mathematical manipulations to simplify (2.6), that the second-order vertical velocity component $w_{2}$ satisfies the inhomogeneous differential equation:

$$
\begin{gathered}
w_{2 z z}+l^{2} w_{2}--\phi f_{\tau}\left[\frac{2 N^{2}-u_{o z z}\left(u_{0}-c_{0}\right)}{\left(u_{0}-c_{0}\right)^{3}}\right]-\frac{\phi^{2} f f_{\xi}}{\left(u_{0}-c_{0}\right)^{4}} \\
{\left[u_{o z z z}\left(u_{0}-c_{o}\right)^{2}-u_{o z} u_{o z z}\left(u_{0}-c_{0}\right)-2\left(N^{2}\right)_{z}\left(u_{o}-c_{o}\right)+3 N^{2} u_{o z}\right]} \\
-\frac{2 N^{2} f f_{\xi}}{g\left(u_{0}-c_{0}\right)^{3}}\left[N^{2} \phi^{2}+\frac{3}{2}\left(\phi_{z}\right)^{2}\left(u_{0}-c_{o}\right)^{2}\right],
\end{gathered}
$$


where subscripts $z, \xi$, and $T$ specify differentiation. Equation (2.8) reduces, in the limit $u_{0} \rightarrow 0$, to Ono's second-order equation for motionless media, but only in the Boussinesq approximation. Consistent with this approximation, the two terms in the last bracket are negligible when compared with the term containing $\left(\mathrm{N}^{2}\right)_{z}$; this reduced form of $(2.8)$ is then exactly analogous to that given by Maslowe and Redekopp (1980) for an incompressible fluid. However, it should be noted that if a shearless lower region is linearly stratified, then $\left(\mathrm{N}^{2}\right)_{z}=0$ and the last two terms can no longer be ignored. In this case, the theory provides a proper description of finite amplitude waves of permanent form only when these non-Boussinesq terms are retained (Long, 1965; Benjamin, 1966). Nevertheless, under most conditions found in the lower atmosphere, these non-Boussinesq terms are small compared with the other terms. Therefore, we can simplify $(2.8)$ to

$$
w_{2 z z}+\ell^{2} w_{2}-G(\phi, f)
$$

where

$$
\begin{aligned}
G(\phi, f) & =\frac{\phi f}{T}-\left[l^{2}+\frac{N^{2}}{\left(u_{0}-c_{0}\right)^{2}}\right] \\
& +\frac{\phi^{2} f f_{\varepsilon}}{\left(u_{0}-c_{0}\right)^{2}}\left[\left(u_{0}-c_{0}\right)\left(l^{2}\right)_{z}+\left(\frac{N^{2}}{u_{0}-c_{0}}\right)_{z}\right] .
\end{aligned}
$$

To derive the equation governing $f$, we apply the following solvability condition: 


$$
\int_{0}^{h}\left(w_{2 z z}+\ell^{2} w_{2}\right) \phi d z-\int_{0}^{h} \phi G(\phi, f) d z,
$$

which, when satisfied, avoids a resonant solution of (2.9). If there are critical levels (i.e., altitudes at which wave speed equals the wind component in the direction of wave propagation), an alternate solution of (2.9) is necessary to avoid singularities (Maslowe and Redekopp, 1980; Tung et al., 1981). We assume in this paper that critical levels do not occur. Integrating the left side of (2.10) by parts and applying the boundary conditions, $\phi=0$ and $w_{2}-0$ at $z-0$, gives

$$
\phi(h) w_{2 z}(h)-w_{2}(h) \phi_{z}(h)-\int_{0}^{h} \phi G(\phi, f) d z .
$$

If $w_{2}(\xi, h, \tau)$, its vertical derivative at the interface, and $\phi(z)$ are all known, (2.11) will give the governing equation for $f(\xi, \tau)$. If $\theta_{\text {vo }}$ or $u_{o z}$ are discontinuous at $z-h$, the required solution can be determined by invoking a dynamic (i.e., matching of pressure p) condition and the continuity of vertical velocity w across the interface (Maslowe and Redekopp, 1980), whereas if $\theta_{v o}$ and $u_{o z}$ are continuous, we simply need to apply the kinematic condition (i.e. matching $w$ and its vertical derivative $w_{z}$ ) at the interface to ensure that $p$ and $w$ are continuous to first order in the perturbation variable. If $\ell^{2}$ is continuous, then $\mathrm{p}$ and $\mathrm{w}$ are continuous to at least second order in the perturbation variables. Because we are applying theory to measured atmospheric parameters, we use analytic profiles for which $l^{2}$ is continuous in height. 


\section{c. The upper-layer equations}

Because the upper layer is weakly stratified, there is no need to stretch one coordinate relative to the other to have vertical scales roughly equal to those in the horizontal. Therefore we adopt the coordinate transformation used by Ono (1975). That is,

$$
X=x-c_{0} t, \quad T-\varepsilon^{2} t, \quad z-z .
$$

Upper-case variables denote solutions in the upper layer. Ono (1975) also noted that because $w(\xi, z, \tau)$ asymptotically approaches $\varepsilon^{2} w_{1}$ as $\varepsilon \rightarrow 0$ and the continuity equation in the coordinate frame $X, z, \tau$ is

$$
\mathrm{U}_{\mathrm{x}}+\mathrm{W}_{\mathrm{z}}-0
$$

the horizontal velocity in the region $z \geq h$ must also asymptotically have an $\varepsilon^{2}$ dependence. These conditions lead us to expand the upper-layer variables in the following form:

$$
\begin{aligned}
& U-u_{0}(z)+\sum_{n=1}^{\infty} \varepsilon^{n+1} U_{n}(X, z, \tau) \\
& W- \\
& P=P_{0}(z)+\sum_{n=1}^{\infty} \varepsilon^{n+1} E_{n}(X, z, \tau) \\
& R=R_{0}(z)+\sum_{n=1}^{\infty} \varepsilon^{n+1} R_{n}(X, z, \tau)
\end{aligned}
$$


Introducing (2.13) into the equations of motion for the coordinate frame defined by (2.12) and applying a transformation to $\mathrm{W}$ similar to (2.5), we find that if $\ell_{u}=0$, W satisfies Laplace's equation

$$
\frac{\partial^{2} W}{\partial X^{2}}+\frac{\partial^{2} W}{\partial z^{2}}=0
$$

to the third order of $\varepsilon$. Equation (2.14) is exactly the same as obtained by Ono (1975) for a statically neutral and motionless upper layer.

We introduced into (2.13) general functions for the background wind and density to show, although we do not present the proof, that (2.14) is satisfied irrespective of the shape of the $u_{0}$ and $R_{0}$ profiles if $\ell_{u}-0$. Thus we need not restrict our analysis to cases in which the upper region is neutrally stable with uniform wind, but to a class of canonical profiles for which $\ell_{u}-0$. However, in general, $\ell_{u}$ is a function of the eigenvalue $c_{0}$ and thus the condition $\ell_{u}-0$ can be satisfied for only one eigenmode, if at all. The solution of the eigenvalue problem may be further complicated if the boundary between the lower and upper layers is not clearly defined. Assuming there is an eigenmode for which $\ell_{u}-0$, a trial and error method can be applied, when $h$ is unknown, in which successively increasing values of $h$ are tried to find the interface above which $\mathrm{dc}_{\mathrm{o}} / \mathrm{dh}$ is small as demonstrated by Noonan and Smith (1985).

A solution of (2.14) can be obtained given the following boundary conditions :

$$
W \rightarrow 0 \text { as } z \rightarrow \infty ; \quad W-w \text { at } z-h \text {, }
$$


where $w$ is the vertical velocity in the lower layer which matches to $O\left(\varepsilon^{3}\right)$ the vertical velocity $W$ in the upper layer at $z=h$. The solution of this Dirichlet problem is

$$
W_{1}=\frac{2}{\varepsilon \pi} P \int_{-\infty}^{+\infty} \frac{\left(X^{\prime} X^{\prime}\right)(z-h) f\left(X^{\prime}, \tau, \varepsilon\right)}{\left[\left(X-X^{\prime}\right)^{2}+(z-h)^{2}\right]^{2}} d X^{\prime}
$$

where $P$ denotes the Cauchy Principal value of the integral.

From this point forward the analysis parallels that given by Ono (1975). Thus we obtain

$$
\phi(0)=0, \quad \phi_{z}(h)=0, \quad w_{2 z}(h)=H_{\xi \xi}(f),
$$

where $H(f)$ is the Hilbert transform of $f$. The boundary conditions on $\phi$ apply to any continuous profile of $\ell^{2}$ and are exactly the same as those for a statically neutral and motionless upper region, the case considered by Ono. If $l^{2}$ asymptotically approaches zero in the limit $h \rightarrow \infty$, the boundary conditions still apply.

d. The equation of wave evolution and the steady-state solution

We finally obtain the desired equation governing $f(\xi, \tau)$ by substituting (2.17) into $(2.11)$ to get

$$
f_{\tau}+\alpha f f_{\xi}-\beta H_{\xi \xi}(f)-0,
$$

where the coefficients of nonlinearity and dispersion, $\alpha\left(\mathrm{m}^{-1}\right)$ and 
$\beta\left(m^{2} s^{-1}\right)$, are

$$
\alpha=\beta \int_{0}^{h} \frac{\phi^{3}}{\left(u_{0}-c_{0}\right)^{2}}\left[\left(u_{0}-c_{0}\right)\left(l^{2}\right)_{z}+\left(\frac{N^{2}}{u_{0}-c_{0}}\right)_{z}\right] d z
$$

and

$$
\beta^{-1}=-\int_{0}^{h} \frac{\phi^{2}}{\left(u_{0}-c_{0}\right)}\left[l^{2}+\frac{N^{2}}{\left(u_{0}-c_{0}\right)^{z}}\right] d z .
$$

This evolution equation, which we refer to as the Benjamin-Davis-Ono (BDO) equation, is analogous to that derived by Ono except that we included the effects of shear and invoked the Boussinesq approximation. The eigenfunction $\phi$ and eigenvalue $c_{0}$ are obtained by solving (2.7a) subject to the boundary conditions (2.17). The Scorer parameter (2.7b) can then be determined and the coefficients $\alpha$ and $\beta$ can be found by evaluating the integral in (2.18).

Steady-state solutions to the governing equation (2.18) result when a dynamical balance exists between the competing effect of nonlinearity (the second term), which steepens the wave, and dispersion (the third term), which flattens the wave. In this case $f(\xi, \tau)$ is a function of $\zeta-\xi-c_{1} \tau$ alone; thus (2.18a) reduces to

$$
{ }^{-c}{ }_{1} f_{\zeta}+\alpha f f_{\zeta}-\beta H_{\zeta \zeta}(f)-0,
$$

where $c_{1}$ is the speed of the wave in the $\xi$ coordinate system, which itself moves toward positive $x$ at a speed $c_{0}$ relative to the stationary earth frame. 
Because the $\xi$ axis is stretched by $\varepsilon$ relative to the $x$ axis, the speed of the wave in the stationary frame is

$$
c=c_{0}+\varepsilon c_{1} .
$$

The solitary wave solution of (2.19) is

$$
f(\zeta)=\frac{\psi_{0} \lambda_{s}^{2}}{\zeta^{2}+\lambda_{s}^{2}} ; \quad \lambda_{s} \equiv \varepsilon \lambda ; \quad 0 \leq z \leq h,
$$

provided that the conditions

$$
\psi_{0}-\frac{4 c_{1}}{\alpha}, \quad \lambda_{s}-\frac{4 \beta}{\alpha \psi_{0}}
$$

are satisfied. Equation (2.21a) is the algebraic solution first discovered by Benjamin (1967). The streamfunction amplitude at $z-h$ and $\zeta-0$ is $\psi_{0}$, and $\lambda_{s}$ is the wavelength in the stretched coordinate $\zeta$. In terms of the unstretched coordinate axis $\tilde{x}$ moving at the speed $c$,

$$
f(\tilde{x})=\frac{\psi_{0} \lambda^{2}}{(\tilde{x})^{2}+\lambda^{2}}, \quad \tilde{x}-(x-c t), \quad \text { for } 0 \leq z \leq h .
$$

The wind components $u, v, w$, to lowest order in $\varepsilon$ and in the $x, y, z$ earth frame are given by

$$
\mathrm{u}-\mathrm{u}_{\mathrm{o}}+\psi_{\mathrm{z}}, \quad \mathrm{v}-\mathrm{v}_{\mathrm{o}}+\mathrm{v}_{\mathrm{oz}} \delta, \quad \mathrm{w}=-\psi_{\mathrm{x}} \quad \text { (2.23a, b, c) }
$$

where 


$$
\psi \equiv \psi^{(l)} \quad-\frac{\varepsilon \psi_{0} \lambda^{2} \phi(z)}{(\tilde{x})^{2}+\lambda^{2}}, \quad 0 \leq z \leq h,
$$

is the stream function in the lower region and $\delta$ is the vertical displacement caused by the wave. Although the wave is two-dimensional, wave-like perturbations will appear in all three wind components if $v_{0}$ has a $z$ dependence. The perturbation in $v$ is caused by the vertical displacement of vertically sheared wind parallel to the wavefront. The largest horizontal winds produced by the wave occur at the earth's surface (if friction is ignored), and consequently this is where the horizontal displacement and shear are largest. Thus, wave passage can enhance preexisting shear causing dynamic instabilities and the formation of Kelvin-Helmholtz (K-H) waves, as observations discussed in Section 3 suggest.

Doviak and Chen (1988) showed that the upper layer solution for steady. state solitary waves is given by the perturbation streamfunction

$$
\psi^{(u)}-\frac{\varepsilon \psi_{0} \lambda(z-h+\lambda)}{\left[(X-c t)^{2}+(z-h+\lambda)^{2}\right]}, \quad z \geq h,
$$

which is exactly the same as for a neutrally stable and motionless upper region, thus showing that shear and static stability in the upper region do not generally alter the shape of a solitary wave of permanent form if $\ell_{u}=0$.

The wave speed $c$ can be expressed in terms of the dispersion parameter $\beta$ by eliminating $\psi_{0}$ between $(2.21 b)$ and $(2.21 c)$. Thus, when $c_{1}$ is substituted into $(2.20)$, we obtain 


$$
c=c_{0}+\beta / \lambda
$$

the speed of the wave relative to the earth frame. In the unstretched coordinate frame, the streamfunction amplitude $\psi(0, z)$ at $z=h$ is equal to $\varepsilon \psi_{O}$. Substituting this and $\lambda_{S}-\varepsilon \lambda$ into (2.21c) gives the expression

$$
\lambda=\frac{4 \beta}{\alpha \psi(0, h)}
$$

which clearly shows the inverse relation between wavelength and amplitude for weakly nonlinear solitary waves. This characteristic scaling for these waves breaks down at larger amplitudes as the effective wavelength starts to increase with the onset of closed circulation in the relative streamline pattern (Davis and Acrivos, 1967; Tung et al., 1982).

\section{Observations}

Our data were obtained from a $444-\mathrm{m}$-tall tower, Doppler radar, a network of meteorological sensors near the ground ( $2 \mathrm{~m} \mathrm{AGL),} \mathrm{and} \mathrm{rawinsondes,} \mathrm{all} \mathrm{in}$ central Oklahoma. For further details on the experimental set-up and location of the various instruments, see Doviak and Ge (1984). Using these data, we were able to deduce both the ambient conditions of the environment through which a wave-like disturbance propagated and as the properties of the disturbance.

We hypothesize that the observed localized perturbations in temperature and wind fields were manifestations of an evolving solitary wave. This hypothesis is supported by the following observational evidence. First, radar 
data (discussed in detail in section 3.c.2) show that the disturbance speed asymptotically approached a ground relative speed of about $12 \mathrm{~m} \mathrm{~s}^{-1}$ in a direction opposing the low-altitude wind (i.e., perturbations were not advected by the wind). This observation alone indicates that the disturbance has the properties of a wave and not a cut-off vortex because, relative to the ambient wind, the translational speed of a vortex should have asymptotically approached zero (Clarke, 1965). Second, the speed of the disturbance-is faster than the winds at any altitude behind the disturbance, thus the disturbance cannot be described as a density current head. Finally, the disturbance disintegrated rapidly when it reached the horizontal boundary of the stable layer (Doviak and Ge, 1984). This provides additional support for the evolving solitary wave hypothesis since the existence of a propagating cut-off vortex does not depend on the presence of a stable layer as numerical simulations show (Drogemeier and Babcock, 1989). The fact that perturbations were not seen at a surface site $10 \mathrm{~km}$ in front of the wave at a time when its amplitude and speed approached a constant value attests to the fact that the disturbance depended on the existence of the stable layer, which acted as a waveguide. Therefore we conclude that the properties of the isolated disturbance are characteristic of those of a wave, and we refer here to the disturbance as an evolving solitary wave.

\section{a. The ambient environment}

Profiles of the ambient wind in which the wave propagates are measured using Doppler radar and tower data, but we did not have a temperature sounding immediately before wave passage. Using data from the nearest sounding, as well as the time-continuous data from the tower and satellite, we can at best 
speculate on the static stability of the ambient air. Figure 1 shows the dry-bulb and dew point temperature profiles from the rawinsonde launched $5 \mathrm{~h}$ earlier and a 10 -min average of data from the tower just before wave passage. At 1800 CST, a well-mixed layer of moist air, brought by southerly winds, extended from the surface to about $1.5 \mathrm{~km}$ AGL.

A stationary cold front was present along the Oklahoma-Kansas border for several hours before wave passage in central Oklahoma, and thunderstorms initiated in western Oklahoma propagated along this frontal boundary. Data from the network and the tower showed that the leading edge of a southwardmoving thunderstorm outflow passed the tower at 2130 , about $1.5 \mathrm{~h}$ before wave passage there. The tower temperature profiles are within this outflow.

It is hypothesized that the outflow lifted the lighter, mixed-layer air above it by at least $7-8 \mathrm{kPa}$ (i.e. about $700-800 \mathrm{~m}$ ). If, at that time, the moist air was as deep as at $1800 \mathrm{CST}$, the lifted air would have become saturated and very unstable. The lack of deep convection over the density current and the sparsity of weather echoes in the $2.5-4 \mathrm{~km}$ height interval (only a few weak echoes of reflectivity factor less than $10 \mathrm{dBZ}$ were observed above and below this interval) suggest that the lifted air was drier at 2130 CST. Furthermore, Doppler radar data show that the ambient wind shifted from southerly to southwesterly $5 \mathrm{~h}$ later, and air from this direction is usually drier in central Oklahoma. Thus we assume neutrally stable air was lifted dry adiabatically so that there was at least a $1.5-\mathrm{km}$-deep layer of neutrally stable air above the first kilometer of dense outflow air, which leads to a vertical profile of $\mathrm{N}^{2}$ given by

$$
N^{2}(z) \approx N^{2}(0) \exp (-b z) \quad z \leq 1 \mathrm{~km},
$$




$$
N^{2}(z)=0 \quad 1 \leq z \leq 2.5 \mathrm{~km},
$$

where $\mathrm{N}^{2}(0) \approx 1.5 \times 10^{-3} \mathrm{~s}^{-2}$ and $\mathrm{b} \approx 2.78 \mathrm{~km}^{-1}$.

The northwesterly $\left(u_{0}\right)$ ambient wind component (Fig. 2a), estimated using only tower and Doppler radar observations, was parallel to the direction of wave travel. The southwesterly wind is not presented because it did not affect wave dynamics. Wind data from anemometers on the tower $(36 \mathrm{~km}$ from the radar at azimuth $356^{\circ}$ ) are plotted in Fig. 2a as 10 -min averages of digitally recorded values spaced $10 \mathrm{~s}$ apart about $2245 \mathrm{CST}$. The tower wind estimates had a standard deviation of less than about $1 \mathrm{~m} \mathrm{~s}^{-1}$ at all levels. The values for $u_{0}$ marked by the filled circles in Fig. 2a are derived from measured Doppler velocities in nonprecipitating regions over an azimuthal sector from $330^{\circ}$ to $30^{\circ}$, and at ranges 30 to $40 \mathrm{~km}$ where an assumed horizontally uniform wind model is least squares fitted to the data. Below about $1 \mathrm{~km}$, Doppler velocities were uniformly observed in this entire volume. However, at higher altitudes, observations were possible at only a few locations, where scattered clouds of moderate reflectivity (less than $10 \mathrm{dBZ}$ ) were capable of providing measurements of Doppler velocities. These clouds were observed with radar beam elevation angles up to $8^{\circ}$ and at ranges from about $20-80 \mathrm{~km}$. An estimate of wind was made whenever a cluster of at least 10 Doppler velocities, spread in range and azimuth by no more than a few kilometers and a few degrees, appeared at about the same range and elevation angle as another cluster-with an azimuthal separation of at least $25^{\circ}$, over which the wind was assumed uniform. The Doppler velocities in each cluster were averaged, and the wind was estimated from pairs of averaged Doppler velocities (i.e., a pairwise fit); these $u_{0}$ data are plotted in Fig. $2 a$ as open circles. 
Since no suitable cluster pairs of Doppler velocities could be found in the 2-4 km height interval, the $u_{0}$ profile is assumed to be linear above 1.5 $\mathrm{km}$ to connect the data at $5 \mathrm{~km}$. The $u_{0}$ data are fitted by the formula

$$
u_{0}=-3.33\left(1-e^{0.417 z} \cos \left(3.63 z+1.97 z^{2}-1.055 z^{3}\right)\right) \mathrm{m} \mathrm{s}^{-1}, 0 \leq z \leq 1.2 \mathrm{~km}
$$

This formula is plotted as the solid line on Fig. 2a. Because this analytical expression results in a discontinuity of $\ell^{2}$ at $z=1.5 \mathrm{~km}$ when extended to that height, we fitted a fifth-order polynomial to match $u_{0}, u_{o z}$, and $u_{o z z}$ at $z-1.2$ and $1.5 \mathrm{~km}$ so that $\ell^{2}$ is a continuous function of height. Although the fitted polynomial gave $u_{0}$ values that differed little from those given by (3.2) in the height interval $1.2-1.5 \mathrm{~km}$ (i.e. a difference less than a few tenths of a meter per second), it notably altered the estimates of $\alpha$ and $\beta$ presented in section 4 (i.e., $\alpha$ decreased by $20 \%$ and $\beta$ increased by $10 \%$ ), indicating a marked estimation sensitivity to the details of the wind profile. Calculated values of $\ell^{2}$ for the height interval $0-2.5 \mathrm{~km}$ are plotted in Fig. $2 \mathrm{~b}$ for measured wave speeds of $12 \mathrm{~m} \mathrm{~s}^{-1}$, the speed at about the time the wave passed the tower, and $25 \mathrm{~m} \mathrm{~s}^{-1}$, an earlier speed when the wave had larger amplitude (section 3.c.2). We note that $h_{s}-1 \mathrm{~km}$ can be taken as a reasonable estimate for the depth of the waveguide layer. As can be seen from Fig. $2 b$, a region where $\ell^{2}$ is negative and hence where upward-propagating waves would be reflected (Gossard and Hooke 1975) is present throughout the lifetime of the disturbance. Thus wave energy should be trapped below $1 \mathrm{~km}$, except for the portion that tunnels through the height interval of imaginary $\ell$ (Charney and Drazin, 19.61). 
Only the extremely long wavelength components of the wave can tunnel through the layer of imaginary $\ell$ to be lost to the upper atmosphere. But data presented in section 3.c.5 suggest this loss is negligible. To lend theoretical support to this observation, we computed the reflection coefficient $R$ at the top of the lower stable layer using the three-layer model and linear theory of Lindzen and Tung (1976) in which the evanescent middle layer of finite thickness is the lifted neutral layer. The stability (i.e., $\mathrm{N}^{2}$ $\approx 1.2 \times 10^{-4} \mathrm{~s}^{-2}$ ) of the topmost layer is derived from the mean gradient of temperature (Fig. 1) above the hydrostatically neutral stable layer where the mean wind is about $10 \mathrm{~m} \mathrm{~s}^{-1}$. A reflection coefficient of 0.85 is considered to form a good wave-guiding duct and is perfect if $\mathrm{R}$ equals unity. The Scorer parameter in each layer is taken to be a constant equal to the average in that layer. Thus, for $c-12 \mathrm{~m} \mathrm{~s}^{-1}$, the average $\ell^{2}$ (Fig.2b) in the first kilometer is about $3 \times 10^{-6} \mathrm{~m}^{-2},-1 \times 10^{-6} \mathrm{~m}^{-2}$ in the $1.5-\mathrm{km}$-thick middle layer, and $30 \times 10^{-6}$ $\mathrm{m}^{-2}$ in the upper layer. Applying the estimated values of the Scorer parameter in each of the regions to the formulation of Lindzen and Tung (1976), we compute $R-0.97$. If $\ell$ above $2.5 \mathrm{~km}$ were zero, $R$ would be equal to 1.0 . Since these calculations indicate the loss of guided energy to the upper atmosphere is negligible, for the sake of simplicity we assume $\mathrm{N}=0$ everywhere above $1 \mathrm{~km}$ so that $\ell$ is also zero above $1.5 \mathrm{~km}$.

\section{b. Wind perturbations observed with tower and surface instruments}

The wave propagated in a southeasterly direction, and its front was oriented along an azimuth of about $45^{\circ}$ at the time it passed the tower (Fig. 1 of Doviak and Ge 1984). A time series of data spaced $10 \mathrm{~s}$ apart is plotted in Fig. 3 to show the temporal dependence of wind components $u$ and $v$ (orthogonal 
to and along the front) at five heights. If we ignore the contribution of storm outflow (i.e., the shaded areas in Fig. 3b; see section 3.b.2), only at lower altitudes (i.e., $z<176 \mathrm{~m}$ ) do these data exhibit a definite, singlecrested velocity perturbation, which is confined almost entirely to the northwest-southeast cross section.

1) THE COMPONENTS OF WIND CHANGE AND THE PLANE OF CIRCULATion

The wind field can be analyzed in terms of six components:

$$
\vec{v}-\vec{v}_{0}+\Delta \vec{v}_{0}+\vec{v}_{2}+\vec{v}_{t o}+\vec{v}_{K H}+\vec{v}_{t}
$$

where $\vec{v}_{0}$ is the ambient wind; $\Delta \vec{v}_{0}$ is the change caused by the two-dimensional wave when it vertically transports different amounts of $\vec{v}_{0}$ momentum because of $\vec{v}_{0}$ shear; $\vec{v}_{2}$ is the residual, two-component (i.e., $u_{2}$ and $w_{2}$ ) wave-induced wind perturbation, including contributions from recirculating flow, which remains after $\Delta \vec{v}_{0}$ components have been removed; $\vec{v}_{\text {to }}$ is the axial (i.e., parallel to the wave crest) component of the trapped thunderstorm outflow; $\vec{v}_{\mathrm{KH}}$ is the velocity of well-defined, short, wave-like perturbations assumed to be $\mathrm{K}-\mathrm{H}$ waves; and $\vec{v}_{t}$ is the contribution from turbulence. In their analysis of buoyancy wave effects of turbulence, Einaudi and Finnigan (1981) made a decomposition of velocity similar to that of (3.3). Although the decomposition of wind into a linear sum is questionable with nonlinear waves, it is reasonable if $\vec{v}_{K H}$ and $\vec{v}_{t}$ are much smaller than $\vec{v}_{2}$, as data suggest they are. Then the $\vec{v}_{2}$ wave should modulate these weaker components, as appears to be evident for the $\mathrm{K}-\mathrm{H}$ waves seen in Fig. $3 \mathrm{a}$ at the $7 \mathrm{~m}$ level. 
Support for the $\mathrm{K}-\mathrm{H}$ wave interpretation is obtained by comparing the theoretical estimate of $\mathrm{K}-\mathrm{H}$ wavelength with that observed. Using the theory presented by Stoeffler (1972), and data in Fig. 2a, we compute a 500-m effective depth of the shear layer and a wavelength for maximum rate of $\mathrm{K}-\mathrm{H}$ wave growth of about $1500 \mathrm{~m}$. This length compares well with the roughly $1300-\mathrm{m}$ wavelength deduced from Fig. 3a (at $z-7 \mathrm{~m}$ ) using the wave speed of $12 \mathrm{~m} \mathrm{~s}$ reported in section 3.c.2.

The weakly nonlinear wave theory presented in section 2 gives an expression for the perturbation u component (i.e., $\psi_{z}$ in eq. (2.23a)) equal to $\mathrm{u}_{2}+\Delta \mathrm{u}_{0}$. Here, however, we separate the contribution from ambient shear because first-order theory assumes that the vertical displacement $\delta$ caused by the wave, although finite, is small and that the ambient wind is essentially uniform in the vertical interval of wave displacement. Because data show that the ambient wind shear is strong and displacements are not small, we estimate $u_{2}$ by subtracting $\Delta_{0}$ from the total wave perturbation (i.e., $u-u_{t}-u_{K H}-u_{0}$ ). We hypothesize that $u_{2}$ estimated in this way gives a more accurate value of wave amplitude for comparison with first-order theory.

The dashed, double-dotted curves (i.e., $u_{0}+\Delta_{0}+u_{2}$ ) in Fig. 3a are subjectively estimated to filter turbulence and the solitary-wave-induced $\mathrm{K}-\mathrm{H}$ disturbances. We calculate $u_{0}+\Delta u_{0}$ and $v_{0}+\Delta v_{0}$ (i.e., those given by the dashed curves) using the actual wind profiles and the displacements determined from equivalent potential temperature $\theta_{e}$ contours displayed by Doviak and Christie (1989, their Fig. 5). These authors provide convincing evidence that the contributions $\Delta u_{0}$ and $\Delta v_{0}$ are due to the vertical displacement of horizontal momentum. Thus, as can be seen in Fig. 3b, practically all the 
perturbation in $v$ is explained by $\Delta v_{0}$ and $v_{t_{0}}$, demonstrating that wave circulation $u_{2}, w_{2}$ lies entirely in the northwest-southeast plane.

\section{2) DYNAMICS OF TRAPPED THUNDERSTORM OUTFLOW}

The shaded areas in Fig. $3 \mathrm{~b}$ are those perturbations hypothesized to be associated with trapped thunderstorm outflow. The arrows in Fig. $3 \mathrm{~b}$ indicate the arrival time of trapped outflow estimated from the $\theta_{e}$ plot of Doviak and Christie (1989, their Fig. 5). The onset of wind perturbations (the shaded areas) agrees perfectly well at all heights with the location of the trapped air inferred from the $\theta_{e}$ contours, thus supporting our contention that the portion of axial flow shaded in Fig. $3 \mathrm{~b}$ is due to trapped thunderstorm outflow.

Two new and interesting features of thunderstorm-generated solitary waves are revealed by the observations presented in Fig. 3b. First, the observed axial component of trapped thunderstorm outflow in the main body of the wave shows that outflow is guided along the axis of the wave away from the thunderstorm. Second, the component $v_{t o}$, lingering after wave passage, gives evidence to show that trapped outflow leaks continuously from the rear of the wave at altitudes below $90 \mathrm{~m}$.

\section{i. $\quad$ Guided Outflow}

We hypothesize that flow of trapped fluid along the wave axis is due primarily to the influence of the Coriolis force. The earth's rotation has only a minor influence on the properties of weakly nonlinear atmospheric waves (Grimshaw, 1985), because the influence of the Coriolis force acting on fluid particles in small-amplitude waves is limited to the relatively short wave 
interaction time, $t \approx 2 \lambda c^{-1}$. This situation is changed substantially, however, when regions of closed circulation appear in the relative streamline pattern of large-amplitude waves. Recirculating air parcels trapped in the interior of the southeastward-propagating wave are now continuously subjected to the Coriolis force, with the result that the trapped air parcels follow a clockwise path (Northern Hemisphere), thus leading to the appearance of an axial outflow component $v_{a}$ in the wind field below $450 \mathrm{~m}$.

The solution of the general transport equations for this problem, which includes the effects of a pressure gradient along the axis of the wave and turbulent frictional damping, is nontrivial. However, if we assume that the pressure gradient and frictional forces can be neglected to first order, an estimate of the axial wind component at the time the wave passed the tower can be determined from $v_{a} \approx-2 \Omega u \Delta \sin \phi_{L}$, where $\Delta t(\approx 53 \mathrm{~min})$ is the time taken for the wave to propagate from its origin to the tower, $a$ is an estimate of the mean trapped outflow wind component in the direction of the ray path, $\Omega$ is the angular velocity of the earth, and $\phi_{L}$ is the latitude. For 0 we substitute the mean wave speed of about $16 \mathrm{~m} \mathrm{~s}^{-1}$, estimated from observations presented in of section $4 \mathrm{a}$, and compute $v_{a} \simeq-4.3 \mathrm{~m} \mathrm{~s}^{-1}$, which is close to the observed value, $v_{\text {to }} \approx-4.0 \mathrm{~m} \mathrm{~s}^{-1}$. We therefore conclude that the observed transport of thunderstorm outflow along the axis of the wave in the southwesterly direction is predominantly a manifestation of the influence of the Coriolis force on the dynamics of the wave. 


\section{ii. Leaking of trapped outflow}

Evidence of leaked thunderstorm outflow can be seen in the wind and temperature data at surface sites displayed in Fig. 4a (see Station C for the key to interpret this figure). The recorded wind and temperature data are 1min averages of samples spaced $10 \mathrm{~s}$ apart. Figure $4 \mathrm{~b}$ shows a sample data set from Station A.

In all cases, temperature did not recover within 30 or more minutes after wave passage, and the flow behind the wave was away from the wave front at all heights in a coordinate frame moving with the wave. This latter observation alone suggests that the shallow pool of denser air is trapped outflow leaking out the rear of the wave and is not a manifestation of a gravity current. Conclusive support for this suggestion is found in the fact that the evolving wave propagates at speeds faster than the current behind it by much more than $10 \mathrm{~m} \mathrm{~s}^{-1}$. This is in sharp contrast with the computed frontal speed of less than $3 \mathrm{~m} \mathrm{~s}^{-1}$ for a density current with the same depth as the shallow pool of cooler air behind the wave (Doviak and Ge, 1984). Thus the wave, in less than $10 \mathrm{~min}$, would have left the density current behind by more than $6 \mathrm{~km}$. However, the position of the advancing temperature front, which marks the leading edge of the shallow pool of denser air near the surface, was observed to correspond with the position of the wave for at least 20 min (i.e., the time it took the wave to propagate from station A to the tower). Thus we are led to conclude that the denser air behind the wave is there because the wave leaked trapped outflow air.

Remarkably, there was no discernible change in temperature at sites $\mathrm{E}$ and $F$, suggesting that all the trapped outflow had leaked from the wave before it reached those two sites. Furthermore, there was little or no residual wind 
change at these sites. Besides losing trapped outflow before reaching sites $\mathrm{E}$ and $F$, the wave had less amplitude than it had at sites farther north. We thus conclude that the continuous depletion of trapped air from the interior of the wave caused a concomitant decrease of wave amplitude. This interpretation is consistent with the laboratory observations of Maxworthy (1980), who showed that recirculating fluid trapped in the interior of a large-amplitude, evolving solitary wave is slowly ejected and deposited at the surface in the wake of the wave.

The fact that a shallow pool of cool air is deposited at the surface in the wake of a large-amplitude solitary wave with recirculating flow suggests it is quite likely that some observed gust fronts, which have traditionally been identified from data obtained with surface instruments as thunderstorm density currents, may in fact be manifestations of one or more evolving largeamplitude solitary waves transporting thunderstorm outflow.

\section{3) EROSION OF THE WAVE GUIDE}

We were unable to discern a solitary wave or any step decrease in temperature in the data from Stations $G$ and $H$. On the contrary, at Station $G$, temperature and windspeed increased (from $22.2^{\circ}$ to $24.2^{\circ} \mathrm{C}$ and from 0 to about $3.5 \mathrm{~m} \mathrm{~s}^{-1}$ from the south) in a step-like way at $2330 \mathrm{CST}$. No changes in wind or temperature were observed at site $\mathrm{H}$. If the wave continued to propagate at the roughly $10 \mathrm{~m} \mathrm{~s}^{-1}$ speed it had when it passed site $D$, it would have arrived at site $\mathrm{G}$ at about $2338 \mathrm{CST}$, or $8 \mathrm{~min}$ after the beginning of the observed step-like temperature increase. Our explanation for the increases in temperature and wind is that they were due to an erosion from above of the layer of dense air that had been deposited at 2142 CST by the outflow from the 
earlier thunderstorms. The northerly outflow density current decreased the temperature at this time by about $3.0^{\circ} \mathrm{C}$. The ambient flow above this density current is southerly. We hypothesize that shear instabilities caused the warm southerly ambient airstream to mix downward and erode the inversion layer in $1 \mathrm{~h}$ and $50 \mathrm{~min}$, thus destroying the stable waveguide layer that is required for the solitary wave to propagate to sites $G$ and $H$.

\section{4) WAVE CHARACTERISTICS FROM SURFACE AND TOWER DATA}

The wind data at surface sites A,C, D, and the tower show evidence of a second, much weaker wave following the leading large-amplitude wave. The time separation of the two waves at stations $A$ and $C$ is about $15 \mathrm{~min}$, whereas at sites $D$ and the tower it is about 25 to $30 \mathrm{~min}$. This increasing separation for wave positions later is expected for nonlinear waves whose speed is faster for larger amplitudes. Furthermore, the Doppler radar display at 2245 CST, shows evidence of a second, much weaker wave displaced about $12 \mathrm{~km}$ northwest of the leading wave. The leading wave was at this position at 2231 CST or about 14 min before the arrival of the second wave, confirming the inference made from the surface station observations.

The separation between the traces $u_{0}+\Delta u_{0}$ and the dashed double-dotted traces $u_{0}+\Delta u_{0}+u_{2}$ in Fig. 3a gives estimates of $u_{2}$, the wave component to be compared with the first-order theory. The amplitude of $u_{2}$ and the wave's half-amplitude width $\ell_{1 / 2}$ are plotted in Fig. 5. The half-amplitude width is determined from the $11.5 \mathrm{~m} \mathrm{~s}^{-1}$ speed (estimated in section 3.c.2) for the wave when it passed the tower. Above the $200-\mathrm{m}$ altitude, the wave amplitude decreased monotonically, as expected from theory (section 2). The decrease in $u_{2}$ below $150 \mathrm{~m}$ is attributed to the drag caused by the terrain or mixing of 
air by convective instabilities that develop when cool air is atop warm ground. Without the effects of drag, wave amplitude at the surface would have been $14.5 \mathrm{~m} \mathrm{~s}^{-1}$ (see dashed curve on Fig. 5). The dashed curve is an extension based on the eigenfunction solution of weakly nonlinear theory and discussed in more detail in section 4. Although the data above $150 \mathrm{~m}$ extend over too short a height interval to give confidence that they confirm the eigenfunction form, the velocity data are at least consistent with this shape. The fact that amplitude, $u_{2}(0, z)$, exceeds the wave propagation speed $c=$ $11.5 \mathrm{~m} \mathrm{~s}^{-1}$ provides additional evidence that trapped air is recirculating when it passes the tower.

Weakly nonlinear wave theory shows that half-amplitude width, $\ell_{1 / 2}=2 \lambda$, should be constant within $h_{s}$, and above the stratified layer it should slowly increase with height [see (2.17) and (2.24d)]. The presence of recirculating denser air will cause the wave to be broader near the surface, and thus the decrease of $l_{1 / 2}$ with height is attributed to the decrease in the amount of trapped outflow. Since first-order theory does not account for the effects of dense recirculating air, we assume that $\ell_{1 / 2}$ measured above the trapped outflow is the wave width to be used when observations are compared with weakly nonlinear wave theory.

c. Wave characteristics observed using Doppler radar

Although the tower data give high-resolution, in situ velocity measurements, Doppler radar can measure the radial component of velocity over a large area and provide a direct measure of wave speed and evolution. 
The observations described by Doviak and Ge (1984) show that the orientation of the slightly curved wave front slowly varies with time as the wave propagates away from the path of the storm. Curved wave fronts complicate our comparison with theory, which assumes that velocity perturbations are in the plane orthogonal to straight wave fronts. To proceed, we need to decide whether to define the wave characteristics at all times along directions perpendicular to the wave front or along the direction defined by the wave circulation plane. Wave characteristics (speed, length, etc.) are crucially dependent on this choice, which can strongly affect our comparison with theory.

It is natural to define wave characteristics along lines orthogonal to the wave front. But if the wave contains flow circulating in planes, then it also seems reasonable to expect energy to propagate in straight lines defined by these planes, because angular inertia of circulation would resist change in direction. There would have to be an external force, other than gravity, acting on these circulations to turn the axis of rotation. The Coriolis force could have a minor influence on the orientation of these circulations. Since gravity alone cannot cause the axis to turn, we hypothesize that, at least to first order, the wave circulations are confined to planes with constant orientation.

Supporting this contention are meteorological data (Fig. 4) at the surface sites, which show that wave circulations remain in a plane whose direction is within a few degrees of the $318^{\circ}$ mean value over the entire sampled length of the wave even though wave-front direction changed by at least $20^{\circ}$. If wave energy travels in planes of circulation with constant 
orientation, then rays of energy paths would be straight, although the speed of energy propagation would depend on the local characteristics (i.e., wave amplitude, inversion thickness, etc.) along the ray. It is not readily apparent from Fig. 3 that the wave circulations are entirely in a plane, but the analysis of wind perturbations given in section 3.b.1 shows that wave circulations are wholly within the northwest-southeast plane, and hence the ray direction appears $A^{\text {to be southeastward. }}$

2) WAVE SPEED

To determine wave speed as a function of time along the southeastward ray passing through the tower site, in Fig. 6 we plotted the wave-front position observed with Doppler radar along this path. The wave speed decreases as the wave propagates, and the rate of decrease is less at later times when the wave speed is approaching a steady-state value slightly less than $12 \mathrm{~m} \mathrm{~s}^{-1}$.

The solitary wave speed given by theory is the group velocity of a wave packet along a ray because the wave comprises a group of spectral components that form the packet. Therefore, the comparison with theory should be made with wave characteristics measured along the ray direction irrespective of the wave-front orientation. Doviak et al. (1989) showed that the wave-front curvature and location of a large amplitude wave can be estimated given the amplitude-dependent speed and the single direction of rays along which the disturbance propagates. The assertion that rays (i.e., energy paths) are not orthogonal to the wave-front might be surprising because the intuitive feeling is that wave-fronts and rays are perpendicular to each other, but this is not necessarily so (e.g., see Whitham, 1974, Ch. 14, Sec. 7.9). 
At 2245:30 CST the radar beam intersects the wave to the northwest at ranges of about $50 \mathrm{~km}$. This is the latest time for which we have digitally recorded Doppler data from which to derive quantitative estimates of velocity profiles along the beam. In Fig. 7 we present the radial component $v_{r}$ of the wind deduced from Doppler velocity measurements along the beam through the wave. Each curve consists of Doppler velocity data averaged over a $4^{\circ}$ sector centered at azimuth $\phi_{C}$ for a beam elevation angle of $0.4^{\circ}$. The averaged profiles are plotted along a distance scale that is relative to the location of the wave's peak reflectivity used to obtain a reference distance. The vertical bars on Fig. 7 denote the standard deviations of the estimates.

To relate tower and radar measurements, we need an estimate of effective beam height at the range $r_{p}$ corresponding to the position of the wave peak. The effective beam height $h_{e}$ is the altitude at which the measured, powerweighted, mean Doppler velocity (Doviak and Zrnic', 1984, Ch. 6) needs to be placed to have agreement with the actual radial velocity at that height. The methods used to deduce $h_{e}$ are given by Doviak and Chen (1988). Values of $h_{e}$, $\phi_{c}$, and $r_{p}$ are tabulated in Table $I$ along with parameters defined in subsequent sections. 
Table I. Wave characteristics along the front.

$\begin{array}{ccccccccc}\phi_{c} & v_{r o} & r_{p} & h_{e} & v_{r p} & u_{2}\left(0, h_{e}\right) & \phi_{F} & l_{1 / 2} & t_{e} \\ (\operatorname{deg}) & \left(\mathrm{m} \mathrm{s}^{-1}\right) & (\mathrm{km}) & (\mathrm{m}) & \left(\mathrm{m} \mathrm{s}^{-1}\right) & \left(\mathrm{m} \mathrm{s}^{-1}\right) & (\mathrm{deg}) & (\mathrm{km}) & (\mathrm{CST})\end{array}$

$\begin{array}{lllllllll}331.2 & 7.1 & 46.8 & 430 & -7.0 & 7.3 & 41 & 3.2 & 2256: 30 \\ 339.8 & 8.0 & 50.3 & 440 & -8.0 & 8.8 & 38 & 3.1 & 2250: 30 \\ 347.0 & 9.8 & 56.2 & 500 & -10.0 & 11.8 & 32 & 3.2 & 2243: 30 \\ \text { TOWER } & \ldots . . & \ldots .- & 444 & \ldots \ldots & 6.4 & \ldots & 3.2 & 2311\end{array}$

4) Estimates of WAVE AMPLitude AND HALF WidTH

Since the tower-measured $u_{0}+\Delta u_{0}$ waveform (dashed line in Fig. 3a) inferred from the $\theta_{\mathrm{e}}$ contours and ambient wind profiles agrees reasonably well with the leading and trailing edges of the observed waveform and then abruptly becomes constant at zero during most of the wave period when $u_{2}$ has appreciable value, we can reasonably expect that the radar-measured $u$ waveform must have similar characteristics. Evidence shows that the wave had larger amplitude earlier, and hence zero-momentum air from low altitudes would have been brought to even greater heights than $444 \mathrm{~m}$. So we should expect that $u_{0}+\Delta u_{0} \approx 0 \approx v_{0}+\Delta v_{0}$ for much of the wave period during the radar observations. Trapped outflow reaches the beam, but its velocity $v_{\text {to }}$ is likely to be less than a few meters per second at the heights where it intersects the beam because Fig. $3 \mathrm{~b}$ shows that $v_{\text {to }}$ decreases rapidly with height. Furthermore, because the beam is directed to the northwest, the weight that $v_{\text {to }}$ gives to the Doppler velocity $v_{r}$ is less than that received from $u_{2}$. Therefore we ignore its contribution to $v_{r}$, so that the observed peak Doppler velocity $v_{r p}$ (Fig. 7) equals approximately the projection of $u_{2}\left(0, h_{e}\right)$ onto the beam direction. Thus we can 
estimate $u_{2}\left(0, h_{e}\right)$ from $v_{r p}$ measurements. These estimates of $u_{2}\left(0, h_{e}\right)$ along each azimuth $\phi_{C}$ are given in Table I. However, the total Doppler velocity change is $v_{r p}+v_{r o}$ where $v_{r o}$ is the ambient Doppler velocity, which also is the perturbation caused by the vertical transport of horizontal momentum.

The half-amplitude width $l_{1 / 2}$ can be estimated from Fig. 7 , in which $u_{2}$ is related to the negative portion of $v_{r}(r)$. Thus from the geometrical arrangement of the sectors centered at azimuth $\phi_{c}$ and the orientation of the wave fronts (see Fig. 6 of Doviak and Christie, 1989), we determine that

$$
\ell_{1 / 2}-\ell_{r} \frac{\sin \left(\phi_{F}-\phi_{C}\right)}{\sin \left(135-\phi_{F}\right)}
$$

where $\ell_{r}$ is the half-amplitude width deduced from Fig. 7 along the azimuth $\phi_{C}$, and $\phi_{F}$ is the azimuth of the wave front at its intersection with the radar beam.

Values of $l_{1 / 2}$ and $\phi_{F}$ (Table I) show that the wave width is relatively constant along the front, whereas $u_{2}\left(0, h_{e}\right)$ shows a significant decrease away from the source. These $l_{1 / 2}$ values agree with those measured at the $444-\mathrm{m}$ tower level (Fig. 5).

\section{5) TIME DEPENDENCE OF WAVE AMPLITUdE}

To further compare tower and radar observations, we note that points along the wave front farther from the storm correspond to older portions of the wave. Thus we can compare wave amplitudes observed at the tower with those observed by radar at 2245:30 CST. An equivalent time scale along the wave front can be deduced by noting that the wave source (i.e., the storm) 
travels in the advecting ambient air at a uniform speed $\left|\vec{V}_{s}^{\prime}\right|$ of about $23.6 \mathrm{~m} \mathrm{~s}^{-1}$ in the direction $\phi_{S}^{\prime}=80.7^{\circ}$ (Doviak and Chen, 1988), and that elements of the wave front travel along straight rays in a southeasterly direction $\left(135^{\circ}\right)$, as inferred in section 3.c.1 (the primes denote variables in a frame advecting with the wind). Then it can be shown that

$$
d t_{e} \simeq \frac{d \ell}{v_{s}^{\prime}} \frac{\sin \left(135-\phi_{\ell}\right)}{\sin \left(135-\phi_{s}^{\prime}\right)}
$$

where $\mathrm{d} l$ is the element of distance along the front, measured relative to the reference point (i.e., the intersection of the front at 2245:30 with the ray passing through the tower site), $d t_{e}$ is the equivalent increment in time, and $\phi_{\ell}$ is the azimuth of a line connecting the reference point to the intersection of the beam (at $\phi_{c}$ ) with the wave front. The equivalent time increment $d t_{e}$ is then measured relative to $2245: 30$, the time the radar observes the peak of the wave-front element that passes through the tower about $25 \mathrm{~min}$ later. Since $\phi_{\ell}$ is roughly a constant equal to about $36^{\circ}$ (see Fig. 6 of Doviak and Christie, 1989), we deduce the equivalent times $t_{e}-2245: 30+d t_{e}$ listed in Table I. The amplitude, $u_{2}(0,444)-6.4 \mathrm{~m} \mathrm{~s}^{-1}$, of the wave as 1 passed the tower is also listed in Table I because the $444-\mathrm{m}$ tower level is almost at the same height as the effective height $h_{e}$ of the Doppler radar observations. For better comparison of wave amplitudes, we plotted $u_{2}\left(0, h_{e}\right)$ on Fig. 8 to show that the wave amplitude decreases with time, losing about one-half its value in less than $30 \mathrm{~min}$. Although we do not have radar data for the time after the wave passes the tower, this figure suggests that wave amplitude is nearly constant after $2300 \mathrm{CST}$, consistent with the observations (Fig. 6). 
4. Comparison with theory

We compared weakly nonlinear wave theory with observations using a direct as well as an indirect approach with the aid of the wind decomposition given in section 3.b.1. In the direct approach, we simply determine $u_{2}+\Delta u_{0}$ from the numerical solution to the eigenvalue problem (2.7) corresponding to the ambient stability and shear profiles specified by (3.1) and (3:2).

In the indirect approach, we solve the simplified eigenvalue problem in the absence of background shear to get a theoretical estimate for $u_{2}$, which then may be compared independently with the observations.

The justification for the indirect approach rests on the assumption that the decomposition of the wind field into the individual components defined by (3.3) is valid. The findings of Tung et al. (1981) (although for a specific example) showed that if the initial condition for the equation of wave evolution is a solitary wave for shearless media, the wave evolution in the presence of shear is, except for a faster linear wave speed, the same as for the shearless case. Since our indirect approach effectively removes the influence of background shear from the problem, this approach may result in better comparisons of observations with weakly nonlinear wave theory when shear is large.

\section{a. Indirect approach}

Because wave amplitude and speed change with time, the wave is obviously not of permanent form. Although Fig. 8 shows $u_{2}(0, h)$ decreasing with time, it appears to approach a steady value asymptotically. We hypothesize that this decrease is caused by the loss of recirculating air that leaks out the rear of the wave. When the reservoir of recirculating fluid is depleted, the wave 
becomes steady if radiation and friction losses are negligible, as Fig. 8 suggests. Thus the wave seems to be nearly of permanent form at the time it passes the tower.

On the other hand, even though there are times when the wave is not steady there is value in comparing observed wave characteristics with those deduced from steady-state theory to determine whether these characteristics adjust as energy is lost in such a way as to be in reasonable accord with theory. If losses are sufficiently small and wave amplitude large, the dynamical balance between the nonlinear and dispersive terms in the equations governing wave properties should continue to be effective. Evidence presented in section 3 points to the fact that wave amplitude is large, but the effect of losses on the dynamical balance is not easily estimated.

Koop and Butler (1981) observed solitary waves in the laboratory and found, for waves of modest amplitude, that energy loss due to viscosity can have a noticeable influence on wave characteristics (principally amplitude). Nevertheless, they showed theoretically that for $\delta_{\mathrm{m}} / \mathrm{h}_{\mathrm{s}} \geq 0.05$, the difference in the inverse relation between wave width and amplitude is $\$ 10 \%$. On estimating the attenuation rate per unit distance traveled normalized to the wavewidth in their laboratory experiment and scaling to our observed atmospheric wave, we find that the atmospheric wave had an attenuation rate about double that of the laboratory one. But our estimate of attenuation due to frictional losses is an upper limit because attenuation is also caused in part by the loss of recirculating outflow. Thus the theoretical relation between wave width, amplitude, and speed is followed if our comparisons refer to $\delta_{m} / h_{s}$ larger than about 0.1 . The wave discussed here satisfies this requirement. 
Substituting (3.1) into (2.7), ignoring $u_{0}$, and applying the boundary conditions (2.17), we obtain a second-order eigenfunction

$$
\phi=1.31-0.26 z-e^{-b z}[0.74 \sin (\pi z / 2)+1.31 \cos (\pi z / 2)],
$$

for $0 \leq z \leq 1 \mathrm{~km}$, and eigenvalue

$$
\Gamma^{2}-\mathrm{N}^{2}(0) / \mathrm{c}_{0}^{2} \simeq 14.2 \mathrm{~km}^{-2}
$$

by employing the iterative technique of Stodola and Vianello, which gives functions that converge to the dominant mode eigenfunction (Hildebrand, 1956). The wave guide or lower layer depth is taken to be $\mathrm{h}-1 \mathrm{~km}$ in accord with (3.1).

Solving (4.2) for $c_{0}$ and using (4.1) in (2.18), we numerically integrate to solve for $\alpha$ and $\beta$ to obtain

$$
\begin{aligned}
& c_{0}=10.3 \quad \mathrm{~ms}^{-1}, \\
& \alpha=2.59 \times 10^{-3} \mathrm{~m}^{-1}, \\
& \beta-3.44 \times 10^{3} \mathrm{~m}^{2} \mathrm{~s}^{-1} .
\end{aligned}
$$

Using (2.23d), (2.25), (2.26), and (2.23a) modified to apply the indirect approach (i.e., $u-\Delta u_{0} \equiv u_{0}+\psi_{z}$ ), we can show that wave speed is given by

$$
c-c_{0}+\frac{\alpha u_{2}(0, z)}{4 \phi_{z}(z)} .
$$


So given measurements of $u_{2}(0, z)$, we can estimate $c$. However, the wave-speed estimate, obtained from weakly nonlinear wave theory for a uniform ambient flow model, must recognize that the wave is advected by the background flow $u_{0}$. At low altitudes, where wave energy is concentrated (Fig. 5), $u_{0}$ is negative (Fig. 2a) and hence the ambient wind can slow the wave's speed relative to the ground. The wave would therefore appear to the observer to propagate more slowly. The velocity at which the wave advects is difficult to estimate because wind is nonuniform in height. However, because most of the wave's energy is confined to the lowest half kilometer of the atmosphere, we assume that an unweighted average of ambient wind in this layer determines advection velocity $u_{o a}$. From Fig. 2a we, estimate $u_{o a}$ to be $\approx-2.5 \mathrm{~m} \mathrm{~s}^{-1}$.

Substituting the value of $\alpha$ given by (4.3b), measurements of $u_{2}$ versus time from Fig. 8, and computed values of $\phi_{z}(z)$ derived from (4.1) into (4.4), we obtain estimates of $c$ as a function of time and these, after correction for advection, are plotted as the dashed line in Fig. 9.

By tracking wave-front position along the ray, we deduced the variation in wave speed $c_{t}$ with time. These values are also illustrated in Fig. 9. In the 1 -h period of observation, $c_{t}$ decreased by a factor of 2 and, at the end of the hour, appeared to have been asymptotically approaching a steady value of about $11.5 \mathrm{~m} \mathrm{~s}^{-1}$. The dashed-dotted line shows clearly the monotonic decrease of $c_{t}$ versus time. We see that observations agree well with weakly nonlinear wave theory estimates that have been corrected for advection.

We next compare the wave perturbation $u_{2}(\bar{x}, z)$ computed from theory and that observed by Doppler radar and tower instruments. Wave amplitude is obtained from (2.23a and d), which give, at $\tilde{x}-0$, 


$$
u_{2}(0, z)=\varepsilon \psi_{O} \phi_{z}(z)
$$

for heights $\mathrm{z}$ below $1 \mathrm{~km}$. $\varepsilon \psi_{\mathrm{O}}$ is obtained by solving (2.21c), in which $\lambda_{S} \equiv \varepsilon \lambda$. Substituting this solution into (4.5) we obtain

$$
u_{2}(0, z)=\frac{4 \beta}{\alpha \lambda} \phi_{z}(z)
$$

which relates the eigenfunction $\phi(z)$ and parameters $\alpha, \beta$ of the stratified layer to $u_{2}$. Hence, $u_{2}(x, z)$ can be estimated if $\lambda$ is known. Substituting our theoretical estimates of $\alpha, \beta$ and $\phi_{z}$ and our experimentally derived estimate of $\lambda$ into (4.6), we find that

$$
\begin{aligned}
u_{2}(0, z)- & 3.32\left(-0.26+e^{-b z}\left[4.12 \sin \left(\frac{\pi z}{2}\right)\right.\right. \\
& \left.\left.+2.48 \cos \left(\frac{\pi z}{2}\right)\right]\right] \mathrm{ms}^{-1},
\end{aligned}
$$

where $z$ is in kilometers. At $z-0$, where the wave amplitude should be largest, (4.7) gives

$$
u_{2}(0,0)-7.3 \mathrm{~m} \mathrm{~s}^{-1} \text {, }
$$

which is in good agreement with the observed value of about $7 \mathrm{~m} \mathrm{~s}^{-1}$ at the surface (see Fig. 5). We recognize, however, that the apparently good fit to the wave amplitude is misleading because the observed perturbation winds are attenuated near the surface by frictional processes and this effect has not 
been accounted for in the theory. For example (see Fig. 5), the observed amplitude at $z-100 \mathrm{~m}$ is nearly $12 \mathrm{~m} \mathrm{~s}^{-1}$, about $50 \%$ higher than that at the ground.

b. Direct approach

We now compare the observations with the predictions of weakly nonlinear wave theory with background shear included in order to assess the merits of the indirect approach and to delineate the influence of shear in the ambient flow on the characteristics of nonlinear waves. In this case, we can no longer determine a tractable analytic solution to the Taylor-Goldstein equation (2.7). We solve (2.7) numerically using a standard shooting technique [Press et al. 1986]. This numerical solution is then used in $(2.18 b)$ and $(2.18 c)$ to evaluate the coefficients $\alpha$ and $\beta$. The results of this calculation are

$$
\begin{array}{ll}
c_{0}-10.0 & \mathrm{~m} \mathrm{~s}^{-1} \\
\alpha-4.47 \times 10^{-3} \mathrm{~m}^{-1} \\
\beta=1.59 \times 10^{3} \mathrm{~m}^{2} \mathrm{~s}^{-1} .
\end{array}
$$

A comparison of the eigenfunctions shows that the energy of the wave is concentrated closer to the surface by the background shear, which is consistent with the results, although for a hyperbolic wind profile, reported by Tung et al. (1981). Furthermore, the introduction of shear given by (3.2) has had, in this case, only a minor influence on the linear wave speed. This result does not hold in general. We have found, in agreement with the work of Tung et al., (1981), that the linear wave speed can change significantly 
depending on the detailed form of the shear profile. On the other hand, the inclusion of the shear has led to a significant change in the coefficients of the evolution equation, and these also depend strongly on the detailed form of the shear profile. For example, an alternative analytical expression, which fitted the wind data at least as well as (3.2), nevertheless produced a $c_{0}$ almost one-half, $\alpha$ twice, and $\beta$ one-quarter those obtained using (3.2). These large differences were found to be related to a discontinuity of $l$ at the interface $z-h$. It is therefore concluded that when shear is present, estimates of the BDO equation coefficients are very sensitive to small changes in the wind profile and thus subject to large uncertainties.

The wave propagation speed c (dotted curve in Fig. 9) is determined from (4.4) by using the parameters in (4.9) and the estimate of the total wave perturbation amplitude $u_{2}\left(0, h_{e}\right)+\Delta u_{0}$ from Figs. $3 a$ and 7 . Thus, the direct approach predicts a faster wave propagation speed than observed. We attribute the faster theoretical speed to the inadequacy of first-order perturbation theory for cases in which shear is strong. That is, the observed perturbation, $u_{2}+\Delta u_{0}$, is larger than what first-order theory predicts.

The fact that half-amplitude width $\ell_{1 / 2}$ remains constant while amplitude changes by a factor of 2 (Table I) is also inconsistent with (2.21c), which indicates $\Lambda^{\text {waves }}$ have half-amplitude widths that depend inversely on amplitude. We attribute these discrepancies to the premise that the wave was strongly nonlinear. Nevertheless, when the wave appeared to approach a steady-state condition with a wave speed of about $11.5 \mathrm{~m} \mathrm{~s}^{-1}$ (Fig. 6), $\lambda$ computed from (2.25) was $1.1 \mathrm{~km}$, in fair agreement with the observed value of $1.6 \mathrm{~km}$. Moreover, we found that when we used an analytically more complicated wind profile, which provided a better fit to the data below $700 \mathrm{~m}$, 
$c_{0}$ and $\beta$ increased $\left(10.2\right.$ and $1.63 \times 10^{3}$ ) so that the theoretically estimated value of $\lambda(1.3 \mathrm{~km})$ was even closer to that observed and in better agreement than the value $(880 \mathrm{~m})$ estimated using parameter values obtained via the indirect method. Thus the direct method appears to give a better estimate of wavelength but a worse estimate of speed.

\section{c. Comparison with strongly nonlinear theory}

The results presented in the preceding section support the view that the wave is strongly nonlinear. Thus, we are naturally led to make comparisons with the few available numerical solutions for large-amplitude waves of permanent form. Tung et al. (1982) presented numerical results that relate stream-function amplitude $\varepsilon \psi_{0} \equiv \psi_{\max }$ to wave speed $c^{\prime}$ (wave speed in the coordinate frame advecting with the fluid) for fluids of various depths $H$, topped by a rigid lid. Although they solved the problem for waves propagating in a medium without shear and in which the vertical profile of density change is described by a hyperbolic tangent function, the results should be similar to those presented in section 4 .a if $H \gg h_{s}$ and if $h_{s}$ and the net change in $N^{2}(z)$ are the same as for our exponential fit to the data. The hyperbolic tangent function that best fits the $\theta_{\text {vo }}$ data is

$$
\theta_{\mathrm{vo}}=294.4\left(1+4.755 \times 10^{-2} \tanh \mathrm{sz}\right)
$$

where

$$
s-2.07 \times 10^{-3} \mathrm{~m}^{-1}, \quad h_{s}-1 / \mathrm{s}
$$

We used the numerically evaluated normalized streamfunction amplitude $\left(\psi_{\max } / \mathrm{c}^{\prime} \mathrm{h}_{\mathrm{s}}\right)$ values in Tung et al. (1982; their Fig. 14, H $\left.-40 \mathrm{~h}_{\mathrm{s}}\right)$ and 
replotted them in Fig. 10 versus the wave speed $c^{\prime}$ normalized to the linear wave speed $c_{0}$ for the hyperbolic tangent profile. Interestingly, the wave speed for strongly nonlinear waves shows a linear dependence on normalized stream function amplitude, and is in good agreement with weakly nonlinear wave theory for normalized amplitudes as large as 1 . The linear dependence can be expressed as

$$
\frac{c^{\prime}}{c_{o}} \simeq 1+0.455 \frac{\psi_{\max }}{c^{\prime} h_{s}}
$$

Thus, given an observed solitary wave speed and ambient conditions (from which eacily

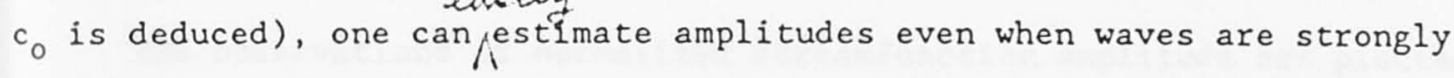
nonlinear.

To place our observations in Fig. 10, we need to estimate $\psi_{\max }$. We assume that as wave amplitude increases, the stream function retains the same functional form suggested by nonlinear wave theory. This may not be a bad assumption because the $u_{2}(0, z)$ data for $z>150 \mathrm{~m}$ are consistent with the $u_{2}(0, z)$ (dashed line on Fig. 5) derived from the eigenfunction form based on weakly nonlinear wave theory. With this assumption we can estimate $\psi_{\max }$ from the $\mathrm{u}_{2}\left(0, \mathrm{~h}_{\mathrm{e}}\right)$ measurements made by radar or tower anemometers.

The amplitude of the perturbation wind given by (2.23a) and (2.23d) is 


$$
u_{2}\left(0, h_{e}\right)=\left.\frac{\partial \psi}{\partial z}\right|_{h_{e}}=\psi_{\max } \phi_{z}\left(h_{e}\right)
$$

where $\phi_{z}\left(h_{e}\right)-s \cdot \operatorname{sech}^{2}\left(s_{e}\right)$. Values of $u_{2}\left(0, h_{e}\right)$ and $h_{e}$, obtained from Table I, are substituted into (4.12) to obtain $\psi_{\max }$ at the equivalent times $t_{e}$ in Table I. Corresponding to these $t_{e}$ we obtain estimates of $c$ from Fig. 9, and these are normalized by the linear wave speed $c_{0}=10.6 \mathrm{~m} \mathrm{~s}^{-1}$ for waves propagating in the medium having the stratification given by (4.10). This $c_{0}$ value compares well with $10.3 \mathrm{~m} \mathrm{~s}^{-1}$ deduced for the exponential profile, attesting to the fact that either the hyperbolic or exponential functions fit the $\theta_{\text {vo }}$ data nearly as well.

The observations of normalized streamfunction amplitude are plotted as a function of normalized wave speed in Fig. 10. If the eigenfunction for the strongly nonlinear wave is well described by the analytical form (4.1a) derived from weakly nonlinear wave theory, then $\psi_{\max }$ estimated from data at $\mathrm{h}_{\mathrm{e}}$ - $444 \mathrm{~m}$ should be equal to the estimate computed from data at $266 \mathrm{~m}$.

Figure 10 shows that they are coincident; at the time the wave passed the tower, the normalized streamfunction amplitude computes to be near unity. This value is supported by the fact that the pressure trace (Chen, 1985; Fig. 4) shows evidence of a flattened crest, in accord with theory for the case in which air is barely recirculating in a steady wave, a condition that occurs at normalized amplitudes just above unity. Furthermore, the data in Figs. 8 and 9 show wave amplitude and speed approaching steady values, which is expected when recirculating fluid is depleted by leakage. This is consistent with the interpretation that recirculation is weak. 
We see fair agreement between observations and strongly nonlinear wave theory, but the data plotted on Fig. 10 suggest that observed wave speed $c$ ' is consistently less than that given by theory. Nevertheless, our observations agree with numerical simulations conducted by Hase and Smith (1989, Part II, Fig. 6) which show that evolving solitary wave-like perturbations of large amplitude have speeds slower than those determined by (4.11). The data points of highest amplitude depart most significantly from the theoretical line.

This discrepancy can be attributed in part to the breakdown of our hypothesis that the eigenfunction waveform is independent of amplitude when the wave is strongly nonlinear. Tung et al. (1982) and Pullin and Grimshaw (1988) show that the eigenfunction form of internal solitary waves of finite amplitude changes at higher amplitudes. In addition, we have not resolved rigorously the influence of shear on the properties of highly nonlinear waves, and thus a correction for shear based on an estimate of the advection speed is subject to some uncertainty. Furthermore, the theory that leads to (4.11) ignores drag between the recirculating and ambient air as well as boundary friction.

Even though weakly nonlinear wave theory is strictly valid for $\psi_{\max } / \mathrm{ch}_{s} \ll 1$, it is surprising that it gives wave speeds that agree so well with numerical results, even for normalized amplitudes as large as 1.0 or, equivalently, displacements $\delta_{m}$ as large as the scale of stratification.

We should not be led to believe that the good agreement on wave speed alone implies that weakly and strongly nonlinear wave theories are in complete accord for displacements as large as $h_{s}$ because we have not considered wave width. Nonlinear waves of permanent form have amplitude, speed, and half amplitude width $2 \lambda$ that are all interdependent, and the good agreement with respect to wave amplitude and speed does not extend to $\lambda$. 
We plotted, in Fig. $11, \lambda$ (dashed line) computed from (2.25) and (4.3).

We also plotted $\lambda$ data from Table $I$. We note that the observed half-width $\lambda$ is significantly larger than that given by weakly nonlinear wave theory. Others (Drake, 1985; Clarke et al., 1981) also found observed values of $\lambda$ to be larger than those computed from weakly nonlinear wave theory. On the other hand, Noonan and Smith (1985) found observed widths $1 / 2$ or $1 / 3$ smaller than first-order theoretical estimates. To show that larger observed $\lambda$ can be explained in terms of strongly nonlinear wave theory, values of $\lambda$ obtained from this theory for the case $\mathrm{H}=4 \mathrm{~h}_{\mathrm{s}}$ are also plotted in Fig. 11. The relatively constant observed half-width independent of wave speed implies that the wave has recirculation, and data fall within the expected speed range for amplitude-independent wavelength suggested by strongly nonlinear wave theory.

Although $\lambda$ data for other values of $H$ were not available, we did manage to estimate $\lambda$ from streamlines given by Tung et al. (1982, Fig. 16b) for the case $H-40 h_{s}$ (the $\otimes$ in Fig. 11). Thus, we find that the observed wavelengths lie between the $\mathrm{H}-4 \mathrm{~h}_{\mathrm{s}}$ and the $\mathrm{H}-40 \mathrm{~h}_{\mathrm{s}}$ estimate. The difference between observations and the prediction from strongly nonlinear wave theory for a deep fluid can be explained by the fact that the nonlinear wave solution is very sensitive to slight changes in the ambient stability profile (Tung et al., 1982). For example, it appears that these differences can be reconciled by assuming that the changes that occur in wavelength estimates between different stability profile models in weakly nonlinear wave theory are accurately represented in estimates based on strongly nonlinear wave theory. Thus, using weakly nonlinear wave theory and dividing $\lambda_{H}$, the calculated length of the wave propagating in a medium having $\theta_{\text {vo }}$ described by the hyperbolic fit $(4.10)$ by $\lambda_{E}$, the wavelength defined by the numerical 
solution (4.1) for the exponential fit (3.1), we obtain $\lambda_{H} / \lambda_{E} \simeq 1.2$. Applying this scaling factor to the highly nonlinear case, the value for the wavelength $\left(\lambda_{H} \approx 2.1 \mathrm{~km}\right)$ given by Tung et al. for $\mathrm{H}=40 \mathrm{~h}_{\mathrm{S}}$ should be corrected to $\lambda_{E}=$ $1.8 \mathrm{~km}$, a value that is closer to the observed wavelength.

d. An evolution model for the generation of solitary waves by rapidly moving storms

To this point we have been concerned primarily with the theoretical description of the leading quasi-stationary, large-amplitude solitary wave that dominated the observed disturbance over most of its lifetime. The observational evidence (Doviak and $\mathrm{Ge}, 1984$ ) shows that this leading solitary wave was followed by a second solitary wave of much smaller amplitude, and that both waves originated in the interaction of a rapidly moving thunderstorm with an existing stable layer created by outflow from previous thunderstorms. It is worth emphasizing again that we have been unable to find any trace of a density current in the wake of these essentially isolated solitary waves. The fact that the observed disturbance consists almost entirely of only two wellseparated solitary waves is an important and unusual feature that seems to be characteristic of the nonlinear wave disturbances generated by the rapidly moving storm.

Previous studies (Christie et al. 1979, 1981; Clarke et al., 1981; Noonan and Smith, 1985) focused primarily on the extensive family of amplitude-ordered solitary waves that develops along the leading edge of an undular bore. Christie (1989), in a detailed study of the time-dependent solutions of the BDO equation both with and without frictional dissipation, showed how internal bore waves in the atmospheric boundary layer are transformed into an ordered sequence of solitary waves. This theoretical 
treatment cannot, however, be applied in its present form to provide an explanation for the formation of solitary waves by rapidly moving storms. The new type of evolution model presented here is based on the numerical solution of the BDO equation for the generation of solitary waves by a thunderstorm moving at supercritical speeds (i.e., faster than $c_{0}$ ).

Because the intense thunderstorm downdraft had a relatively short horizontal dimension (about $5 \mathrm{~km}$ ) and the storm was propagating rapidly $\left(\mathrm{v}_{\mathrm{S}}=\right.$ $24 \mathrm{~m} \mathrm{~s}^{-1}$ ), the stably stratified boundary layer was perturbed at a given point by the passing storm for only a short period of time. Because the storm velocity exceeded the speed of propagation of solitary waves, the wave fronts of nonlinear waves that are generated by the disturbance are aligned at an angle to the storm path given by a modified Mach relation (Doviak and Chen, 1988). Wave energy is then propagated (section 3.c.1) away from the storm path along the ray direction. Since the characteristics of the disturbance are to be measured along the ray direction, it is along this direction that we follow the development of the disturbance as it evolves in time according to the governing equation (2.18), subject initially to prescribed boundary conditions determined by the characteristics of the storm.

We consider only a very simple model for this evolution process; losses caused by both boundary friction and radiation into the upper atmosphere are ignored. This simple model, nevertheless, captures essential features observed in the evolution of this disturbance. The initial perturbation of the boundary-layer waveguide is accounted for by assuming that the onset of outflow at the surface from the passing thunderstorm forces the height of the inversion layer at the origin of a ray path to increase linearly over a period of time $t_{i}$ from the ambient value $h-1000 m$ to a height $h+A_{0}$. We then 
suppose that this elevated inversion level is maintained over a period $t_{m}$ by storm outflow. Finally, the perturbation is terminated as the storm departs from the origin of the ray path in question by allowing the inversion to return linearly over a period of time $t_{f}$ to the original unperturbed level.

This process is incorporated into the theoretical framework described by the BDO equation (2.18) by integrating the equation numerically on the halfline subject to the application of these boundary conditions to the first grid point at the left-hand side of the computation domain over the period when the thunderstorm perturbation is active. The results of this calculation are presented in dimensional form in Fig. 12 in an absolute frame of reference. The amplitudes and number of solitary waves depend primarily on the perturbation amplitude, $A_{0}$, and the mean time over which the source perturbation is active; the detailed form of the perturbation has only a minor influence on the properties of the excited waves. The results illustrated in Fig. 12 are based on the evolution equation coefficients given in (4.9) with $A_{0}-300 \mathrm{~m}, t_{1}-1.5 \mathrm{~min}, t_{m}-2.0 \mathrm{~min}$, and $t_{f}-1.5 \mathrm{~min}$. Since the actual value of the source perturbation amplitude $A_{0}$ is unknown, we have chosen $A_{0}$ such that the average speed of the leading solitary wave shown in Fig. 12 is comparable with the observed average propagation speed during the first hour. The transient thunderstorm perturbation of the inversion waveguide layer results in the formation of a large-amplitude solitary wave followed by a second solitary wave of much smaller auplitude, in excellent agreement with the observations. The observed 15 min separation between the two solitary waves at site A (Fig. 4b) and a 29 min separation at the tower site (Doviak and $\mathrm{Ge}, 1984$, their Fig. 13) is in good agreement with the time separation 
(about $15 \mathrm{~min}$ and $23 \mathrm{~min}$, respectively) indicated by the results of this evolution model calculation.

This calculation also reveals two further features that appear to characterize solitary waves created by relatively short-lived perturbations associated with thunderstorms. First, it is evident that solitary waves form very rapidly and attain large amplitudes in the immediate neighborhood of thunderstorms; second, these waves can have amplitudes that are more that twice the amplitude of the disturbance created initially by the transient thunderstorm outflow.

Although this model appears to give a fairly good description of the principal features in the time evolution of this disturbance, it fails to account for the observed decrease in propagation speed and amplitude with time as the waves propagate away from the source region. We attribute these variations in speed and amplitude to energy losses due to frictional dissipation, the leaking of trapped outflow air, and possibly vertical propagation of wave energy to the upper atmosphere. Nevertheless, despite its limitations, the evolution model described here is in remarkably good agreement with the observations. Thus we conclude that the nonlinear wave theory developed in section 2 provides a reasonably good description of the formation and evolution of solitary waves formed by a rapidly moving thunderstorm that disturbed a stable layer.

\section{Summary and conclusions}

We adapted Ono's (1975) theory for the evolution of weakly nonlinear waves in an incompressible motionless fluid to a compressible fluid with shear and applied this theory to observations of an atmospheric solitary wave. We 
show that Ono's boundary conditions, at the interface of a motionless statically stable lower layer and a neutrally stable upper layer, are exactly the same when shear exists in both the lower and upper layers provided that the Scorer parameter for the upper region is zero.

Analytic solutions to the resulting eigenvalue equation are not easily obtained, especially for vertical profiles of environmental stability and shear encountered in practice. We therefore developed an indirect method that allowed us to separately account for the wind perturbations caused by vertical transport of horizontal momentum in the presence of shear, and perturbations in the absence of shear. This method allowed us to solve, to good approximation, the simpler shearless eigenfunction equation to determine wave parameters.

Observed wave amplitude near the ground and wave speed are in fairly good agreement with estimates from weakly nonlinear wave theory. However, from additional data at higher altitudes we infer that the apparent agreement with first-order theory is fortuitous and can be attributed to the influence of surface friction, which is ignored in the theory. Furthermore, the wave was actually strongly nonlinear.

We then compared our observations and weakly nonlinear wave theory with the numerical solution described by Tung et al. (1982) for strongly nonlinear waves in a shearless environment. The linear relationship between wave amplitude and wave speed given by weakly nonlinear wave theory, agrees surprisingly well with the numerical results for strongly nonlinear waves, even for peak wave displacements as large as 1.3 times the scale of stratification $h_{s}$ (Fig. 10). At larger amplitudes, the solitary wave speed c from numerical solutions is slower than that computed from theory but faster 
than that observed. Although observed wave widths are in reasonable accord with numerical results for strongly nonlinear waves, they are much larger than those given by weakly nonlinear wave theory.

The observations show the evolving solitary wave had trapped recirculating thunderstorm air that was denser than the environment and slowly leaked out from the rear of the wave, leaving a shallow layer of denser air at the surface. The recirculating thunderstorm air remained within the wave for distances of at least $60 \mathrm{~km}$ from the storm. Thus, large-amplitude solitary waves formed by the interaction of a storm's downdraft with a stable layer can transport thunderstorm air faster and with greater momentum over longer distances than an outflow density current would in the absence of the stable layer. We emphasize that the pool of denser outflow air deposited at the surface behind the wave can complicate the interpretation of measurements if they are made only with instruments near the ground, because observations of a temperature drop and wind shift might be interpreted as evidence of an advancing density current.

The observations also show that a portion of the trapped thunderstorm outflow is guided by the wave along its axis and away from the storm. Thus, thunderstorm outflow deposited in a shallow layer at the ground in the wake of the wave is continually replenished by outflow fed into the wave at its origin. A plausible relationship between this axial component and the Coriolis force has been demonstrated.

Finally, we developed a new type of evolution model for solitary waves generated by a rapidly propagating thunderstorm. The interaction of the storm with the existing stable layer is accounted for in the numerical solution of the governing BDO equation by introducing an appropriate time-dependent 
boundary condition at the origin of a ray path. The results of this model calculation show that only two solitary waves, one of very large amplitude followed at some distance by a second of much smaller amplitude, are generated by the passing storm, in excellent agreement with observations.

In a separate case, Doviak et al. (1989) reported Doppler radar and surface array observations of the formation and evolution of two solitary waves along the leading edge of a thunderstorm density current. These waves propagated away from the initiating current, eventually leaving it behind, in good agreement with theoretical calculations. A third case study of evolving solitary waves were also observed to propagate ahead of an initiating thunderstorm density current was recently described by Fulton et al. (1990). The observations presented here are unique in that data from the surface sites shown in Fig. 4 provide no evidence of a thunderstorm driven surface current behind the waves, even $4 \mathrm{~h}$ after wave passage.

The results of this study suggest a scenario for the evolution of the solitary waves (Fig. 13). A rapidly propagating thunderstorm downdraft generates locally a limited outflow current with a horizontal rotor at its leading edge. If the outflow current and rotor invade a stable layer, they excite one or more solitary wave disturbances that propagate at speeds determined by wave amplitude and the vertical profiles of wind and virtual potential temperature of the ambient environment. Support for this suggested mechanism is provided by the recent numerical model results reported by Haase and Smith (1989), who found that density currents, which invade stable layers under supercritical conditions (i.e., frontal speeds that are, in absence of a stable layer, faster than the linear wave speed), are transformed directly into nonlinear waves of large amplitude. Much of the energy from the storm 
perturbation appears to go into the formation of solitary waves. A weak, dissipating density current may, however, be left behind in the wake of these waves.

As the leading solitary wave propagates away from the storm path, it can drag a vortex of storm outflow along with it, separating the vortex from its source. The fact that an atmospheric solitary wave can transport a mass of recirculating air can be explained by first noting that when recirculation is present the pressure distribution becomes double peaked. These pressure peaks are associated with the stagnation points in front of and behind the vortex. We hypothesized that in the coordinate frame moving with the wave, the trailing pressure peak provides the pressure-gradient force that balances the horizontal drag force and thus keeps the vortex stationary (i.e., moving with the wave).

If the part of the troposphere that overlies the inversion layer has a positive Scorer parameter, wave energy will be radiated away from the inversion layer. In the Appendix, we derive conditions under which nonlinear waves of permanent form can propagate even if the upper troposphere is statically stable and supports wave propagation in the vertical direction.

As noted in the introduction, Chimonas and Nappo (1987) give a different interpretation for the solitary wave disturbance described in this paper. They conclude that all the properties of this wave are consistently modeled by linear wave theory as a thunderstorm-generated bow wave, ducted in the atmospheric boundary layer. Their analysis is based on the observations reported by Doviak and Ge (1984). It is hoped that the new additional data, the more rigorous analysis of these data, and thorough comparisons with non- 
linear buoyancy wave theories reported herein resolve some of the features of the data that puzzled Chimonas and Nappo as well as the present authors.

\section{Acknowledgments .}

Many thanks to Susumu Kato, Director of the Radio Atmospheric Science Center, Kyoto University, for inviting Richard Doviak to his Center from January to March 1987, a time during which much of the research reported here was accomplished in an environment conducive to study. Bob Davies-Jones of NSSL, and Qin Xu, Zina Kogan, Misha Rancic, and Edwin Kessler of the University of Oklahoma provided helpful comments during the preparation of this paper. We are particularly indebted to Bob Maddox, who helped us interpret the wind profiles, and to Carmen Nappo and Roger K. Smith, who carefully reviewed the manuscript and provided valuable criticisms. Michael Eilts and Kevin Thomas contributed to the presentation of tower data, Jerry Wardius provided data from the surface network, and Steven Smith provided the radarderived wind profiles. Dusan S. Zrnic was most helpful in emphasizing the Importance of axial flow in the wave, and Pravas Mahapatra, of the Indian Institute of Science in Bangelore, India, provided a dynamical basis for the propagation of trapped air. This work was partially supported by the Federal Aviation Administration under Contract DTFAO1-80-Y-10524 and by the Air Force Office of Scientific Research under Contract AFOSR-83-0045. 
Appendix: Conditions for solitary waves to have permanent form in presence of vertical propagation

Early theoretical treatments (e.g., Benjamin 1967; Ono 1975) of

nonlinear internal buoyancy waves start with the assumption that horizontally propagating disturbances are guided by a stable layer of motionless fluid that lies beneath a neutrally stable layer of infinite depth. This configuration forms a wave guide in which buoyancy wave energy propagates within the stable layer, whereas the upper region is incapable of supporting propagation for waves of any length.

Crook (1986) showed that an infinitesimally small amplitude sinusoidal wave with horizontal wave number $k$ will remain trapped in the lower layer only if $\mathrm{k}$ is larger than the Scorer parameter $\ell_{\mathrm{u}}$ of the upper troposphere. A solitary wave, however, is a finite-amplitude, single-crested disturbance and therefore contains spectral energy in a continuum of wave numbers that extend to the limit $\mathrm{k}-0$. Thus, large amounts of wave energy are contained in the long-wavelength portion of the wave-number spectrum. If $\ell_{u}$ is positive in the upper troposphere, as it always is for weakly curved flow, waves of sufficiently long length may propagate upward and result in energy loss to the horizontally ducted disturbance or, more likely, prevent its formation unless $\ell_{u}$ is sufficiently small. Therefore, we seek general conditions on $\ell_{u}$ such that nonlinear waves can be ducted with little or no loss of energy when the upper troposphere is weakly stratified with shear. Maslowe and Redekopp (1980) derived the more restrictive conditions required for the propagation of ducted waves of finite amplitude when shear is absent in the weakly stratified upper layer.

For purposes of argument, we assume that the region above the strongly stratified layer has a Scorer's parameter much smaller than that in the lower 
region. To obtain an estimate of how small $\ell_{\mathrm{u}}$ must be, consider a solitary wave disturbance that has the bulk of its energy in the wave-number range $\mathrm{k}>\mathrm{k}_{\ell}$

The upper-region eigenfunction equation, for a spectral component with horizontal wave number $\mathrm{k}$ is

$$
\phi^{\prime \prime}+\left(l^{2}-k^{2}\right) \phi-0
$$

If $\ell_{\mathrm{u}}$ is a constant, then spectral components having $\mathrm{k}<\ell_{\mathrm{u}}$ will propagate upward as well as horizontally, thus removing energy from the disturbance in the lower layer. The condition required for a spatially localized disturbance to propagate without significant attenuation is that the spectral components that constitute the bulk of the wave's energy must not radiate through the upper layer. Thus, $k_{\ell}$ must be larger than $\ell_{u}$ :

$$
k_{\ell}>\ell_{u} \text {. }
$$

It is straightforward to show that the amplitude spectrum of a weakly nonlinear solitary wave $(2.22)$ is given by

$$
A(k)=\frac{h_{s}^{2}}{2} e^{-|k| \lambda} ; \quad-\infty<k<+\infty \text {, }
$$

where $h_{s}$ is the scale of vertical stratification and $\lambda$ the half width of the wave at half-amplitude. Although this spectral form is exact for a Lorentzian distribution of vertical displacements, any disturbance producing a localized, 
single-crested displacement would have roughly similar form. The energy spectrum is proportional to the square of (A3a), and so a percentage $P$, where

$$
P=e^{-2 k} \ell^{\lambda} \times 100 \%
$$

of the wave's energy is contained in waves having wave numbers larger than $k_{\ell}$. Thus $k_{\ell} \lambda \ll 1$ is the condition for which the bulk of the wave's energy is contained in spectral components $\mathrm{k}>\mathrm{k}_{\ell}$.

Combining this condition and (A2) we see that a solitary wave of quasipermanent form will be trapped in the waveguide with little leakage of its energy into the upper troposphere, provided

$$
\ell_{u} \ll \lambda^{-1}
$$

Under this condition the disturbance will experience little attenuation, even though the upper troposphere may be stable throughout.

Using the amplitude-wave width relation, $\delta_{m} \lambda / h_{s}^{2} \simeq 0(1)$, for steady. state, weakly nonlinear solitary waves, we can express condition (A4a) as

$$
\frac{\delta_{\mathrm{m}}}{\mathrm{h}_{\mathrm{s}}} \gg \mathrm{h}_{\mathrm{s}}{ }^{\mathrm{u}} \text {. }
$$

Thus, if $h_{s} \ell_{u}>0.1$, a solitary wave must be strongly nonlinear in order for it to propagate with quasi-permanent form.

Tung et al. (1982) and Pullin and Grimshaw (1988) showed that very

large amplitude solitary waves can also attain permanent form, but the 
amplitude-width relation of weakly nonlinear theory is no longer satisfied. As waves become strongly nonlinear, the decrease in wave width with increases in amplitude is less than that described by weakly nonlinear wave theory. As long as wavelength continues to decrease with increasing amplitude, losses due to radiation into the upper atmosphere will be smaller for solitary waves of larger amplitude. This situation is altered when wave amplitude is large enough for the appearance of closed circulation in the relative streamline flow pattern. Further increases in amplitude now lead to increases in wave width, which in turn result in an increase in the rate of energy loss into the upper atmosphere. We therefore conclude that strongly nonlinear solitary waves with amplitudes near the minimum required to support recirculating flow $\left(\delta_{m} / h_{s} \approx 1\right.$, Davis and Acrivos 1967; Tung et al. 1982) are less subject to energy losses because of vertical propagation than are waves with either larger or smaller amplitudes.

If the upper region has negligible wind, then

$$
\ell_{u} \simeq \frac{N_{u}}{c_{o}},
$$

where $\mathrm{N}_{\mathrm{u}}$ is the $\mathrm{B}-\mathrm{V}$ frequency in the region above the stable layer. Since the speed of the wave is roughly given by

$$
c_{0} \simeq N_{c} h_{s}
$$

where $\mathrm{N}_{c}$ is the characteristic B-V frequency of the lower layer, the above conditions can be expressed as 


$$
\frac{\mathrm{N}_{\mathrm{u}}}{\mathrm{N}_{\mathrm{c}}} \ll \frac{\mathrm{h}_{\mathrm{s}}}{\lambda}
$$

or, for weakly nonlinear waves,

$$
\frac{\mathrm{N}_{\mathrm{u}}}{\mathrm{N}_{\mathrm{c}}} \ll \frac{\delta_{\mathrm{m}}}{\mathrm{h}_{\mathrm{s}}} .
$$

Condition (A7b) is similar to that deduced by Maslowe and Redekopp (1980), who used a different approach and limited their analysis to wave propagation in the absence of shear in the upper region; their condition gives a weaker inequality than ( $A 7 \mathrm{~b})$. We emphasize that condition ( $A 7 \mathrm{~b})$, which assumes the upper tropospheric winds are negligible, is usually too restrictive when applied to realistic atmospheric conditions. The structure of the upper tropospheric winds usually determines the degree of radiation damping and the condition for finite-amplitude stable waves. In general, (A4a) would be the appropriate condition determining the permanency of the wave when the upper troposphere is stable and has sheared flow. 
REFERENCES

Benjamin, T.B., 1966: Internal waves of finite amplitude and permanent form. J. Fluid Mech. 25, 241-270.

Benjamin, T.B., 1967: Internal waves of permanent form in fluids of great depth. J. Fluid Mech. 29, 559-592.

Carbone, R.E., J.W. Conway, N.A. Crook and M.W. Moncrieff, 1990: The generation and propagation of a nocturnal squall line. Part I:

Observations and implications for mesoscale predictability. Mon. Wea. Rev. $118,26-49$.

Charney, J.G. and P.G. Drazin, 1961: Propagation of planetary-scale disturbances from the lower into the upper atmosphere. J. Geophys. Res. $66,83-109$

Chen, S., 1985: A theoretical analysis and comparison of the theory and observation of wind and pressure fields in an atmospheric solitary wave. M.S. Thesis, School of Meteorology, University of Oklahoma, Norman, OK.

Chimonas, G., and C.J. Nappo, 1987: A thunderstorm bow wave. J. Atmos. Sci. 44, $533-541$

Christie, D.R. 1989: Long nonlinear waves in the lower atmosphere. J.Atmos. Sci. $46,1462-1491$.

Christie, D.R., and K.J. Muirhead, 1983: Solitary waves: a hazard to aircraft operating at low altitudes. Aust. Meteor. Mag. 31, 97-109.

Christie, D.R., K.J. Muirhead and A.L. Hales, 1978: On solitary waves in the atmosphere. J.Atmos. Sci. 35, 805-825. 
Christie, D.R., K.J. Muirhead and A.L. Hales, 1979: Intrusive density flows in the lower troposphere: A source of atmospheric solitons. J. Geophys. Res. 84, 4959-4970.

Christie, D.R., K.J. Muirhead and R.H. Clarke, 1981: Solitary waves in the lower atmosphere. Nature 293, 46-49.

Clarke, R. H., 1965: Horizontal mesoscale vortices in the atmosphere. Aust. Met. Mag. 50, 1-26.

Clarke, R.H., R.K. Smith and D.G. Reid, 1981: The morning glory of the Gulf of Carpentaria: an atmospheric undular bore. Mon. Wea. Rev. 109, $1726-1750$.

Crook, N.A., 1986: The effect of ambient stratification and moisture on the motion of atmospheric undular bores. J. Atmos. Sci. 43, 171-181.

Crook, N.A., 1988: Trapping of low-level internal gravity waves. J. Atmos. Sci. $45,1533-1541$.

Davis, R.E., and A. Acrivos, 1967: Solitary internal waves in deep water. J. Fluid Mech. 29, 593-607.

Dodd, R.K., J.C. Ellbeck, J.D. Gibbon, and H.C. Morris, 1982: Solitons and nonlinear wave equations. Academic Press, Orlando, Florida, 630 pp.

Doviak, R.J., and S. Chen, 1988: Observations of a thunderstorm generated gust compared with solitary wave theory. Final Report, DOT/FAA/SA88/1, Dept. of Transportation, FAA, Advanced System Acquisition Service, Washington, DC, 20590, 135 pp.

Doviak, R.J., and D.R. Christie, 1989: Thunderstorm-generated solitary waves: a wind shear hazard. J. Aircraft 26, 423-431. 
Doviak, R.J., K.W. Thomas and D.R. Christie, 1989: The wavefront shape, position and evolution of a great solitary wave of translation. IEEE Trans. Geosci. and Remote Sensing., 27, 658-665.

Doviak, R.J., and R.S. Ge, 1984: An atmospheric solitary gust observed with a Doppler radar, a tall tower and a surface network. J. Atmos. Sci. 41, $2559-2573$.

Doviak, R.J., and D.S. Zrnić, 1984: Doppler Radar and Weather Observations. Academic Press, Orlando, Florida, 458 pp.

Drake, V.A., 1985: Solitary wave disturbances of the nocturnal boundary layer revealed by radar observations of migrating insects. Boundary-Layer Meteor. 31, 269-286.

Drogemeier, K. K. and M. R. Babcock, 1989: Numerical simulations of microburst downdrafts: Application to on-board and look-ahead sensor technology. AIAA 27th Aerospace Science Meeting. AIAA-89-0821, Reno, 12pp.

Einaudi, F., and J.J. Finnigan, 1981: The interaction between an internal gravity wave and the planetary boundary layer. Part I: The linear analysis. Quart. J. Roy. Meteor. Soc. 107, 793-806.

Fulton, R., D.S. Zrnic and R.J. Doviak, 1990: Initiation of a solitary wave family in the demise of a nocturnal thunderstorm density current. J. Amos. Sci. 47, 319-337.

Gossard, E.E., and W.H. Hooke, 1975: Waves in the Atmosphere. Elsevier Scientific Publ. Co., New York, 456 pp.

Grimshaw, R., 1980/81: Solitary waves in a compressible fluid. Pure Appl. Geophys. 119, 780-797.

Grimshaw, R., 1985: Evolution equations for weakly nonlinear long internal waves in rotating fluid. Stud. in Appl. Math. 73, 1-33. 
Haase, S.P., and R.K. Smith, 1989: The numerical simulation of atmospheric gravity currents, Part II. Environments with stable layers. Geophys. Astro. Phys. Fluid Dyn. 46, 35-51.

Hildebrand, F.B., 1956: Advanced Calculus for Engineers. Prentice Hall, Englewood Cliffs, New Jersey, 594 pp.

Koop, C.G., and G. Butler, 1981: An investigation of internal solitary waves in a two-fluid system. J. Fluid Mech. 112, 225-251.

Lindzen, R.S. and K.K. Tung, 1976: Banded convective activity and ducted gravity waves. Mon. Wea. Rev. 104, 1602-1617.

Long, R.R., 1965: On the Boussinesq approximation and its role in the theory of internal waves. Tellus 17, 46-52.

Maslowe, S.A., and L.G. Redekopp, 1980: Long nonlinear waves in stratified shear flows. J. Fluid Mech. 101, 321-348.

Maxworthy, T., 1980: On the formation of nonlinear internal waves from gravitational collapse of mixed regions in two and three dimensions. J. Fluid Mech. 96, 47-64.

Miesen, R. H. M., 1990: Internal Solitary waves in compressible fluids. Dissertation, Physics Dept., Eindhoven University of Tech., Netherlands. 131pp.

Noonan, J.A., and R.K. Smith, 1985: Linear and weakly nonlinear internal wave theories applied to "morning glory" waves. Geophys. Astrophys. Fluid Dyn. 33, 123-143.

Ono, H., 1975: Algebraic solitary waves in stratified fluids. J. Phys. Soc. Japan 39, 1082-1091.

Ostrovsky, L.A. and Yu.A. Stepanyants, 1989: Do internal solitons exist in the ocean? Rev. of Geophys. 27, 293-310. 
Press, W.H., B.P. Flannery, S.A. Teukolsky, and W.T. Vetlerling, 1986:

Numerical Recipes. The Art of Scientific Computing. Cambridge

University Press, Cambridge, 818 pp.

Pullin, D.I., and R.H.J. Grimshaw, 1988: Finite-amplitude solitary waves at the interface between two homogeneous fluids, Phys. Fluids, 31, 3550 3559.

Scorer, R.S., 1949: Theory of waves in lee of mountains. Quart. J. Roy. Meteorol. Soc. 75, 41-56.

Scorer, R.S., 1954: Theory of airflow over mountains: III - airstream characteristics. Quart. J. Roy. Meteor. Soc. 80, 417-428.

Smith, R.K., 1988: Travelling waves and bores in the lower atmosphere: the 'morning glory' and related phenomena. Earth-Sci. Rev. 25, 267-290.

Spiegel, E.A., and G. Veronis, 1960: On the Boussinesq Approximation for a compressible fluid. Astrophys. J. (2), 131, 442-447.

Stoeffler, R.C., 1972: Additional research on instabilities in atmospheric flow systems associated with clear air turbulence. NASA Rept. CR-1985, Wash. D.C. $71 \mathrm{pp}$.

Tung, K.K., D.R.S. Ko and J.J. Chang, 1981: Weakly nonlinear internal waves in shear. Stud. Appl. Math. 65, 189-221.

Tung, K.K., T.F. Chan and T. Kubota, 1982: Large-amplitude internal waves of permanent form. Stud. Appl. Math. 66, 1-44.

Whitham, G.B., 1974: Linear and Nonlinear Waves. Wiley \& Sons, New York, 636, pp.

Zhang, D.L. and Fritsch, J.M., 1987: Numerical simulation of the Meso- $\beta$ scale structure and evolution the 1977 Johnstown flood. Part III: Internal gravity waves and the squall line. J.Atmos. Sci., 45, 1252-1268. 
List of Figures

Fig. 1. Dry-bulb $(\mathrm{T})$ and dewpoint $\left(\mathrm{T}_{\mathrm{d}}\right)$ temperatures from a rawinsonding and from instruments on a 500 -m tower.

Fig. 2. Vertical profiles of (a) the u component of wind (northwest wind) in the direction of wave travel (circa 2245 CST), (b) the Scorer parameter squared.

Fig. 3. u component of wind vs. time at five tower levels; (b) $v$ component of wind vs. time. The dots are observed data, and the shaded areas are winds attributed to trapped thunderstorm outflow. The solid lines are subjective fits to the data; $u_{0}$ and $v_{0}$ are the ambient wind components parallel and orthagonal to the propagation direction; $\Delta u_{0}$ and $\Delta v_{0}$ are calculated changes in the wind caused by vertical transport of sheared flow.

Fig. 4. (a) Wind observed at surface sites A to F before (solid arrows) and after (dashed arrows) wave passage. The $2 \mathrm{~m} \mathrm{~s}^{-1}$ vector scales the wind. The magnitude $\left|\bar{v}_{p}\right|$ and direction $D_{p}$ are the wave's peak perturbation. Site B (open triangle) marks the location of the tall tower. $\Delta \mathrm{T}$ is the drop in dry-bulb temperature caused by leaked outflow occurring $\Delta t$ after wavefront arrival. The double dashes at sites $E, F$ and $G$ denote that data are unavailable because the wave or leaked outflow were not observed. (b) Dry-bulb temperature $T_{D B}$ and wind components east $\left(u^{\prime}\right)$ and north $\left(v^{\prime}\right)$ recorded at station $A$ from 2230 to 2325 CST.

Fig. 5. Wave amplitude, $u_{2}$, and half-amplitude width, $\ell_{1 / 2}$, vs. height. Circled $x$ is $\ell_{1 / 2}$ estimated from radar data. The dashed line is an 
Fig. 5. Wave amplitude, $\mathbf{u}_{2}$, and half-amplitude width, $\ell_{1 / 2}$, vs. height. Circled $\mathrm{x}$ is $l_{1 / 2}$ estimated from radar data. The dashed line is an extrapolation based on the eigenfunctions of weakly nonlinear wave theory and ignoring friction at the surface.

Fig. 6. Position of the leading edge of the wave perturbation along a ray path passing through the tall-tower site.

Fig. 7. Observed average radial (Doppler) velocity along azimuth $\phi_{c}$ vs. radial distance. $v_{\text {ro }}$ and $v_{r p}$ are the radial components of the ambient wind in advance of the wave and at the wave peak, respectively.

Fig. 8. Horizontal velocity amplitude $\mathrm{u}_{2}\left(0, \mathrm{~h}_{e}\right)$ of the wave at about $450 \mathrm{~m}$ AGL versus time.

Fig. 9. Wave speed c versus time. The + symbols are wave speed $c_{t}$ deduced by tracking wavefront positions. The unfilled circles are wave-speed estimates based on amplitude $\mathrm{u}_{2}$ data and weakly nonlinear wave theory with ambient shear omitted. The filled circles are wavespeed estimates based on theory when shear is included.

Fig. 10. Normalized wave speed versus normalized stream-function amplitude. $C^{\prime}$ is the observed wave speed relative to the ambient air that advects northward. The solid line is the relation obtained from weakly nonlinear wave theory, and the dashed line is a linear fit to the theoretical results.

Fig. 11. Normalized wave speed vs. wave half-width. 
Fig. 12. Numerical solution of the Benjamin-Davis-ono equation illustrating the evolution and propagation of two solitary waves formed by the rapidly propagating thunderstorm.

Fig. 13. A scenario for the evolution of thunderstormgenerated solitary waves 


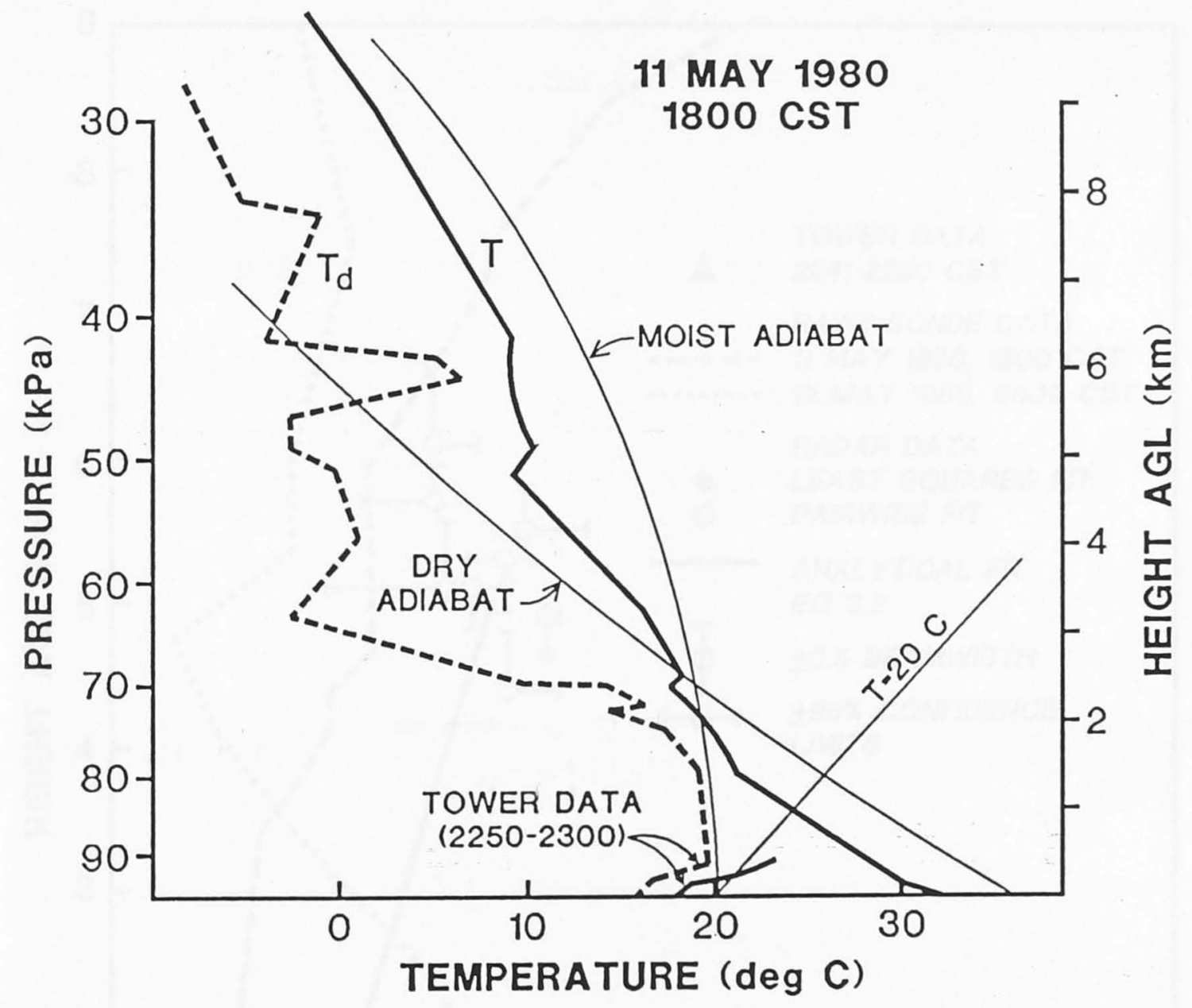

Fig. 1 


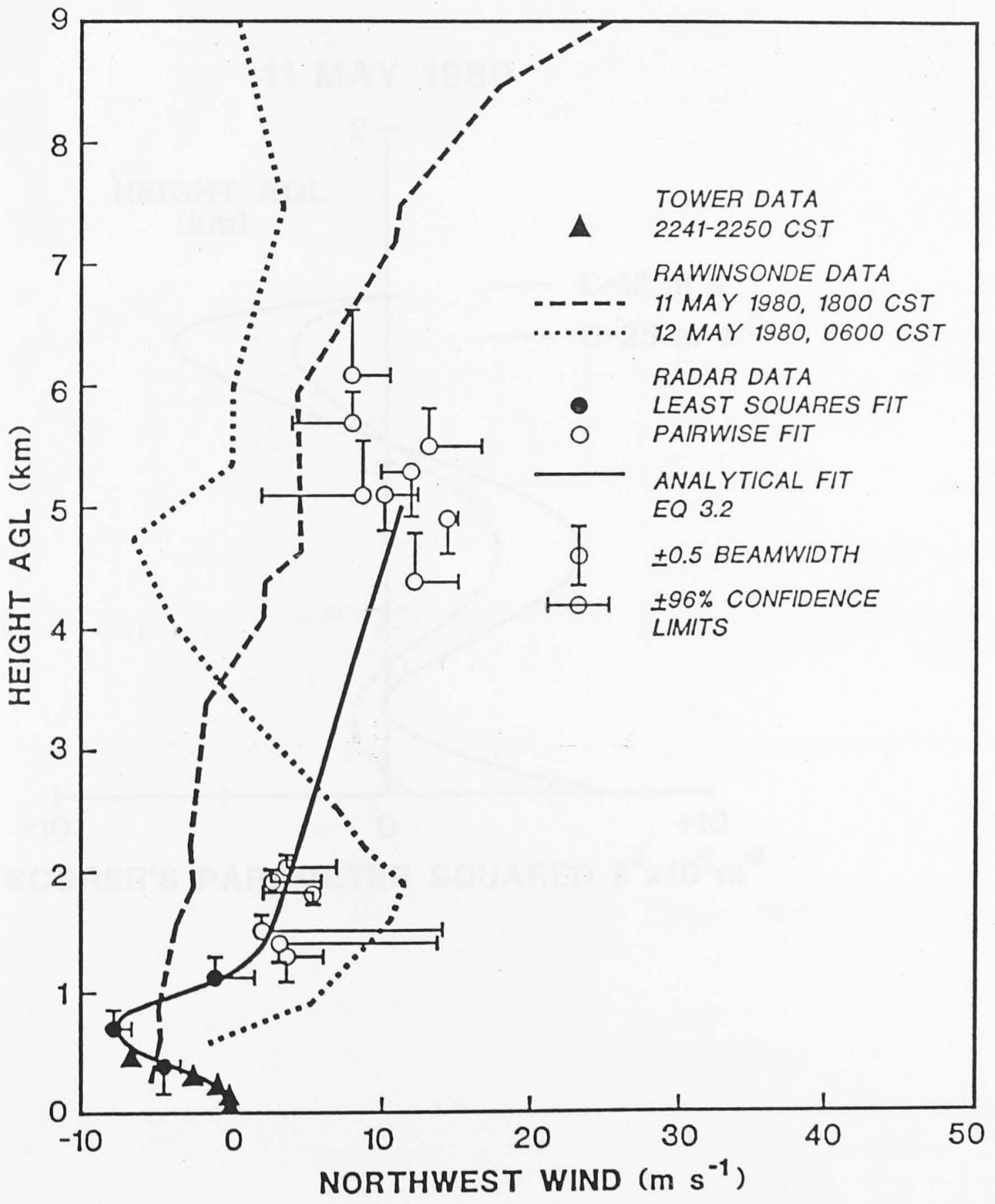


11 MAY 1980

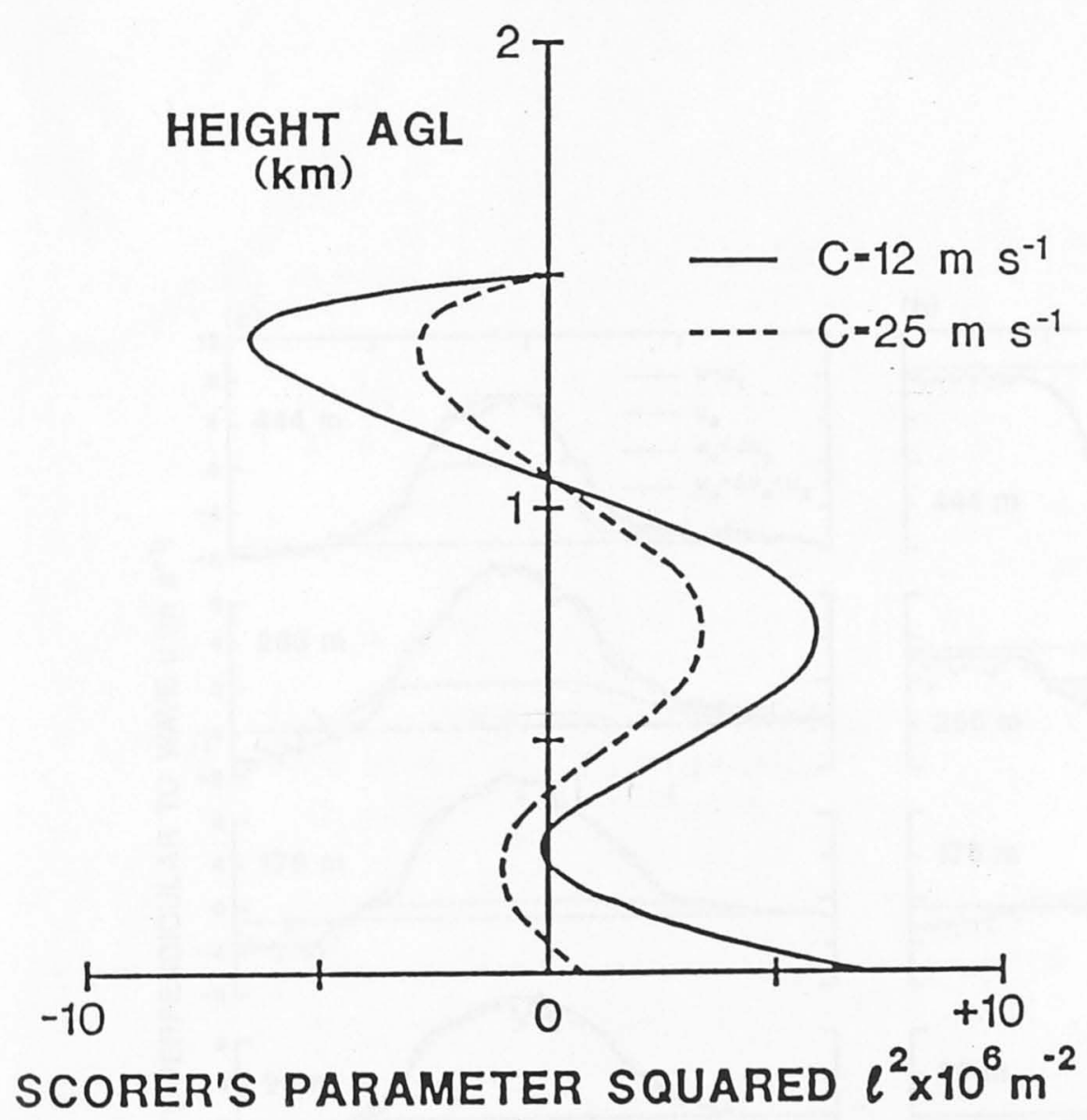




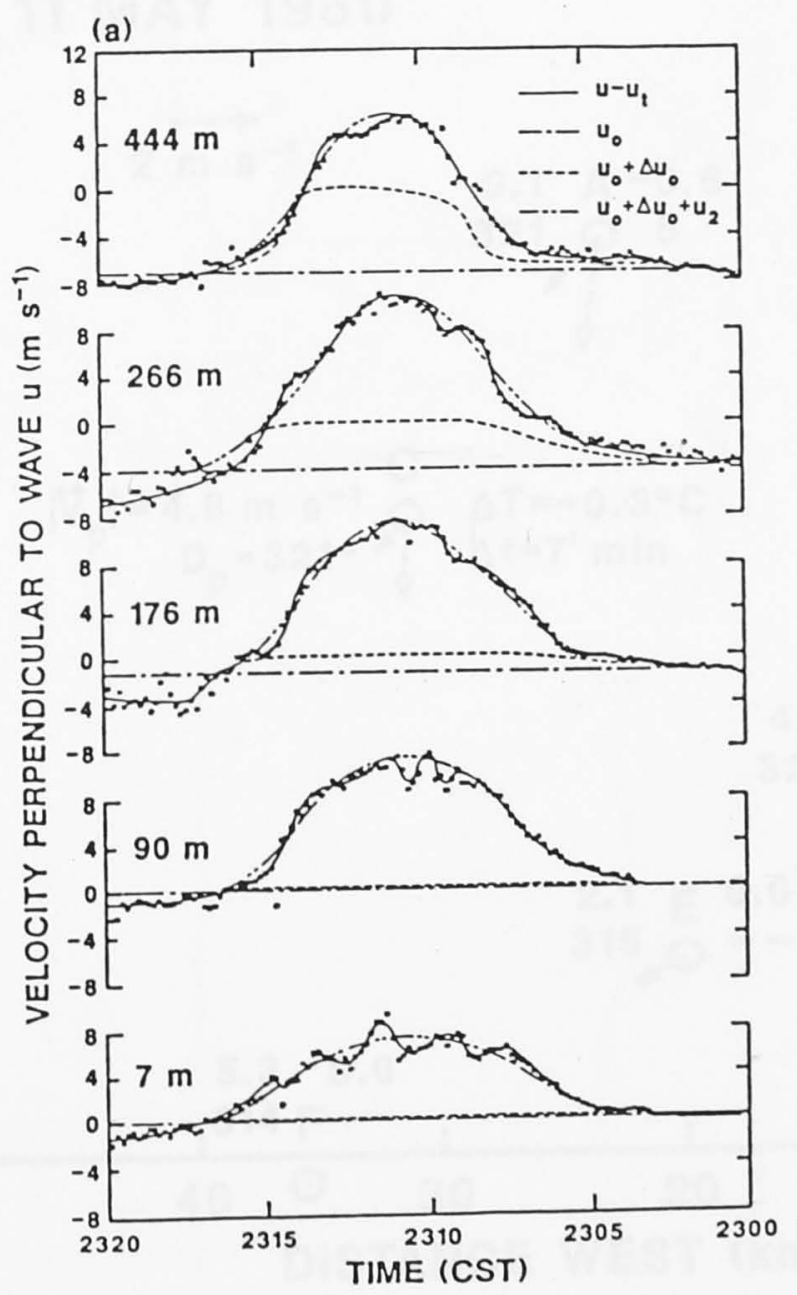

(b)
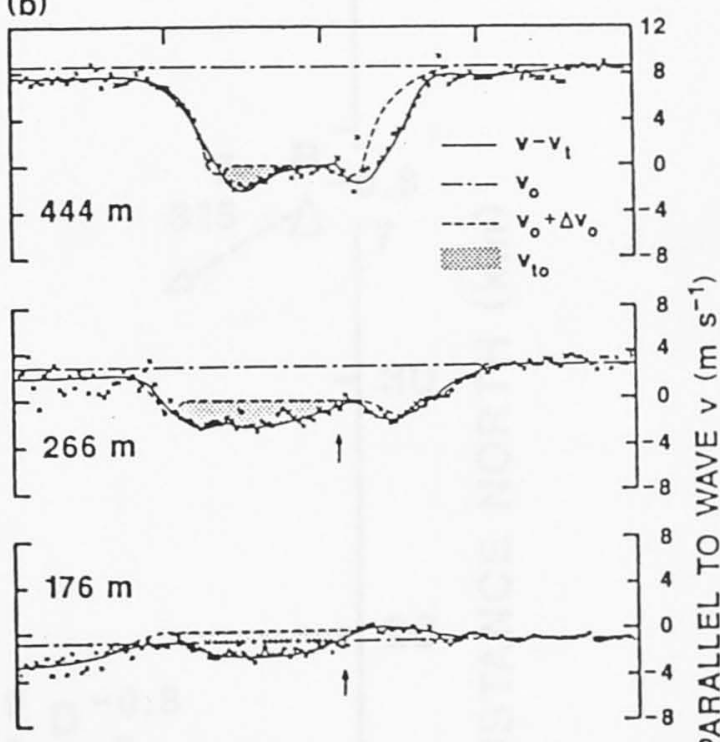

은
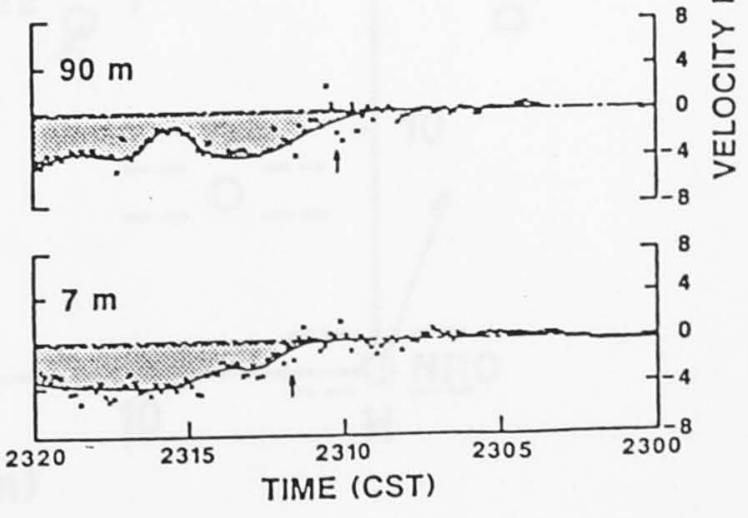

Fig. 3 
11 MAY 1980

$$
\begin{aligned}
& 2 \overrightarrow{\mathrm{m} \mathrm{s}}^{-1} \\
& 9.1 \text { A }-0.6 \\
& 321 \bigotimes_{i} 5 \\
& \nabla_{p} \mid=\begin{array}{c}
4.8 \mathrm{~m} \mathrm{~s}^{-1} \stackrel{\mathrm{C}}{D_{p}=321 \circ} \Delta \mathrm{T}=-0.3^{\circ} \mathrm{C} \\
\Delta t=7 \mathrm{mln}
\end{array}
\end{aligned}
$$

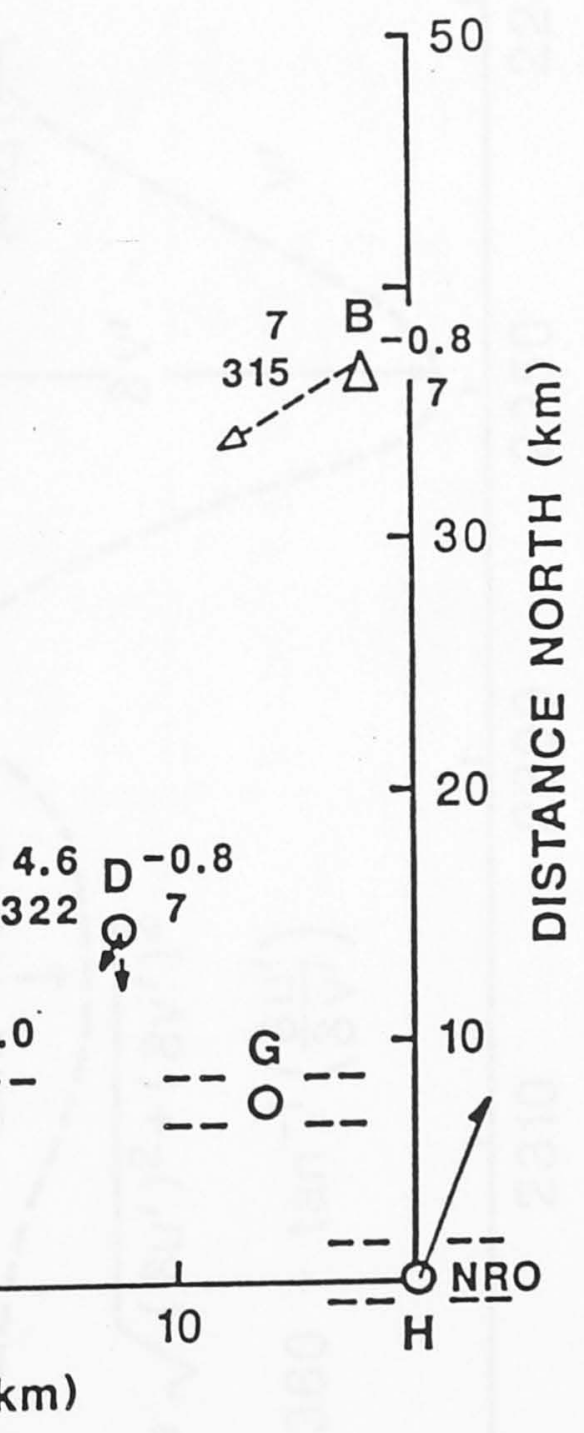




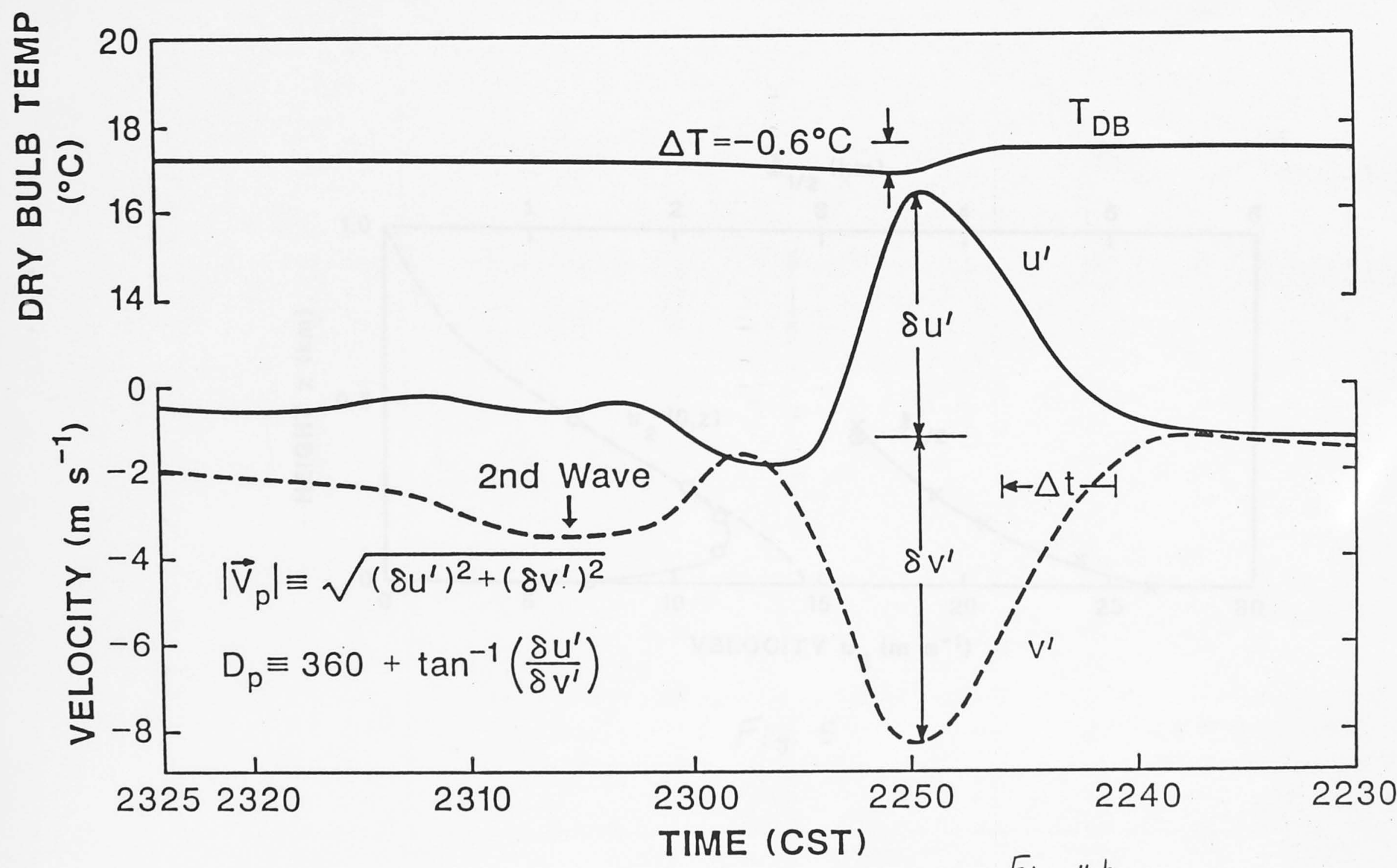

Fig. $4 b$ 


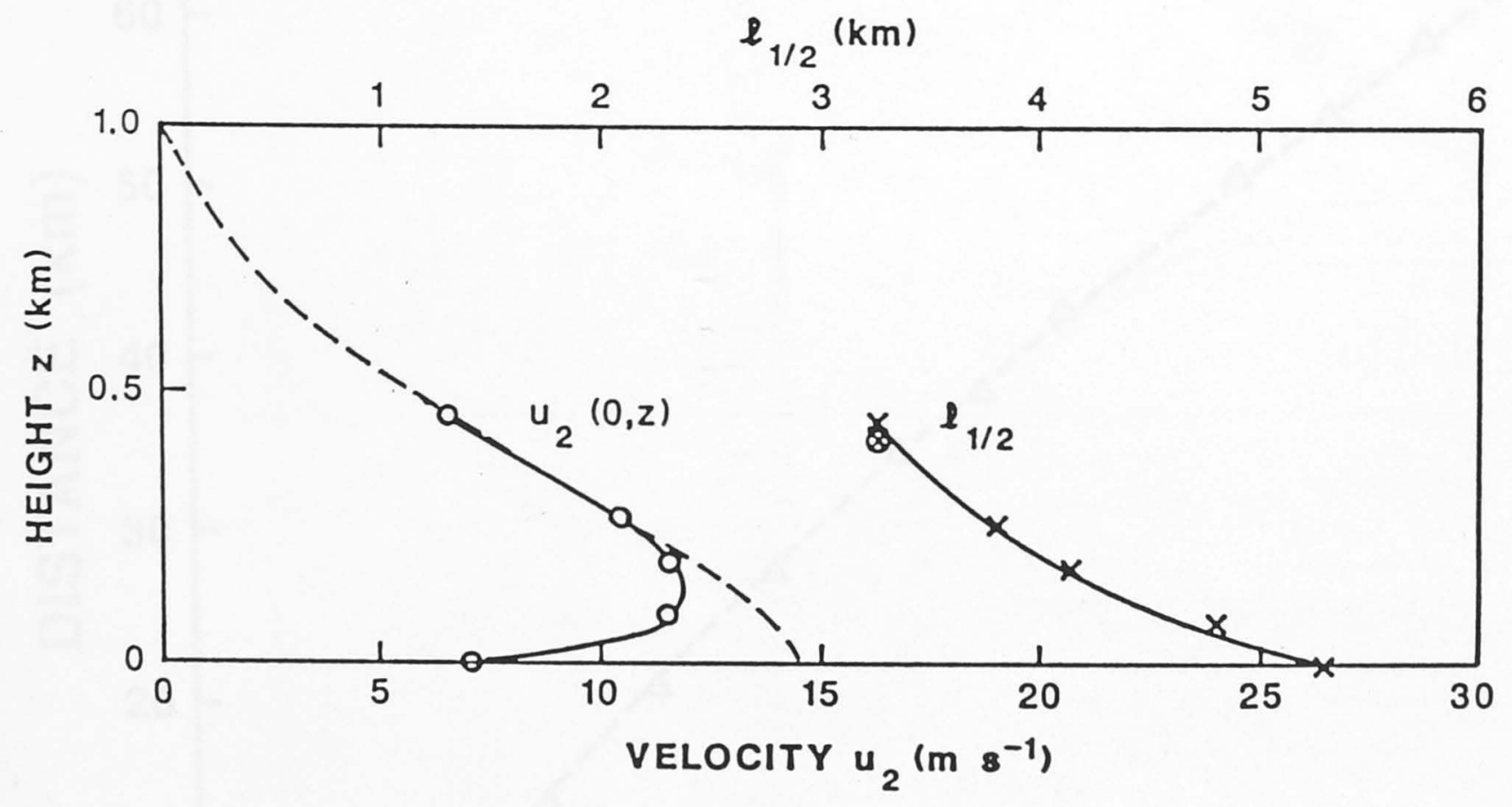

Fig. 5 
Fig. 6

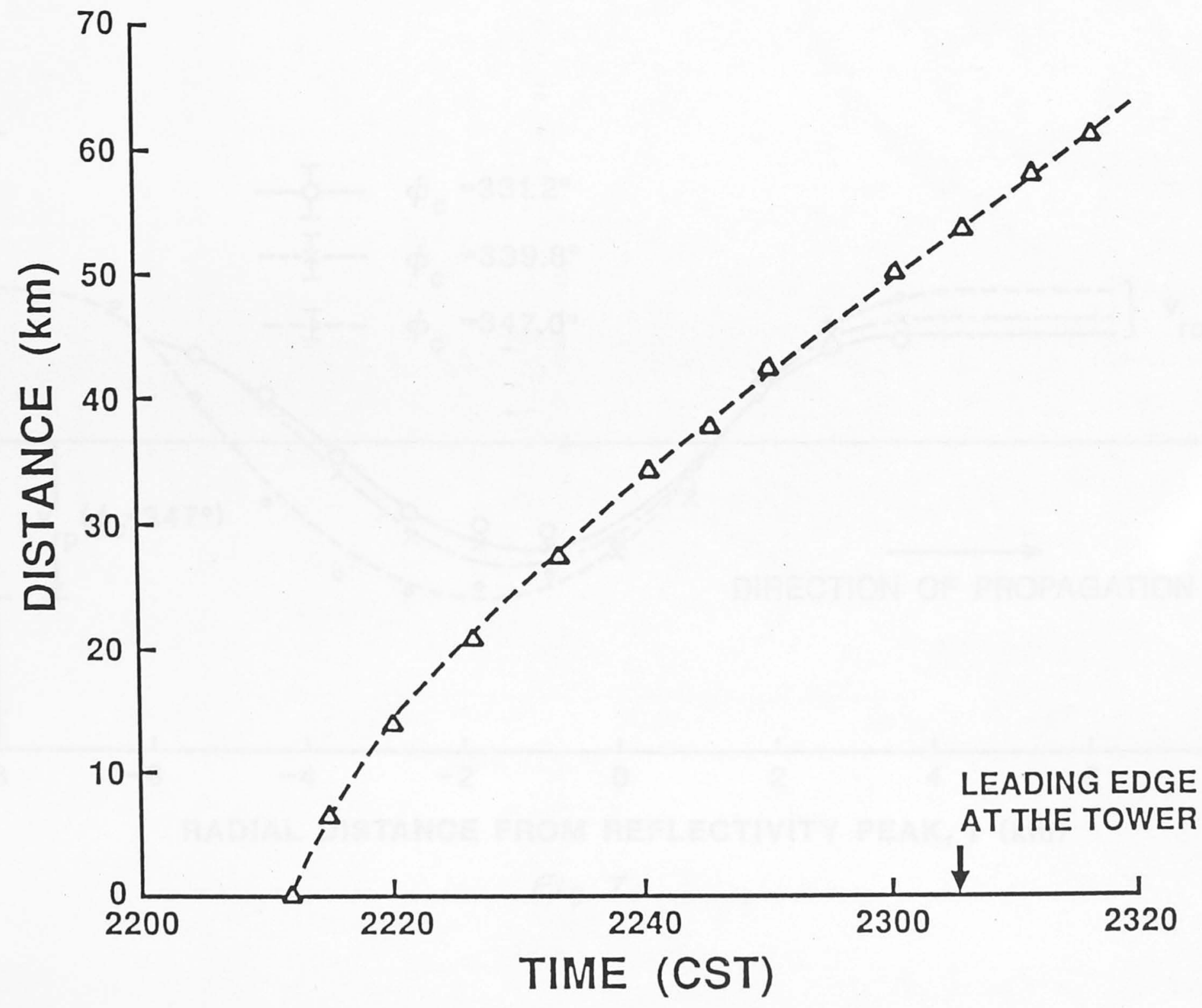




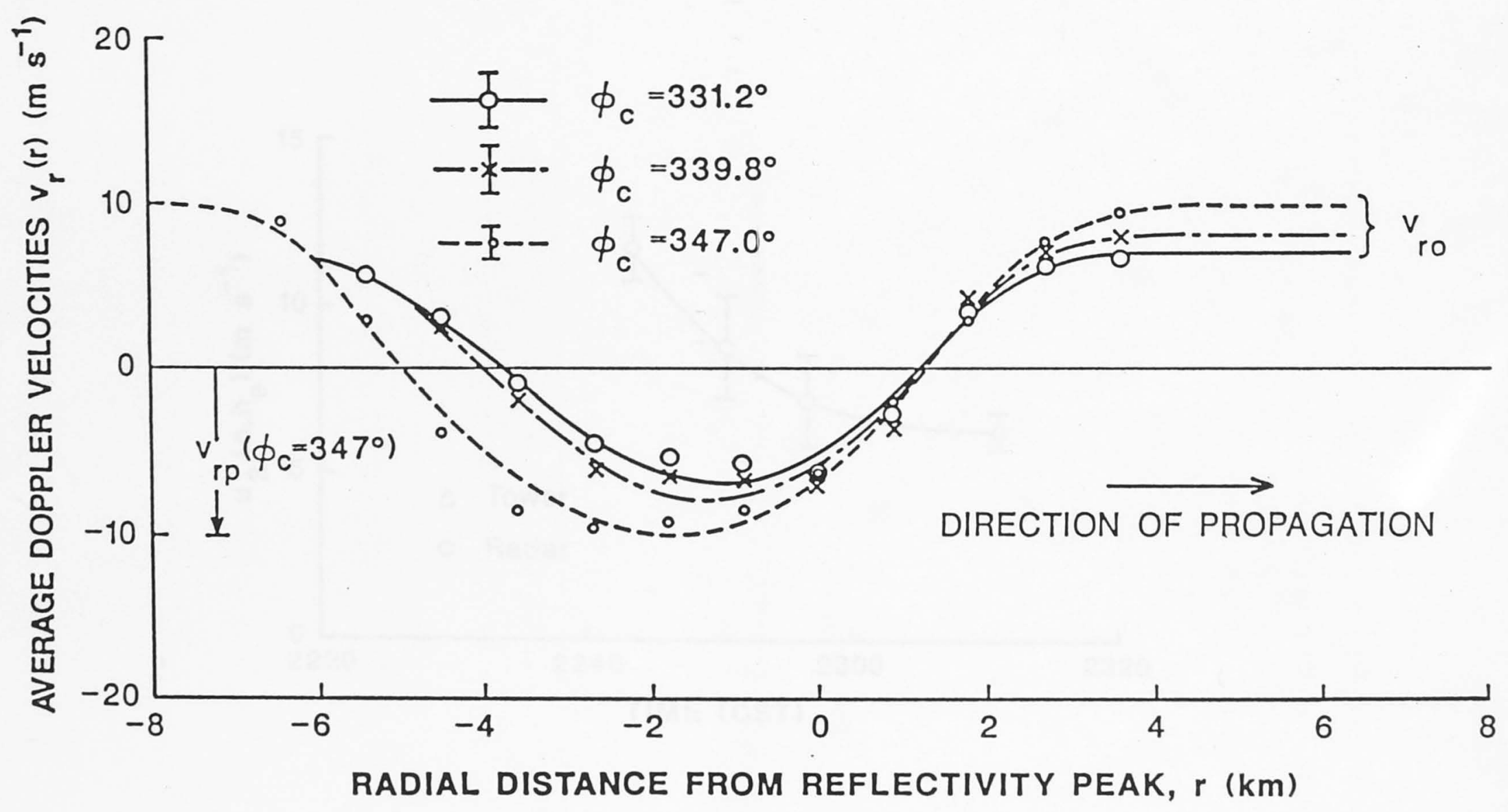

$$
\text { Fig. } 7
$$




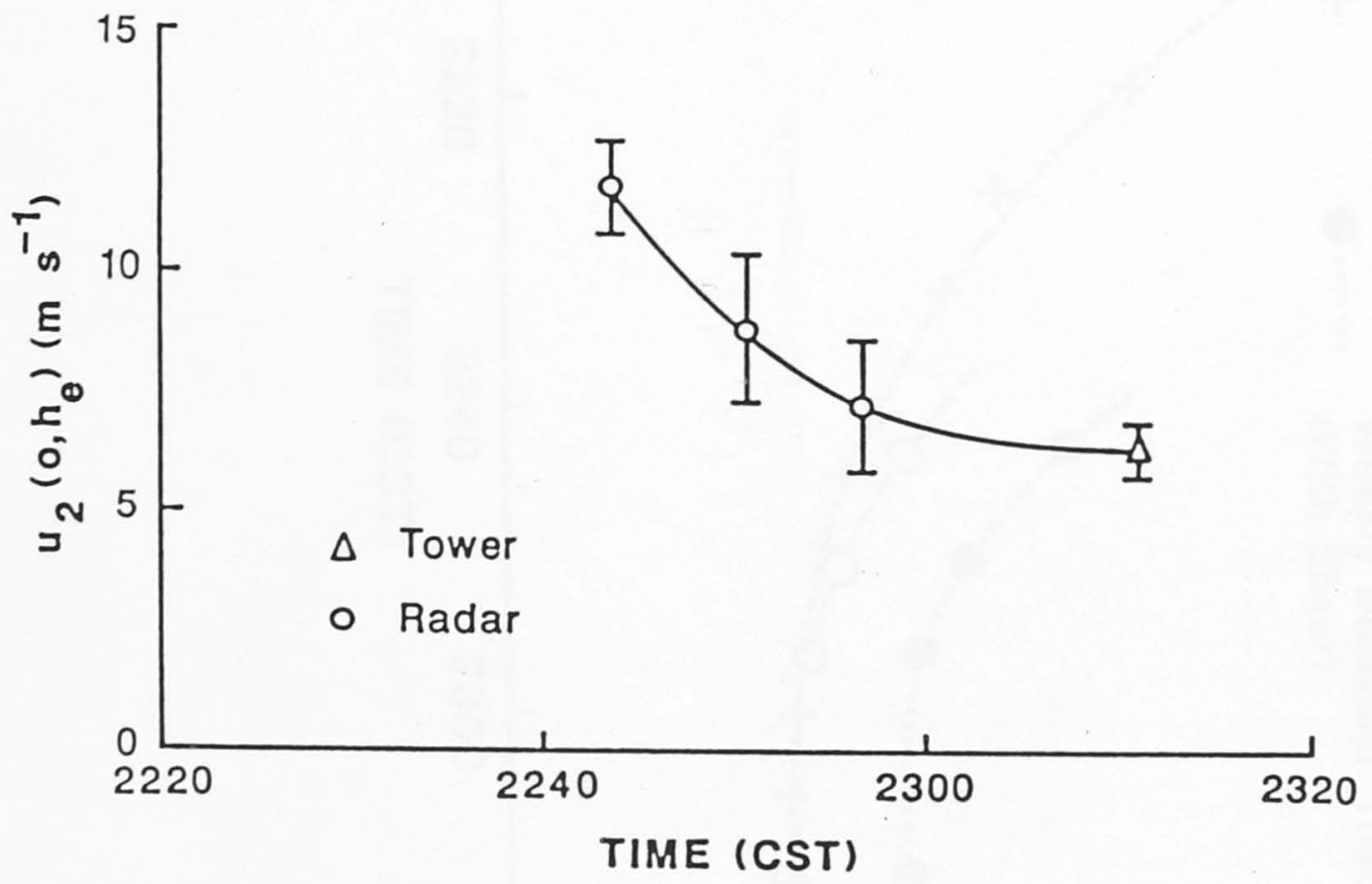

Fig. 8 


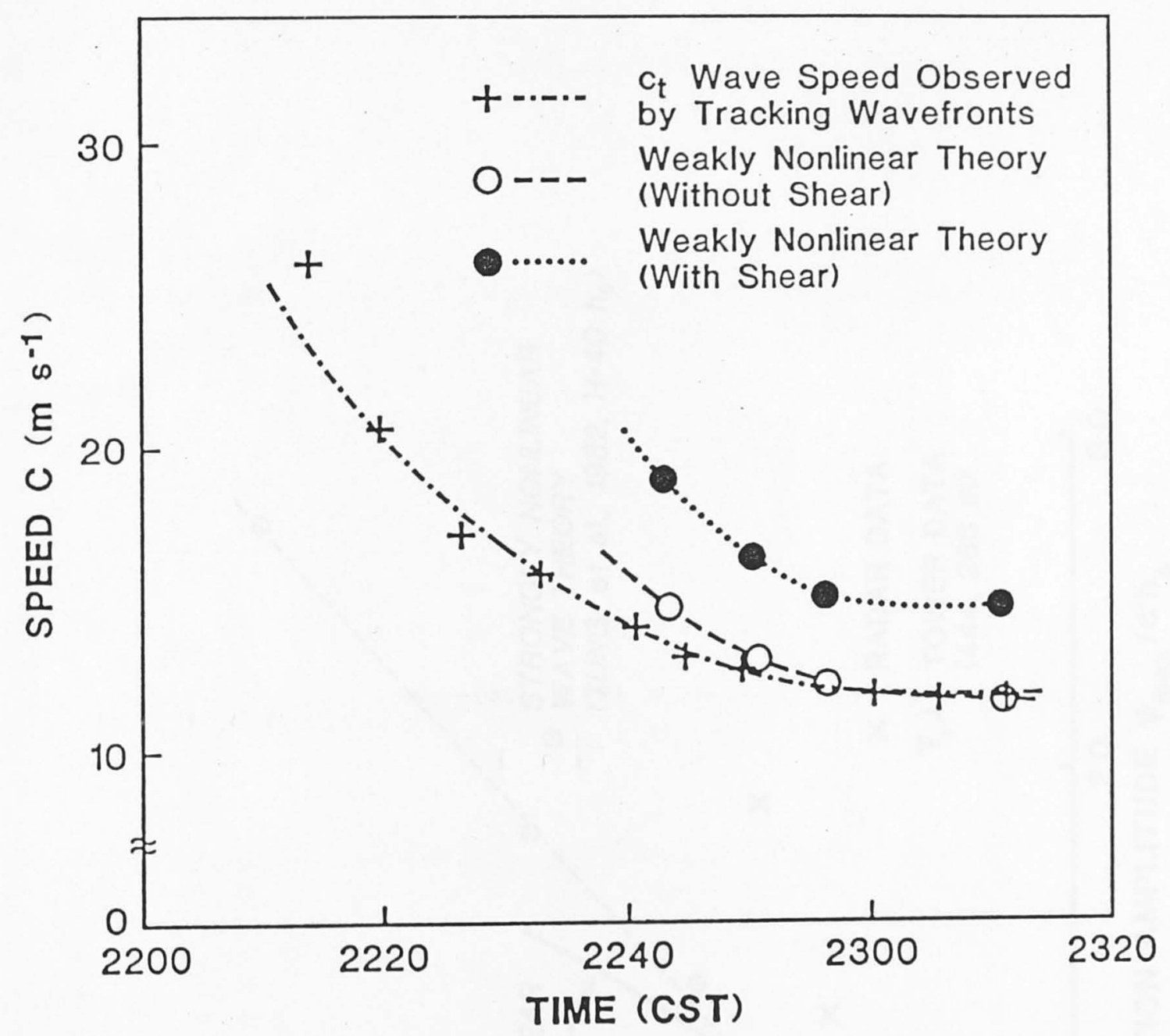




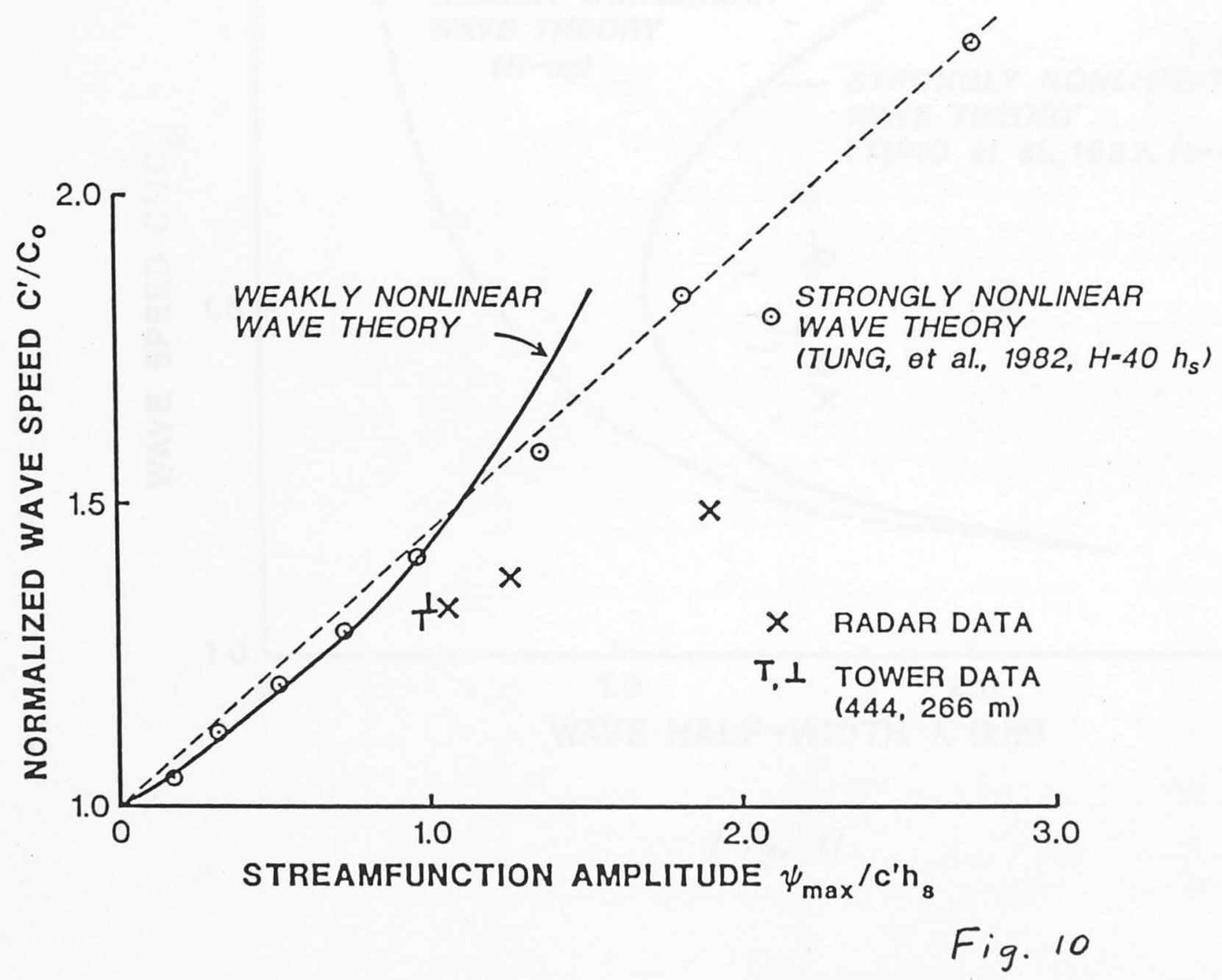




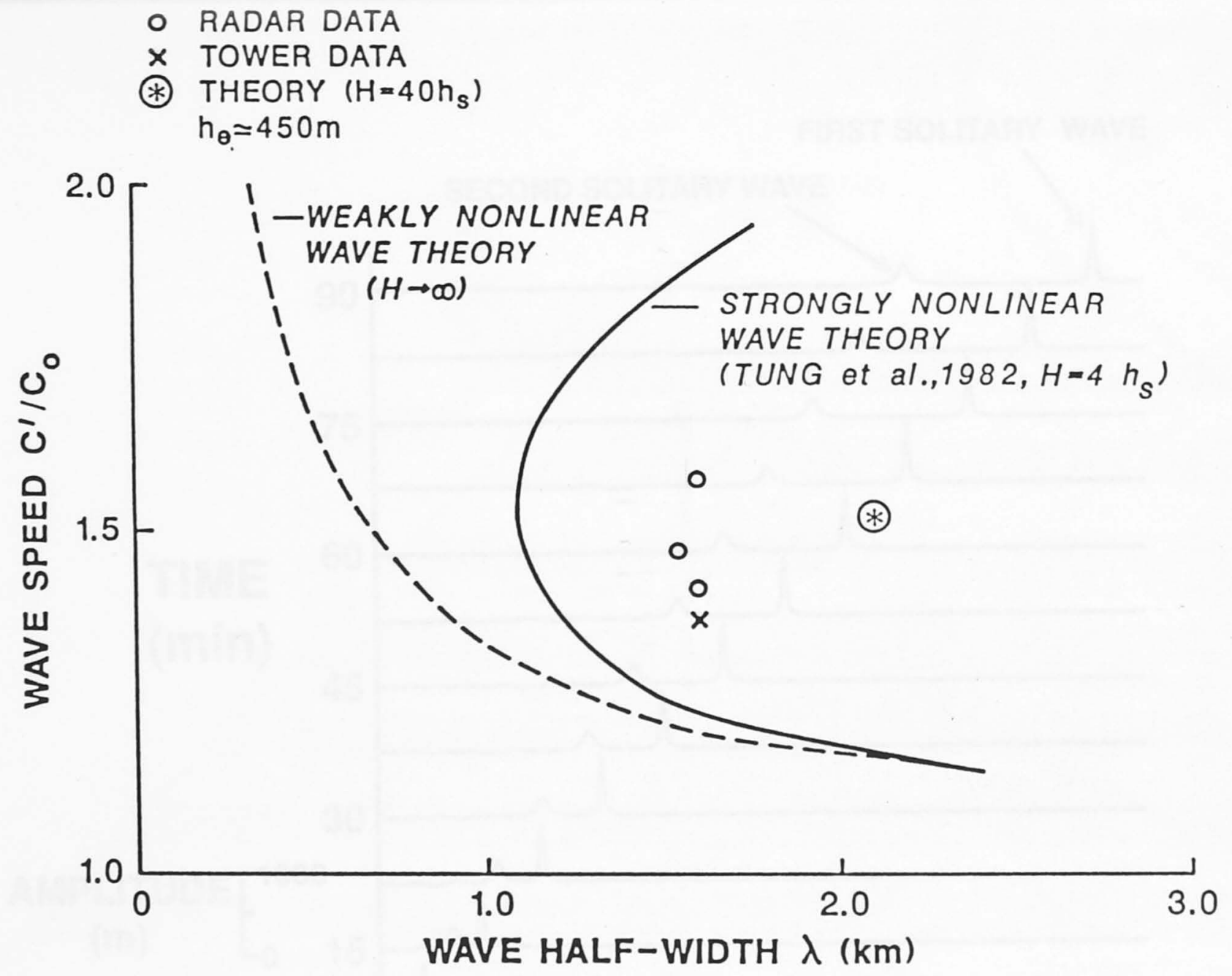

Fig. II 


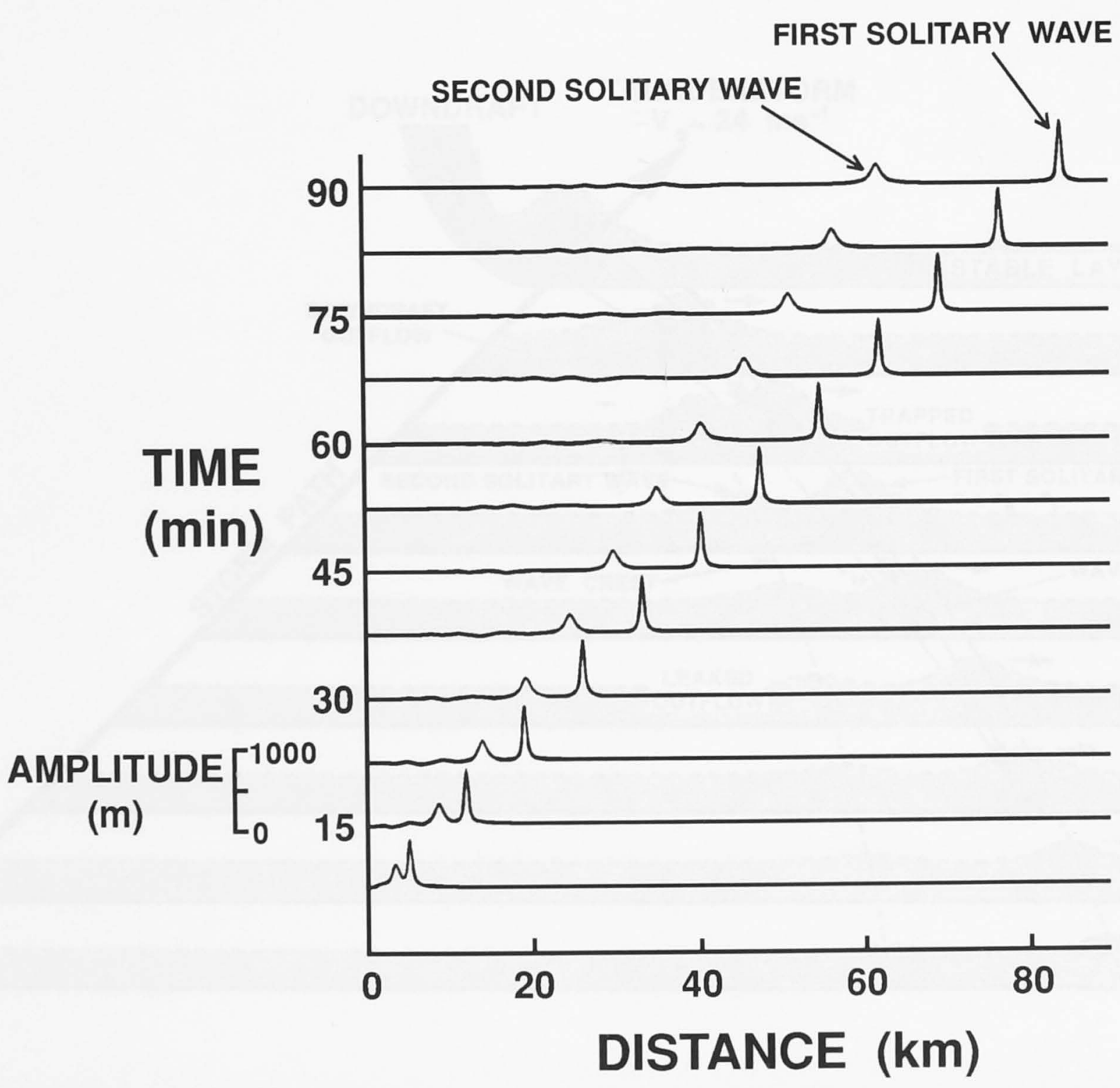




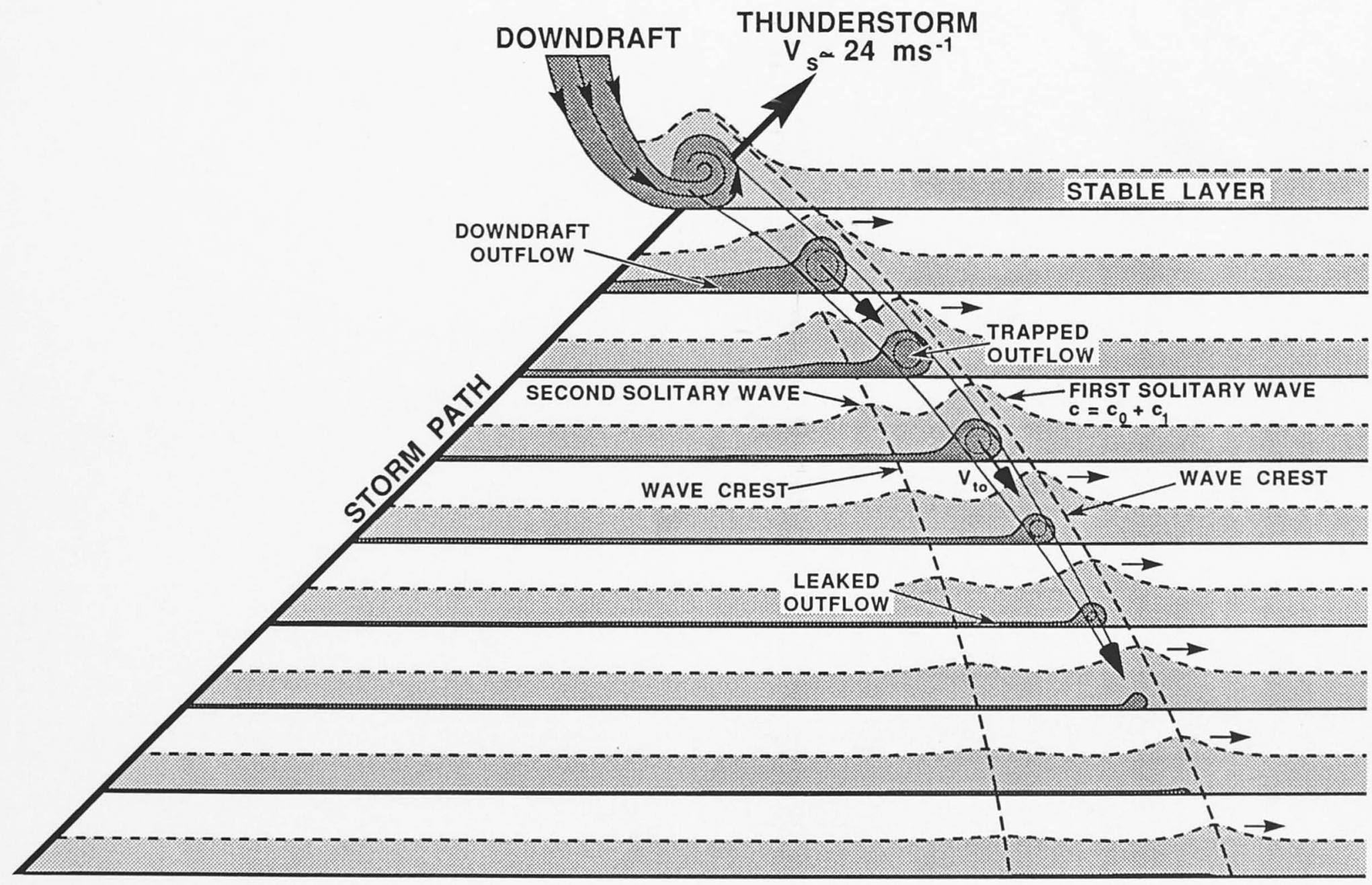

